\title{
Synthesis of Terminal Transition Metal Pnictide Complexes by Activation of Small Molecules
}

\section{Dissertation}

Zur Erlangung des mathematisch-naturwissenschaftlichen Doktorgrades

Doctor Rerum Naturalium

Im Promotionsprogramm der Georg-August-University School of Science (GAUSS)

vorgelegt von

M. Sc. Josh Abbenseth

aus Bremervörde 


\section{Betreuungsausschuss}

Prof. Dr. Sven Schneider

Institut für Anorganische Chemie, Georg-August-Universität Göttingen

Prof. Dr. Franc Meyer

Institut für Anorganische Chemie, Georg-August-Universität Göttingen

\section{Mitglieder der Prüfungskommission}

Referent: Prof. Dr. Sven Schneider

Institut für Anorganische Chemie, Georg-August-Universität Göttingen

Co-Referent: Prof. Dr. Franc Meyer

Institut für Anorganische Chemie, Georg-August-Universität Göttingen

Weitere Mitglieder der Prüfungskommision

Prof. Dr. Manuel Alcarazo

Institut für Organische und Biomolekulare Chemie, Georg-August-Universität Göttingen

Prof. Dr. JÖrg Behler

Institut für Physikalische Chemie, Georg-August-Universität Göttingen

Jun.-Prof. Dr. Nathalie Kunkel

Institut für Anorganische Chemie, Georg-August-Universität Göttingen

Dr. Christian Sindlinger

Institut für Anorganische Chemie, Georg-August-Universität Göttingen 


This thesis originated in the time between January 2016 and June 2019 at the Institute for Inorganic Chemistry of the Georg-August-University Göttingen.

I am deeply grateful to my supervisor

Prof. Dr. Sven Schneider

Thank you Sven for the trust you put in me and my skills, the constant support, the opportunity to present my work on several conferences and the freedom to pursue my own scientific ideas.

Our inspiring and sometimes heated discussions taught me how to properly address scientific problems.

I also want to express my gratitude to

Prof. Dr. Franc Meyer

for being my second supervisor and the fruitful discussions. 

Furthermore, my special thanks go to:

The examination board for their time.

Dr. Christian Würtele and Dr. Markus Finger for teaching me how to perform and interpret X-ray diffraction and density functional theory studies and their constant support during my whole stay.

Dr. A. Claudia Stückl and Dr. Serhiy Demeshko for EPR and SQUID measurements, respectively.

My B. Sc. students Sarah Carolin Bete and Marc Christian Neben for a fantastic time in the lab and their incredible effort to support my research. Without you some crucial results would have never been accomplished.

Florian Wätjen, Jan Gerkens and Richt van Alten for the great atmosphere in the lab and their reliability.

All members of the Schneider workgroup for the scientific discussions, great group activities and several barbeques.

Prof. Dr. Max C. Holthausen, Dr. Martin Diefenbach, Prof. Dr. Bas de Bruin, Prof. Dr. Moniek Tromp, Bas Venderbosch, Jean-Pierre Oudsen, Prof. Dr. Jose M. Goicoechea, Dr. Alexander Hinz, Dr. Christian Herwig, Prof. Dr. Christian Limberg, Dr. Gleb Silantyev, Bastian Schluschaß and Daniel Delony for the several successful collaborations.

Felix Schneck, Florian Wätjen and Dr. Stefan Resch for helpful corrections.

The members of the analytical laboratories, the mass department and NMR facility for their efforts in measuring highly air sensitive samples under various conditions.

Dalila Griffin and Dr. Claudia Stückl for help with administrative affairs. 



\section{Contents}

1 Introduction $\quad 1$

1.1 Physical and Chemical Characteristics of Pnictogens . . . . . . . . . . . 1

1.2 Terminal Transition Metal Pnictide Complexes . . . . . . . . . . . . 6

1.2.1 Heavy Analogues of Nitride Complexes - First Indications . . . . . 6

1.2.2 Synthesis of Terminal Pnictide Complexes from the Elements . . . 8

1.2.3 Alternative Heavy Pnictide Sources . . . . . . . . . . . . . . . . 13

1.2.4 Transition Metal - Pnictogen Multiple Bonding . . . . . . . . . . . 15

1.2.5 Reactivity of Transition Metal Pnictide Complexes . . . . . . . . . 18

1.3 Generation of Dipnictogens in the Condensed Phase . . . . . . . . . . . . 21

1.4 Phosphinyl Radicals and Phosphinidenes . . . . . . . . . . . . . . . . . . 24

1.5 Scope of this Work . . . . . . . . . . . . . . . . . . . 27

2 Os(II/III) and Re(III) PNP Pincer Complexes 29

2.1 Possible Platforms for the Stabilization of Low-Valent Os Complexes . . . 29

2.2 A Square-Planar Os(II) Complex . . . . . . . . . . . . . . . . . . . . . 32

$2.3 \operatorname{Re}(\mathrm{III}) \mathrm{PNP}$ Pincer Halide Complexes . . . . . . . . . . . . . . . . . . . . . . . . 37

2.4 Five- and Six-Coordinate Os Bipyridine Complexes . . . . . . . . . . . . . 40

2.5 Re and Os PNP Pincer Complexes Ligated by a Hemilabile Chelate . . . 45

2.6 Conclusion . . . . . . . . . . . . . . . . . . . . . 48

3 Os Nitride and Imide Complexes in Various Coordination Spheres $\quad 51$

3.1 Four-and Five-Coordinate Os(IV) Nitrides and Imide Complexes . . . . . 51

3.2 Bipyridine Ligated Os Nitride Complexes . . . . . . . . . . . . . . . . . 61

3.3 Conclusion . . . . . . . . . . . . . . . . . . . . 64

4 Transition Metal Complexes with Metal-Pnictogen Multiple Bonds $\quad 67$

$4.1 \mathrm{P}_{2}$ and $\mathrm{As}_{2}$ Complexes from Coupling of Terminal Re Pnictides . . . . . . 67

4.2 Oxygenation of a Terminal Re Phosphide Complex . . . . . . . . . . . . 77

4.3 Interconversion of Os PHMes* and PMes* Complexes by PCET . . . . . 85

4.4 An Isolable Terminal Chlorophosphinidene Complex . . . . . . . . . . . . 92

4.5 Conclusion . . . . . . . . . . . . . . . . . . . . . . 101

5 Proton-Induced Splitting of Dinitrogen by Dimeric Mo PNP Pincer $\begin{array}{ll}\text { Complexes } & 105\end{array}$

$\begin{array}{llr}6 & \text { Summary and Outlook } & 117\end{array}$ 
7 Experimental Section $\quad 123$

7.1 General Comments . . . . . . . . . . . . . . . . . . . . . . . . 123

7.2 Materials and Methods . . . . . . . . . . . . . . . . . . . 123

$7.3 \mathrm{Os}(\mathrm{II} / \mathrm{III})$ and $\operatorname{Re}(\mathrm{III}) \mathrm{PNP}$ Pincer Complexes . . . . . . . . . . . . . . 127

7.3.1 A Square-Planar Os(II) Complex - Computational Details . . . . . 127

7.3.2 Five- and Six-Coordinate Os Bipyridine Complexes - Computational Details . . . . . . . . . . . . . . . . . 128

7.3.3 Crystallographic Details . . . . . . . . . . . . . . . . . . . 131

7.3 .4 Syntheses . . . . . . . . . . . . . . . . . . . . . . 144

7.4 Os Nitride and Imide Complexes in Various Coordination Spheres . . . . 151

7.4.1 Computational Details . . . . . . . . . . . . . . . . . 151

7.4.2 Crystallographic Details . . . . . . . . . . . . . . . . . 153

7.4 .3 Synthesis . . . . . . . . . . . . . . . . . . 163

7.5 Transition Metal Complexes with Metal-Pnictogen Multiple Bonds . . . . 169

7.5.1 $\mathrm{P}_{2}$ and $\mathrm{As}_{2}$ Complexes from Coupling of Terminal Re Pnictides Computational Details . . . . . . . . . . . . . . 169

7.5.2 Oxygenation of a Terminal Re Phosphide Complex - Computational Details . . . . . . . . . . . . . . . . . . 175

7.5.3 Interconversion of Os PHMes* and PMes* Complexes by PCET Computational Details . . . . . . . . . . . . . . 180

7.5.4 Crystallographic Details . . . . . . . . . . . . . . . . 192

7.5.5 Isothermal Titration Calorimetry . . . . . . . . . . . . . . . 209

7.5 .6 Syntheses . . . . . . . . . . . . . . . . . . . . . 212

7.6 Proton-Induced Splitting of Dinitrogen by Dimeric Mo PNP Pincer Complexes . . . . . . . . . . . . . . . . . . . . . . . 224

7.6.1 Computational Details . . . . . . . . . . . . . . . . . . . . . 224

7.6.2 Crystallographic Details . . . . . . . . . . . . . . . . . 230

7.6 .3 Syntheses . . . . . . . . . . . . . . . . . . 235

8 Appendix $\quad 239$

8.1 Os(II) and $\operatorname{Re}(\mathrm{III}) \mathrm{PNP}$ Halide Complexes . . . . . . . . . . . . . . . 239

8.2 Os Nitride and Imide Complexes in Various Coordination Spheres . . . . 244

8.3 Transition Metal Complexes with Metal-Pnictogen Multiple Bonds . . . . 249

8.4 Proton-Induced Splitting of Dinitrogen by Dimeric Mo PNP Pincer Com-

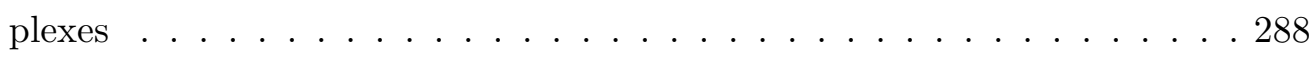

9 Used Abbreviations $\quad 295$

$\begin{array}{lr}\text { Literature } & 299\end{array}$ 




\section{Introduction}




\section{Introduction}

\subsection{Physical and Chemical Characteristics of Pnictogens}

The elements belonging to group 15 of the periodic table (nitrogen, phosphorus, arsenic, antimony, bismuth and moscovium) are knows as the pnictogens. The name is derived from the Greek word $\pi v \imath \gamma$ - (to strangle, to choke) and was first proposed by the Dutch chemist Anton Eduard van Arkel.[1]

Nitrogen is an essential element to all biological life. ${ }^{[2]}$ Biological and industrial utilization of nitrogen is achieved upon nitrogen fixation towards ammonia. The Haber-Bosch process facilitates the conversion of $\mathrm{N}_{2}$ and $\mathrm{H}_{2}$ towards $\mathrm{NH}_{3}$ at elevated temperatures using metal catalysts. Due to the high demand of ammonia in the fertilizer and chemical industry this highly optimized industrial process operates on a scale of hundreds of megatons per year. ${ }^{[3],[4]}$ The generally accepted mechanism consists of initial splitting of the dinitrogen molecule on the catalyst surface to form metal nitrides which are further converted by chemisorbed dihydrogen to afford ammonia (Scheme 1.1, left). The harsh reaction conditions $\left(400 \mathrm{bar}, 480^{\circ} \mathrm{C}\right.$ ) and the required production of highly pure dihydrogen leads to an overall energy demand of over $1 \%$ of the global energy production. ${ }^{[5],[6]}$ In nature, nitrogen fixation is mediated at ambient conditions by enzymes called nitrogenases. ${ }^{[7]}$ The most active representative of this class is the Fe-Mo nitrogenase which was structurally characterized (Scheme 1.1, right). ${ }^{[8]-[10]}$

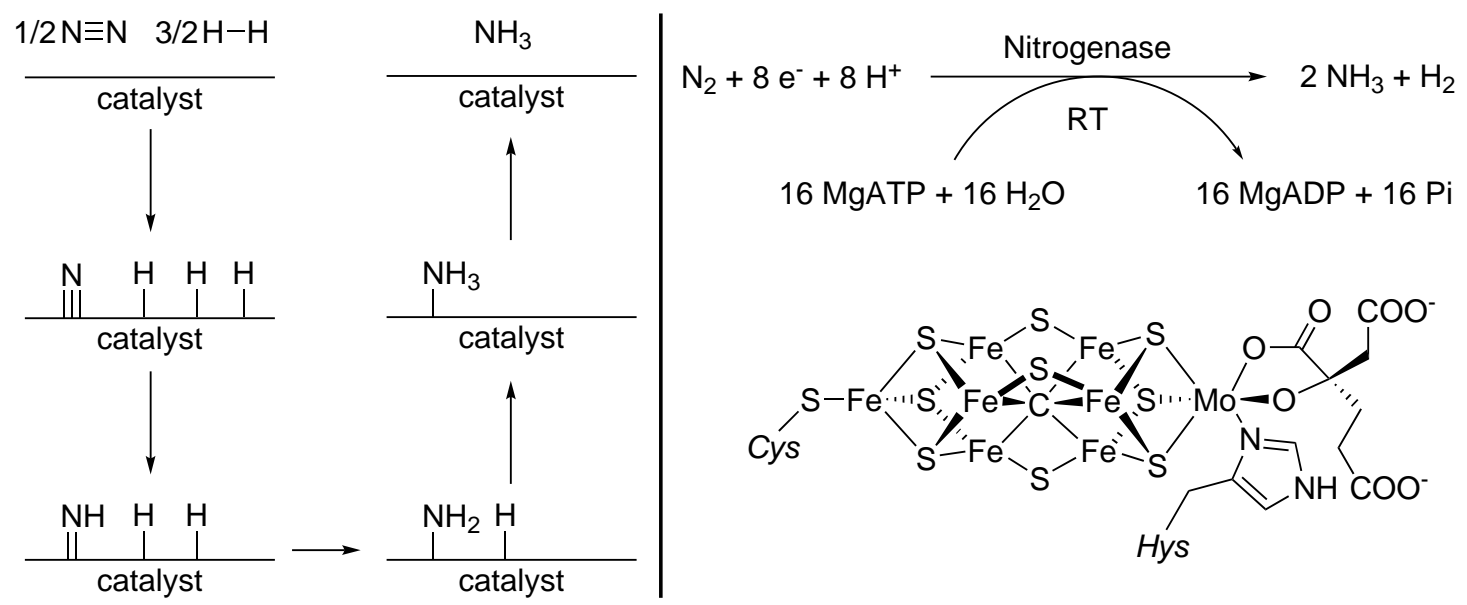

Scheme 1.1: Left: Mechanism of ammonia production by the Haber-Bosch process; Right: Nitrogen fixation by the Fe-Mo nitrogenase. ${ }^{[5]-[7]}$

While the exact mechanism of nitrogen fixation by Fe-Mo-Nitrogenase is not fully understood yet, the overall stoichiometry requires 16 equivalents of adenosine triphosphate (ATP), rendering this process highly energy demanding. ${ }^{[2],[11]}$ This phosphorus containing molecule is commonly called the energy currency of biological life. ${ }^{[12]}$ Besides 
this, phosphorus also plays an important role as phosphate in the construction of bones, phospholipids, DNA and RNA. ${ }^{[12]}$ Therefore, phosphates are important components of fertilizers. White phosphorus $\left(\mathrm{P}_{4}\right)$, mainly produced by electrothermal conversion of $\mathrm{Ca}_{3}\left(\mathrm{PO}_{4}\right)_{2} \cdot \mathrm{CaF}_{2}$, is an essential building block for the production of phosphorus containing chemicals. ${ }^{[13]}$ The heavier elements of the pnictogens do not exhibit comparable relevance due to their higher toxicity and/or lower abundancy. ${ }^{[14]}$ Typical uses are fabrication of alloys (As, $\mathrm{Sb}, \mathrm{Bi}$ ), insecticides (As), flame retardants ( $\mathrm{Sb}$ ), pharmaceuticals and cosmetics $(\mathrm{Bi}) \cdot{ }^{[12]}$

At ambient conditions nitrogen is encountered as gaseous dinitrogen. In this molecule the nitrogen atoms are bound via a short $\mathrm{N} \equiv \mathrm{N}$ triple bond $(1.0977 \AA)$ consisting of one $\sigma$ and two $\pi$-bonds (Scheme 1.2). The large HOMO-LUMO gap of $10.82 \mathrm{eV}$, the missing dipole moment and the resulting large bond dissociation energy of $225.0 \mathrm{kcal} \cdot \mathrm{mol}^{-1}$ render $\mathrm{N}_{2}$ kinetically and thermodynamically highly stable. Thus, the Haber-Bosch process requires the rather drastic conditions described above. ${ }^{[13]}$ The high stability of the triple bond can be attributed to the almost identical radii of the $2 \mathrm{~s}$ and $2 \mathrm{p}$ orbitals allowing for efficient overlap and hybridization (Scheme 1.2, Figure 1.1). This is attributed to the high degree of penetration of the $2 \mathrm{p}$ orbitals into the core.

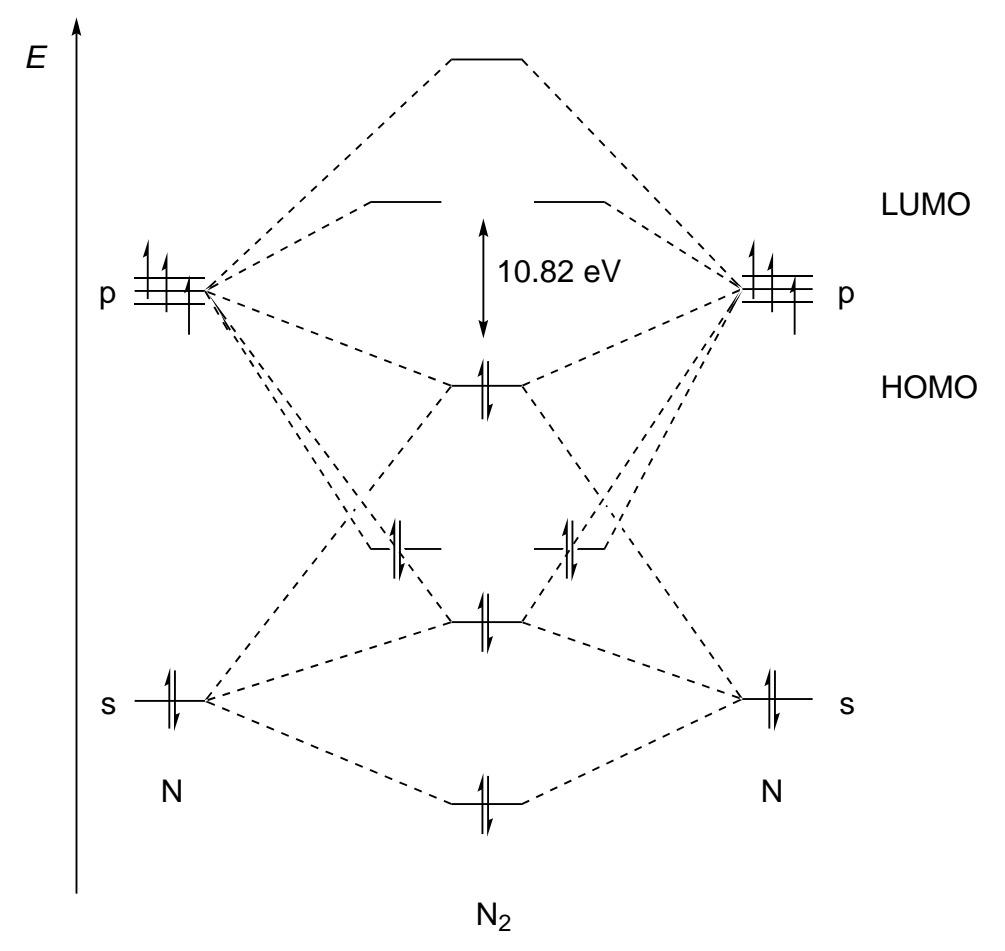

Scheme 1.2: Qualitative MO scheme of the dinitrogen molecule. ${ }^{[13]}$ 


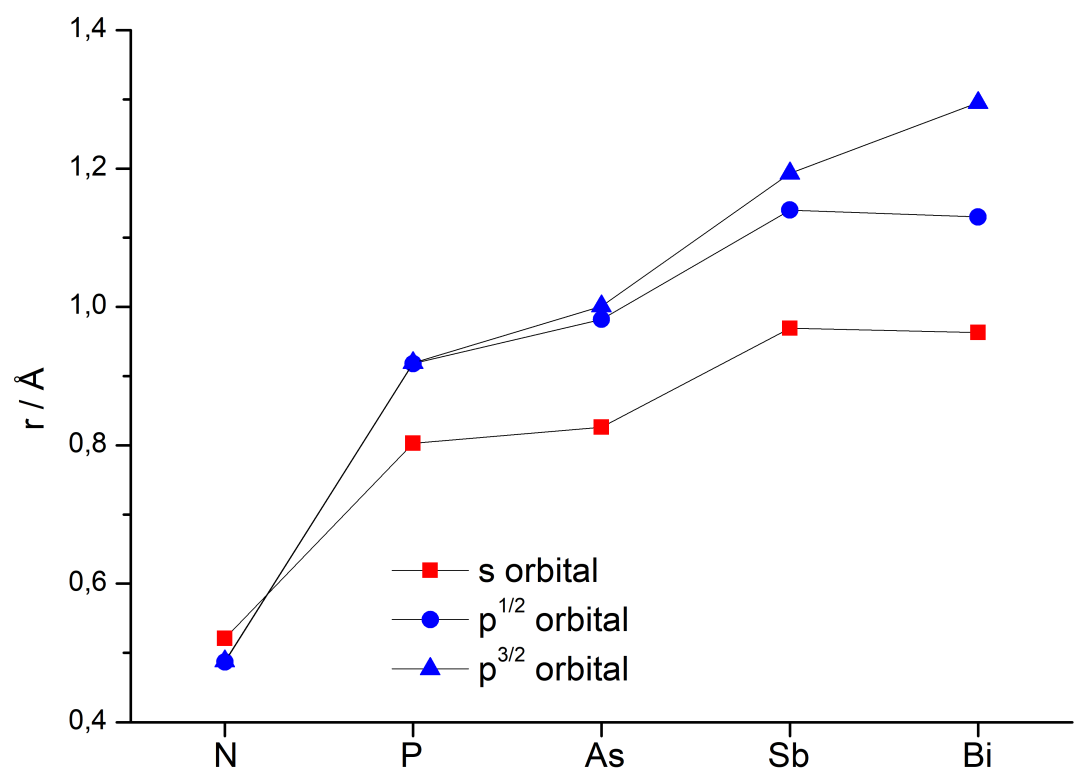

Figure 1.1: Radii of the pnictogens and p orbitals. ${ }^{[15]}$

This changes drastically when moving to the heavier elements that favor formation of tetrahedral $\mathrm{Pn}_{4}$. Diphosphorus and its heavier analogues can only be generated at temperatures above $1000 \mathrm{~K}$ in the gas phase due to the high reactivity and low bond dissociation energy (Table 1.1). ${ }^{[13],[16]}$ Furthermore, the $\mathrm{Pn} \equiv \mathrm{Pn}$ bond distance elongates by ca. $0.8 \AA$ when moving from nitrogen to phosphorus. ${ }^{[16]}$ While for $2^{\text {nd }}$ row elements the $\mathrm{s}$ and $\mathrm{p}$ orbitals exhibit almost identical radii, the $\mathrm{np}$ orbitals with $n \geq 3$ encounter Pauli repulsion with other $\mathrm{p}$ core orbitals resulting in a significant increase of the radii compared to the corresponding ns orbitals (Figure 1.1). ${ }^{[15],[17]}$

\begin{tabular}{ccc} 
Table 1.1: Physical properties of $\mathrm{Pn}_{2}, \mathrm{Pn}=\mathrm{N}, \mathrm{P}, \mathrm{As}, \mathrm{Sb}, \mathrm{Bi}^{[16}$ \\
\hline Compound & Bond distance $/ \AA$ & $D_{e} / \mathrm{kcal} \cdot \mathrm{mol}^{-1}$ \\
\hline $\mathrm{N}_{2}$ & 1.0977 & 225.0 \\
$\mathrm{P}_{2}$ & 1.8934 & 116.1 \\
$\mathrm{As}_{2}$ & 2.1026 & 91.3 \\
$\mathrm{Sb}_{2}$ & 2.3415 & 71.3 \\
$\mathrm{Bi}_{2}$ & 2.6596 & 46 \\
\hline
\end{tabular}

Older textbooks rationalize the lower stability of $\mathrm{P}_{2}$ and its heavier homologues by arguing that $\pi$-bonding in heavier elements is considerably weaker due to poorer $\mathrm{p}(\pi)$ orbital overlap when compared to $2^{\text {nd }}$ row elements. However, these statements are not in agreement with quantum chemical calculations that predict the more diffuse $\mathrm{p}(\pi)$ orbitals to allow for similar overlap as the lighter elements. ${ }^{[18]}$ Energy decomposition analysis $(\mathrm{EDA})^{[19]}$ further elucidates this bonding situation. The attractive interaction 

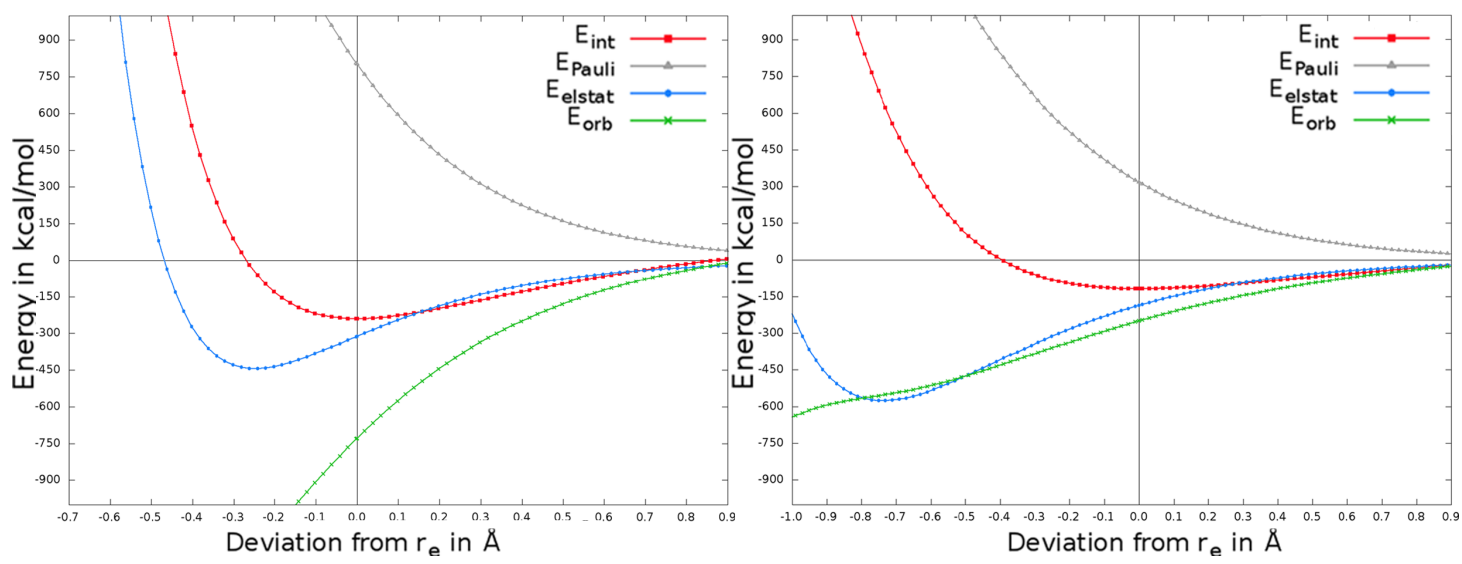

Figure 1.2: Calculated energy terms of the EDA at different bond length of $\mathrm{N}_{2}$ (left) and $\mathrm{P}_{2}$ (right), Copyright (C) 2014, Springer-Verlag Berlin Heidelberg.[18]

between the nitrogen atoms in the dinitrogen molecule mainly arises from covalent orbital interactions $\Delta E_{\text {orb }}(70 \%)$ with smaller contribution from the electrostatic coulombic attraction $\Delta E_{\text {elstat }}(30 \%$, Figure 1.2$)$.

These contributions are more alike in $\mathrm{P}_{2}\left(\Delta E_{\text {orb }} / \Delta E_{\text {elstat }}: 57 \% / 43 \%\right)$. If the orbital interactions are further separated into $\sigma$ - and $\pi$-bonding contributions it becomes evident that the stabilization by $\pi$-bonding in diphosphorus is even greater than in $\mathrm{N}_{2}$, disproving the general assumption that weak $\pi$-interactions are the reason for the tendency of heavier elements to form single bonds with other elements. Further quantum chemical investigation shows that the equilibrium bond distance of the diatomic pnictogens is rather dictated by the Pauli repulsion $\Delta E_{\text {Pauli }}$ since the attractive terms $\Delta E_{\text {orb }}$ and $\Delta E_{\text {elstat }}$ become larger when the $\mathrm{Pn} \equiv \mathrm{Pn}$ bond gets shortened with respect to the equilibrium distance $r_{e}$ (Figure 1.2). The higher stability of $\mathrm{P}_{4}$ over $\mathrm{P}_{2}$ can therefore be linked to the different radii ratios of the $\mathrm{s}$ and $\mathrm{p}$ orbitals for second row elements and their heavier analogues. Due to Pauli repulsion, $\mathrm{N}_{2}$ is favored over $\mathrm{N}_{4}$ whereas $\mathrm{P}_{4}$ is more stable than $\mathrm{P}_{2}$. This statement also holds true for heavier elements of group 15 of the periodic table.

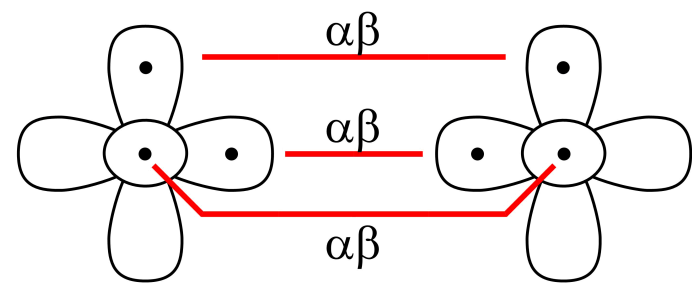

molecular

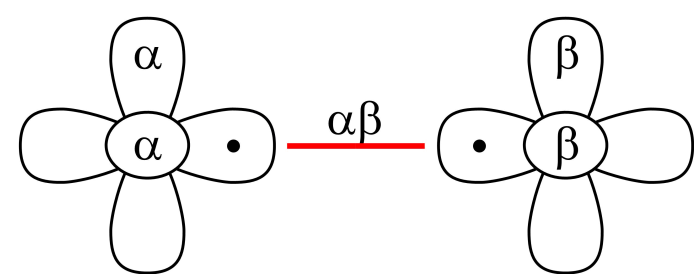

quasi atomic

Figure 1.3: Schematic representation of important spin couplings in $\mathrm{Pn}_{2} \cdot{ }^{[20]}$ 
Dinitrogen is commonly described as the prototype of an unpolar, covalent triple bond as a product of three singlet spin functions that show almost perfect paring (molecular coupling). Generalized valence bond (GVB) $)^{[21]}$ theory suggests that the heavier analogues differ from this description and that the formulation of quasi-atomic coupling become increasingly important in describing the bond. ${ }^{[20]}$ In this formulation two electrons are coupled to a singlet in a $\sigma$-bond. The remaining electrons resulting in the two $\pi$-bonds adopt triplet states which are antiferromagnetically coupled (Figure 1.3). In $\mathrm{As}_{2}$, both couplings exhibit the same weight in describing the bonding. The latter one is therefore expected to become dominant in heavier analogues. 


\subsection{Terminal Transition Metal Pnictide Complexes}

Parts of this chapter were adapted from: J. Abbenseth, S. C. Bete, M. Finger, C. Volkmann, C. Würtele, S. Schneider, Organometallics 2018, 37, 802-811. Copyright 2018 American Chemical Society.

\subsubsection{Heavy Analogues of Nitride Complexes - First Indications}

While terminal transition metal nitride complexes are a well-studied class of compounds, isolation of the heavier analogues was only achieved in 1995. ${ }^{[22],[23]}$ Prior to this finding the possible formation of triply bonded heavy transition metal pnictide species was a subject of much speculation. ${ }^{[24]}$ Becker and co-workers first suggested the formation of a terminal tungsten phosphide species in 1985 upon studying the reaction of $\left[\mathrm{W}_{2}\left(\mathrm{O}^{t} \mathrm{Bu}\right)_{6}\right]$ (I, Scheme 1.3) and ${ }^{t} \mathrm{BuC} \equiv \mathrm{P} .{ }^{[25],[26]} \mathrm{A}$ four-membered $\mathrm{WC}_{2} \mathrm{P}$ heterocycle was identified as one of the reaction product as well as an unstable $\mathrm{WCP}_{2}$ ring compound which was characterized by ${ }^{31} \mathrm{P}$ NMR spectroscopy. The proposed mechanism involves a breaking of the $\mathrm{W} \equiv \mathrm{W}$ triple bond to form transient alkylidyne and phosphide species (II, Scheme 1.3) which form four-membered rings upon addition of another equivalent of ${ }^{t} \mathrm{BuC} \equiv \mathrm{P}$. A $1,3-\mathrm{O}^{t} \mathrm{Bu}$ migration step yields the final products III and IV.

This mechanistic proposal is supported by the formation of alkylidynes and nitrides when alkynes or nitriles are used instead of the phosphaalkyne ${ }^{t} \mathrm{BuC} \equiv \mathrm{P}$. Scheer and co-workers further confirmed this mechanistic proposal upon performing the reaction in the presence of Lewis-acidic transition metal carbonyl compounds which allowed for the isolation of $\left[\left({ }^{t} \mathrm{BuO}\right)_{3} \mathrm{~W} \equiv \mathrm{P}-[\mathrm{M}]\right] .{ }^{[27]}$ Later, several additional reports also proposed the possible formation of terminal transition metal phosphides. ${ }^{[24]}$ The first terminal

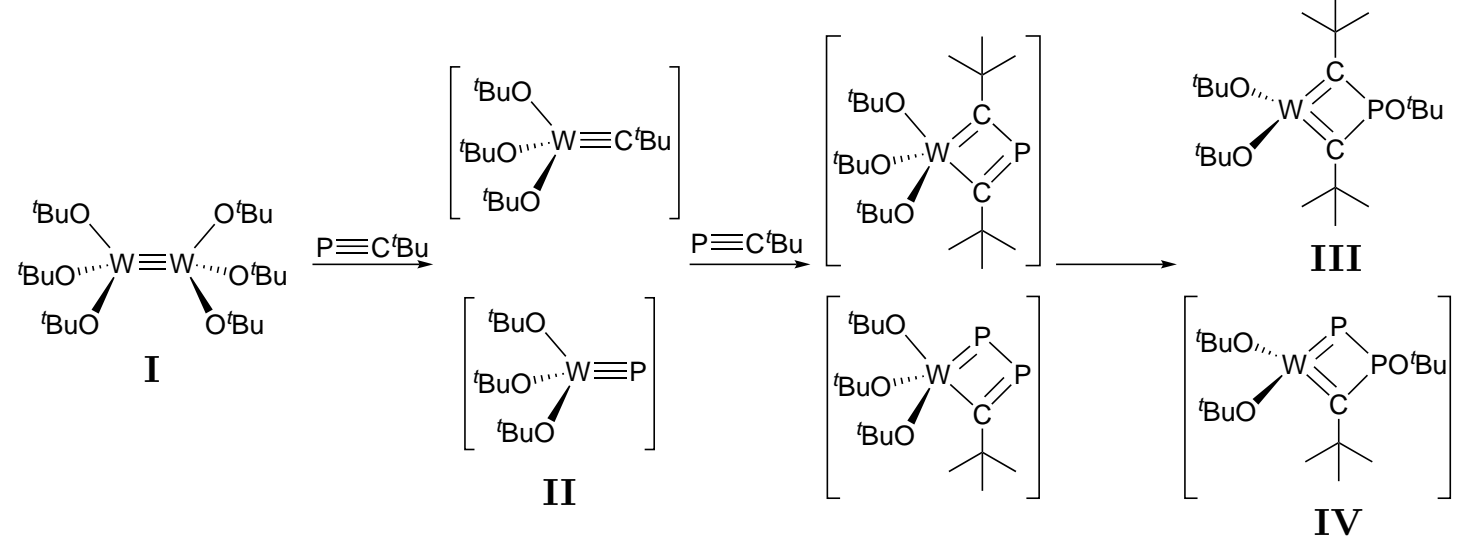

Scheme 1.3: Proposed reactivity of $\mathbf{I}$ to give the transient terminal tungsten phosphide II. [25], [26] 
arsenide complex was postulated by Ziegler and co-workers in 1989 based on the results obtained for the thermolysis and co-photolysis of arsenide capped tris-Mo complex $\left[\left\{\mathrm{MoCp}(\mathrm{CO})_{2}\right\}_{3}\left(\mu^{3}-\mathrm{As}\right)\right]$. The reaction yields a triply bonded Mo $\equiv$ Mo complex which suggests formation of the arsenide $\mathbf{V}$. This was further substantiated by the addition of $\left[\mathrm{CoCp}(\mathrm{CO})_{2}\right]$ which yielded complexes containing a single arsenic atom (Scheme 1.4). $[28],[29]$
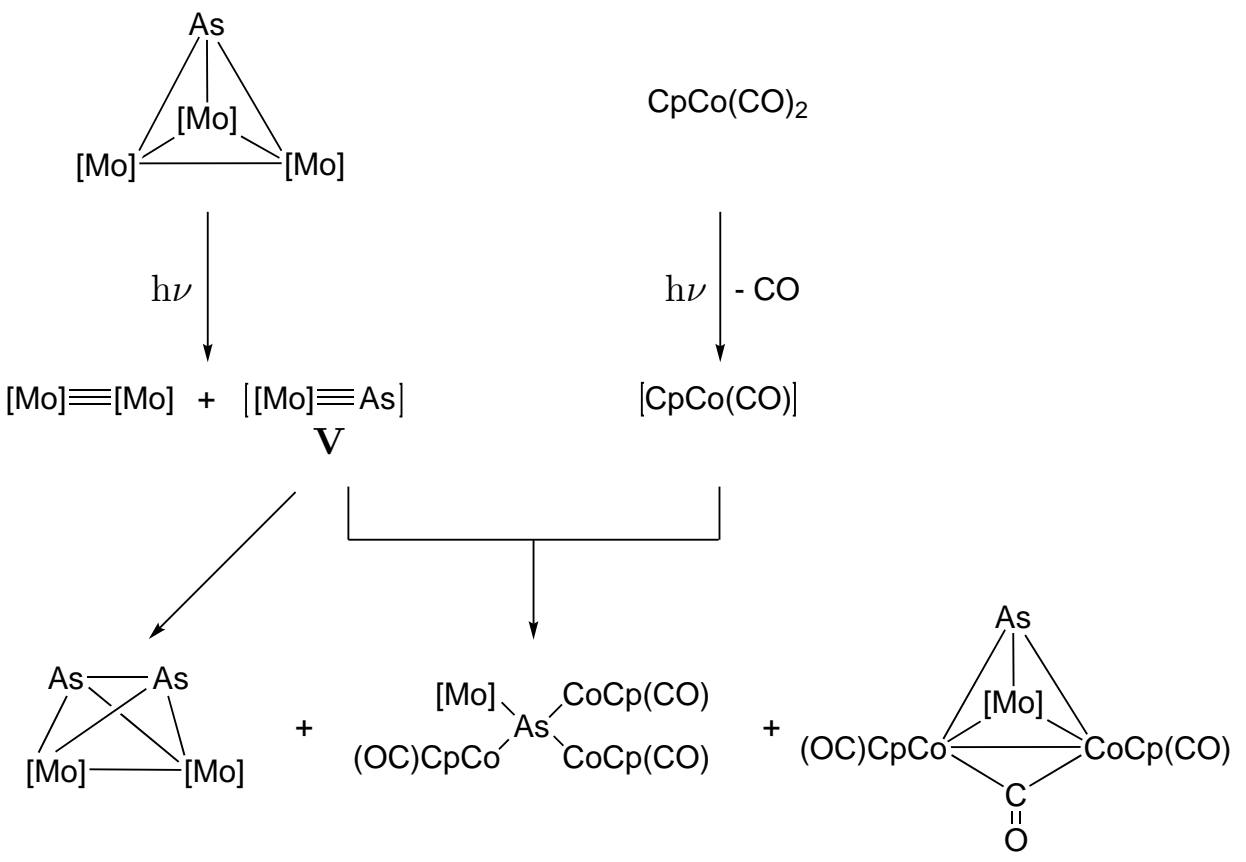

Scheme 1.4: Proposed mechanism of the photolysis of $\left[\left\{\mathrm{MoCp}(\mathrm{CO})_{2}\right\}_{3}\left(\mu^{3}-\mathrm{As}\right)\right]$ and $\left[\mathrm{CoCp}(\mathrm{CO})_{2}\right]$ to form the postulated arsenide complex $\mathbf{V}$ and subsequent reactivity with $[\mathrm{CoCp}(\mathrm{CO})],[\mathrm{Mo}]=\left[\mathrm{MoCp}(\mathrm{CO})_{2}\right] .^{[28]}$

Spectroscopic evidence for the formation of terminal stibide complexes was provided by Rheingold and co-workers. The gas-phase reaction of transition metal carbonyl complexes with $\mathrm{Sb}_{2}^{-}$allowed for the observation of $\left[\mathrm{MSb}(\mathrm{CO})_{\mathrm{n}}\right]^{-}(\mathrm{M}=\mathrm{Fe}, \mathrm{n}=3 ; \mathrm{M}=$ $\mathrm{Cr}, \mathrm{Mo}, \mathrm{W} ; \mathrm{n}=4$ ) by Fourier-transform ion cyclotron resonance mass spectrometry (FT-ICR). ${ }^{[30]}$ 


\subsubsection{Synthesis of Terminal Pnictide Complexes from the Elements}

The functionalization of dinitrogen still represents a major challenge in synthetic chemistry. As described in the previous chapter, dinitrogen is a kinetically and thermodynamically highly stabilized molecules which necessitates rather forcing conditions to facilitate full bond cleavage. Today, several systems are available that facilitate the splitting of $\mathrm{N}_{2}$ into terminal nitrides (dominated by Mo complexes) and even the catalytic conversion of dinitrogen to ammonia and trimethylsilyl amine has been accomplished, while more functionalized nitrogen containing molecules can be generated stoichiometrically. ${ }^{[31]-[39]}$ In this context, photochemical and electrocatalytic approaches have also been investigated, however a complete survey of this field is beyond the scope of this thesis. ${ }^{[31],[35],[40]-[45]}$ Understanding the molecular interactions of transition metal complexes and the dinitrogen molecule in dimeric complexes is crucial for further developments towards more efficient platforms capable of splitting and functionalization of dinitrogen and will be the focus of this chapter.

Complete $\mathrm{N} \equiv \mathrm{N}$ bond scission was first reported for the molybdenum trisanilide complex $\left[\mathrm{Mo}(\mathrm{NRAr})_{3}\right]\left(\mathbf{V I}, \mathrm{R}=\mathrm{C}\left(\mathrm{CD}_{3}\right)_{2} \mathrm{Me}, \mathrm{Ar}=3,5-\mathrm{Me}_{2} \mathrm{C}_{6} \mathrm{H}_{3}\right.$, Scheme 1.5) in 1995. Storing a solution of VI at $-35^{\circ} \mathrm{C}$ under a dinitrogen atmosphere yields the $\mathrm{N}_{2}$-bridged complex $\left[\left\{\mathrm{Mo}(\mathrm{NRAr})_{3}\right\}_{2}\left(\mu-\mathrm{N}_{2}\right)\right]$ (VII) which splits the dinitrogen bond homolytically upon warming to room temperature. The resulting nitrido complex $\left[\mathrm{MoN}(\mathrm{NRAr})_{3}\right]$ (VIII) is formed via a zig-zag transition state. ${ }^{[46]-[48]}$ Consideration of the MO scheme of the $\{$ MoNNMo $\}$ core, constructed by the Mo $d$ and nitrogen $\mathrm{p}$ orbitals, gives valuable insights into the required electronic structure to facilitate splitting of dinitrogen (Scheme 1.5). The $S_{6}$-symmetric dimer VII features $10 \pi$-electrons (formally: $2 \cdot \operatorname{Mo}(\mathrm{III})=6$; $\mathrm{N}_{2}=4$ ) giving rise to an overall triplet configuration. The zig-zag transition state lifts the degeneracy of the $2 \mathrm{e}_{\mathrm{u}} \pi$-MOs and allows for mixing with the antibonding $\sigma^{*}$-orbital which is forbidden in the case of VII. This interaction, besides the overall $\pi$-electron count, seems to be crucial for scission of the $\mathrm{N}_{2}$ bond since it allows for the population of the $\sigma$-bonding orbital set $1 b_{u}$ in the reaction products. ${ }^{[31]}$ This is showcased by the triamidoamine complex $\left[\mathrm{Mo}\left(\mathrm{R}^{\prime} \mathrm{NCH}_{2} \mathrm{CH}_{2}\right)_{3}\left(\mu-\mathrm{N}_{2}\right)\right]\left(\mathbf{I X}, \mathrm{R}^{\prime}={ }^{t} \mathrm{BuMe}_{2} \mathrm{Si}\right)$ which would feature an identical $\pi$-electron count within the $\{\mathrm{MoNNMo}\}$ core and high similarities in bond distances and angles. However, the ligand in trans-position to the dinitrogen bridge destabilizes the $\sigma^{*}$-MO leading to unfavorable kinetic and thermodynamic parameters for splitting into terminal nitrides. ${ }^{[4]}$ Furthermore, the rigidity of the chelate ligand prevents rotation of the amide donors which is proposed to lead to increased charge transfer in the transition state favoring the splitting of $\mathrm{N}_{2} \cdot{ }^{[50],[51]}$ 


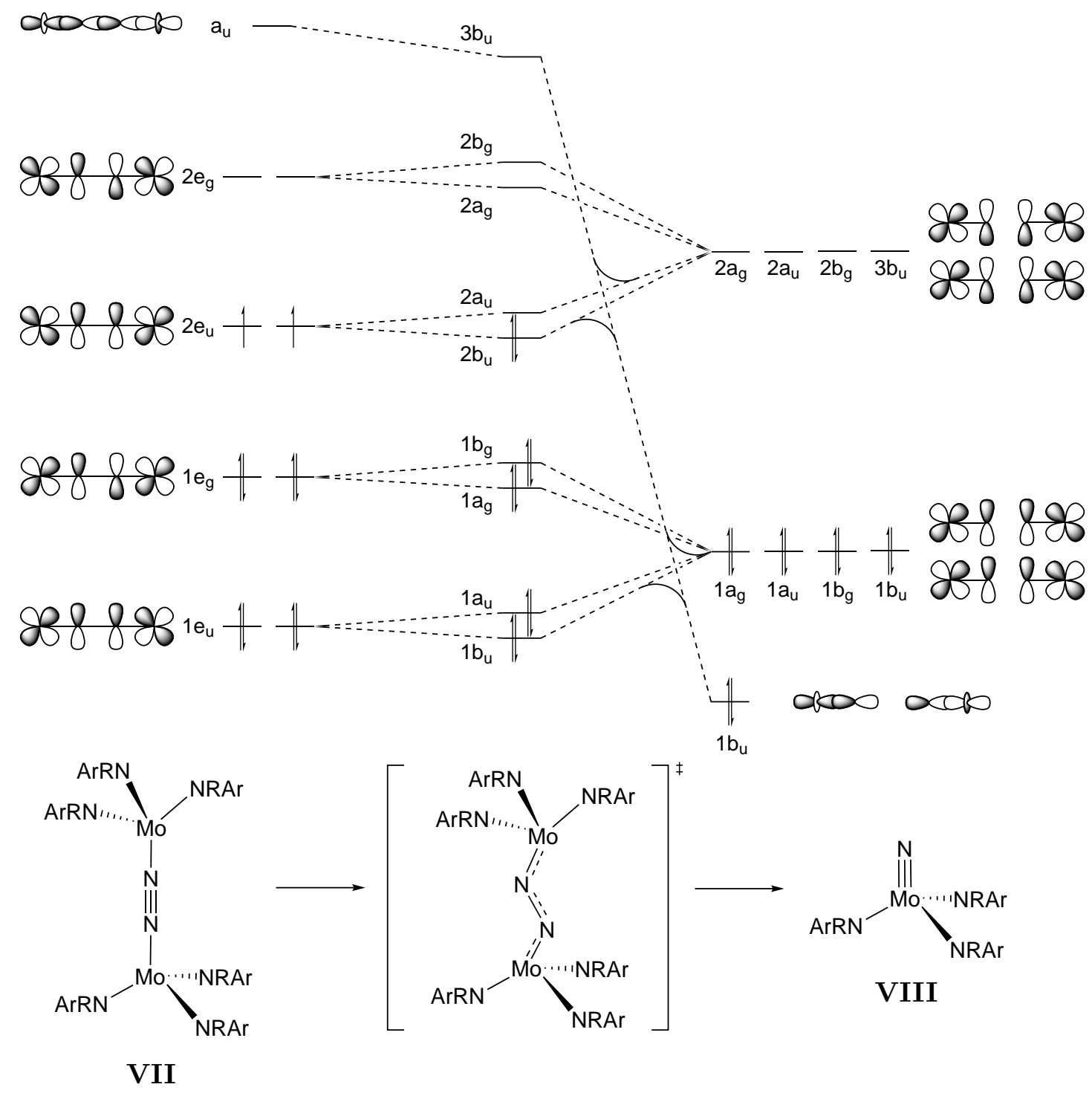

Scheme 1.5: MO scheme for $\mathrm{N}_{2}$-cleavage by VII towards VIII. ${ }^{[31]}$

This $\mathrm{MO}$ consideration also explains why strong $\mathrm{N}_{2}$ activation does not necessarily lead to bond scission. VII can be oxidized to cationic $\mathbf{X}$ and dicationic $\mathbf{X I}$ which feature a doublet and a singlet state, respectively. Increased $\mathrm{N}_{2}$ bond activation is observed by Raman spectroscopy $\left(\tilde{\nu}_{\mathrm{NN}}: 1630 \mathrm{~cm}^{-1}\right.$ (VII), $\left.1503 \mathrm{~cm}^{-1}(\mathbf{X}), 1349 \mathrm{~cm}^{-1}(\mathbf{X I})\right)$ due to depopulation of the N-N bonding orbitals sets $2 \mathrm{e}_{\mathrm{u}}$ upon oxidation. Consequently, no dinitrogen splitting is observed since the decreased amount of $\pi$-electrons prevents the formation of stable nitride complexes. ${ }^{[47]}$ 


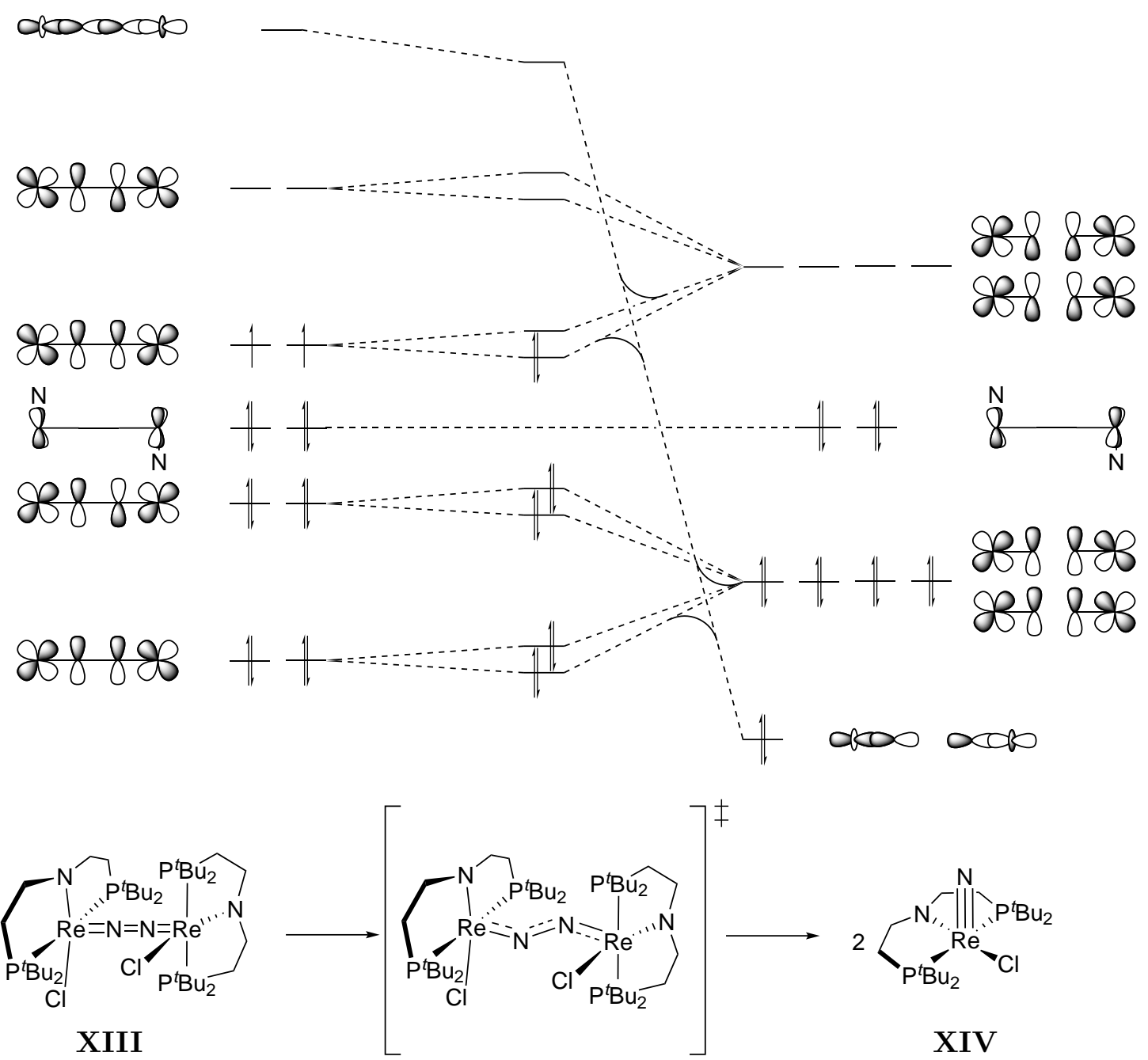

Scheme 1.6: MO scheme for $\mathrm{N}_{2}$-cleavage by XIII towards XIV. ${ }^{[52]}$

Schneider and co-workers reported the first example of dinitrogen splitting to a terminal nitride by a well-defined transition metal complex containing a central atom beyond group 6. Upon one-electron reduction of the $\mathrm{Re}^{\mathrm{III}}$ dichloride complex $\left[\operatorname{ReCl}_{2}\left(\mathrm{~L}^{1}\right)\right]$ (XII, Scheme 1.6, $\left.\mathrm{L}^{1}=\mathrm{N}\left(\mathrm{CH}_{2} \mathrm{CH}_{2} \mathrm{P}^{t} \mathrm{Bu}_{2}\right)_{2}\right)$, dinitrogen is bound within the dimer $\left[\left\{\operatorname{ReCl}\left(\mathrm{L}^{1}\right)\right\}_{2}\left(\mu-\mathrm{N}_{2}\right)\right]$ (XIII) which could be characterized crystallographically. ${ }^{[40]}$ Splitting towards the $\mathrm{Re}^{\mathrm{V}}$ nitride $\left[\operatorname{ReNCl}\left(\mathrm{L}^{1}\right)\right](\mathbf{X I V})$ is observed in high yield upon warming to room temperature. ${ }^{[53]}$ The obtained nitride could be further functionalized to allow for the synthesis of nitrogen containing organic molecules. ${ }^{[33],[53]}$ Due to the change of the overall geometry of the obtained dinitrogen dimer from $S_{6}$ towards $C_{2}$, non-bonding, $\delta$ symmetric orbitals have to be considered. The overall increased electron count within the \{ReNNRe\} core when compared to Cummins' system results in a $\pi^{10}\left(\delta^{4}\right)$-configuration (Scheme 1.6). This result shows that dinitrogen splitting might also be accomplished 


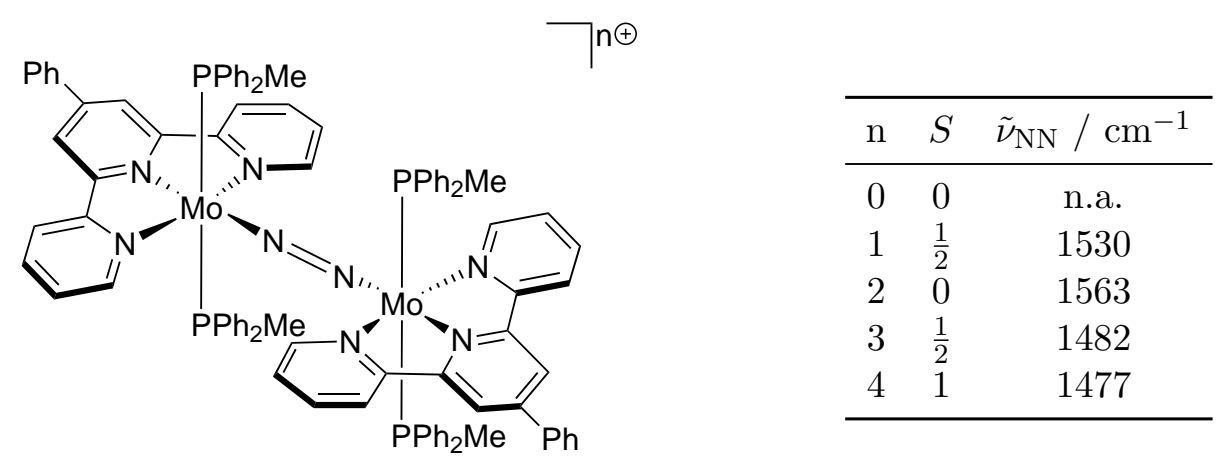

XV

Figure 1.4: Redox series of $\mathbf{X V}^{\mathbf{n}+} .{ }^{[55]}$

with late transition elements if a suitable coordination geometry and electronic configuration is provided. The formed nitrides should be more reactive due to successive weakening of the $\mathrm{M} \equiv \mathrm{N}$ bond along the periodic table. ${ }^{[54]}$

The participation of ligand centered orbitals can alter the overall picture of bond activation drastically as shown for the redox series $\left[\left\{\mathrm{Mo}\left({ }^{\mathrm{Ph}} \mathrm{Tpy}\right)\left(\mathrm{PPh}_{2} \mathrm{Me}\right)_{2}\right\}_{2}\left(\mu-\mathrm{N}_{2}\right)\right]^{\mathrm{n}+}$ $\left(\mathbf{X V}^{\mathbf{n}+}, \mathbf{n}=0-4,{ }^{{ }^{h}}\right.$ Tpy $=4$ '-phenyl-2,2':6',2"-terpyridine). ${ }^{[55]}$ For $\mathbf{X V}^{\mathbf{2 +}}$, an overall $\pi^{10}\left(\delta^{4}\right)$-configuration arises, however degeneracy of the $\mathrm{N}_{2}$-bonding orbital is lifted due to coupling with the redox active ligand (Figure 1.4). As expected, oxidation leads to stronger dinitrogen activation, however this effect is also observed upon reduction due to population of ligand-based orbitals. ${ }^{[55]}$

Up to this date, isolable terminal heavy transition metal pnictide complexes are still scarce and only known up to group $6 .{ }^{[60]-[65]}$ This can partly be attributed to the synthetic problems associated with transferring single $\mathrm{P}$ or $\mathrm{As}$ atoms from $\mathrm{P}_{4}$ and
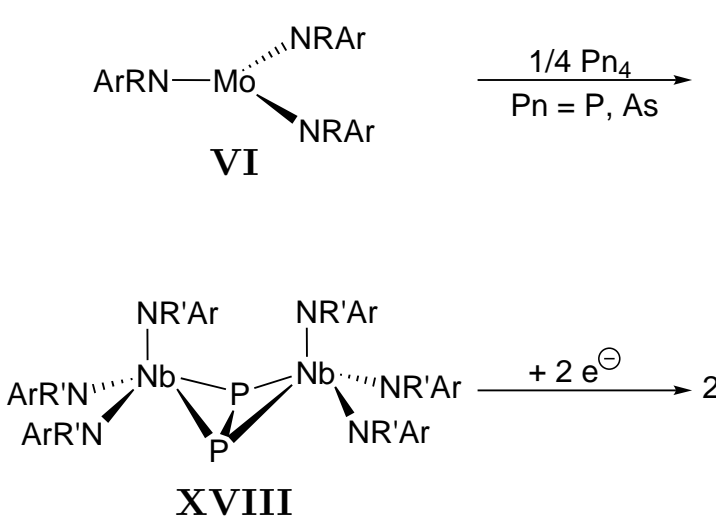

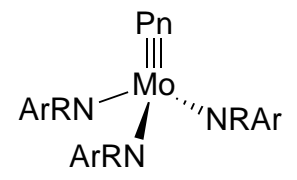

XVI $\quad(P n=P)$

XVII $\quad(\mathrm{Pn}=\mathrm{As})$

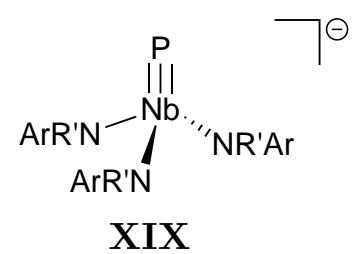

Scheme 1.7: Synthesis of phosphides and arsenides from the elements, $\mathrm{Ar}=3,5-\mathrm{Me}_{2} \mathrm{C}_{6} \mathrm{H}_{3}, \mathrm{R}$ $=\mathrm{C}\left(\mathrm{CD}_{3}\right)_{2} \mathrm{Me}, \mathrm{R}^{\prime}=$ neopentyl. ${ }^{[23],[56]-[59]}$ 
$\mathrm{As}_{4}$, respectively. Besides the terminal coordination of group 15 elements, various reports feature stabilization of the reactive triple bonds by metal coordination or metalheterocumulene formation. However, a full coverage of this field is beyond the scope of this thesis. ${ }^{[63]}$

Cummins and co-workers showed that white phosphorus can be reacted with the Mo trisanilide complex VI to give $\left[\mathrm{MoP}(\mathrm{NRAr})_{3}\right]$ (XVI) representing the first isolable, terminal transition metal phosphide complex (Scheme 1.7). ${ }^{[23]}$ Later, it was shown that yellow arsenic can be utilized in the same manner yielding $\left[\mathrm{MoAs}(\mathrm{NRAr})_{3}\right]$ (XVII) ${ }^{[56]}$ The splitting of a $\mathrm{P}_{2}^{4-}$ moiety towards a terminal phosphide complex was shown to be accessible upon reduction of the $\mathrm{P}_{4}$-derived dimeric $\mathrm{Nb}^{\mathrm{V}}$ complex $\left[\left\{\mathrm{Nb}(\mathrm{NRAr})_{3}\right\}_{2}\left(\mu-\mathrm{P}_{2}\right)\right]$ (XVIII, $\mathrm{Ar}=3,5-\mathrm{Me}_{2} \mathrm{C}_{6} \mathrm{H}_{3}, \mathrm{R}=\mathrm{CH}_{2}{ }^{t} \mathrm{Bu}$ ) which gave the terminal Nb phosphide complex $\left[\mathrm{NbP}(\mathrm{NRAr})_{3}\right]^{-}$(XIX, Scheme 1.8). ${ }^{[58],[59]}$ 


\subsubsection{Alternative Heavy Pnictide Sources}

The first terminal arsenide $\mathbf{X X}$ and stibide $\mathbf{X X I}$ complexes were reported by Scheer and co-workers upon reacting trimethylsilyl substituted pnictide transfer reagents with the tungsten trisamido amine complex $\left[\mathrm{WCl}\left(\mathrm{N}\left\{\mathrm{CH}_{2} \mathrm{CH}_{2} \mathrm{NSiMe}_{3}\right\}_{3}\right)\right]$ (XXII, Scheme 1.7). ${ }^{[57],[66]}$ Additional examples have been reported for Ta and Nb. ${ }^{[63]}$ A synthesis of terminal stibide complexes from antimony has not been reported so far.

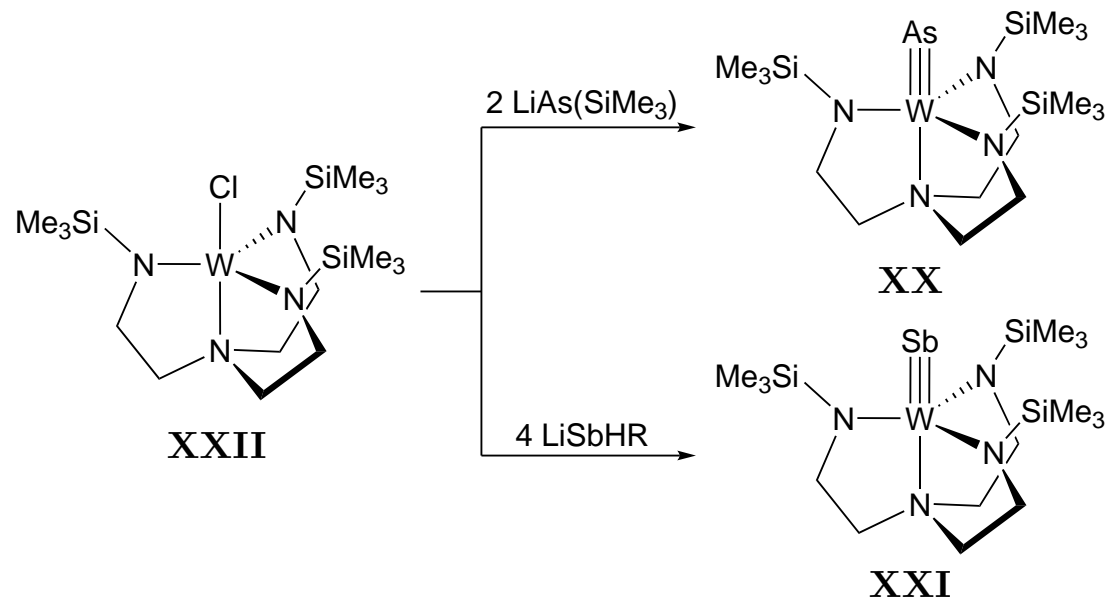

Scheme 1.8: Synthesis of the first arsenide $(\mathbf{X X})$ and stibide $(\mathbf{X X})$ complexes, $\mathrm{R}=$ $\mathrm{CH}\left(\mathrm{SiMe}_{3}\right)_{2} \cdot{ }^{[57],[66]}$

The recently reported phosphorus transfer reagents CIPA (chloro-substituted dibenzo$7 \lambda^{3}$-phosphanorbornadiene, $\mathbf{A}=$ anthracene $^{[67]}$ and $\left[\mathrm{Na}(\operatorname{diox})_{\mathrm{x}}\right][\mathrm{PnCO}]($ diox $=$ dioxane, $\mathrm{Pn}=\mathrm{P}, \mathrm{As})^{[68],[69]}$ offer the possibility to introduce single phosphorus atoms more conveniently and gave rise to the isolation of the first terminal pnictide complexes featuring $\mathrm{d}$ electrons. The $\mathrm{PnCO}^{-}$anions have extensively been used in organic and inorganic chemistry to synthesize phosphorus and arsenic containing heterocycles, $\operatorname{Pn}_{2}^{\mathrm{n}-}(\mathrm{n}=2,4)$ bridged transition metal complexes and new materials. ${ }^{[68],[70]-[74]}$ Upon substitution of nitrogen for its heavier congeners, the carbon-pnictogen bond dissociation energy drops drastically, resulting in easier cleavage of the Pn-C bond. ${ }^{[75]}$

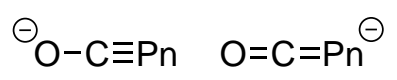

$\begin{array}{lll}\mathrm{Pn}=\mathrm{N} & 61 \% & 30 \% \\ \mathrm{Pn}=\mathrm{P} & 51 \% & 38 \% \\ \mathrm{Pn}=\mathrm{As} & 46 \% & 36 \%\end{array}$

Figure 1.5: Resonance forms and their relative weights for $\mathrm{NCO}^{-}, \mathrm{PCO}^{-}$and $\mathrm{AsCO}^{-} .{ }^{[76]}$

Natural resonance theory of $\mathrm{NCO}^{-}, \mathrm{PCO}^{-}$and $\mathrm{AsCO}^{-}$reveals two major resonance structure (Figure 1.5). The heavier analogues of isocyanate feature an increased contribution 
to the HOMO, as expected for less electronegative molecules. ${ }^{[76]}$ Therefore, orbital controlled reactions favor Pn-coordination to the metal center, whereas charged controlled processes result in O-coordination, e.g. in the case of f-block elements. ${ }^{[71]}$

Cummins and co-workers reported a series of terminal tungsten pnictide complexes upon decarbonylation of $\mathrm{PnCO}^{-}(\mathrm{Pn}=\mathrm{N}, \mathrm{P}, \mathrm{As})$ by reaction with $\left[\mathrm{W}(\mathrm{ODipp})_{4}\right]($ XXIII $)$ to give $\left[\mathrm{WPn}(\mathrm{ODipp})_{4}\right]^{-}(\mathbf{X X I V}(\mathrm{Pn}=\mathrm{N}), \mathbf{X X V}(\mathrm{Pn}=\mathrm{P}), \mathbf{X X V I}(\mathrm{Pn}=\mathrm{As})$, Scheme 1.8). ${ }^{[65]}$ Oxidative addition of a $\mathrm{PCl}$ moiety, stemming from $\mathrm{ClPA}$, by the $\mathrm{Mo}^{0}$ dinitrogen complex $\left[\mathrm{Mo}\left(\mathrm{N}_{2}\right)(\mathrm{PCP})\right]$ (XXVII, PCP = 2,2"-bis(diisopropylphos-phaneyl)-1,1':4',1"terphenyl) gave the $\mathrm{Mo}^{\mathrm{IV}}$ phosphide $[\mathrm{MoPCl}(\mathrm{PCP})]$ (XXVIII, Scheme 1.9).

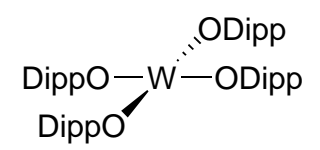

XXIII

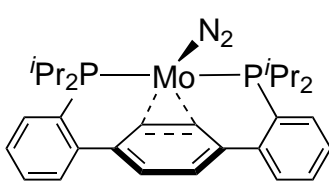

XXVII

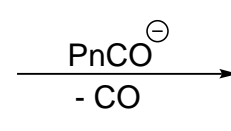

$$
\begin{array}{cl}
\text { XXIV } & (P n=N) \\
\text { XXV } & (P n=P) \\
\text { XXVI } & (P n=A s)
\end{array}
$$

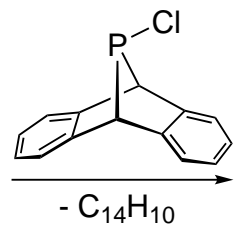

Scheme 1.9: Synthesis of the tungsten series XXIV - XXVI upon decarbonylation of $\mathrm{PnCO}^{-}$ $(\mathrm{Pn}=\mathrm{N}, \mathrm{P}, \mathrm{As})$ and synthesis of XXVIII upon addition of ClPA to XXVII. ${ }^{[64],[65]}$ 


\subsubsection{Transition Metal - Pnictogen Multiple Bonding}

The Grey-Ballhausen (GB) model rationalizes transition metal oxo bonding in the prevalent octahedral and square-pyramidal geometries. ${ }^{[77],[78]}$ These simple d orbital splitting considerations can be expanded to other two-faced, $\pi$-donating, six-electron donors, such as a terminal pnictides (Scheme 1.10, left). Only one d orbital remains $\sigma$ - and $\pi$-nonbonding in character. Therefore, electronic configurations beyond $\mathrm{d}^{2}$ are beyond the "oxo/nitrido wall" due to the destabilizing population of antibonding molecular orbitals. As a consequence, + VI is the dominating oxidation state for group 8 nitrido complexes. ${ }^{[22]}$ In turn, isolable low-valent group 8 nitrides and terminal nitride complexes beyond group 8 are rare and generally exhibit lower coordination numbers, such as square-planar geometry, to evade $\pi$-bonding conflicts (Scheme 1.10, right).[79] Stabilization of this exotic coordination environment can be achieved upon utilization of meridionally coordinating pincer ligands, e.g. PNP pincer and PDI ligands, and was shown for Ru, Os, Rh (transient) and Ir. ${ }^{[80]-[86]}$

Nitride ligands bound to early-transition metal ions are generally susceptible to electrophilic attack. This nucleophilicity is attributed to the polarization of $\mathrm{M}^{\delta^{+}} \mathrm{N}^{\delta^{-}} \pi$ bonding and is therefore attenuated along the transition series with dropping metal $\mathrm{d}$ orbital energies (Scheme 1.11). ${ }^{[54]}$
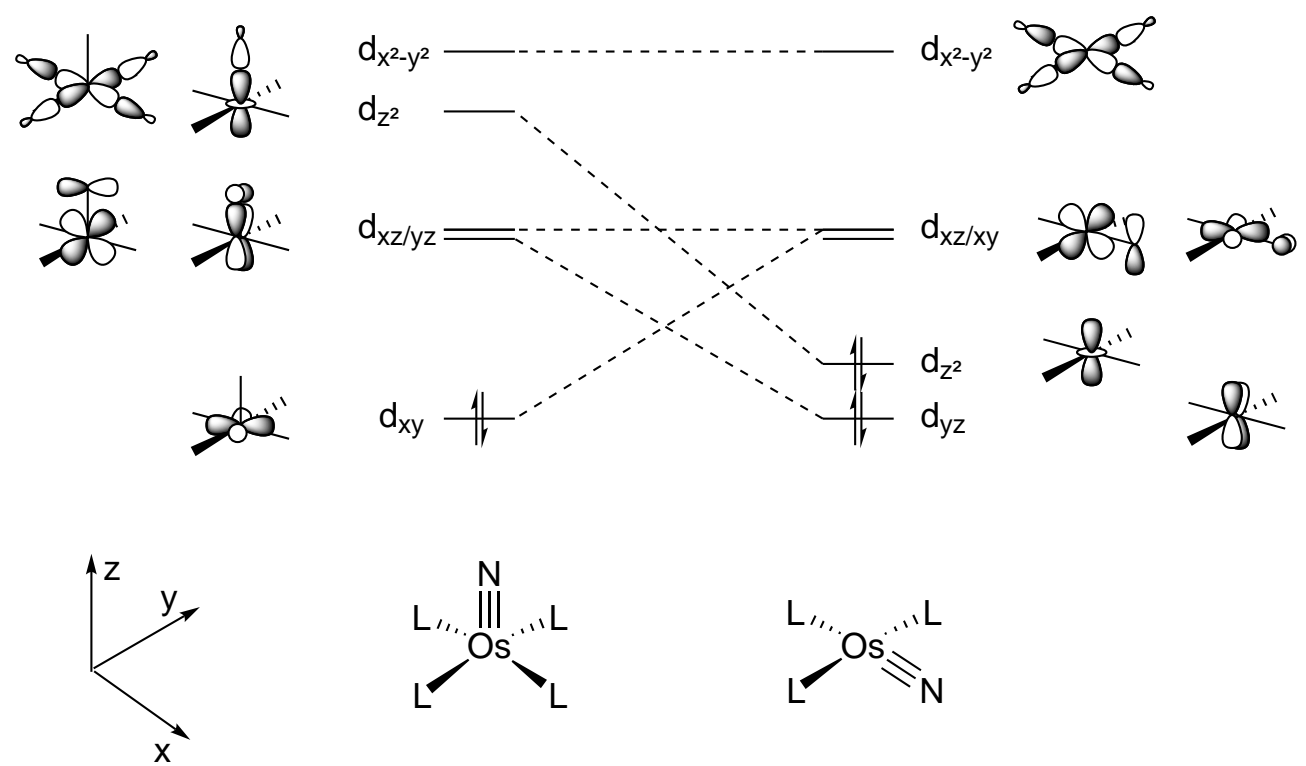

Scheme 1.10: Qualitative d orbital splitting for square-pyramidal Os ${ }^{\mathrm{VI}}$ and square-planar $\mathrm{Os}^{\mathrm{IV}}$ nitride complexes. ${ }^{[77],[78],[80]}$ 


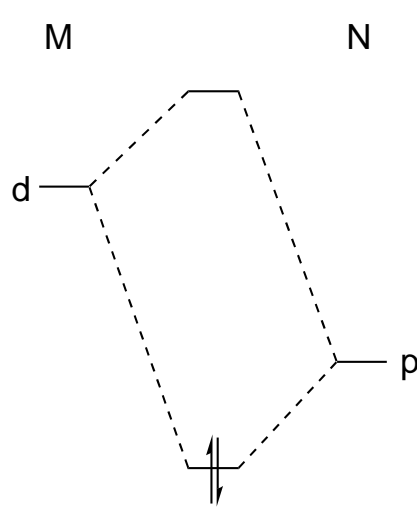

nucleophilic nitride
M

$\mathrm{N}$

early transition metals

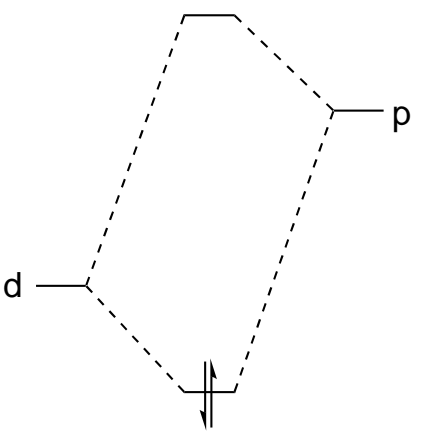

electrophilic nitride

late transition metals

Scheme 1.11: Qualitative orbital interaction of transition metal d orbitals with nitrogen $\mathrm{p}$ orbitals resulting in either nucleophilic or electrophilic nitride reactivity.

Since the electronegativity of elements drops drastically when moving from the $2^{\text {nd }}$ period to heavier elements, the transition metal pnictide triple bond is expected to become less polarized and should exhibit electrophilic reactivity. ${ }^{[87],[88]}$ In fact, computations of the terminal pnictide series $\left[\mathrm{WPn}\left(\mathrm{N}\left\{\mathrm{CH}_{2} \mathrm{CH}_{2} \mathrm{NSiMe}_{3}\right\}_{3}\right)\right](\mathbf{X X I X}: \mathrm{Pn}=\mathrm{P}, \mathbf{X X}$ : Pn = As, XXI: Pn $=$ Sb, XXX: Pn $=\mathrm{Bi}$ ) reveal only weak polarization towards the pnictide ligand in all cases. ${ }^{[66]}$ The $\sigma$-bond carries considerably s-character of the tungsten center which increases for the heavier pnictides while the group 15 elements construct the bond via p orbitals. The overall high covalency is reflected in the computed partial charges which render the pnictogen atoms almost neutral or slightly positive. NBO calculations of XXV and XXVI yield a similar s-character for the pnictide ligands while the s-character of the tungsten center is drastically reduced in a five-fold coordination environment. Frenking and co-workers perfomed EDA calculations on the series $\left[\mathrm{MPn}(\mathrm{OH})_{3}\right]$ $(\mathrm{M}=\mathrm{Mo}, \mathrm{W} ; \mathrm{Pn}=\mathrm{N}, \mathrm{P}, \mathrm{As}, \mathrm{Sb}, \mathrm{Bi}) .{ }^{[88]} \mathrm{NBO}$ analysis reveals that the $\sigma$-bonds are always polarized towards the pnictogen atoms as also described above. The $\pi$-bonds are slightly polarized towards the metal center for the heavier analogues of nitrides. While these bond are calculated to be highly covalent, the electrostatic contributions to the triple bond become dominant for the heavier pnictogens accompanied by severe weakening of the bond dissociaton energies. The tungsten species are predicted to be more stable than the molybdenum analogues. 
Table 1.2: Natural bond orbital analysis of the pnictide series XLV, XXV-Ph, XX, XXVI$\mathrm{Ph}, \mathbf{X X I}, \mathbf{X X X}, \mathrm{NBO}$ partial charges, NBO occupancies (occ) \% W/Pn: bond polarizations, $\%$ s: contribution of the s orbital, WBI: Wiberg bond index, $D_{e}$ : Bond dissociation energies in $\mathrm{kJ} \mathrm{mol}^{-1} \cdot[65],[66]$

\begin{tabular}{|c|c|c|c|c|c|c|c|c|c|}
\hline Compound & Partial charge & & occ & $\% \mathrm{~W}$ & $\% \mathrm{~s}$ & $\% \mathrm{Pn}$ & $\% \mathrm{~s}$ & WBI & $D_{e}$ \\
\hline XLV & $\begin{array}{l}\mathrm{W}: 0.84 \\
\mathrm{P}:-0.03\end{array}$ & $\begin{array}{l}\sigma: \\
\pi: \\
\pi:\end{array}$ & $\begin{array}{l}1.917 \\
1.652 \\
1.664\end{array}$ & $\begin{array}{l}42.2 \\
46.9 \\
47.3\end{array}$ & 43.5 & $\begin{array}{l}57.8 \\
53.1 \\
52.7\end{array}$ & 23.2 & 2.38 & 481.9 \\
\hline & & & & & 16.8 & & 17.3 & & \\
\hline XX & $\begin{array}{l}\text { W: } 0.81 \\
\text { As: } 0.01\end{array}$ & $\begin{array}{l}\sigma: \\
\pi: \\
\pi:\end{array}$ & $\begin{array}{l}1.913 \\
1.636 \\
1.636\end{array}$ & $\begin{array}{l}42.8 \\
47.3 \\
48.2\end{array}$ & 44.6 & $\begin{array}{l}57.2 \\
52.7 \\
51.8\end{array}$ & 20.1 & 2.35 & 429.0 \\
\hline $\begin{array}{l}\text { Pho } \\
\text { XXVI }\end{array}$ & & & & & 18.6 & & 14.3 & & \\
\hline $\mathrm{X}$ & $\begin{array}{l}\text { W: } 0.73 \\
\text { Sb: } 0.11\end{array}$ & $\begin{array}{l}\sigma: \\
\pi: \\
\pi:\end{array}$ & $\begin{array}{l}1.890 \\
1.591 \\
1.643\end{array}$ & $\begin{array}{l}45.8 \\
47.3 \\
48.2\end{array}$ & 47.6 & $\begin{array}{l}54.2 \\
52.7 \\
50.6\end{array}$ & 17.4 & 2.28 & 333.4 \\
\hline XXX & $\begin{array}{l}\text { W: } 0.71 \\
\text { Bi: } 0.15\end{array}$ & $\begin{array}{l}\sigma: \\
\pi: \\
\pi:\end{array}$ & $\begin{array}{l}1.887 \\
1.664 \\
1.555\end{array}$ & $\begin{array}{l}46.0 \\
51.4 \\
47.2\end{array}$ & 47.8 & $\begin{array}{l}54.0 \\
48.6 \\
52.8\end{array}$ & 14.2 & 2.27 & 293.3 \\
\hline
\end{tabular}




\subsubsection{Reactivity of Transition Metal Pnictide Complexes}

The reactivity of transition metal nitride complexes and their electronic structure has been extensively studied due to their relevance for important transformations, such as nitrogen fixation or nitrogen group transfer. Besides the above mentioned direct generation from gaseous dinitrogen, the synthesis via salt metathesis of halide ligands for azides or oxidative deprotonation of coordinated ammonia are typical synthetic approaches. ${ }^{[22],[89]}$ As described above, the reactivity (nucleophilic/electrophilic) of the $\mathrm{M} \equiv \mathrm{N}$ triple bond is dependent on the metal. In the case of group 9, electrophilic reacitivty was observed. ${ }^{[80],[84],[85],[90],[91]}$ Group 8 nitrides apparently define a transition. Several high-valent $\mathrm{Ru}^{\mathrm{IV}}$, $\mathrm{Os}^{\mathrm{VI}}$ and $\mathrm{Fe}^{\mathrm{IV}}$ nitride complexes exhibit reactivity that is attributed to nucleophilic attack at the nitride ligand. ${ }^{[92]-[96]}$ Mayer and co-workers demonstrated that the philicity of $\mathrm{Os}^{\mathrm{VI}}$ nitrides can be tuned by subtle changes of the coordination environment, enabling ambiphilic nitride reactivity. The Os ${ }^{\mathrm{VI}}$ nitrides XXXI and XXXII show different reactivity when reacted with the Lewis-acidic borane $\mathrm{B}\left(\mathrm{C}_{6} \mathrm{~F}_{5}\right)_{3}$. While XXXII forms the adduct XXXIII, substitution of the phenyl groups for chloride ligands allows for splitting of the boron carbon bond upon addition of $\mathrm{B}\left(\mathrm{C}_{6} \mathrm{~F}_{5}\right)_{3}$ to XXXI yielding the amide complex XXXIV (Scheme 1.12). [92], [93] This change in reactivity was rationalized by the different donor-properties of the ancillary ligands affecting the LUMO energy. This multifaceted reactivity renders group 8 nitrides particular attractive for nitride funtionalization.
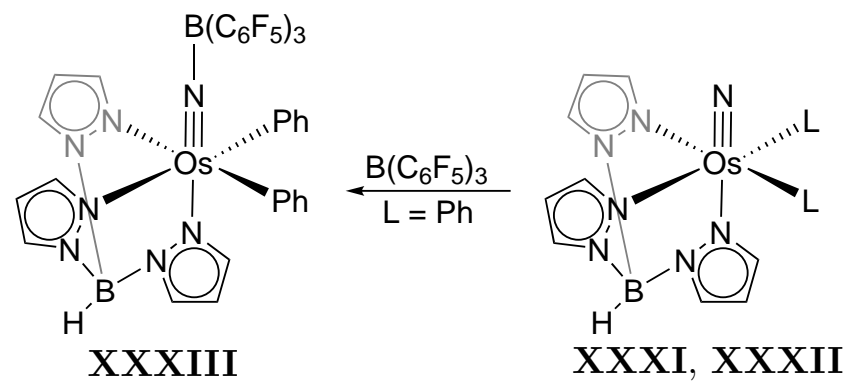

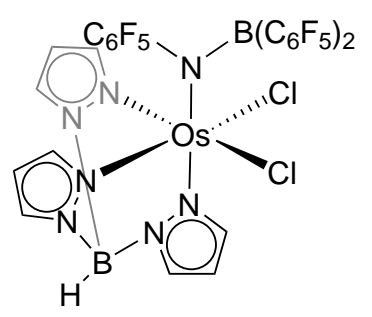

XXXIV

Scheme 1.12: Tuning of the reactivity of Os nitride complexes by variation of the ancillary ligands. ${ }^{[92],[93]}$

In the case of $\mathrm{Os}^{\mathrm{IV}}$ nitrido complexes, only two examples have been reported so far. While $\left[\mathrm{OsN}\left(\mathrm{L}^{1}\right)\right]\left(\mathbf{X X X V}, \mathrm{L}^{1}=\mathrm{N}\left(\mathrm{CH}_{2} \mathrm{CH}_{2} \mathrm{P}^{t} \mathrm{Bu}_{2}\right)_{2}\right)$ exhibits square-planar geometry, the reaction of the $\mathrm{Os}^{\mathrm{II}}$ iodide complex $\left[\mathrm{OsI}\left(\mathrm{L}^{\mathrm{Si}}\right)\right](\mathbf{X X X V I})$ with $\mathrm{Me}_{3} \mathrm{NO}$ or pyridine$\mathrm{N}$-oxide affords the tetrahedral nitride complex $\left[\mathrm{OsNI}\left(\mathrm{L}^{\mathrm{SiO}}\right)\right]$ (XXXVII, Scheme 1.13, $\left.\mathrm{L}^{\mathrm{SiO}}: \mathrm{O}\left(\mathrm{SiMe}_{2} \mathrm{CH}_{2} \mathrm{P}^{t} \mathrm{Bu}_{2}\right)_{2}\right) \cdot{ }^{[97]}$ The nitride complex $\mathbf{X X X V}$ exhibits ambiphilic reactivity, as demonstrated by $\mathrm{TMS}^{+}$and $\mathrm{PMe}_{3}$ addition to the nitride complex. However, the low coordination number gives rise to additional reaction patterns in comparison with 


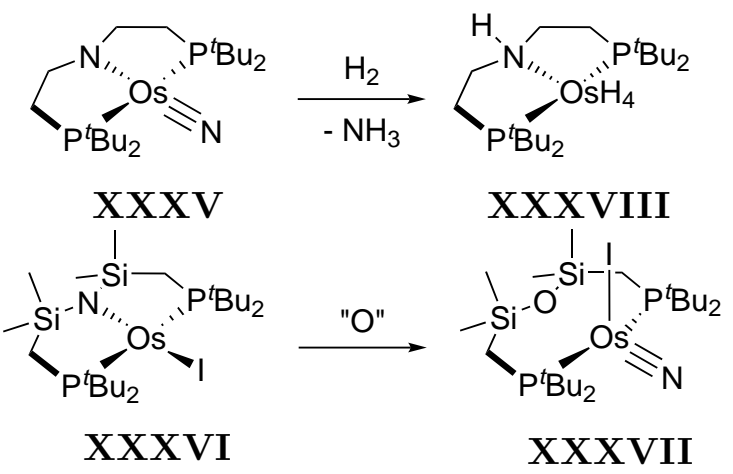

Scheme 1.13: Top: Hydrogenolysis of XXXV towards XXXVIII; Bottom: Synthesis of XXXVII from XXXVI. ${ }^{[83],[97]}$

"traditional" nitride chemistry. For example, the reaction with a Brønsted acid leads to reversible metal protonation and nitride hydrogenolysis affords the tetrahydride complex $\left[\mathrm{OsH}_{4}\left(\mathrm{~L}^{1 \mathrm{H}}\right)\right]$ (XXXVIII, Scheme 1.13) and ammonia in almost quantitative yield. ${ }^{\left[{ }^{[3]} \mathrm{In}\right.}$ analogy to the respective $\mathrm{Ru}^{\mathrm{IV}}$ nitride, this reaction presumably proceeds via initial $\mathrm{H}_{2}$ heterolysis facilitated by pincer ligand cooperativity. ${ }^{[81]}$ These observations emphasize

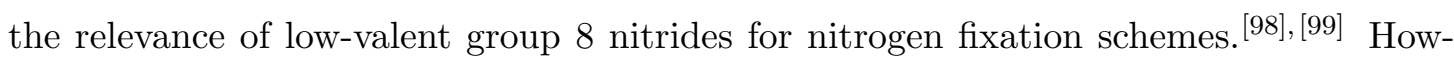
ever, the reactivity of $\mathrm{Os}^{\mathrm{IV}}$ nitrides in general remains scarcely examined.

The investigation of the reactivity of heavy terminal pnictide complexes is still at a nascent stage due to the limited number of isolated examples. XIX was reacted with Mes*NPCl to give $\left[\mathrm{Nb}(\mathrm{NRAr})_{3}\left(\mathrm{P}_{2} \mathrm{NMes}^{*}\right)\right]$ (XXXIX, $\mathrm{Ar}=3,5-\mathrm{Me}_{2} \mathrm{C}_{6} \mathrm{H}_{3}, \mathrm{R}=$ neopentyl) which releases diphosphorus at elevated temperatures (Scheme 1.14). Trapping experiments proved successful $\mathrm{P}_{2}$ generation upon addition of 1,3-cyclohexadiene. ${ }^{[100]}$

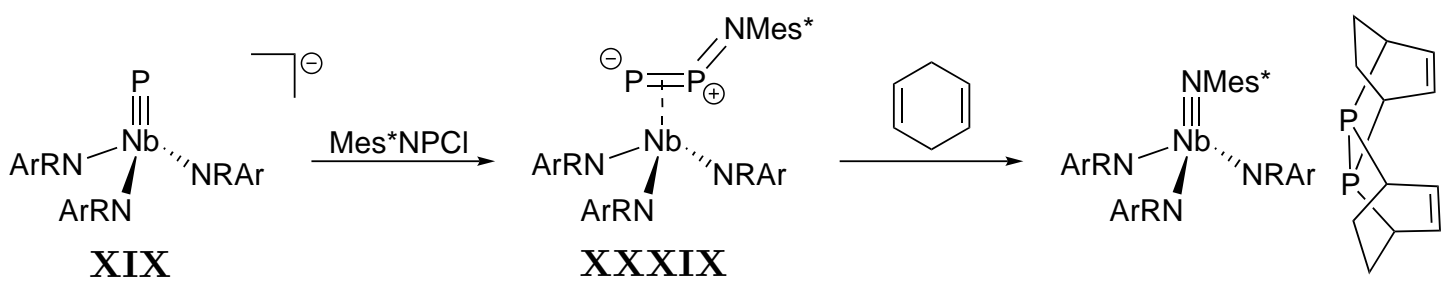

Scheme 1.14: $\mathrm{P}_{2}$ generation by reaction of XIX and $\mathrm{Mes} * \mathrm{NPCl}, \mathrm{Ar}=3,5-\mathrm{Me}_{2} \mathrm{C}_{6} \mathrm{H}_{3}, \mathrm{R}=$ neo-pentyl ${ }^{[100]}$

Due to the strong pnictogen-chalcogen bonds and the electrophilicity of heavy pnictide ligands, several reports feature the synthesis of heterocumulenes from heavy terminal transition metal pnictide complexes. The oxygenation of the terminal molybdenum phosphide $\mathbf{X V I}$ yielded the unprecedented terminal phosphorus monoxide complex $\left[\mathrm{Mo}(\mathrm{PO})(\mathrm{NArR})_{3}\right]\left(\mathbf{X L}\right.$, Scheme 1.15, $\mathrm{Ar}=3,5-\mathrm{Me}_{2} \mathrm{C}_{6} \mathrm{H}_{3}, \mathrm{R}=$ tert-butyl) reminiscent 
of known transition metal nitride reactivity. ${ }^{[22],[90],[101]} \mathbf{X L}$ could be further converted to the diphosphenido complex $\left[\mathrm{Mo}\left(\mathrm{P}_{2} \mathrm{SR}_{3}\right.\right.$ ") $\left.(\mathrm{NArR})_{3}\right](\mathbf{X L I}, \mathrm{R} "=$ iso-propyl) upon reaction with $\left[\mathrm{Nb}\left(\mathrm{PSiR}_{3}{ }^{\prime}\right)\left(\mathrm{NArR}_{3}{ }^{\prime}\right)\right]$ (XLII, Scheme 1.15, R' = neo-pentyl). ${ }^{[102]}$ The $\mathrm{Mo} \equiv \mathrm{P}$ triple bond was also susceptible towards functionalization with elemental sulfur and $\mathrm{N}_{3}$ Mes* which yielded the phosphorous monosulfide complex $\left[\mathrm{Mo}(\mathrm{PS})(\mathrm{NArR})_{3}\right]$ $(\mathbf{X L I I I})$ and $\left[\mathrm{Mo}\left(\mathrm{PNMes}^{*}\right)(\mathrm{NArR})_{3}\right]$ (XLIV), respectively (Scheme 1.15). ${ }^{[23]}$

Similar reactivity was reported for the terminal phosphide and arsenide complexes $\left[\mathrm{WPn}\left(\mathrm{N}\left\{\mathrm{CH}_{2} \mathrm{CH}_{2} \mathrm{NSiMe}_{3}\right\}_{3}\right)\right](\mathrm{Pn}=\mathrm{P}(\mathbf{X L V}), \mathrm{Pn}=\mathrm{As}(\mathbf{X X}))$. The PS and AsS ligated complexes $\left[\mathrm{W}(\mathrm{PnS})\left(\mathrm{N}\left\{\mathrm{CH}_{2} \mathrm{CH}_{2} \mathrm{NSiMe}_{3}\right\}_{3}\right)\right](\mathrm{Pn}=\mathrm{P},(\mathbf{X L V I}), \mathrm{Pn}=\mathrm{As}(\mathbf{X L V I I})$ were obtained via reaction with cyclohexane sulfide (Scheme 1.15). A theoretical investigation of the bonding patterns indicate a suitable description as two 3c4e bonds.

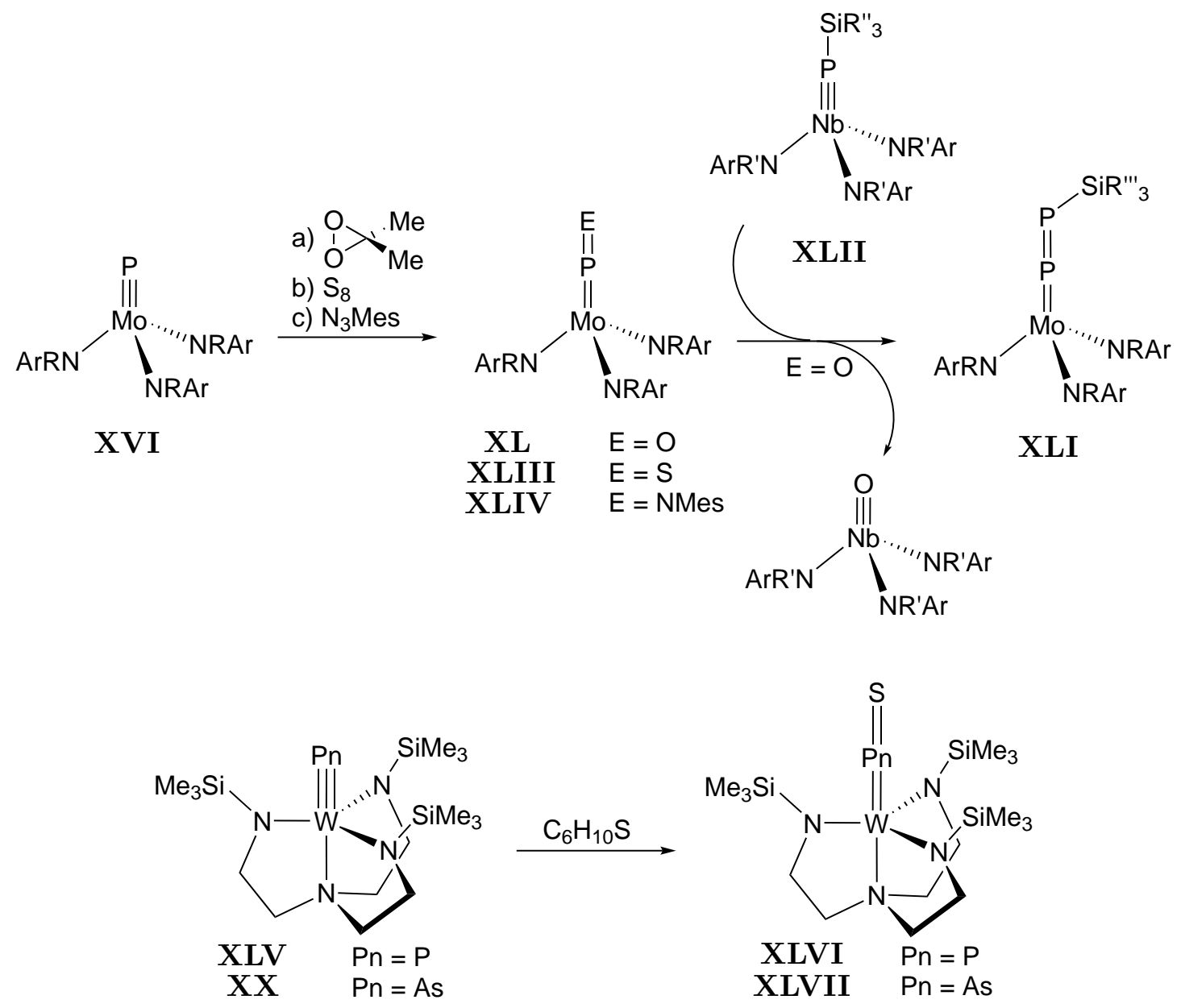

Scheme 1.15: Selected functionalization reaction of heavy terminal transition metal pnictide complexes, $\mathrm{Ar}=3,5-\mathrm{Me}_{2} \mathrm{C}_{6} \mathrm{H}_{3}, \mathrm{R}=$ tert-butyl, $\mathrm{R}^{\prime}=$ neo-pentyl, $\mathrm{R} "=$ iso-propyl. ${ }^{[23],[101]-[103]}$ 


\subsection{Generation of Dipnictogens in the Condensed Phase}

The reverse reaction of dinitrogen splitting, the coupling of terminal transition nitrides towards dinitrogen complexes, is commonly observed for octahedral $\mathrm{M}^{\mathrm{V}}$ nitrides $(\mathrm{M}=$ $\mathrm{Fe}, \mathrm{Ru}, \mathrm{Os}$ ) which are accessible by photolysis of azides, reduction of stable $\mathrm{M}^{\mathrm{VI}}$ nitrides or oxidation of ammine complexes. ${ }^{[104]-[108]}$ This is easily understood by considering the amount of $\pi$-electrons within the $\{\mathrm{MNNM}\}$ unit as described above. However, detailed studies on the electronic structure and the associated coupling kinetics of these systems remain scarce and closed-shell nitrides are also known to perform coupling reactions indicating that the electronic configuration of such systems is not an axiomatic predictor. ${ }^{[94],[109]-[112]}$

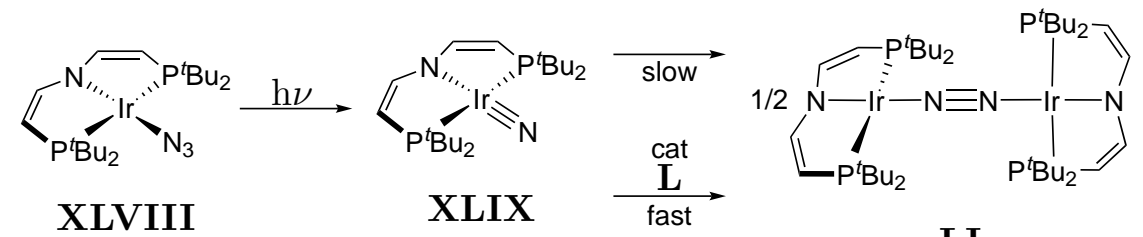

LI

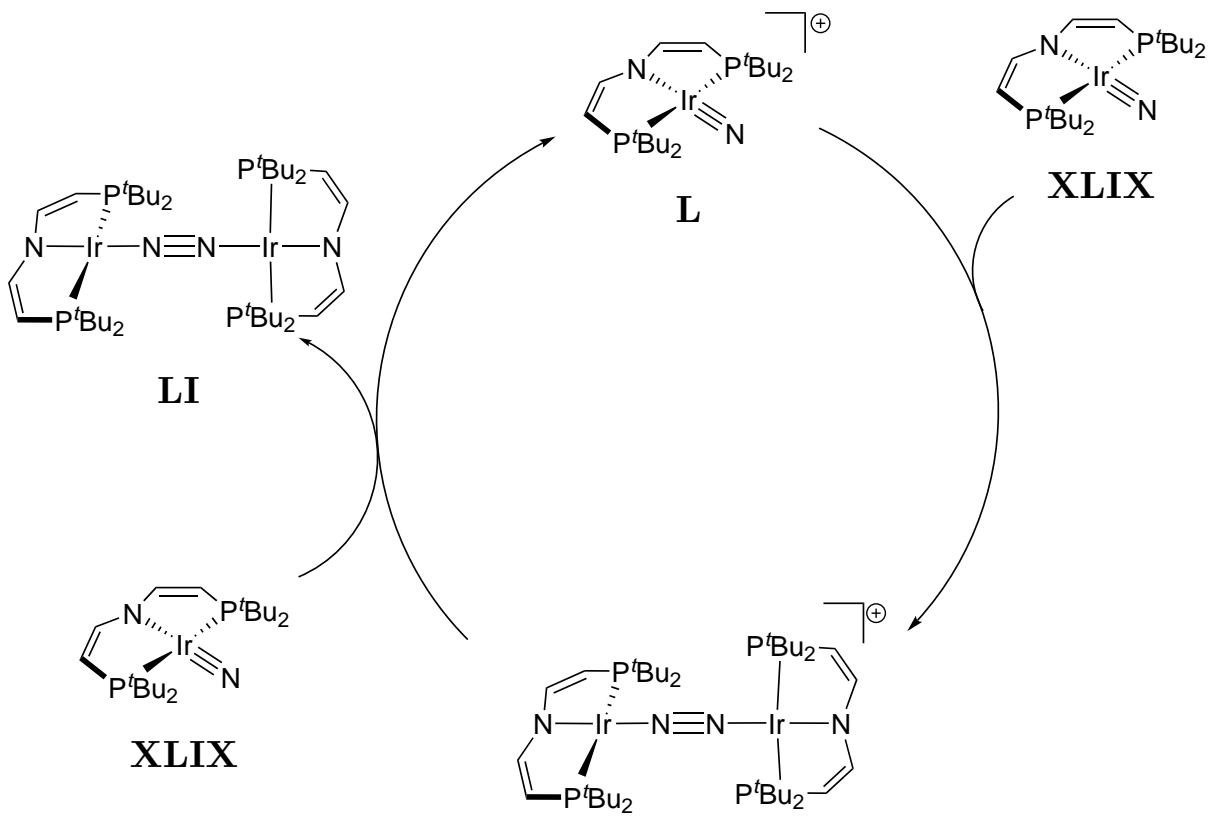

LII

Scheme 1.16: Nitride-Coupling reactions of the $\left\{\operatorname{Ir}\left(\mathrm{L}^{3}\right)\right\}$ pincer platform. ${ }^{[80],[113]}$

Studying the reverse reaction of dinitrogen splitting can give insights into the associated thermodynamic and kinetic parameters of this important reaction. The ligand platform $\mathrm{L}^{3}\left(\mathrm{~L}^{3}=\mathrm{N}\left(\mathrm{CHCHP}^{t} \mathrm{Bu}_{2}\right)_{2}\right)$ has proven to be a suitable platform for the isolation of unusual coordination numbers and exotic bonding motifs. In the case of Ir, the square- 
planar azide complex $\left[\operatorname{Ir}\left(\mathrm{L}^{3}\right)\left(\mathrm{N}_{3}\right)\right]$ (XLVIII) was isolated which could be photolyzed or oxidized yielding open shell $\operatorname{Ir}^{\mathrm{IV}}$ (XLIX) and closed shell $\operatorname{Ir}^{\mathrm{V}}(\mathbf{L})$ species, respectively (Scheme 1.16). ${ }^{[80]}$ While $\mathbf{L}$ is an isolable species, radical homo-coupling of XLIX yields the dimeric dinitrogen complex $\left[\left\{\operatorname{Ir}\left(\mathrm{L}^{3}\right)\right\}_{2}\left(\mu-\mathrm{N}_{2}\right)\right](\mathbf{L I})$. In the case of Rh, similar reactivity was observed but no isolable nitride could be obtained. ${ }^{[82]}$ A mechanistic study revealed that hetero-coupling of XLIX and $\mathbf{L}$ towards $\left[\left\{\operatorname{Ir}\left(\mathrm{L}^{3}\right)\right\}_{2}\left(\mu-\mathrm{N}_{2}\right)\right]^{+}(\mathbf{L I I})$ is basically barrierless, allowing for redox catalytic nitride coupling (Scheme 1.16). ${ }^{[113]}$ Additionally, the dicationic dimeric $\mathrm{N}_{2}$ complex $\left[\left\{\operatorname{Ir}\left(\mathrm{L}^{3}\right)\right\}_{2}\left(\mu-\mathrm{N}_{2}\right)\right]^{2+}(\mathbf{L I I I})$ could be isolated which features a $\pi^{10}\left(\delta^{4}\right)$ configuration. However, no splitting of dinitrogen was observed since the overall thermochemistry does not allow for nitride formation, as shown by computational analysis. ${ }^{[113]}$

As outlined in Chapter 1.1, the heavier homologues of dinitrogen exhibit significantly reduced bond dissociation energies which render them highly reactive. The homocoupling of an isolable terminal heavy transition metal pnictide towards a well-defined $\mathrm{Pn}_{2}$ complex is unknown. The generation of diphosphorus and diarsenic in the condensed phase has been shown by extrusion from the above described diphosphaazide complex XXXIX, white phosphorus, the $\mathrm{P}_{2}$-bisanthracene adduct LIV and the Ni dipnictide complexes $\left[\{\mathrm{NiL}(\mathrm{CO})\}_{2}\left(\mu-\mathrm{Pn}_{2}\right)\right](\mathbf{L V}, \mathbf{L V I}, \mathrm{L}=1,3$-bis $(2,4,6$-trimethylphenyl)imidazol-2-ylidene, Pn $=$ P, As, Figure 1.6). ${ }^{[67],[73],[100],[114]}$ Recent reports showcase different strategies to stabilize $\mathrm{Pn}_{2}$ fragments, e.g. with N-heterocyclic carbene ligands upon reductive coupling of $\mathrm{PCl}_{3}$ (LVII, Figure 1.7, left) and $\mathrm{AsCl}_{3}$ or via extrusion from white phosphorus. ${ }^{[15]-[117]}$ This area of research strongly benefited from the introduction of the previously mentioned pnictogen transfer reagents $\mathrm{PCO}^{-}, \mathrm{AsCO}^{-}$and $\mathrm{ClPA}$. The (photo)decarbonylative coupling of the heavier isocyanate analogues led to a variety of phosphanyl, germanyl, cyclopropenyl and stannyl stabilized diphosphene/diarsene $(\mathrm{RPn}=\mathrm{PnR})$ products. ${ }^{[118]-[122]}$

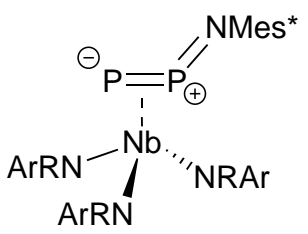

XXXIX

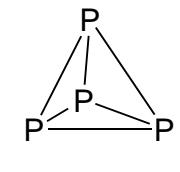

LIV

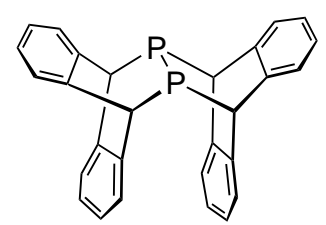

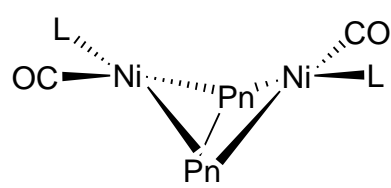

LV/LVI

Figure 1.6: Isolable sources of $\mathrm{Pn}_{2}$ in the condensed phase, $\mathrm{Ar}=3,5-\mathrm{Me}_{2} \mathrm{C}_{6} \mathrm{H}_{3}, \mathrm{~L}=1,3$ -

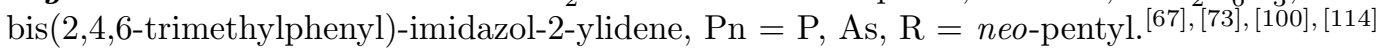

Transition metal coordination provides an alternative strategy to stablize dipnictide fragments. $\mathrm{P}_{4}$ activation has led to several side-on bridged complexes with $\mathrm{M}_{2} \mathrm{P}_{2}$ butterflycore (XVIII, Figure 1.7, middle) and in rare cases end-on bridging diphosphinidene 
species $\mathrm{M}=\mathrm{P}-\mathrm{P}=\mathrm{M}$, contrasting the rich chemistry of end-on dinitrogen bridged coordination compounds. ${ }^{[31],[60],[123],[124]}$ The decarbonylative coupling of $\mathrm{PCO}^{-}$gave bridging $\mathrm{P}_{2}^{4-}$ and $\mathrm{P}_{2}^{2-}$ ligands, e.g. $\left[\{\operatorname{Ir}(\mathrm{PDI})(\mathrm{CO})\}_{2}\left(\mu-\mathrm{P}_{2}\right)\right]$ (LVIII, Figure 1.7, right). ${ }^{[70], ~[73], ~[74]}$ Terminal phosphide complexes can be assumed as key intermediates for these P-P coupling reactions but direct observation of oxidative $\mathrm{M} \equiv \mathrm{P}$ coupling or the reverse reduction remains scarcely examined.

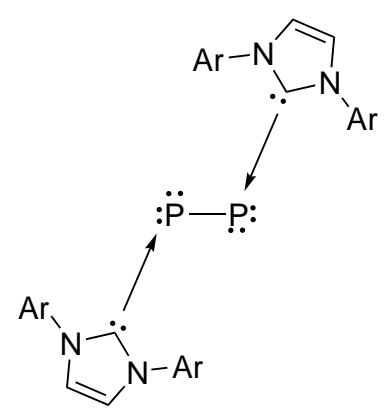

XCV

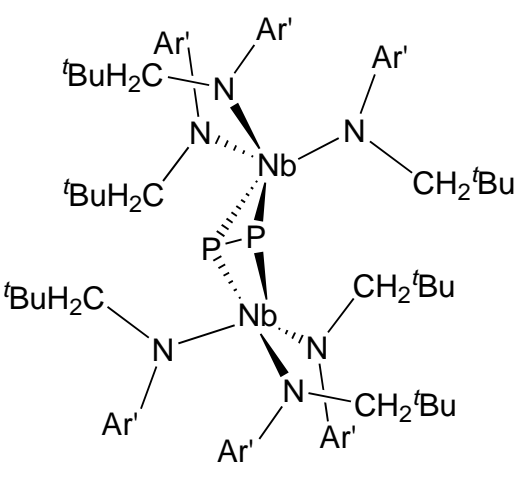

XXXIX

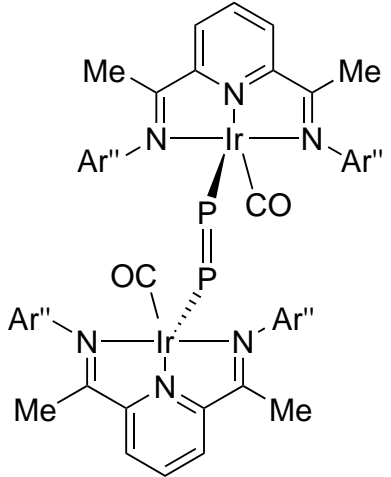

LVIII

Figure 1.7: Examples for isolable diphosphene compounds generated by $\mathrm{PCl}_{3}$ reduction $(\mathbf{X C V}$, left), $\mathrm{P}_{4}$ activation (XXXIX, middle) and PCO decarbonylation (LVIII, right), $\mathrm{Ar}=2,6$ ${ }^{i} \mathrm{Pr}_{2} \mathrm{C}_{6} \mathrm{H}_{3}, \mathrm{Ar} '=3,5-\mathrm{Me}_{2} \mathrm{C}_{6} \mathrm{H}_{3}, \mathrm{Ar} "=2,6-\mathrm{Me}_{2} \mathrm{C}_{6} \mathrm{H}_{3} \cdot{ }^{[58],[74],[115]}$

As mentionened above, heavy transition metal terminal pnictide complexes are restricted to group 5 and 6 and mostly feature a $\mathrm{d}^{0}$ electron configuration, preventing oxidative functionalization. One-electron oxidation of XXVIII ( $\mathrm{d}^{2}$ configuration) results in the formation of the isolable open-shell complex $[\mathrm{MoPCl}(\mathrm{PCP})]^{+}(\mathbf{L I X})$ which undergoes unprecedented oxidative phosphide coupling towards dimeric $\left[\{\mathrm{MoCl}(\mathrm{PCP})\}_{2}\left(\mu-\mathrm{P}_{2}\right)\right]$ (LX) at temperatures above $-10^{\circ} \mathrm{C}$ (Scheme 1.17). Although LX could not be isolated, the generated $\mathrm{P}_{2}^{4-}$ fragment was trapped chemically. ${ }^{[64]}$ The coupling reactivity of terminal arsenide complexes has not been investigated so far.

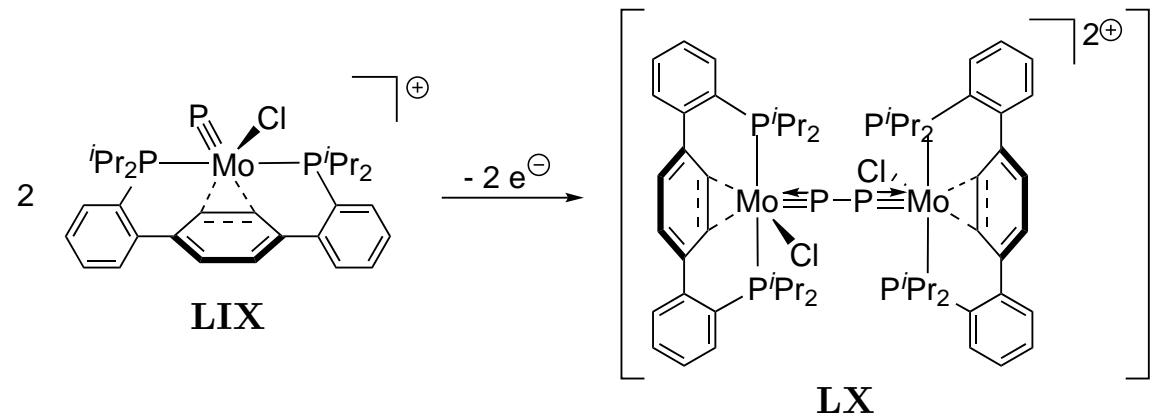

Scheme 1.17: Proposed coupling reactivity of LIX towards LX. ${ }^{[64]}$ 


\subsection{Phosphinyl Radicals and Phosphinidenes}

Parts of this chapter were adapted from: J. Abbenseth, D. Delony, M. C. Neben, C. Würtele, B. de Bruin, S. Schneider, Angew. Chem. Int. Ed. 2019, 58, 6338-6341, with permission from WILEY-VCH Verlag GmbH \& Co. KGaA, Weinheim.

Phosphinyl radicals possess seven valence electrons and carry a lone-pair and an unparied electron at the phosphorus center. Over the last 50 years phosphinyl radicals have been proposed to play a key role in $\mathrm{P}-\mathrm{C}$ bond formation reactions, like olefin phosphination. ${ }^{[125]-[129]}$ Nevertheless, stabilization by metal coordination has only been scarcely examined and, so far, did not lead to isolable compounds. The $\mathrm{PPh}_{2}$ radical was first observed in 1963 after photolysis of $\mathrm{HPPh}_{2}$ or $\left(\mathrm{Ph}_{2} \mathrm{P}\right)_{2} \cdot{ }^{[130]}$ Further EPR studies afforded a full characterization of this radical. ${ }^{[131]-[136]}$ Due to the forcing irradiation conditions, complex product mixture were generated. This was partly overcome by the utilization of spin traps. ${ }^{[137]-[140]}$ Irradiation of metal carbonyl phosphine complex single-crystals to give phosphinyl radicals has also been reported. In all studied cases, the unpaired electron mainly resides at the phosphorus center with minor delocalization towards the metal center. ${ }^{141]}$ More recently, the $\mathrm{PPh}_{2}$ radical was generated from $\mathrm{HPPh}_{2}$ by proton coupled electron transfer (PCET) mediated by 2,2-diphenyl-1-picrylhydrazyl which represents the first non-irradiative method for the production of this species (Scheme 1.18).[142], [143]

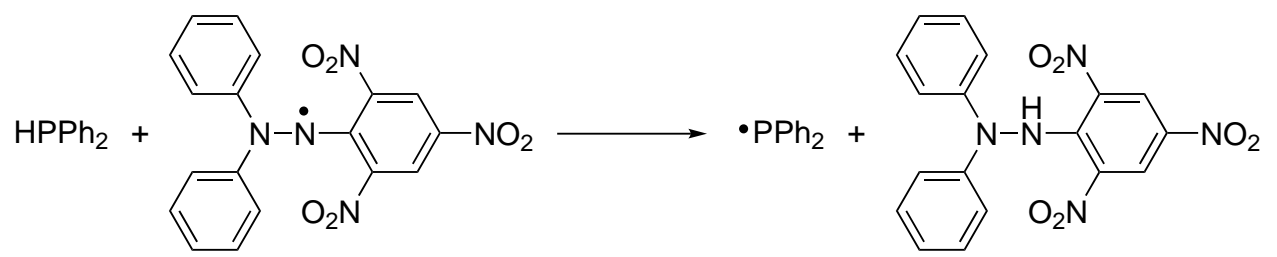

Scheme 1.18: Generation of the $\mathrm{PPh}_{2}$ phosphinyl radical by PCET. ${ }^{[143]}$

Over the last years, several isolable phosphinyl radicals have been reported. Structural and spectroscopic characterization of free and coordinated phosphinyl radicals facilitated the examination of (electronic) structure/reactivity relationships. ${ }^{[141],[144]-[158]}$ Isolable free phosphinyl radicals however require stabilization by bulky ligands and do not feature $\mathrm{P}-\mathrm{H}$ bonds. Stabilization of such highly reactive species by transition metal coordination offers an attractive pathway to investigate this class of compounds. A possible access consists of the generation of open shell species by redox-chemical conversion of terminal phosphanide complexes. Terminal phosphanide complexes are still scarce due to their high reactivity owing to their available lone pair and radicals generated this way undergo rapid follow-up reactivity. ${ }^{[157]}$ In comparison, free phosphinidenes (PR) 
could only very recently be sufficiently stabilized.[118], [159] A variety of transition metals form isolable phosphinidene complexes $(\mathrm{M}=\mathrm{PR})$ with promising stoichiometric reactivity, such as $\mathrm{P}-\mathrm{C}$ bond formation. ${ }^{[160]-[163]}$ However, catalytic phosphinidene transfer protocols remain exceedingly rare, compared to, e.g. nitrene transfer which has emerged as a powerful method for $\mathrm{C}-\mathrm{N}$ bond formation. ${ }^{[164]-[168]}$

The scarcity of catalytic PR-transfer might in part be attributed to a lack of suitable oxidizing PR-transfer reagents, such as analogs of iminoiodane $(\mathrm{ArI}=\mathrm{NR})$ or azide $\left(\mathrm{RN}_{3}\right)$ nitrene sources. Primary phosphines are attractive precursors to phosphinyl radicals as well as phosphinidenes from a thermochemical point of view due to lower intrinsic $\mathrm{P}-\mathrm{H}$ vs. N-H bond dissociation (free) energies (BD(F)Es, Scheme 1.19). ${ }^{[16],[169]-[172]}$ Further chemical conversion represents an attractive strategy to utilize primary phosphines as $\mathrm{P}_{1}$-building blocks.

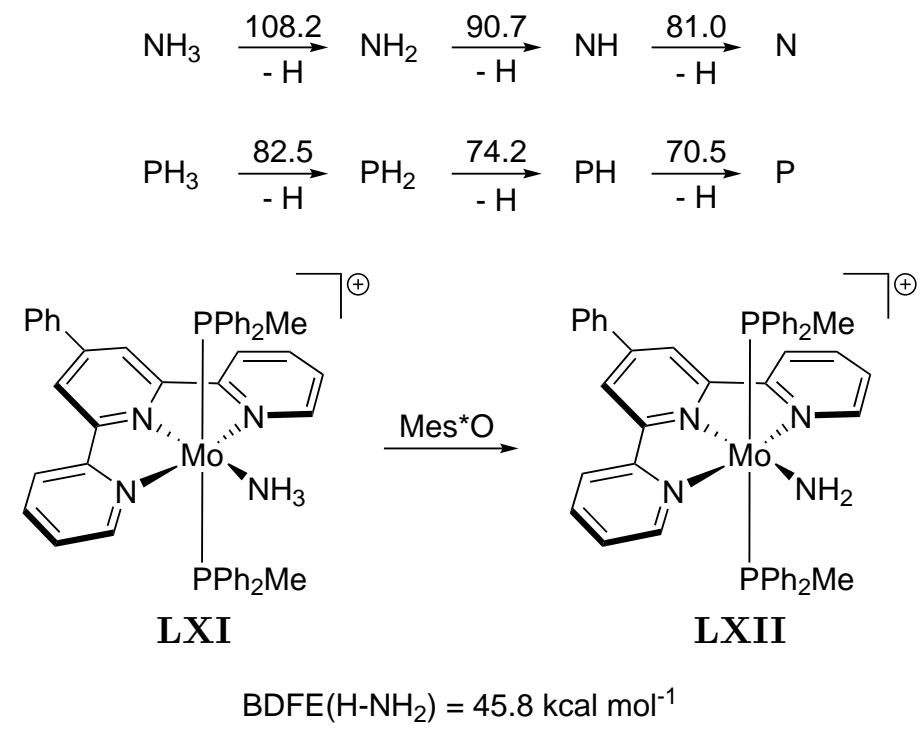

Scheme 1.19: Top: $\mathrm{N}-\mathrm{H}$ and $\mathrm{P}-\mathrm{H}$ bond dissociation energies (BDEs) in the gas phase (kcal . $\left.\mathrm{mol}^{-1}\right)$, The $\mathrm{BDE}\left(\mathrm{NH}_{2}\right)$ was calculated via a Hess cycle: $\mathrm{BDE}\left(\mathrm{NH}_{2}\right)=\Delta_{\mathrm{f}} \mathrm{H}_{\text {gas }}^{\circ}(\mathrm{N})+2 \Delta_{\mathrm{f}} \mathrm{H}_{\text {gas }}^{\circ}(\mathrm{H})-$ $\mathrm{BDE}(\mathrm{NH})-\Delta_{\mathrm{f}} \mathrm{H}_{\text {gas }}^{\circ}\left(\mathrm{NH}_{2}\right) ;{ }^{[16],[169]-[172]}$ Bottom: Severe weakening of the $\mathrm{N}-\mathrm{H}$ bond in ammonia by $\mathbf{L X I}$ and its PCET reactivity towards $\mathbf{L X I I}, \mathrm{Mes}^{*}=2,4,6-{ }^{t} \mathrm{Bu}_{3} \mathrm{C}_{6} \mathrm{H}_{2} \cdot{ }^{[173]}$

The $\mathrm{P}-\mathrm{H}$ bonds might be further weakened upon coordination to a metal complex as was demonstrated for coordinated $\mathrm{H}_{2} \mathrm{O}$ and $\mathrm{NH}_{3}$ ligands. ${ }^{[174]}$ The group of Chirik and co-workers showed that the bond dissociation free energy of ammonia is severely weakened when coordinated to the $\mathrm{Mo}^{\mathrm{I}}$ complex $\left[\mathrm{Mo}(\mathrm{NNN})\left(\mathrm{PPh}_{2} \mathrm{Me}\right)_{2}\left(\mathrm{NH}_{3}\right)\right]^{+}(\mathbf{L X I})$ allowing for synthesis of the amido complex $\left[\mathrm{Mo}\left(\mathrm{NH}_{2}\right)\left({ }^{\mathrm{Ph}_{\mathrm{Tpy}}}\right)\left(\mathrm{PPh}_{2} \mathrm{Me}\right)_{2}\right]^{+}(\mathbf{L X I I}$, Scheme 1.19) by PCET. ${ }^{[173]}$ Since the N-H BDFE of the coordinated ammonia molecule is smaller than the BDFE of dihydrogen, $\mathrm{H}_{2}$ formation was observed when heating LXI to $60^{\circ} \mathrm{C}$. However, while amide ligand $\mathrm{N}-\mathrm{H}$ bond activation by PCET is an active 
field of research, phosphide $\mathrm{P}-\mathrm{H}$ homolysis was only reported by Hillhouse and coworkers. ${ }^{[79],[175]-[185]}$ The open-shell phosphanide complex LXIII reacts with Mes*O to form the phosphinidene LXIV (Scheme 1.20).[186],[187] This result showcases the potential of transition metal complexes to stabilize open-shell phosphorus centers with decreased steric bulk, therefore allowing for generation of radicals possessing a P-H moiety that allows for interconversion of phosphinyl radicals and phosphinidene complexes via proton coupled electron transfer. Coordinatively unsaturated, electron-rich transition metal complexes, e.g. square-planar $\mathrm{Os}^{\mathrm{II}}$, represent privileged starting platforms to facilitate oxidative addition of phosphines to yield a phosphanide complex which might be further converted to the targeted species.

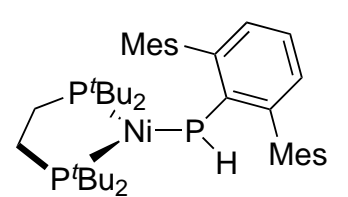

LXIII

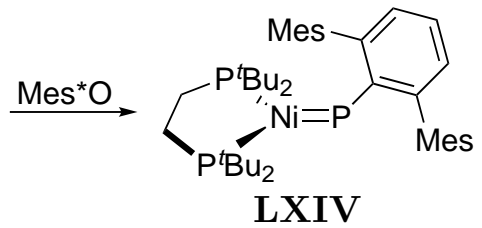

LXIV

Scheme 1.20: Generation of a nickel phosphinidene complex by PCET reported by Hillhouse and co-workers. ${ }^{[186],[187]}$ 


\subsection{Scope of this Work}

The aim of this thesis is to investigate and develop suitable platforms capable of forming unprecedented $\mathrm{M} \equiv \mathrm{Pn}$ species and to further elucidate the electronic and structural requirements to access these compounds.

- A low-valent, square-planar Os halide pincer complex with a $\mathrm{d}^{6}$ configuration is targeted. This complex should feature an interesting electronic structure due to its isolobal-relationship with octahedral $\mathrm{Re}^{\mathrm{III}}$ which is well-known to feature effects of temperature independent paramagnetism. The system will be further derivatized by chelating neutral and anionic ligands to tune the overall redox potentials. Additionally, prior reported $\mathbf{R} \mathbf{e}^{\mathbf{I I I}}$ pincer complexes will be characterized by magnetic measurements and the influence of the ancillary ligands on the magnetic behaviour will be evaluated by derivatization.

- Synthesis of square-planar Os nitride complexes will be targeted by reacting the prior obtained Os halide complex with azide salts. The reactivity will be examined upon addition of typical nucleophiles and electrophiles. Furthermore, possible redox-mediated dinitrogen coupling reactions will be evaluated.

- Transition metal complexes featuring multiple bonds with heavy pnictogens will be targeted by the following approaches:

- The phosphorus and arsenic atom transfer reagents $\mathrm{PnCO}^{-}(\mathrm{Pn}=\mathrm{P}, \mathrm{As})$ and ClPA will be reacted with the obtained Re PNP pincer complexes to access unprecedented terminal Re pnictide complexes and investigate their reactivity.

- The low-valent Os halide complex should allow for the activation of electron rich, primary phosphines to access terminal Os phosphanide complexes which represent an interesting starting point to investigate the effect of metalcoordination on the P-H bond strength. The interconversion of Os-PHR and Os-PR complexes by proton coupled electron transfer will be investigated.

- The possible formation of terminal Os phosphide/phosphinidene complexes by addition of $\mathrm{PCO}^{-}$and ClPA to the low-valent Os halide complex will be evaluated.

- Dimeric molybdenum dinitrogen complexes will be investigated by spectroscopic methods to gain additional insights into the electronic structure of this class of compounds and how the degree of dinitrogen activation changes upon oxidation of the metal center. 


\title{
Os(II/III) and Re(III) PNP Pincer Complexes
}

Parts of this chapter were adapted from:

J. Abbenseth, M. Diefenbach, S. C. Bete, C. Würtele, C. Volkmann, S. Demeshko, M. C. Holthausen*, S. Schneider*,

Chem. Commun. 2017, 53, 5511-5514.

\author{
Author contributions \\ J.A. Synthesis, spectroscopy, theoretical calculations (Chapter 2.4) and \\ crystallography $(\mathbf{6}, \mathbf{1 0})$ \\ M.D. Theoretical calculations (Chapter 2.2, supporting) \\ S.B. Synthesis and spectroscopy (Chapter 2.2, supporting) \\ C.W. Crystallography \\ C.V. Crystallography (Chapter 2.2, scsupporting) \\ S.D. SQUID \\ M.H. Theoretical calculations (Chapter 2.2)
}




\section{Os(II/III) and Re(III) PNP Pincer Complexes}

\subsection{Possible Platforms for the Stabilization of Low-Valent Os Complexes}

A simple access to low-valent, square-planar Os nitride complexes featuring a $\mathrm{d}^{4}$ electron configuration is a substitution reaction of a halide ligated $\mathrm{d}^{6}$ complex with an azide salt and subsequent dinitrogen extrusion to yield a nitride complex. Utilization of tridentate, meridionally coordinating pincer ligands allows for the possible synthesis of suitable halide precursors. The control of pincer complex reactivity is often achieved by varying the substituents in their electronic and steric properties at the terminal donor positions. However, typical bond activation reactions occur trans to the central pincer donor so variations of the donor properties of this moieties have a significant higher impact on reactivity. ${ }_{[}[188]-[190]$ Slight variation can not only alter the reactivity but also offer the possibility to change the multiplicity of the studied system.
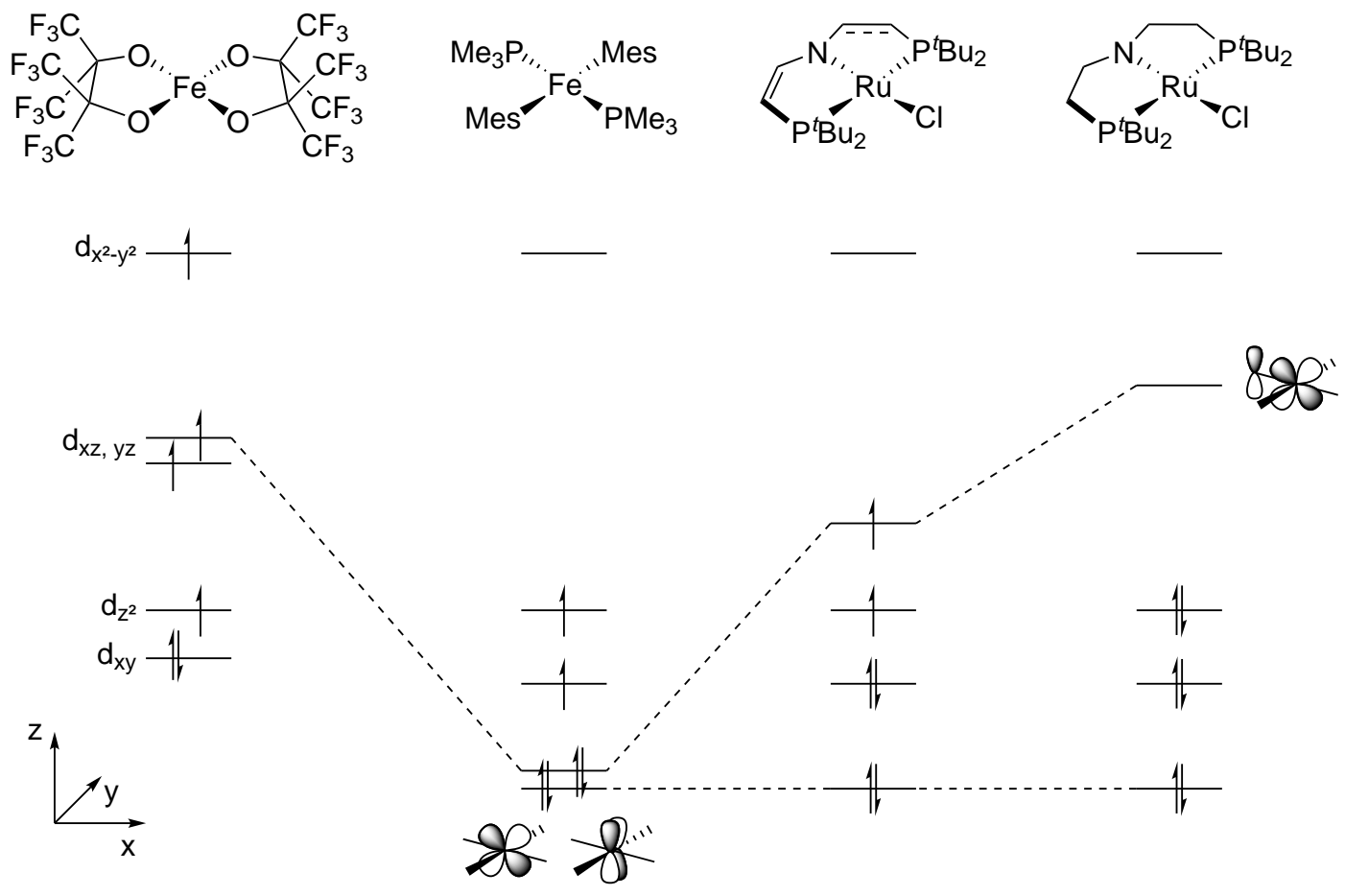

Figure 2.1: Qualitative d orbital splitting of square-planar $\mathrm{d}^{6}$ complexes with strongly $\pi$ donating (left), pure $\sigma$-donating ligands (middle left) and Ru-pincer systems LXV - LXVII (right). ${ }^{[191]}$

For square-planar group 8 complexes with a $\mathrm{d}^{6}$-configuration, three possible spin states have to be considered $(S=0,1,2)$. In ideal $D_{4 \mathrm{~h}}$-symmetry, the d orbital manifold is split into a set of three non-bonding $\left(\mathrm{d}_{\mathrm{xy}, \mathrm{xz}, \mathrm{yz}}, \mathrm{e}_{\mathrm{g}}\right.$ and $\left.\mathrm{b}_{2 \mathrm{~g}}\right)$, a weakly antibonding $\left(\mathrm{d}_{\mathrm{z}^{2}}, \mathrm{a}_{1 \mathrm{~g}}\right)$ 
and a strongly antibonding orbital $\left(\mathrm{d}_{\mathrm{x}^{2}-\mathrm{y}^{2}}, \mathrm{~b}_{1 \mathrm{~g}}\right)$. For $1^{\text {st }}$ row transition-metals usually the intermediate-spin configuration $\left(\mathrm{e}_{\mathrm{g}}\right)^{4}\left(\mathrm{~b}_{2 \mathrm{~g}}\right)^{1}\left(\mathrm{a}_{1 \mathrm{~g}}\right)^{1}\left(\mathrm{~b}_{1 \mathrm{~g}}\right)^{0}$ arises (Figure 2.1, middle left). When strongly $\pi$-donating ligands are employed the $\mathrm{e}_{\mathrm{g}}$ set is raised in energy which can result in a high-spin configuration (Figure 2.1, left). For heavier elements the ligand field splitting is increased significantly which favors intermediate- and low-spin configurations. Schneider and co-workers examined the reactivity of coordinatively unsaturated metal complexes with unusual electronic structures. The use of related diphosphineamido pincer ligands (Figure 2.2) enabled the isolation and characterization of square-planar $\mathrm{d}^{6}$ complexes like $\left[\mathrm{CoCl}\left(\mathrm{L}^{3}\right)\right]^{+},\left[\operatorname{IrCl}\left(\mathrm{L}^{3}\right)\right]^{+}$and the $\mathrm{Ru}^{\mathrm{II}}$ series $\left[\mathrm{RuCl}\left(\mathrm{L}^{1,2,3}\right)\right](\mathbf{L X V}, \mathbf{L X V I}$, LXVII, Figure 2.2, $\left.\mathrm{L}^{2}: \mathrm{N}\left(\mathrm{CHCHP}^{t} \mathrm{Bu}_{2}\right)\left(\mathrm{CH}_{2} \mathrm{CH}_{2} \mathrm{P}^{t} \mathrm{Bu}_{2}\right)\right) \cdot{ }^{[191]-[194]} \mathbf{L X V}$ exhibits a singlet ground-state due to the strong $\pi$-donation of the pincer ligands which raises the $\mathrm{d}_{\mathrm{xz}}$ orbital in energy (Figure 2.1 right). Weakening this interaction by successive dehydrogenation of the pincer ligand results in LXVI and LXVII which are best described by an intermediate spin-state ( $S=1$, Figure 2.1 middle right). Both compounds exhibit sharp signals in the ${ }^{1} \mathrm{H}$ NMR spectrum which show a linear shift over a temperature range of $\Delta T=140^{\circ} \mathrm{C}$, indicating the presence of an energetically well separated ground state. ${ }^{[191]}$ The latter also exhibit large magnetic anisotropy as a consequence of mixing with excited state through spin-orbit coupling (SOC) resulting in unusual high $D$ values (axial component of the magnetic dipole-dipole interaction) of over $200 \mathrm{~cm}^{-1}$.

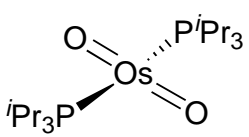

LXVIII

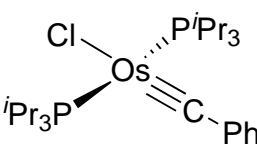

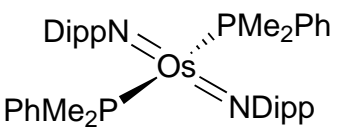

LXIX<smiles>N#[Pb]O[Pb](=[Pb])CCN1CC[Pb]C1</smiles>

XXXV
LXX

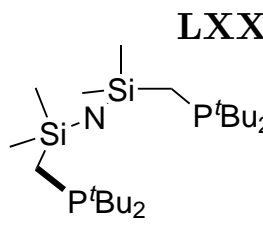

$\mathrm{L}^{\mathrm{Si}}$<smiles>[PH3+][Pb](Cl)([Pb])[PbH]</smiles>

LXXI

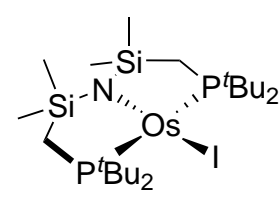

XXXVI<smiles>CCCCCCCCCCCCCCCCCCCCCCCCCCC</smiles>

$\mathrm{L}^{1}$<smiles>CCCCCCCCCCCCCCCCCCCCCCCCCC</smiles>

$\mathrm{L}^{2}$

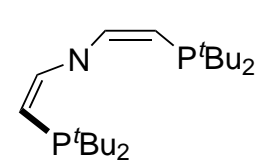

$\mathrm{L}^{3}$

Figure 2.2: Square-planar osmium complexes reported in the literature and the anionic PNP ligands used by the Caulton and Schneider group. ${ }^{[83],[97],[195]-[198]}$ 
The coordination chemistry of osmium is strongly dominated by six-coordinate $\mathrm{Os}^{\mathrm{II}}$. While low-coordinate species are known for high valent osmium featuring strong $\pi$ donating ligands (e.g. $\mathrm{OsO}_{4}$ ), square-planar species are rare. There are a number of $\mathrm{Os}^{\mathrm{IV}}$ complexes reported in literature which feature oxo (LXVIII), imido (LXIX), nitrido $(\mathbf{X X X V})$ or carbyne ligands $(\mathbf{L X X})$ and for (formal) $\mathrm{Os}^{0}$ with the $\pi$-acceptor ligand NO (LXXI) (Figure 2.2). ${ }^{[83],[195]-[198]}$ The only square-planar $\mathrm{d}^{6}$ system is represented by the $\mathrm{Os}^{\mathrm{II}}$ halide complex $\left[\mathrm{OsI}\left(\mathrm{L}^{\mathrm{Si}}\right)\right](\mathbf{X X X V I}) .{ }^{[97]}$ Although the pincer resonances in the ${ }^{1} \mathrm{H}$ NMR of XXXVI are sharp and coupling towards the phosphorus atoms is resolved, no ${ }^{31} \mathrm{P}$ NMR signal was detected and a non-magnetic triplet ground state was proposed which is unparalleled in osmium chemistry. Theoretical computations predict the singlet state to be $1.7 \mathrm{kcal} \cdot \mathrm{mol}^{-1}$ higher in energy than the assigned electronic ground state which lies in the error of the employed method. ${ }^{[199]}$ The $\mathrm{Ru}$ analogue $\left[\mathrm{RuI}\left(\mathrm{L}^{\mathrm{Si}}\right)\right]$ (LXXII) was also reported, however no coupling patterns in the ${ }^{1} \mathrm{H}$ NMR were observed and the triplet state was calculated to be favored by an energetic difference of $12.8 \mathrm{kcal} \cdot \mathrm{mol}^{-1}$. These results indicate that $3 \mathrm{rd}$ row metal complexes like XXXVI could exhibit unique electronic structures and magnetic properties. Moreover, the electronic structure of square-planar $\mathrm{Os}^{\mathrm{II}}$ might not be accurately described by DFT and should be investigated by more elaborate computational methods. Since a systematic study of the ligand set $\mathrm{L}^{1}-\mathrm{L}^{3}$ is available in the case of ruthenium, the corresponding osmium analogues are attractive synthetic targets to gain further insight into the electronic structure of heavy group 8 square-planar halide complexes. Additionally, Schneider and co-workers recently reported the synthesis of the five-coordinate Re ${ }^{\mathrm{III}}$ dichloride complex $\left[\mathrm{ReCl}_{2}\left(\mathrm{~L}^{1}\right)\right]$ (LXXIII) which served as a starting platform for dinitrogen fixation. ${ }^{[52]}$ In contrast to the targeted Os system, LXXIII features a saturated pincer ligand which is expected to result in stronger splitting of states due to its stronger $\pi$-donating properties. ${ }^{[191]}$ Furthermore, the complex $\left[\operatorname{ReCl}_{2}\left(\mathrm{~L}^{3}\right)\right](\mathbf{L X X I V})$ has been synthesized. ${ }^{1}$ The effect of successive PNP pincer desaturation or halide exchange has not been investigated thoroughly until this point.

${ }^{1}$ Unpublished results. See the Ph.D. thesis of Isabel Klopsch for additional details. 


\subsection{A Square-Planar Os(II) Complex}

Schneider and co-workers reported the synthesis of the $\mathrm{Os}^{\mathrm{IV}}$ complex $\left[\mathrm{OsCl}_{2} \mathrm{H}\left(\mathrm{L}^{1}\right)\right]$ (LXXV) upon coordination of ${ }^{\mathrm{H}} \mathrm{L}^{1}$ to $\left[\mathrm{OsCl}_{2}\left(\mathrm{PPh}_{3}\right)\right] .{ }^{[83]}$ All attempts to synthesize $\left[\mathrm{OsCl}\left(\mathrm{L}^{1}\right)\right]$ by elimination of $\mathrm{HCl}$ resulted in unselective decomposition, even at low temperatures. ${ }^{[200]}$ The divinylamide pincer ligand $\mathrm{L}^{3}$ can be introduced by application of a template ligand dehydrogenation strategy which has been successfully employed in the case of Co and Ni.[192], [201]

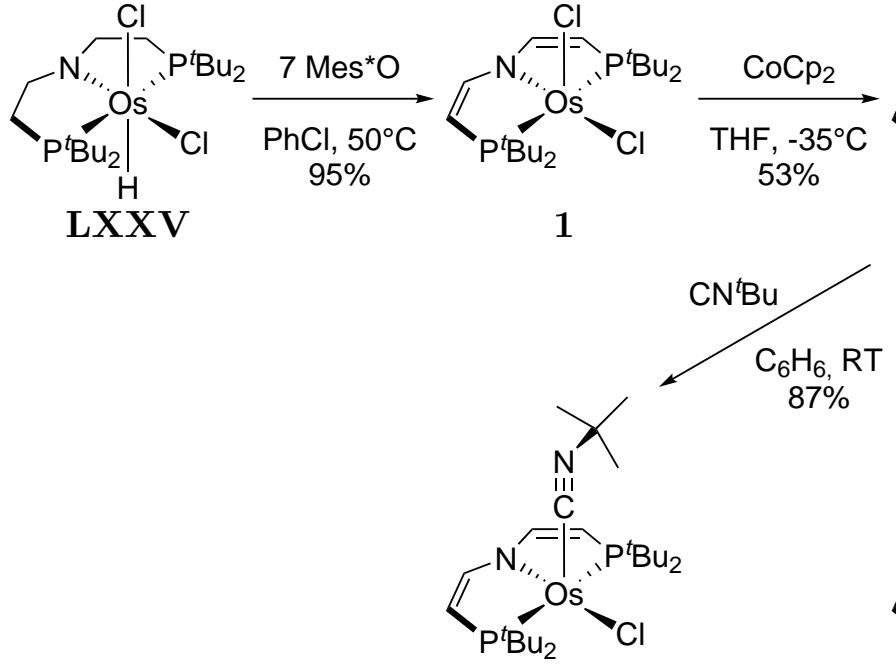

3

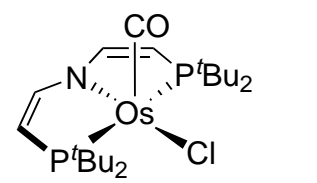

4

Scheme 2.1: Synthesis of 1 by five-fold $\mathrm{H}$ atom abstraction via Mes*O and reduction towards 2 followed by coordination of $\mathrm{CN}^{t} \mathrm{Bu}(\mathbf{3})$ and $\mathrm{CO}(4)$.

Reaction of LXXV with excess $\mathrm{Mes}^{*} \mathrm{O}$ at $50^{\circ} \mathrm{C}$ in chlorobenzene affords the fivecoordinate $\mathrm{Os}^{\mathrm{III}}$ complex $\left[\mathrm{OsCl}_{2}\left(\mathrm{~L}^{3}\right)\right](\mathbf{1}$, Scheme 2.1) in almost quantitative yield. Single-crystal X-ray analysis confirms complete ligand dehydrogenation (Figure 2.3, C1C2: 1.323(9) $\AA$ ). The coordination geometry is best described as square-pyramidal with the Cl1 ligand in apical position $(\tau=0.02) .^{[202]}$ In contrast to the solid state, $C_{2 \mathrm{v}^{-}}$ symmetry is observed on the NMR timescale in solution as indicated by three strongly paramagnetically shifted and broadened ${ }^{1} \mathrm{H}$ NMR resonances $\left(\delta_{t} \mathrm{Bu}=-0.06 \mathrm{ppm} ; \delta_{\mathrm{CH}}\right.$ $=43.9 \mathrm{ppm}$ and $-97.7 \mathrm{ppm})$. The magnetic moment of $\mathbf{1}$ derived by Evans' method at room temperature $\left(\mu_{\mathrm{eff}}=1.65 \mu_{\mathrm{B}}\right)$ is close to the spin-only value for an $S=1 / 2$ ground state. Furthermore, the broad, rhombic EPR powder spectrum with large $g$-anisotropy $\left(g_{x}=1.215, g_{y}=2.000, g_{z}=2.220\right.$, Appendix 8.1.1) is in agreement with a metal centered radical. The redox chemistry of $\mathbf{1}$ was examined by cyclic voltammetry (Appendix 8.1.2). Quasi-reversible oxidation was found at $E_{1 / 2}=0.04 \mathrm{~V}$ vs. $\mathrm{Fc}^{+/ 0}$ besides two reduction waves at $E_{\text {p.c. }} \approx-1.28 \mathrm{~V}$ and $E_{\text {p.c. }} \approx-2.05 \mathrm{~V}$ that remained irreversible 


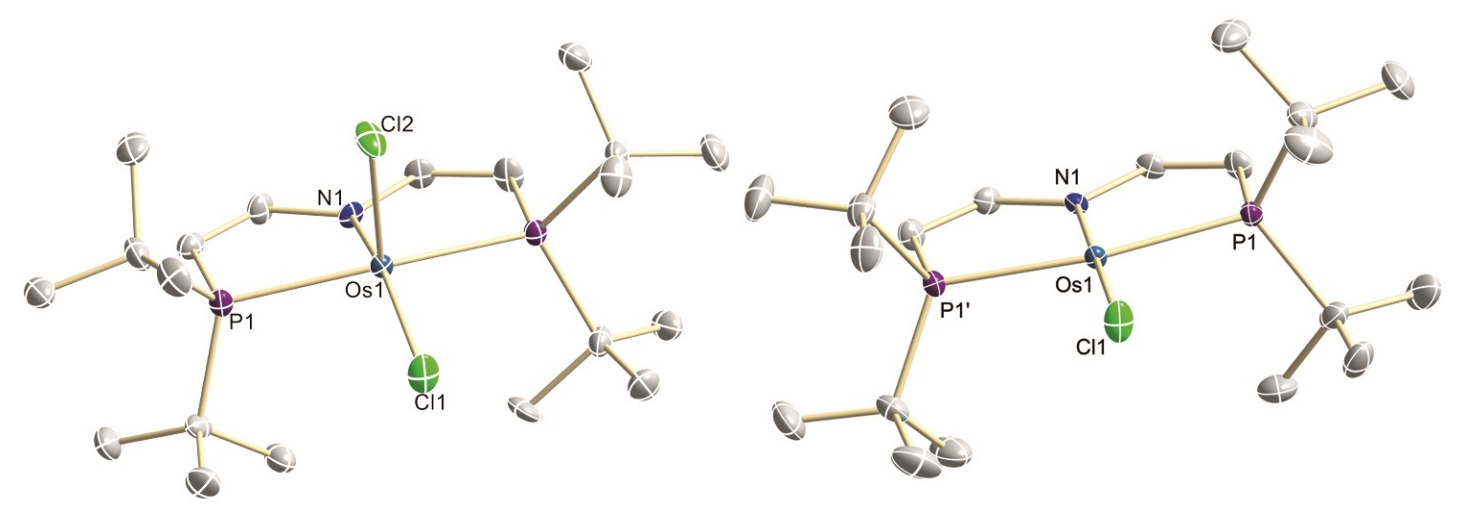

Figure 2.3: Molecular structure of complex 1 and $\mathbf{2}$ from single-crystal X-ray diffraction (thermal ellipsoids drawn at the $50 \%$ probability level); hydrogen atoms are omitted for clarity. Selected bond lengths $[\AA]$ and angles $\left[^{\circ}\right]$ : 1 Os1-N1 1.966(6), Os1-Cl1 2.380(3), Os1-Cl2 2.319(2), N1-C1 1.409(6), C1-C2 1.323(9), N1-Os1-Cl1 160.16(19), N1-Os1-Cl2 106.82(19), Cl1-Os1-Cl2 93.02(10); 2 Os1-N1 1.980(2), Os1-Cl1 2.3542(7), N1-C1 1.403(2), C1-C2 1.333(3)).

up to scan rates of $1 \mathrm{~V} \cdot \mathrm{s}^{-1}$, suggesting successive chloride elimination upon reduction. Accordingly, chemical reduction of $\mathbf{1}$ with $\mathrm{CoCp}_{2}$, extraction with pentane at $-30^{\circ} \mathrm{C}$ and crystallization overnight at $-35^{\circ} \mathrm{C}$ allows for the isolation of dark purple, crystalline $\left[\mathrm{OsCl}\left(\mathrm{L}^{3}\right)\right]$ (Scheme 2.1, 2) in isolated yield over 50\%. 2 is temperature sensitive and decomposes unselectively over the course of $24 \mathrm{~h}$. NMR spectroscopic detection of isobutene points towards intramolecular decomposition pathways, which also prevented reliable characterization by elemental analysis (Appendix 8.1.4). However, crystallographic, spectroscopic, and mass spectrometric data confirm the proposed structure.

In the solid state, the osmium ion exhibits square-planar coordination with some distortion due to the pincer bite angle (Figure 2.3). The bond lengths around the metal center are close to parent $\mathbf{1}$ and no indications for intermolecular interactions could be found. While no ${ }^{31} \mathrm{P}$ NMR signal was reported in the case of XXXVI, the ${ }^{31} \mathrm{P}\left\{{ }^{1} \mathrm{H}\right\}$ spectrum of $\mathbf{2}$ features a signal with an unusual chemical shift for a trialkylphosphine $\left(\delta_{\mathrm{P}}\right.$ $=-978.2 \mathrm{ppm})$. In addition, three strongly shifted $\left(\delta_{t_{\mathrm{Bu}}}=-4.2 \mathrm{ppm} ; \delta_{\mathrm{CH}}=18.4 \mathrm{ppm}\right.$ and $-35.3 \mathrm{ppm}$ ), yet sharp signals with resolved ${ }^{1} \mathrm{H}$ and ${ }^{31} \mathrm{P} J$-coupling are observed in the ${ }^{1} \mathrm{H}$ NMR spectrum (Appendix 8.1.3). Between -55 to $-15^{\circ} \mathrm{C}$ the chemical shifts exhibit non-Curie behavior, i.e. a linear $(\delta \propto T)$ and very small $(\Delta \delta<0.4 \mathrm{ppm})$ temperature dependence (Appendix 8.1.5). This observation suggest temperature independent magnetism (TIP) indicating an energetically well separated $\left(\gg k_{\mathrm{B} T}\right)$ ground state. ${ }^{[203]}$ This interpretation is further supported by SQUID magnetometry of a powdered sample (Figure 2.4, top right). In the range of $2-295 \mathrm{~K}$ constant susceptibility $\chi_{\mathrm{M}}(\mathrm{TIP})=1030 \cdot 10^{-6} \mathrm{~cm}^{3} \mathrm{~mol}^{-1}$ is observed. Hence, the magnetic properties are in line with the exclusive thermal population of a ground state, which experiences only second order paramagnetism through mixing with excited states. In comparison, the 
ruthenium analogue LXVII exhibits strongly temperature dependent NMR spectra and magnetic susceptibility in these temperature ranges as a consequence of smaller separations of the lowest spin-orbit states. ${ }^{[191]}$ This reflects the significant higher spinorbit coupling constant of osmium ( $\left.\zeta_{\mathrm{Os}} \approx 3000 \mathrm{~cm}^{-1}\right)$ compared to that of ruthenium $\left(\zeta_{\mathrm{Ru}} \approx 1000 \mathrm{~cm}^{-1}\right) .{ }^{[204]}$ Large TIP is typically found for (octahedrally coordinated) heavy $\mathrm{d}^{4}$ ions like $\mathrm{Re}^{\mathrm{III}}$ (vide infra) which share an isolobal relationship with 2 . $^{[205]}$ Further information is obtained from computational analysis of $\mathbf{2}$. Correlated single reference $a b$ initio calculations, ONIOM-(CCSD(T)-F12:PBE0D), predict a triplet ground state separated from the lowest singlet by merely $1.5 \mathrm{kcal} \cdot \mathrm{mol}^{-1}$. The singly occupied molecular orbitals are computed to be mainly metal centered with minor admixtures of the nitrogen pincer atom and the chloride ligand in the case of the $\mathrm{d}_{\mathrm{yz}}$ orbital (Figure 2.4, left). Additional multireference computations were performed to get insights into state mixing through SOC. A state-averaged NEVPT2/CAS $(16,10)$ approach (Figure 2.4 , bottom right) also indicates a triplet ground state with a $\left(\mathrm{d}_{\mathrm{z}^{2}}\right)^{1}\left(\mathrm{~d}_{\mathrm{xz}}\right)^{1}$ configuration (94\% weight). The first excited state lies $6.2 \mathrm{kcal} \cdot \mathrm{mol}^{-1}$ above the triplet and corresponds to the lowest singlet state $\left(\mathrm{d}_{\mathrm{z}^{2}}\right)^{2}\left(\mathrm{~d}_{\mathrm{xz}}\right)^{0}$ configuration ( $86 \%$ weight). Hence, these results qualitatively reflect the single reference ONIOM calculations. However, after QDPT/SOC treatment on the NEVPT2/CASSCF energies the ground-state triplet root is stabilized by $\Delta E(\mathrm{SOC})=-9.2 \mathrm{kcal} \cdot \mathrm{mol}^{-1}$. Furthermore, it splits into three sublevels, where the lowest eigenstate derives from a triplet contribution with significant admixtures $(16 \%)$ of the lowest singlet state. The two eigenstates above originate directly from the lowest triplet state. This state-energy diagram supports the rationalization of the magnetic susceptibility measurements with an energetically isolated ground-state. In an effective spin-Hamiltonian picture this would translate to large positive axial zero field splitting with the corresponding three ground-state magnetic sublevels of a $S=1$ spin system. However, this model breaks down and should not be applied if the spin-orbit coupling matrix elements approach the same order of magnitude of the associated nonrelativistic excitation energies. ${ }^{[206]}$ The Os ${ }^{\mathrm{II}}$ pincer platform was further characterized by the coordination of spectroscopic probes. As was reported for Caulton's complex XXXVI, the coordinatively unsaturated, low-valent complex 2 undergoes facile coordination of additional $\pi$-acceptor ligands. ${ }^{[97]}$ Addition of one equivalent of $\mathrm{CN}^{t} \mathrm{Bu}$ to in situ prepared 2 results in an immediate color change to dark blue and isolation of $\left[\mathrm{OsCl}\left(\mathrm{L}^{3}\right)\left(\mathrm{CN}^{t} \mathrm{Bu}\right)\right](\mathbf{3}$, Scheme 2.1) in high yield after column chromatography. The band at $\tilde{\nu}_{\mathrm{CN}^{t} \mathrm{Bu}}=1931 \mathrm{~cm}^{-1}$ in the IR spectrum (Appendix 8.1.6), assignable to the $\mathrm{C}=\mathrm{N}$ stretching vibration, indicates strong back bonding. This is confirmed by single crystal X-Ray diffraction (Figure 2.5). 

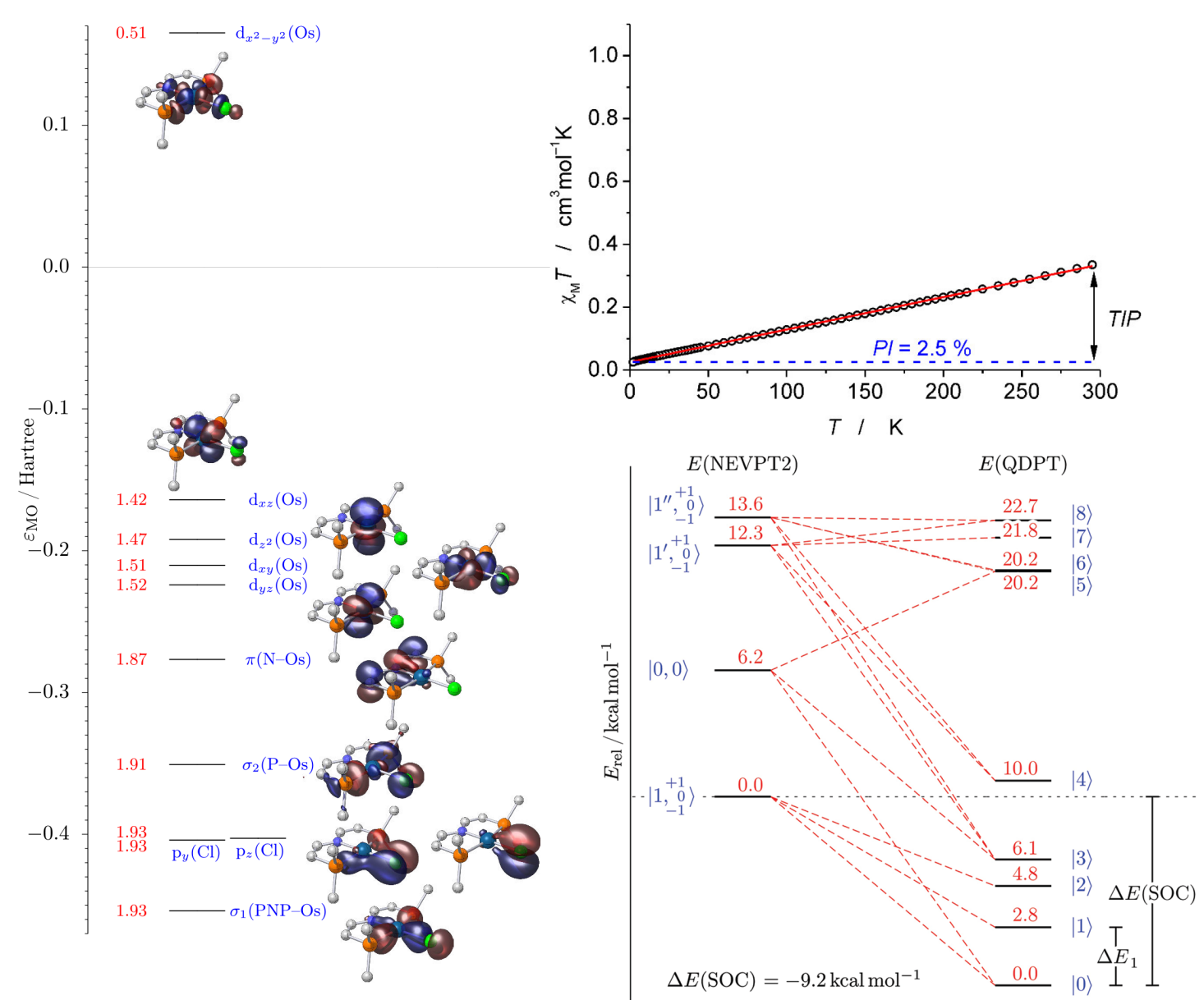

Figure 2.4: Left: Active $\mathrm{MO}$ scheme computed at the $C_{2}$-symmetric triplet geometry of 2 for a $\operatorname{SA}-\mathrm{CASSCF}(16,10)$ wavefunction, state-averaged over the 5 quintet, 45 triplet, and 50 singlet CSFs, which arise from the local $5 \mathrm{~d}^{6}$ configuration of the formal $\mathrm{Os}^{\mathrm{II}}$ center; average occupation numbers are given in red. Top right: $\chi_{\mathrm{M}} T$ versus $T$ plot for $2, \mathrm{PI}=2.5 \%, S=$ 1, $\chi_{\mathrm{M}}($ TIP $)=1030 \cdot 10^{-6} \mathrm{~cm}^{3} \mathrm{~mol}^{-1}$. Bottom right: State-energy diagram for $\mathbf{2}$ based on NEVPT2/SA-CASSCF $(16,10)$ computations. Non-relativistic energies of the lowest four states are shown with their corresponding state labels (left). The lowest nine spin-orbit states (right) are complemented with selected contributions (weights $>10 \%$ ) of the corresponding spin-free states (dashed red lines).

The isonitrile ligand of $\mathbf{3}$ is situated in the apical position of the square-pyramidal structure and features strong bending (C21-N2-C22: $\left.144.4(4)^{\circ}\right)$ to an extent which is rarely observed. ${ }^{[53],[207]}$ Furthermore, the Os- $\mathrm{N}$ bond is slightly elongated with respect to parent $\mathbf{2}(\Delta d=0.07 \AA)$ indicating reduced $\pi$-donation by the pincer ligand.

In analogy, exposure of in situ prepared $\mathbf{2}$ to one equivalent of $\mathrm{CO}$ gives the pink compound $\left[\mathrm{OsCl}\left(\mathrm{L}^{3}\right)(\mathrm{CO})\right](\mathbf{4}$, Scheme 2.1$)$. When treated with one atmosphere of carbon monoxide the formation of several side products is observed, e.g. a dicarbonyl complex which decomposes quickly. As in the case of complex $\mathbf{3}$, the ${ }^{1} \mathrm{H}$ NMR spectrum is in agreement with $C_{\mathrm{S}}$-symmetry on the NMR timescale. Complex $\mathbf{4}$ was fully characterized 


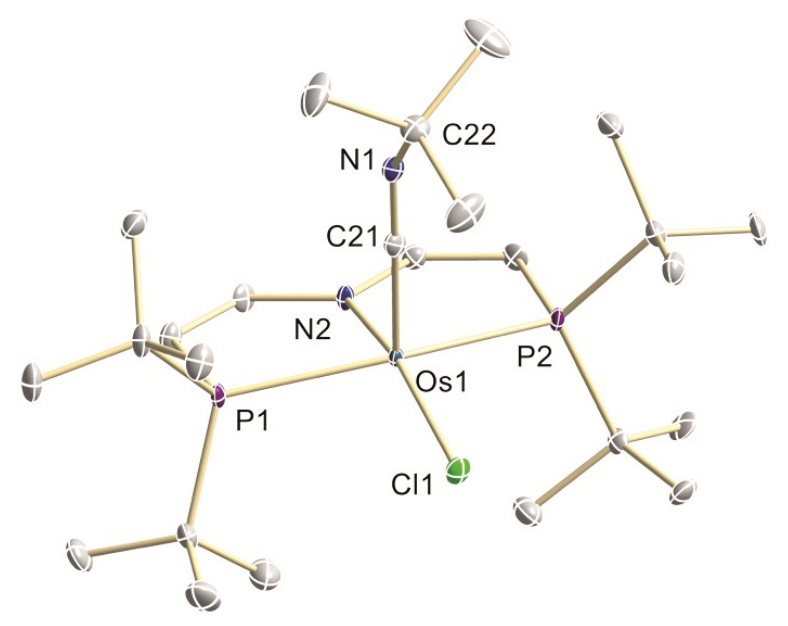

Figure 2.5: Molecular structure of complex 3 from single-crystal X-ray diffraction (thermal ellipsoids drawn at the $50 \%$ probability level); hydrogen atoms are omitted for clarity. Selected bond lengths $[\AA]$ and angles $\left[^{\circ}\right]$ : 3 Os1-N2 2.050(3), Os1-C21 1.827(4), Os1-Cl1 2.4046(9), C21-N1 1.197(5), C21-N2-C22 144.4(4).

including single-crystal X-Ray diffraction (Due to significant disorder the bond lengths will not be discussed in detail). The CO ligand exhibits a rather unusual high-field shift in the ${ }^{13} \mathrm{C}$ NMR spectrum $\left(\delta_{\mathrm{CO}}=168.3 \mathrm{ppm}\right)$. The $\mathrm{CO}$ stretching vibration is observed at $\tilde{\nu}_{\mathrm{CO}}=1893 \mathrm{~cm}^{-1}$ in the IR spectrum (Appendix 8.1.7), almost identical with Caulton's $\left[\mathrm{OsI}\left(\mathrm{L}^{\mathrm{Si}}\right)(\mathrm{CO})\right]\left(\mathbf{L X X V I}, 1892 \mathrm{~cm}^{-1}\right) .{ }^{[97]}$ These values confirm the electron rich nature of the $\{\mathrm{OsX}(\mathrm{PNP})\}$ platform and increased back bonding in comparison with the $\mathrm{Ru}^{\mathrm{II}}$ analogue $\left[\mathrm{RuCl}\left(\mathrm{L}^{3}\right)(\mathrm{CO})\right]\left(\mathbf{L X X V I I}, \tilde{\nu}_{\mathrm{CO}}=1916 \mathrm{~cm}^{-1}\right) .{ }^{[191]}$ Importantly, the NMR data of complexes $\mathbf{3}$ and $\mathbf{4}$ indicate the removal of the TIP experienced by parent 2 upon coordination of a fifth ligand $\left(3: \delta_{\mathrm{P}}=55.6, \delta_{\mathrm{H}}=7.04-1.19 \mathrm{ppm} ; 4: \delta_{\mathrm{P}}=65.1\right.$, $\left.\delta_{\mathrm{H}}=6.92-1.16 \mathrm{ppm}\right)$. 


\subsection{Re(III) PNP Pincer Halide Complexes}

Based on the results obtained for $\mathrm{Os}^{\mathrm{II}}$, a reinvestigation of isoelectronic LXXIII and LXXIV is promising. Furthermore, the influence of dehydrogenation of the ligand backbone and substitution of the halide ligands could provide additional insights into magnetic property - structure relationships. Stirring LXXIII with an excess of NaI results in the complete exchange of the chloride ligands resulting in the formation of $\left[\operatorname{ReI}_{2}\left(\mathrm{~L}^{1}\right)\right]$ (5, Scheme 2.2) in $91 \%$ isolated yield. The NMR signals are unusually shifted with ${ }^{1} \mathrm{H}$ NMR signals in the range of $\delta_{\mathrm{H}}=3.31--0.71$ and a ${ }^{31} \mathrm{P}$ shift of $\delta_{\mathrm{P}}=-164.2 \mathrm{ppm}$. The complex can be further reacted with an excess of Mes*O at elevated temperatures to give the $\mathrm{L}^{3}$ ligated system $\left[\operatorname{ReI}_{2}\left(\mathrm{~L}^{3}\right)\right](\mathbf{6}$, Scheme 2.2$)$ in $70 \%$ yield.

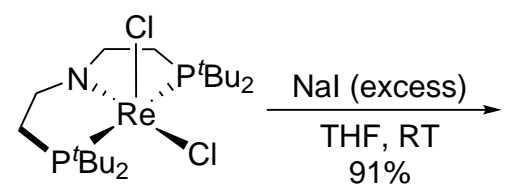

LXXIII

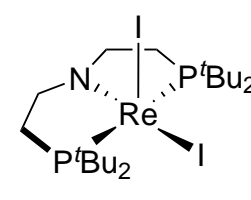

5

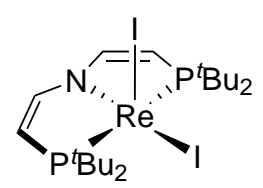

6

Scheme 2.2: Synthesis of $\mathbf{5}$ by salt metathesis and its dehydrogenation towards 6 .
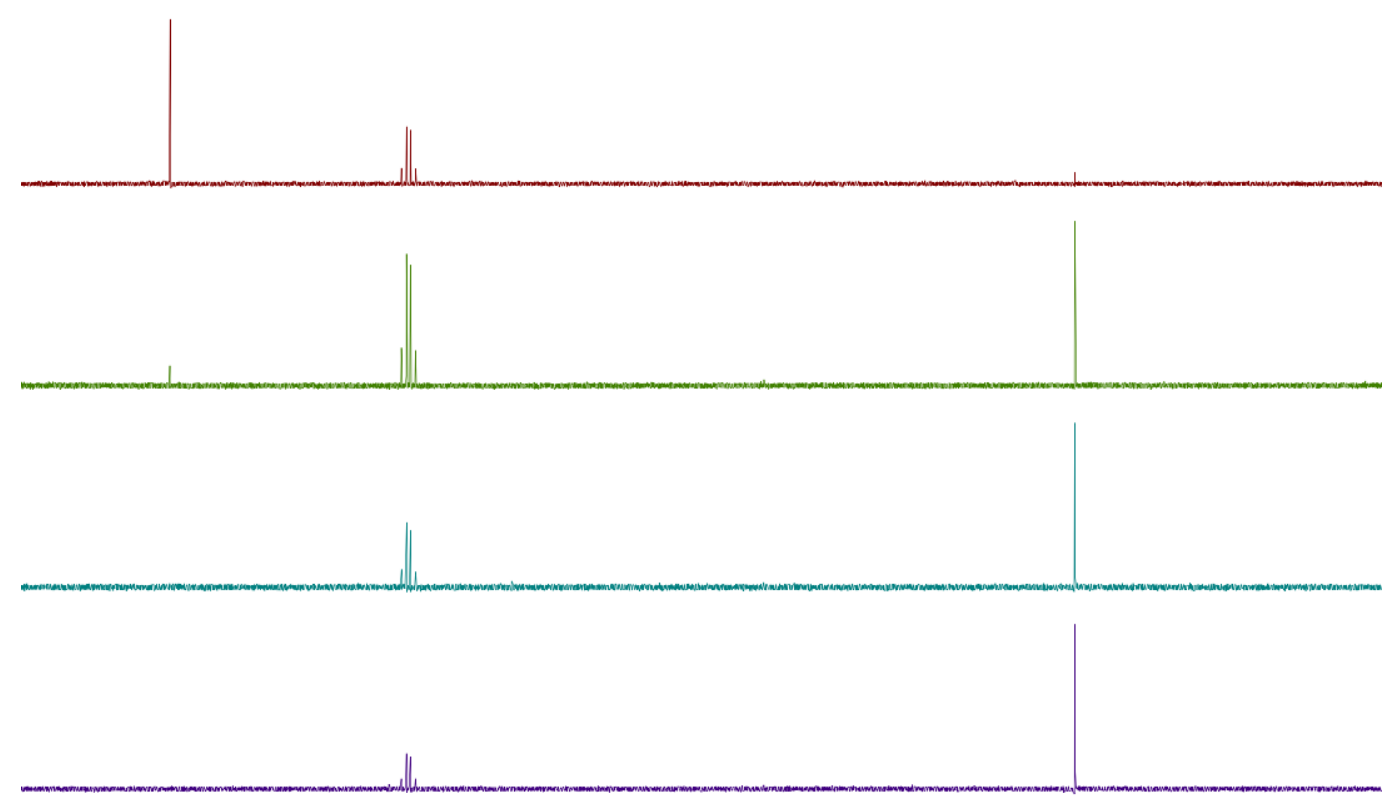

$\begin{array}{lllllllllllllllllllllllllllllllllll}-120 & -140 & -160 & -180 & -200 & -220 & -240 & -260 & -280 & -300 & -320 & -340 & -360 & -380 & -400 & -420 & -440 & -460 & -480 & -500 & -520 & -540 & -560 & -580 & -600 & -620 & -640\end{array}$

Figure 2.6: Dehydrogenation of 5 with Mes* ${ }^{*}$ (5.0 eq.), $\mathrm{C}_{6} \mathrm{D}_{6}, 50^{\circ} \mathrm{C}$, after $30 \mathrm{~min}$ (red), $2 \mathrm{~h}$ (green), $18 \mathrm{~h}$ (turquoise), $24 \mathrm{~h}$ (purple). 


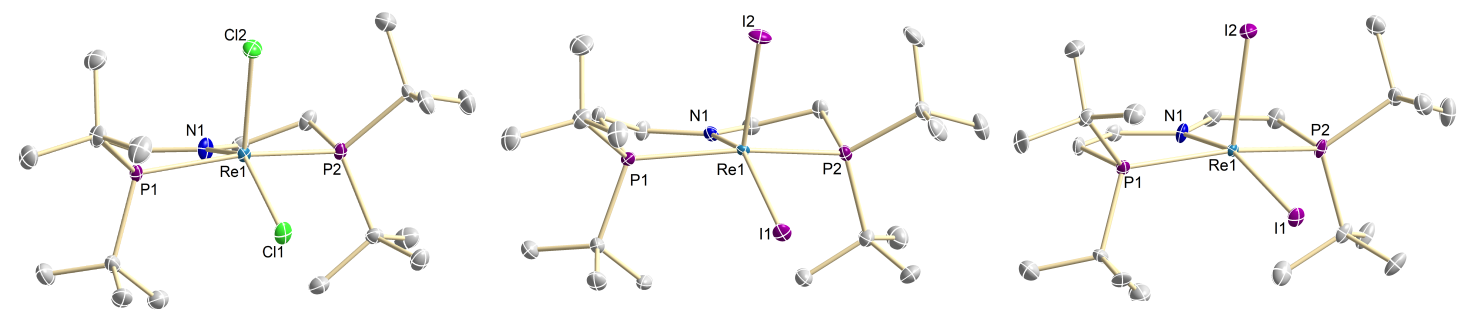

Figure 2.7: Molecular structure of complex LXXIII, 5 (middle) and 6 (right) from singlecrystal X-ray diffraction (thermal ellipsoids drawn at the $50 \%$ probability level); hydrogen atoms are omitted for clarity. Selected bond lengths $[\AA]$ and angles $\left[^{\circ}\right]$ : LXXIII ${ }^{[52]} \operatorname{Re} 1-\mathrm{Cl1} 2.364(2)$, Re1-Cl2 2.331(2), Re1-N1 1.923(7), Re1-P1 2.402(2), Re1-P2 2.407(3), N1-Re1-Cl1 140.8(3), P1-Re1-P2 163.04(9), $\tau=0.37 ; 5$ Re1-I1 2.7156(2), Re1-I2 2.6382(2), Re1-N1 1.926(2), Re1-P1 2.4277(8), Re1-P2 2.4172(8); N1-Re1-I1 151.09(7), N1-Re1-I2 107.70(7), P1-Re1-P2 161.62(3), $\tau=0.18 ; 6$ Re1-I1 2.6336(3), Re1-I2 2.6898(3), Re1-N1 1.994(4), Re1-P1 2.4470(12), Re1-P2 2.4568(12), N1-Re1-I1 159.21(11), N1-Re1-I2 100.39(11), P1-Re1-P2 158.12(4), $\tau=0.02$.

Over the course of the reaction, the half-saturated complex $\left[\operatorname{ReI}_{2}\left(\mathrm{~L}^{2}\right)\right]$ can be observed as an intermediate by ${ }^{31} \mathrm{P} \mathrm{NMR}$, as indicates by the detection of two inequivalent phosphorus atoms $\left(\delta_{\mathrm{P}}=-263.6,-266.5 \mathrm{ppm},{ }^{2} J_{\mathrm{PP}}=240 \mathrm{~Hz}\right.$, Figure 2.6$)$, that cleanly convert to 6 . The ${ }^{1} \mathrm{H}$ and ${ }^{31} \mathrm{P}$ NMR values are further shifted with respect to $\mathbf{5}\left(\delta_{\mathrm{H}}=4.30\right.$ $\left.-7.08, \delta_{\mathrm{P}}=-526.9 \mathrm{ppm}\right)$. In the solid state, LXXIII and $\mathbf{5}$ exhibit almost identical bond lengths between the metal center and the PNP pincer scaffold, however $\mathbf{5}$ adopts a more square-pyramidal geometry as indicated by its $\tau$-value (Figure 2.7). The molecular structure of $\mathbf{6}$ reveals a shortening of the Re1-I1 distance with respect to $\mathbf{5}$ due to the

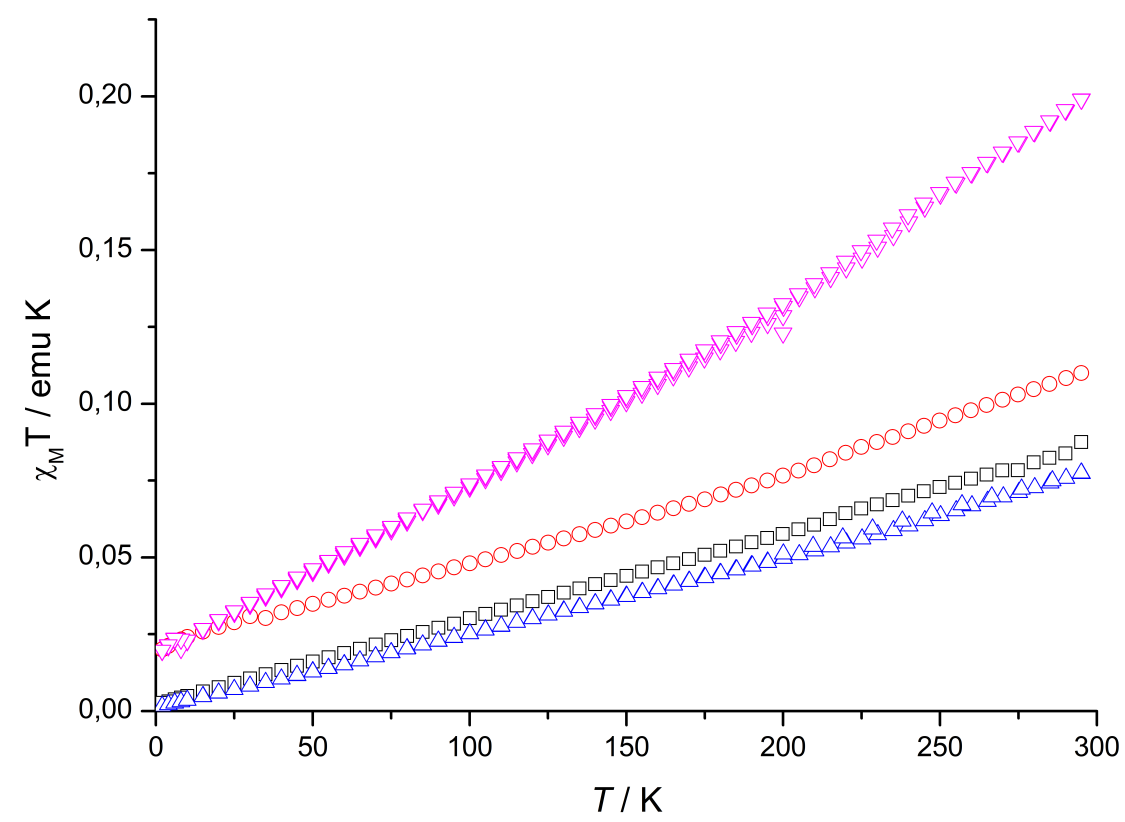

Figure 2.8: Top: $\chi_{\mathrm{M}} T$ vs. $T$ plot of LXXIII (black), LXXIV (red), 5 (blue) and 6 (pink). 
reduced bite angle and the decreased $\pi$-donating properties of the pincer framework. The overall coordination mode is close to ideal square-pyramidal $(\tau=0.02)$. The Re-N1 bond is elongated by $\Delta d=0.07 \AA$ which is close to the isoelectronic Os complex 2 . The observed ${ }^{31} \mathrm{P}$ NMR chemical shift values become more negative upon substitution of the chloride for iodide $\left(\Delta \delta_{\mathrm{P}}=112.7 \mathrm{ppm}\right)$ and ligand dehydrogenation $\left(\Delta \delta_{\mathrm{P}}=224.1(\mathrm{Cl})\right.$, $251.9 \mathrm{ppm}$ (I), Figure 2.8, Table 2.1). SQUID magnetometry reveals that the observed chemical shifts do not correlate with the magnetic moment of the substances (Figure 2.8, Table 2.1). All complexes feature linear behavior in the temperature region of $2-300 \mathrm{~K}$, indicating no thermal population of excited states, as observed for the square-planar Os ${ }^{\mathrm{II}}$ complex 2. For LXXIII, LXXIV and $\mathbf{5}$ similar $\chi_{\mathrm{M}} T$ values are obtained whereas $\mathbf{6}$ differs significantly. A possible explanation lies in the reduced donor properties of iodide that lead to reduced ligand field splitting. Furthermore, $\mathrm{L}^{3}$ also reduces the energetic gap between the ground state and excited states. A combination of both factors apparently leads to an increase in the magnetic moment suggesting that $\mathbf{6}$, like $\mathbf{2}$, can be described with a non-magnetic ground state which strongly mixes with excited states via SOC. Nevertheless, multi-reference computations are needed when such effects are observed since $S$ is not a good quantum number and rather $J$ should be used, as discussed above.

Table 2.1: ${ }^{31} \mathrm{P}$ NMR and SQUID values of LXXIII, LXXIV, 5 and 6.

\begin{tabular}{crrrr}
\hline & LXXIII & LXXIV & \multicolumn{1}{c}{$\mathbf{5}$} & \multicolumn{1}{c}{$\mathbf{6}$} \\
\hline$\delta / \mathrm{ppm}$ & -51.5 & -275.6 & -164.2 & -527.5 \\
$\chi_{\mathrm{M}}(\mathrm{TIP}) / 10^{-6} \mathrm{~cm}^{3} \mathrm{~mol}^{-1}$ & 282.6 & 296.5 & 248.4 & 569.2 \\
\hline
\end{tabular}




\subsection{Five- and Six-Coordinate Os Bipyridine Complexes}

A common strategy to vary the redox-potential of complexes is the exchange or attachment of ancillary ligands. 2,2'-bipyridine is particular attractive in case of $\mathbf{2}$ since it is a planar chelate ligand which should afford a sterically encumbered octahedral platform, without inducing too much steric strain on the PNP ligand. Additionally, this chelate is one of the most prominent ligands in photochemistry which could allow for light induced reactions. ${ }^{[208]}$ For example, the $\operatorname{Ir}^{I I I}$ hydride $\left[\operatorname{Ir}\left(\mathrm{Cp}^{*}\right) \mathrm{H}(\text { bipy })\right]^{+}$(LXXVIII, Scheme 2.3 ) is known to engage in multiple photochemical transformations. ${ }^{[209]-[214]}$ A hypothetical isoelectronic, octahedral Os ${ }^{\mathrm{II}}$ hydride complex ligated by $\mathrm{L}^{3}$ and bipy might exhibit rich photochemistry allowing for new transformations. For example, unprecedented photochemical $\mathrm{CO}_{2}$ activation was reported for $\left[\mathrm{NiH}\left(\mathrm{L}^{3}\right)\right](\mathbf{L X X I X})$ to give a $\left[\mathrm{Ni}(\mathrm{COOH})\left(\mathrm{L}^{3}\right)\right]$ (Scheme 2.3, LXXX). ${ }^{[215]}$ Therefore, a six-coordinate $\mathrm{Os}^{\mathrm{II}}$ hydride complex is an attractive synthetic target.

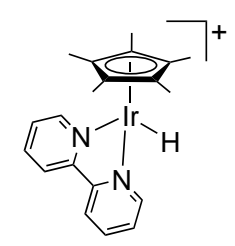

LXXVIII

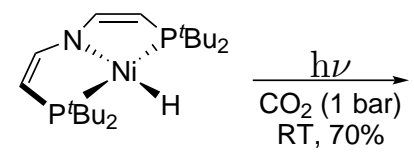

LXXIX

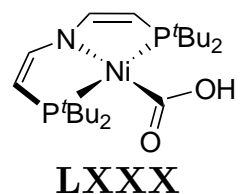

LXXX

Scheme 2.3: Extensively studied $\mathrm{Ir}^{\mathrm{III}}$ photocatalyst LXXVIII and photochemical induced fixation of $\mathrm{CO}_{2}$ by LXXIX to give LXXX. ${ }^{[209]-[215]}$

As described above, the square-planar halide $\mathbf{2}$ can be prepared in situ by addition of $\mathrm{CoCp}_{2}$ to1. The addition of 2,2'-bipyridine in benzene allows for the isolation of $\left[\mathrm{OsCl}\left(\mathrm{L}^{3}\right)\right.$ (bipy)] (7, Scheme 2.4$)$ in high yield. 7 does not show any signs of significant spin-orbit coupling contributions to the ${ }^{1} \mathrm{H}$ NMR signals as indicated by well-resolved coupling constants and shift values similar to complexes obtained by the addition of monodentate $\pi$-acceptors to $\mathbf{2}$ (Chapter 2 ).

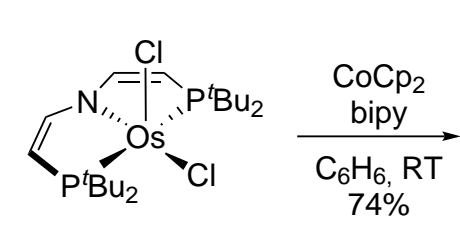

1

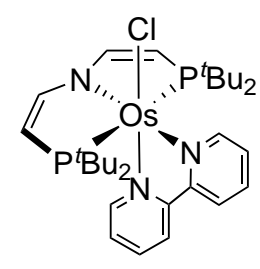

7

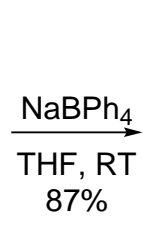

$87 \%$

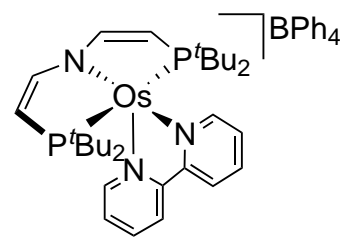

8

Scheme 2.4: Synthesis of $\mathbf{7}$ by addition of 2,2'-bipyridine to in situ generated $\mathbf{2}$ and successive chloride removal yielding 8 . 
In the solid state, $\mathbf{7}$ exhibits a distorted octahedral coordination sphere (Figure 3.6). In comparison to $\mathbf{2}$, the Os- $\mathrm{N}$ distance of the pincer ligand is significantly elongated due to the more electron rich $\mathrm{Os}^{\mathrm{II}}$ center. The bipyridine ligand remains planar suggesting no significant charge transfer by the metal center. Furthermore, the metal-chloride distance is elongated by $\Delta d \approx 0.1 \AA$ with respect to 2 . Addition of $\mathrm{NaBPh}_{4}$ results in the clean formation of $\left[\mathrm{Os}\left(\mathrm{L}^{3}\right)(\right.$ bipy $\left.)\right]\left[\mathrm{BPh}_{4}\right](\mathbf{8}$, Scheme 2.4) in almost quantitative yield. The solid-state structure of $\mathbf{8}$ reveals a distorted square-pyramidal complex $(\tau=0.30)$ with a slightly shortened Os1-N1 distance due to increased $\pi$-donation of the pincer ligand. The ${ }^{1} \mathrm{H}$ NMR signals of $\mathbf{8}$ reveal $C_{2 \mathrm{v}}$-symmetry on the NMR timescale suggesting that the solid-state structure is not preserved in solution due to a flexible bipy ligand.
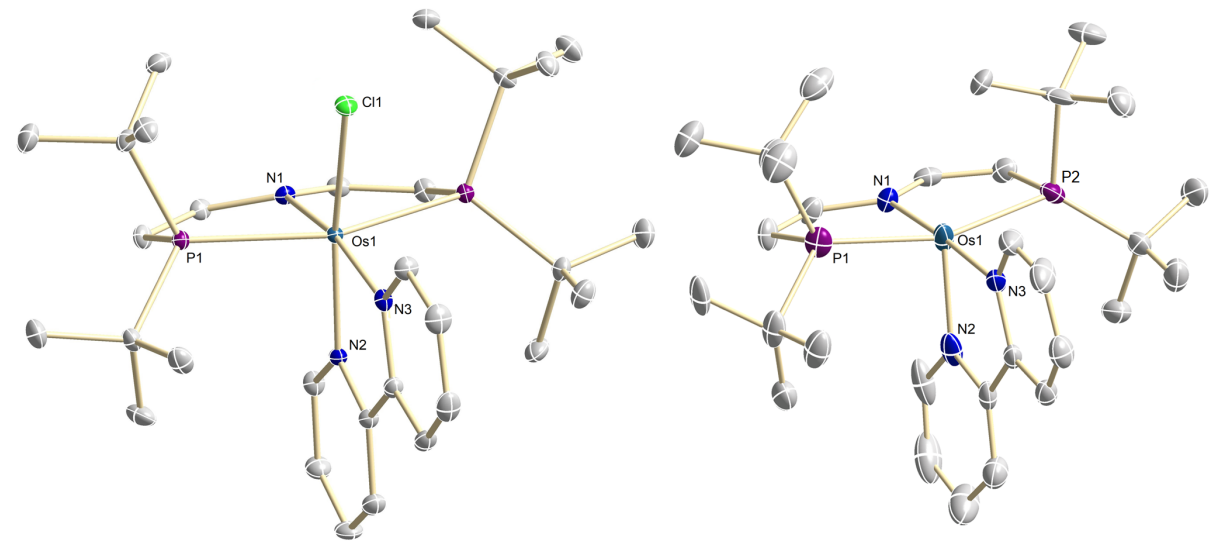

Figure 2.9: Molecular structure of $\mathbf{7}$ (left) and 8 from single-crystal X-ray diffraction (thermal ellipsoids at the $50 \%$ probability level); hydrogen atoms, solvent and anion molecules are omitted for clarity. Selected bond lengths $[\AA]$ and angles [ $\left.{ }^{\circ}\right]$ : 7 Os1-Cl1 2.4557(6), Os1-N1 2.120(2), Os1N2 2.031(2), Os1-N3 2.060(2), Os1-P1 2.4352(5), N1-Os1-Cl1 107.00(6), P1-Os1-P1\# 156.05(2); 8 Os1-N1 2.015(4), Os1-N2 2.039(3), Os1-N3 2.036(2), Os1-P1 2.3676(7), Os1-P2 2.3852(7), N1-Os1-N3 178.02(16), P1-Os1-P2 160.29(2), $\tau=0.30$.

With $\mathrm{L}^{3}$ and bipy attached to the Os center, the question arises if electrons can be stored on either of the two ligands since $\mathrm{L}^{3}$ exhibits redox non-innocent behavior in the case of 3d metals. ${ }^{[192],[201]}$ Additionally, clearly defined bipy ${ }^{-}$ligand are scarce due to the difficult discrimination between strong back-bonding and electron transfer in such systems. ${ }^{[216],[217]}$ The cyclic voltammogram of $\mathbf{8}$ features two reductive redox events (Figure 2.10). The first wave is reversible with $E_{1 / 2}=-1.83 \mathrm{~V}$ while the second redox event at $E_{\text {p.c. }} \approx-2.59 \mathrm{~V}$ vs. $\mathrm{Fc}^{0 /+}$ appears pseudo-reversible. When 8 is reacted with an excess of $\mathrm{NaHg}$ in THF a color change from brown to dark red can be observed. The ${ }^{31} \mathrm{P}$ NMR spectrum features no detectable signals indicating the formation of a paramagnetic species. Over the course of several minutes the formation of asymmetric diamagnetic products can be observed in the ${ }^{31} \mathrm{P}$ NMR which were not further investigated (Appendix 8.2.4). 

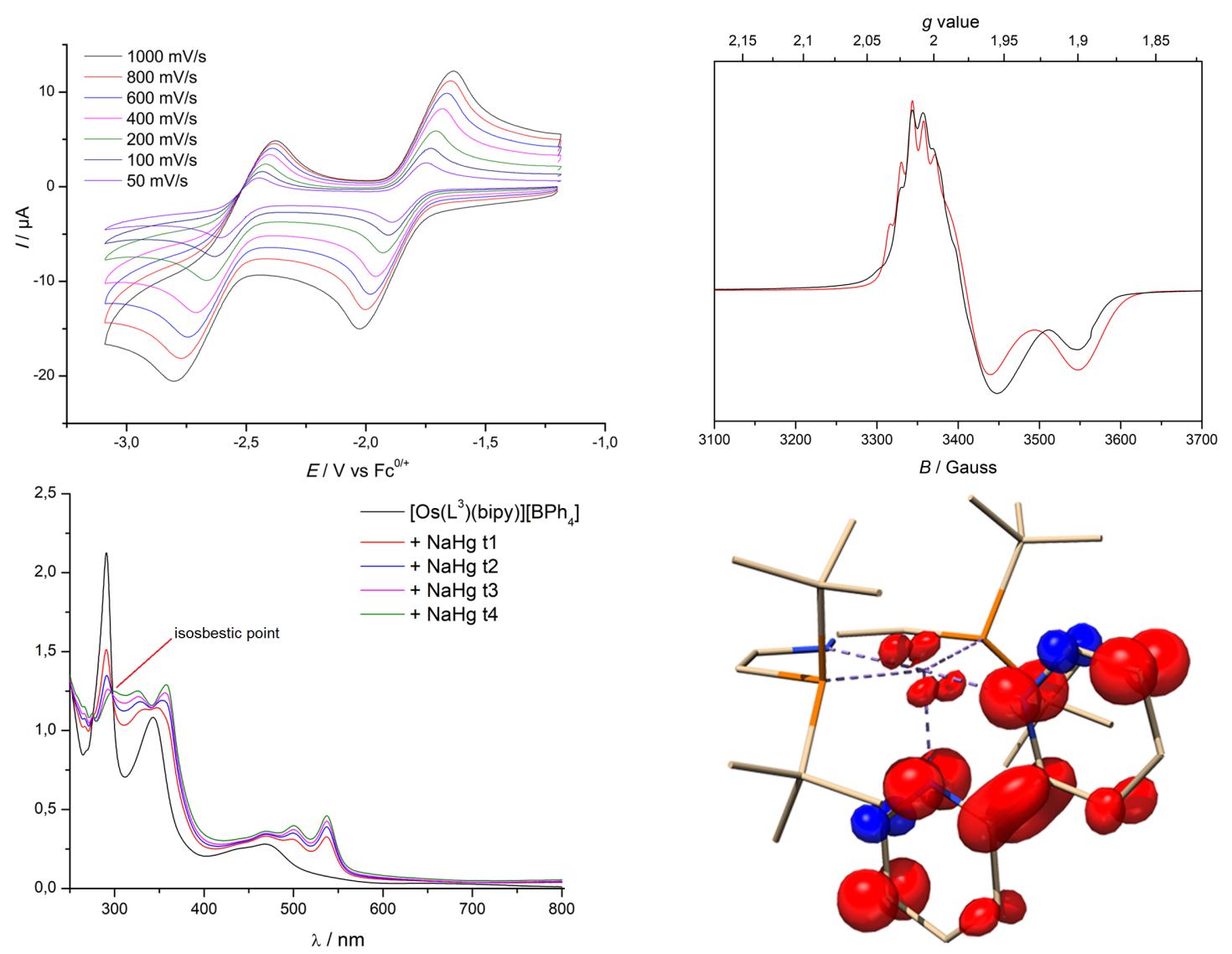

Figure 2.10: Top left: Cyclovoltammogramm of $\mathbf{8}, 0.1 \mathrm{mM}$, THF, $0.1 \mathrm{M} \mathrm{NBu}_{4} \mathrm{PF}_{6}$, RT; Top right: EPR spectrum of $\mathbf{9}, 140 \mathrm{~K}, 9.434 \mathrm{GHz}$, Simulation: $g_{x}=1.898, g_{y}=1.976, g_{z}=2.015$, $\mathrm{A}_{1 / 2}\left({ }^{14} \mathrm{~N}\right)=-,-, 40 \mathrm{MHz}$; Bottom left: UV/vis spectrum of 8 and its reduction with $\mathrm{NaHg}$, $5 \cdot 10^{-5} \mathrm{mM}$, THF, RT, 30 s intervals; Bottom right: Spin-density of $\mathbf{9}$.

Keeping the reaction mixture at low temperatures did no lead to formation of single crystals suitable for X-ray diffraction. Following the reduction of $\mathbf{8}$ with $\mathrm{NaHg}$ by UV/vis spectroscopy reveals an isosbestic point at $\lambda=298 \mathrm{~nm}$ suggesting clean initial reduction (Figure 2.10). An EPR spectrum of the reaction mixture shows a narrow, rhombic EPR signal $(\Delta B=300 \mathrm{G})$ with resolved ${ }^{14} \mathrm{~N}$ super-hyperfine interaction for one $\mathrm{g}$ value. Based on this results, DFT calculations were performed to clarify if $\left[\mathrm{Os}\left(\mathrm{L}^{3}\right)(\right.$ bipy)$]$ (9) is indeed formed as the initial reaction product. Theory predicts the electron to be mainly located at the bipy ligand with only minor contributions of the Os center which features a spin density of $4.1 \%$. The computed EPR parameters reproduce the experimental findings and confirm that nitrogen hyperfine interaction, caused by spin polarization, is only observed at one of the principal axis which is in agreement with the observed spectrum (Appendix 8.1). Other orientations of the hyperfine interaction tensor could not be quantified due to significant line broadening. However, combination of spectroscopy and theory supports the assumption that $\mathbf{9}$ is observable and exhibits 
a certain lifetime at room temperature. The two-electron reduction of $\mathbf{8}$ was extremely sluggish and produced multiple products which were not further investigated. $\mathbf{7}$ and $\mathbf{8}$ should exhibit a high tendency to bind anionic ligands such as azide or hydride due to the labile chloride ligand and the cationic charge, respectively.

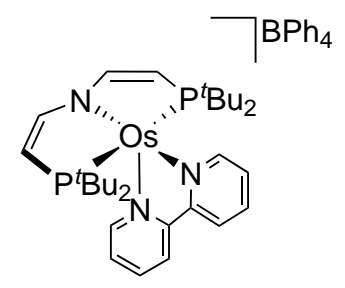

8

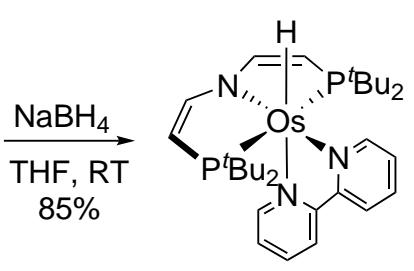

10

Scheme 2.5: Synthesis of 10 by addition of $\mathrm{NaBH}_{4}$ to 8 .

By reacting 8 with an excess of $\mathrm{NaBH}_{4}$ the hydride complex $\left[\mathrm{OsH}\left(\mathrm{L}^{3}\right)(\right.$ bipy) $]$ (10, Scheme 2.5 ) can be isolated in $85 \%$ yield. Although confirmation of the existence of the hydride ligand was not accurately possible by X-ray crystallography (Figure 2.11), the complex is easily identified by its characteristic ${ }^{1} \mathrm{H}$ NMR and IR signals $\left(\delta_{\mathrm{H}}=-11.0 \mathrm{ppm}\right.$, $\left.\tilde{\nu}_{\mathrm{OsH}}=2074 \mathrm{~cm}^{-1}\right) . \quad 10$ is a dark powder which easily dissolves in polar and apoloar solvents with a dark greenish color, hinting towards multiple electronic absorptions in the UV/vis region. In fact, the electronic absorption spectrum exhibits multiple intense absorptions in the visible range (Figure 2.11). Time-dependent functional theory is in good agreement with the experimental spectrum accompanied by a typical offset to higher energies. ${ }^{[218]}$ The absorptions with the most prominent oscillator strengths were visualized by their difference densities (Figure 2.11). The highest energy absorption at $\lambda=248 \mathrm{~nm}$ (a) is caused by a $\pi \rightarrow \pi^{*}$ transition of the bipy ligand. The band at $\lambda=290 \mathrm{~nm}$ consists of multiple intense transitions. While (b) and (c) are bipy centered $\pi \rightarrow \pi^{*}$ transitions, band (d) is caused by an MLCT process of the Os- $\mathrm{N}_{\mathrm{PNP}}$ unit to the pincer backbone.

A similar process is found within the broad absorption at $\lambda=386 \mathrm{~nm}$ (e) with small admixtures of the bipy $\pi$-orbitals accompanied by an Os-bipy MLCT process (f). The shoulder at $\lambda=435 \mathrm{~nm}$ and the broad transition at $\lambda=575 \mathrm{~nm}$ are caused by transitions of the metal $d$ orbitals into the $\pi^{*}$-orbitals of the bipy ligand $(\mathrm{g}, \mathrm{h})$. Since the transitions appear well-separated, 10 could represent an attractive compound for future band-selective hydride photochemistry. 

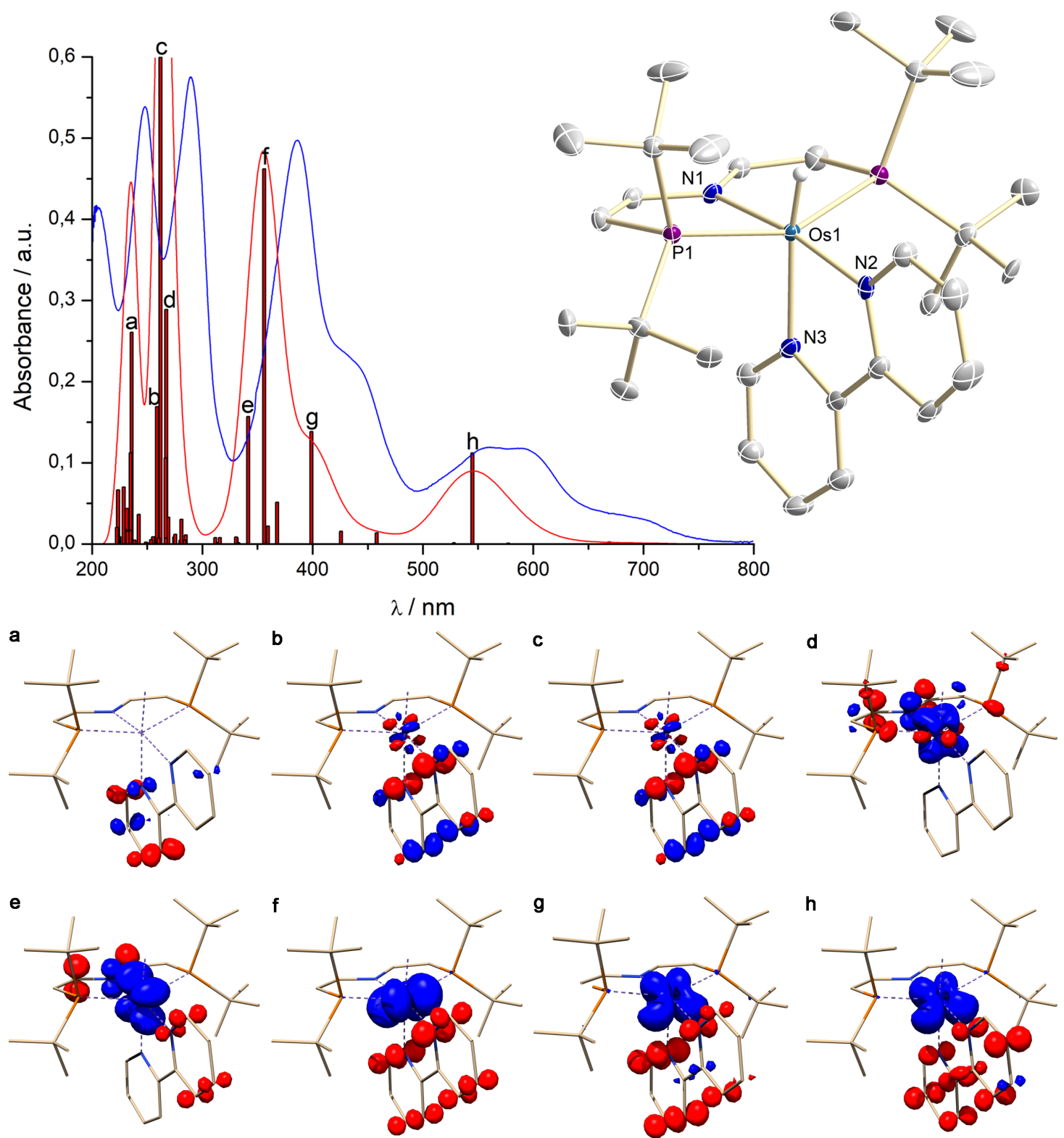

Figure 2.11: Top left: Experimental (blue) and computed (red, sticks and broadened by $2500 \mathrm{~cm}^{-1}$ ) UV/vis spectrum of $\mathbf{1 0}, 3 \cdot 10^{-5} \mathrm{M}$, pentane, RT; Top right: Molecular structure of $\mathbf{1 0}$ from single-crystal X-ray diffraction (thermal ellipsoids at the $50 \%$ probability level); hydrogen atoms, except the hydride ligand, are omitted for clarity. Selected bond lengths $[\AA]$ and angles [ $\left.{ }^{\circ}\right]$ : 10: Os1-N1 2.103(5), Os1-N2 2.021(5), Os1-N3 2.096(5), Os1-P1 2.3621(11), N1-Os1N2 170.1(2), P1-Os1-P1\# 152.17(5); Bottom: Selected computed difference densities (loss and gain of electron density is depicted in blue and red, respectively). 


\subsection{Re and Os PNP Pincer Complexes Ligated by a Hemilabile Chelate}

Heavy pnictide ligands presumably exhibit a strong trans-influence. The presence of a ligand in trans-position would therefore result in a destabilization of the multiple bond. The higher reactivity might lead to unwanted follow-up reactivity and thermal instability. However, the pnictide transfer reagents $\mathrm{PnCO}^{-}(\mathrm{Pn}=\mathrm{P}, \mathrm{As})$ also release carbon monoxide upon triple-bond formation which could also bind to the starting material, inhibiting further reactivity, or destabilize the reaction product. Consequently, a system is to be designed that does not react with carbon monoxide but also allows for the generation of isolable $\mathrm{M} \equiv \mathrm{Pn}$ species. Linkage isomerism is an attractive strategy in this particular case. The system needs to be flexible enough to form stable compounds in monodentate and chelating coordination modes, preferably upon binding to different donor sites of the chelate ligand. Therefore, $3-\left(1 H\right.$-pyrazol-3-yl)-pyridine $\left({ }^{\mathrm{H}} \mathrm{PyrPz}\right)$ was introduced as a possible hemilabile ligand by reaction with $\mathbf{5}$ under basic conditions yielding $\left[\operatorname{ReI}\left(\mathrm{L}^{1}\right)\left(\kappa^{2} N^{1}, N^{3}-\mathrm{PyrPz}\right)\right](\mathbf{1 1}$, Scheme 2.6). 11 exhibits an octahedral coordination mode in the solid state with the iodide in the apical position. The chelate ligand is fully coordinated with the pyrazole unit in trans-position to the iodide ligand which slightly elongates when compared to 5 (Figure 2.12, Table 2.2).

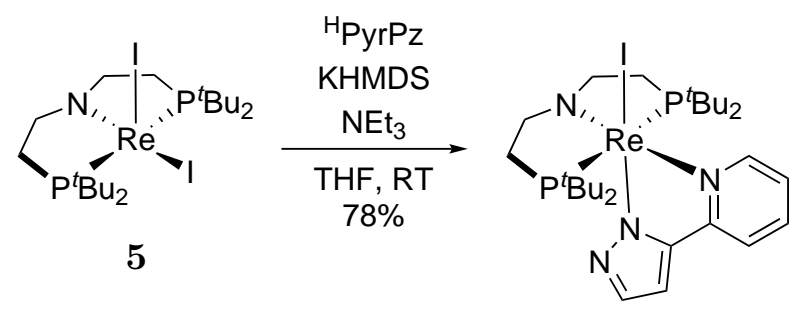

11

Scheme 2.6: Synthesis of $\mathbf{1 1}$ by reaction of $\mathbf{5}$ with ${ }^{\mathrm{H}} \mathrm{PyrPz}$.

In analogy to the $\mathrm{Re}^{\mathrm{III}}$ iodide $\mathbf{1 1}$, a six-coordinate Os complex featuring the $\mathrm{PyrPz}$ ligand could give rise to more selective $\mathrm{Os}=\mathrm{P}(\mathrm{R})$ chemistry. Stirring 1 with ${ }^{\mathrm{H}} \mathrm{PyrPz}$ results in the clean formation of the octahedral $\mathrm{Os}^{\mathrm{III}}$ complex $\left[\mathrm{OsCl}\left(\mathrm{L}^{3}\right)\left(\kappa^{2} N^{1} N^{3}-\mathrm{PyrPz}\right)\right]$ (12, Scheme 4.13) in 90\% isolated yield. 12 features a rhombic EPR signal with pronounced g-anisotropy $\left(g_{x}=1.498, g_{y}=2.095, g_{z}=2.530\right)$ and no resolved hyperfine coupling, in analogy to the values obtained for $\mathbf{1}$ (Scheme 2.13). In the solid state, $\mathbf{1 2}$ features a slightly distorted octahedral coordination geometry. In comparison with $\mathbf{1 1}$, the Os$\mathrm{N}_{\text {PNP }}$ distance is slightly elongated $(\Delta d \approx 0.1 \AA)$ due to the higher d electron count with the remaining bonding parameters being almost identical (Table 2.2). 


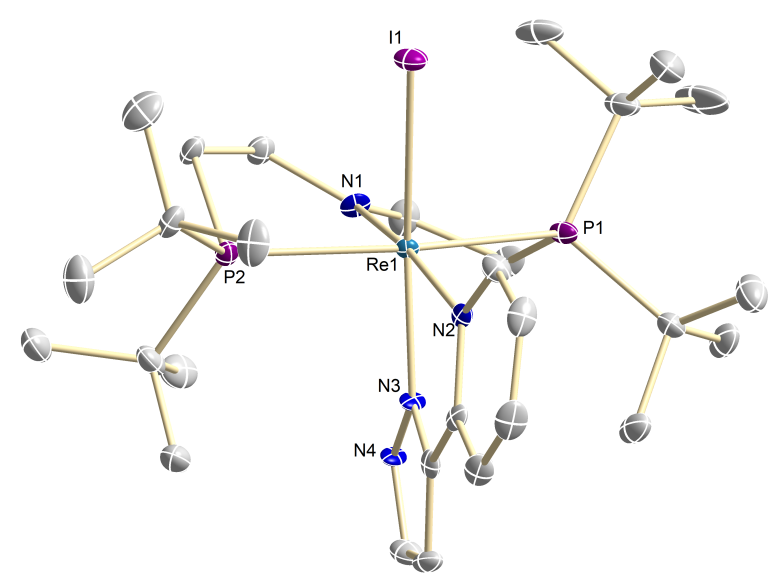

Figure 2.12: Solid state structure of 11 from single-crystal X-ray diffraction (thermal ellipsoids drawn at the $50 \%$ probability level); hydrogen atoms are omitted for clarity. Selected bond lengths $[\AA]$ and angles $\left[^{\circ}\right]$ : 11 Re1-I1 2.7966(4), Re1-N1 1.900(4), Re1-N2 2.213(4), Re1-N3 2.081(4), Re1-P1 2.4532(12), Re1-P2 2.4713(13), N1-Re1-N2 173.58(16), P1-Re1-P2 160.03(4).

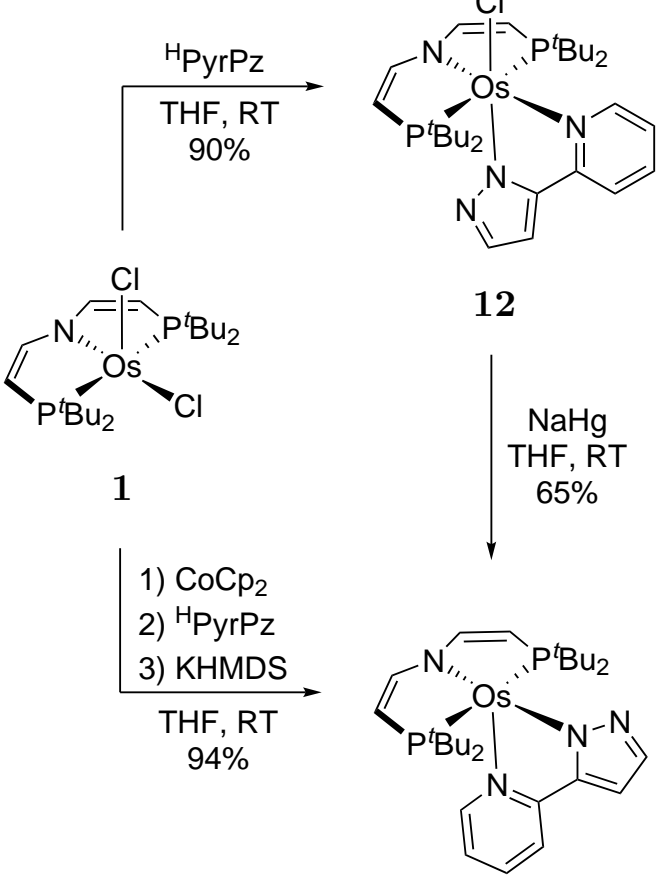

13
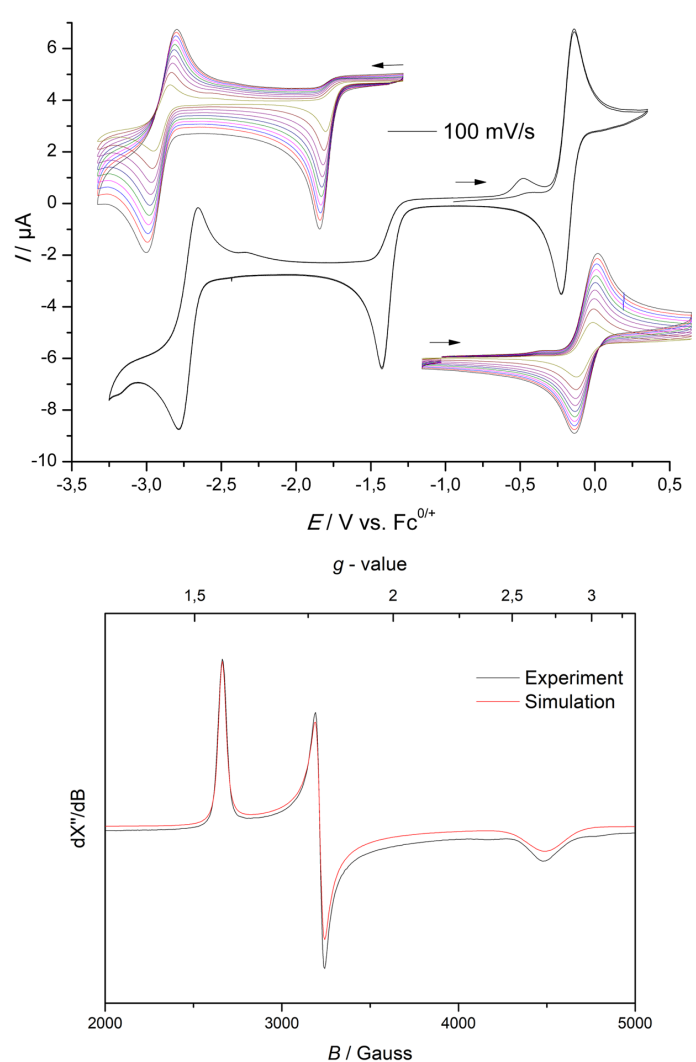

Figure 2.13: Left: Synthesis of 12 and 13 starting from 1, respectively; Right: Cyclic voltammogram (top) of 12, $100 \mathrm{mV} \cdot \mathrm{s}^{-1}, 1 \mathrm{mM}, 0.1 \mathrm{M} \mathrm{NBu}_{4} \mathrm{PF}_{6}$, THF, RT; Inset: Isolated scans of oxidative and reductive regions, $1 \mathrm{~V} \cdot \mathrm{s}^{-1}-100 \mathrm{mV} \cdot \mathrm{s}^{-1}$; EPR spectrum (bottom) of 12, $g_{x}=1.498$, $g_{y}=2.095, g_{z}=2.530$, toluene, $150 \mathrm{~K}, 9.4324 \mathrm{GHz}$ 


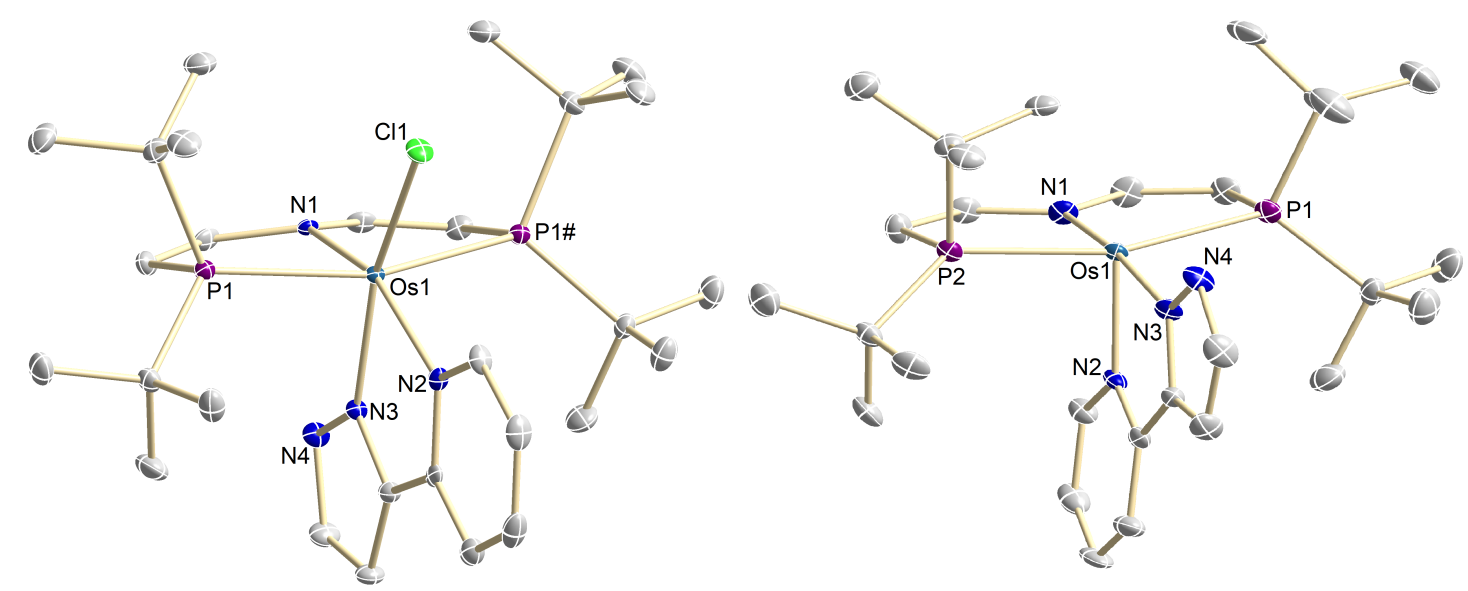

Figure 2.14: Molecular structure of complex 12 (left) and 13 (right) from single-crystal Xray diffraction (thermal ellipsoids drawn at the $50 \%$ probability level); hydrogen atoms are omitted for clarity. Selected bond lengths $[\AA]$ and angles $\left[^{\circ}\right]$ : 12 Os1-N1 2.017(3), Os1-N2 2.161(3), Os1-N3 2.063(3), Os1-Cl1 2.3837(9), Os1-P1 2.4297(6), N1-Os1-N2 157.83(11), P1Os1-P1\# 157.74(3); 13: Os1-N1 2.087(3), Os1-N2 2.041(3), Os1-N3 2.065(3), Os1-P1 2.3937(9), Os1-P2 2.3129(9), N1-Os1-N3 171.21(12), P1-Os1-P2 158.92(3).

Table 2.2: Comparison of the $\mathrm{PyrPz}$ ligated Re and Os Systems 11, 12, 13, and the corresponding dihalide compounds $\mathbf{5}$ and $\mathbf{1}$.

\begin{tabular}{ccccc}
\hline & $\mathrm{M}-\mathrm{N}_{\mathrm{PNP}}$ & $\mathrm{M}-\mathrm{N}_{\text {pyrazole }}$ & $\mathrm{M}-\mathrm{N}_{\text {pyridine }}$ & $\mathrm{M}-\mathrm{X}_{\text {apical }}$ \\
\hline $\mathbf{5}$ & $1.926(2)$ & & & $2.6382(2)$ \\
$\mathbf{1 1}$ & $1.900(4)$ & $2.081(4)$ & $2.213(4)$ & $2.7966(4)$ \\
$\mathbf{1}$ & $1.980(2)$ & & & $2.319(2)$ \\
$\mathbf{1 2}$ & $2.017(3)$ & $2.063(3)$ & $2.161(3)$ & $2.3837(9)$ \\
$\mathbf{1 3}$ & $2.087(3)$ & $2.065(3)$ & $2.041(3)$ & \\
\hline
\end{tabular}

The six-fold coordination environment results in a minor elongation of the $\mathrm{Os}-\mathrm{N}_{\mathrm{PNP}}$ and the $\mathrm{Os}-\mathrm{Cl}_{\text {apical }}$ bond distance. Cyclic voltammetry reveals a reversible oxidation at $E_{1 / 2}=-0.18 \mathrm{~V}$ and an irreversible followed by a pseudo-reversible reductive redox event at $E_{\text {p.c. }} \approx-1.40$ and $E_{1 / 2}=-2.71 \mathrm{~V}$ vs. $\mathrm{Fc}^{0 /+}$ suggesting initial chloride loss upon reduction (Figure 2.13). In fact, one-electron reduction of $\mathbf{1 2}$ with $\mathrm{NaHg}$ results in the clean formation of $\left[\mathrm{Os}\left(\mathrm{L}^{1}\right)\left(\kappa^{2} N^{1} N^{3}-\mathrm{PyrPz}\right)\right]$ (13, Figure 2.13). The complex can alternatively be synthesized in almost quantitative yield by successive addition of $\mathrm{CoCp}_{2}$, ${ }^{\mathrm{H}} \mathrm{PyrPz}$ and KHMDS to $\mathbf{1}$. In the solid state, $\mathbf{1 3}$ features a slightly distorted squarepyramidal coordination sphere (Figure 4.16). The chelate ligand rearranged itself upon reduction with the pyrazole moiety in the equatorial position. The Os- $\mathrm{N}_{\mathrm{PNP}}$ distance is slightly elongated with respect to $\mathbf{1 2}$ due to the increase in oxidation state $(\Delta d \approx$ $0.07 \AA)$. The NMR spectroscopic signals suggest no significant admixture of excited states $\left(\delta_{\mathrm{P}}=24.6, \delta_{\mathrm{H}}=8.42-0.64 \mathrm{ppm}\right)$. 


\subsection{Conclusion}

In summary, the isolation and full characterization of a square-planar $\mathrm{Os}^{\mathrm{II}}$ species was presented. Spectroscopic, crystallographic and magnetic characterization of $\mathbf{2}$ indicates an energetically separated ground state, which is subject to large TIP. A correlated single-reference $a b$ initio formalism on the basis of $\operatorname{CCSD}(\mathrm{T})$ expansions predicts a triplet ground state energetically close to an excited singlet state. Multi-reference computations corroborate the experimental picture and assign an electronic triplet ground state, which is strongly stabilized by mixing with excited states through SOC. Additionally, isoelectronic, five-coordinate Re ${ }^{\mathrm{III}}$ complexes were investigated. The $\mathrm{L}^{3}$ scaffold in combination with iodide ligands enforces strong TIP to occur in the case of Re. Moreover, SQUID measurements show that the observed NMR shifts give no direct indications of the magnitude of this effect.

The synthesis and characterization of a new PNP pincer bipy system was demonstrated. The complexes $\mathbf{7}$ and $\mathbf{8}$ can be isolated in high yield and represent suitable platforms for the synthesis of octahedral Os ${ }^{\mathrm{II}}$ complexes by addition of anionic nucleophiles. Furthermore, one-electron reduction yields the neutral radical complex $\mathbf{9}$ with the electron density mainly located at the bipy ligand which possesses a limited live-time at room temperature. The hydride complex $\mathbf{1 0}$ could be isolated in high yield and represents a promising entry into Os hydride photochemistry based on multiple well-separated absorptions in the visible spectrum.

The hemilabile ligand 3-(1H-pyrazol-3-yl)-pyridine $\left({ }^{\mathrm{H}} \mathrm{PyrPz}\right)$ was utilized to obtain the $\mathrm{Re}^{\mathrm{III}}$ complex 11. Additionally, the Os ${ }^{\mathrm{III}}$ complex 12 was synthesized which can be cleanly converted to the five-coordinate $\mathrm{Os}^{\mathrm{II}}$ species 13 . Both platforms represent interesting entries for the synthesis of multiply bonded pnictide species due to the possible linkage isomerism of the $\mathrm{PyrPz}$ ligand which accounts for the expected strong transinfluence of these ligands. 



\section{Os Nitride and Imide Complexes in Various Coordination Spheres}

Parts of this chapter were adapted from:

J. Abbenseth, S. C. Bete, M. Finger, C. Volkmann, C. Würtele, S. Schneider*, Organometallics 2018, 37, 802-811.

Copyright 2018 American Chemical Society.

\section{Author contributions}

J.A. Synthesis, spectroscopy and theoretical calculations

S.B. Synthesis and spectroscopy (supporting)

M.F. Relaxed surface scan calculation

C.V. Crystallography (Chapter 3.1, supporting)

C.W. Crystallography 


\section{Os Nitride and Imide Complexes in Various Coordina- tion Spheres}

\subsection{Four-and Five-Coordinate Os(IV) Nitrides and Imide Complexes}

Based on the successful isolation of $\mathbf{2}$, the synthesis and follow-up chemistry of squareplanar nitride complexes will be evaluated. The reaction of in situ generated $\mathbf{2}$ with $\left[\mathrm{N}\left(\mathrm{PPh}_{3}\right)_{2}\right] \mathrm{N}_{3}\left([\mathrm{PPN}] \mathrm{N}_{3}\right)$ at room temperature results in an immediate color change from purple to brown. From this mixture the square-planar $\mathrm{Os}^{\mathrm{IV}}$ nitride $\left[\mathrm{OsN}\left(\mathrm{L}^{3}\right)\right]$ (14, Scheme 3.1) can be isolated in over $60 \%$ yield. No intermediate azide complex was detected by NMR or IR spectroscopy indicating rapid dinitrogen elimination after salt metathesis. Alternatively, 1 undergoes clean chloride-azide exchange by reacting with $\mathrm{TMSN}_{3}$ to generate a putative open shell nitride which is subsequently oxidized by the trityl cation, which is not able to oxidize parent $\mathbf{1}$, to generate the $\mathrm{Os}^{\mathrm{VI}}$ nitride $\left[\mathrm{OsNCl}\left(\mathrm{L}^{3}\right)\right]\left[\mathrm{PF}_{6}\right]$ (15, Scheme 3.1). A characterization of intermediate $\left[\mathrm{OsNCl}\left(\mathrm{L}^{3}\right)\right]$ was not targeted since rapid dinitrogen coupling is expected to proceed without oxidant

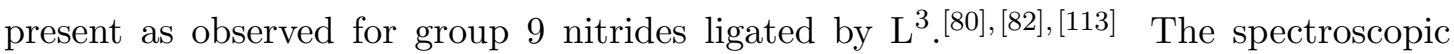
and crystallographic characterization of $\mathbf{1 5}$ confirms the typical square-pyramidal ( $\tau=$ $0.14)^{[202]}$ coordination geometry found for $\mathrm{Os}^{\mathrm{VI}}$ with the nitride ligand in the apical position (Figure 3.1). Treatment of $\mathbf{1 5}$ with an excess of $\mathrm{NaHg}$ allows for the isolation of $\mathbf{1 4}$ in $95 \%$ yield. The cyclic voltammogram features an irreversible redox event at $E_{\text {p.c. }} \approx-1.48 \mathrm{~V}$ (an oxidative event was not observed, Appendix 8.2.2).
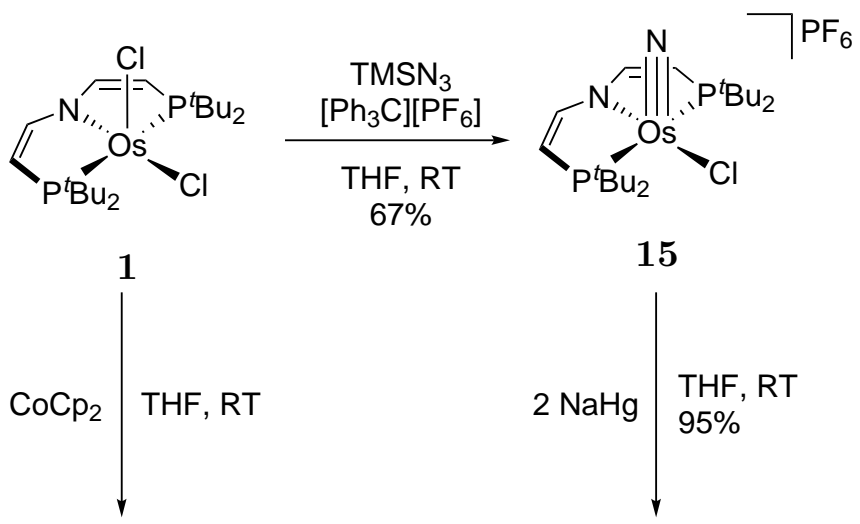

15

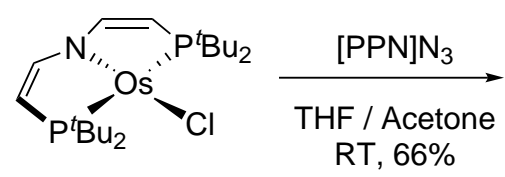

2

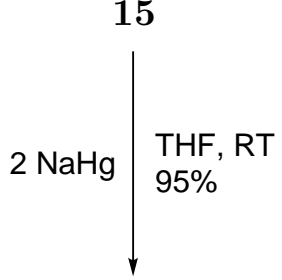

Scheme 3.1: Synthesis of square-planar $\mathrm{Os}^{\mathrm{IV}}$ nitride $\mathbf{1 4}$. 

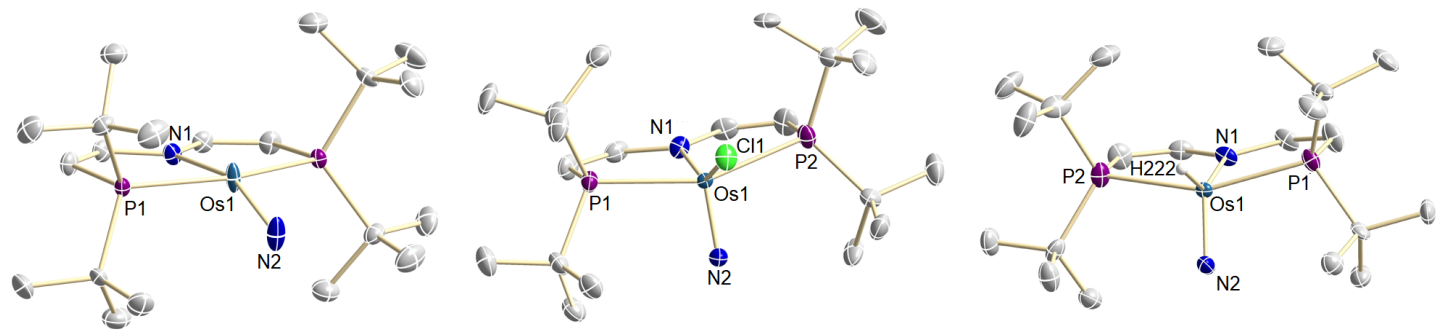

Figure 3.1: Molecular structure of complex 14 (left), 15 (middle) and 16 (right) derived by single-crystal X-ray diffraction (thermal ellipsoids drawn at the $50 \%$ probability level); hydrogen atoms except H222 and anions are omitted for clarity. Selected bond lengths $[\AA]$ and angles $\left[^{\circ}\right]$ : 14 Os1-N1 2.137(3), Os1-N2 1.735(5), Os1-P1 2.3697(7), N1-Os1-N2 152.8(2), P1-Os1-P1\# 158.60(4); 15 Os1-Cl1 2.3277(18), Os1-N1 1.977(3), Os1-N2 1.629(4), Os1-P1 2.4400(12), Os1P2 2.4408(11), N1-Os1-N2 107.55(19), P1-Os1-P2 155.35(4), $\tau=0.14 ; 16$ Os1-H222 1.67(4), Os1-N1 2.029(3), Os1-N2 1.630(2), Os1-P1 2.4118(6), Os1-P2 2.4277(6), N2-Os1-H222 97.2(15), N1-Os1-H222 143.4(15), N1-Os1-N2 119.45(13), P1-Os1-P2 154.54(2), $\tau=0.19$.

Multinuclear NMR characterization of diamagnetic 14 indicates $C_{2 \mathrm{v}}$-symmetry on the NMR timescale at room temperature $\left(\delta_{t} \mathrm{Bu}=1.54 \mathrm{ppm}, 36 \mathrm{H}\right)$. However, the molecular structure in the solid state features a distinctively bent $\mathrm{Os} \equiv \mathrm{N}$ unit with respect to the pincer backbone (N1-Os1-N2: $\left.152.8(2)^{\circ}\right)$. Such bending was also found for the previously reported nitride $\mathbf{X X X V}$, albeit less pronounced (N1-Os1-N2: $\left.168.02(11)^{\circ}\right)$ as well as for the $\mathrm{Ru}^{\mathrm{IV}}$ nitrides $\left[\mathrm{RuN}\left(\mathrm{L}^{\mathrm{Si} / 1}\right)\right]\left(\mathrm{N} 1-\mathrm{Ru} 1-\mathrm{N} 2: 155.86(13) / 165.3(3)^{\circ}\right) .{ }^{[81],[83],[86]}$ In fact, computational analysis of XXXV indicated very flat N-M-N bending potentials as a result of two mutually opposing effects. Bending of the strong donor ligand nitride out of the plane defined by the pincer ligand stabilizes the HOMO with mainly $\mathrm{d}_{\mathrm{z}^{2}}$ character (Scheme 3.3). In turn, the Os $\equiv \mathrm{N}$ triple bond length is slightly weakened due to reduced $\pi$-bonding, which is manifested by the longer nitride bond of 14 (Os1-N2: 1.735(5) $\AA$ ) versus more planar XXXV (Os1-N2: 1.6832(18) $\AA$ ). ${ }^{[83]}$ The nitride bond length is also considerably longer in comparison with Os ${ }^{\mathrm{VI}}$ nitrides such as 15 (Os1-N2: 1.629(4) $\AA$, Figure 3.1) which is attributed to the lower formal oxidation state and trans-influence of the pincer ligand.

The electron rich $\mathrm{Os}^{\mathrm{IV}}$ nitride 14 readily reacts with electrophiles, such as Brønsted acids and boranes. In analogy to $\mathbf{X X X V}$, protonation results in electrophilic attack at the HOMO with mainly $\mathrm{d}_{\mathrm{z}^{2}}$ character (Figure 3.3). Using Brookhart's acid, $\left[\mathrm{H}\left(\mathrm{OEt}_{2}\right)_{2}\right]\left[\mathrm{BArF}_{24}\right]\left(\mathrm{BarF}_{24}^{-}=\mathrm{B}\left(\mathrm{C}_{6} \mathrm{H}_{3}-3,5-\left(\mathrm{CF}_{3}\right)_{2}\right)_{4}^{-}\right),\left[\mathrm{OsNH}\left(\mathrm{L}^{3}\right)\right]\left[\mathrm{BarF}_{24}\right]$ (16, Scheme 3.2 ) can be isolated in over $80 \%$ yield. The hydride ligand of $\mathbf{1 6}$ is detected at $\delta_{\mathrm{H}}=$ $0.47 \mathrm{ppm}$ by ${ }^{1} \mathrm{H}$ NMR spectroscopy. As expected for $\mathrm{Os}^{\mathrm{VI}}$, square-pyramidal osmium coordination $(\tau=0.19)$ is observed in the solid state with the strong donor ligand nitride in the apical position (Figure 3.1). The formal metal oxidation and the vacant site trans to the nitride results in decrease of the metal nitride bond length by more than $0.1 \AA$ 


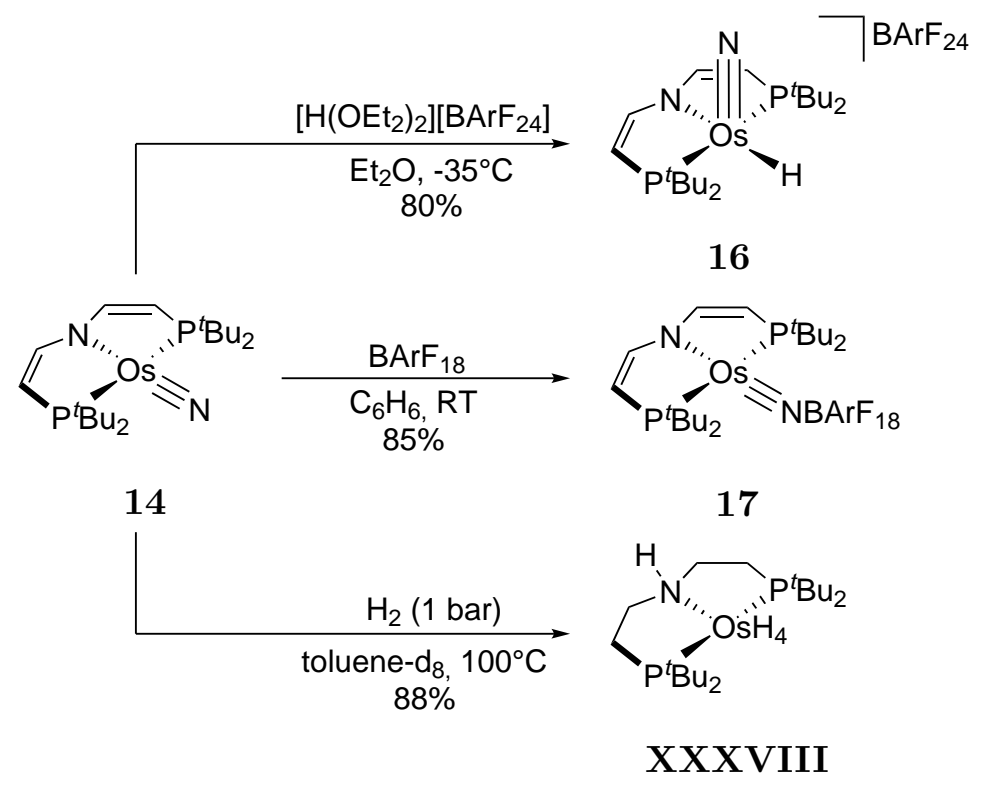

Scheme 3.2: Reactions of 14 with electrophiles to give 16 and 17 and hydrogenolysis of $\mathbf{1 4}$.

(Os1-N2: $1.60(2) \AA$ ) in comparison to 14. In contrast to Brønsted acids, the sterically demanding Lewis acid $\mathrm{B}\left(\mathrm{C}_{6} \mathrm{H}_{3}-3,5-\left(\mathrm{CF}_{3}\right)_{2}\right)_{3}\left(\mathrm{BArF}_{18}\right)$ attacks the lone pair of the nitride ligand and the borane adduct $\left[\mathrm{Os}\left\{\mathrm{NB}\left(\mathrm{C}_{6} \mathrm{H}_{3}-3,5-\left(\mathrm{CF}_{3}\right)_{2}\right)_{3}\right\}\left(\mathrm{L}^{3}\right)\right](\mathbf{1 7}$, Scheme 3.2) was isolated in over $80 \%$ yield. Solution NMR characterization is in agreement with $C_{2 \mathrm{v}^{-}}$ symmetry. The structure is further confirmed by single-crystal X-ray analysis (Figure 3.2). The considerably reduced nitride bending of $\mathbf{1 7}$ (N1-Os1-N2: $\left.174.6(2) / 176.7(2)^{\circ}\right)$ in comparison to parent $\mathbf{1 4}$ is accompanied by slight shortening of the osmium-nitride bond distance $(\Delta d=0.03 \AA)$. In contrast to $\mathbf{X X X V}$, which is selectively silylated at nitride lone pair with TMSBr, the reaction of $\mathbf{1 4}$ with trimethysilyl halides is surprisingly sluggish, giving several products.

In previous work it was demonstrated that hydrogenolysis of $\left[\mathrm{MN}\left(\mathrm{L}^{1}\right)\right](\mathrm{M}=\mathrm{Ru}$, Os $)$ with dihydrogen yields ammonia and polyhydride complexes in high yield. ${ }^{[81], ~[83] ~ C o m-~}$ putational analysis of the $\mathrm{Ru}$ system suggests metal-ligand cooperative $\mathrm{H}_{2}$ heterolysis across the metal-amide bond as the initial step of the reaction sequence. For the divinyl amide ligand $\mathrm{L}^{3}$, not the amine nitrogen but the remote $\mathrm{sp}^{2}$ carbon bound to phosphorus generally represents the basic pincer site. ${ }^{[192],[201]}$ Exposure of $\mathbf{1 4}$ to $\mathrm{H}_{2}$ (1 bar) at elevated temperatures results in clean conversion over $48 \mathrm{~h}$ with release of $\mathrm{NH}_{3}$ in about $88 \%$ yield as determined by indophenolic titration (Scheme 3.2, Figure 3.3). In addition to ammonia, the known tetrahydride XXXVIII is obtained as in the case of XXXV (Appendix 8.2.1). The full hydrogenation of the oxidized ligand $\mathrm{L}^{3}$ to parent amine $\mathrm{HL}^{1}$ suggests cooperative dihydrogen activation also for the unsaturated ligand $\mathrm{L}^{3}$. The reaction was followed by $\mathrm{NMR}$ at $100^{\circ} \mathrm{C}$ to obtain further insights into the 


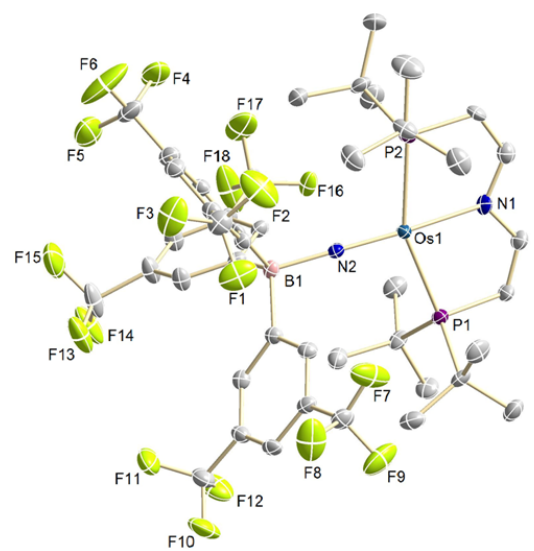

Figure 3.2: Molecular structure of complex 17 derived by single-crystal X-ray diffraction (thermal ellipsoids drawn at the $50 \%$ probability level); hydrogen atoms are omitted for clarity. Selected bond lengths $[\AA]$ and angles $\left[^{\circ}\right]$ : 17 (one of two independent molecules) B1-N2 1.593(9), Os1-N1 2.087(5), Os1-N2 1.700(5), Os1-P1 2.4282(16), Os1-P2 2.3991(17), B1-N2-Os1 173.6(4), N1-Os1-N2 174.6(2), P1-Os1-P2 156.07(6).

reaction mechanism. A fast $1^{\text {st }}$ order decay of the starting material is observed with $k_{\text {obs }}=0.353(3) \mathrm{h}^{-1}$. Over the course of the reaction, two intermediates are observed which feature an asymmetric PNP pincer ligand due to the detected coupling patterns of the PNP phosphorus atoms (Figure 3.3). This can most likely be attributed to partial hydrogenation of the ligand backbone which occurs in several steps. ${ }^{[192]}{ }^{1} \mathrm{H}$ NMR spectroscopy did not give further insights into the structure of the intermediates due to broadened signals at $100^{\circ} \mathrm{C}$. The formed product also exhibits broadened NMR signals at elevated temperatures, even in the ${ }^{31} \mathrm{P}\left\{{ }^{1} \mathrm{H}\right\} \mathrm{NMR}$, due to dynamic processes. ${ }^{[83]}$

Lewis acidic reactivity, such as the addition of a fifth ligand, might at first be anticipated for coordinatively unsaturated 16-valence-electron nitride complexes such as XXXV and 14. However, five-coordinate $\mathrm{d}^{4}$ nitrides are predicted to be unstable by the GB model. ${ }^{[77],[78],[219]}$ In fact, both XXXV and 14 exhibit relatively high-lying LUMOs with mainly $\pi^{*}$-antibonding character with respect to the metal nitride moiety, suggesting weak electrophilicity (Figure 3.3). Accordingly, $\mathrm{PMe}_{3}$ attacks at the nitride ligand rather than the metal center of $\mathbf{X X X V}$, forming an equilibrium with the corresponding phosphoraneiminato complex. ${ }^{[83]}$ However, the addition of phosphines to nitrides is somewhat ambiguous due to synergistic donor/acceptor character of phosphines in the transition state. ${ }^{[220]}$ In contrast to XXXV, complex 14 does not react with phosphines $\left(\mathrm{PMe}_{3}, \mathrm{PPh}_{3}\right)$ or stronger nucleophiles such as Grignard reagents. The apparently reduced electrophilicity of $\mathbf{1 4}$ versus XXXV correlates with a longer osmium-nitride bond and stronger bending of the nitride ligand. This observation suggests that reduced covalency in metal-nitride bonding contributes to the shifted reactivity. 

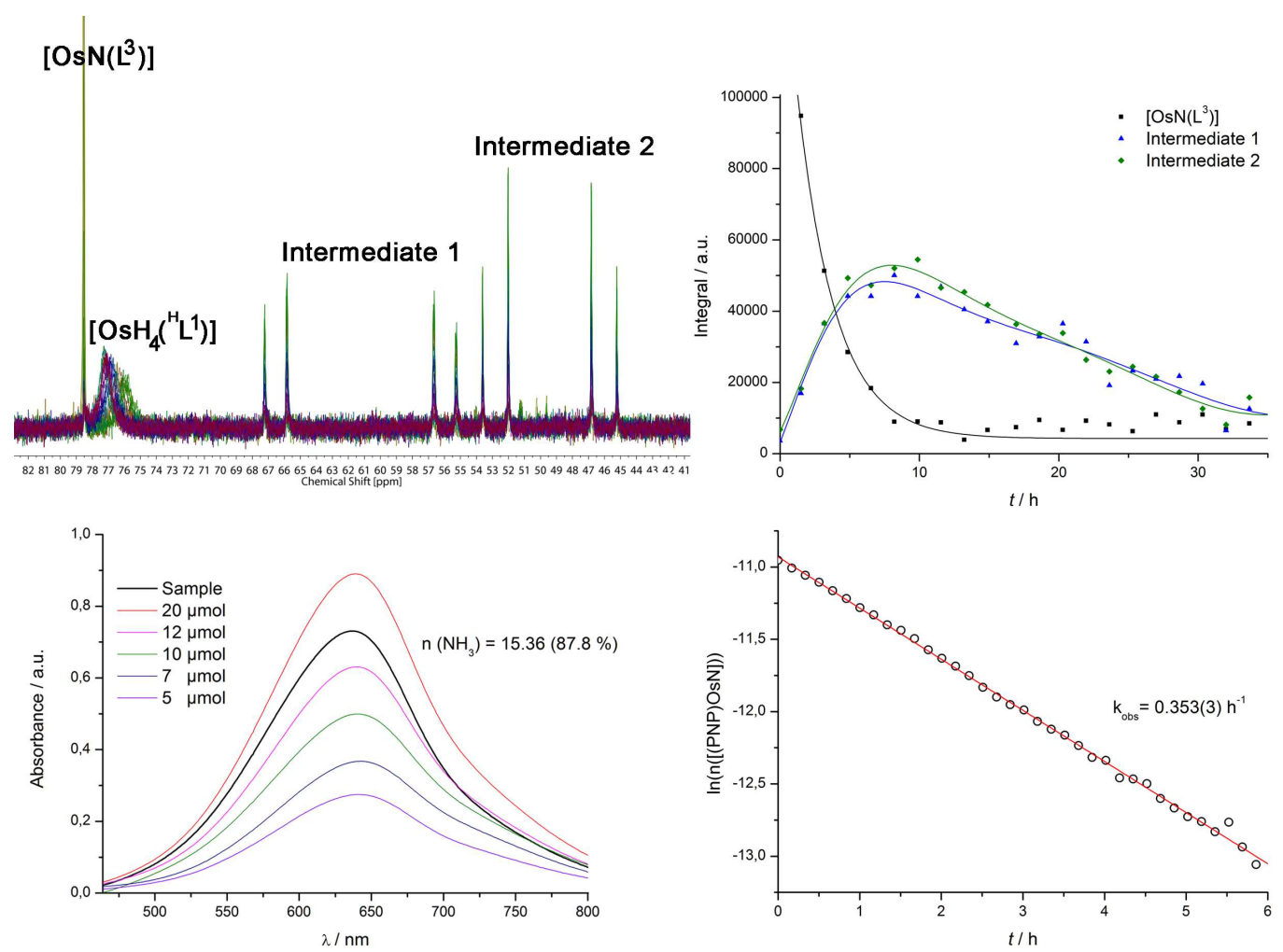

Figure 3.3: Top left: Stacked ${ }^{31} \mathrm{P}\left\{{ }^{1} \mathrm{H}\right\}$ NMR spectra of the hydrogenation of $\mathbf{1 4}$ to XXXVIII, toluene- $d_{8}, 100^{\circ} \mathrm{C}$.; Top right: Integral-time plot of the decay of $\mathbf{1 4}$ and rise and fall of intermediate $1 / 2$ over the course of the reaction; Bottom left: UV/vis spectrum of the indophenolic titration experiment to determine the amount of generated ammonia; Bottom right: $1^{\text {st }}$ order plot of the decay of $\mathbf{1 4}$.

In contrast to phosphines, $\mathbf{1 4}$ immediately reacts with $\mathrm{CN}^{t} \mathrm{Bu}$, even at low temperatures, as indicated by a color change from brown to bright green. Surprisingly, NMR spectroscopic characterization at $-50^{\circ} \mathrm{C}$ and single-crystal $\mathrm{X}$-ray analysis reveal the formation of an unprecedented five-coordinate $\mathrm{Os}^{\mathrm{IV}}$ nitride, the thermally unstable complex $\left[\mathrm{OsN}\left(\mathrm{L}^{3}\right)\left(\mathrm{CN}^{t} \mathrm{Bu}\right)\right](\mathbf{1 8}$, Scheme 3.3). A particular striking structural feature of squarepyramidal $18(\tau=0.03)$ is the basal coordination of the nitride ligand (Figure 3.2). This contrasts with all other square-pyramidal nitrides, which favor axial nitride coordination as a consequence of the strong trans influence. Furthermore, the isonitrile ligand exhibits an unusual tilt (Os1-C21-N3: 165.16(8)/164.27(18) ${ }^{\circ}$. Marked bending within the isonitrile ligand and the distinct bathochromic shift of the $\mathrm{C} \equiv \mathrm{N}$ stretching vibration $\left(\tilde{\nu}_{\mathrm{CN}^{t} \mathrm{Bu}}=2007 \mathrm{~cm}^{-1}\right)$ support significant back-donation by the $\left\{\mathrm{OsN}\left(\mathrm{L}^{3}\right)\right\}$ platform. However, both values and the $\mathrm{C} \equiv \mathrm{N}$ bond lengths (C21-N3: 1.175(3)/1.180(3) $\AA$ ) still favor the isonitrile over the azavinylidene resonance representation. ${ }^{[53],[207]}$ Interestingly, coordination of the isonitrile ligand keeps the nitride bond distance and angle almost unchanged (Os1-N2: 1.6995(18) A, N1-Os1-N2: $\left.152.69(8)^{\circ}\right)$. 


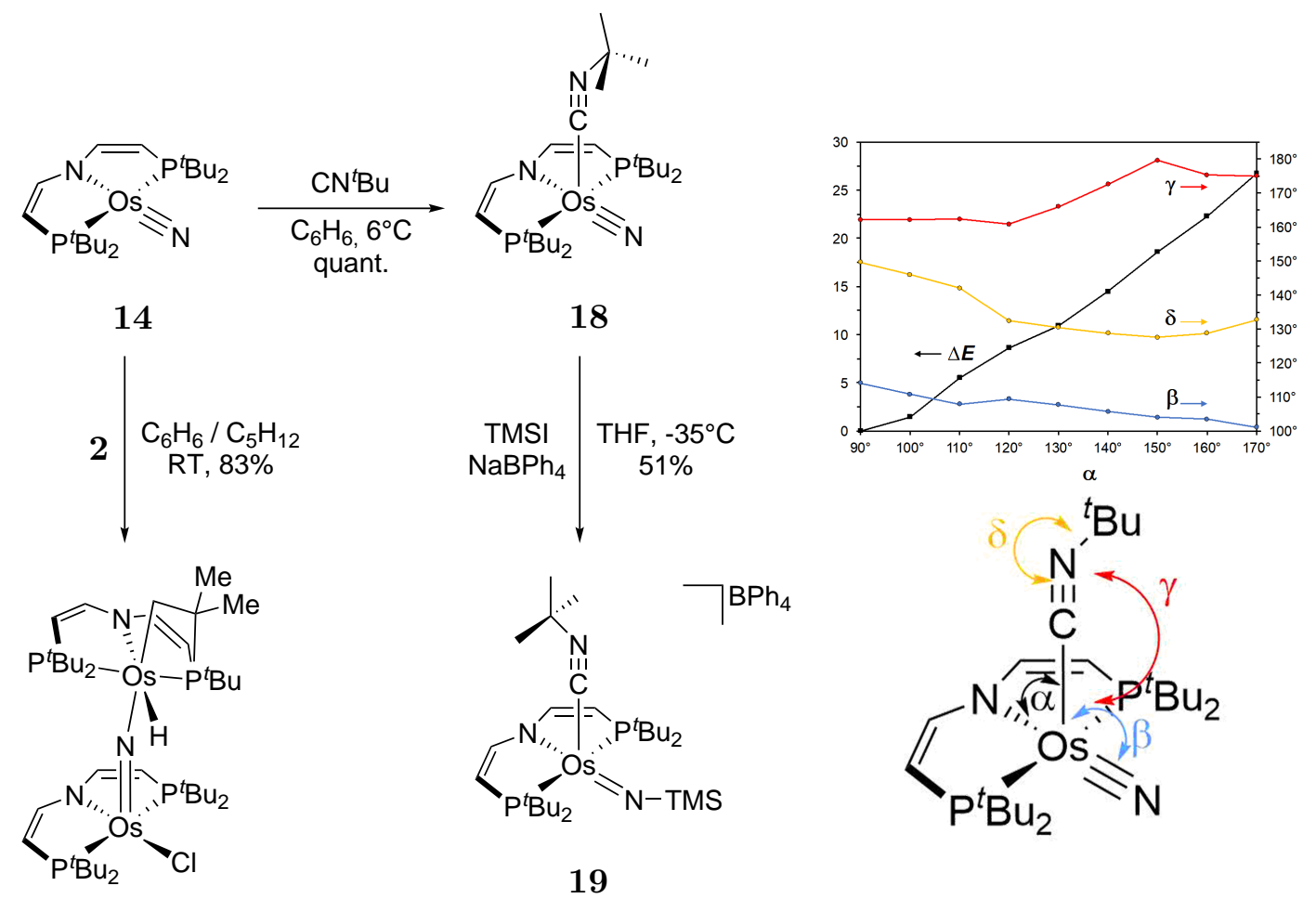

20
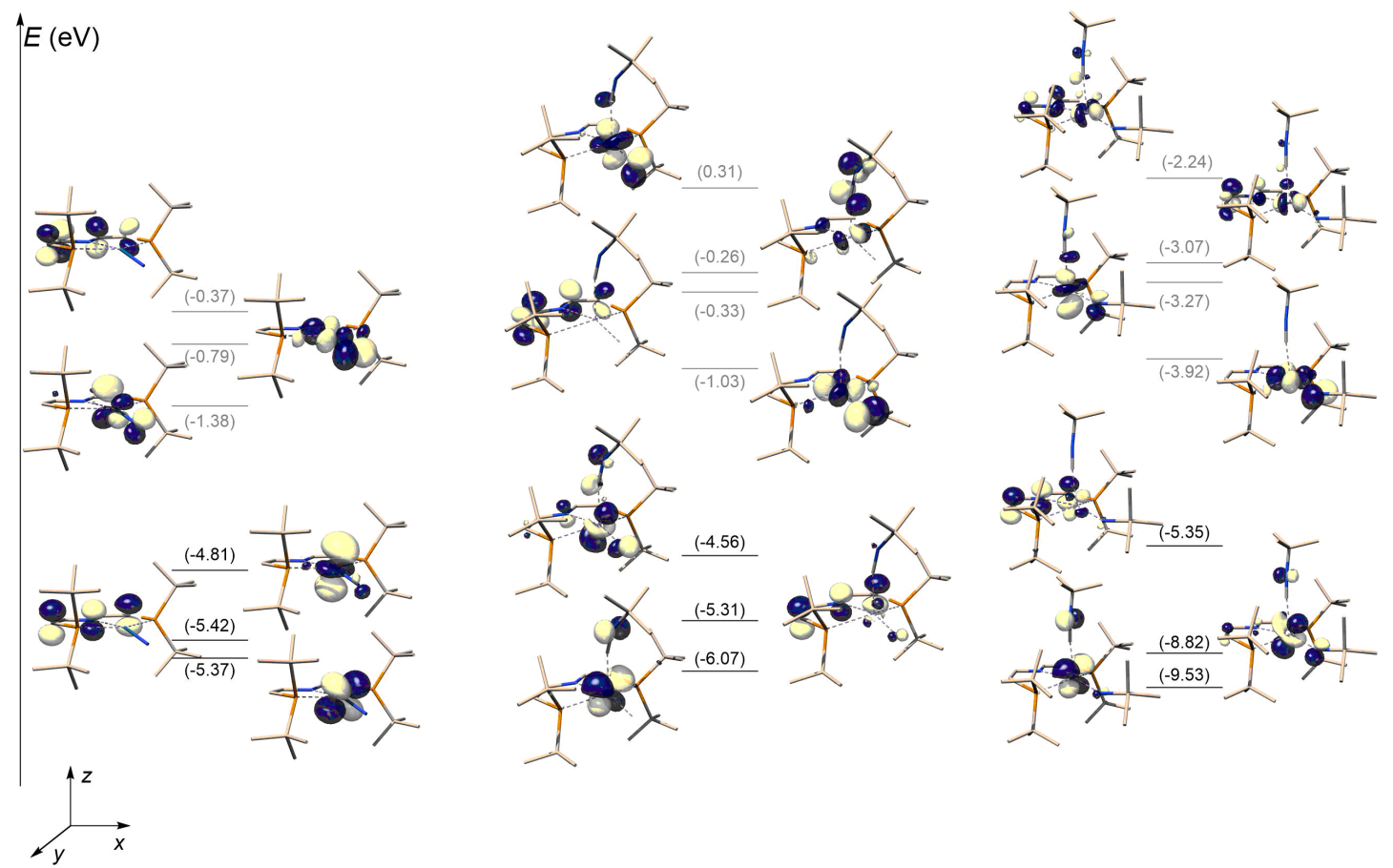

Scheme 3.3: Top left: Syntheses of five-coordinate $\mathrm{Os}^{\mathrm{IV}}$ nitrido $(\mathbf{1 8})$ and imido $(\mathbf{1 9}, \mathbf{2 0})$ complexes; Top-right: Relaxed surface scan for the $\left({ }^{t} \mathrm{BuNC}\right)-\mathrm{Os}-\mathrm{N}_{\text {amide }}$ angle $(\alpha)$ and selected structural parameters (y-axis right), energies given in units of $\mathrm{kcal} \cdot \mathrm{mol}^{-1}$ (y-axis left); Bottom: Computed MO scheme of 14 (left), 18 (middle) and 19 (right). Unoccupied MOs are drawn in grey and the $\mathrm{MO}$ energies are given in $\mathrm{eV}$. 

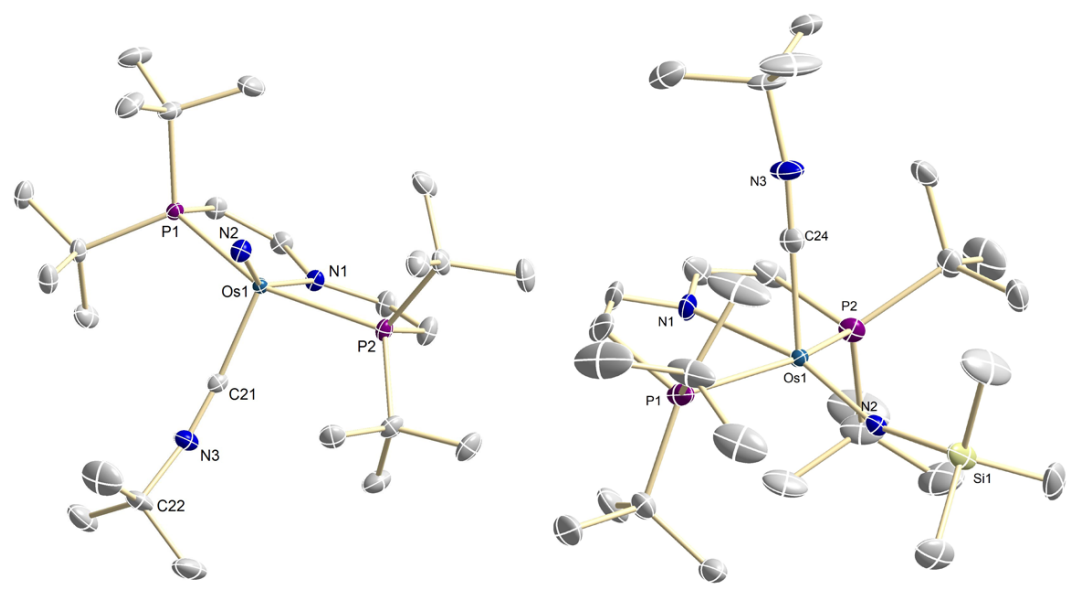

Figure 3.4: Molecular structure of complex 18 (left) and 19 (right) derived by single-crystal X-ray diffraction (thermal ellipsoids drawn at the $50 \%$ probability level); hydrogen atoms are omitted for clarity. Selected bond lengths $[\AA]$ and angles $\left[^{\circ}\right]: \mathbf{1 8}$ (one of two independent molecules) C21-N3 1.175(3), Os1-C21 1.941(2), Os1-N1 2.2423(17), Os1-N2 1.6995(18), Os1-P1 2.4022(5), Os1-P2 2.4027(5), C21-N3-C22 160.7(2), N1-Os1-C21 91.25, N2-Os1-C21 116.05(9), N1-Os1-N2 152.69(8), P1-Os1-P2 150.970(18); 19 C24-N3 1.151(6), Os1-N1 2.237(4), Os1-N2 1.754(4), Os1-C24 1.933(5), Os1-P1 2.4169(12), Os1-P2 2.4232(13), C24-N3-C25 170.9(6), N1Os1-C24 74.38(19), N2-Os1-C24 122.4(2), N1-Os1-N2 163.18(17), Os1-C24-N3 179.0(4), P1-Os1P2 148.28(5), Os1-N2-Si1 156.8(3).

DFT computations were carried out to rationalize the stability and unusual structural features of complex 18. The DFT model reproduces basal nitride and tilted isonitrile coordination (Os1-C21-N3: $162.1^{\circ}$ ). In fact, a local minimum for axial nitride coordination could not be found and a relaxed surface scan reveals a relatively steep energy surface that is around $25 \mathrm{kcal} \cdot \mathrm{mol}^{-1}$ above the ground state for such a structure (Scheme 3.3 right). The computed frontier orbital MO scheme of $\mathbf{1 8}$ indicates considerable mixing of the metal in-plane interactions with the nitride and the isonitrile (Figure 3.3). A more localized bonding picture is provided by natural bond orbital (NBO) analysis (Scheme 3.4). The natural localized molecular orbital (NLMO) that represents the isonitrile/metal $\sigma$-interaction reveals donation of the carbon lone pair into the metal $\mathrm{d}_{\mathrm{xz}}$ orbital. The Os $\equiv \mathrm{N}$ antibonding character of this orbital results in a three-center/fourelectron interaction of the metal with the isocyanide $(\sigma)$ and the nitride $(\pi)$ ligand, in addition to the two cleanly defined 2c2e $\sigma$ - and $\pi$-Os $\equiv \mathrm{N}$ bonds. However, this geometry also enables back-donation from the high-lying $\mathrm{d}_{\mathrm{z}^{2}}$ orbital into the isonitrile $\pi^{*}$-acceptor MO. Within this bonding picture, the isonitrile-tilt is a consequence of avoiding an unfavorable $\sigma$-overlap with the filled $\mathrm{d}_{\mathrm{z}^{2}}$ orbital but instead favorable back-donation from this orbital. Consequently, weak multicenter isonitrile $\sigma$-bonding results, which is counterbalanced by strong back-donation. Therefore, it is fully consistent that the weakest $\sigma$-donor and strong $\pi$-acceptor isonitrile coordinates apically, rather than the nitride ligand as in case of $\mathrm{d}^{2}$ nitride complexes. These considerations suggest that such a five- 
coordinate $\mathrm{d}^{4}$ nitrides that are formally situated beyond the "oxo wall" can be stabilized with a weak $\sigma$-donor/strong $\pi$-acceptor ligand.
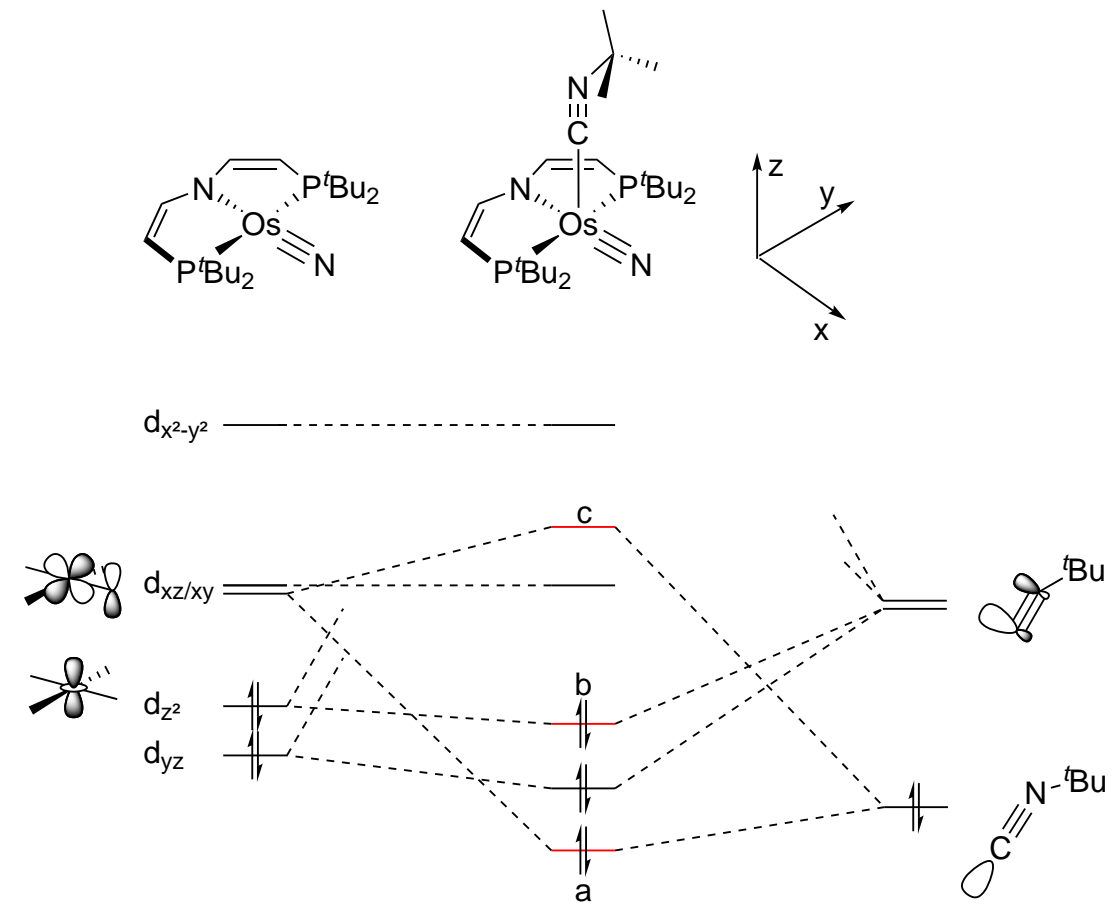

a)
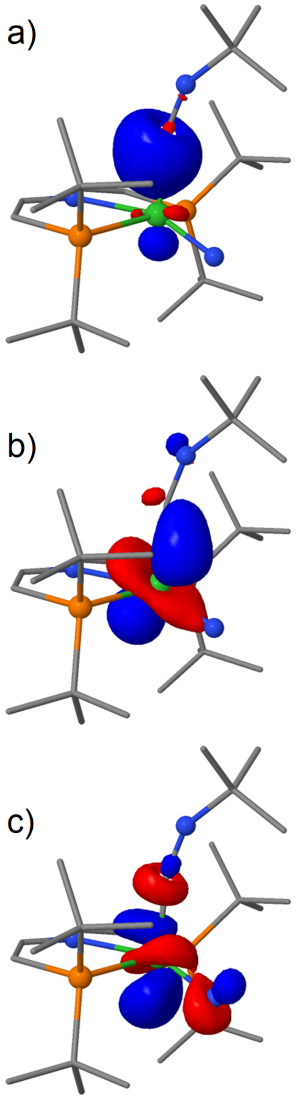

Scheme 3.4: Left: Qualitative MO interactive diagram of 14 with the isonitrile ligand; Right: NLMO representation of the $\sigma$ - (top) and $\pi$-bonding (middle) interactions and the antibonding $\sigma$-interaction (bottom) of the metal with the isonitrile.

In comparison to parent $\mathbf{1 4}$, five-coordinate $\mathbf{1 8}$ exhibits much cleaner reactivity with silyl halide electrophiles. NMR spectroscopic monitoring reveals selective in situ nitride silylation with TMSI. The silylimide complex $\left[\mathrm{Os}(\mathrm{NTMS})\left(\mathrm{L}^{3}\right)\left(\mathrm{CN}^{t} \mathrm{Bu}\right)\right]\left[\mathrm{BPh}_{4}\right](\mathbf{1 9}$, Scheme 3.3) can be isolated in around $50 \%$ yield after anion exchange with $\mathrm{NaBPh}_{4}$. In contrast to nitride $\mathbf{1 8}$, the five-coordinate $\mathrm{Os}^{\mathrm{IV}}$ imido complex is thermally stable at room temperature in both solution and solid state. Monomeric $\mathrm{d}^{4}$ imido complexes are rare, due to the same general considerations discussed for nitrides. ${ }^{[196],[221]}$ Meyer and coworkers structurally characterized octahedral $\mathrm{Os}^{\mathrm{IV}}$ imide complexes upon addition of cyanide, azide, amines or thiols to the respective Os ${ }^{\mathrm{VI}}$ nitride. ${ }^{[222]-[225]}$ Electron delocalization resulting from the heterocumulene character of these ligand probably contributes to their stability. Furthermore, they exhibit distinct bending of the $\mathrm{M}=\mathrm{N}-\mathrm{R}$ moiety, which evades the in-plane $\pi$-bonding conflict with the low-valent metal center. 
In contrast, simple five- or six-coordinate $\mathrm{d}^{4}$ alkyl-, aryl-, or silylimides have not been characterized to date, rendering 19 the first example, but might be intermediates in the addition of nucleophiles to electrophilic $\mathrm{d}^{2}$ nitrides. ${ }^{[92],[93],[226],[227]}$ NMR spectroscopic characterization of $\mathbf{1 9}$ is in agreement with a $C_{\mathrm{S}^{-}}$symmetric structure in solution. The molecular structure in the solid state confirms slightly more distorted square-pyramidal geometry in comparison with 18 (Figure 3.2, $\tau=0.25$ ). However, the overall relatively small structural perturbations that result from nitride silylation are reflected in the similarities of the computed frontier molecular orbital scheme (Scheme 3.3). The distinct tilt of the isocyanide ligand is not observed for 19 (Os1-C24-N3: 179.0(4) ${ }^{\circ}$, suggesting reduced $\mathrm{d}_{\mathrm{z}^{2}} \rightarrow \pi^{*}(\mathrm{C} \equiv \mathrm{N})$ back-donation. This interpretation is supported by enhanced linearity of the isonitrile ligand (C24-N3-C25: $\left.170.9(6)^{\circ}\right)$, a slightly shortened $\mathrm{C} \equiv \mathrm{N}$ bond $\left(\mathrm{C} 24-\mathrm{N} 3:\right.$ 1.151(6) $\AA$ ) and a hypsochromic shift of the $\mathrm{C} \equiv \mathrm{N}^{t} \mathrm{Bu}$ stretching vibration by about $\Delta \tilde{\nu}=120 \mathrm{~cm}^{-1}$ in the IR spectrum. Instead, the isocyanide ligand of 19 exhibits an incline towards the amide ligand (N1-Os1-C24: $\left.74.38(19)^{\circ}\right)$ to maintain isonitrile $\sigma$-donation within the ${ }^{t} \mathrm{BuNC}-\mathrm{Os} \equiv \mathrm{N} \sigma / \pi 3 \mathrm{c} 4 \mathrm{e}$ interaction as detailed for 18 . Furthermore, bending of the imido moiety (Os1-N2-Si1: $\left.156.8(3)^{\circ}\right)$ reduces the in-plane $\pi$-interaction of the metal with the nitride accompanied by elongation of the metal nitride bond (Os1-N2: 1.754(4) $\AA$ ). The unusual basal imide coordination is therefore again attributed to a preference for the weakest $\sigma$-donor and strongest $\pi$-acceptor to coordinate in the apical position.

Another way of avoiding $\pi$-bonding conflicts of low valent $\mathrm{Os}^{\mathrm{IV}}$ in five-fold coordination geometry with a nitride ligand is represented by forming metalloimido species. The Schneider group recently reported the formation of nitride bridged ruthenium complexes featuring the ligands $\mathrm{L}^{1}$ and $\mathrm{L}^{3}$. In this report it was discussed that double bonding of the nitride ligand to both metal centers effectively prevents repulsive $\pi$-interactions which has also been proposed for a few other ruthenium and osmium $\mu$-nitrido species. ${ }^{[228]-[230]}$ However, a detailed bonding analysis of such species is yet to be done.

The reaction of a freshly prepared solution of $\mathbf{2}$ with either an equimolar amount of 14 or 0.5 equivalents of $\mathrm{TMSN}_{3}$ allows for the isolation of the bright green bridging nitride $\left[\left(\mathrm{L}^{3}\right) \mathrm{ClOs}(\mu-\mathrm{N}) \mathrm{OsH}\left\{\mathrm{N}\left(\mathrm{CHCHP}^{t} \mathrm{Bu}_{2}\right)\left(\mathrm{CHCHP}^{t} \mathrm{BuCMeCH}_{2}\right)\right\}\right]$ (20, Scheme 3.3) in over $80 \%$ yield after column chromatography. In contrast to the analogous ruthenium platform, ${ }^{[228]}$ spectroscopic and crystallographic characterization of $\mathbf{2 0}$ reveals cyclometallation of one of the $\mathrm{P}^{t} \mathrm{Bu}$ substituents, resulting in an $\left\{\mathrm{Os}^{\mathrm{IV}}(\mu-\mathrm{N}) \mathrm{Os}^{\mathrm{IV}}\right\}$ core that is close to linearity (Figure 3.5, Os1-N3-Os2: $170.11(16)^{\circ}$ ). The significant higher metal basicity of $5 \mathrm{~d}$ over $4 \mathrm{~d}$ metals is most likely responsible for the observed structural differences. The two Os-PNP fragments are twisted toward each other by about $90^{\circ}$ as a result of the bulky phosphine substituents. Computational examination supports the $\mathrm{Os}^{\mathrm{IV} / \mathrm{IV}}$ redox state assignment. 


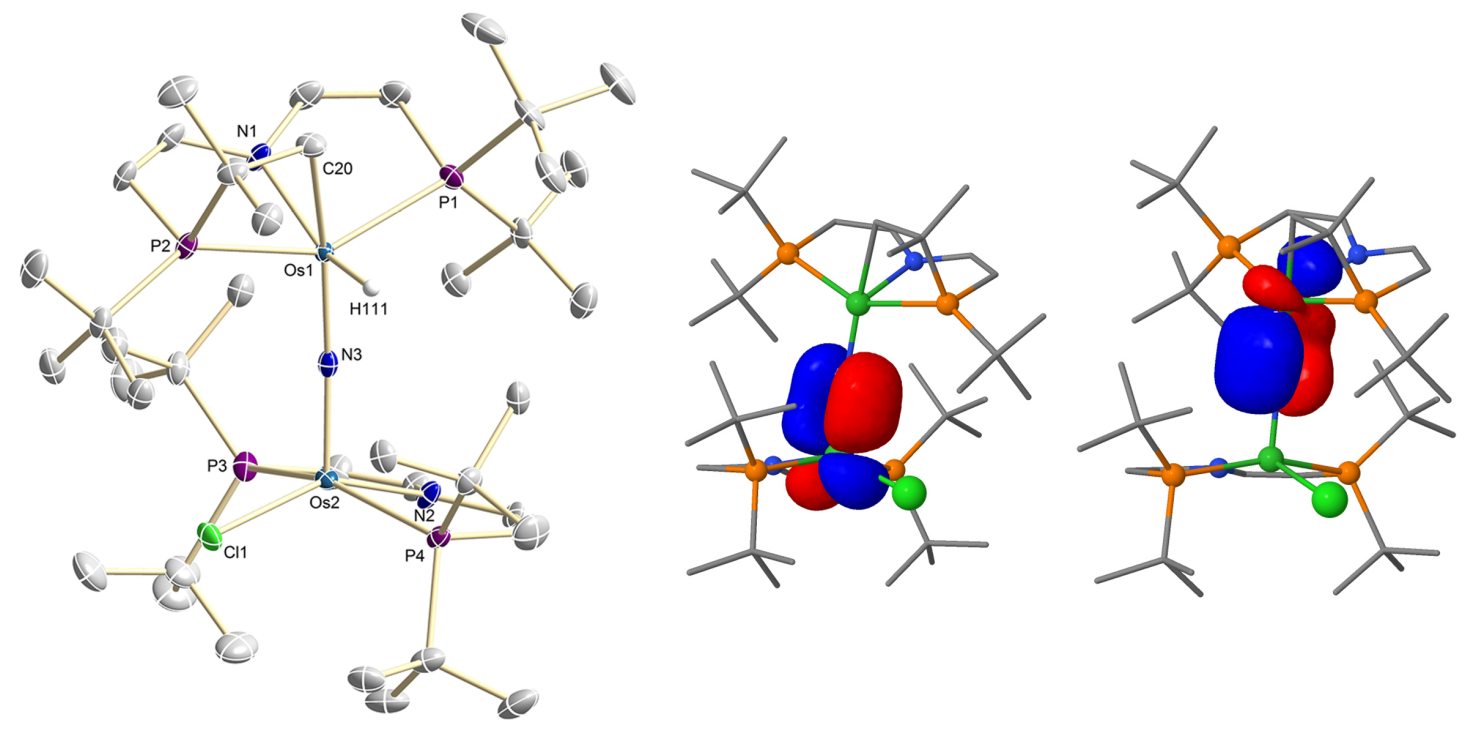

Figure 3.5: Left: Molecular structure of complex 20 from single-crystal X-ray diffraction (thermal ellipsoids drawn at the $50 \%$ probability level); hydrogen atoms except the hydride ligand are omitted for clarity. Selected bond lengths $[\AA]$ and angles $\left[{ }^{\circ}\right]$ : 20 Os1-H111 1.53(4), Os1N1 2.126(3), Os1-N3 1.839(3), Os1-C20 2.220(3), Os1-P1 2.3778(8), Os1-P2 2.4502(8), Os2-N2 2.034(4), Os2-N3 1.796(3), Os2-Cl1 2.3648(9), Os2-P3 2.4271(19), Os2-P4 2.4404(9); Os1-N3Os2 170.11(16), P2-Os1-P1 150.09(3), P3-Os2-P4 155.95(4); Right: NBO representation of the Osmium/nitride $\pi$-bonding interactions in $\mathbf{2 0}$.

The computed frontier orbitals show a high degree of interaction between the metal center resulting in rather complex orbitals which cannot be easily assigned in terms of simple bonding considerations (Appendix 8.2.3). Therefore, NBO analysis was again employed to get insight into the bonding situation of the $\{$ OsNOs\} core. NBO analysis features one $\pi$-bond of the bridging nitride with each osmium center, which are orthogonal to each other (Figure 3.5). Consequently, the nitride effectively serves as a single-faced $\pi$-donor or metalloimido four-electron donor ligand for both Os fragments. As a result, the $\mathrm{d}^{4}$ configuration is easily sustained for five- and six-coordinate geometry and the $\mu$-nitrido ligand occupies the apical site of the square-pyramidal fragment. 


\subsection{Bipyridine Ligated Os Nitride Complexes}

Since square-planar $\mathbf{2}$ does not allow for the substitution of the chloride ligand to give stable azides and exhibited no discrete redox chemistry, 2,2'-bipyridine (bipy) is chosen as a spectator ligand with the potential of high $\pi$-backbonding or even charge transfer to stabilize open-shell nitrides. Reacting 7 with $\mathrm{TMSN}_{3}$ results in the formation of $\left[\mathrm{Os}\left(\mathrm{L}^{3}\right)\left(\mathrm{N}_{3}\right)\right.$ (bipy)] (21, Scheme 3.5) in 85\% isolated yield. The NMR resonances are in line with a $C_{\mathrm{S}}$-symmetric species in solution and are only minorly shifted with respect to 7 as expected for a pseudohalogenide complex. Successful addition of azide is confirmed by IR spectroscopy $\left(\tilde{\nu}_{\mathrm{N}_{3}}=2072 \mathrm{~cm}^{-1}\right)$ and single crystal X-ray crystallography (Figure $3.6)$

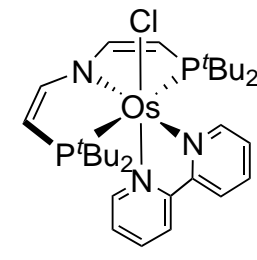

7

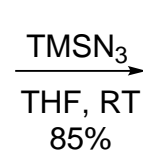

$85 \%$

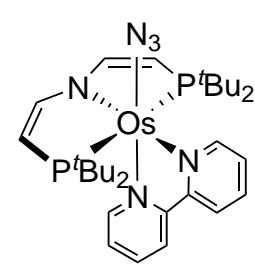

21

Scheme 3.5: Synthesis of 21 from 7 by ligand exchange.

The solid-state structure exhibits almost identical bonding parameters between metal and bipy/PNP ligand with respect to 7 and a slightly bent azide ligand which shows equivalent N-N distances (N4-N5: 1.179(4) $\AA$, N5-N6: 1.177(4) $\AA$, N4-N5-N6: 175.9(3) ${ }^{\circ}$. $\mathbf{2 1}$ is stable at room temperature and does not show any sign of decomposition when exposed to light in solution or in the solid state. Irradiation of $\mathbf{2 1}$ should lead to release

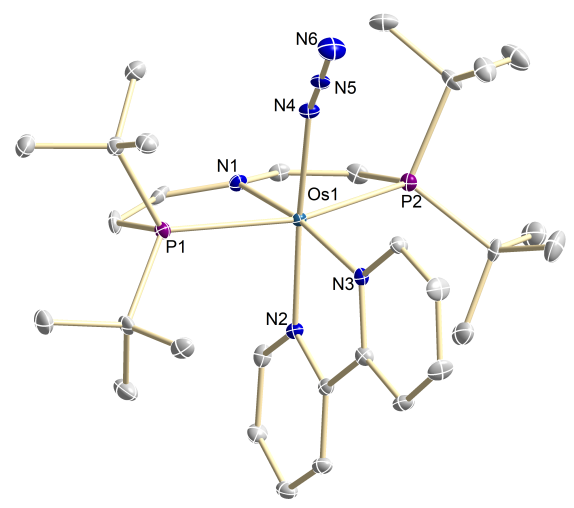

Figure 3.6: Molecular structure of $\mathbf{2 1}$ from single-crystal X-ray diffraction (thermal ellipsoids at the $50 \%$ probability level); hydrogen atoms are omitted for clarity. Selected bond lengths $[\AA]$ and angles $\left[{ }^{\circ}\right]$ : 21 Os1-N1 2.110(2), Os1-N2 2.032(3), Os1-N3 2.064(2), Os1-N4 2.112(3), Os1-P1 2.4627(8), Os1-P2 2.4329(8), N4-N5 1.179(4), N5-N6 1.177(4), N1-Os1-N4 98.95(10), P1-Os1-P2 158.44(3), Os1-N4-N5 142.3(2), N4-N5-N6 175.9(3). 
of dinitrogen and formation of an octahedral $\mathrm{Os}^{\mathrm{IV}}$ nitrene complex which is not expected to be isolable due to the high d electron count. Nitride coupling and formation of $\mathbf{9}$ could be a possible follow-up reaction pathway. However, irradiation experiments yielded no isolable reaction products to be detected by NMR or EPR spectroscopy (Appendix 8.2.4). This indicates that the photo-product is too reactive to be accurately studied. The cyclic voltammogram of $\mathbf{2 1}$ shows a reversible oxidative wave at $E_{1 / 2}=-0.74 \mathrm{~V}$ and a second irreversible oxidative wave at $E_{\text {p.a. }} \approx 0.21 \mathrm{~V}$ vs. $\mathrm{Fc}^{0 /+}$ which suggest that $\mathrm{Os}^{\mathrm{V} / \mathrm{VI}}$ nitride species could be isolable upon oxidation of $\mathbf{2 1}$ (Appendix 8.2.5).

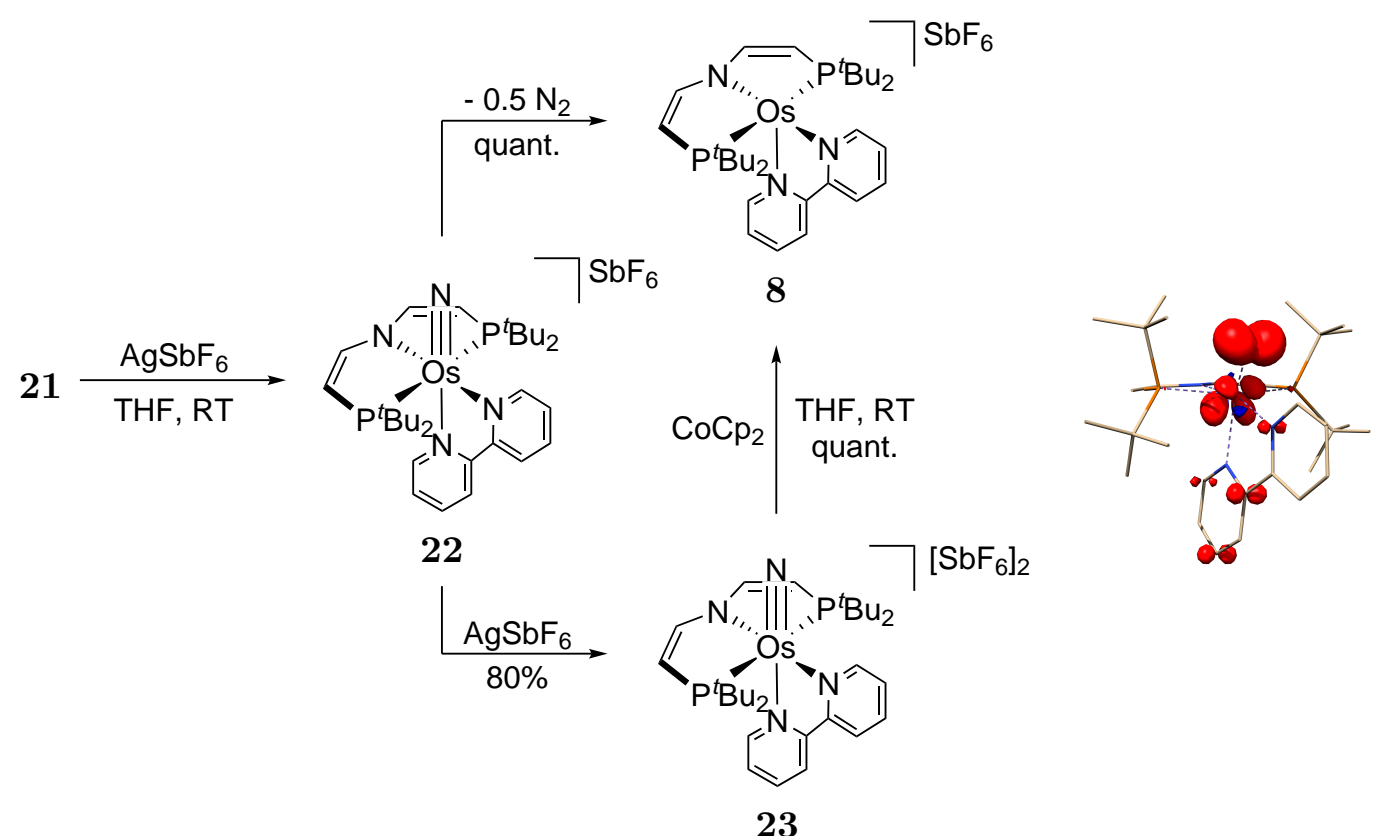

Scheme 3.6: Left: Fate of the transient open-shell nitride 22; Right: Spin-density of 22.

An instant color change from red to dark brown is detected accompanied by silver precipitation when $\mathbf{2 1}$ is oxidized by one equivalent of $\mathrm{AgSbF}_{6}$. NMR spectroscopy reveals 8 to be the sole reaction product (Scheme 3.6, Appendix 8.2.7). Since the starting material is stable in solution, oxidation most likely triggers dinitrogen extrusion to yield the open-shell $\mathrm{Os}^{\mathrm{V}}$ nitride species $\left[\mathrm{OsN}\left(\mathrm{L}^{3}\right)(\right.$ bipy $\left.)\right]\left[\mathrm{SbF}_{6}\right](\mathbf{2 2})$. As stated in the introduction, these species are prone to undergo oxidative nitride coupling towards dinitrogen complexes. Following the reaction at lower temperatures by NMR or EPR did not give any further information about possible intermediates of this reaction indicating rapid coupling reactivity of $\mathbf{2 2}$. Reacting $\mathbf{2 1}$ with two equivalents of $\mathrm{AgSbF}_{6}$ results in the formation of a $C_{\mathrm{S}}$-symmetric species, accompanied by minor formation of an asymmetric side product and organic impurities, as indicated by NMR spectroscopy (Appendix 8.2.6). The procedure is highly sensitive to the scale of the reaction and could only be performed on a $6.7 \mu \mathrm{M}$ scale in acceptable purity which prevented full characteriza- 
tion. However, mass-spectrometry suggests that $\left[\mathrm{OsN}\left(\mathrm{L}^{3}\right)(\right.$ bipy $\left.)\right]\left[\mathrm{SbF}_{6}\right]_{2}\left(\mathbf{2 3}, \frac{m}{z}=359.1\right.$ $\left.\left[\mathrm{M}^{2+}\right]\right)$ is formed. Prolonged standing in solution or heating did not result in any further conversion of 23. The addition of stochiometric amounts of $\mathrm{CoCp}_{2}$ gives $\mathbf{8}$ quantitatively (Appendix 8.2.7). This results reaffirms the presence of $\mathbf{2 2}$ prior to dinitrogen coupling. DFT computations of $\mathbf{2 2}$ provide a possible explanation for the observed reactivity. The SOMO is represented by an Os- $\mathrm{N} \pi^{*}$-orbital with $60 \%$ and $9.2 \%$ spin-density located at the nitride nitrogen and the osmium center, respectively (Scheme 3.6). Although there are reasonable arguments for the existence of $\mathbf{2 2}$, the experimental results lack a direct spectroscopic observation of the compound. The problems associated with isolating $\mathbf{2 3}$ prevented an alternative access via reduction. Based on these results the system was not further investigated in terms of kinetics of homo-/hetero- coupling reactions of $\mathbf{2 2} / \mathbf{2 3}$. 


\subsection{Conclusion}

In summary, this chapter exemplarily showcases different strategies to stabilize lowvalent transition-metal nitrido and imido complexes. Terminal nitrides of $\mathrm{d}^{4}$ ions such as $\mathrm{Os}^{\mathrm{IV}}$ in square-pyramidal or octahedral geometry are formally beyond the "nitrido wall" defined by the GB bonding model. Population of the M-N $\pi^{*}$-antibonding molecular orbitals renders them inherently unstable. This $\pi$-conflict can be avoided by reduction of the coordination number. In analogy to $\mathrm{Os}^{\mathrm{IV}}, \mathrm{Ru}^{\mathrm{IV}}$ and $\mathrm{Ir}^{\mathrm{V}}$ nitrides with bulky pincer ligands, the four-coordinate nitride complex $\mathbf{1 4}$ can be synthesized. Nucleophilic reactivity of the metal and the nitride is observed with Brønsted and Lewis acids. Nitride hydrogenolysis gives ammonia and the tetrahydride XXXVIII in high yield. Surprisingly, the addition of $\mathrm{CN}^{t} \mathrm{Bu}$ allows for the isolation of the five-coordinate, thermally labile $\mathrm{Os}^{\mathrm{IV}}$ nitride 18. The highly unusual preference of the isonitrile instead of the strong donor nitride for axial coordination is attributed to the high metal valence electron count and the unexpected binding mode of the isocyanide. On one hand, tilting about the Os-C-N angle allows for stabilization of the filled $\mathrm{d}_{\mathrm{z}^{2}}$ orbital by $\pi$-backdonation. On the other hand, mixing of the isonitrile lone pair with the in-plane Os $\equiv \mathrm{N} \pi$-interaction results in an overall stabilizing $3 \mathrm{c} 4 \mathrm{e}$ interaction. This synergistic binding mode explains why the strong $\pi$-acceptor and comparatively weak $\sigma$-donor isocyanide is placed in the axial position. Nitride silylation further stabilizes this compound and allows for the isolation of the first $\mathrm{d}^{4}$ silylimido complex beyond four-coordination. Electrophilic attack of low-valent nitrides provides a new synthetic route to such electron-rich imidos. In analogy to the five-coordinate nitride $\mathbf{1 8}, 3 \mathrm{c} 4 \mathrm{e}$ bonding of the isonitrile in combination with reduced in-plane $\pi$-donation by imide bending stabilizes silylimide 19. Finally, stabilization arises from binding of two low-valent metal ions. The bridging nitride ligand within the $\left\{\mathrm{Os}^{\mathrm{IV}}=\mathrm{N}=\mathrm{Os}^{\mathrm{IV}}\right\}$ core of dinuclear complex 20 forms one $\pi$-bond with each metal ion. The resulting Os-N double bond character also avoids $\pi$-bonding conflicts. Accordingly, the metalloimido ligand is in the apical position of the $\mathrm{OsCl}(\mathrm{PNP})$ platform, as is usually observed for high-valent group 8 nitrido and imido complexes.

The azide complex $\mathbf{2 1}$ was obtained which could be oxidized by one and two electrons to give transient $\mathbf{2 2}$, which exhibits rapid nitride coupling reactivity, and stable the nitride complex 23, respectively. Kinetics of homo-/hetero-coupling reactions could not be obtained since clean complex synthesis was hampered by low selectivity at increased reaction scales. 



\title{
Transition Metal Complexes with Metal-Pnictogen Multiple Bonds
}

\author{
Parts of this chapter were adapted from:
}

J. Abbenseth, M. Diefenbach, A. Hinz, L. Alig, C. Würtele, J. M. Goicoechea*, M. C. Holthausen*, S. Schneider*, 2019, 58, 10966-10970.

J. Abbenseth, D. Delony, M. C. Neben, C. Würtele, B. de Bruin*,

S. Schneider*, Angew. Chem. Int. Ed. 2019, 58, 6338-6341.

with permission from WILEY-VCH Verlag GmbH \& Co. KGaA, Weinheim

\section{Author contributions}

J.A. Synthesis, spectroscopy, crystallography $(\mathbf{2 5}, \mathbf{3 1}, 34$ and 44) and theoretical caluclations (Chapter 4.2 and 4.4)

M.D. Theoretical calculations (Chapter 4.1, supporting)

A.H. Synthesis of $\left[\mathrm{Na}(\operatorname{diox})_{\mathrm{x}}\right][\mathrm{PnCO}]$

L.A. Crystallography (24, supporting)

C.W. Crystallography

D.D. Isothermal titration calorimetry

M.N. Synthesis and spectroscopy (Chapter 4.3, supporting)

B.B. Theoretical Calculations (Chapter 4.3)

M.H. Theoretical Calculations (Chapter 4.1) 


\section{Transition Metal Complexes with Metal-Pnictogen Mul- tiple Bonds}

\section{1 $\mathrm{P}_{2}$ and $\mathrm{As}_{2}$ Complexes from Coupling of Terminal Re Pnictides}

LXXIII proved to be an efficient platform for nitrogen fixation to yield terminal nitrides upon dinitrogen splitting, indicating strong pnictide bonding. ${ }^{[52]}$ Therefore, this complex provides a promising platform to also stabilize higher homologues. LXXIII was reacted with $\left[\mathrm{Na}(\text { diox })_{1.39}\right][\mathrm{PCO}]$ yielding unselective conversion to multiple products as indicated by ${ }^{31} \mathrm{P}$ NMR spectroscopy, but also afforded a $\mathrm{Re} \equiv \mathrm{P}$ species suggested by signals above $\delta_{\mathrm{P}}=1000 \mathrm{ppm}$ (Figure 4.1). The reaction of LXXIII with $\mathrm{PCO}^{-}$yields a $C_{\mathrm{S}}$-symmetric species as the main product $\left(\delta_{\mathrm{P}}=31.5 \mathrm{ppm}\right)$. IR spectroscopy gave an intense absorption at $\tilde{\nu}_{\mathrm{CO}}=1875 \mathrm{~cm}^{-1}$ suggesting carbon monoxide coordination as the dominant side reaction. This was proven by independent synthesis of $\left[\mathrm{ReCl}_{2}\left(\mathrm{~L}^{1}\right)(\mathrm{CO})\right]$ upon reaction of LXXIII with carbon monoxide $\left(\mathbf{2 4}\right.$, Scheme $4.1, \delta_{\mathrm{P}}=31.5 \mathrm{ppm}$, Appendix 8.3.2). The different products could not be separated by crystallization or column chromatography. Therefore, $\boldsymbol{5}$ was reacted with $\left[\mathrm{Na}(\operatorname{diox})_{1.39}\right][\mathrm{PCO}]$ since the more electron withdrawing properties should hinder carbon monoxide coordination. In fact, no CO-complex is formed over the course of the reaction and a ${ }^{31} \mathrm{P}$ NMR signals above $\delta_{\mathrm{P}}=1000 \mathrm{ppm}$ suggest phosphide complex formation (Figure 4.1), however the side products could not be removed by crystallization or other purification methods. To prevent CO trapping and increase selectivity the vacant coordination site was to be blocked as a synthetic strategy. Multiply bonded heavy pnictide ligands presumably exhibit a strong trans-influence. Therefore, 3-(1H-pyrazol-3-yl)-pyridine ( $\left.{ }^{\mathrm{H}} \mathrm{PyrPz}\right)$ was introduced as a possible hemilabile ligand (Chapter 2).

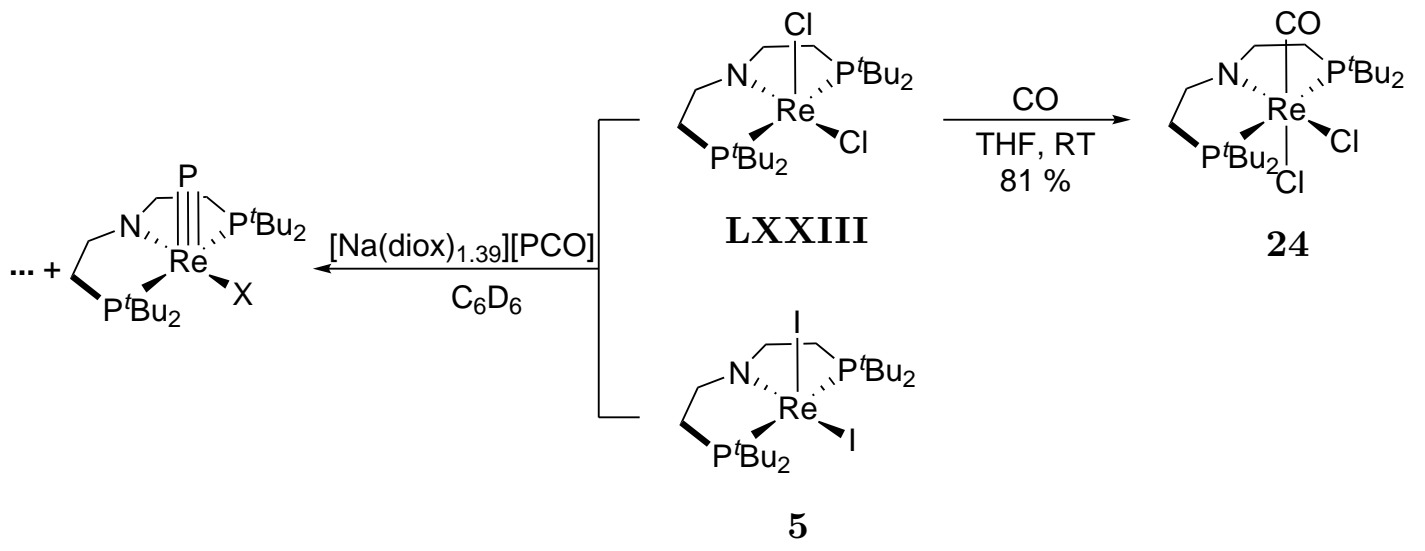

Scheme 4.1: Reactivity of LXXIII and 5 towards $\mathrm{PCO}^{-}$and synthesis of $\mathbf{2 4}$ by addition of CO to LXXIII. 

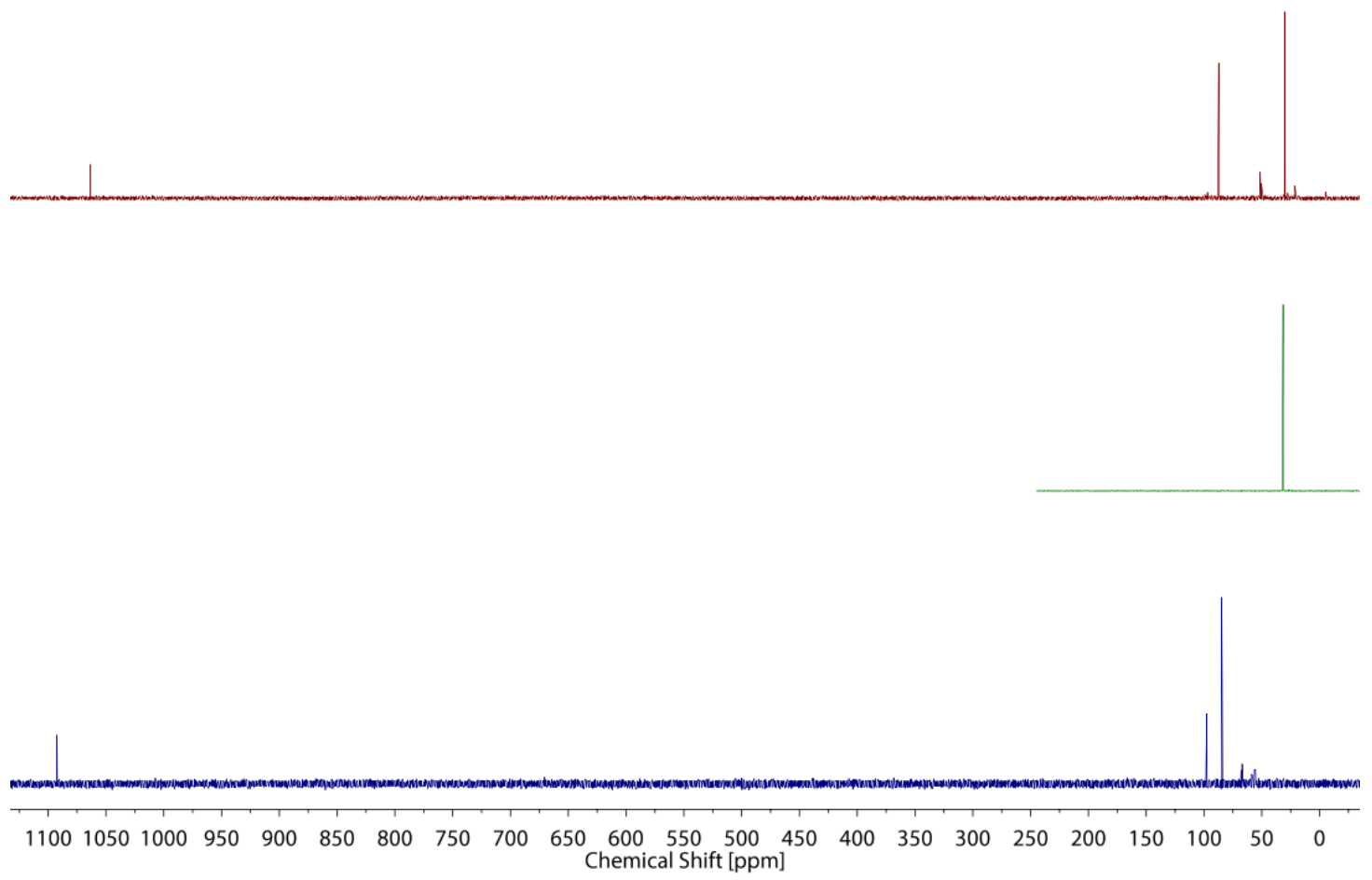

Figure 4.1: ${ }^{31} \mathrm{P}\left\{{ }^{1} \mathrm{H}\right\}$ NMR spectra of the reaction of LXXIII and $\left[\mathrm{Na}(\text { diox })_{1.39}\right][\mathrm{PCO}]$ (top), $\mathrm{C}_{6} \mathrm{D}_{6}$; pure 24 (middle), $\mathrm{CD}_{2} \mathrm{Cl}_{2}$; reaction of 5 and $\left[\mathrm{Na}(\text { diox })_{1.39}\right][\mathrm{PCO}]$ (bottom), $\mathrm{C}_{6} \mathrm{D}_{6}$, all RT.

Inspired by the work of Cummins and co-workers, $\mathbf{1 1}$ was reacted with $\mathrm{NCO}^{-}$to access a terminal nitride complex upon decarbonylation. ${ }^{[65]}\left[\operatorname{Re}\left(\mathrm{L}^{1}\right)(\mathrm{NCO})\left(\kappa^{2} N^{1}, N^{3}-\mathrm{PyrPz}\right)\right]$ (25, Scheme 4.2) can be isolated after salt metathesis as indicated by an intense IR absorption at $\tilde{\nu}_{\mathrm{NCO}}=2234 \mathrm{~cm}^{-1}$ (Appendix 8.3.2) and a ${ }^{13} \mathrm{C}$ NMR resonance of $\delta_{\mathrm{NCO}}=$ $159.6 \mathrm{ppm}$. The isocyanate ligand is slightly tilted due to the high d electron count of the Re center (see crystallographic details). $\mathbf{2 5}$ did not show any signs of decarbonylation after irradiation or heating to $80^{\circ} \mathrm{C}$ over an extended period of time, suggesting that nitride formation might be thermodynamically unfavorable.

Reaction of $\mathbf{1 1}$ with $\left[\mathrm{Na}(\operatorname{diox})_{1.39}\right][\mathrm{PCO}]$ at low temperatures results in formation of transient $\left[\operatorname{Re}\left(\mathrm{L}^{1}\right)(\mathrm{PCO})\left(\kappa^{2} N^{1}, N^{3}-\mathrm{PyrPz}\right)\right](\mathbf{2 6}$, Scheme 4.2$)$ as indicated by its ${ }^{31} \mathrm{P}$ NMR signals $\left(\delta_{\mathrm{P}}=1.92,-268.8 \mathrm{ppm}\right.$, Appendix 8.3.6). The characteristic proton resonance of the pyridine $o-\mathrm{CH}$ unit is in line with $\mathrm{PyrPz}$ ligand still acting as a chelate ligand $\left(\delta_{\mathrm{H}}=9.81 \mathrm{ppm}\right.$, Appendix 8.3.6 - 8.3.9). Upon warming, a clean conversion towards the terminal phosphide complex $\left[\operatorname{ReP}\left(\mathrm{L}^{1}\right)\left(\kappa N^{2}-\mathrm{PyrPz}\right)\right](\mathbf{2 7}$, Scheme 4.2$)$ is observed. The phosphide ligand exhibits a characteristic ${ }^{31} \mathrm{P}$ NMR shift of $\delta_{\mathrm{P}}=1069 \mathrm{ppm}$ that arises from second-order paramagnetic shielding contribution. ${ }^{231]}$ 


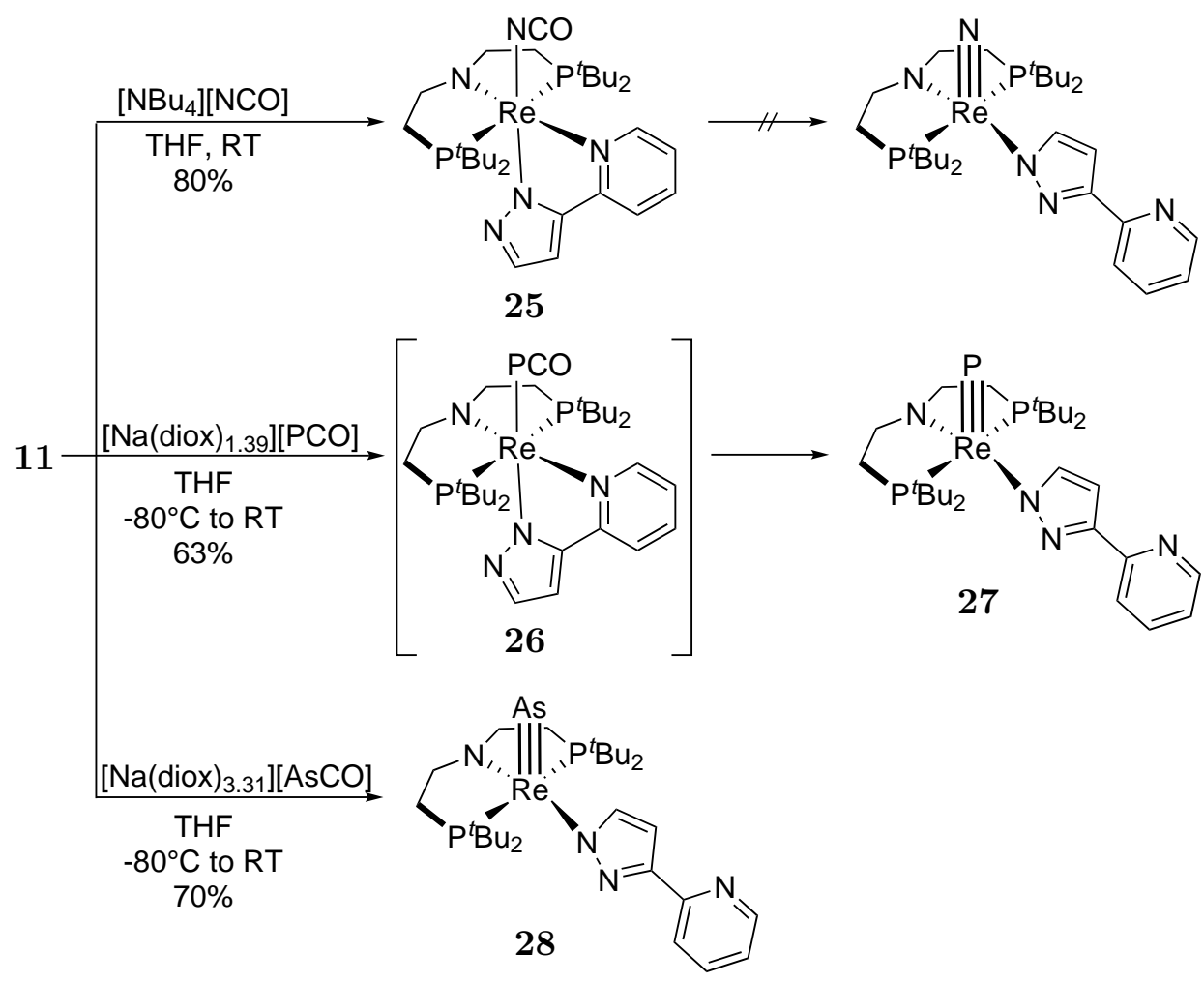

Scheme 4.2: Access to the isocyanate complex 25 by salt metathesis and synthesis of the terminal heavy pnictide complexes $\mathbf{2 7}$ and $\mathbf{2 8}$ upon decarbonylation of [ $\left.\mathrm{Na}(\operatorname{diox})_{1.39}\right][\mathrm{PnCO}]$.

When $\mathbf{1 1}$ is reacted with $\left[\mathrm{Na}(\operatorname{diox})_{3.31}\right][\mathrm{AsCO}]$, an $\mathrm{AsCO}^{-}$ligated complex could not be identified but direct conversion to the terminal arsenide $\left[\operatorname{ReAs}\left(\mathrm{L}^{1}\right)\left(\kappa N^{2}-\mathrm{PyrPz}\right)\right](\mathbf{2 8}$, Scheme 4.2) was observed. Both pnictide complexes could be fully characterized including X-ray diffraction studies (Figure 4.2). The ${ }^{1} \mathrm{H}$ NMR resonances of the PyrPz ligand are considerably broadened for both complexes at room temperature (Appendix 8.3.3), but sharpening of all signals is observed at $0^{\circ} \mathrm{C}$ which is readily explained by the solid state structure that reveals the $\mathrm{PyrPz}$ ligand to be partly decoordinated, most likely due to the trans-influence of the $\mathrm{Re} \equiv \mathrm{Pn}$ bonds. The Re $\equiv \mathrm{Pn}$ bond distances (Re1-P3: 2.0939(6) $\AA$, Re1-As1: 2.2021(3) $\AA$ ) are in the range found for terminal group 6 pnictide complexes. ${ }^{[63]-[65]}$

A complementary quantum-chemical study of the electronic structure of $\mathbf{2 7}$ and $\mathbf{2 8}$ corroborates the notion of triply bound terminal rhenium pnictides. For both species a natural bond order (NBO) analysis reveals a set of three bonding, natural localized molecular orbitals (NLMOs) which correspond to $\sigma^{2} \pi^{4}$ triple bonds complemented by a lone-pair localized at the pnictide end in support of a genuine $\mathrm{Re} \equiv \mathrm{Pn} \mid$ Lewis-picture (Figure 4.3; Wiberg bond index: $2.23(\mathbf{2 7}), 2.17(\mathbf{2 8})$ ). 

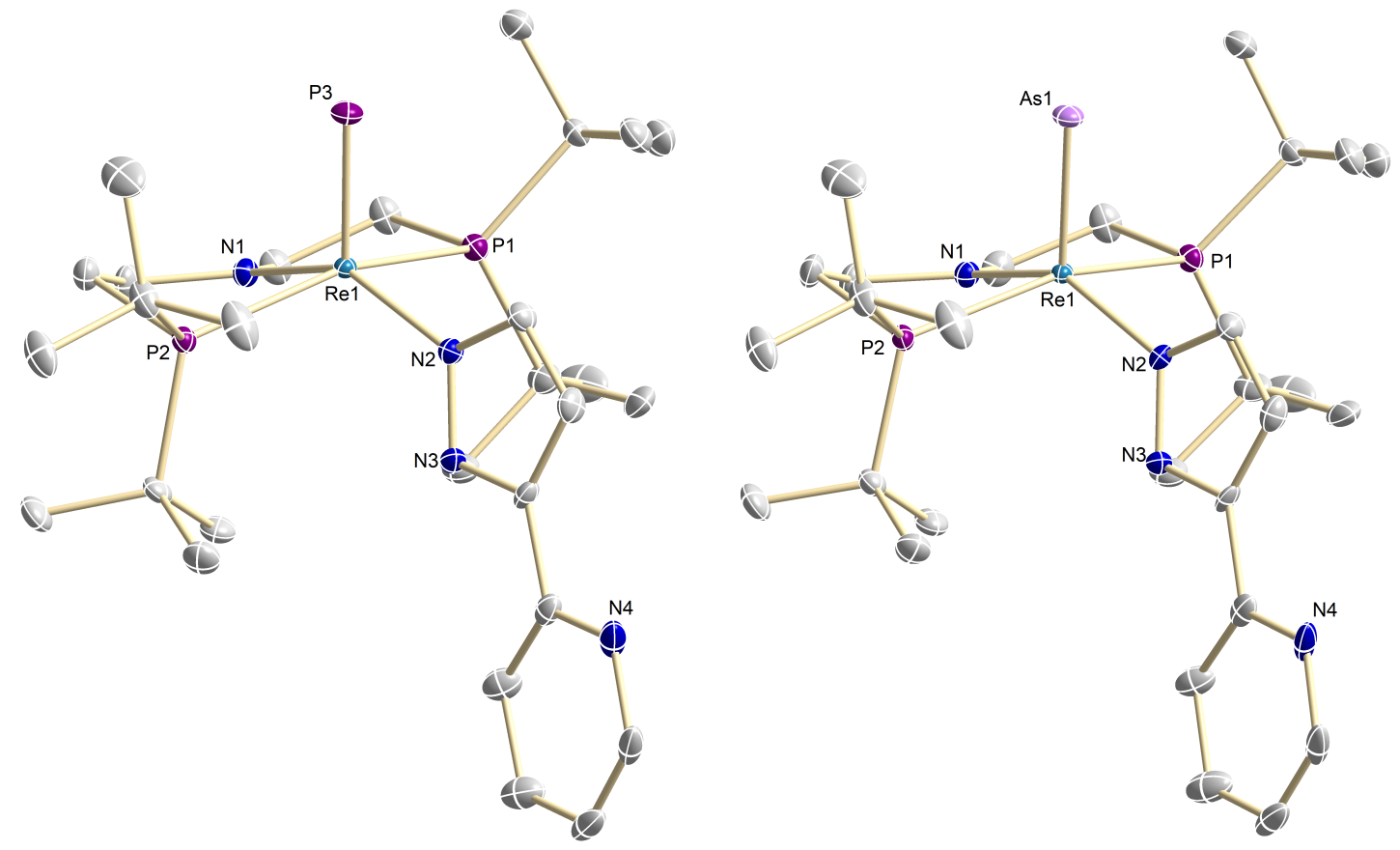

Figure 4.2: Solid state structure of $\mathbf{2 7}$ (left) and $\mathbf{2 8}$ (right) from single-crystal X-ray diffraction (thermal ellipsoids drawn at the $50 \%$ probability level); hydrogen atoms are omitted for clarity. Selected bond lengths $[\AA]$ and angles $\left[{ }^{\circ}\right]$ : 27 Re1-N1 1.9894(17), Re1-N2 2.1208(17), Re1-P1 2.4223(6), Re1-P2 2.4280(6), Re1-P3 2.0939(6), N1-Re1-N2 148.29(7), N1-Re1-P3 110.66(5), P1-Re1-P2 154.820(19), $\tau=0.11 ; 28$ Re1-N1 1.986(2), Re1-N2 2.123(2), Re1-P1 2.4227(8), Re1-P2 2.4288(7), Re1-As1 2.2021(3), N1-Re1-N2 148.60(9), N1-Re1-As1 110.60(7), P1-Re1-P2 $154.86(3), \tau=0.10$.

In both cases, the $\sigma$ - and one of the $\pi$-bonds are distributed almost equally while the other $\pi$-bond carries increased Re d orbital character. This is substantiated by topological analysis of the computed electron densities by means of Bader's quantum theory of atoms in molecules (QTAIM).

The Re $\equiv \mathrm{P}$ interaction classifies as a strong, covalent and polar bond with corresponding values at the bcp for the electron density $\rho\left(\mathbf{r}_{\mathrm{b}}\right)=1.07 \mathrm{e} \AA^{-3}$, the relative total energy density $H\left(\mathbf{r}_{\mathrm{b}}\right) / \rho\left(\mathbf{r}_{\mathrm{b}}\right)=-0.68$ au and the relative kinetic energy density $G\left(\mathbf{r}_{\mathrm{b}}\right) / \rho\left(\mathbf{r}_{\mathrm{b}}\right)=$ $0.56 \mathrm{au}$, all slightly weaker but remarkably close to those for the homonuclear and lighter diatomic congener $\mathrm{P} \equiv \mathrm{P}$ (Appendix 8.3.23, 8.3.22). Furthermore, the cylindrical electron density at the bcp with a bond ellipticity of $\epsilon=0.04$, as well as a delocalization index of $\delta(\operatorname{Re}, \mathrm{P})=2.47$, support the formulation as a triply bonded $\mathrm{Re} \equiv \mathrm{P}$ moiety. The arsenide draws a close parallel to this description, however the bond exhibits reduced covalency due to the more diffuse electron density of arsenic $\left(\rho\left(\mathbf{r}_{\mathrm{b}}\right)=0.95 \mathrm{e} \AA^{-3}, H\left(\mathbf{r}_{\mathrm{b}}\right) / \rho\left(\mathbf{r}_{\mathrm{b}}\right)=\right.$ $\left.-0.61 \mathrm{au}, G\left(\mathbf{r}_{\mathrm{b}}\right) / \rho\left(\mathbf{r}_{\mathrm{b}}\right)=0.65 \mathrm{au}, \epsilon=0.06, \delta(\operatorname{Re}, \mathrm{As})=2.40\right)$. 


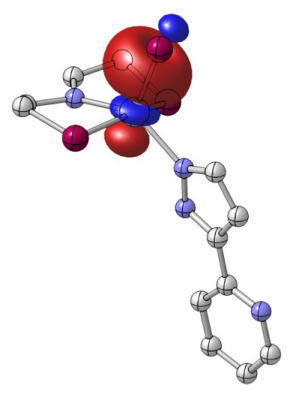

(47\%) Re1-P3 (50\%)

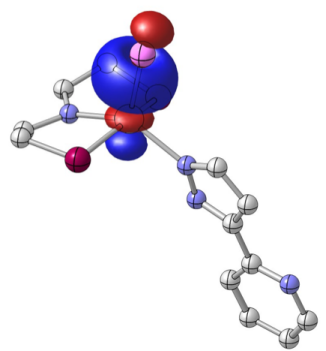

(48\%) Re1-As1 (50\%)

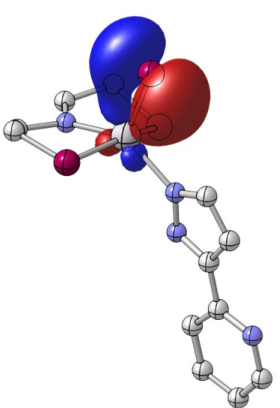

(47\%) Re1-P3 (51\%)

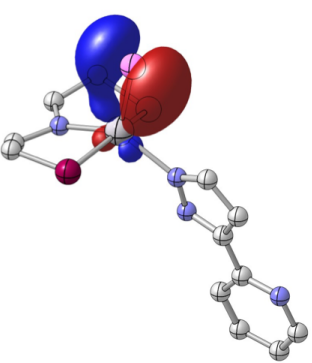

(46\%) Re1-As1 (51\%)

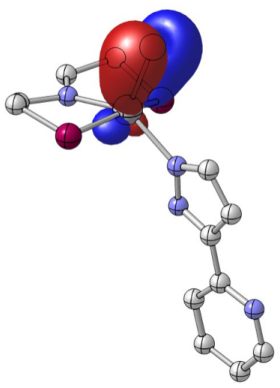

(67\%) Re1-P3 (31\%)

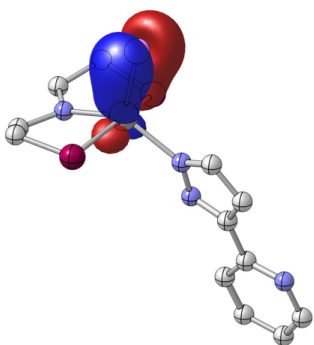

(69\%) Re1-As1 (28\%)

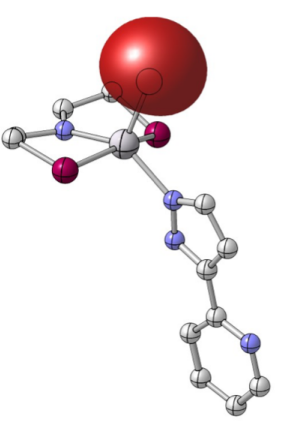

(99\%) P3 sp 0.28

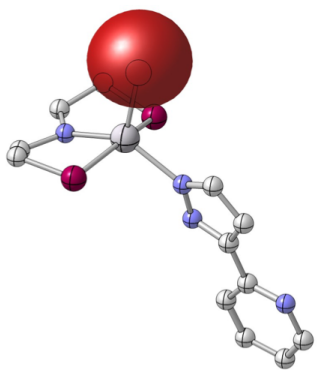

(99\%) As1 $s p^{0.23}$

Figure 4.3: NLMOs ( $\sigma, \pi$ and lone-pairs) representing the bonding in $\mathbf{2 7}$ (top) and $\mathbf{2 8}$ (bottom).
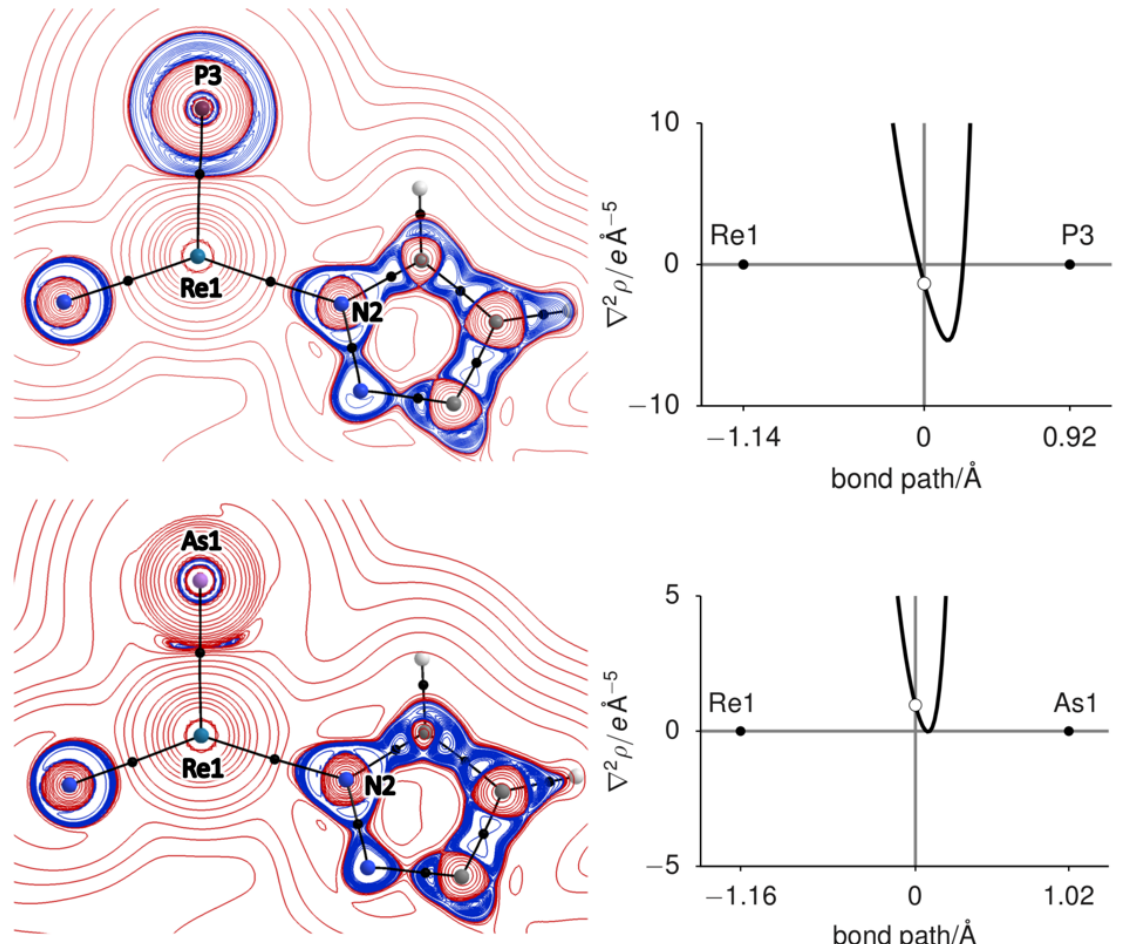

Figure 4.4: Bonding analysis of 27 (left) and 28; Left: 2D plots of $\nabla^{2} \rho(r)$, charge concentrations (blue) and depletion (red), bond paths (black lines), and bond critical points (black dots); Right: 1D Laplacian profiles along the bond paths. 

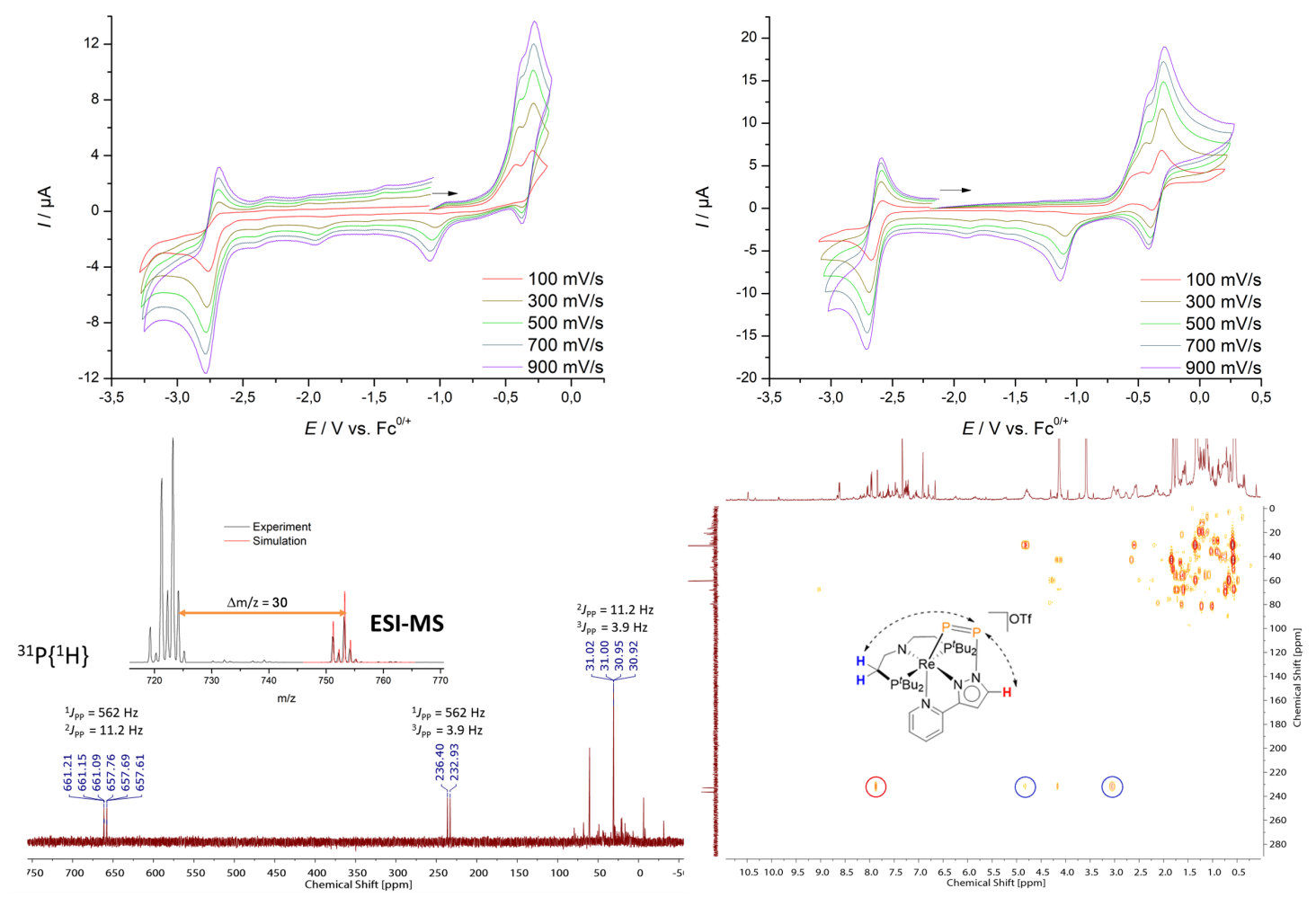

Figure 4.5: Top left: Cyclic voltammogram of $\mathbf{2 7}, 1 \mathrm{mM}, 0.1 \mathrm{M} \mathrm{NBu}_{4} \mathrm{PF}_{6}$, THF, RT; Top right: Cyclic voltammogram of $\mathbf{2 8}, 1.3 \mathrm{mM}, 0.1 \mathrm{M} \mathrm{NBu}_{4} \mathrm{PF}_{6}$, THF, RT; Bottom left: ${ }^{31} \mathrm{P}\left\{{ }^{1} \mathrm{H}\right\}$ NMR spectrum of the oxidation of $\mathbf{2 7}$ with [Fc][OTf], THF, RT; Inset: ESI spectrum of the oxidation of $\mathbf{2 7}$ with [Fc][OTf] (Main peak: 27+ $\mathrm{H}^{+}$; Simulation: 29); Bottom right: ${ }^{31} \mathrm{P}\left\{{ }^{1} \mathrm{H}\right\}-{ }^{1} \mathrm{H} H M B C$ NMR spectrum of the oxidation of $\mathbf{2 7}$ with [Fc][OTf], THF, $-38^{\circ} \mathrm{C}$.

The cyclic voltammogram of $\mathbf{2 7}$ features an irreversible oxidation at $E_{\text {p.a. }} \approx-0.42 \mathrm{~V}$ vs. $\mathrm{Fc}^{0 /+}$ closely followed by a quasi-reversible wave at $E_{1 / 2}=-0.31 \mathrm{~V}$, indicating rapid reactivity that follows oxidation. For $\mathbf{2 8}$ a similar result is obtained with increased reversibility for the quasi-reversible redox event (Figure 4.5). Cathodic reverse scans reveal the same irreversible, reductive feature just below $E=-1.0 \mathrm{~V}$ vs. $\mathrm{Fc}^{0 /+}$ for both pnictides, suggesting at least partial pnictide loss after initial oxidation (Appendix 8.3.4, 8.3.5). Chemical oxidation of $\mathbf{2 7}$ and $\mathbf{2 8}$ with [Fc][OTf] at room temperature lead to the formation of several products. Mass spectrometry of the reaction solution hints towards the formation of $\left[\operatorname{Re}\left(\mathrm{Pn}_{2}\right)\left(\mathrm{L}^{1}\right)(\mathrm{PyrPz})\right](\mathbf{2 9}(\mathrm{Pn}=\mathrm{P})$, Figure 4.5, Appendix 8.3.14, 30 $(\mathrm{Pn}=\mathrm{As})$, Appendix 8.3.19) as major oxidation products. The respective yields are determined by NMR to be $33-44 \%$. In addition, 27 exhibits two ${ }^{31} \mathrm{P}$ NMR signals at $\delta_{\mathrm{P}}=569,235 \mathrm{ppm}$ which correspond to a single $\mathrm{P}$ atom, respectively, when compared to the pincer resonance $\left(\delta_{\mathrm{P}}=31.0 \mathrm{ppm}\right)$. The observed chemical shifts and their large mutual $J$-coupling $\left({ }^{1} J_{\mathrm{PP}}=560 \mathrm{~Hz}\right)$ indicate phosphide radical coupling upon oxidation, yielding a product featuring a P-P multiple bonded moiety that is unsymmetrically 
bound to the Re center, as suggested by the largely different $\mathrm{P}-\mathrm{P}_{\mathrm{PNP}}$ coupling constants $\left({ }^{2} J_{\mathrm{PP}}=11.8 \mathrm{~Hz},{ }^{3} J_{\mathrm{PP}}=3.9 \mathrm{~Hz}\right)$. The proton resonances are sharpened when compared to 27 indicating full coordination of the $\mathrm{PyrPz}$ chelate ligand. ${ }^{31} \mathrm{P}\left\{{ }^{1} \mathrm{H}\right\}-{ }^{1} \mathrm{H}$ HMBC spectroscopy reveals indicative cross peaks for the $\mathrm{P}$ atom at $\delta_{\mathrm{P}}=235 \mathrm{ppm}$ and the pincer $\mathrm{PCH}_{2}$ unit as well as for a $\mathrm{CH}$ group of the pyrazole moiety suggesting the presence of a $\mathrm{Re}-\mathrm{P}=\mathrm{P}-\mathrm{N}$ bonding motif (Figure 4.5). The oxidation of $\mathbf{2 8}$ yields almost identical NMR parameters $\left({ }^{1} \mathrm{H},{ }^{13} \mathrm{C}\right.$ and $\left.{ }^{31} \mathrm{P}\right)$ and ESI spectrometry further supports the formation of an isostructural $\mathrm{As}_{2}$ complex (Appendix 8.3.16 - 8.3.19).

While 29 could not be isolated due to gradual degradation during workup, even at $-40^{\circ} \mathrm{C}$, the crystallographic characterization of $\mathbf{3 0}$ confirms the structural assignment (Scheme 4.3). The $\mathrm{PyrPz}$ ligand rearranges over the course of the reaction with the pyrazole ring in trans-position to the pincer backbone, since the Re-Pn bond strength is considerably lowered. The $\mathrm{As}_{2}$ unit of $\mathbf{3 0}$ bridges the metal center and the pyrazole N4 atom. The As=As bond distance of 2.2422(7) $\AA$ suggests double bonding. ${ }^{[232]}$ This structural assignment is in full agreement with the multinuclear NMR features of both pnictide oxidation products. Furthermore, spin-orbit relativistic NMR calculations ac-
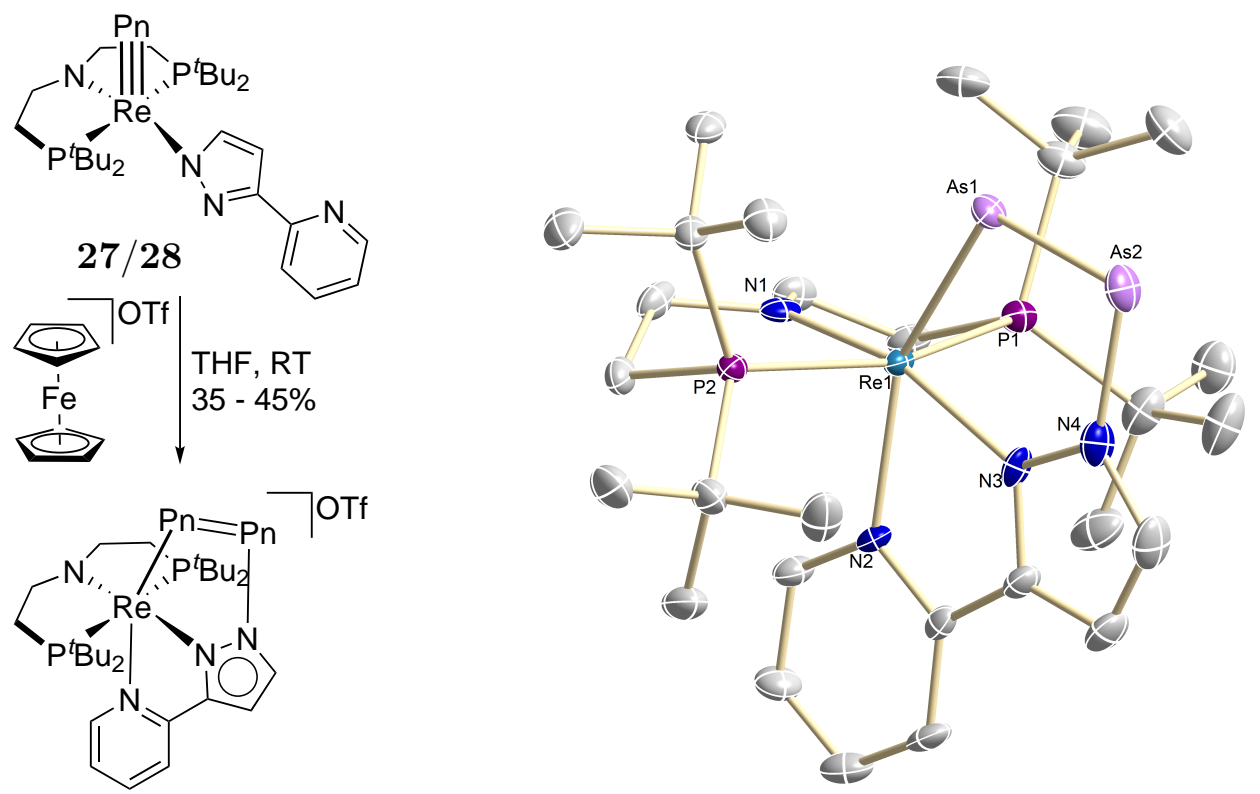

$29 / 30$

Scheme 4.3: Left: Synthesis of $\mathbf{2 9}(\mathrm{Pn}=\mathrm{P})$ and $\mathbf{3 0}(\mathrm{Pn}=\mathrm{As})$ by oxidation of $\mathbf{2 7}$ and $\mathbf{2 8}$, respectively; Right: Solid state structure of $\mathbf{3 0}$ (one of the complexes in the asymmetric unit) from single-crystal X-ray diffraction (thermal ellipsoids drawn at the $50 \%$ probability level); solvent molecules, anions and hydrogen atoms are omitted for clarity. Selected bond lengths $[\AA]$ and angles $\left[{ }^{\circ}\right]$ : 30 Re1-As1, 2.4421(5), Re1-N1 1.951(4), Re1-N2 2.173(3), Re1-N3 2.139(3), Re1P1 2.4482(12), Re1-P2 2.4743(12), As1-As2 2.2422(7), As2-N4 1.943(4), Re1-As1-As2 109.83(2), As1-As2-N4 93.75(11), N1-Re1-N2 90.98(13), N1-Re1-As1 115.31(10), P1-Re1-P2 160.86(4). 
curately reproduce the ${ }^{31} \mathrm{P}$ chemical shifts and coupling constants of $29\left(\delta_{\mathrm{P} 1 / 2}^{\mathrm{DFT}}=30 \mathrm{ppm}\right.$, $\left.\delta_{\mathrm{P} 3}^{\mathrm{DFT}}=664 \mathrm{ppm}, \delta_{\mathrm{P} 4}^{\mathrm{DFT}}=212 \mathrm{ppm},{ }^{1} J_{\mathrm{P} 3 / 4}^{\mathrm{DFT}}=-400 \mathrm{~Hz}\right)$. Identification of $\mathbf{2 9}$ and $\mathbf{3 0}$ as the main reaction products involves the formal loss of a $\left[\operatorname{Re}\left(\mathrm{L}^{1}\right)(\mathrm{PyrPz})\right]^{+}$unit.

All attempts to synthesize this complex were unsuccessful and gave multiple products, as observed for the oxidation of $\mathbf{2 7} / \mathbf{2 8}$. Oxidizing $\mathbf{2 7}$ at $-80^{\circ} \mathrm{C}$ and instant freezing led to the observation of a weak, rhombic EPR signal, suggesting that most of the immediate $S=\frac{1}{2}$ oxidation product has decayed, possibly by P-P coupling (Appendix 8.3.15). Interestingly, slow warming of the solution to room temperature did not give 29 in detectable amounts. The stabilization of a putative $\left\{\operatorname{Re}_{2} \mathrm{P}_{2}\right\}$ intermediate might be associated with the kinetic barrier for $\kappa^{1}$ - to $\kappa^{2}$-rearrangement of the $\mathrm{PyrPz}$ ligand.

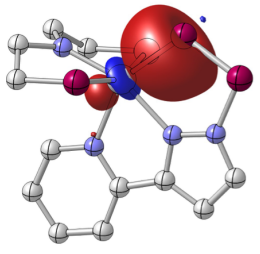

(37\%) Re1-P3 (58\%)

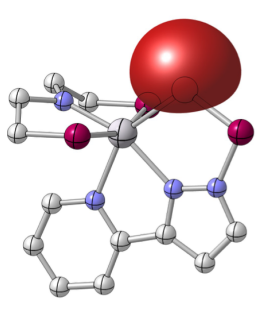

$(94 \%) \mathrm{P} 3 \mathrm{sp}^{0.59}$

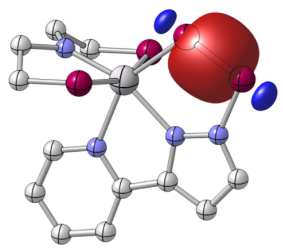

(49\%) P3-P4 (48\%)

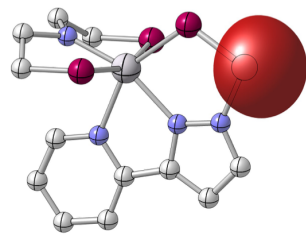

(98\%) P4 sp 0.41

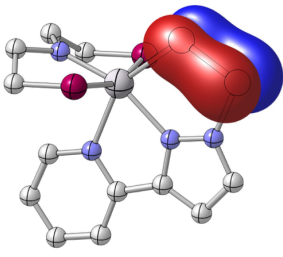

(39\%) P3-P4 (58\%)

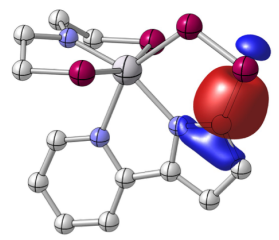

(20\%) P4-N4 (79\%)

Figure 4.6: Bonding analysis of 29: a) NLMOs representing the Re1-P3, P3=P4 and P4-N4 bonds and the two lone pairs at P3 and P4 (isosurfaces at $\pm 0.05 \mathrm{a}_{0}^{-3 / 2}$ ).

NBO analysis of the computed electron density of $\mathbf{2 9}$ yields four NLMOs, which correspond to a $\mathrm{P}=\mathrm{P}$ double bond and a $\sigma$-type lone-pair at each phosphorus atom (Figure 4.6). Two additional NLMOs represent significantly polarized, localized 2c2e single bonds between Re1-P3 and P4-N4. Accordingly, alternating atomic charges are obtained by natural population analysis (Re1: 0.21, P3: -0.03 , P4: 0.36, N4: -0.41 ). From this considerations it is not obvious if the dipnictide moiety is best described as a neutral $\mathrm{Pn}_{2}$ unit, a cis-dipnictogene or a metalla-diazadipnictole (Scheme 4.4). 


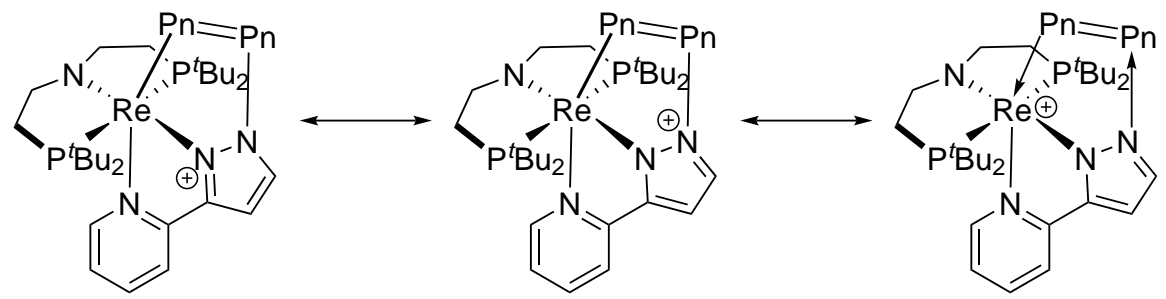

Scheme 4.4: Possible mesomeric structures of 29 and 30.

Further insight is provided by QTAIM analysis of the computed electron density of 29. All topological criteria point towards an electron-sharing, covalent P-P interaction that closely mirrors diphosphene, $\mathrm{P}_{2} \mathrm{H}_{2}$ (Appendix 8.3.23). The computed delocalization index $\delta(\mathrm{P} 3, \mathrm{P} 4)=1.71$ and the ellipticity $\epsilon=0.36$ indicates the presence of a formal $\mathrm{P}=\mathrm{P}$ double bond. The symmetric, one-dimensional Laplacian distribution profile between
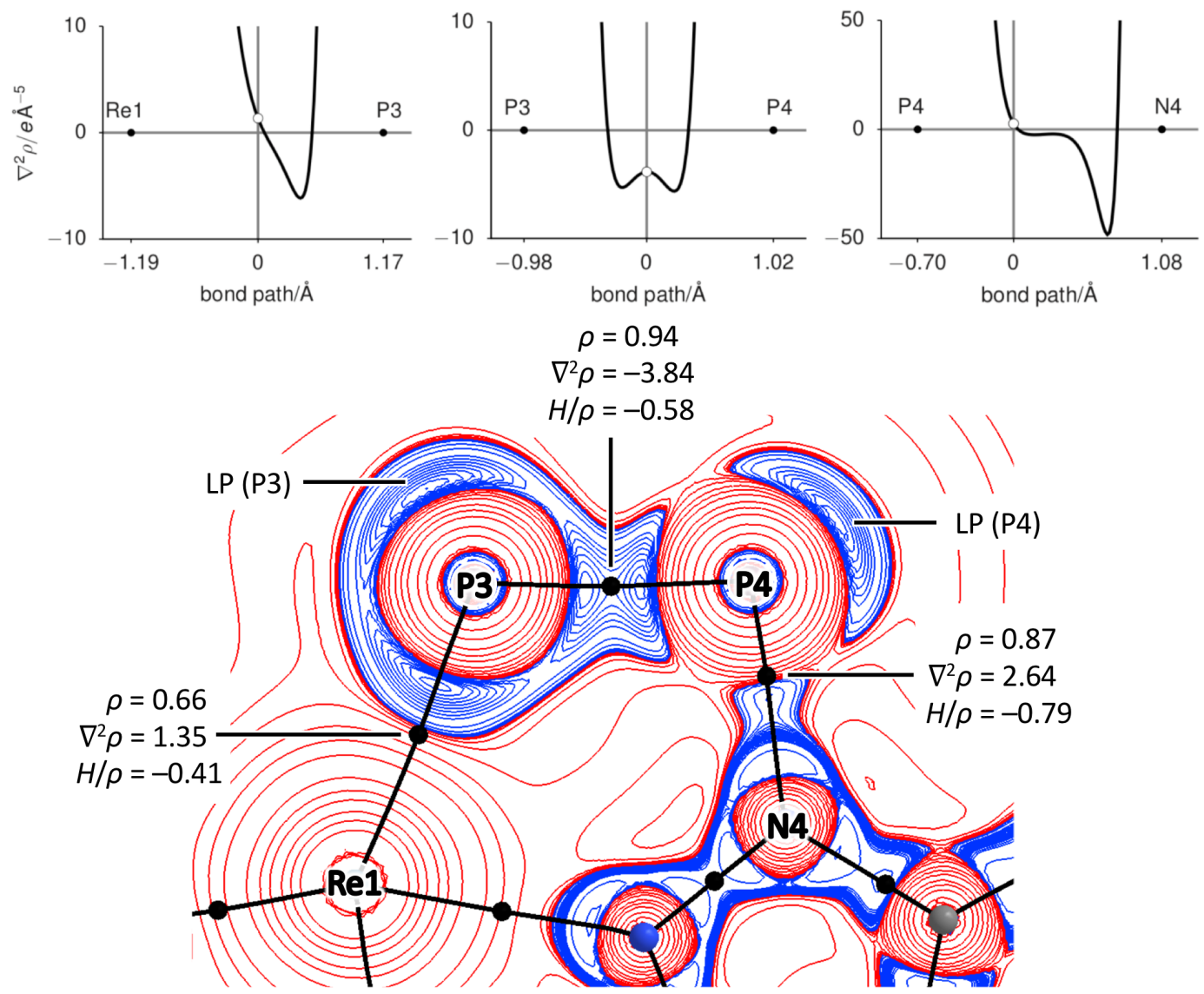

Figure 4.7: Top: 1D Laplacian profiles along the R1-P3 (left), the $\mathrm{P} 3=\mathrm{P} 4$ (middle) and the P4-N4 (right) bond paths; Bottom: 2D plot of $\nabla^{2} \rho$; charge concentration (blue) and depletion (red), bond paths (black lines), and bond critical points (black dots) with selected characteristic properties at the bcp ( $\rho$ in $\mathrm{e} \AA^{-3}, \nabla^{2} \rho$ in $\mathrm{e}^{-5}$, relative total energy density $H / \rho$ in au). 
P3 and P4 shows two valence shell charge concentrations (VSCC), originating from the overlapping valance shells of the interacting atoms, with two minima on both sides of the bcp, suggesting essentially homopolar covalent bonding (Figure 4.7). In contrast, the profiles along the Re1-P3 and the P4-N4 bond paths exhibit typical features of dative covalent interactions. ${ }^{[233]-[235]}$ In both cases, the bcp resides close to the nodal surface in the Laplacian distribution such that the sign of $\nabla^{2} \rho$ at the bcp is unsuitable for a bond classification (Figure 4.7). ${ }^{[236], ~[237]}$ As commonly found for transition metals, the diffuse outer valence shell of Re lies in a region of charge depletion and a VSCC is present only in the P3 donor atomic basin. Similarly, the profile along the P4-N4 bond path exhibits a pronounced VSCC in the vicinity of the more electronegative nitrogen donor atom and a shallow shoulder corresponding to the second VSCC near P4, both residing within the N4 atomic basin. The computed delocalization indices $\delta(\operatorname{Re} 1, \mathrm{P} 3)=1.11$ and $\delta(\mathrm{P} 4, \mathrm{~N} 4)=0.79$ further document the covalent nature of these interactions. A very similar bonding situation is found for the arsenide analog 30 (Appendix 8.3.24). The covalency is slightly reduced by the more diffuse arsenic electron density $(\delta(\operatorname{Re} 1, \mathrm{As} 1)=$ $1.07 ; \delta(\operatorname{As} 2, \mathrm{~N} 4)=0.77)$, however As=As double bonding is evident due to $\delta($ As1,As2 $)=$ 1.70 and $\epsilon=0.27$.

The NBO and QTAIM results for both complexes are therefore best represented by a formal $\mathrm{Re} \leftarrow \mathrm{Pn}=\mathrm{Pn} \leftarrow \mathrm{N}$ bonding pattern corresponding to push-pull stabilized neutral $\mathrm{P}_{2}^{0}$ and $\mathrm{As}_{2}^{0}$-ligands with $\mathrm{Pn}=\mathrm{Pn}$ double bonds. 


\subsection{Oxygenation of a Terminal Re Phosphide Complex}

The aerobic oxidation of white phosphorus $\left(\mathrm{P}_{4}\right)$ was first performed by Brandt in 1680 in search of the Philosopher's stone. Upon burning, a greenish glow is observed which coined the term phosphorescence. ${ }^{[238]}$ Until today, combustion of $\mathrm{P}_{4}$ and organophosphorus compounds (OPCs) is still not well understood. Investigating these processes on a molecular level is of great interest in the context of chemical warfare agent disposal such as Sarin or VX. ${ }^{[239]}$ Observed phosphorus containing species in these reaction are lower phosphorus oxides and hydroxides $\left(\mathrm{PO}, \mathrm{PO}_{2}, \mathrm{HOPO}\right.$ and $\left.\mathrm{HOPO}_{2}\right) \cdot{ }^{[240]-[242]}$ In general, lower (charged) phosphorus oxides can only be generated and studied under carefully controlled experimental conditions. ${ }^{[243],[244]}$ However, several experimental and computational studies are available providing bond distances, ionization potentials and further characteristic data of $\mathrm{P}_{\mathrm{x}} \mathrm{O}_{\mathrm{y}}^{\mathrm{n}+/-}$ species. ${ }^{[241]-[252]}$

While low molecular oxides of nitrogen are ubiquitous in nature, easily synthesized and well-studied ligands in coordination chemistry, preparing the phosphorus analogues is hindered by suitable precursors. ${ }^{[253]-[255]}$ In this context, rare terminal phosphide transition metal complexes are particularly attractive since they represent $\mathrm{P}_{1}$ building blocks which can be functionalized stoichiometrically, providing an entry into low-valent phosphorus chemistry. ${ }^{[63]}$

In the case of phosphorus monoxide, a molecule only found in interstellar clouds, several $\mu^{3}$-type complexes have been described for various transition metals across the periodic table, however terminal phosphorus monoxide transition metal complexes remain extremely scarce. ${ }^{[243],[256]}$ The oxygenation of the terminal molybdenum phosphide XVI gave the unprecedented terminal phosphorus monoxide complex $\mathbf{X L}$, as described in the introduction of this thesis. The phosphorus monoxide ligand is coordinated almost linearly suggesting no antibonding interaction of the metal center and the $\pi$-system and a retention of the $\mathrm{Mo}^{\mathrm{VI}}$ oxidation state. ${ }^{[257]}$

Other low-molecular phosphorus oxides are limited to three examples. The push-push stabilized diphosphorus compound $\mathbf{X C V}$ was oxygenated by molecular $\mathrm{O}_{2}$ yielding an NHC stabilized neutral $\mathrm{P}_{2} \mathrm{O}_{4}$ compound LXXXI, while the oxygenation of the FeCo-P cluster LXXXII gave a $\mathrm{P}_{2} \mathrm{O}$ ligand encumbered within the cluster LXXXIII (Scheme 4.5). ${ }^{[258],[259]}$ A recent report features the preparation of $\mathrm{PO}_{3}^{2-}$ upon heating $\mathrm{ReI}_{3}$ with red phosphorus and sodium cyanate and subsequent hydrolysis yielding the cluster $\mathrm{Na}_{8}\left[\left\{\mathrm{Re}_{4}(\mathrm{PO})_{3}\left(\mathrm{PO}_{2}\right)\right\}(\mathrm{CN})_{12}\right]$ (LXXXIV, Scheme 4.5).

Oxygenation of $\mathbf{2 7}$ could enable access to new $\mathrm{P}_{\mathrm{x}} \mathrm{O}_{\mathrm{y}}^{\mathrm{n}-}$ species and will be evaluated in the following. 


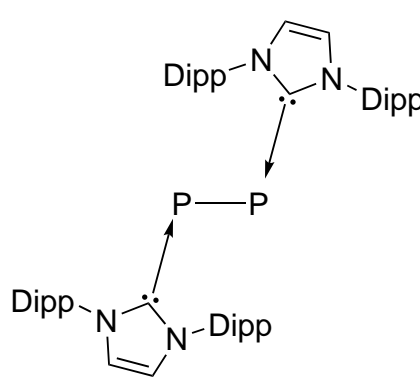

$\mathrm{XCV}$

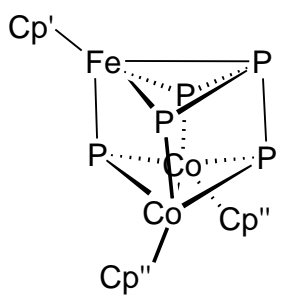

\section{LXXXII}

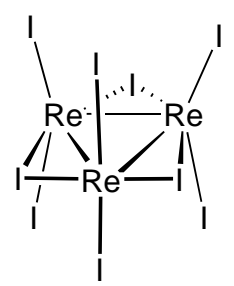

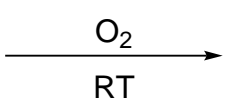

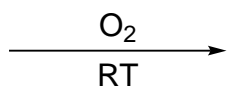

1) $\mathrm{P}_{\text {red }}+\mathrm{NaCN}$

2) $\mathrm{H}_{2} \mathrm{O}$

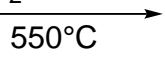

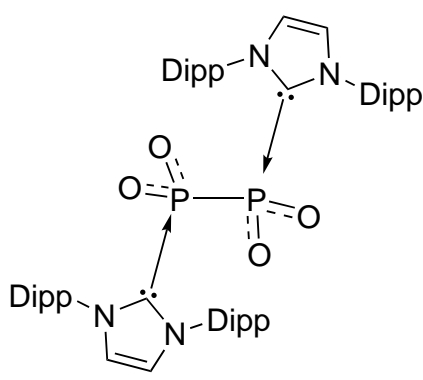

LXXXI

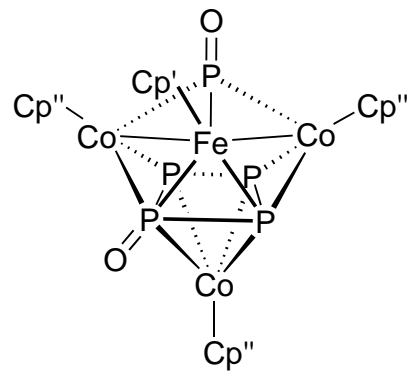

\section{LXXXIII}

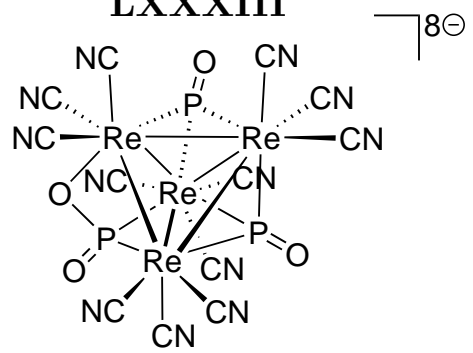

LXXXIV

Scheme 4.5: Oxygenation of XCV and LXXXII to give LXXXI and LXXXIII, respectively, $\mathrm{Cp}^{\prime}=\mathrm{C}_{5} \mathrm{Me}_{5}, \mathrm{Cp}^{\prime \prime}=1,3{ }^{t} \mathrm{Bu}_{2} \mathrm{C}_{5} \mathrm{H}_{3}$, and synthesis of the cluster compound LXXXIV from $\operatorname{ReI}_{3} \cdot{ }^{[258]-[260]}$

Table 4.1: $\mathrm{P}-\mathrm{O}$ bond lengths in low-molecular phosphorus oxide ligands.

\begin{tabular}{ccc}
\hline Compound & Ligand & Bond distance $/ \AA$ \\
\hline XL & $\mathrm{PO}$ & $1.49(2)$ \\
& & \\
LXXXI & $\mathrm{P}_{2} \mathrm{O}_{4}$ & $1.466(3)$ \\
& & $1.470(3)$ \\
LXXXIII & $\mathrm{P}_{2} \mathrm{O}$ & $1.509(6)$ \\
& & \\
LXXXIV & $\mathrm{PO}_{2}^{3-}$ & $1.58(3)$ \\
& & $1.50(3)$ \\
\hline
\end{tabular}


Heating 27 with two equivalents of pyridine- $N$-oxide in benzene at elevated temperatures results in the clean formation of dimeric $\left[\left\{\operatorname{Re}\left(\mathrm{L}^{1}\right)\left(\kappa^{2} N^{1} N^{2}-\mathrm{PyrPz}\right)\right\}_{2}\left\{\mu-P^{1} P^{2}-\mathrm{P}_{2} \mathrm{O}_{4}\right\}\right]$ (31, Scheme 4.6) in 77\% isolated yield. 31 features an unprecedented dianionic $\mathrm{P}_{2} \mathrm{O}_{4}^{2-}$ fragment with a planar $\mathrm{P}_{2} \mathrm{O}_{2}$ unit bridging both $\mathrm{Re}{ }^{\mathrm{III}}$ centers (Figure 4.8), in stark contrast to LXXXI. The oxygenation reaction results in a significant elongation of the $\mathrm{Re}-\mathrm{P}$ bond by $\Delta d=0.36 \AA$ with respect to 27 . The $\mathrm{PyrPz}$ ligand rearranges over the course of the reactions, reminiscent of the iodide complex 11, since the strong trans-influence of the phosphide ligand is lost upon oxygenation, allowing for chelation, resulting in the overall observed octahedral coordination sphere. An intense band at $\tilde{\nu}_{\mathrm{PO}}=1112 \mathrm{~cm}^{-1}$ is observed for the antisymmetric stretching vibration of the $\mathrm{P}_{2} \mathrm{O}_{4}^{2-}$ ligand, redshifted by $\Delta \tilde{\nu}_{\mathrm{PO}}=167 \mathrm{~cm}^{-1}$ when compared to LXXXI. Two signals are detected by ${ }^{31} \mathrm{P}\left\{{ }^{1} \mathrm{H}\right\}$ NMR spectroscopy at $\delta_{\mathrm{P}}=245.7$ and 13.2 with a mutual coupling constant of ${ }^{2} J_{\mathrm{PP}}=20.7 \mathrm{~Hz}$. When the reaction is carried out with equimolar amounts of oxygen atom transfer reagent, $50 \%$ conversion towards 31 is observed by ${ }^{31} \mathrm{P}$ NMR, suggesting that a putative phosphorus monoxide complex is too reactive to be isolated under this reaction conditions. A DFT geometry optimization of the hypothetical complex $\left[\operatorname{Re}(\mathrm{PO})\left(\mathrm{L}^{1}\right)\left(\kappa^{2} N^{1} N^{2}-\mathrm{PyrPz}\right)\right](\mathbf{3 2})$ reveals a short Re-P bond distance and a highly bent Re-P-O unit, contrasting with linear $\mathrm{XL}$, suggesting $\mathrm{Re}=\mathrm{P}$ double bond character (Re-P: $2.16 \AA$, P-O: $1.52 \AA$, Re-P-O: $146.4^{\circ}$; for an NBO analysis see: Appendix 8.3.25).

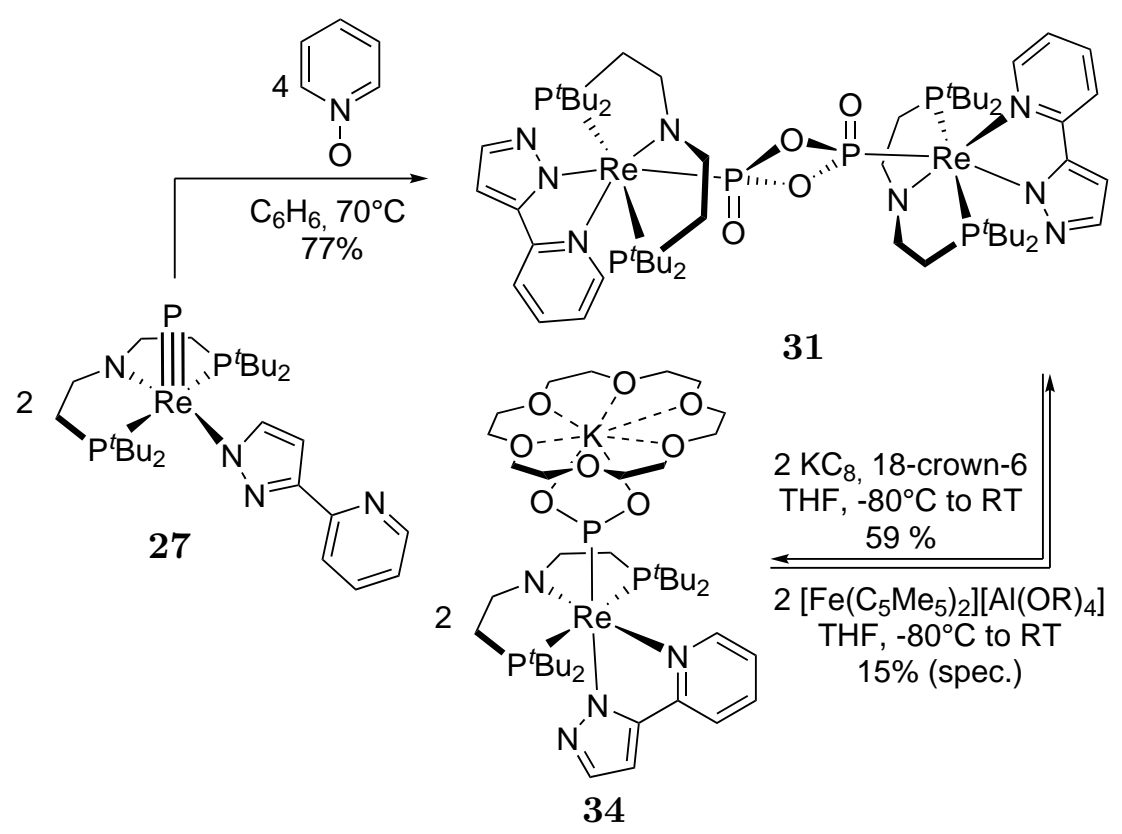

Scheme 4.6: Oxygenation of 27 to give 31 and its redox-chemical interconversion with $34, \mathrm{R}$ $=\mathrm{C}\left(\mathrm{CF}_{3}\right)_{3}$. 

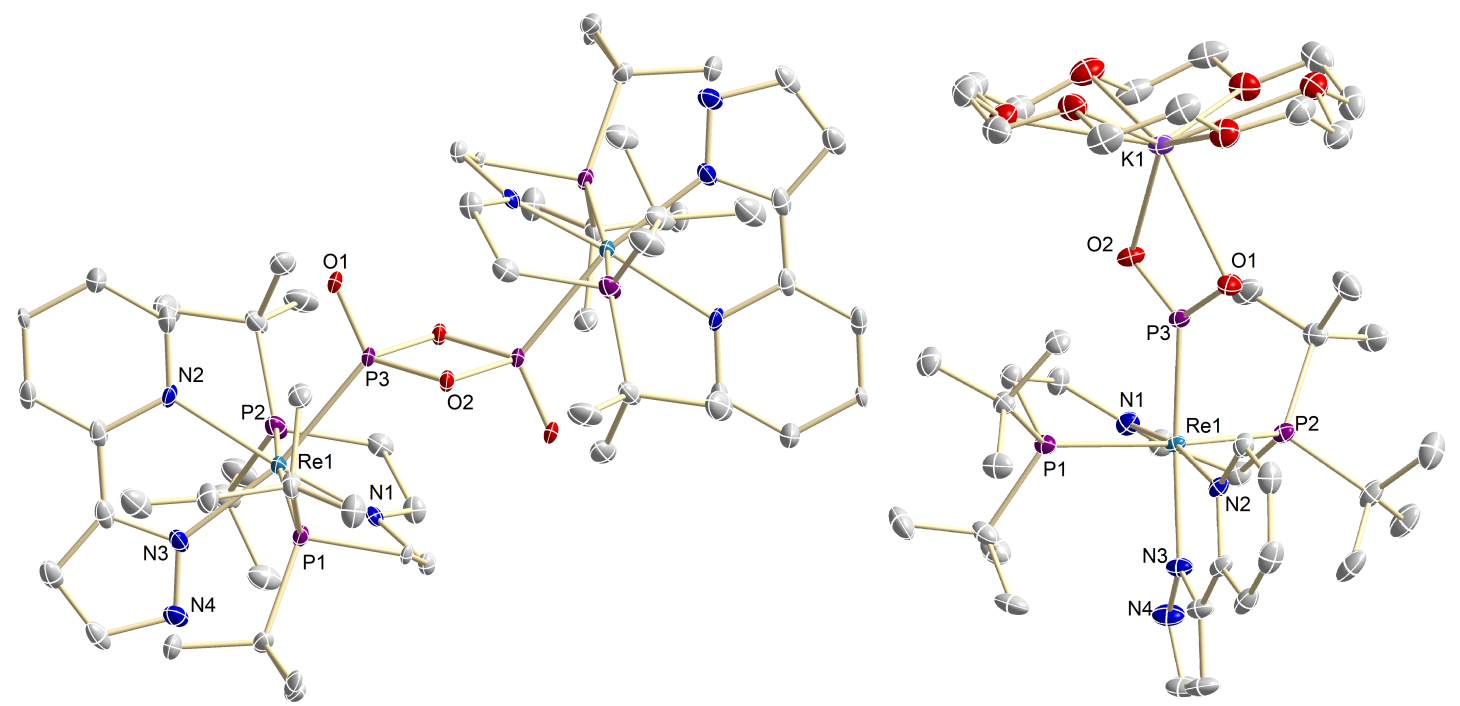

Figure 4.8: Solid state structure of $\mathbf{3 1}$ (left) and $\mathbf{3 4}$ (right) from single-crystal X-ray diffraction (thermal ellipsoids drawn at the $50 \%$ probability level); solvent molecules and hydrogen atoms are omitted for clarity. Selected bond lengths $[\AA]$ and angles $\left[^{\circ}\right]$ : 31 Re1-N1 1.900(5), Re1-N2 2.263(5), Re1-N3 2.143(6), Re1-P1 2.4457(19), Re1-P2 2.4528(18), Re1-P3 2.4564(18), P3-O1 1.484(5), P3-O2 1.685(5), N1-Re1-P3 82.75(16), P1-Re1-P2 163.84(16), O2-P3-O2\# 82.8(2); 34 Re1-N1 2.082(2), Re1-N2 2.141(2), Re1-N3 2.162(2), Re1-P1 2.3977(7), Re1-P2 2.4332(7), Re1P3 2.2545(7), P3-O1 1.517(2), P3-O2 1.512(2), O1-K1 2.730(2), O1-K2 2.731(2), N1-Re1-P3 94.31(9), P1-Re1-P2 155.70(2), O1-P3-O2 110.26(12).

The cyclic voltammogram of $\mathbf{3 1}$ features an irreversible oxidative wave at $E_{\text {p.a. }} \approx 0.23 \mathrm{~V}$, a reversible reduction at $E_{1 / 2}=-1.92 \mathrm{~V}$ and an irreversible reductive event at $E_{\text {p.c. }} \approx$ $-3.02 \mathrm{~V}$ vs. $\mathrm{Fc}^{0 /+}$ (Figure 4.10, Appendix 8.3.26). A new wave at $E_{\text {a.c. }} \approx 1.71 \mathrm{~V}$ emerges from the irreversible reductive event, suggesting an overall $E E C$ mechanism. The decrease of the peak current at $E_{\text {p.c. }} \approx 1.71 \mathrm{~V}$ upon application of slower scan rates is indicative for a chemical follow-up reaction of the two-electron reduction product of 31. ${ }^{[261]}$ Stirring 31 with one equivalent of NaHg and 15-crown-5 in THF results in a slow color change from orange to red. The obtained product slowly decomposes at room temperature in THF and toluene, indicated by a color change to brown. The cyclic voltammogram suggests that the one-electron reduction of 31 towards $\left[\left\{\operatorname{Re}\left(\mathrm{L}^{1}\right)\left(\kappa^{2} N^{1} N^{2}\right.\right.\right.$ $\left.\mathrm{PyrPz})\}_{2}\left\{\mu-P^{1} P^{2}-\mathrm{P}_{2} \mathrm{O}_{4}\right\}\right]^{-}(\mathbf{3 3})$ is reversible on the timescale of the experiment, indicating a certain lifetime. The formation of a paramagnetic species is confirmed by EPR spectroscopy (Figure 4.10). The unpaired electron resides mainly on the metal center since only ${ }^{185 / 187}$ Re hyperfine splitting is detected $\left(A_{\text {iso }}\left({ }^{185 / 187} \operatorname{Re}\right)=756 \mathrm{MHz}\right.$, Figure 4.10). No additional (super)-hyperfine interactions could be resolved. The sign of $A_{\text {iso }}$ can be derived by the different broadenings of the observed transitions. For an $S=\frac{1}{2}$ systems, the peak-to-peak linewidths $\Delta B_{p-p}$ for the anisotropically splitted signal can be expressed as a polynome dependent on $m_{I}:^{[262]}$ 


$$
\Delta B_{p-p}=A+B m_{I}+C m_{I}^{2}+D m_{I}^{3}
$$

$\mathrm{A}, \mathrm{B}, \mathrm{C}$ and $\mathrm{D}$ are constant and positive. $\mathrm{D}$ is usually close to zero and therefore neglected. From this equation it is obvious that signals with positive $m_{I}$ values will be splitted the most, while negative values lead to smaller splitting and thereby easier isotropic averaging at room temperature. The term $B m_{I}$ is usually dominant and therefore allows to determine the sign of $A_{\text {iso }}$. Since the transition at higher fields are broadened the most, a positive sign is derived for the hyperfine coupling constant (Figure 4.9). A possible rationalization of the positive value lies in electron-electron exchange interactions at the Re center resulting in spin-polarization of the s orbitals. ${ }^{[263]-[266]}$ Nevertheless, spin-orbit coupling and other effects could also play an important role and a more thorough theoretical analysis of the EPR spectrum would be required to fully interpret this finding which is beyond the scope of this thesis.

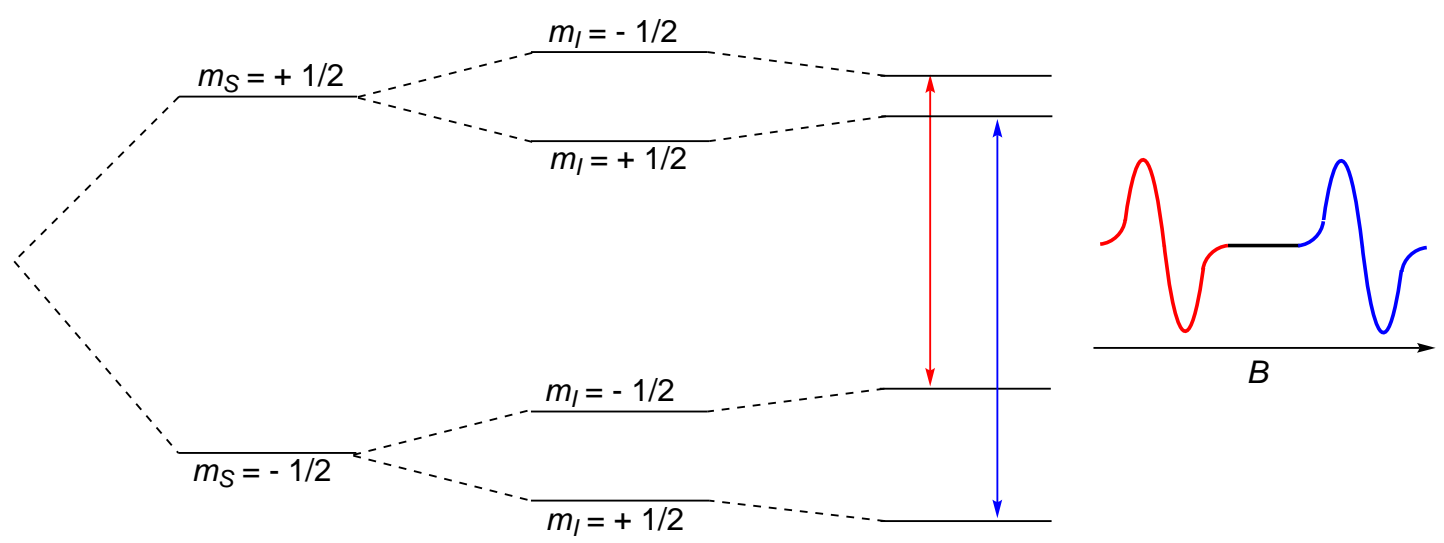

Figure 4.9: State-splitting for an $S=\frac{1}{2}$ system coupled with an $I=\frac{1}{2}$ nucleus and a positive hyperfine splitting value $A$.

IR spectroscopy reveals an intense band at $\tilde{\nu}_{\mathrm{PO}}=1124 \mathrm{~cm}^{-1}$. The minor shift of the stretching vibration when compared to $\mathbf{3 1}$ suggests no significant alteration of the bonding situation in the $\mathrm{P}_{2} \mathrm{O}_{4}^{2-}$ fragment upon reduction, in line with the results of the EPR spectroscopy. Single-crystals suitable for X-ray diffraction experiments could not be obtained.

When 31 is reacted with an excess of $\mathrm{KC}_{8}$ in THF, at low temperatures in the presence of 18-crown-6, a color change from orange over red to deep purple can be observed upon slow warming to room temperature. The disappearance of all ${ }^{31} \mathrm{P}$ NMR signals suggests paramagnetism of the reaction product. Single crystal X-ray diffraction reveals that the $\mathrm{P}_{2} \mathrm{O}_{4}^{2-}$ ligand is split homolytically resulting in the formation of $\left[\mathrm{K}\left(\mathrm{C}_{12} \mathrm{H}_{24} \mathrm{O}_{6}\right)\right]\left[\operatorname{Re}\left(\mathrm{L}^{1}\right)\left(\mathrm{PO}_{2}\right)\left(\kappa^{2} N^{1} N^{3}-\mathrm{PyrPz}\right)\right]$ (34, Scheme 4.6, Figure 4.8), which rep- 
resents the first example of a $\mathrm{PO}_{2}^{-}$ligand bound to a transition metal. 34 features an octahedral coordination mode with a twisted PNP ligand. The $\mathrm{P}-\mathrm{O}$ bond lengths of the trigonal planar $\mathrm{PO}_{2}^{-}$moiety are almost identical (P3-O1: 1.517(2) $\left.\AA, \mathrm{P} 3-\mathrm{O} 2: 1.512(2) \AA\right)$, close to the shorter bond in LXXXIV (P-O: $1.50(3) \AA$ ) and slightly elongated when compared to the experimental value of free $\mathrm{PO}_{2}^{-}$(P-O: $\left.1.50 \pm 0.01 \AA\right) .[245],[260]$
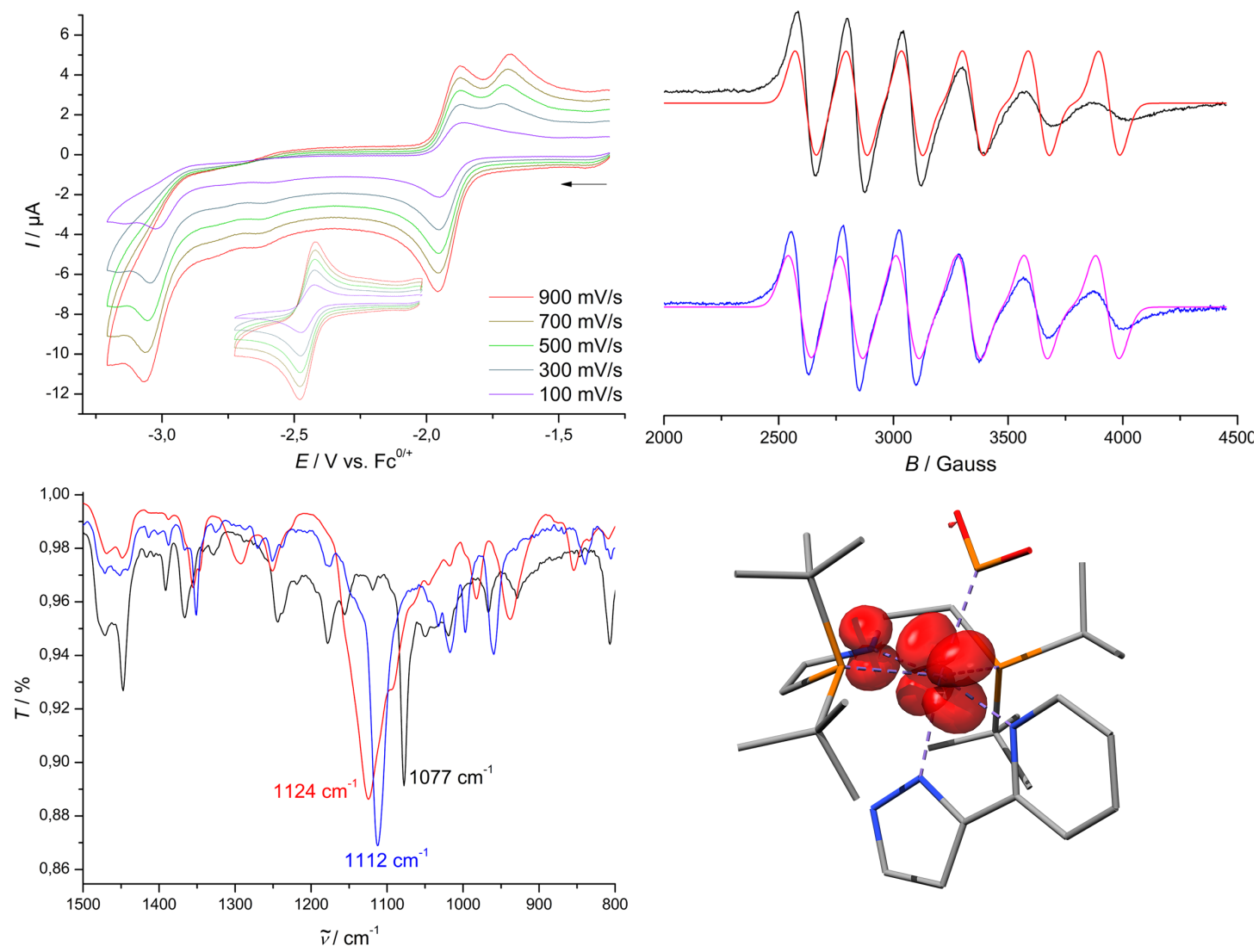

Figure 4.10: Top-Left: Cyclic voltammogram of 31, 0.7 mM, 0.1 M NBu $\mathrm{PF}_{6}$, THF, RT; Inset: First reduction; Top-right: EPR spectrum of 33, Toluene, $260 \mathrm{~K}, 9.4277 \mathrm{GHz}, g_{\text {iso }}=2.038$, $A_{\text {iso }}\left({ }^{185 / 187} \mathrm{Re}\right)=756 \mathrm{MHz}\left(\right.$ top, simulation in red), 34, THF, $300 \mathrm{~K}, 9.4169 \mathrm{GHz}, g_{\text {iso }}=2.046$, $A_{\text {iso }}\left({ }^{185 / 187} \mathrm{Re}\right)=770 \mathrm{MHz}$ (bottom, simulation in pink); Bottom-left: IR spectra of 31 (blue), 33 (red), 34, ATR, RT; Bottom-right: Computed spin-density of $\mathbf{3 4}$.

The Re-P bond length of $d_{\mathrm{ReP}}=2.4564(18) \AA$ and its torsion angle (N1-Re1-P3-O2: $\left.22.6(3)^{\circ}\right)$ suggest no $\pi$-interaction between the metal center and the phosphorus atom. Interestingly, no pure compound can be obtained when no crown ether is added, in line with the cyclic voltammogram. An intense band for the asymmetric stretching vibration is detected at $\tilde{\nu}_{\mathrm{PO}_{2}}=1077 \mathrm{~cm}^{-1}$ (Figure 4.10). The EPR spectrum of $\mathbf{3 4}$ shows high similarities with 33, accompanied by more effective isotropic averaging. While the hyperfine interaction value is almost identical, $g_{\text {iso }}$ shows a minor shift of ca. 0.01 . This suggests that the singly occupied molecular orbitals of $\mathbf{3 3}$ and $\mathbf{3 4}$ are highly similar in 
shape with no significant participation of the $\mathrm{P}_{2} \mathrm{O}_{4}^{2-}$ and $\mathrm{PO}_{2}^{-}$ligands, respectively.

Chemical reversibility of the splitting reaction could be shown by reacting $\mathbf{3 4}$ with one equivalent of $\left[\mathrm{Fe}\left(\mathrm{C}_{5} \mathrm{Me}_{5}\right)_{2}\right]\left[\mathrm{Al}\left(\mathrm{O}\left(\mathrm{C}\left(\mathrm{CF}_{3}\right)_{3}\right)_{4}\right]\right.$ at $-80^{\circ} \mathrm{C}$, which resulted in $33 \%$ conversion towards 31 accompanied by several dia- and paramagnetic side products (Scheme 4.6, Appendix 8.3.28). This result suggests that coupling of the neutral complex $\mathrm{PO}_{2}$ complex $\left[\operatorname{Re}\left(\mathrm{L}^{1}\right)\left(\mathrm{PO}_{2}\right)\left(\kappa^{2} N^{1} N^{3}-\mathrm{PyrPz}\right)\right](\mathbf{3 5})$ towards 31 is feasible at low temperatures but unselective. Therefore, oxygenation of $\mathbf{2 7}$ towards $\mathbf{3 1}$ is likely to proceed via a different pathway, e.g. coupling of a phosphorus monoxide complex towards a dimeric $\mathrm{P}_{2} \mathrm{O}_{2}^{2-}$ complex which is further oxygenated towards $\mathbf{3 1}$.

The electronic structure is further elucidated by DFT calculations. Spin-density calculations reveal a minor density of $1.3 \%$ at the $\mathrm{PO}_{2}^{-}$-phosphorus atom while $74.6 \%$ is located at the Re ion (Figure 4.10). The Re s orbital contribution is calculated to be $10 \%$, in line with the observed sign of the hyperfine coupling constant. In accordance with the computed SOMO, which represents an antibonding $\mathrm{N}_{\mathrm{PNP}}-\mathrm{Re} \pi^{*}$-orbital, the PNP-nitrogen atom exhibits a spin-density of $28.7 \%$ (Appendix 8.3.27). To clarify the bonding situation between the metal center and the $\mathrm{PO}_{2}^{-}$ligand NBO/NLMO computations and Mayer bond orders were considered to obtain a representative Lewis-structure. The calculated bond order of 1.19 of the $\mathrm{Re}-\mathrm{P}$ unit and the shape of the bonding Re-P NLMOs reveal only $\sigma$-bonding interactions while $\pi$-interactions seem to be small, as in nitrogen-bound $\mathrm{NO}_{2}^{-}$ligands, in line with the molecular orbital diagram of free $\mathrm{PO}_{2}^{-}$ (Figure 4.11, Appendix 8.3.29). ${ }^{[253]}$ The Re-P bond features $66 \% \mathrm{P}$ character. However, an energy decomposition analysis of this bonding situation could provide a more detailed description of the Re- $\mathrm{PO}_{2}$ interaction. ${ }^{[19]}$ Bond analysis of the $\mathrm{PO}_{2}^{-}$fragment reveals $\mathrm{P}-\mathrm{O}$ bond order of 1.63 and 1.69. Three lone-pairs are found for each oxygen atom while the phosphorus atom features an unoccupied valence orbital. One of the lone pairs of each oxygen atoms is depleted of electron density which can be located at the phosphorus center (Figure 4.11). Based on this, the $\mathrm{PO}_{2}^{-}$ligand is best described as an anionic $\sigma$-donor ligand which features a phosphorus atom that is resonance-stabilized by the oxygen lone-pairs, resulting in the Lewis-structure shown in Figure 4.11, in line with the computed NPA charges. Due to the identical contributions of $\alpha$ and $\beta$ spin NLMOs to the fragments considered in the $\mathrm{PO}_{2}^{-}$unit, it can be concluded that the unpaired electron has no significant influence on this bonding situation. 


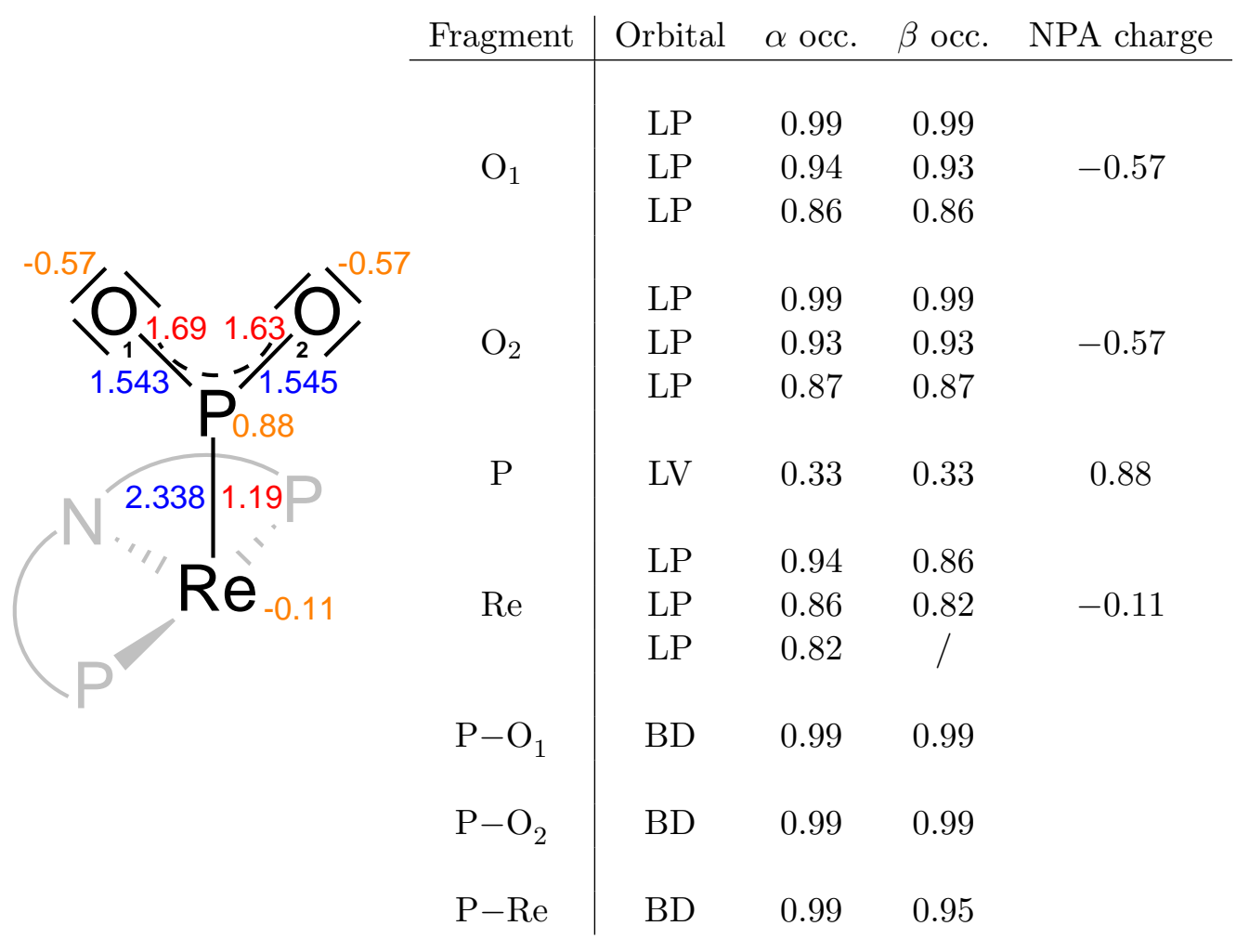
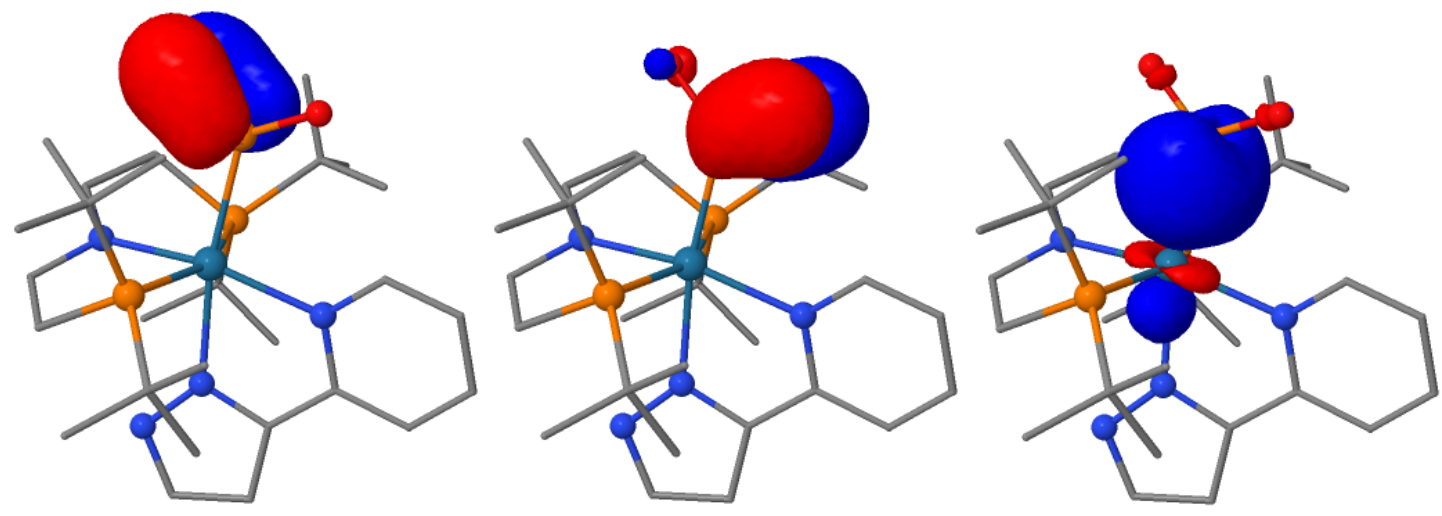

\begin{tabular}{c|ccc} 
Fragment & $\mathrm{O} / \%$ & $\mathrm{P} / \%$ & $\mathrm{Re} / \%$ \\
\hline $\mathrm{O}_{1} \stackrel{\pi}{\rightarrow} \mathrm{P}$ & 82.9 & 16.1 & \\
$\mathrm{O}_{2} \stackrel{\pi}{\rightarrow} \mathrm{P}$ & 85.5 & 11.7 & \\
$\mathrm{P} \stackrel{\sigma}{\rightarrow} \mathrm{Re}$ & & 62.5 & 34.0
\end{tabular}

Figure 4.11: Top left: Lewis-structure of $\mathbf{3 4}$ derived by NBO/NLMO and Mayer bond order calculations (calculated bond distances in blue, bond orders in red, NPA charges in orange); Top right: $\mathrm{NBO}$ occupations for $\alpha$ and $\beta$ spin orbitals, $\mathrm{LP}=$ lone pair, $\mathrm{LV}=$ lone vacancy, $\mathrm{BD}$ $=2 \mathrm{c} 2 \mathrm{e}$ bond and calculated NPA charges; Middle: Computed $\alpha$ spin NLMOs representing the resonance stabilization of the phosphorus atom (left, middle) and $\sigma$-donation of the ligand to the $\operatorname{Re} \mathrm{d}_{\mathrm{z}^{2}}$ orbital (right); Bottom: Atom contributions to the Re-P $\sigma$ and $\pi$ NLMOs. 


\subsection{Interconversion of Os PHMes* and PMes* Complexes by PCET}

The reactivity of $\mathbf{2}$ towards oxidative addition of primary phosphines was initially probed by reaction with $\mathrm{PH}_{2} \mathrm{Ph}$. When a freshly prepared solution of 2 is reacted with $\mathrm{PH}_{2} \mathrm{Ph}$, clean formation of green phosphine complex $\left[\mathrm{OsCl}\left(\mathrm{L}^{3}\right)\left(\mathrm{PH}_{2} \mathrm{Ph}\right)\right](\mathbf{3 6}$, Scheme 4.7) can be accomplished in $80 \%$ isolated yield. 36 exhibits a distorted square-pyramidal coordination sphere in the solid state with the phosphine ligand in the apical position (Figure 4.12). Due to coordination, the ${ }^{31} \mathrm{P}$ NMR signal of $\mathrm{PH}_{2} \mathrm{Ph}$ is downshifted to $\delta_{\mathrm{P}}=-62.2 \mathrm{ppm}$ with a coupling constant of ${ }^{2} J_{\mathrm{PP}}=18.7 \mathrm{~Hz}$. No conversion to an $\mathrm{Os}^{\mathrm{IV}}$ species was observed upon heating indicating that oxidative addition might be thermodynamically unfavorable.

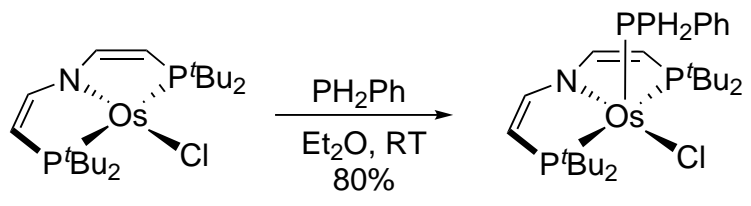

2

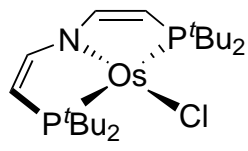

2

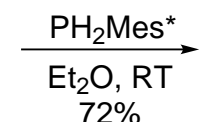

$72 \%$
36

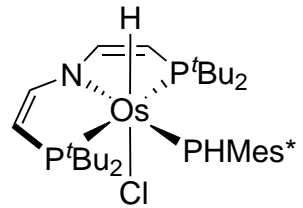

37

Scheme 4.7: Reaction of $\mathbf{2}$ with the primary phosphines $\mathrm{PH}_{2} \mathrm{Ph}$ and $\mathrm{PH}_{2} \mathrm{Mes}^{*}$ to yield $\mathbf{3 6}$ and 37, respectively.

Therefore, the more electron-rich phosphine $\mathrm{PH}_{2}$ Mes* was utilized. Complex 2 reacts readily with the primary phosphine to the dark-blue oxidative addition product [OsCl$\mathrm{H}\left(\mathrm{L}^{3}\right)$ (PHMes*) (37, Scheme 4.7). The ${ }^{31} \mathrm{P}$ signal of the PHMes* moiety is observed at $\delta_{\mathrm{P}}=103.9 \mathrm{ppm}$ excluding simple phosphine coordination. Upon mixing an instant color change to green is observed which is tentatively assigned to initial phosphine coordination. 37 decomposed over the course of a few hours in solution at room temperature but could be fully characterized including single crystal X-ray diffraction. The NMR signals at $-30^{\circ} \mathrm{C}$ are in agreement with $C_{1}$-symmetry, indicating phosphanide to $\mathrm{Os}^{\mathrm{IV}}$ $\pi$-donation. Accordingly, the phosphide P-atom exhibits trigonal-planar coordination in the solid state (Figure 4.12). 

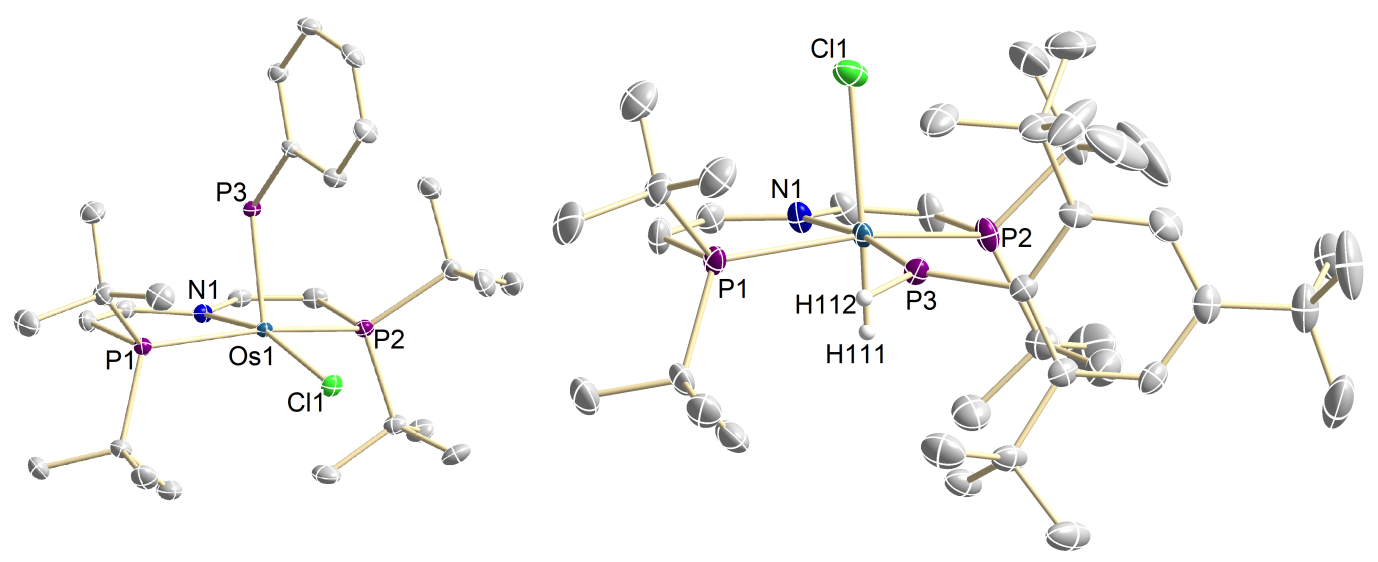

Figure 4.12: Molecular structure of complex 36 (left) and 37 (right) from single-crystal X-ray diffraction (thermal ellipsoids drawn at the $50 \%$ probability level); solvent molecules, hydrogen atoms, except hydrides and the $\mathrm{P}-\mathrm{H}$ of $\mathbf{3 7}$ are omitted for clarity. Selected bond lengths $[\AA]$ and angles [ [ ${ }^{\circ}$ : 36 Os1-Cl1 2.3865(5), Os1-N1 3.0754(18), Os1-P1 2.3604(6), Os1-P2 2.3602(6), Os1-P3 2.1928(6), N1-Os1-Cl1 162.89(6), P1-Os1-P2 160.39(2), $\tau=0.04$; 37 Os1-Cl1 2.4912(11), Os1-H111 1.54(2), Os1-N1 2.078(3), Os1-P1 2.4004(11), Os1-P2 2.4475(10), Os1-P3 2.1919(11), P3-H112, 1.35(3), N1-Os1-P3 171.59(8), P1-Os1-P2 156.14(5).

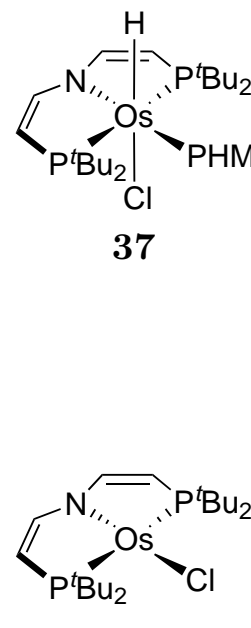

2

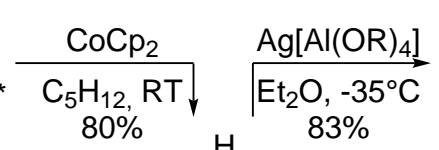
$80 \%$

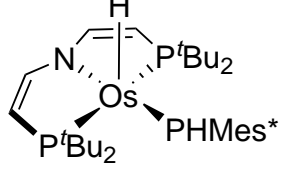

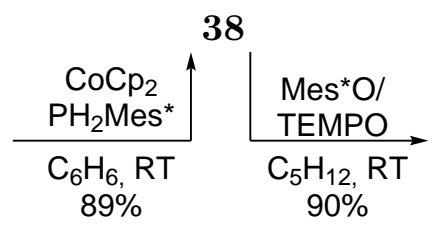
$89 \%$

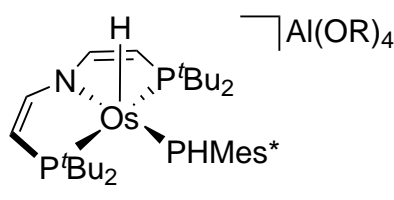

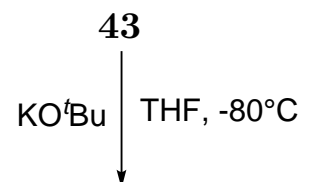

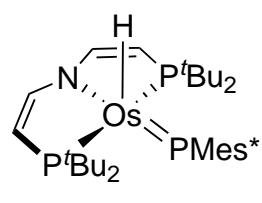

39

Scheme 4.8: Synthesis of $\mathbf{3 8}$ starting from $\mathbf{3 7}$ or $\mathbf{2}$ and its conversion towards $\mathbf{4 3}$ and $\mathbf{3 9}, \mathrm{R}=$ $\mathrm{C}\left(\mathrm{CF}_{3}\right)_{3}$.

Reduction of $\mathbf{3 7}$ with $\mathrm{CoCp}_{2}$ affords the radical complex $\left[\mathrm{OsH}\left(\mathrm{L}^{3}\right)\left(\mathrm{PHMes}^{*}\right)\right](\mathbf{3 8}$, Scheme $4.8)$ in $80 \%$ isolated yield after column chromatography. The magnetic moment in solution $\left(\mu_{\mathrm{eff}}=1.51 \mu_{\mathrm{B}}\right)$ derived by Evan's method is in agreement with an $S=\frac{1}{2}$ system with unquenched orbital momentum. $\mathbf{3 8}$ can also be directly synthesized from 2 by one-pot oxidative addition and subsequent reduction. Characteristic bands for the PHMes* and hydride ligands were detected in the IR spectrum $\left(\tilde{\nu}_{\mathrm{PH}}=2345 \mathrm{~cm}^{-1}\right.$; 

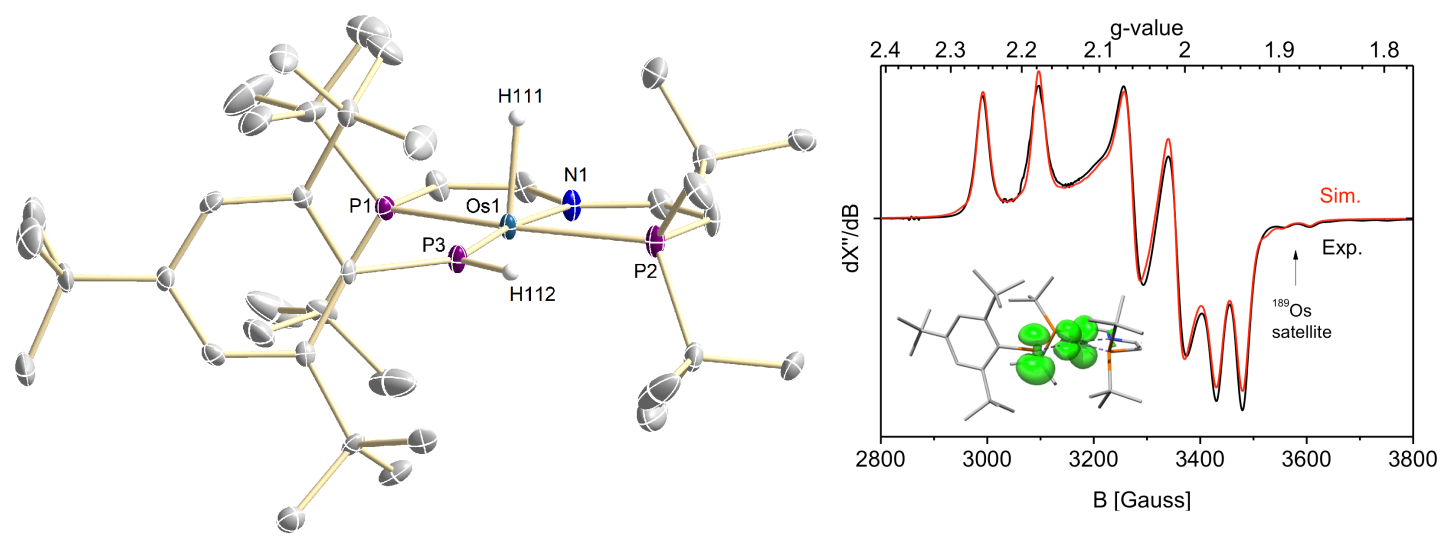

Figure 4.13: Left: Molecular structure of complex 38 (left) from single-crystal X-ray diffraction (thermal ellipsoids drawn at the 50\% probability level); solvent molecules, hydrogen atoms, except hydrides and the $\mathrm{PH}$ protons of $\mathbf{3 8}$ are omitted for clarity. Selected bond lengths $[\AA]$ and angles [ $\left.{ }^{\circ}\right]$ : 38 Os1-H111 1.96(6), Os1-N1 2.069(4), Os1-P1 2.3723(12), Os1-P22.3537(13), Os1-P3 2.2301(13), P3-H112 1.35(5), N1-Os1-P3 172.17(11), P1-Os1-P2 160.20(4), $\tau=0.2$; Right: EPR spectrum of 38, $9.4366 \mathrm{GHz}$, toluene, $148 \mathrm{~K}$, simulation (red) with the following parameters (DFT computed values in brackets): $g_{x}=1.952(1.951), g_{y}=2.033(2.024)$, $g_{z}=2.214(2.230) ; A_{x}\left({ }^{31} \mathrm{P}\right)=+57 \mathrm{Mhz}(+58 \mathrm{MHz}), A_{y}\left({ }^{31} \mathrm{P}\right)=+105 \mathrm{Mhz}(+95 \mathrm{MHz}), A_{z}\left({ }^{31} \mathrm{P}\right)=$ $+440 \mathrm{Mhz}(+441 \mathrm{MHz})$; Euler angles: $\alpha=-73(-74.3), \beta=+137(+142.7), \gamma=-7.5(-7.5)$; $A_{x}\left({ }^{189} \mathrm{Os}\right)=-240 \mathrm{MHz}(-277 \mathrm{MHz})$. Inset: Spin-density plot.

$\tilde{\nu}_{\mathrm{OsH}}=2180 \mathrm{~cm}^{-1}$, Appendix 8.3.36). In the solid state, complex $\mathbf{3 8}$ features a distorted square-pyramidal coordination geometry with the hydride in the apical position (Figure 4.12). The Os-P bond to the PHMes* ligand is slightly elongated by $\Delta d=0.04 \AA$ upon reduction from $\mathbf{3 7}$ to 38 .

The EPR spectrum of $\mathbf{3 8}$ in frozen toluene exhibits a rhombic signal that is in agreement with an $S=\frac{1}{2}$ low-spin configuration (Figure 4.13). The g-anisotropy $\left(g_{x}=1.952\right.$, $\left.g_{y}=2.033, g_{z}=2.214\right)$ is considerably smaller than that of typical Os ${ }^{\mathrm{II}}$ complexes, e.g. 1 and $\mathbf{1 2}$, in line with reduced spin-orbit interaction due to ligand redox noninnocence. ${ }^{[267]}$ Accordingly, large and slightly rhombic hyperfine interaction (HFI) with one ${ }^{31} \mathrm{P}$ nucleus is observed in all principal directions of the $g$-tensor $\left(A_{x}=+57 \mathrm{MHz}\right.$, $A_{y}=+105 \mathrm{MHz}, A_{z}=+440 \mathrm{MHz}$ ), supporting considerable spin delocalization to the PHMes* ligand. Partially resolved ${ }^{189}$ Os satellite HFIs are detected as well. The free phosphinyl radical $\mathrm{PPh}_{2}$ exhibits axial ${ }^{31} \mathrm{P}-\mathrm{HFI}$ with an isotropic coupling constant $\left(A_{\text {iso }}\left({ }^{31} \mathrm{P}\right)=260 \mathrm{MHz}\right)$ close to that of $\mathbf{3 8}\left(A_{\text {iso }}\left({ }^{31} \mathrm{P}\right)=201 \mathrm{MHz}\right)$. The higher isotropic HFI $\left(A_{\text {iso }}\left({ }^{31} \mathrm{P}\right)=499 \mathrm{MHz}\right)$, yet reduced dipolar coupling, found for the transient phosphinyl radical complex $\left[\mathrm{W}\left(\mathrm{PPh}_{2}\right)(\mathrm{CO})_{5}\right]$ was attributed to $\mathrm{p}_{\mathrm{P}} \rightarrow \mathrm{d}_{\mathrm{W}}$ spin delocalization $\left(\rho_{\mathrm{P}}=75 \%\right)$ and concomitant polarization of the $\mathrm{P}$ lone-pair. ${ }^{[141]}$ For 38, the higher $g$-anisotropy and smaller isotropic and anisotropic contributions of the HFI tensor are consistent with increased $\mathrm{P} \rightarrow \mathrm{M}$ spin delocalization. Comparing the isotropic $\left(A_{\text {iso }}=201 \mathrm{MHz}\right)$ and anisotropic $\left(T_{x}=-144 \mathrm{MHz}, T_{y}=-96 \mathrm{MHz}, T_{z}=+239 \mathrm{MHz}\right)$ 
contributions to the ${ }^{31} \mathrm{P}$-HFI with atomic parameters allows for a rough estimate of phosphorus spin densities $\left(\rho_{3 \mathrm{~s}} \approx 2 \%, \rho_{3 \mathrm{p}} \approx 42 \%\right)$ when treating the HFI approximately axial $\left(T_{\perp}=\left(T_{x}+T_{y}\right) / 2\right)$. ${ }^{[267]}$ DFT calculations fully support this interpretation. Both the experimental molecular structure and the EPR parameters were reproduced computationally $\left(g_{x}^{\mathrm{DFT}}=1.951, g_{y}^{\mathrm{DFT}}=2.024, g_{z}^{\mathrm{DFT}}=2.230 ;{ }^{31} \mathrm{P}-\mathrm{HFI}: A_{x}^{\mathrm{DFT}}=+58 \mathrm{MHz}\right.$, $\left.A_{y}^{\mathrm{DFT}}=+95 \mathrm{MHz}, A_{z}^{\mathrm{DFT}}=+441 \mathrm{MHz}\right)$. The $\mathrm{SOMO}$ of $\mathbf{3 8}$ represents an antisymmetric $\left(\pi^{*}\right)$ combination of the metal $\mathrm{d}_{\mathrm{yz}}$ orbital and a phosphorus p orbital (Appendix 8.3.37). Reduced $\pi$-bonding is expressed by the Os-PHMes* Mayer bond index (1.46). In consequence, the computed spin density is almost equally distributed between the phosphorus $\left(\rho_{\mathrm{P}}=47 \%\right)$ and osmium atoms $\left(\rho_{\mathrm{Os}}=50 \%\right)$. The spectroscopic and computational evaluation supports considerable redox non-innocence of the PHMes* ligand. Complex 38 represents an unprecedented, isolable phosphanide complex with large phosphinyl radical-character $\left(\mathrm{Os}^{\mathrm{II}}-\mathrm{PR}_{2}\right)$. Therefore, possible $\mathrm{PCET}$ reactivity of the radical ligand, specifically as an entry to phosphinidene chemistry, was examined. Two experimental methods are widely used to estimate $\mathrm{E}-\mathrm{H} \mathrm{BD}(\mathrm{F}) \mathrm{Es}$ of organometallic compounds, i.e. (a) bracketing based on hydrogen transfer reagents with reference H-donor/acceptor reagents and (b) quantification via the square-scheme formalism, i.e. a thermochemical redox/protonation cycle. ${ }^{[268]}$

Complex 38 shows no reactivity with the H-atom donor reagent TEMPO-H (TEMPO $=2,2,6,6$-tetramethylpiperidinyloxyl; $\left.\mathrm{BDFE}_{\mathrm{OH}} \approx 66 \mathrm{kcal} \cdot \mathrm{mol}^{-1[268]}\right)$, indicating weak $\mathrm{P}-\mathrm{H}$ bonds for the hypothetical $\mathrm{Os}^{\mathrm{II}}$ phosphine complex $\left[\mathrm{OsH}\left(\mathrm{L}^{3}\right)\left(\mathrm{PH}_{2} \mathrm{Mes}^{*}\right)\right]$. Kinetic reasons are unlikely for the lack of reactivity as $\mathbf{3 8}$ readily undergoes $\mathrm{P}-\mathrm{H}$ transfer with TEMPO. In turn, $\mathbf{3 8}$ readily reacts with the $\mathrm{H}$-atom acceptors Mes*O and TEMPO. The purple product that is obtained in high yields around $90 \%$ exhibits low thermal stability at room temperature even in the solid state but could be characterized by NMR spectroscopy at $-30^{\circ} \mathrm{C}$ (Appendix 8.3.38). Retention of the hydride ligand is indicated by a ${ }^{1} \mathrm{H}$ NMR signal at $\delta_{\mathrm{H}}=-15.9 \mathrm{ppm}$. Formation of the phosphinidene complex $\left[\mathrm{Os}\left(\mathrm{PMes}^{*}\right) \mathrm{H}\left(\left(\mathrm{L}^{3}\right)\right] \quad(\mathbf{3 9}\right.$, Scheme 4.8$)$ is evidenced by the ${ }^{31} \mathrm{P}$ NMR signal at $\delta_{\mathrm{P}}=$ $825 \mathrm{ppm}$, assignable to the PMes* group. While suitable crystals for X-ray diffraction could not be obtained, the structural integrity of the osmium pincer phosphinidene framework is supported by derivatization (Scheme 4.9).

A solution of in situ prepared 39 in THF selectively converts over the course of some hours to a mixture of the two diastereomers of $\mathrm{Os}^{\mathrm{II}}$ phosphine complex $\mathbf{4 0}$ as indicated by LIFDI-MS and NMR spectroscopy (Figure 4.14, Appendix 8.3.41). Electrophilic phosphinidene insertion into $\mathrm{C}-\mathrm{H}$ bonds has previously been reported. ${ }^{[161],[269]}$ Furthermore, addition of carbon monoxide to $\mathbf{3 9}$ gives the five-coordinate phosphanide complex $\left[\mathrm{Os}\left(\mathrm{L}^{3}\right)\left(\mathrm{PHMes}^{*}\right)(\mathrm{CO})\right]$ (41, Scheme 4.9) after Os-H reductive elimination, which could be crystallographically characterized (Figure 4.15). 

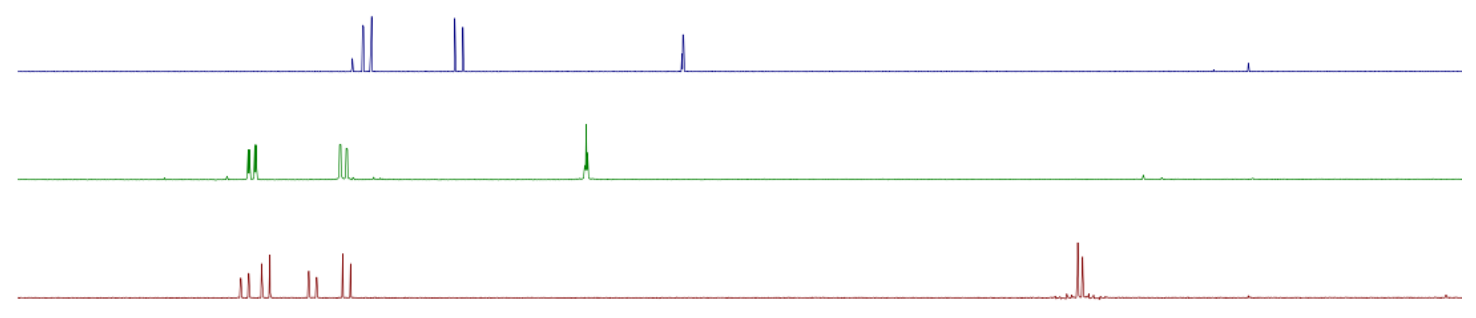

$\begin{array}{lllllllllllllllllllllll}0 & 90 & 80 & 70 & 60 & 50 & 40 & 30 & 20 & 10 & 0 & -10 & -20 & -30 & -40 & -50 & -60 & -70 & -80 & -90 & -100 & -11\end{array}$

Figure 4.14: ${ }^{31} \mathrm{P}\left\{{ }^{1} \mathrm{H}\right\}$ NMR spectrum of the reaction of 39 with $\mathrm{PhCCH}$ yielding $42, \mathrm{C}_{6} \mathrm{D}_{6}$ (top), 41, THF-d $\mathrm{d}_{8}$ (middle) and 40, $\mathrm{C}_{6} \mathrm{D}_{6}$ (bottom).

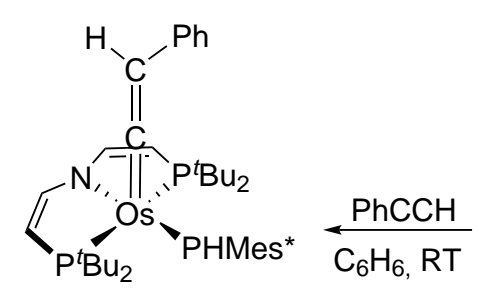

42

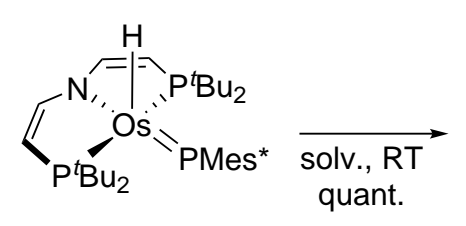

39

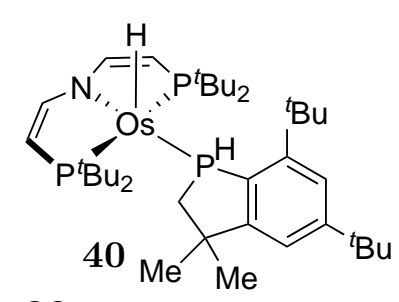

$\mathrm{CO}$

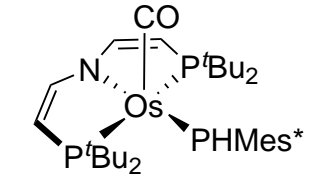

41

Scheme 4.9: Conversion of 39 towards 40 and reaction with $\mathrm{CO}$ and $\mathrm{PhCCH}$ to give $\mathbf{4 1}$ and 42 , respectively.

The Os-PHMes* distance shows an elongation by $\Delta d=0.034 \AA$ with respect to $\mathbf{3 8}$, accompanied by a non-planar phosphorus atom which can be attributed by further population of the repelling Os d orbital. Considerable $\pi^{*}$-backbonding is indicated by $\tilde{\nu}_{\mathrm{CO}}=1888 \mathrm{~cm}^{-1}$ (Appendix 8.3.42). Since the phosphinidene shows electrophilic reactivity, the terminal alkyne $\mathrm{PhCCH}$ was reacted with 39 to investigate if heterocycle formation occurs. Full conversion towards a $C_{1}$-symmetric species is observed by ${ }^{31} \mathrm{P}$ NMR. As for 41, a PHMes* moiety is detected in the ${ }^{1} \mathrm{H}$ NMR $\left(\delta_{\mathrm{H}}=9.39 \mathrm{ppm}\right.$, ${ }^{1} J_{\mathrm{HP}}=308.7,{ }^{3} J_{\mathrm{HP}}=12.3,5.3,{ }^{4} J_{\mathrm{HP}}=1.6 \mathrm{~Hz}$ ) suggesting reductive elimination of the hydride ligand. This is further supported by the phosphorus NMR shift of the PHMes* moiety $\left(\delta_{\mathrm{P}}=3.52 \mathrm{ppm}\right.$, Figure 4.14$) .{ }^{13} \mathrm{C}$ NMR shows that the alkyne isomerized over the course of the reaction yielding a vinylidene ligand as commonly observed for the addition of alkynes to low-valent metal centers $\left(\delta_{\mathrm{Os}=\mathrm{C}=\mathrm{C}}=274.8,113.2 \mathrm{ppm}\right) .{ }^{[270]}$ The reaction was accompanied by the formation of side products which could not be removed 
by common purification techniques preventing characterization by combustion analysis. Due to high solubility in organic solvents, even at $-80^{\circ} \mathrm{C}$, no single crystals suitable for $\mathrm{X}$-ray diffraction could be obtained. However, the indicative NMR signals suggest that the vinylidene complex $\left[\mathrm{Os}(\mathrm{CCHPh})\left(\mathrm{L}^{3}\right)\left(\mathrm{PHMes}^{*}\right)\right](\mathbf{4 2})$ was formed. The observed metal centered reactivity likely occurs due to the high Lewis-basicity of Os center in combination with the steric bulk shielding the phosphinidene phosphorus atom.
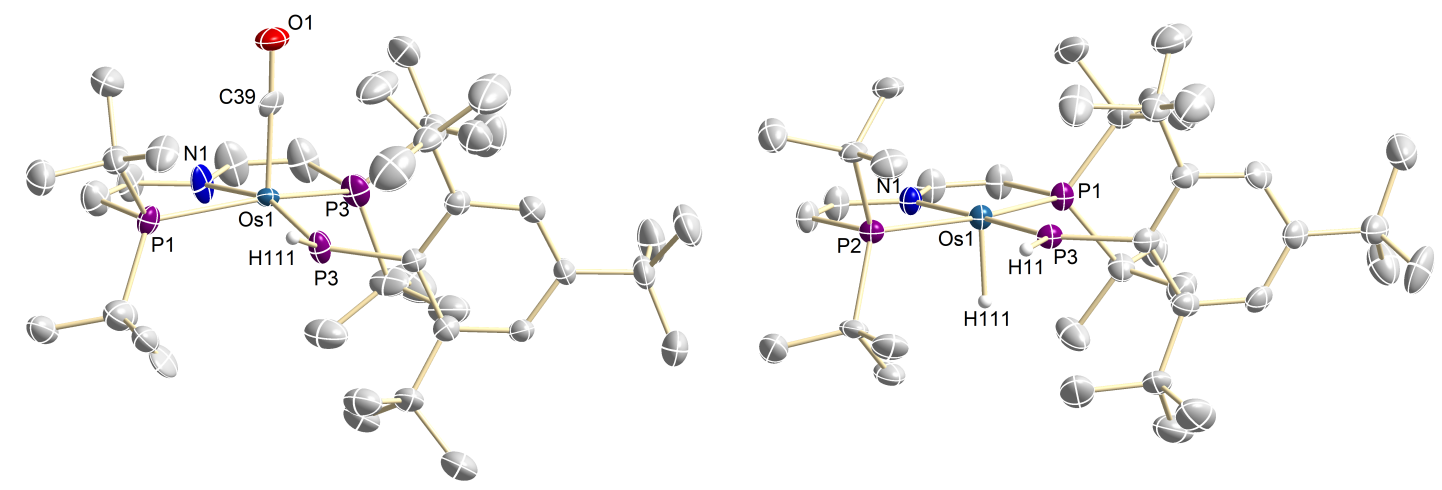

Figure 4.15: Molecular structure of complex 41 (left) and 43 (right) from single-crystal X-ray diffraction (thermal ellipsoids drawn at the $50 \%$ probability level); hydrogen atoms, except the $\mathrm{PH}$ protons and hydride ligands, and anions are omitted for clarity. Selected bond lengths $[\AA]$ and angles $\left[{ }^{\circ}\right]$ : 41 Os1-C39 17.761(16), C39-O1 1.24(2), Os1-N1 2.141(9), Os1-P1 2.443(3), Os1P2 2.372(3), Os1-P3 2.264(3), P3-H111 1.31(12), N1-Os1-P3 161.4(3), P1-Os1-P2 155.39(13), $\tau=0.1 ; 43$ Os1-H111 1.59(2), Os1-N1 2.004(5), Os1-P1 2.4011(15), Os1-P2 2.3927(15), Os1-P3 2.1943(15), P3-H11 1.27(7), N1-Os1-P3 175.14(15), P1-Os1-P2 161.03(5), $\tau=0.24$.

Parent 38 offers two potential sites for hydrogen atom abstraction. The generation of phosphinidene 39 therefore indicates higher Os-H over $\mathrm{P}-\mathrm{H}$ bond strength if the reaction proceeds under thermodynamic control. BDE quantification was attempted by stepwise oxidation and deprotonation of $\mathbf{3 8}$. The cyclic voltammogram of $\mathbf{3 8}$ reveals quasi-reversible reduction at $E_{\text {p.c. }} \approx-2.06 \mathrm{~V}$ and reversible oxidation at $E_{1 / 2}=-0.88 \mathrm{~V}$ vs. $\mathrm{FeCp}_{2}^{+/ 0}$ (Appendix 8.3.31).

Chemical oxidation with $\mathrm{Ag}\left[\mathrm{Al}(\mathrm{OR})_{4}\right]\left(\mathrm{R}=\mathrm{C}\left(\mathrm{CF}_{3}\right)_{3}\right)$ at $-35^{\circ} \mathrm{C}$ immediately gives the deep blue $\mathrm{Os}^{\mathrm{IV}}$ phosphanide complex $\left[\mathrm{OsH}\left(\mathrm{L}^{3}\right)\left(\mathrm{PHMes}^{*}\right)\right]\left[\mathrm{Al}(\mathrm{OR})_{4}\right]$ (43, Scheme 4.8). Complex 43 readily decomposes at room temperature but could be fully characterized at low temperatures including crystallography which features square-pyramidal coordination and a shortening of the Os-PHMes* by $\Delta d=0.036 \AA$ with respect to 38 . Retention of the hydride and PHMes* proton is indicated by their distinctive ${ }^{1} \mathrm{H}$ NMR signals $\left(\delta_{\mathrm{H}}=18.3,-21.7 \mathrm{ppm}\right) . \mathbf{3 8}, \mathbf{4 1}$ and $\mathbf{4 3}$ represent an unprecedented series of fivecoordinate Os complexes with a terminally bound phosphanide ligand in three oxidation states (Figure 4.15). Deprotonation of in situ prepared 43 with $\mathrm{KO}^{t} \mathrm{Bu}$ at $-80^{\circ} \mathrm{C}$ gives the phosphinidene complex $\mathbf{3 9}$ in almost quantitative yield. However, the low thermal stability of $\mathbf{4 3}$ severely hampered reliable $\mathrm{p} K_{\mathrm{a}}$ derivation. 
The $\mathrm{P}-\mathrm{H}$ bond strength of $\mathbf{3 8}$ was therefore derived by isothermal titration calorimetry (ITC) using a PCET reagent. Titration of $\mathbf{3 8}$ with $\mathrm{Mes}^{*} \mathrm{O}$ in benzene or THF afforded the reaction enthalpies for $\operatorname{HAT}\left(\Delta_{\mathrm{r}} H^{\mathrm{C}_{6} \mathrm{H}_{6}}=-16.5 \mathrm{kcal} \cdot \mathrm{mol}^{-1}, \Delta_{\mathrm{r}} H^{\mathrm{THF}}=-17 \mathrm{kcal}\right.$. $\left.\mathrm{mol}^{-1}\right)$ and consequently the $\mathrm{BDE}_{\mathrm{PH}}$ of $\mathbf{3 8}\left(\mathrm{BDE}_{6} \mathrm{H}_{6}=65.1 \pm 1 \mathrm{kcal} \cdot \mathrm{mol}^{-1}, \mathrm{BDE}^{\mathrm{THF}}=\right.$ $\left.67 \pm 1 \mathrm{kcal} \cdot \mathrm{mol}^{-1}\right)$. Computational evaluation confirmed the calorimetric results. DFT analysis of $\mathbf{3 8}$ predicted a $\mathrm{BDE}$ of the phosphanide $\mathrm{P}-\mathrm{H}$ bond (calibrated $\mathrm{BDE}_{\mathrm{PH}}=$ $67.5 \mathrm{kcal} \cdot \mathrm{mol}^{-1}$; non-calibrated: $\left.64.0 \mathrm{kcal} \cdot \mathrm{mol}^{-1}\right)$, i.e. considerably lower than the Os $-\mathrm{H}$ bond (calibrated $\mathrm{BDE}_{\mathrm{OsH}}=74.2 \mathrm{kcal} \cdot \mathrm{mol}^{-1}$; non-calibrated: $70.1 \mathrm{kcal} \cdot \mathrm{mol}^{-1}$, for calibration details see experimental section), indicating that phosphinidene $\mathbf{3 9}$ is the thermodynamic PCET product.

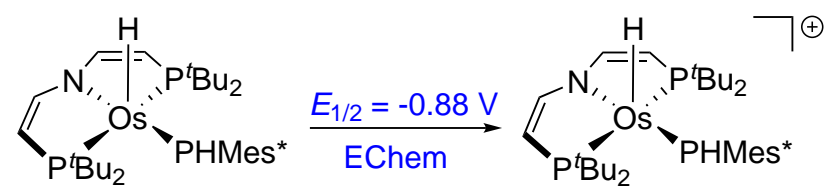

38

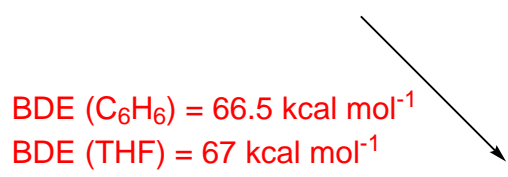

43

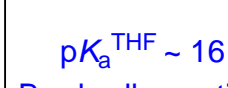

Bordwell equation

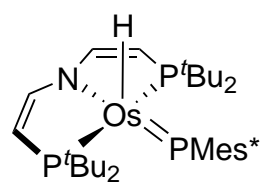

39

Scheme 4.10: Thermochemical square-scheme of 38,43 and 39 .

The calorimetric and electrochemical data also allows for calculation the $\mathrm{p} K_{\mathrm{a}}$ of $\mathbf{4 3}$ $\left(\mathrm{p} K_{\mathrm{a}}^{\mathrm{THF}}=16\right)$ from a thermochemical square-scheme by using the Bordwell equation (Scheme 4.10, Equation 4.2).

$$
\operatorname{BDE}_{\mathrm{THF}}(\mathrm{X}-\mathrm{H})=1.37 \mathrm{p} K_{\mathrm{a}}+23.06 E^{\circ}+\mathrm{C}_{\mathrm{H}, \mathrm{THF}}, \quad \text { where } \mathrm{C}_{\mathrm{H}, \mathrm{THF}}=66 \mathrm{kcal} \cdot \mathrm{mol}^{-1}
$$




\subsection{An Isolable Terminal Chlorophosphinidene Complex}

In Chapter 4.1 it was demonstrated that the phosphaethynolate anion is a competent phosphorus transfer reagent to access the terminal Re phosphide complex $\mathbf{2 7}$. The question arises if this approach can be transferred to group 8 systems to further extend the realm of heavy pnictide multiple bonds. Besides this possible entry to low valent osmium phosphorus chemistry, utilization of the recently reported chloro-substituted 7-phosphadibenzonorbornadiene (ClPA, Scheme 4.11), which has served as an attractive precursor for the synthesis of phosphorus transfer reagents, can be considered. ${ }^{[67],[271]-[273]}$ A recent report features the transfer of a $\mathrm{P}-\mathrm{Cl}$ unit to a $\mathrm{Mo}^{0}$ complex resulting in a $\mathrm{Mo}^{\mathrm{IV}}$ phosphide chloride complex (For details see the introduction of this thesis). ${ }^{[64]}$ Additionally, it was demonstrated that ClPA can serve as a phosphorus atom source when reacted with the anionic vanadium nitride $\mathbf{L X X X V}$ yielding the transient unprecedented $\mathrm{N} \equiv \mathrm{P}$ ligated complex $\mathbf{L X X X V I}$ which quickly aggregates towards the cyclo- $\mathrm{P}_{3}$ trimeric complex LXXXVII (Scheme 4.11). ${ }^{[274]}$

The existence of LXXXVI was verified by addition of peri-1,8-naphthalene disulfide yielding compound LXXXVIII (Scheme 4.11). Addition of ClPA to low valent, coor-

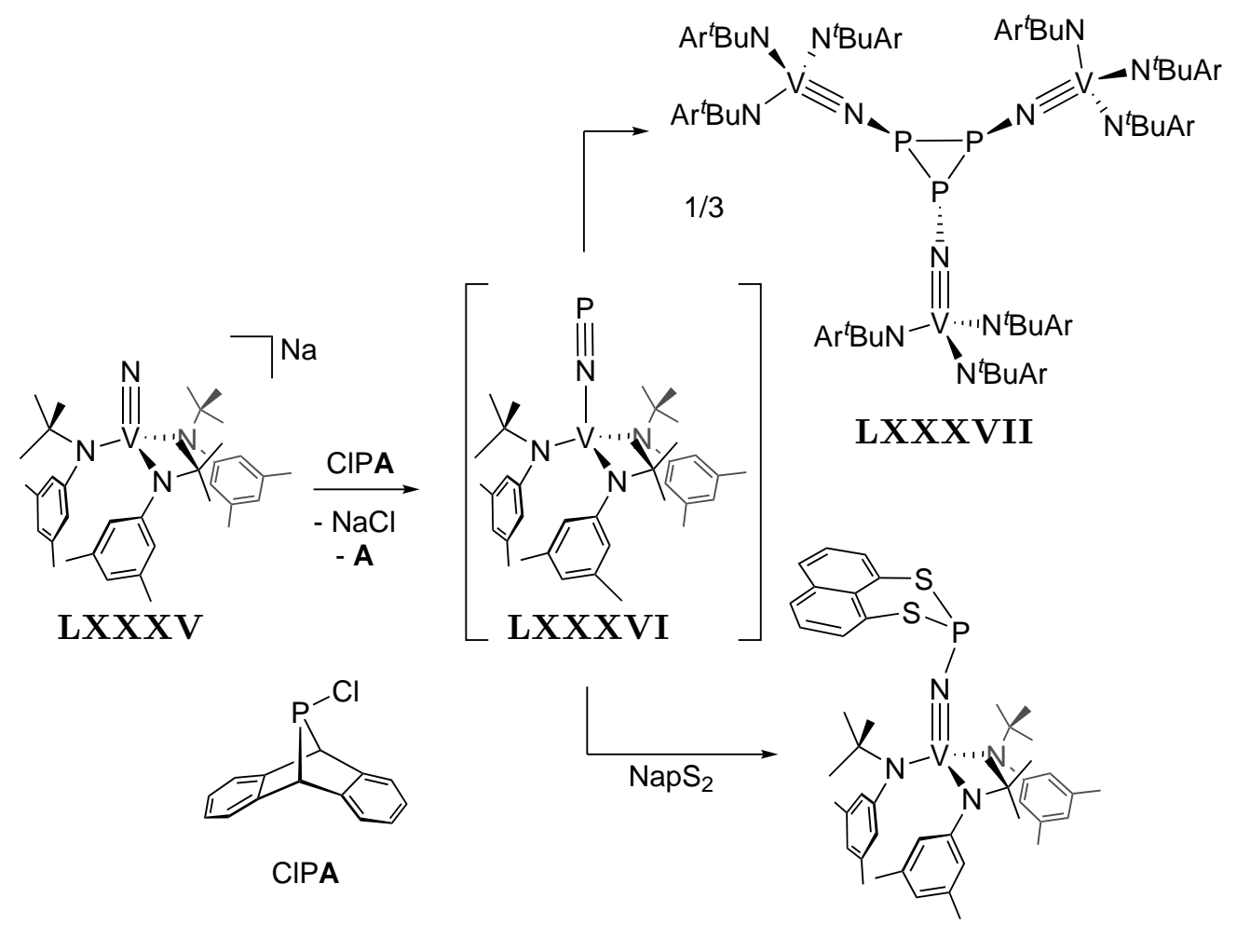

LXXXVIII

Scheme 4.11: Phosphorus atom transfer of ClPA towards LXXXV. ${ }^{[274]}$ 
dinatively unsaturated transition metal complexes could also give rise to unprecedented chlorophosphinidene complexes which lack a general synthetic procedure. Chlorophosphinidene complexes represent highly desired synthetic targets since they are expected to be strong electrophiles and therefore more reactive than the well-known alkyl- and aryl-substituted phosphinidenes in terms of follow-up P-C coupling reactions. ${ }^{[275],[276]}$ In addition to this, the $\mathrm{P}-\mathrm{Cl}$ moiety allows for diverse derivatization to access a variety of different phosphines, most desirable in a catalytic process, which are ubiquitous ligands in transition metal chemistry. ${ }^{[277]}$ Heating of the tungsten chlorophosphole complex LXXXIX and dimethyl acetylenedicarboxylate (DMAD) yielded the 7phosphanorbornadiene complex $\mathbf{X C}$ which served as a source of the transient chlorophosphinidene XCI. Trapping by addition of tolane or cyclohexene gave XCII and, exclusively cis-configured, XCIII, respectively (Scheme 4.12). ${ }^{[275]}$ This observed reactivity was attributed to a phosphorus centered, low lying LUMO resulting in pronounced electrophilicity. Although no mechanistic or computational studies concerning the reaction pathway were presented that indicate the actual formation of the $\mathrm{PCl}$-complex, this results nevertheless demonstrate the possible potential of chlorophosphinidenes in synthetic phosphorus chemistry. Employing transition metals beyond group 7 are particular attractive since the $\mathrm{M} \equiv \mathrm{P}$ bond strength is expected to decrease successively along the periodic table, when going to more electron rich systems, which might favor transfer of the $\mathrm{PCl}$ moiety as a whole. ${ }^{[54]}$

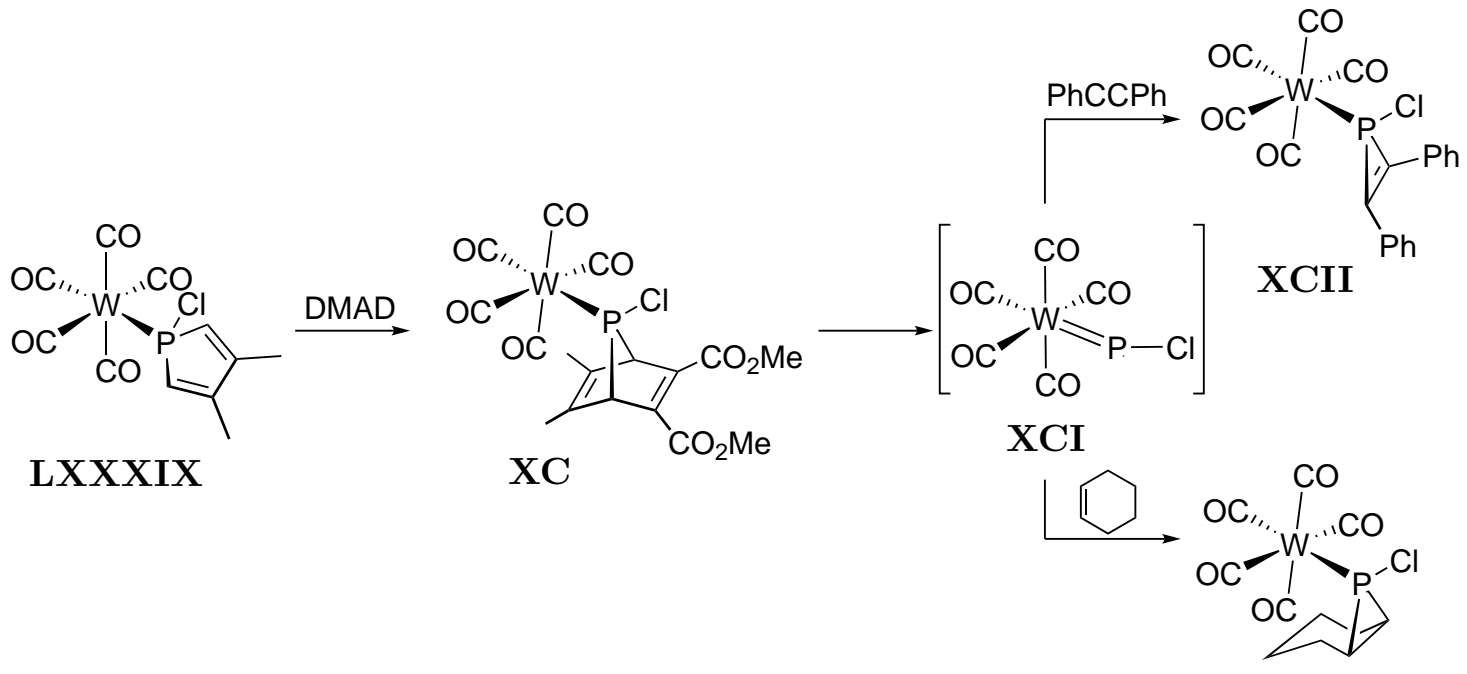

XCIII

Scheme 4.12: Formation and follow-up reactivity of the proposed chlorophosphinidene complex XCI. ${ }^{[275]}$ 
Stirring 1 with one equivalent of $\left[\mathrm{Na}(\operatorname{diox})_{1.39}\right][\mathrm{PCO}]$ in THF results in an immediate color change to deep purple. Quantitative formation of $\mathbf{2}$ is confirmed by NMR spectroscopy (Scheme 4.13). If $\mathbf{7}$ is used instead, a color change towards deep red accompanied by no detectable ${ }^{31} \mathrm{P}$ NMR signals is observed, suggesting formation of $\mathbf{9}$ (Scheme 4.13). One-electron reduction is also observed for 12 (Scheme 4.13). The products obtained after the reduction reactions show no further reactivity towards $\mathrm{PCO}^{-}$ which renders the studied systems unsuitable as a whole.

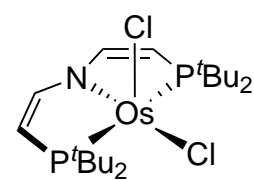

1

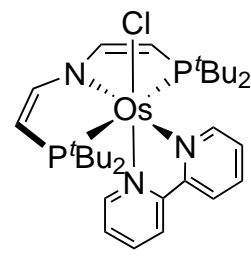

7

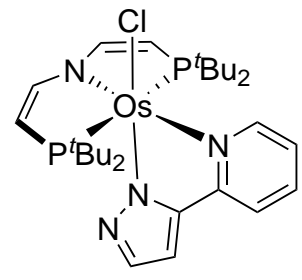

12

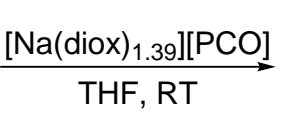
$\underset{\mathrm{THF}, \mathrm{RT}}{\stackrel{\mathrm{Na}\left(\text { diox }_{1.39}\right][\mathrm{PCO}]}{\longrightarrow}}$

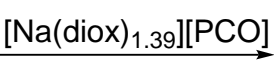
THF, RT

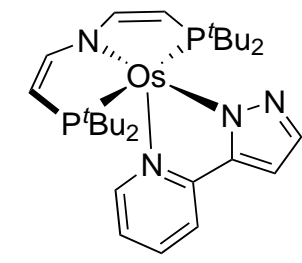

13

Scheme 4.13: Reduction of $\mathbf{1}, \mathbf{7}$ and $\mathbf{1 2}$ with $\left[\mathrm{Na}(\operatorname{diox})_{1.39}\right][\mathrm{PCO}]$ towards $\mathbf{2}, \mathbf{9}$ and $\mathbf{1 3}$, respectively.

ClPA is another possible transfer reagent for phosphorus atoms. The square-planar Os ${ }^{\mathrm{II}}$ complex 2 represents an ideal substrate since the low coordination number and high electron count should favor $\mathrm{PCl}$ extrusion from the sterically demanding 7-phosphanorbornadiene moiety. Furthermore, the four-electron oxidation upon formation of an $\mathrm{Os} \equiv \mathrm{P}$ and an $\mathrm{Os}-\mathrm{Cl}$ bond would result in an overall oxidation state of + VI which is a suitable electron configuration for the stabilization of terminal pnictide complexes. 


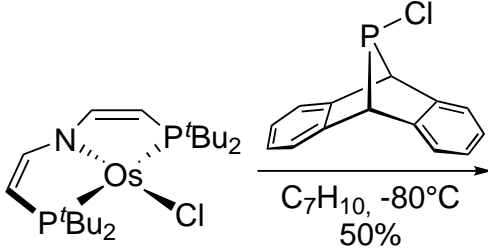

2

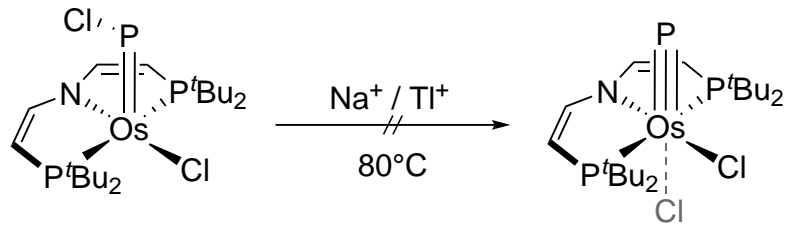

44

Scheme 4.14: Transfer of a PCl moiety to 2 upon addition of ClPA to yield 44.

When a freshly prepared solution of $\mathbf{2}$ is reacted with one equivalent of ClPA at $-80^{\circ} \mathrm{C}$ and slowly warmed to room temperature, a slow color change from purple to reddishbrown is observed. ${ }^{31} \mathrm{P}$ NMR spectroscopy indicates full and quantitative conversion to a new diamagnetic species with phosphorus signals suggesting formation of a phosphinidene species over the course of the reaction $\left(\delta_{\mathrm{P}}=854.8,70.4 \mathrm{ppm}\right)$. The ${ }^{31} \mathrm{P} \mathrm{NMR}$ signals are significantly broadened at room temperature hinting towards a flexible phosphorus substituent. The obtained molecular structure in the solid state shows that the chlorophosphinidene complex $\left[\mathrm{Os}(\mathrm{PCl}) \mathrm{Cl}\left(\mathrm{L}^{3}\right)\right](\mathbf{4 4}$, Scheme 4.14) is indeed obtained. 44 features a distorted square-pyramidal coordination sphere and an $\mathrm{Os}=\mathrm{P}$ bond length of $d_{\mathrm{OsP}}=2.1759(12) \AA$. This result is remarkable since it demonstrates the first example of a transition metal chlorophosphinidene complex which is isolable and stable at room temperature. Furthermore, this implicates that the generation of the terminal phosphide dichloride complex $\left[\mathrm{OsPCl}\left(\mathrm{L}^{3}\right)\right]$ is associated with a high kinetic barrier or thermodynamically unfeasible or both.
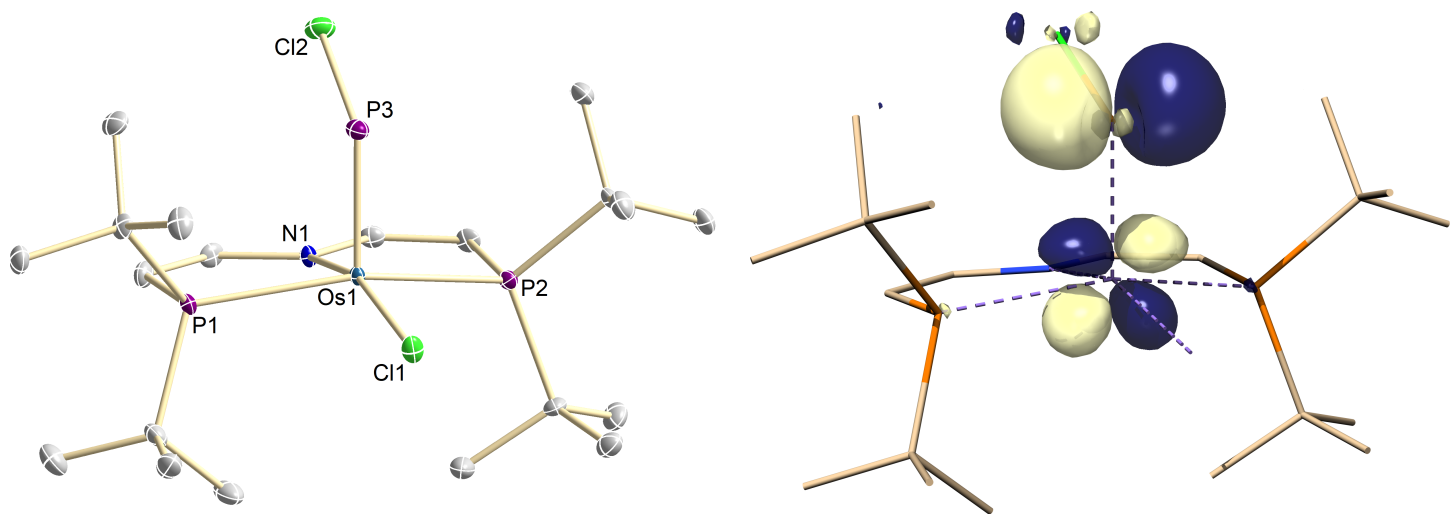

Figure 4.16: Left: Molecular structure of complex 44 from single-crystal X-ray diffraction (thermal ellipsoids drawn at the $50 \%$ probability level); hydrogen atoms are omitted for clarity. Selected bond lengths $[\AA]$ and angles $\left[^{\circ}\right]$ : 44 Os1-Cl1 2.387(3), Os1-N1 2.056(3), Os1-P1 2.3942(9), Os1-P2 2.4073(9), Os1-P3 2.1759(12), P3-Cl2 2.1759(12), N1-Os1-Cl1 162.46(8), P1Os1-P2 157.34(3), N1-Os1-P3 93.69(8); Right: Computed LUMO of 44. 
44 can be heated to $80^{\circ} \mathrm{C}$ in benzene without any signs of decomposition. Additionally, the addition of halide abstracting agents such as sodium or thallium salts did not result in any observed transformation of the compound. DFT computation show that the $\mathrm{P}-\mathrm{Cl}$ moiety can rotate easily on a relatively flat energy surface, in line with the broadened ${ }^{31} \mathrm{P}$ NMR signals, to a rotamer featuring syn-orientation of the chloride atoms $\left(\Delta G_{\text {Rot. }}^{\ddagger}=\right.$ $9.85 \mathrm{kcal} \cdot \mathrm{mol}^{-1}, \Delta G_{\text {Rot. }}=5.05 \mathrm{kcal} \cdot \mathrm{mol}^{-1}$, Figure 4.17$)$. However, the computed kinetic and thermodynamic values for the further rearrangement towards a terminal phosphide dichloride complex are calculated to be highly unfavorable which can be explained by the successive weakening of the transition metal pnictide multiple bonds when moving right in the periodic table $\left(\Delta G_{\text {Ox.Ad. }}^{\ddagger}=39.30 \mathrm{kcal} \cdot \mathrm{mol}^{-1}, \Delta G_{\text {Ox.Ad. }}=35.38 \mathrm{kcal} \cdot \mathrm{mol}^{-1}\right.$, Figure 4.17).

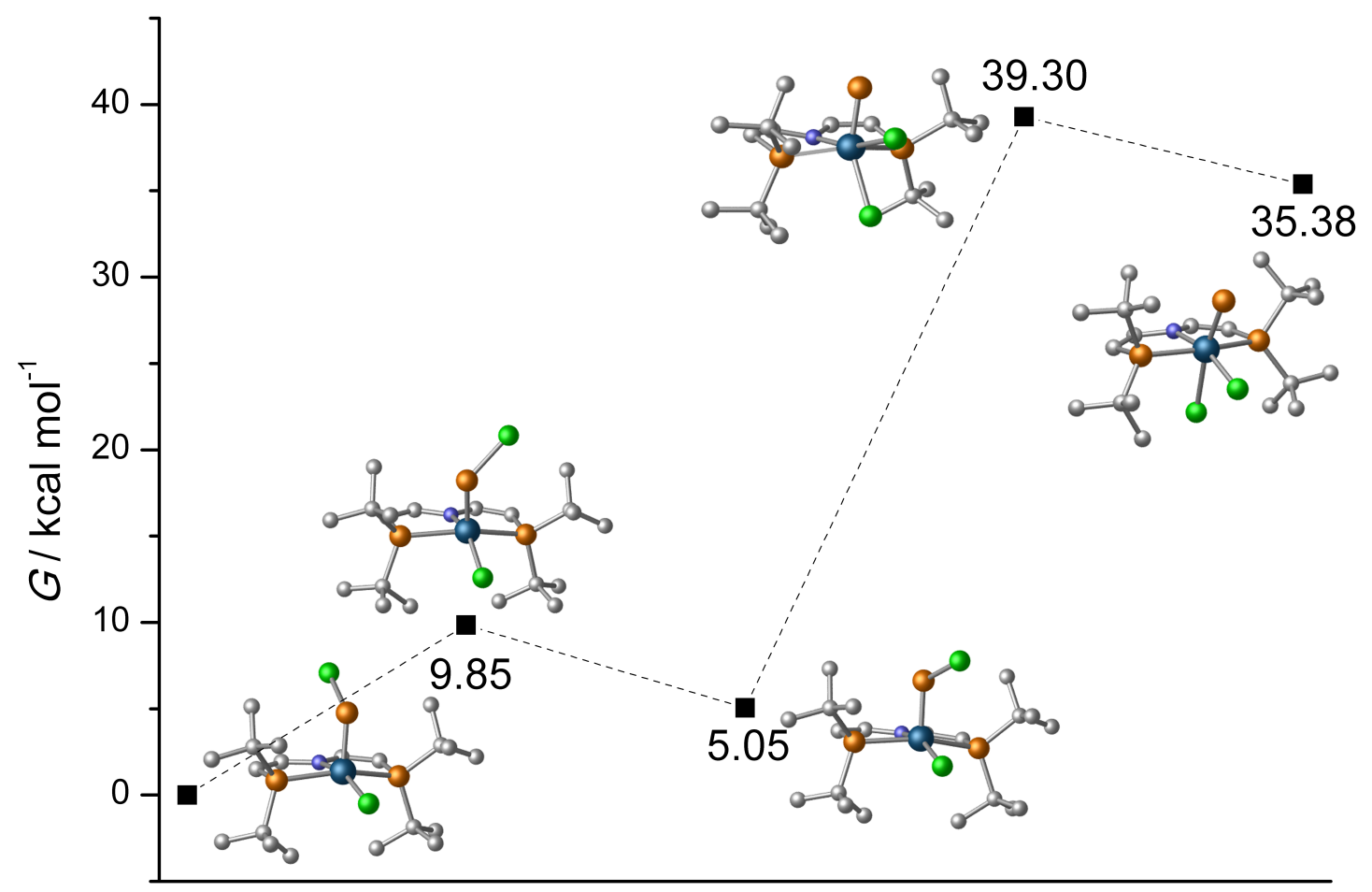

reaction coordinate

Figure 4.17: Computed reaction pathway of the isomerization of $\mathbf{4 4}$ towards the terminal phosphide $\left[\mathrm{OsCl}_{2}\left(\mathrm{~L}^{3}\right) \mathrm{P}\right]$.

Chlorophosphinidenes could serve as suitable species to perform $\mathrm{P}-\mathrm{C}$ coupling reactions with dienes and diynes yielding chloro-substituted cyclic phosphines. Theoretical calculations demonstrate that the LUMO of $\mathbf{4 4}$ is mainly P-centered and should therefore serve as an electrophilic attack site for nucleophilic unsaturated substrates as tolane, cyclohexene or dimethyl butadiene (Figure 4.16). However, no reactivity with any of the substrates was observed, even at elevated temperatures up to $80^{\circ} \mathrm{C}$ in benzene for 

BONDS

hours. Therefore, DFT computations of the hypothetical reaction of $\mathbf{4 4}$ and tolane to give the phosphirene complex $\left[\mathrm{OsCl}\left(\mathrm{L}^{3}\right)\left(\mathrm{PClR}_{2}\right)\right]\left(\mathbf{4 5}, \mathrm{PClR}_{2}=1\right.$-chloro-2,3-diphenyl$1 H$-phosphirene) were carried out to identify the main obstacles for this reaction. The overall reaction is calculated to be thermoneutral with a relative energetic difference of only $\Delta G=-0.67 \mathrm{kcal} \cdot \mathrm{mol}^{-1}$. The kinetic barrier however is calculated to be $\Delta G^{\ddagger}=33.5 \mathrm{kcal} \cdot \mathrm{mol}^{-1}$. In the transition state $\mathbf{4 6}{ }^{\mathrm{tBu}}$, significant steric repulsion of the tert-butyl groups of the phosphorus donor sites and the substrate is indicated by the short distance between one of the phenyl and the tert-butyl groups and a long P3-C2 bond distance (Figure 4.18, Table 4.2). To clarify if repulsive steric interactions are the main obstacle for the reaction to proceed, the tert-butyl groups were substituted by iso-propyl substituents and the reaction was again investigated by DFT. The transition state $46^{\mathrm{iPr}}$ exhibits almost identical bond distances around the metal center but the bond distances and angles of the $\mathrm{P}-\mathrm{Cl}$ moiety and the tolane unit are strikingly different. While the chloride is situated almost directly above the nitrogen pincer atom in the sterically more demanding model, it shows a significant twist in the iso-propyl case preventing a Y-shaped phosphorus centrum in transition state. Additionally, the phenyl substituent of the tolane molecule can approach the $\mathrm{P}-\mathrm{Cl}$ moiety more easily as indicated by the shortened P3-C2 distance and shows no direct hints for strong steric repulsion due to the long $\mathrm{C} 3-\mathrm{C} 4$ distance which leads to a computed activation barrier of $\Delta G^{\ddagger}=14.9 \mathrm{kcal} \cdot \mathrm{mol}^{-1}$ and by that renders the reaction to be feasible even at room temperature. The decreased steric bulk also results in a stabilization of the reaction product yielding an overall exergonic reaction $\left(\Delta G=-22.6 \mathrm{kcal} \cdot \mathrm{mol}^{-1}\right.$, Figure 4.18).

Table 4.2: Computed bond lengths of $46^{\mathrm{iPr}}$ and $46^{\mathrm{tBu}}$.

\begin{tabular}{lccc}
\hline & $\mathbf{4 6}^{\mathbf{i P r}}$ & $\mathbf{4 6}^{\mathbf{\text { tBu}}}$ & $\boldsymbol{\Delta}$ \\
\hline Os1-N1 & 2.065 & 2.092 & 0.027 \\
Os1-C11 & 2.419 & 2.430 & 0.011 \\
Os1-P1 & 2.358 & 2.413 & 0.055 \\
Os1-P2 & 2.377 & 2.422 & 0.045 \\
Os1-P3 & 2.155 & 2.155 & 0.000 \\
P3-C12 & 2.151 & 2.157 & 0.006 \\
P3-C1 & 1.844 & 1.856 & 0.012 \\
P3-C2 & 2.258 & 2.342 & 0.084 \\
P3-C3 & 4.639 & 5.435 & 0.796 \\
C3-C4 & 3.848 & 3.162 & 0.686 \\
C1-C2-C3 & 167.42 & 158.02 & 9.400 \\
Cl2-P3-Os1-N1 & 55.02 & 7.09 & 47.93 \\
\hline
\end{tabular}




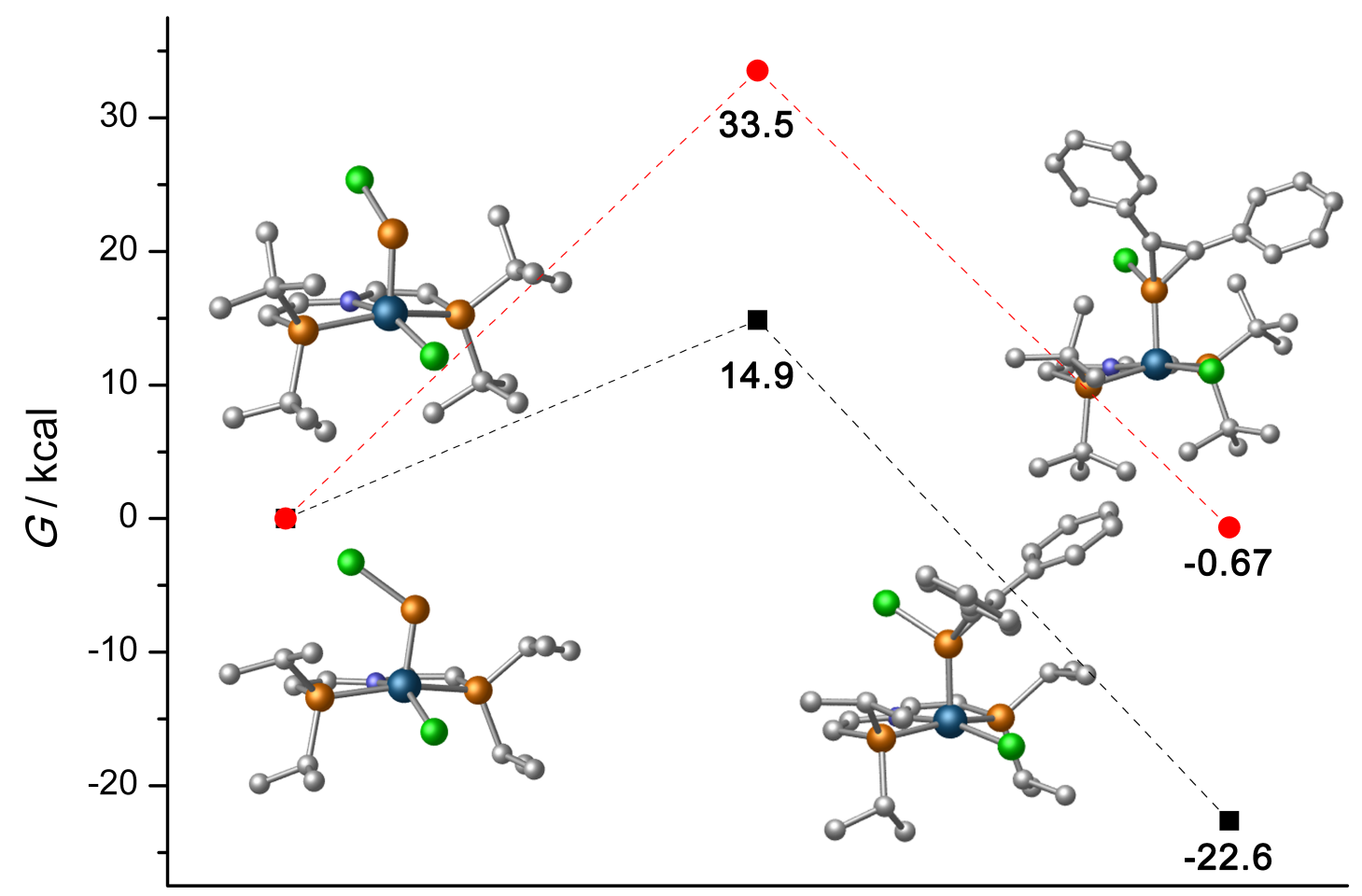

reaction coordinate
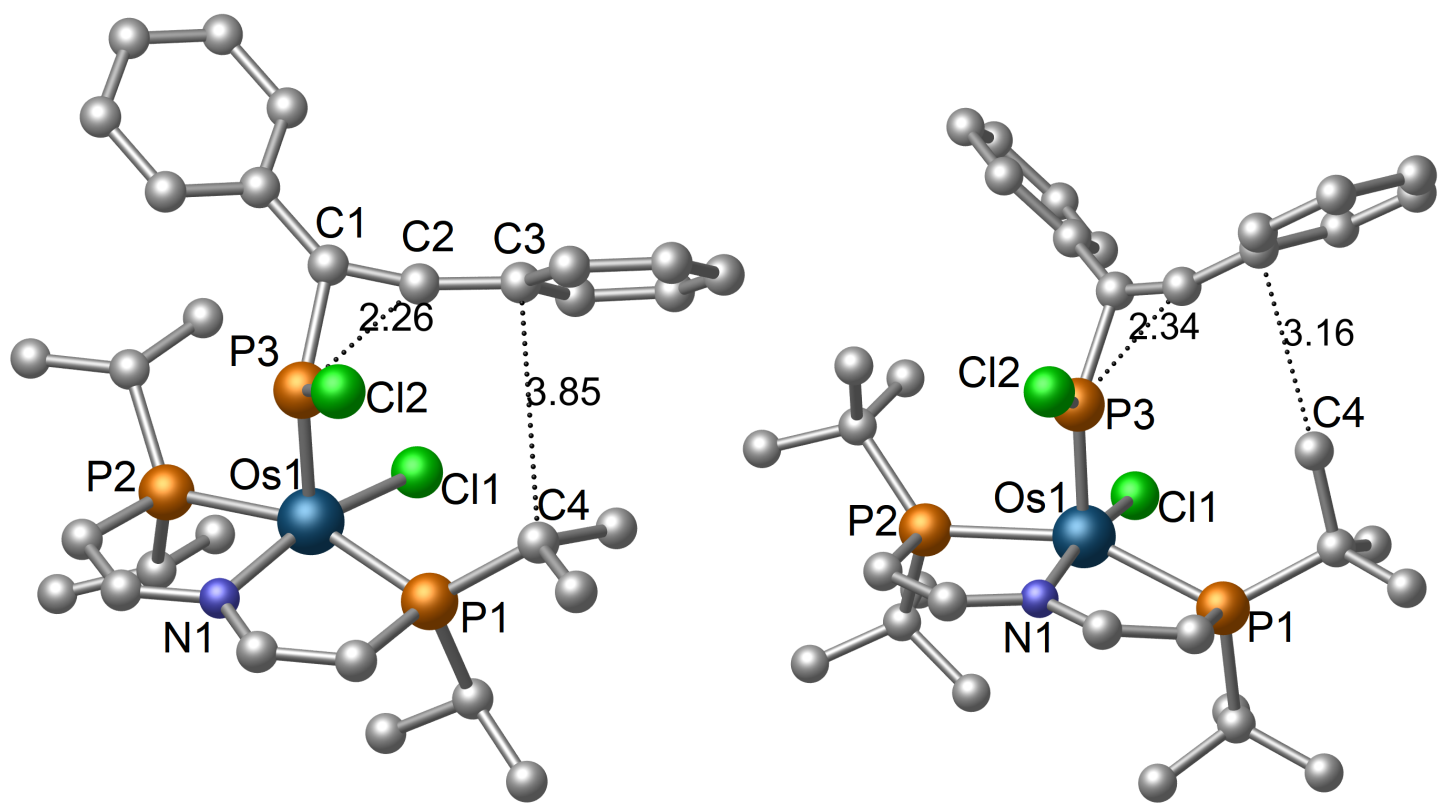

Figure 4.18: Top: Computed reaction pathway of the cyclization of tolane by 44 to yield 45 with differently substituted phosphorus donor sites (isopropyl in black; tert-butyl in red); Bottom: Computed structures of the transition states. 
As outlined above, phosphorus atom transfer from CIPA can be realized when reacted with the anionic vanadium nitride complex $\mathbf{X X X V}$ to give a transient $\mathrm{N} \equiv \mathrm{P}$ ligated complex. Since $\mathbf{4 4}$ features a $\mathrm{PCl}$ ligand, the substitution of chloride for nitrogen is targeted to obtain a phosphorus-bound $\mathrm{P} \equiv \mathrm{N}$ ligand. When $\mathbf{4 4}$ is reacted with azides such as $\mathrm{TMSN}_{3}$ or $\mathrm{TBAN}_{3}\left(\mathrm{TBA}=\mathrm{NBu}_{4}\right)$ slow reaction towards multiple products is observed which were not further investigated.

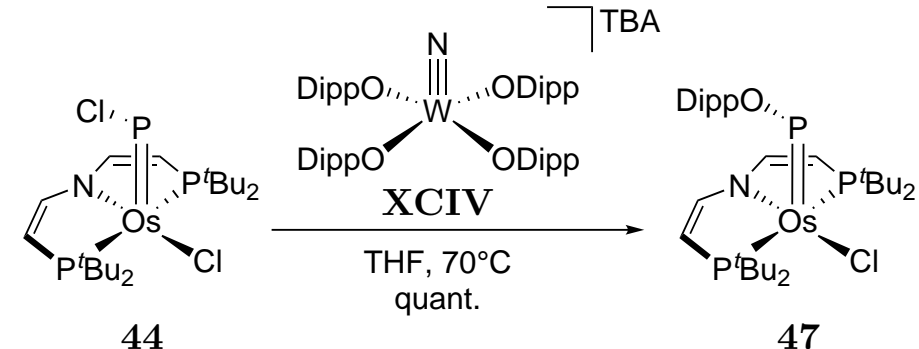

Scheme 4.15: Reaction of 44 and XCIV yielding 47.

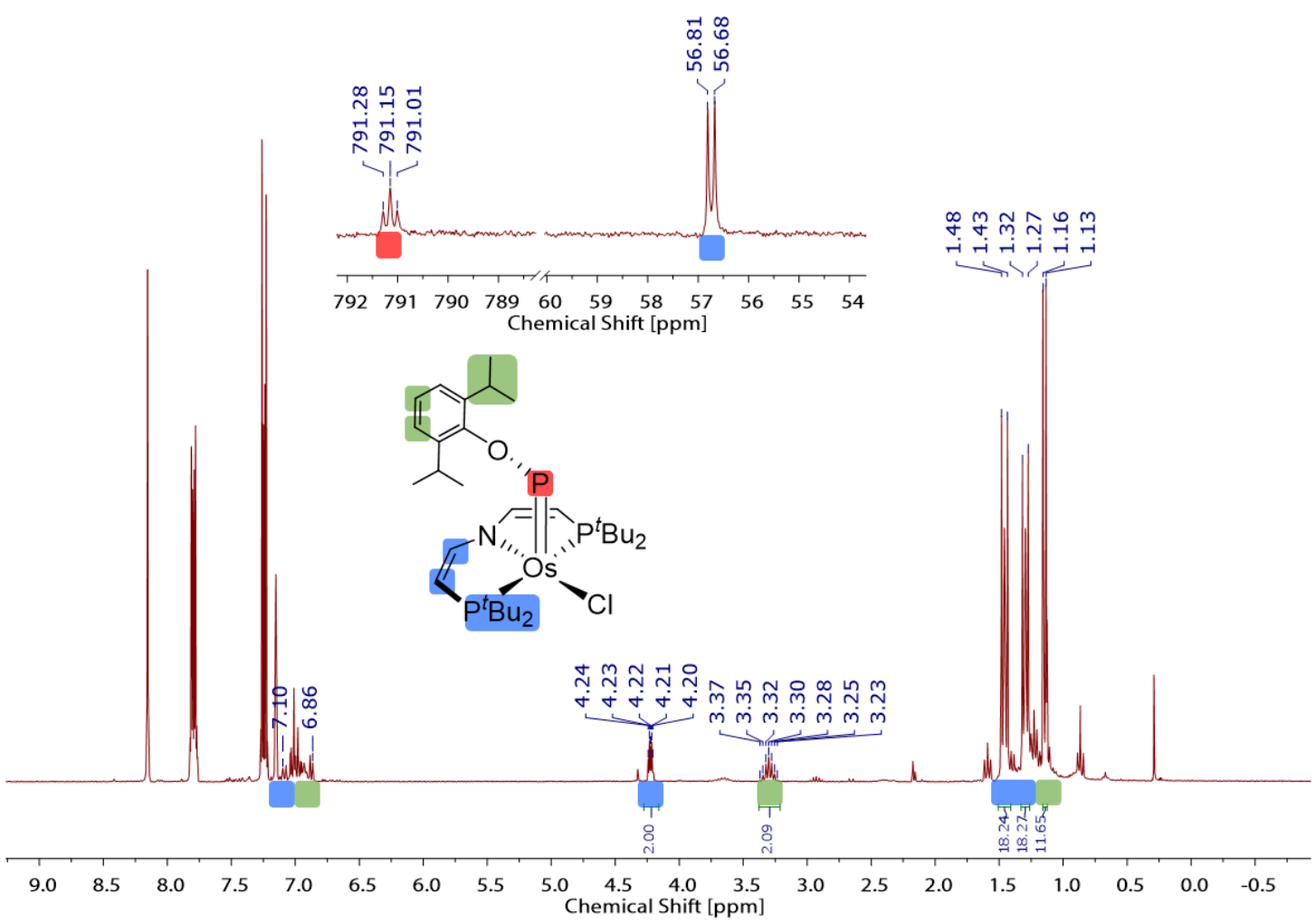

Figure 4.19: ${ }^{1} \mathrm{H}$ NMR spectrum of the reaction of 44 and XCIV after purification, $\mathrm{C}_{6} \mathrm{D}_{6}, \mathrm{RT}$; Inset: ${ }^{31} \mathrm{P}\left\{{ }^{1} \mathrm{H}\right\}$ NMR spectrum, $\mathrm{C}_{6} \mathrm{D}_{6}$, RT (since $\mathbf{4 4}$ was prepared in situ anthracene is still present in the purified sample). 
$\mathrm{N} \equiv \mathrm{P}$ is expected to be a highly reactive molecule which may necessitate a more thorough steric protection. Therefore, synthesis by coupling of $\mathbf{4 4}$ with the anionic nitride complex $[\mathrm{TBA}]\left[\mathrm{WN}(\mathrm{ODipp})_{4}\right](\mathbf{X C I V})$ was attempted. Upon mixing at room temperature, no conversion was detected over the course of days, however heating the solution to $70^{\circ} \mathrm{C}$ for $24 \mathrm{~h}$ gave complete conversion to a new diamagnetic phosphinidene complex $\left(\delta_{\mathrm{P}}=791.3\right.$, $56.7 \mathrm{ppm}$, Figure 4.19). The sharpened signals and observed mutual coupling $\left({ }^{2} J_{\mathrm{PP}}=\right.$ $16.8 \mathrm{~Hz}$ ) suggest the formation of an $\mathrm{Os}=\mathrm{PR}$ species which is not as flexible as 44. Based on ${ }^{1} \mathrm{H}$ NMR spectroscopy it can be concluded that no P-N coupling occurs and rather an ODipp ligand is transferred yielding the phosphinidene complex [Os(PODipp) $\mathrm{Cl}\left(\mathrm{L}^{3}\right)$ ] (47, Figure 4.19, Scheme 4.15) quantitatively, accompanied by minor organic impurities stemming from the disassembly of XCIV. Although additional spectroscopy was not performed, the indicative NMR signatures sufficiently proof the formation of $\mathbf{4 7}$. 


\subsection{Conclusion}

This chapter demonstrated the synthesis of the first terminal heavy pnictide complexes beyond group 6 ( $\mathbf{2 7}$ and $\mathbf{2 8}$ ). The species were fully characterized and DFT calculations reveal a highly covalent bonding between the Re center and the Pn ligands. Upon oneelectron oxidation, coupling reactivity towards the $\mathrm{Pn}_{2}$-species $\mathbf{2 9}$ and $\mathbf{3 0}$ was observed and the products were characterized by NMR, mass spectrometry and XRD studies. QTAIM analysis reveals an unprecedented donor-acceptor interaction between diphosphorus and diarsenic with the $\mathrm{Re}^{\mathrm{III}}$ ion and the PyrPz ligand, representing the first isolable example of neutral $\mathrm{Pn}_{2}^{0}$ coordinated by a metal center. The push-pull interaction leads to a formal reduction of the bond order resulting in the observed double bond which contrasts with prior reported NHC stabilized $\mathrm{Pn}_{2}^{0}$ compounds XCV and XCVI (Figure 4.20).[115],[116],[278], [279] In this case, the donation of the electron rich carbene results in the formation of a $\mathrm{Pn}-\mathrm{Pn}$ single bond due to push-push stabilization (Figure $4.20)$.

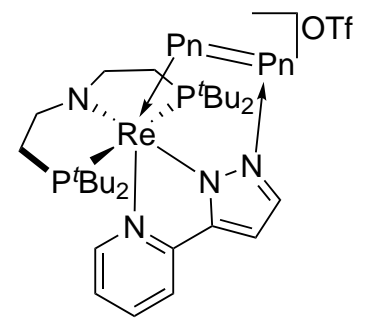

$29 / 30$

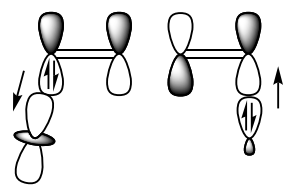

Pull - Push stabilized $\mathrm{Pn}_{2}{ }^{0}$
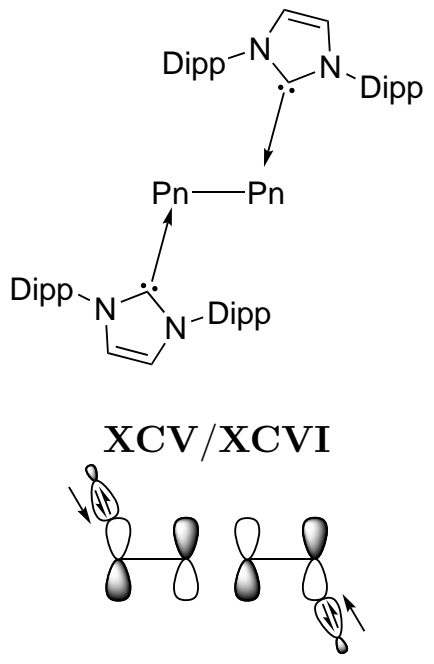

Push - Push stabilized $\mathrm{Pn}_{2}{ }^{0}$

Figure 4.20: Left: Push-pull stabilization of $\mathrm{Pn}_{2}$ by the $\left\{\operatorname{Re}\left(\mathrm{L}^{1}\right)(\mathrm{PyrPz})\right\}$ platform; Right: Push-push stabilization of $\mathrm{Pn}_{2}$ by XCV and XCVI $(\mathrm{Pn}=\mathrm{P}, \mathrm{As}) .{ }^{[115],[116]}$

The oxygenation of the terminal Re phosphide $\mathbf{2 7}$ with pyridine- $N$-oxide was presented. Upon addition of two oxygen atoms the dinuclear species $\mathbf{3 1}$ is obtained which features an unprecedented bridging $\mathrm{P}_{2} \mathrm{O}_{4}^{2-}$ moiety. Single addition of an oxygen atom could not be accomplished. 31 was fully characterized and cyclic voltammetry revealed a possible two electron reduction which results in the formation of the open-shell complex 34, representing the first example of a $\mathrm{PO}_{2}^{-}$ligand coordinated to a transition metal. 
$\mathrm{PO}_{2}^{-}$acts as a $\sigma$-donor ligand with delocalized P-O bonds, as shown by $\mathrm{NBO}$ analysis. One-electron oxidation of $\mathbf{3 4}$ results in the reformation of $\mathbf{3 1}$.

The first spectroscopically and crystallographically characterized isolable phosphanide complex with large phosphinyl radical character was accomplished. The rhombic ${ }^{31} \mathrm{P}$ HFI tensor and the DFT model are in line with even spin delocalization over the Os-P core. Versatile access to an electrophilic phosphinidene complex that undergoes intramolecular $\mathrm{C}-\mathrm{H}$ activation was demonstrated by $\mathrm{P}-\mathrm{H}$ PCET. Thermochemical analysis by means of ITC was particularly useful as the thermal instability of $\mathbf{4 3}$ prevented experimental $\mathrm{p} K_{\mathrm{a}}$ determination. The data indicates that concerted or stepwise ET/PT are both viable routes to access phosphinidenes from phosphanide complexes.

Several Os PNP platforms were tested towards possible substitution reactions with the $\mathrm{PCO}^{-}$anion which led to clean one-electron reduction chemistry. The obtained reduction products exhibited no further reactivity with the reagent. The reaction of ClPA with the square-planar $\mathrm{Os}^{\mathrm{II}}$ chloride $\mathbf{2}$ gives the first isolable chlorophosphinidene complex 44 in $50 \%$ isolated yield. Attempts to substitute the chloride with nitrogen failed. The test reactions with unsaturated substrates were unsuccessful since no reactivity was observed even at elevated temperatures. A computational investigation of the reaction of $\mathbf{4 4}$ with tolane suggests that the steric demand of the tert-butyl substituents leads

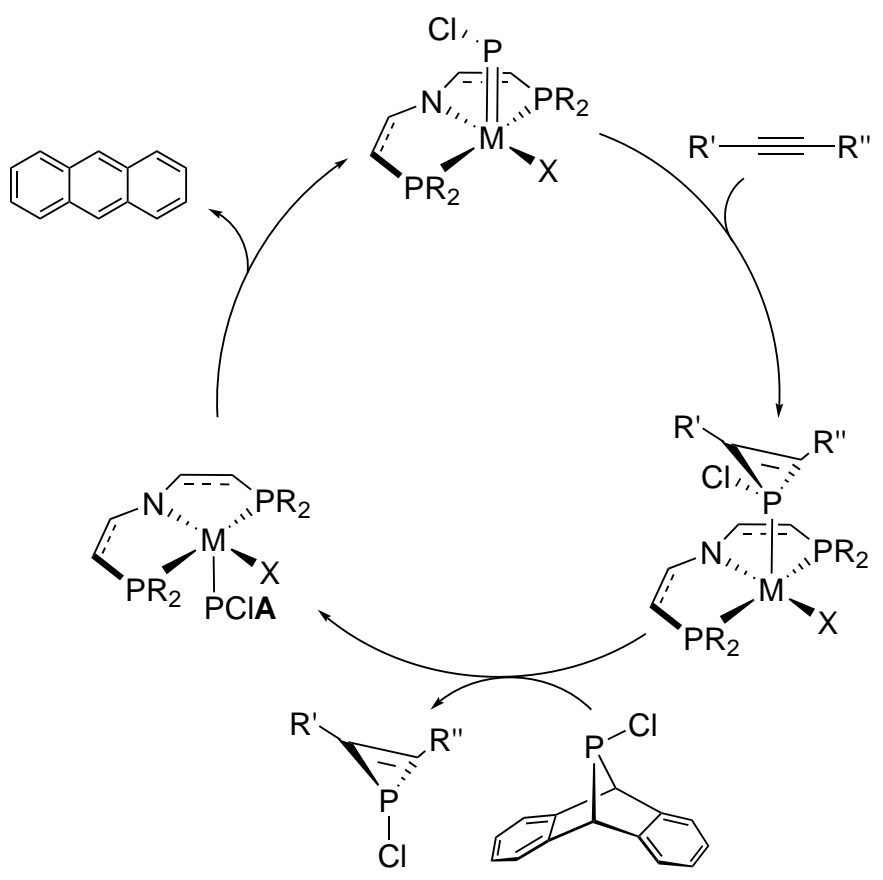

Scheme 4.16: Possible formation of chloro-substituted phospirenes by catalytic PCl transfer. 
to an unfavorable transition state. Comparison with a computed iso-propyl substituted system yields a significantly lowered kinetic barrier and an overall exergonic reaction.

These results show that $\mathrm{PCl}$ transfer from ClPA to mid- and late transition metals might offer a unique possibility to access chlorophosphinidene complexes. Increased reactivity towards unsaturated substrates, e.g. alkynes, could be realized by introduction of smaller substituents at the phosphorus donor atoms. In this context also catalytic $\mathrm{PCl}$ transfer might be possible. The choice of appropriate phosphorus substituents might however be challenging since the activation barrier for cyclization has to allow for heterocycle formation but thereby also facilitate the decoordination of the product to enable the metal to re-enter the catalytic cycle (Scheme 4.16). 


\title{
Proton-Induced Splitting of Dinitrogen by Dimeric Mo PNP Pincer Complexes
}

J. Abbenseth, Serhiy Demeshko, Markus Finger, Maximilian Fritz, Christian Herwig, Jean-Pierre Oudsen, Bas Venderbosch, Christian Würtele, Moniek Tromp, Sven Schneider*

\author{
Author contributions \\ J.A. Synthesis and spectroscopy \\ S.D. SQUID \\ M.Fi. Theoretical calculations \\ M.Fr. Crystallography (supporting) \\ C.H. rRaman \\ J.O. XAS \\ B.V. XAS \\ M.T. XAS \\ C.W. Crystallography
}




\section{Proton-Induced Splitting of Dinitrogen by Dimeric Mo PNP Pincer Complexes}

Dinitrogen functionalization is an active field of research in the Schneider group and dinitrogen splitting into terminal nitrides was demonstrated for Mo and Re systems which extended this process up to group 7. ${ }^{[33],[35],[40],[52],[53],[280]}$

The $\mathrm{N}_{2}$-bridged Mo complex $\left[\left\{\mathrm{MoCl}\left(\mathrm{L}^{1}\right)\right\}_{2}\left(\mathrm{~N}_{2}\right)\right]$ (XCVII) is thermally stable and does not convert to nitride complexes upon heating or irradiation. Although this process was computed to be thermodynamically favored, the associated kinetic barrier of $\Delta G_{\mathrm{DFT}}^{\ddagger}=$ $37 \mathrm{kcal} \cdot \mathrm{mol}^{-1}$ rationalized the non-observed splitting. However, twofold protonation towards $\left[\left\{\mathrm{MoCl}\left({ }^{\mathrm{H}} \mathrm{L}^{1}\right)\right\}_{2}\left(\mathrm{~N}_{2}\right)\right][\mathrm{OTf}]_{2}$ (XCVIII) at low temperatures and successive warming to room temperature results in complete $\mathrm{N} \equiv \mathrm{N}$ bond scission yielding the open-shell nitride [MoNCl $\left.\left({ }^{\mathrm{H}} \mathrm{L}^{1}\right)\right][\mathrm{OTf}]$ (XCIX, Scheme 5.1). ${ }^{[280]}$ Due to $C_{2}$-symmetry of XCVII, a set of degenerate $\delta$-bonding orbitals with respect to the dinitrogen bridge have to be taken into account which results in an overall $\pi^{8}\left(\delta^{4}\right)$-configuration (Scheme 5.1). When XCVII is protonated by two equivalents of triflic acid the $\pi$-donor capabilities of the $\mathrm{L}^{1}$ ligands are switched off, resulting in a stabilization of the $\sigma^{*}$ - and $\pi_{2}$-orbital, which allows for the generation of a $S=\frac{3}{2}$ state for XCVIII. Furthermore, mixing of the $\sigma^{*}$ and $\pi_{2}$-orbital in the zig-zag transition state becomes feasible, allowing for dinitrogen splitting.

XCVII was characterized electrochemically yielding no reductive but reversible oxidative waves at $E_{1 / 2}=-1.14$ and $-0.64 \mathrm{~V}$ vs. $\mathrm{Fc}^{0 /+}$. This chapter aims to provide a comprehensive study of one- and two-electron oxidized derivatives of XCVII. The effect of depopulation of $\delta$-symmetric orbitals with respect to the $\mathrm{N}_{2}$ bridge has not been investigated for any dimeric molybdenum dinitrogen systems. In addition, characterization of XCVIII was restricted to Evan's method and UV/vis spectroscopy in combination with DFT computations due to thermal instability. X-ray absorption spectroscopy will be utilized to validate the initial structural and electronic hypotheses concerning XCVIII and derive experimental structural parameters. 

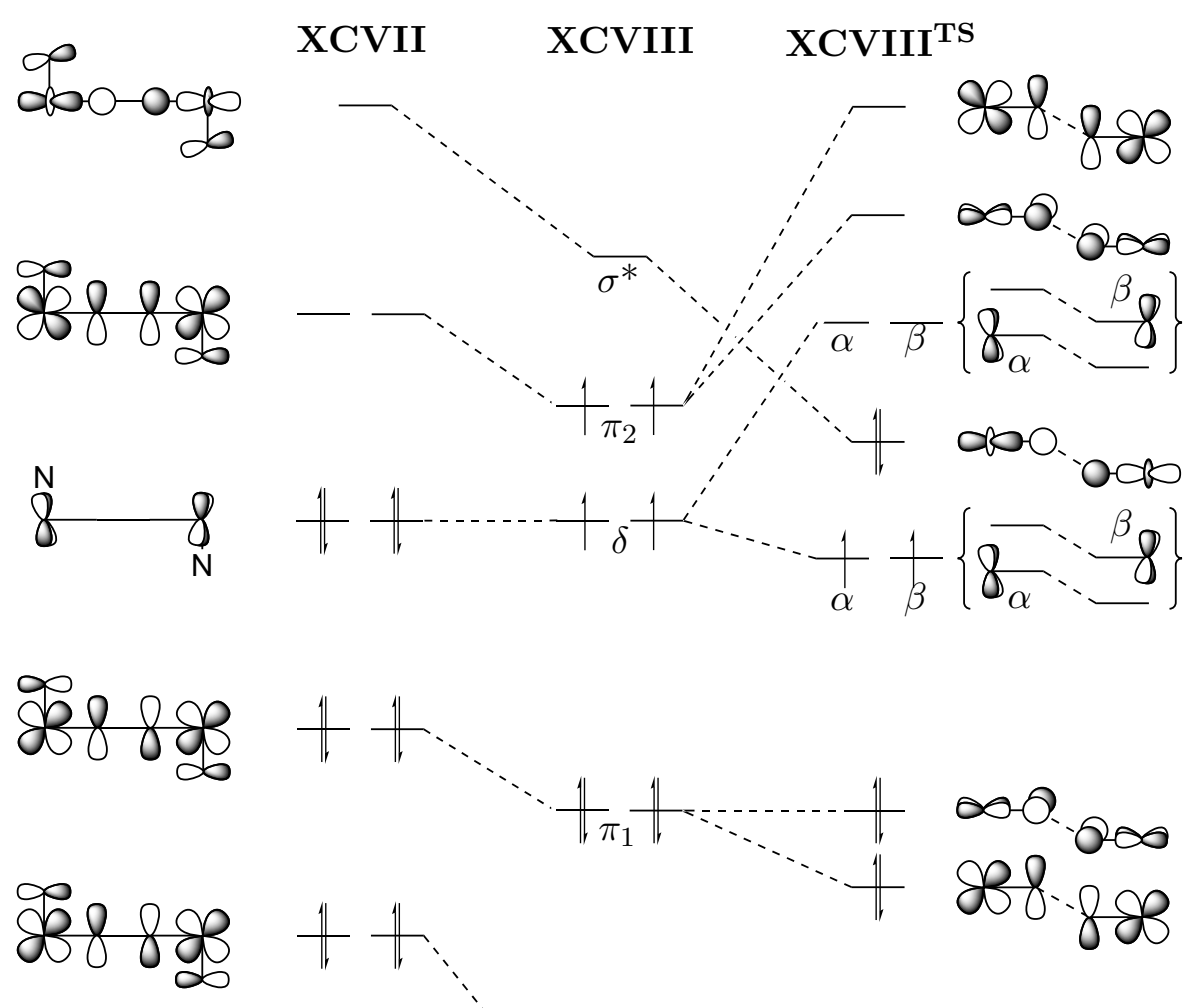

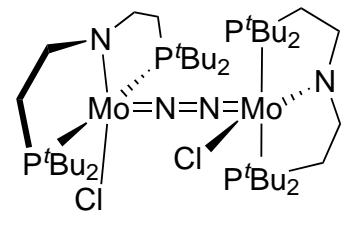

XCVII
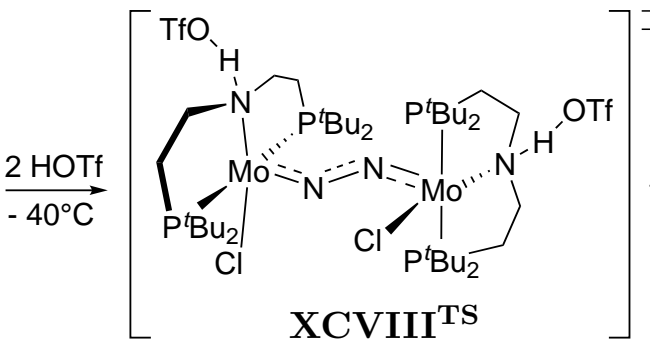

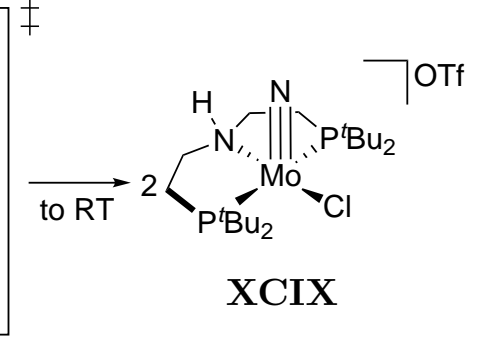

Scheme 5.1: MO scheme for the protonation of XCVII (left) to XCVIII (middle) and its zig-zag transition state (right) resulting in splitting towards XCIX. ${ }^{[280]}$

Based on electrochemical studies of XCVII, one- and two-electron oxidations towards dimeric species were targeted. Mixing XCVII with $\mathrm{Ag}\left[\mathrm{Al}(\mathrm{OR})_{4}\right]\left(\mathrm{R}=\mathrm{C}\left(\mathrm{CF}_{3}\right)_{3}\right)$ in benzene results in the clean formation of $\left[\left\{\mathrm{MoCl}\left(\mathrm{L}^{1}\right)\right\}_{2}\left(\mathrm{~N}_{2}\right)\right]\left[\mathrm{Al}(\mathrm{OR})_{4}\right]_{\mathrm{n}}(\mathbf{4 8}, \mathrm{n}=1 ; \mathbf{4 9}$, $\mathrm{n}=2$ ) in $88 \%$ and $69 \%$ isolated yield, respectively (Scheme 5.2). This synthetic procedure allows for the clean separation of the mono- and dicationic species since $\mathbf{4 8}$ exhibits minor benzene solubility due to the large non-coordinating anion. Both complexes exhibit paramagnetically shifted ${ }^{1} \mathrm{H}$ NMR signals suggesting a $S=\frac{1}{2}$ state for 48 and a $S=1$ state for 49 , respectively. The number of detected peaks and their corresponding integrals are in line with the preservation of the molecular structure upon oxidation with rapid electron transfer between the metal centers in the case of $\mathbf{4 8}$ (Appendix 8.4.1). 


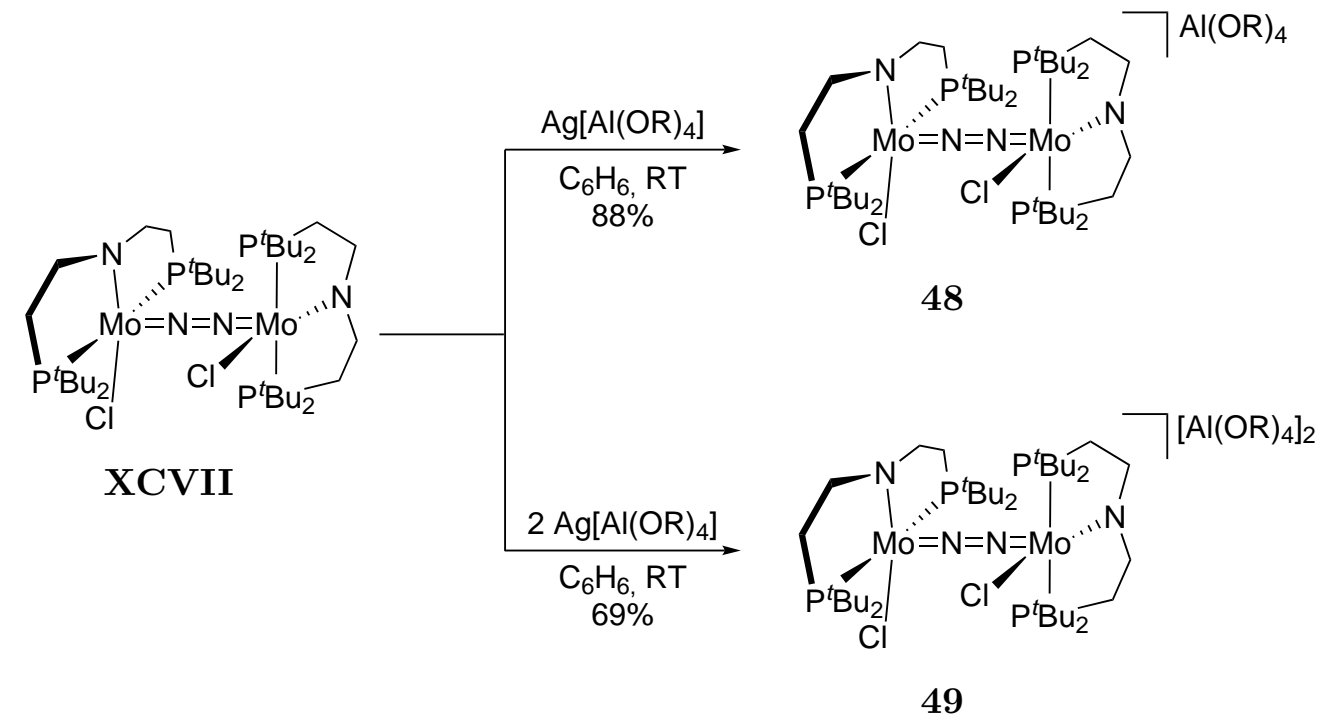

Scheme 5.2: Synthesis of 48 and 49 by oxidation of XCVII, $\mathrm{R}=\mathrm{C}\left(\mathrm{CF}_{3}\right)_{3}$.
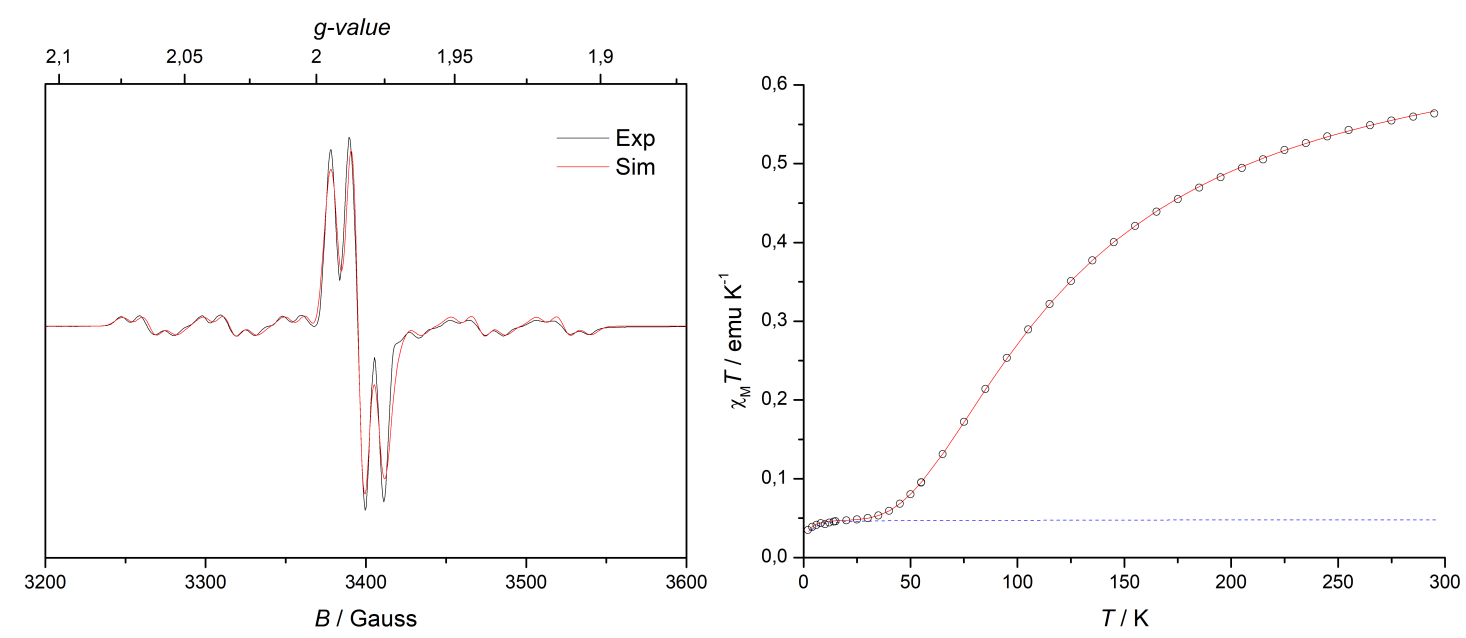

\begin{tabular}{ccccc}
\hline Species & $\tilde{\nu}\left({ }^{14} \mathrm{~N}_{2}\right)$ & $\tilde{\nu}\left({ }^{15} \mathrm{~N}_{2}\right)$ & $\Delta \tilde{\nu}\left(\mathrm{N}_{2}\right)$ & $\Delta \tilde{\nu}_{\text {theo. }}$ \\
\hline XCVII & 1343 & 1300 & 43 & 45 \\
$\mathbf{4 8}$ & 1397 & 1350 & 47 & 47 \\
$\mathbf{4 9}$ & 1390 & 1343 & 47 & 47 \\
\hline
\end{tabular}

Figure 5.1: Top: EPR spectrum of 48 (left), $9.43087 \mathrm{GHz}$, THF, RT, $g=1.9846, A\left({ }^{95 / 97} \mathrm{Mo}\right)=$ $142 \mathrm{MHz} ; A\left({ }^{31} \mathrm{P}\right)=33.7 \mathrm{MHz}$; SQUID data of 49 (right), $g_{1 / 2}=1.921, J_{1 / 2}=-75.672 \mathrm{~cm}^{-1}$; Bottom: ${ }^{14} \mathrm{~N}_{2} /{ }^{15} \mathrm{~N}_{2}$ rRaman data of XCVII,${ }^{[280]} 48$ and 49; $\Delta \tilde{\nu}_{\text {theo. }}$ : Expected shift of $\tilde{\nu}\left(\mathrm{N}_{2}\right)$ in the harmonic oscillator approximation upon labelling. 
EPR spectroscopy of $\mathbf{4 8}$ in $\mathrm{PhCl}$ reveals a Mo-centered radical with hyperfine interaction towards both phosphorus atoms of the PNP pincer ligand (Figure 5.1). According to the Robin-Day classification, $\mathbf{4 8}$ can be described as a class II/III system with a rate of inner sphere electron transfer between the timescales of NMR and EPR spectroscopy at room temperature $\left(10^{-4}-10^{-7} \mathrm{~s}^{-1}\right)$. ${ }^{[281],[282]}$ SQUID measurement reveals that 49 is best described as two antiferromagnetically coupled $S=\frac{1}{2}$ systems $\left(J=-75.672 \mathrm{~cm}^{-1}\right.$, Figure 5.1) which is reasonable since the SOMOs consist of degenerate Mo-centered, $\delta$ symmetric, non-bonding orbitals with respect to the dinitrogen bridge (Scheme 5.1). To probe the degree of activation of the bridging dinitrogen ligand vibrational spectroscopy was employed.

Resonance Raman measurements show that one-electron oxidation leads to a blue shift of the $\mathrm{N}=\mathrm{N}$ stretching vibration by $\Delta \tilde{\nu}_{\mathrm{NN}}=54 \mathrm{~cm}^{-1}$. Two-electron oxidation does not further strengthen the dinitrogen bond but leads to a minor weakening when compared to $48\left(\Delta \tilde{\nu}=9 \mathrm{~cm}^{-1}\right.$, Figure 5.1$)$. Overall, these changes are significantly smaller when compared to the series VII - XI reported by Cummins and co-workers.

Although both dimers can be purified by crystallization, no suitable singe-crystals for X-ray diffraction could be obtained when $\mathrm{Ag}\left[\mathrm{Al}(\mathrm{OR})_{4}\right]$ was used as the oxidant. The reaction of XCVII with silver salts containing more coordinating anions such as $\mathrm{PF}_{6}^{-}$, $\mathrm{BF}_{4}^{-}, \mathrm{SbF}_{6}^{-}$and $\mathrm{BPh}_{4}^{-}$gave sluggish reaction mixtures which prevented characterization

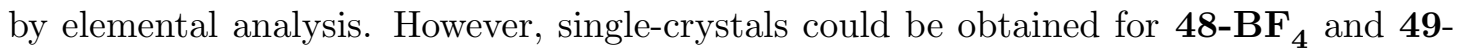
$\left(\mathbf{S b F}_{\mathbf{6}}\right)_{\mathbf{2}}$ (Figure 5.2). The overall structural changes upon oxidation are minor since $\delta$-symmetric orbitals with respect to the $\mathrm{N}=\mathrm{N}$ bond are depopulated. Applying a confidence interval of $95 \%(2 \sigma)$ shows that the data quality is not sufficient to discuss structural changes of the $\{\mathrm{MoNNMo}\}$ moiety upon oxidation since the error bars overlap in all selected bond lengths (Figure 5.2). Furthermore, the bond distances might be altered by packing effects. ${ }^{[283]-[285]}$

$\mathrm{X}$-ray absorption spectroscopy is particularly useful in this case since it allows for the determination of bond distances in solution via extended X-ray absorption fine structure spectroscopy (EXAFS)). ${ }^{[286]-[288]}$ As outlined in the introduction, the doubly protonated dimer XCVIII leads to dinitrogen splitting into the terminal nitride XCIX. XAS spectroscopy could allow to gain insight into the bond distances and overall electronic structure of XCVIII which are currently only accessible by theoretical calculations. ${ }^{[280]}$ 

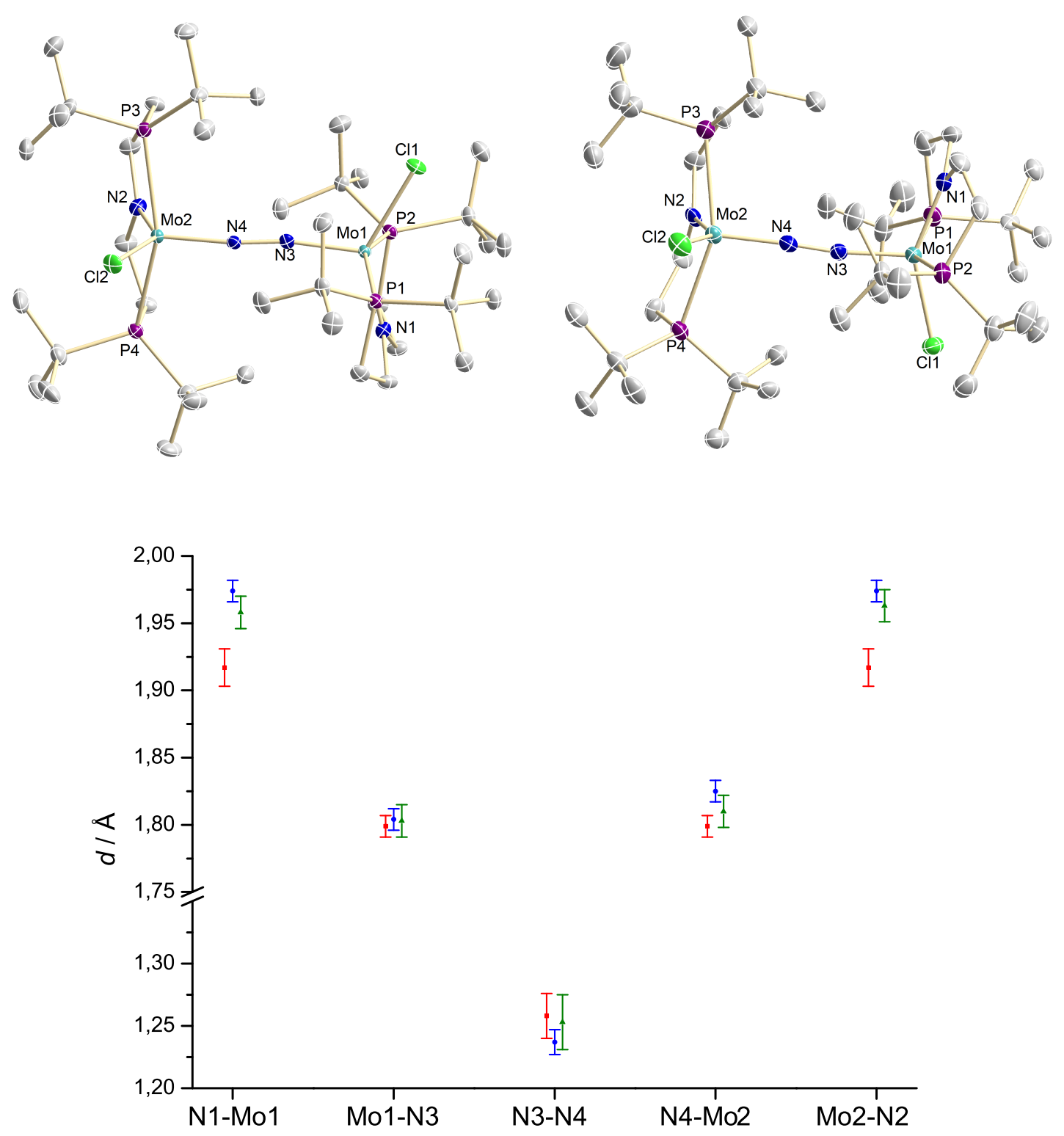

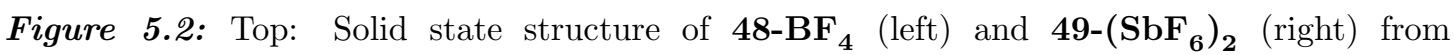
single-crystal X-ray diffraction (thermal ellipsoids drawn at the $50 \%$ probability level); solvent molecules, anions and hydrogen atoms, are omitted for clarity. Selected bond lengths $[\AA]$ and angles []: 48 Mo1-Cl1 2.3519(11), Mo1-N1 1.974(4), Mo1-N3 1.804(4), Mo1-P1 2.5387(12), Mo1-P2 2.5387(12) Mo2-Cl2 2.4515(11) Mo2-N2 1.974(4), Mo2-N4 1.825(4), Mo2-P3 2.4966(12), Mo2-P4 2.4921(12), N3-N4 1.237(5), N1-Mo1-N3 113.24(17) P1-Mo1-P2 155.84(4), Mo1-N3-N4 169.6(3), N2-Mo2-N4 109.51(16), P3-Mo2-P4 154.34(4), Mo2-N4-N3 170.9(3); 49 Mo1-Cl1 2.352(3), Mo1N1 1.958(8), Mo1-N3 1.803(8), Mo1-P1 2.532(3), Mo1-P2 2.583(3), Mo2-Cl2 2.349(3), Mo2N2 1.963(8), Mo2-N4 1.810(8), Mo2-P3 2.534(3), Mo2-P4 2.574(3), N3-N4 1.253(11), N1-Mo1N3 108.9(3), P1-Mo1-P2 151.29(9), Mo1-N3-N4 171.0(7), N2-Mo2-N4 108.1(3), P3-Mo2-P4 152.10(9), Mo2-N4-N3 168.7(7); Bottom: Selected bond lengths of XCVII (red), ${ }^{[280]} 48$ (blue) and 49 (green) from single-crystal X-ray diffraction, $2 \sigma$ error bars. 
Table 5.1: Experimental structural parameters of XCIX, XCVII, 48, 49 and XCVIII in solution derived by Mo-K edge EXAFS spectroscopy and single crystal X-ray diffraction in $\AA$, $\mathrm{THF}, 100^{\circ} \mathrm{C}$, for $\mathbf{X C V I I I}$ three set of computed bond lengths are given for the considered spin states, [a]: For XCIX the nitride bond length is given. ${ }^{[280]}$

\begin{tabular}{|c|c|c|c|c|c|c|c|c|}
\hline & \multicolumn{2}{|c|}{ Mo-N $\mathrm{N}_{\mathrm{PNP}}$} & \multicolumn{2}{|c|}{$\mathrm{Mo}-\mathrm{N}_{2}[\mathrm{a}]$} & \multicolumn{2}{|c|}{ Mo-Mo } & \multicolumn{2}{|c|}{$\mathrm{N}-\mathrm{N}$} \\
\hline & XRD & XAS & XRD & XAS & XRD & XAS & XRD & XAS \\
\hline XCIX & $2.209(2)$ & $2.11(3)$ & $1.646(2)$ & $1.62(1)$ & & & & \\
\hline XCVII & $1.917(7)$ & $1.99(2)$ & $1.799(4)$ & $1.80(1)$ & $4.846(1)$ & $4.74(2)$ & $1.258(9)$ & $1.27(4)$ \\
\hline 48 & $\begin{array}{l}1.974(4) \\
1.974(4)\end{array}$ & $1.99(2)$ & $\begin{array}{l}1.804(4) \\
1.825(4)\end{array}$ & $1.82(3)$ & $4.846(1)$ & $4.74(1)$ & $1.237(5)$ & $1.22(4)$ \\
\hline 49 & $\begin{array}{l}1.958(8) \\
1.963(8)\end{array}$ & $1.99(2)$ & $\begin{array}{l}1.803(8) \\
1.810(8)\end{array}$ & $1.82(3)$ & $4.847(2)$ & $4.74(8)$ & $1.25(2)$ & $1.20(1)$ \\
\hline & DFT & XAS & DFT & XAS & DFT & XAS & DFT & XAS \\
\hline XCVIII & & & & & & & & \\
\hline$S=0$ & 2.30 & & 1.82 & & 4.88 & & 1.27 & \\
\hline$S=1$ & 2.34 & $2.30(9)$ & 1.932 & $1.87(2)$ & 5.05 & $5.17(3)$ & 1.22 & n.a. \\
\hline$S=2$ & 2.32 & & 1.94 & & 5.08 & & 1.22 & \\
\hline
\end{tabular}

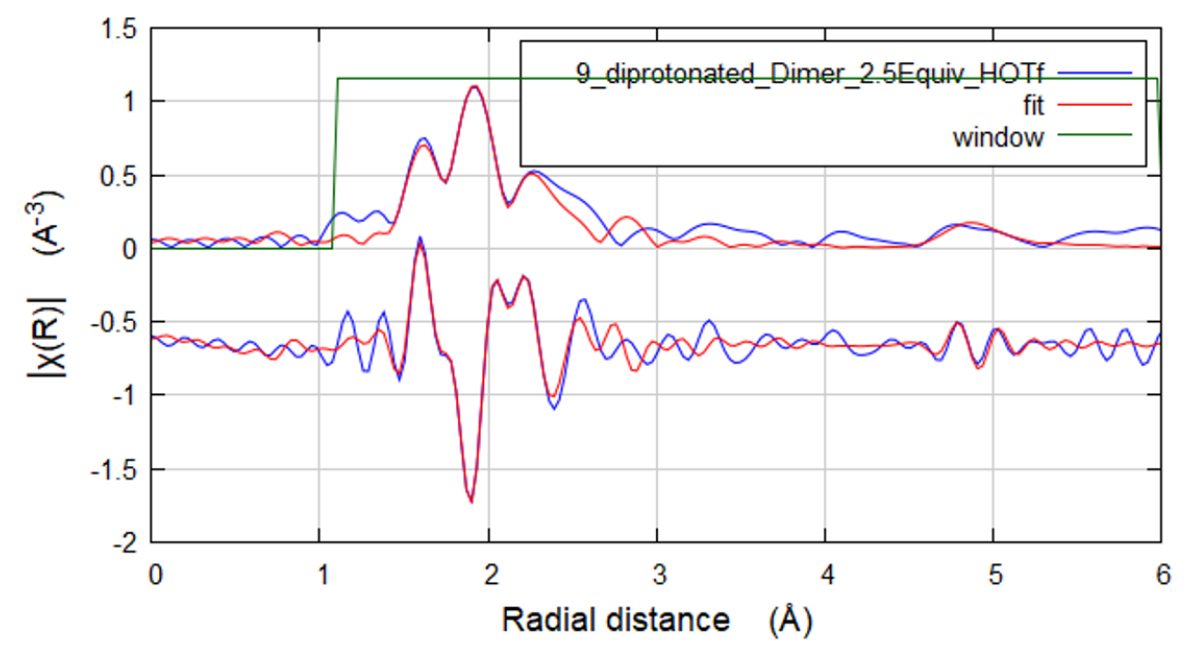

Figure 5.3: EXAFS spectrum of XCVIII, R space, fitted as mixture of XCVIII / XCIX: $75 \% / 25 \%$ (refined with fixed nitride complex bond lengths).

The molecular geometries of XCVII, 48, 49, XCIX and XCVIII in THF solution were derived by Mo-K edge EXAFS spectroscopy (Table 5.1). The lower data resolution when compared to single-crystal X-ray diffraction prevents the discussion of trends within the dimer redox series, however both utilized techniques give similar results for the bond distances around the metal center. The Mo-Mo bond distances are consid- 
erably shortened in solution, possibly owing to packing effect in the solid state. A significantly shorter Mo- $\mathrm{N}_{\mathrm{PNP}}$ bond length is obtained for the nitride complex XCIX by EXAFS spectroscopy which may be attributed to non-quantitative triflate coordination to the protonated pincer complex in solution. Structural information about the proposed diprotonated dimer XCVIII were obtained via protonation of XCVII with 2.5 equivalents of triflic acid at $-80^{\circ} \mathrm{C}$ and successive EXAFS spectroscopy. The resulting spectrum could be satisfactory fitted as a mixture of a dimeric Mo dinitrogen complex with small amount of the splitting product XCIX (Figure 5.3). Since no solidstate structure could be obtained, Schneider and co-workers calculated different possible spin states for XCVIII $(S=0,1,2)$ in a prior report as discussed in the introduction to this chapter. ${ }^{[280]}$ Although the resolution of the obtained data set was not as high as for the isolable compounds, a Mo-Mo multiple scattering pathway could be identified resulting in Mo-Mo distance of 5.17(3) $\AA$. The formation of a dimer in a quintet state prior to dinitrogen splitting can be confirmed due to the computed Mo-Mo bond length of $d_{\text {MoMo }}=5.08 \AA$ that agrees well with the experimental value (experimental and computed values show a decent agreement for XCVII, 48 and 49: Table 8.3)
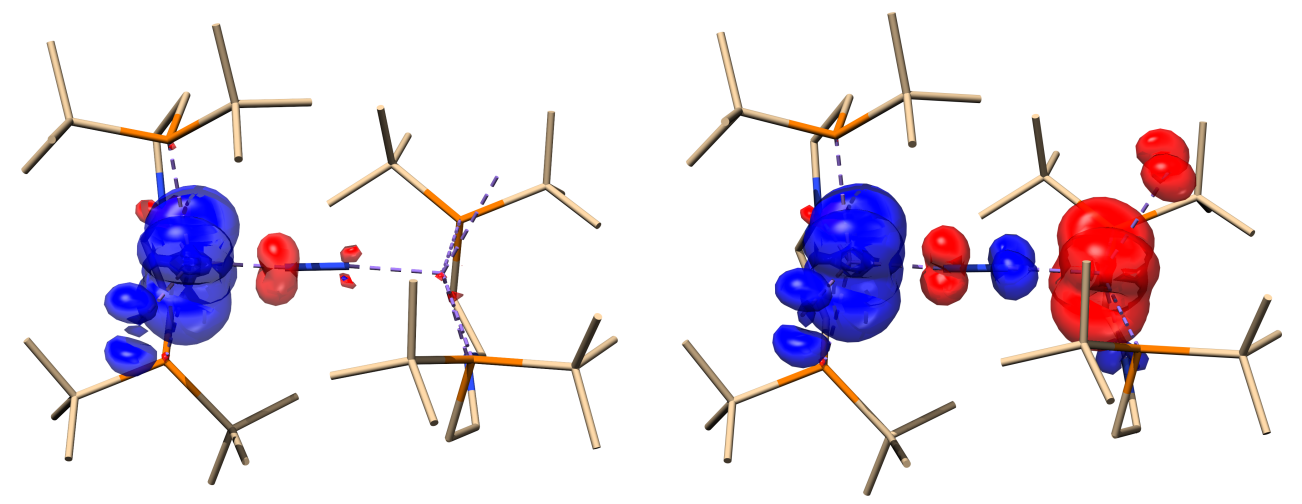

Figure 5.4: Spin-density plot of 48 (left) and 49 (right), positive densities given in blue.

Table 5.2: Selected bond orders of XCVII, 48 and 49.

\begin{tabular}{lccc}
\hline & \multicolumn{3}{c}{ Bond order } \\
Bond & XCVII & $\mathbf{4 8}$ & $\mathbf{4 9}$ \\
\hline N1-Mo1 & 1.08 & 1.09 & 1.15 \\
Mo1-N3 & 1.54 & 1.60 & 1.49 \\
N3-N4 & 1.25 & 1.39 & 1.47 \\
N4-Mo2 & 1.54 & 1.37 & 1.49 \\
Mo2-N2 & 1.08 & 1.15 & 1.15 \\
\hline
\end{tabular}


The results are further corroborated by DFT calculations. Spin-density calculations reveal the SOMO of $\mathbf{4 8}$ to be a metal centered $\delta$-symmetric orbital with respect to the dinitrogen bridge without extensive delocalization, in line with the obtained EPR spectrum (Figure 5.4). An antiferromagnetically coupled singlet ground state is predicted for 49. The calculated spin-density reveals similar localization features as for $\mathbf{4 8}$ per metal center. Since only non-bonding orbitals with respect to the bridging dinitrogen ligand are depopulated, the effect on the overall bonding situations are minor as shown by the computed bond orders (Table 5.2). Upon oxidation, an increase in the $\mathrm{Mo}_{\mathrm{N}} \mathrm{N}_{\mathrm{N}_{2}}$ bond order for one metal center is observed. The second oxidation re-symmetrizes the bonding situation, yielding identical values for both Mo atoms. The bond order of the dinitrogen ligands shows a gradual increase per oxidation step. The overall minor changes upon oxidation are in line with the MO considerations described in the introduction and the observed changes are likely to be attributed to electrostatic effect since the depopulated orbitals are not part of the $\pi$-core of the $\{$ MoNNMo $\}$ moiety.

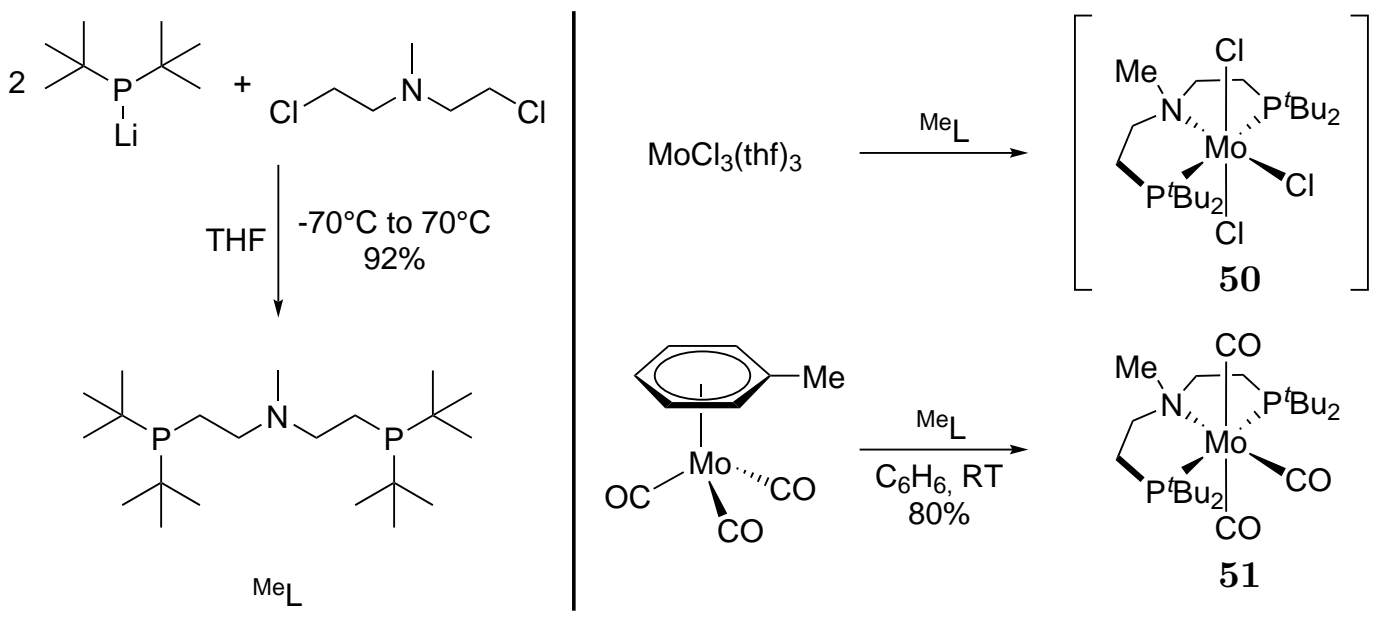

Scheme 5.3: Synthesis of ${ }^{\mathrm{Me}} \mathrm{L}$ and its reaction towards $\left[\mathrm{MoCl}_{3}(\mathrm{THF})_{3}\right]$ and $\left[\mathrm{Mo}(\mathrm{CO})_{3}(\right.$ toluene $\left.)\right]$ yielding 51 .

As outlined in the introduction, protonation of the pincer backbone is necessary to allow for splitting of dinitrogen. If the nitrogen atom of the pincer ligand was alkylated, the destabilization of the $\sigma^{*}$-and $\pi_{2}$-orbital would be prevented and direct splitting of $\mathrm{N}_{2}$ by a suitable Mo halide complex upon reduction could be feasible. The methylated pincer ligand $(\mathrm{Me}) \mathrm{N}\left(\mathrm{CH}_{2} \mathrm{CH}_{2} \mathrm{P}^{t} \mathrm{Bu}_{2}\right)_{2}\left({ }^{\mathrm{Me}} \mathrm{L}\right)$ has been reported by Prechtl and co-workers by coupling of in situ generated $\mathrm{LiP}^{t} \mathrm{Bu}_{2}$ and $(\mathrm{Me}) \mathrm{N}\left(\mathrm{CH}_{2} \mathrm{CH}_{2} \mathrm{Cl}\right)_{2}$. Besides a low yield, the published procedure does not allow for isolation of the pure ligand (69\% yield, $67 \%$ purity). The authors claim that the lithiation of $\mathrm{PH}^{t} \mathrm{Bu}_{2}$ is unselective and produces impurities that cannot be removed. ${ }^{[289]}$ Therefore, a new synthetic approach via lithiation of $\mathrm{PCl}^{t} \mathrm{Bu}_{2}$ and successive coupling with $(\mathrm{Me}) \mathrm{N}\left(\mathrm{CH}_{2} \mathrm{CH}_{2} \mathrm{PCl}\right)_{2}$ was developed (Scheme 


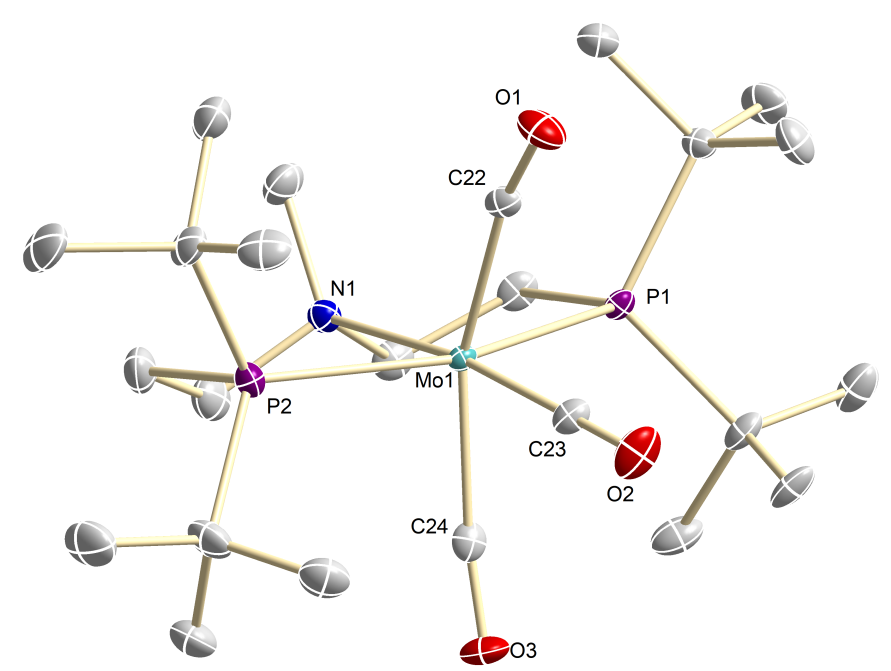

Figure 5.5: Solid state structure of $\mathbf{5 1}$ (one complex molecule of the asymmetric unit) from single-crystal X-ray diffraction (thermal ellipsoids drawn at the $50 \%$ probability level); hydrogen atoms are omitted for clarity. Selected bond lengths $[\AA]$ and angles [ $\left.{ }^{\circ}\right]$ : 51 Mo1-C22 2.012(4), Mo1-C23 1.933(4), Mo1-C24 1.994(4), Mo1-N1 2.401(3), Mo1-P1 2.5154(10), Mo1-P2 2.4959(11), C22-Mo1-C24 156.06(17), C23-Mo1-N1 172.02(15), P1-Mo1-P2 157.00(4).

5.3) which gives ${ }^{\mathrm{Me}} \mathrm{L}$ in $92 \%$ isolated yield. Sivasankar and co-workers independently published an identical protocol and obtained a yield of $65 \%$. ${ }^{[290]}$

Synthesis of $\left[\mathrm{MoCl}_{3}\left({ }^{\mathrm{Me}} \mathrm{L}\right)\right](\mathbf{5 0})$ was attempted by complexation of $\left[\mathrm{MoCl}_{3}(\mathrm{THF})_{3}\right]$. In apolar solvents like pentane, benzene and diethyl ether, precipitation of solid and a complex mixture of dia- and paramagnetic products was observed. Stirring $\left[\mathrm{MoCl}_{3}(\mathrm{THF})_{3}\right]$ and ${ }^{\mathrm{Me}} \mathrm{L}$ in $\mathrm{PhCl}$ at $50^{\circ} \mathrm{C}$ and $\mathrm{DCM} / \mathrm{THF}(1: 2)$ at room temperature both yield a paramagnetic product, accompanied by organic and paramagnetic side products (Appendix 8.4.5). The number of signals and their relative intensity suggest the formation of a $C_{S^{-}}$ symmetric complex, however the complex proved to be unstable in solution for extended periods of time. Mass spectrometry gave no conclusive results and no single-crystals suitable for X-ray diffraction were obtained which prevented full characterization. Complexation by a $\mathrm{Mo}^{0}$ carbonyl precursor was chosen as an alternative entry since the carbon monoxide ligand should be partly exchangeable upon oxidation/irradiation. Stirring $\left[\mathrm{Mo}(\mathrm{CO})_{3}\right.$ (toluene) $]$ and ${ }^{\mathrm{Me}} \mathrm{L}$ in benzene at room temperature gives the tricarbonyl complex $\left[\mathrm{Mo}(\mathrm{CO})_{3}\left({ }^{\mathrm{Me}} \mathrm{L}\right)\right]$ in high yield $(\mathbf{5 1}$, Scheme 5.3). Due to the low oxidation state, significant $\pi^{*}$-backdonation of the CO ligands is observed by three intense band in the IR spectrum, in line with the formation of a $C_{\mathrm{S}^{-}}$symmetric complex $\left(\tilde{\nu}_{\mathrm{CO}}=1911 \mathrm{~cm}^{-1}\right.$, $\left.1805 \mathrm{~cm}^{-1}, 1784 \mathrm{~cm}^{-1}\right)$. In the solid state, $\mathbf{5 1}$ features an octahedral coordination geometry with a mer-coordinated ${ }^{\mathrm{Me}} \mathrm{L}$ ligand (Figure 5.5). The Mo-N bond distance is considerably elongated when compared to $\mathrm{L}^{1,2,3}$ ligated systems due to the absence of $\pi$-donating properties (Mo1-N1: 2.401(3) $\AA$ ). Cyclic voltammetry shows that $\mathbf{5 1}$ can be 
reversibly oxidized at $E_{1 / 2}=-0.33 \mathrm{~V}$ vs. $\mathrm{Fc}^{0 /+}$ (Appendix 8.4.7). One-electron oxidation followed by ligand exchange could offer the possibility to access $\mathrm{Mo}^{\mathrm{III}}$, however further experiments are yet to be done.

\section{Conclusion}

The dinitrogen bridged molybdenum dimer XCVII could be successfully oxidized by one and two electrons to afford cationic 48 and 49. Both species were completely characterized, including single-crystal X-ray diffraction and extended X-ray absorption fine structure spectroscopy. Upon oxidation, only minor changes in the bond distances and overall geometry are observed. The cationic complex features a $S=\frac{1}{2}$ ground-state with a high degree of charge localization on one metal center. The dinitrogen bond get strengthened by $\Delta \tilde{\nu}=50 \mathrm{~cm}^{-1}$ due to polarization effects. Further oxidation leads to a minor weakening of the $\mathrm{N}_{2}$ bond and restores the overall symmetry of the complex. An $S=1$ ground-state for $\mathbf{4 9}$ was derived by SQUID magnetometry. In general, the depopulation of $\delta$-symmetric metal-based orbitals with respect to the dinitrogen bridge does not result in significant weakening of the dinitrogen bond. The prior postulated diprotonated dimer XCVIII was investigated by EXAFS spectroscopy. The acquired resolution was sufficient to identify a Mo-Mo multiple scattering pathway which yielded a bond distance of $d_{\mathrm{MoMo}}=5.17(3) \AA$, confirming the predicted formation of a quintet ground-state prior to dinitrogen splitting. ${ }^{[280]}$ Dinitrogen splitting without the need for protonation was attempted by synthesizing the methylated analogue of $\mathrm{L}^{1}$. Coordination towards $\mathrm{Mo}(\mathrm{III})$ did not result in the formation of a pure compound. However, $\mathrm{L}^{\mathrm{Me}}$ could be successfully coordinated to $\mathrm{Mo}(0)$ carbonyl precursor complexes which might represent an entry into dinitrogen chemistry upon oxidative removal of the carbonyl ligands. 

Summary 


\section{Summary and Outlook}

The main goal of this thesis was to investigate the bonding between transition metals and pnictogen containing ligands as well as terminal pnictides in unprecedented coordination spheres and analyze their electronic structures. Therefore, several Os and Re starting platforms were synthesized.

The first well-defined, square-planar $\mathrm{Os}^{\mathrm{II}}$ complex $\mathbf{2}$ with a triplet ground state could be synthesized and completely characterized. $\mathbf{2}$ exhibits large temperature-independent paramagnetism which was investigated by coupled-cluster calculations that yielded a suitable electronic structure description as a thermally isolated ground state which mixes with excited states through spin-orbit coupling. The platform was reacted with different $\pi$-accepting ligands which resulted in adduct formation and the negation of spinorbit coupling effects, yielding classical singlet ground state systems. The addition of the chelating ligands 2,2 '-bipyridine and 3-(1H-pyrazol-3-yl)-pyridine lead to octahedral

Table 6.1: Comparison of bond lengths and redox potentials of Os pincer platforms synthesized in this thesis, potentials given in V vs. $\mathrm{Fc}^{0 /+}$ and bond lengths in $\AA$.

\begin{tabular}{|c|c|c|c|}
\hline Complex & $\mathrm{M}-\mathrm{N}_{\mathrm{PNP}}$ & $\mathrm{M}-\mathrm{Cl}_{\text {apical }}$ & $E_{\text {Red }}$ \\
\hline & $1.966(6)$ & $2.380(3)$ & -1.28 \\
\hline & $2.120(2)$ & $2.4557(6)$ & -1.40 \\
\hline 2 & $1.980(2)$ & & -2.05 \\
\hline & $2.017(3)$ & $2.3837(9)$ & -2.71 \\
\hline & $2.015(4)$ & & -1.83 \\
\hline 8 & & & \\
\hline
\end{tabular}


platforms. In these cases, the $\mathrm{M}-\mathrm{N}_{\mathrm{PNP}}$ and $\mathrm{M}-\mathrm{Cl}_{\text {apical }}$ bond distances elongated due to the increased electron density at the metal center (Table 6.1). This is also reflected in a negative shift of the reduction potentials. The synthesis of the cationic, five-coordinate bipy complex 8 allowed to shift the redox potential of $\mathrm{Os}^{\mathrm{II}}$ to more positive values. This results show that $\mathbf{2}$ represents an attractive platform to access a variety of $\mathrm{L}^{3}$ ligated species with, dependent on the added ligand, tuneable redox potentials.

2 proved to be a competent platform for the synthesis of the $\mathrm{Os}^{\mathrm{VI}}$ nitrides $\mathbf{1 6}, \mathbf{1 5}$ and the $\mathrm{Os}^{\mathrm{IV}}$ nitride $\mathbf{1 4}$. In contrast to the prior reported system ligated by $\mathrm{L}^{1}, \mathbf{1 4}$ did not exhibit ambiphilic reactivity. Upon addition of ${ }^{t} \mathrm{BuCN}$, the five coordinate adduct $\mathbf{1 8}$ was obtained which represents the first $\mathrm{d}^{4}$ configured nitride complex in a five-fold coordination sphere. Although the Gray-Ballhausen scheme predicts such compounds not to be stable, $3 \mathrm{c} 4 \mathrm{e}$ bonding interactions circumvent this general rule. Additionally, stabilization of $\mathrm{d}^{4}$ nitrides was shown upon silylation to give the first isolable $\mathrm{d}^{4}$ configured silylimido complex 19 and heterocumulene formation with 2, respectively. Interestingly, the Os $\equiv \mathrm{N}$ bond lengths changed only minorly upon adduct formation $(\mathbf{1 7}, \mathbf{1 8})$ with respect to parent $\mathbf{1 4}$ (Table 6.2). In contrast, imide complex formation results in an elongation up to $\Delta d \approx 0.06 \AA(\mathbf{1 9}, \mathbf{2 0})$. Investigation of oxidative nitride coupling of bipy ligated systems was unsuccessful.

Table 6.2: Comparison of bond lengths of complexes with $\mathrm{M} \equiv \operatorname{Pn}(\mathrm{R})$ bonds and a $\mathrm{d}^{4}$ electron count synthesized in this thesis, bond lengths given in $\AA$.

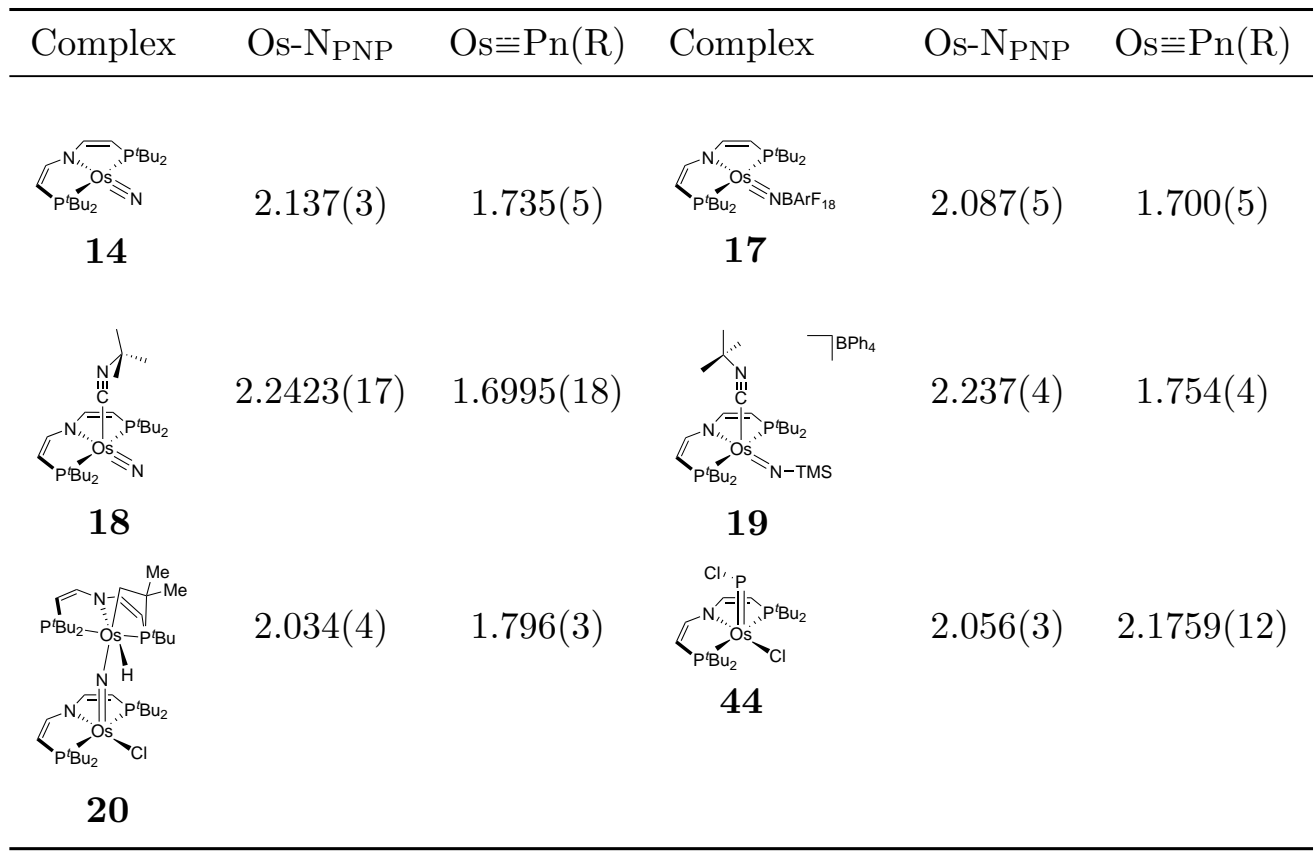


Re PNP pincer complexes were investigated by means of magnetic measurements and significant contributions of temperature independent paramagnetism were observed by SQUID magnetometry. The combination of iodide substituents and the dehydrogenated pincer ligand $\mathrm{L}^{3}$ exhibited the most prominent mixing of thermally non-accessible, excited electronic states to the ground state. An examination of the corresponding bromide compound could give valuable insight into the overall effect of halide ligands on spin-orbit coupling effect in these systems. Additionally, a comprehensive study on the dependencies of the redox potentials of the systems upon variation of halide ligands and degree of dehydrogenation of the pincer backbone is yet to be done.

The hemilabile ligand 3-(1H-pyrazol-3-yl)-pyridine was utilized to obtain the Re $\mathrm{e}^{\mathrm{III}}$ complex 11. 11 showed selective formation of the terminal pnictide complexes $\mathbf{2 7}$ and $\mathbf{2 8}$ upon decarbonylation of $\mathrm{PCO}^{-}$and $\mathrm{AsCO}^{-}$, respectively, which represent the first examples of heavy transition metal pnictide complexes beyond group 6 . Both species were completely characterized and resulted in the formation of donor-acceptor stabilized $\mathrm{P}_{2}$ and $\mathrm{As}_{2}$ complexes upon one-electron oxidation. This bonding description was substantiated by QTAIM analysis. Furthermore, the phosphide complex showed unprecedented reactivity towards pyridine- $\mathrm{N}$-oxide which allowed for the isolation of the $\mathrm{P}_{2} \mathrm{O}_{4}^{2-}$ bridged dimeric compound 31. Two-electron reduction gave the $\mathrm{PO}_{2}^{-}$-ligated complex 34 which represents the first transition metal complex of such kind. The bonding situation could be elucidated by quantum chemical calculation and revealed that this ligand is best described as a $\sigma$-donor with delocalized P-O bonds. The observed reactivity represents an attractive starting point to access low-valent phosphorus species from terminal phosphides as $\mathrm{P}_{1}$ building blocks.

The triple bond distances of the isoelectronic complexes 15, 16, 27 and 28 show a significant change in bond lengths upon comparing nitride and phosphide/arsenide complexes (Table 6.3). These systems feature an occupied d orbital which is non-bonding with respect to the triple bond. However, this does not severely affect the observed bond distances when compared to $\mathrm{d}^{0}$ systems. ${ }^{[22],[63]}$ Theoretical calculations revealed that the heavy triple bonds show a high degree of covalency with minor polarization. As outlined in the introduction, the Pauli repulsion is likely to be the main factor to rationalize the increase in bond distance when moving from nitrogen to phosphorus and arsenic. While oxidation and reduction potentials of $\mathbf{1 5}$ are separated by at least $3 \mathrm{~V}$, the heavier pnictides exhibit significantly decreased, similar spacing between the redox events $(\Delta=2.32 \mathrm{~V}(\mathrm{P}), 2.30 \mathrm{~V}(\mathrm{As}))$. A smaller HOMO-LUMO gap for the heavy multiple bonds can be derived by this, owing to less effective orbital splitting. 
Table 6.3: Comparison of bond lengths and redox potentials of five-coordinate transition metal pnictide complexes with a $\mathrm{d}^{2}$ electron count synthesized in this thesis, potentials given in $\mathrm{V}$ vs. $\mathrm{Fc}^{0 /+}$ and bond lengths in $\AA$.

\begin{tabular}{|c|c|c|c|}
\hline Complex & $\mathrm{M} \equiv \mathrm{Pn}$ & $E_{\mathrm{Ox}}$ & $E_{\text {Red }}$ \\
\hline
\end{tabular}

16

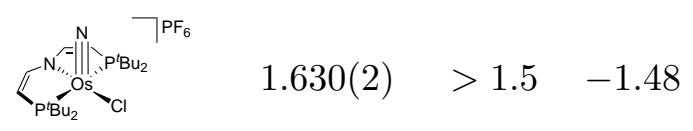

15

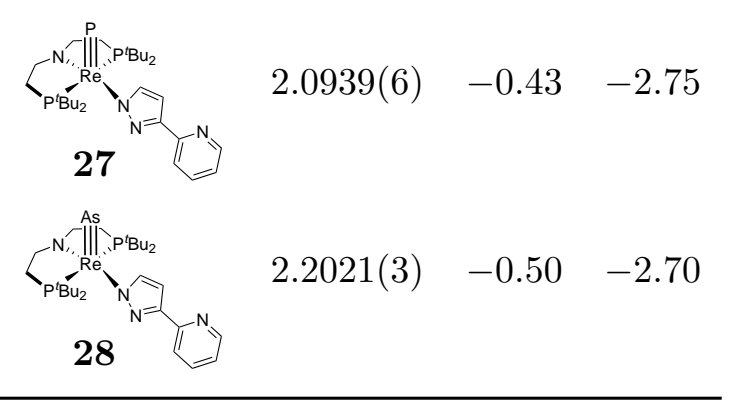

The electron rich $\mathrm{Os}^{\mathrm{II}}$ platform 2 allowed for the oxidative addition of the primary phosphine $\mathrm{PH}_{2}$ Mes* which resulted in the formation of the first isolable transition metal phosphinyl radical complex 38. EPR spectroscopy and theoretical calculation suggest an equal distribution of the unpaired electron along the Os-P bond. The P-H bond could be split homolytically via proton coupled electron transfer to give the corresponding phosphinidene complex 39. The associated bond dissociation energy was derived by isothermal titration calorimetry which allowed to extract additional thermodynamic parameters of the system. The low BDE value of the $\mathrm{P}-\mathrm{H}$ bond suggests that tailored systems could utilize primary phosphines as electrophilic $\mathrm{P}_{1}$ building blocks.

Based on the results obtained for the Re system, the preparation of novel Os $=P(R)$ species was attempted upon reaction of $\mathrm{PCO}^{-}$and $\mathrm{ClPA}$ with different platforms. The heavier analogue of isocyanate was not compatible with all examined systems and resulted in reduction of the metal complex or no reaction at all. The reaction of ClPA with the square-planar halide complex 2 resulted in the formation of the first isolable and remarkably stable chlorophosphinidene complex 44. In comparison to other $\mathrm{Os}^{\mathrm{IV}}$ systems covered in this thesis, the Os- $\mathrm{N}_{\mathrm{PNP}}$ bond length is comparable while the Os=P double bond distance features a value of over $2.1 \AA$ (Table 6.2). Cyclization reactions with unsaturated organic substrates were examined but no reactivity was observed in all 
cases. A DFT study revealed that the steric bulk of the tert-butyl substituents induced a high kinetic barrier which might be overcome upon substitution with sterically less demanding ${ }^{i}$ Pr-groups. Further studies should focus on the preparation of such complexes to eventually allow for (catalytic) phosphinidene transfer to unsaturated substrates.

The prior reported dimeric molybdenum dinitrogen complex XCVII was successfully oxidized by one and two electrons to give the cationic species 48 and 49 , respectively. Both complexes were completely characterized to study the effect of depopulation of $\delta$-symmetric, metal-centered orbitals with respect to the dinitrogen bridge. Beside polarization effects which slightly affect the $\mathrm{N}=\mathrm{N}$ bond strength, no severe structural alterations were observed. This was further confirmed by EXAFS spectroscopy which also served as an experimental technique to investigate the prior postulated diprotonated molybdenum dimeric complex XCVIII. Based on the obtained Mo-Mo distance, a quintet ground state was derived upon comparison with quantum chemical calculations. Since protonation of XCVII is required to allow for dinitrogen splitting towards nitrides, the methylated PNP pincer ligand $\mathrm{L}^{\mathrm{Me}}$ was synthesized to skip this reaction step and directly access a suitable orbital configuration in dimeric dinitrogen complexes to enable splitting of dinitrogen upon reduction of suitable precursors. However, complexation of $\mathrm{Mo}^{\mathrm{III}}$ halide precursors proved to be difficult and a $\mathrm{Mo}^{0}$ carbonyl complex was prepared instead. The compound exhibits promising redox chemistry which might allow for successive decarbonylation to access halide complexes.

In summary, this thesis demonstrated the synthesis of different Re and Os halide platforms which allowed for rich follow-up chemistry and featured unique magnetic properties. New ways of stabilizing nitride complexes beyond the "nitrido wall" were demonstrated. Heavier analogues of nitride complexes, namely phosphides and arsenides, were successfully synthesized and depict the first group 7 representatives of this compound class. Furthermore, different strategies to utilize terminal transition metal pnictides, primary phosphines and ClPA as $\mathrm{Pn}_{1}$ building block were evaluated. New insight into the mechanism of dinitrogen splitting by Mo PNP pincer complexes were obtained. 


\section{Experimental Section}




\section{Experimental Section}

\subsection{General Comments}

For all published computed structures the corresponding XYZ-Files can be obtained free of charge via the publisher. Therefore, this data will not be presented in this section.

If a transition metal compound could not be characterized by means of combustion analysis the full NMR characterization will be given in the Appendix.

\subsection{Materials and Methods}

All experiments were carried out using Schlenk (argon atmosphere) and glovebox (argon atmosphere) techniques. All solvents were dried by passing through columns packed with activated alumina. Deuterated solvents were obtained from Euriso-Top GmbH, dried over Na/K (THF, Toluene, Benzene) or $\mathrm{CaH}_{2}\left(\mathrm{CD}_{2} \mathrm{Cl}_{2}\right)$, distilled by trap-to-trap transfer in vacuo, and degassed by three freeze-pump-thaw cycles, respectively. Silica gel 60 silanized and activated carbon were heated at $120^{\circ} \mathrm{C}$ in vacuo for 5 days prior to use.

Table 7.1: List of used chemicals.

\begin{tabular}{l|l|l} 
Compound & Supplier & Purification \\
\hline $18-\mathrm{C}-6$ & Sigma-Aldrich & Recrystallization \\
$15-\mathrm{C}-5$ & Sigma-Aldrich & \\
2,2 '-Bipyridine & abcr & \\
$\mathrm{Ag}\left[\mathrm{Al}\left(\mathrm{OC}\left(\mathrm{CF}_{3}\right)_{3}\right)_{4}\right]$ & Iolitec & \\
$\mathrm{AgBF}_{4}$ & Sigma-Aldrich & \\
$\mathrm{AgSbF}_{6}$ & Sigma-Aldrich & \\
$\mathrm{B}\left(\mathrm{C}_{6} \mathrm{H}_{3}-3,5-\left(\mathrm{CF}_{3}\right)_{2}\right)_{3}$ & Sigma-Aldrich & \\
$\mathrm{nBuli} 2.5 \mathrm{M}$ in hexane & Sigma-Aldrich & \\
$\mathrm{C}$ & Thermo-Fisher & \\
$\mathrm{ClPA}$ & {$[67]$} & \\
$\mathrm{CN}{ }^{t} \mathrm{Bu}$ & Sigma-Aldrich & \\
$\mathrm{CO}$ & Linde & \\
$\mathrm{CoCp}$ & abcr & \\
$\mathrm{Cyclohexene}$ & TCI & \\
$\mathrm{Dimethylbutadiene}$ & Sigma-Aldrich & \\
{$\left[\mathrm{Fe}\left(\mathrm{C}_{5} \mathrm{H}_{5}\right)_{2}\right]$} & Sigma-Aldrich & Sublimation \\
{$\left[\mathrm{Fe}\left(\mathrm{C}_{5} \mathrm{Me}_{5}\right)_{2}\right]$} & Sigma-Aldrich & Sublimation \\
$\mathrm{Hg}$ & Sigma-Aldrich & \\
{$\left[\mathrm{H}(\mathrm{OEt})_{2}\right]\left[\mathrm{B}\left(\mathrm{C}_{6} \mathrm{H}_{3}-3,5-\left(\mathrm{CF}_{3}\right)_{2}\right)_{4}\right]$} & {$[291]$} \\
$\mathrm{HOTf}$ & Sigma-Aldrich & \\
${ }_{\mathrm{H}} \mathrm{PNP}$ & {$[292]$} \\
${ }_{\mathrm{H}} \mathrm{PyrPz}$ & TCI \\
$\mathrm{K}$ & Sigma-Aldrich & \\
$\mathrm{KO}{ }^{t} \mathrm{Bu}$ & Sigma-Aldrich & Sublimation \\
& &
\end{tabular}



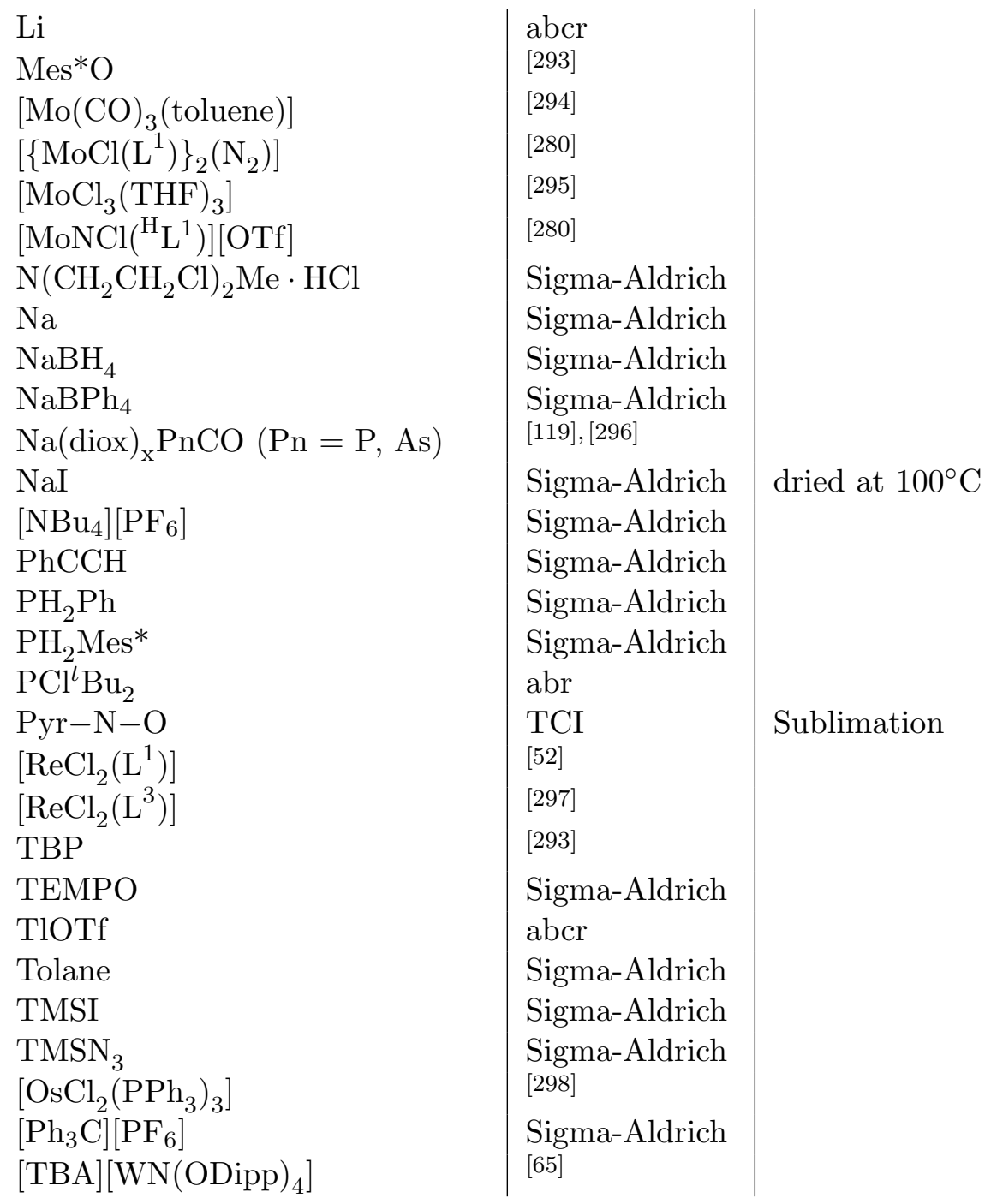

Cyclic voltammograms were recorded with a Metrohm Autolab PGSTAT101 using $\mathrm{Ag} / \mathrm{Ag}^{+}$reference-, glassy-carbon working- and Pt-wire counterelectrodes.

Experimental X-band EPR spectra were recorded on a Bruker ELEXSYS-II E500 CW-EPR. The spectra were simulated by iteration of the anisotropic g-values, (super)hyperfine coupling constants, and line widths using the EPR-simulation program W95EPR developed by Prof. Dr. Frank Neese and EasySpin.

IR spectra were recorded with a Thermo Scientific Nicolet iZ10 FT/IR spectrometer at room temperature.

Magnetic susceptibility measurements were performed with a Quantum Design MPMSXL-5 SQUID magnetometer in the temperature range from 295 to $2 \mathrm{~K}$ at $0.5 \mathrm{~T}$ applied field. The powdered sample was contained in a Teflon bucket and fixed in a non-magnetic sample holder. Each raw data point for the measured magnetic moment of the sample was corrected for the diamagnetic contribution by subtraction of the experimentally de- 
termined magnetic measurement of the Teflon bucket. The molar susceptibility data were corrected for the diamagnetic contribution using the Pascal constants and the increment method according to Haberditzl. ${ }^{[299]}$ Experimental data were modelled with the $j u l X$ program. ${ }^{[300]}$

Elemental analyses were obtained from the analytical laboratories at the GeorgAugust University on an Elementar Vario EL 3.

NMR spectra were recorded on Bruker Avance III 300, Bruker Avance III 300, Bruker Avance III 400 and Bruker Avance III HD 500 and were calibrated to the residual solvent proton resonance. $\left(\mathrm{C}_{6} \mathrm{D}_{6}=7.16 \mathrm{ppm}\right.$, THF- $\mathrm{d}_{8}=3.58 \mathrm{ppm}$, toluene- $\mathrm{d}_{8}=2.08 \mathrm{ppm}$, $\left.\mathrm{CD}_{2} \mathrm{Cl}_{2}=5.32 \mathrm{ppm}\right)$.

LIFDI spectrometry was performed on a Joel AccuTOF spectrometer under inert conditions.

UV/vis spectra were recorded with an Agilent Technologies Cary $300 \mathrm{UV} /$ vis spectrometer.

Indophenolic titration was used for the quantification of ammonia. ${ }^{[301]}$

Electrospray ionization (ESI) mass spectra were collected on a Bruker HCTultra instrument. The moisture and oxygen sensitive samples were prepared in a glovebox (MBRAUN UNIlab) under an argon atmosphere and injected into the Bruker HTCultra instrument via a direct Peek $^{\top M}$ tubing connection to a syringe using dry and degassed solvents.

Suitable single crystals for X-ray structure determination were selected from the mother liquor under an inert gas atmosphere and transferred in protective perfluoro polyether oil on a microscope slide. The selected and mounted crystals were transferred to the cold gas stream on the diffractometer. The diffraction data were obtained at $100 \mathrm{~K}$ on a Bruker D8 three-circle diffractometer, equipped with a PHOTON 100 CMOS detector and an INCOATEC microfocus source with Quazar mirror optics (Mo-K $\alpha$ radiation, $\lambda=0.71073 \AA$ ). The data obtained were integrated with SAINT and a semi-empirical absorption correction from equivalents with SADABS was applied. The structure was solved and refined using the Bruker SHELX 2014 software package. ${ }^{2}$ All non-hydrogen atoms were refined with anisotropic displacement parameters. All C-H hydrogen atoms were refined isotropically on calculated positions by using a riding model with their Uiso values constrained to $1.5 \mathrm{U}_{\text {eq. }}$ of their pivot atoms for terminal $\mathrm{sp}^{3}$ carbon atoms and 1.2 times for all other atoms.

Mo K-edge XAS measurements were performed at the B18 beamline at the Diamond Light Source in Didcot, United Kingdom. All measurements were performed in fluores-

${ }^{2}$ a) APEX3 v2016.9-0 (SAINT/SADABS/SHELXT/SHELXL), Bruker AXS Inc., Madison, WI, USA, 2016. b) George M. Sheldrick, Acta Cryst., 2015, A71, 3. c) George M. Sheldrick, Acta Cryst., 2015, C71, 3. d) George M. Sheldrick, Acta Cryst., 2008, A64, 112. 
cence mode, unless otherwise stated. Measurements at the Diamond Light Source were performed with a $\mathrm{Si}(311)$ double crystal monochromator in combination with a 9 element Ge Solid State detector. A typical measurement required around 90 minutes; a minimum of 20 scans were required to obtain good signal-to-noise in the data. All acquired spectra were calibrated to a Mo metal foil. EXAFS data processing and analysis was performed using the Demeter software package in Athena and Artemis respectively. ${ }^{[302]}$ The amplitude reduction factor was determined using the Mo foil and was 0.95. Samples were prepared in J-Young NMR tubes and cooled (for setup see Appendix 8.4.3) 


\subsection{Os(II/III) and Re(III) PNP Pincer Complexes}

Parts of this chapter were adapted from:

J. Abbenseth, M. Diefenbach, S. C. Bete, C. Würtele, C. Volkmann, S. Demeshko, M. C. Holthausen, S. Schneider Chem. Commun. 2017, 53, 5511-5514.

with permission from The Royal Society of Chemistry.

\subsubsection{A Square-Planar Os(II) Complex - Computational Details}

The molecular geometries of two singlet and two triplet isomers of $\mathbf{2}$ in $C_{2}$ and $C_{\mathrm{s}}$ symmetry were optimized under gas-phase conditions using the Gaussian09 program ${ }^{[303]}$ with the PBE0 hybrid functional ${ }^{[304],[305]}$ in conjunction with Grimme's 3rd generation atom-pairwise dispersion correction including Becke-Johnson damping (D3BJ). ${ }^{[306]}$ The def2-TZVP orbital basis set ${ }^{[307]}$ was employed with the quasi-relativistic ECP60MWB pseudopotential, ${ }^{[308]}$ which replaces the 60 core electrons in osmium. This level of DFT is abbreviated as PBE0D/TZVP. The optimized geometries were identified as minima through analysis of the eigenvalues of the Hessian matrix. Further calculations were conducted on a smaller model system, in which the four $t \mathrm{Bu}$ groups of the pincer were replaced by methyl groups $(\mathbf{2}, \mathbf{M e})$. For each fully optimized electronic state of the real system 2, the model systems $\mathbf{2}, \mathbf{M e}$ were constructed in constrained geometries where only the $\mathrm{C}-\mathrm{H}$ bond lenghts of the twelve newly added hydrogen atoms (which replace the twelve methyl fragments of the four $t$ Bu groups) were allowed to relax, i.e., all angles and dihedrals are kept fixed and all remaining coordinates are unaltered.

Relative energies from correlated wavefunction theory were computed at the DFT molecular geometries for the electromers of the 2,Me model system using the Molpro2015.1 program. ${ }^{[309]}$ The explicitly correlated coupled-cluster ansatz with single and double excitations and perturbative triples, $\operatorname{CCSD}(\mathrm{T})-\mathrm{F} 12 \mathrm{~b},{ }^{[310],[311]}$ was employed in combination with the F12-optimized correlation-consistent polarized double- and triple-zeta orbital and auxiliary basis sets of the cc-pVnZ-F12 family ${ }^{[312]-[314]}$ on non-metal atoms and the aug-cc-pVnZ-PP orbital basis set which includes the relativistic ECP60MDF pseudopotential on $\mathrm{Os}^{[315]}$ in conjunction with the auxiliary def2-QZVPP/JKfit basis sets ${ }^{\text {? }}$ for the many-electron integrals (CABS) as well as for the density-fitting of the Fock and exchange matrices (JKfit) and with the aug-cc-pV ${ }^{[316]}$ for the remaining integrals. The $\operatorname{CCSD}(\mathrm{T})-\mathrm{F} 12$ energies were extrapolated to the complete basis set limit CBS(D,T) according to the procedure of Hill et al. ${ }^{[317]}$ Final energies given in the text are based on an ONIOM(CCSD(T)-F12:PBE0D) approach according to $E_{\text {tot }}(\mathbf{2})=E_{\mathrm{CCSD}(\mathrm{T})-\mathrm{F} 12}(\mathbf{2}, \mathbf{M e})$ $-E_{\mathrm{PBE} 0 \mathrm{D}}(\mathbf{2}, \mathbf{M e})+E_{\mathrm{PBE} 0 \mathrm{D}}(\mathbf{2})$.

Quasi-degenerate perturbation theory (QDPT) was used to calculate spin-orbit eigenstates for the real system 2. Computations were performed on the DFT-optimized geometry of the lowest-energy $C_{2}$-symmetric ${ }^{3} \mathrm{~B}$ state based on a CASSCF wavefunction within the ORCA program. ${ }^{[318]}$ The ZORA approximation ${ }^{[319]}$ for all elements. The active space comprises the five Os-based $5 \mathrm{~d}$ orbitals and five occupied ligand-metal based orbitals, giving rise to a CAS $(16,10)$ expansion. In the CASSCF calculations the orbitals were optimized by the average of 5 quintet, 45 triplet and 50 singlet roots arising from the formal $\mathrm{d}^{6}$ configuration of the osmium(II) center. The RI and RIJCOSX ${ }^{[320]}$ approximations were used along with the corresponding def2TZVP/C auxiliary basis sets $^{[321]}$ and a fine grid (GridX6 in ORCA convention), respectively. The final energies 
are obtained from NEVPT2 calculations, ${ }^{[322]-[324]}$ and the energies that enter QDPT treatment via a full SOMF operator. ${ }^{[325]}$

For a complete list of all computed results see the originial publication.

\subsubsection{Five- and Six-Coordinate Os Bipyridine Complexes - Computational Details}

DFT calculations were carried out with the ORCA program package using the PBE

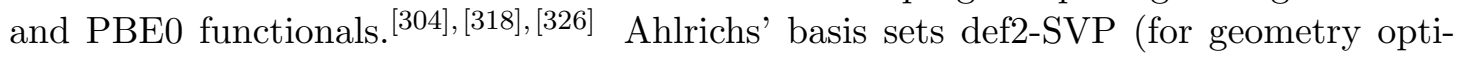
mization and frequency calculation) and def2-TZVPP (for single-point energies) were used with a full basis for all elements but Os for which the Stuttgart-Dresden 60 electron core potential was chosen to replace the inner shell 1s-4f orbitals. ${ }^{[307],[308]}$ The RI-J (PBE) approximation in combination with the corresponding auxiliary basis sets of Alrichs was utilized to reduce computational costs in the geometry optimization and frequency calculations. ${ }^{[327]-[329]}$ Grimme's model (D3) with Becke-Johnson damping was used to account for dispersion with the PBE or PBE0 functionals. ${ }^{[306],[330]}$ No symmetry restraints were imposed and the optimized structures were defined as minima (no negative eigenvalues) by vibrational analyses. TDDFT/EPR calculations were performed with ORCA and the CPCM solvation model was used (pentane, THF). ${ }^{[331]}$ Relativistic treatment was introducted by ZORA. ${ }^{[332]}$ Geometries were analyzed and visualized with Avogadro and molecular orbitals, spin-densities and difference densities were visualized with Chimera. ${ }^{[333],[334]}$

Table 7.2: XYZ-coordinates of computed complex 9.

\begin{tabular}{c|lll|l|lll} 
Atom & $\mathrm{x}$ & $\mathrm{y}$ & $\mathrm{z}$ & $\mathrm{Atom}$ & $\mathrm{x}$ & $\mathrm{y}$ & $\mathrm{z}$ \\
\hline $\mathrm{C}$ & -0.25646 & 2.68861 & -1.70132 & $\mathrm{C}$ & 1.57293 & 2.24456 & 2.16366 \\
$\mathrm{C}$ & -0.78199 & 1.66259 & -2.44998 & $\mathrm{H}$ & 1.1423 & 2.03454 & 3.16711 \\
$\mathrm{~N}$ & -0.22282 & 0.40738 & -2.48361 & $\mathrm{H}$ & 2.3253 & 1.45975 & 1.94644 \\
$\mathrm{H}$ & -1.68978 & 1.81869 & -3.06568 & $\mathrm{H}$ & 2.09951 & 3.216 & 2.23448 \\
$\mathrm{C}$ & -0.7903 & -0.57084 & -3.26458 & $\mathrm{C}$ & -0.53291 & 3.41432 & 1.3894 \\
$\mathrm{C}$ & -0.26806 & -1.83819 & -3.36223 & $\mathrm{H}$ & -0.99859 & 3.26451 & 2.38808 \\
$\mathrm{H}$ & -1.70313 & -0.29082 & -3.82658 & $\mathrm{H}$ & -1.34943 & 3.43222 & 0.64012 \\
$\mathrm{P}$ & 1.18605 & -2.17453 & -2.341 & $\mathrm{H}$ & -0.04812 & 4.40657 & 1.3985 \\
$\mathrm{H}$ & -0.74534 & -2.58927 & -4.00753 & $\mathrm{C}$ & 2.41662 & 3.71205 & -0.86497 \\
$\mathrm{C}$ & 0.52491 & -3.37571 & -0.98223 & $\mathrm{C}$ & 1.92861 & 5.0117 & -0.20697 \\
$\mathrm{C}$ & 2.41051 & -3.12747 & -3.47674 & $\mathrm{H}$ & 0.93371 & 5.32441 & -0.58212 \\
$\mathrm{C}$ & -0.21921 & -2.44152 & -0.01512 & $\mathrm{H}$ & 1.87908 & 4.93521 & 0.89609 \\
$\mathrm{H}$ & 0.4906 & -1.72744 & 0.45515 & $\mathrm{H}$ & 2.64354 & 5.82758 & -0.45028 \\
$\mathrm{H}$ & -0.68658 & -3.02385 & 0.80827 & $\mathrm{C}$ & 2.58529 & 3.96054 & -2.37452 \\
$\mathrm{H}$ & -1.01663 & -1.86444 & -0.52563 & $\mathrm{H}$ & 1.62436 & 4.22109 & -2.86017 \\
$\mathrm{C}$ & -0.47337 & -4.41635 & -1.50922 & $\mathrm{H}$ & 3.00405 & 3.08233 & -2.89599 \\
$\mathrm{H}$ & -0.89916 & -4.97885 & -0.6502 & $\mathrm{H}$ & 3.29196 & 4.80568 & -2.52168 \\
$\mathrm{H}$ & -0.00871 & -5.15364 & -2.18792 & $\mathrm{C}$ & 3.77344 & 3.29072 & -0.2733 \\
$\mathrm{H}$ & -1.315 & -3.93361 & -2.04421 & $\mathrm{H}$ & 4.52133 & 4.08939 & -0.46802 \\
$\mathrm{C}$ & 1.68055 & -4.05382 & -0.23155 & $\mathrm{H}$ & 4.14823 & 2.36002 & -0.74024 \\
$\mathrm{H}$ & 1.28416 & -4.5651 & 0.67235 & $\mathrm{H}$ & 3.7311 & 3.13043 & 0.82011
\end{tabular}




\begin{tabular}{l|lll|l|lll}
$\mathrm{H}$ & 2.44182 & -3.31964 & 0.10135 & $\mathrm{Os}$ & 1.58283 & 0.01106 & -1.41521 \\
$\mathrm{H}$ & 2.18567 & -4.82429 & -0.84601 & $\mathrm{~N}$ & 2.90027 & 0.55229 & -2.83916 \\
$\mathrm{C}$ & 1.92742 & -4.54363 & -3.82546 & $\mathrm{C}$ & 2.54372 & 1.01854 & -4.06471 \\
$\mathrm{H}$ & 0.91944 & -4.5425 & -4.28733 & $\mathrm{C}$ & 3.46897 & 1.36989 & -5.04324 \\
$\mathrm{H}$ & 1.90879 & -5.21483 & -2.94624 & $\mathrm{H}$ & 1.46058 & 1.10209 & -4.22956 \\
$\mathrm{H}$ & 2.62704 & -4.98968 & -4.56568 & $\mathrm{C}$ & 4.85716 & 1.22612 & -4.75329 \\
$\mathrm{C}$ & 2.53158 & -2.3198 & -4.78169 & $\mathrm{H}$ & 3.11155 & 1.74649 & -6.01019 \\
$\mathrm{H}$ & 3.22686 & -2.85081 & -5.46701 & $\mathrm{C}$ & 5.23917 & 0.75027 & -3.51121 \\
$\mathrm{H}$ & 2.94159 & -1.30967 & -4.60894 & $\mathrm{H}$ & 5.61598 & 1.48813 & -5.50492 \\
$\mathrm{H}$ & 1.55538 & -2.20873 & -5.29243 & $\mathrm{C}$ & 4.27058 & 0.40661 & -2.5206 \\
$\mathrm{C}$ & 3.79053 & -3.18206 & -2.79897 & $\mathrm{H}$ & 6.30323 & 0.62606 & -3.26546 \\
$\mathrm{H}$ & 3.78607 & -3.75578 & -1.85438 & $\mathrm{C}$ & 4.54581 & -0.08261 & -1.21018 \\
$\mathrm{H}$ & 4.51581 & -3.67053 & -3.48515 & $\mathrm{~N}$ & 3.40763 & -0.35892 & -0.43458 \\
$\mathrm{H}$ & 4.16929 & -2.16601 & -2.57761 & $\mathrm{C}$ & 3.57715 & -0.81943 & 0.8244 \\
$\mathrm{P}$ & 1.17118 & 2.26188 & -0.67566 & $\mathrm{C}$ & 4.82316 & -1.05025 & 1.4097 \\
$\mathrm{H}$ & -0.73234 & 3.67989 & -1.70421 & $\mathrm{H}$ & 2.64678 & -1.01029 & 1.3841 \\
$\mathrm{C}$ & 0.45062 & 2.26693 & 1.11655 & $\mathrm{C}$ & 5.98643 & -0.78286 & 0.63501 \\
$\mathrm{C}$ & -0.32892 & 0.94391 & 1.18699 & $\mathrm{H}$ & 4.88334 & -1.42798 & 2.43842 \\
$\mathrm{H}$ & -0.80805 & 0.83057 & 2.18343 & $\mathrm{C}$ & 5.8437 & -0.30386 & -0.65674 \\
$\mathrm{H}$ & 0.35495 & 0.07845 & 1.05511 & $\mathrm{H}$ & 6.98872 & -0.95276 & 1.05552 \\
$\mathrm{H}$ & -1.12315 & 0.88727 & 0.41535 & $\mathrm{H}$ & 6.72973 & -0.08719 & -1.26968
\end{tabular}

Table 7.3: XYZ-coordinates of computed complex 10.

\begin{tabular}{c|lll|l|lll} 
Atom & $\mathrm{x}$ & $\mathrm{y}$ & $\mathrm{z}$ & Atom & $\mathrm{x}$ & $\mathrm{y}$ & $\mathrm{z}$ \\
\hline $\mathrm{C}$ & 3.67567 & 12.2056 & 17.07189 & $\mathrm{C}$ & 4.72961 & 11.70545 & 10.62787 \\
$\mathrm{H}$ & 4.16669 & 13.11147 & 17.48067 & $\mathrm{H}$ & 5.66813 & 11.26421 & 10.22802 \\
$\mathrm{C}$ & 3.41457 & 11.12063 & 17.87501 & $\mathrm{H}$ & 4.91638 & 12.78665 & 10.7826 \\
$\mathrm{H}$ & 3.70878 & 11.12541 & 18.93438 & $\mathrm{H}$ & 3.95807 & 11.58527 & 9.84261 \\
$\mathrm{C}$ & 4.25603 & 8.41827 & 16.95894 & $\mathrm{C}$ & 1.5809 & 12.40904 & 11.61798 \\
$\mathrm{C}$ & 5.48783 & 9.23527 & 16.53131 & $\mathrm{C}$ & 2.07372 & 13.55979 & 10.7229 \\
$\mathrm{H}$ & 6.34252 & 8.54617 & 16.36069 & $\mathrm{H}$ & 2.8759 & 13.2686 & 10.02464 \\
$\mathrm{H}$ & 5.77582 & 9.97111 & 17.30636 & $\mathrm{H}$ & 2.42843 & 14.41835 & 11.32713 \\
$\mathrm{H}$ & 5.28841 & 9.78894 & 15.59381 & $\mathrm{H}$ & 1.21854 & 13.92468 & 10.11346 \\
$\mathrm{C}$ & 4.56497 & 7.76741 & 18.31536 & $\mathrm{C}$ & 1.09732 & 11.22188 & 10.77178 \\
$\mathrm{H}$ & 5.50719 & 7.18439 & 18.22493 & $\mathrm{H}$ & 0.21865 & 11.52767 & 10.16305 \\
$\mathrm{H}$ & 3.77794 & 7.06078 & 18.64173 & $\mathrm{H}$ & 0.78337 & 10.37115 & 11.41093 \\
$\mathrm{H}$ & 4.72277 & 8.52054 & 19.11319 & $\mathrm{H}$ & 1.87264 & 10.86259 & 10.06853 \\
$\mathrm{C}$ & 3.99021 & 7.33067 & 15.90551 & $\mathrm{C}$ & 0.40173 & 12.94938 & 12.4428 \\
$\mathrm{H}$ & 3.84674 & 7.79255 & 14.90967 & $\mathrm{H}$ & -0.32095 & 13.44783 & 11.76156 \\
$\mathrm{H}$ & 3.10097 & 6.71349 & 16.13701 & $\mathrm{H}$ & 0.73529 & 13.69206 & 13.19473 \\
$\mathrm{H}$ & 4.86758 & 6.64978 & 15.84721 & $\mathrm{H}$ & -0.13365 & 12.14011 & 12.96672 \\
$\mathrm{C}$ & 1.40283 & 8.96845 & 18.15634 & $\mathrm{C}$ & 0.26919 & 12.79207 & 15.94472 \\
$\mathrm{C}$ & 1.81297 & 8.9246 & 19.6384 & $\mathrm{H}$ & 1.21247 & 13.28271 & 16.22254 \\
$\mathrm{H}$ & 2.64387 & 8.23099 & 19.84798 & $\mathrm{C}$ & -0.96876 & 13.36951 & 16.20448 \\
$\mathrm{H}$ & 0.94033 & 8.5906 & 20.24127 & $\mathrm{H}$ & -1.01463 & 14.34798 & 16.70302
\end{tabular}




\begin{tabular}{l|lll|l|lll}
$\mathrm{H}$ & 2.09477 & 9.93055 & 20.00709 & $\mathrm{C}$ & -2.14368 & 12.68779 & 15.81952 \\
$\mathrm{C}$ & 0.21017 & 9.93163 & 18.04312 & $\mathrm{H}$ & -3.13706 & 13.11757 & 16.01118 \\
$\mathrm{H}$ & -0.26932 & 9.87922 & 17.05062 & $\mathrm{C}$ & -2.01368 & 11.45176 & 15.1881 \\
$\mathrm{H}$ & 0.51363 & 10.98162 & 18.22793 & $\mathrm{H}$ & -2.90718 & 10.8946 & 14.87556 \\
$\mathrm{H}$ & -0.55432 & 9.65377 & 18.79975 & $\mathrm{C}$ & -0.732 & 10.91222 & 14.94799 \\
$\mathrm{C}$ & 0.96976 & 7.57939 & 17.65997 & $\mathrm{C}$ & -0.48002 & 9.63126 & 14.29925 \\
$\mathrm{H}$ & 0.71363 & 7.59933 & 16.5804 & $\mathrm{C}$ & -1.49811 & 8.76655 & 13.84686 \\
$\mathrm{H}$ & 0.0638 & 7.25168 & 18.21383 & $\mathrm{H}$ & -2.54769 & 9.06171 & 13.98185 \\
$\mathrm{H}$ & 1.74912 & 6.80842 & 17.81992 & $\mathrm{C}$ & -1.1847 & 7.55179 & 13.24083 \\
$\mathrm{C}$ & 3.75925 & 13.284 & 14.97055 & $\mathrm{H}$ & -1.97582 & 6.87489 & 12.88943 \\
$\mathrm{H}$ & 4.2541 & 14.12425 & 15.49788 & $\mathrm{C}$ & 0.18018 & 7.22282 & 13.09431 \\
$\mathrm{C}$ & 3.57369 & 13.3179 & 13.60858 & $\mathrm{H}$ & 0.49602 & 6.28089 & 12.6239 \\
$\mathrm{H}$ & 3.935 & 14.17082 & 13.01678 & $\mathrm{C}$ & 1.1504 & 8.10339 & 13.55324 \\
$\mathrm{C}$ & 4.36349 & 10.98495 & 11.93307 & $\mathrm{H}$ & 2.22008 & 7.88292 & 13.4518 \\
$\mathrm{C}$ & 4.03872 & 9.51094 & 11.6434 & $\mathrm{~N}$ & 3.36816 & 12.2188 & 15.73713 \\
$\mathrm{H}$ & 3.14139 & 9.38255 & 11.008 & $\mathrm{~N}$ & 0.41002 & 11.58759 & 15.32867 \\
$\mathrm{H}$ & 3.87368 & 8.96875 & 12.59411 & $\mathrm{~N}$ & 0.86462 & 9.29968 & 14.15717 \\
$\mathrm{H}$ & 4.89612 & 9.03754 & 11.11746 & $\mathrm{H}$ & 3.54829 & 9.73307 & 14.44161 \\
$\mathrm{C}$ & 5.57931 & 11.04849 & 12.87289 & $\mathrm{P}$ & 2.77662 & 9.67929 & 17.00147 \\
$\mathrm{H}$ & 6.42541 & 10.49133 & 12.41663 & $\mathrm{P}$ & 2.89311 & 11.80612 & 12.90293 \\
$\mathrm{H}$ & 5.34478 & 10.58753 & 13.85069 & $\mathrm{Os}$ & 2.24379 & 10.63407 & 14.88051 \\
$\mathrm{H}$ & 5.90253 & 12.09117 & 13.05456 & & & &
\end{tabular}




\subsubsection{Crystallographic Details}

CCDC-1534960 (1), CCDC-1534959 (2), CCDC-1534961 (3) and CCDC-1534958 (4) contain the supplementary complete crystallographic data for this chapter. This data can be obtained free of charge via http://www.ccdc.cam.ac.uk/ products/csd/request/ 

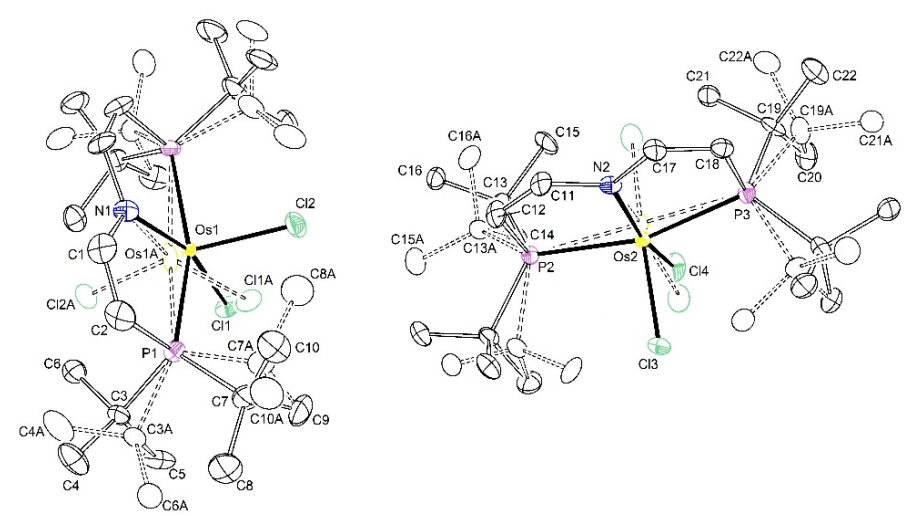

Figure 7.1: Thermal ellipsoid plot of 1 with the anisotropic displacement parameters drawn at the $50 \%$ probability level. The asymmetric unit contains two half complex molecules. Both complex molecules are disordered over two positions. The first disordered complex molecule was refined with population of $0.910(3)$ on the main domain using some restraints (SADI) and constraints (EADP). The second disordered complex molecule was refined with population of $0.502(6)$ on the main domain using some restraints (SADI, RIGU) and constraints (EADP).

Empirical formula
Formula weight
Temperature
Wavelength
Crystal system
Space group
Unit cell dimensions

Volume

Z

Density (calculated)

Absorption coefficient

$\mathrm{F}(000)$

Crystal size

Crystal shape and color

Theta range for data collection

Index ranges

Reflections collected

Independent reflections

Completeness to theta $=25.242^{\circ}$

Max. and min. transmission

Refinement method

Data / restraints / parameters

Goodness-of-fit on $\mathrm{F}^{2}$

Final R indices [I>2sigma(I)]

$\mathrm{R}$ indices (all data)

Largest diff. peak and hole

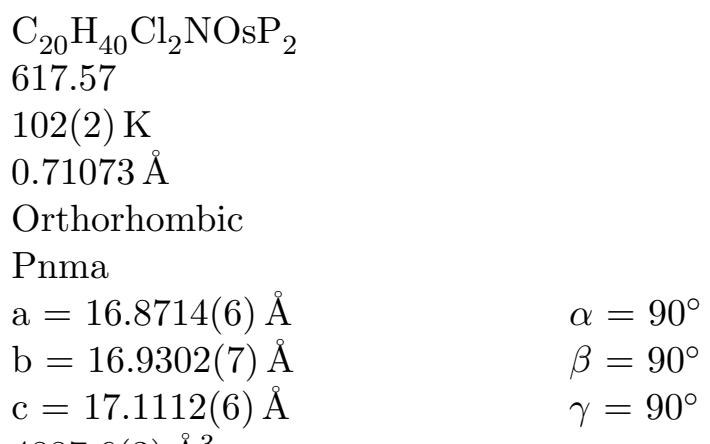

Orthorhombic

Pnma

$\begin{array}{ll}\mathrm{a}=16.8714(6) \AA & \alpha=90^{\circ} \\ \mathrm{b}=16.9302(7) \AA & \beta=90^{\circ} \\ \mathrm{c}=17.1112(6) \AA & \gamma=90^{\circ}\end{array}$

4887.6(3) $\AA^{3}$

8

$$
\begin{aligned}
& 1.679 \mathrm{Mg} / \mathrm{m}^{3} \\
& 5.573 \mathrm{~mm}^{-1} \\
& 2456
\end{aligned}
$$

$0.154 \times 0.097 \times 0.056 \mathrm{~mm}^{3}$

Block, clear intense green

2.079 to $26.731^{\circ}$

$-21<=\mathrm{h}, \mathrm{k}, \mathrm{l}<=21$

70085

$5375[\mathrm{R}(\mathrm{int})=0.0474]$

$99.9 \%$

0.7454 and 0.5878

Full-matrix least-squares on $\mathrm{F}^{2}$

5375 / 64 / 357

1.549

$\mathrm{R} 1=0.0392$,

$\mathrm{R} 1=0.0459$,

1.331 and $-2.360 \mathrm{e}^{-3}$
$\mathrm{wR} 2=0.0761$

$\mathrm{wR} 2=0.0784$ 


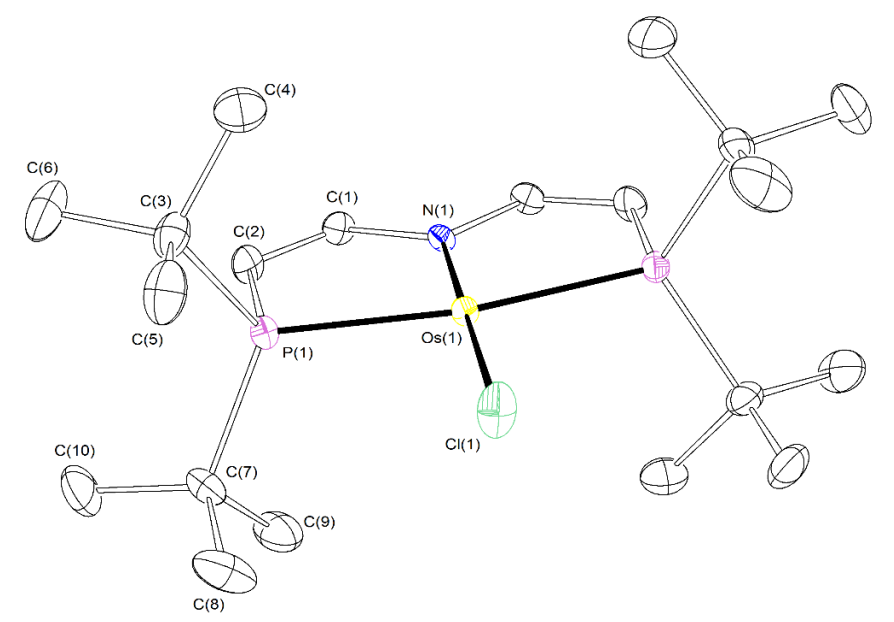

Figure 7.2: Thermal ellipsoid plot of $\mathbf{2}$ with the anisotropic displacement parameters drawn at the $50 \%$ probability level. The asymmetric unit contains a half complex molecule.

Empirical formula

Formula weight

Temperature

Wavelength

Crystal system

Space group

Unit cell dimensions

Volume

Z

Density (calculated)

Absorption coefficient

$\mathrm{F}(000)$

Crystal size

Crystal shape and color

Theta range for data collection

Index ranges

Reflections collected

Independent reflections

Completeness to theta $=25.242^{\circ}$

Max. and min. transmission

Refinement method

Data / restraints / parameters

Goodness-of-fit on $\mathrm{F}^{2}$

Final $\mathrm{R}$ indices $[\mathrm{I}>2 \operatorname{sigma}(\mathrm{I})]$

$\mathrm{R}$ indices (all data)

Largest diff. peak and hole
$\mathrm{C}_{20} \mathrm{H}_{40} \mathrm{ClNOsP}_{2}$

582.12

$112(2) \mathrm{K}$

$0.71073 \AA$

Monoclinic

$\mathrm{P} 2 / \mathrm{c}$

$\mathrm{a}=11.4519(6) \AA$

$\alpha=90^{\circ}$

$\mathrm{b}=8.5831(4) \AA$

$\beta=113.000(2)^{\circ}$

$\mathrm{c}=13.3683(6) \AA$

$1209.55(10) \AA^{3}$

2

$1.598 \mathrm{Mg} / \mathrm{m}^{3}$

$5.518 \mathrm{~mm}^{-1}$

580

$0.187 \times 0.157 \times 0.072 \mathrm{~mm}^{3}$

Block, clear intense purple

2.373 to $28.366^{\circ}$

$-15<=\mathrm{h}<=15$

$-10<=\mathrm{k}<=11$

$-17<=\mathrm{l}<=17$

36752

$3027[\mathrm{R}(\mathrm{int})=0.0469]$

$100.0 \%$

0.7457 and 0.6334

Full-matrix least-squares on $\mathrm{F}^{2}$

$3027 / 0 / 121$

1.122

$\mathrm{R} 1=0.0161$,

$\mathrm{R} 1=0.0269$,

$\mathrm{wR} 2=0.0301$

0.484 and $-0.634 \mathrm{e}^{-3}$ 


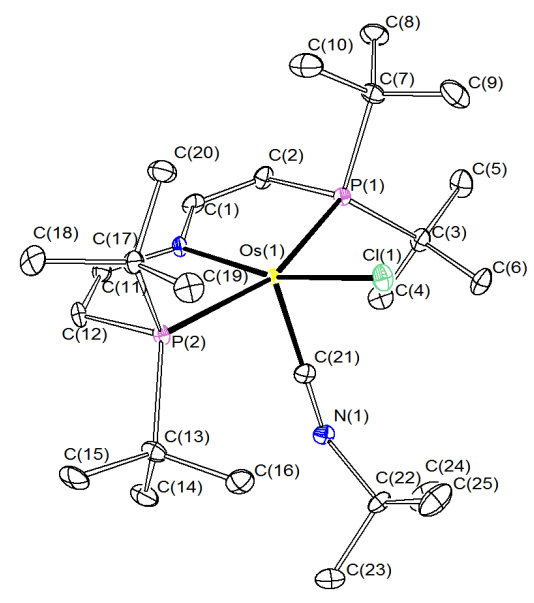

Figure 7.3: Thermal ellipsoid plot of $\mathbf{3}$ with the anisotropic displacement parameters drawn at the $50 \%$ probability level. The structure was refined using two constrains (EADP).

Empirical formula

Formula weight

Temperature

Wavelength

Crystal system

Space group

Unit cell dimensions

Volume

Z

Density (calculated)

Absorption coefficient

$\mathrm{F}(000)$

Crystal size

Crystal shape and color

Theta range for data collection

Index ranges

Reflections collected

Independent reflections

Completeness to theta $=25.242^{\circ}$

Max. and min. transmission

Refinement method

Data / restraints / parameters

Goodness-of-fit on $\mathrm{F}^{2}$

Final $R$ indices [I $>2 \operatorname{sigma}(\mathrm{I})]$

$\mathrm{R}$ indices (all data)

Largest diff. peak and hole
$\mathrm{C}_{25} \mathrm{H}_{49} \mathrm{ClN}_{2} \mathrm{OsP}_{2}$

665.25

100(2) K

$0.71073 \AA$

Monoclinic

$\mathrm{P} 21 / \mathrm{c}$

$\mathrm{a}=15.2391(7) \AA$

$\mathrm{b}=10.9698(5) \AA$

$\mathrm{c}=16.9742(8) \AA$

$2817.4(2) \AA^{3}$

4

$1.568 \mathrm{Mg} / \mathrm{m}^{3}$

$4.750 \mathrm{~mm}^{-1}$

1344

$0.947 \times 0.766 \times 0.556 \mathrm{~mm}^{3}$

Block, clear intense blue

2.215 to $27.197^{\circ}$

$-19<=\mathrm{h}<=19$

$-14<=\mathrm{k}<=14$

$-21<=\mathrm{l}<=21$

76131

$6264[\mathrm{R}(\mathrm{int})=0.0694]$

$100.0 \%$

0.7455 and 0.1448

Full-matrix least-squares on $\mathrm{F}^{2}$

6264 / 0 / 283

1.067

$\mathrm{R} 1=0.0290, \quad \quad \mathrm{wR} 2=0.0755$

$\mathrm{R} 1=0.0349$,

$\mathrm{wR} 2=0.0797$

$$
\begin{aligned}
& \alpha=90^{\circ} \\
& \beta=96.832(2)^{\circ} \\
& \gamma=90^{\circ}
\end{aligned}
$$

1.645 and $-2.020 \mathrm{e}^{-3}$ 


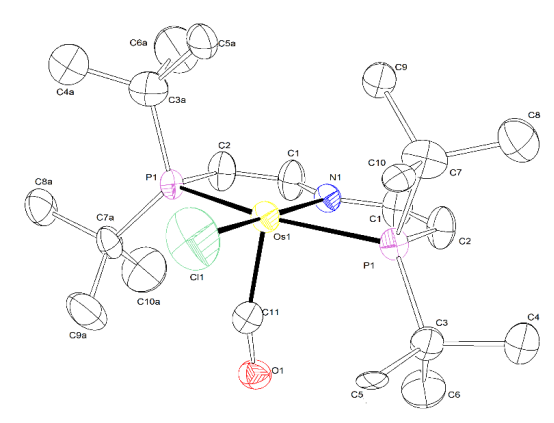

Figure 7.4: Thermal ellipsoid plot of 4 with the anisotropic displacement parameters drawn at the $50 \%$ probability level. The asymmetric unit contains a half disordered complex molecule. The disorder was refined with site occupation factors of 0.25 for both sites using PART commands and some restraints (SADI, RIGU) and constraints (EADP). The structure was refined as an inversion twin using the twin law -100 0-10 00-1 (BASF: 0.50(3)).

Empirical formula

Formula weight

Temperature

Wavelength

Crystal system

Space group

Unit cell dimensions

Volume

Z

Density (calculated)

Absorption coefficient

$\mathrm{F}(000)$

Crystal size

Crystal shape and color

Theta range for data collection

Index ranges

Reflections collected

Independent reflections

Completeness to theta $=25.242^{\circ}$

Max. and min. transmission

Refinement method

Data / restraints / parameters

Goodness-of-fit on $\mathrm{F}^{2}$

Final $R$ indices $[\mathrm{I}>2 \operatorname{sigma}(\mathrm{I})]$

$\mathrm{R}$ indices (all data)

Absolute structure parameter

Largest diff. peak and hole
$\mathrm{C}_{21} \mathrm{H}_{40}$ ClNOOsP $_{2}$

610.13

100(2) K

$0.71073 \AA$

Tetragonal

I4

$\mathrm{a}=12.0393(7) \AA$

$\alpha=90^{\circ}$

$\mathrm{b}=12.0393(7) \AA$

$\beta=90^{\circ}$

$\mathrm{c}=8.5145(5) \AA$

1234.13(16) $\AA^{3}$

2

$1.642 \mathrm{Mg} / \mathrm{m}^{3}$

$5.415 \mathrm{~mm}^{-1}$

608

$0.309 \times 0.180 \times 0.142 \mathrm{~mm}^{3}$

Block, dark brown

2.392 to $33.138^{\circ}$

$-17<=\mathrm{h}<=18$

$-18<=\mathrm{k}<=18$

$-13<=\mathrm{l}<=12$

28153

$2296[\mathrm{R}(\mathrm{int})=0.0545]$

$100.0 \%$

0.7466 and 0.5604

Full-matrix least-squares on $\mathrm{F}^{2}$

2296 / 310 / 201

1.066

$\mathrm{R} 1=0.0355, \quad \mathrm{wR} 2=0.0641$

$\mathrm{R} 1=0.0520, \quad \mathrm{wR} 2=0.0685$

$0.50(3)$

1.354 and $-1.026 \mathrm{e} \AA^{-3}$ 


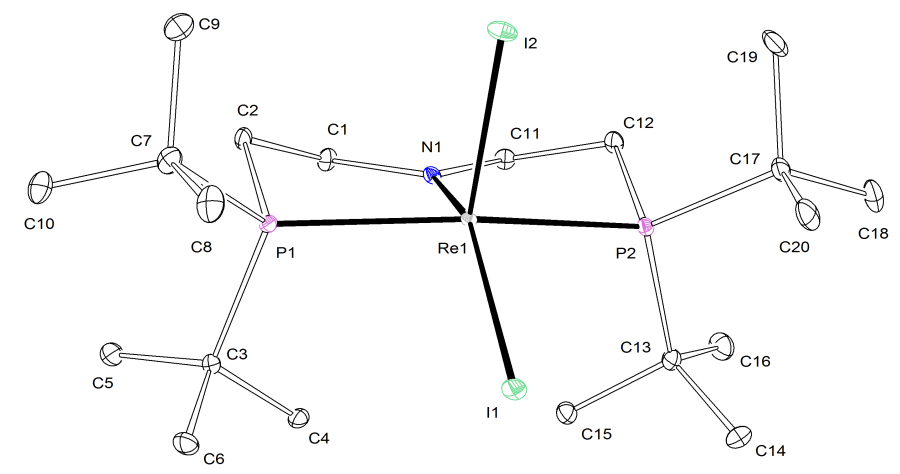

Figure 7.5: Thermal ellipsoid plot of 5 with the anisotropic displacement parameters drawn at the $50 \%$ probability level. The asymmetric unit contains one complex molecule.

Empirical formula
Formula weight
Temperature
Wavelength
Crystal system
Space group
Unit cell dimensions

Volume

Z

Density (calculated)

Absorption coefficient

$\mathrm{F}(000)$

Crystal size

Crystal shape and color

Theta range for data collection

Index ranges

Reflections collected

Independent reflections

Completeness to theta $=25.242^{\circ}$

Refinement method

Data / restraints / parameters

Goodness-of-fit on $\mathrm{F}^{2}$

Final $R$ indices [I $>2 \operatorname{sigma}(\mathrm{I})]$

$\mathrm{R}$ indices (all data)

Largest diff. peak and hole
$\mathrm{C}_{20} \mathrm{H}_{44} \mathrm{I}_{2} \mathrm{NP}_{2} \mathrm{Re}$

800.50

$100(2) \mathrm{K}$

$0.71073 \AA$

Monoclinic

$\mathrm{P} 2{ }_{1} / \mathrm{n}$

$\mathrm{a}=11.9420(6) \AA$

$\alpha=90^{\circ}$

$\mathrm{b}=13.8096(6) \AA$

$\beta=105.337(2)^{\circ}$

$\mathrm{c}=16.7699(7) \AA$

2667.1(2) $\AA^{3}$

4

$1.994 \mathrm{Mg} / \mathrm{m}^{3}$

$6.997 \mathrm{~mm}^{-1}$

1528

$0.163 \times 0.143 \times 0.115 \mathrm{~mm}^{3}$

Block, clear intense red-brown

2.303 to $28.391^{\circ}$

$-15<=\mathrm{h}<=15$

$-18<=\mathrm{k}<=18$

$-22<=\mathrm{l}<=22$

97793

$6666[\mathrm{R}(\mathrm{int})=0.0755]$

$100.0 \%$

Full-matrix least-squares on $\mathrm{F}^{2}$

6666 / 0 / 247

1.053

$\mathrm{R} 1=0.0237$,

$\mathrm{wR} 2=0.0349$

$\mathrm{R} 1=0.0342$,

$\mathrm{wR} 2=0.0367$ 


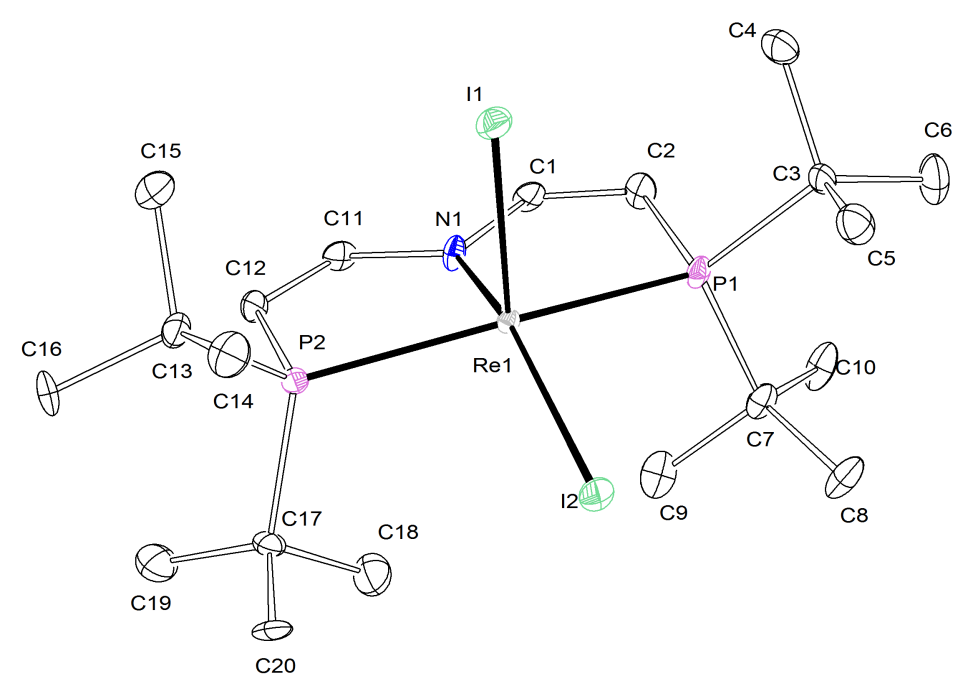

Figure 7.6: Thermal ellipsoid plot of 6 with the anisotropic displacement parameters drawn at the $50 \%$ probability level. The asymmetric unit contains one complex molecule.

Empirical formula

Formula weight

Temperature

Wavelength

Crystal system

Space group

Unit cell dimensions

Volume

Z

Density (calculated)

Absorption coefficient

$\mathrm{F}(000)$

Crystal size

Crystal shape and color

Theta range for data collection

Index ranges

Reflections collected

Independent reflections

Completeness to theta $=25.242^{\circ}$

Refinement method

Data / restraints / parameters

Goodness-of-fit on $\mathrm{F}^{2}$

Final $R$ indices $[\mathrm{I}>2 \operatorname{sigma}(\mathrm{I})]$

$\mathrm{R}$ indices (all data)

Largest diff. peak and hole
$\mathrm{C}_{20} \mathrm{H}_{40} \mathrm{I}_{2} \mathrm{NP}_{2} \mathrm{Re}$

796.47

$100(2) \mathrm{K}$

$0.71073 \AA$

Monoclinic

$\mathrm{P} 21 / \mathrm{n}$

$\mathrm{a}=8.1344(2) \AA$

$\mathrm{b}=26.6172(7) \AA$

$\mathrm{c}=12.6060(4) \AA$

$2589.76(13) \AA^{3}$

4

$2.043 \mathrm{Mg} / \mathrm{m}^{3}$

$7.206 \mathrm{~mm}^{-1}$

1512

$0.333 \times 0.206 \times 0.170 \mathrm{~mm}^{3}$

Block, clear intense brown

2.289 to $30.605^{\circ}$.

$-11<=\mathrm{h}<=11$

$-38<=\mathrm{k}<=37$

$-18<=\mathrm{l}<=17$

103794

$7942[\mathrm{R}(\mathrm{int})=0.1614]$

$100.0 \%$

Full-matrix least-squares on $\mathrm{F}^{2}$

7942 / 0 / 247

1.084

$\mathrm{R} 1=0.0456, \quad \quad \mathrm{wR} 2=0.0531$

$\mathrm{R} 1=0.0786, \quad \mathrm{wR} 2=0.0576$ $\alpha=90^{\circ}$

$\beta=108.4060(10)^{\circ}$

$\gamma=90^{\circ}$

1.188 and $-1.544 \mathrm{e}^{-3}$ 


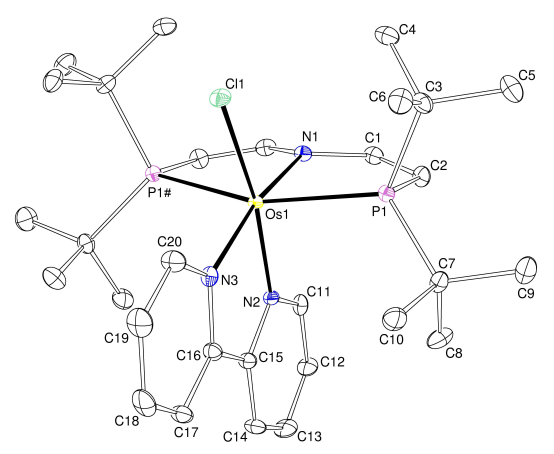

Figure 7.7: Thermal ellipsoid plot of $\mathbf{7}$ with the anisotropic displacement parameters drawn at the $50 \%$ probability level. The asymmetric unit contains only a half complex molecule. The reflections 200 and 040 are removed from the refinement using OMIT commands.

Empirical formula

Formula weight

Temperature

Wavelength

Crystal system

Space group

Unit cell dimensions

Volume

Z

Density (calculated)

Absorption coefficient

$\mathrm{F}(000)$

Crystal size

Crystal shape and color

Theta range for data collection

Index ranges

Reflections collected

Independent reflections

Completeness to theta $=25.242^{\circ}$

Max. and min. transmission

Refinement method

Data / restraints / parameters

Goodness-of-fit on $\mathrm{F}^{2}$

Final $R$ indices $\left[\mathrm{I}_{\iota} 2 \operatorname{sigma}(\mathrm{I})\right]$

$\mathrm{R}$ indices (all data)

Largest diff. peak and hole
$\mathrm{C}_{30} \mathrm{H}_{40} \mathrm{ClN}_{3} \mathrm{OsP}_{2}$

738.30

100(2) K

$0.71073 \AA$

Orthorhombic

Pnma

$\mathrm{a}=14.1295(11) \AA$

$\alpha=90^{\circ}$

$\mathrm{b}=14.0882(11) \AA$

$\beta=90^{\circ}$

$\mathrm{c}=15.2802(11) \AA$

3041.7(4) $\AA^{3}$

4

$1.612 \mathrm{Mg} / \mathrm{m}^{3}$

$4.410 \mathrm{~mm}^{-1}$

1488

$0.488 \times 0.157 \times 0.155 \mathrm{~mm}^{3}$

Block, dark red

1.963 to $30.585^{\circ}$

$-20<=\mathrm{h}<=20$

$-20<=\mathrm{k}<=20$

$-21<=\mathrm{l}<=21$

64224

$4830[\mathrm{R}(\mathrm{int})=0.0743]$

$99.9 \%$

0.7461 and 0.4071

Full-matrix least-squares on $\mathrm{F}^{2}$

4830 / 0 / 196

1.066

$\mathrm{R} 1=0.0191, \mathrm{wR} 2=0.0420$

$\mathrm{R} 1=0.0250, \mathrm{wR} 2=0.0447$

0.981 and $-1.545 \mathrm{e}^{-3}$ 


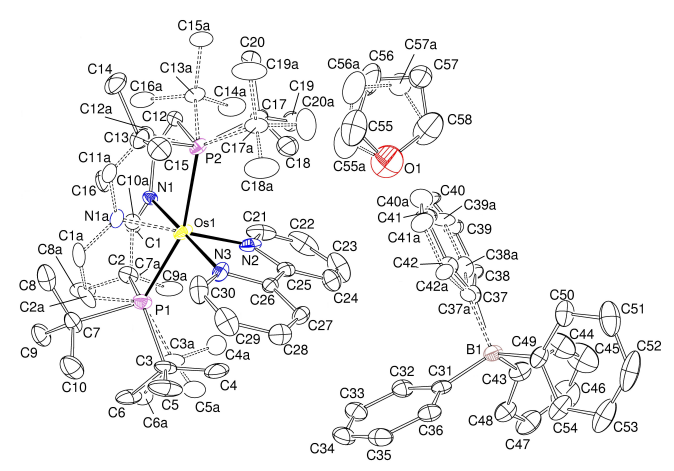

Figure 7.8: Thermal ellipsoid plot of 8 with the anisotropic displacement parameters drawn at the $50 \%$ probability level. The asymmetric unit contains one disordered complex molecule, one disordered THF solvent molecule and one disordered $\mathrm{BPh} 4$ anion. All disorders were refined with site occupation factors of 0.5 for both sites using PART commands and some restraints and constrains (SADI, RIGU, EADP).

Empirical formula

Formula weight

Temperature

Wavelength

Crystal system

Space group

Unit cell dimensions

Volume

Z

Density (calculated)

Absorption coefficient

$\mathrm{F}(000)$

Crystal size

Crystal shape and color

Theta range for data collection

Index ranges

Reflections collected

Independent reflections

Completeness to theta $=25.242^{\circ}$

Max. and min. transmission

Refinement method

Data / restraints / parameters

Goodness-of-fit on $\mathrm{F}^{2}$

Final R indices [I $\_$2sigma(I)]

$\mathrm{R}$ indices (all data)

Largest diff. peak and hole
$\mathrm{C}_{58} \mathrm{H}_{76} \mathrm{BN}_{3} \mathrm{OOsP}_{2}$

1094.16

$100(2) \mathrm{K}$

$0.71073 \AA$

Triclinic

P-1

$\mathrm{a}=11.5963(6) \AA$

$\alpha=93.881(2)^{\circ}$

$\mathrm{b}=13.7010(7) \AA$

$\beta=94.957(2)^{\circ}$

$\mathrm{c}=16.6621(10) \AA$

2614.5(2) $\AA^{3}$

2

$1.390 \mathrm{Mg} / \mathrm{m}^{3}$

$2.542 \mathrm{~mm}^{-1}$

1128

$0.678 \times 0.476 \times 0.220 \mathrm{~mm}^{3}$

Plate, clear pale brown-orange

2.062 to $30.637^{\circ}$

$-16<=\mathrm{h}<=16$

$-19<=\mathrm{k}<=19$

$-23<=\mathrm{l}<=23$

131156

$16066[\mathrm{R}(\mathrm{int})=0.0897]$

$99.9 \%$

0.7461 and 0.5082

Full-matrix least-squares on $\mathrm{F}^{2}$

16066 / 89 / 847

1.049

$\mathrm{R} 1=0.0368$,

$\mathrm{wR} 2=0.0649$

$\mathrm{R} 1=0.0622$,

$\mathrm{wR} 2=0.0736$ 


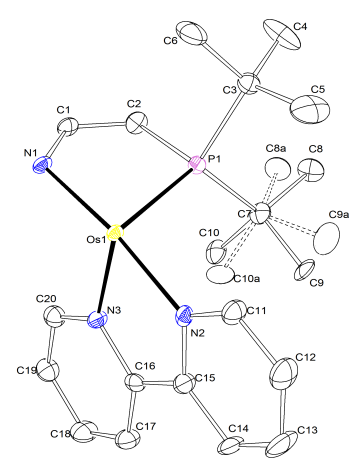

Figure 7.9: Thermal ellipsoid plot of $\mathbf{1 0}$ with the anisotropic displacement parameters drawn at the $50 \%$ probability level. The asymmetric unit contains one disordered complex molecule. The disordered complex molecule was refined with population of 0.60(1) on the main domain using some restraints and constraints (SADI, EADP). The hydride hydrogen atom could not be refined to give a stable solution and was therefore introduced by the DFIX command.

Empirical formula
Formula weight
Temperature
Wavelength
Crystal system
Space group
Unit cell dimensions

Volume

$\mathrm{Z}$

Density (calculated)

Absorption coefficient

$\mathrm{F}(000)$

Crystal size

Crystal shape and color

Theta range for data collection

Index ranges

Reflections collected

Independent reflections

Completeness to theta $=25.242^{\circ}$

Refinement method

Data / restraints / parameters

Goodness-of-fit on $\mathrm{F}^{2}$

Final R indices $\left[\mathrm{I}_{i} 2 \operatorname{sigma}(\mathrm{I})\right]$

$\mathrm{R}$ indices (all data)

Largest diff. peak and hole

$$
\begin{aligned}
& \mathrm{C}_{30} \mathrm{H}_{49} \mathrm{~N}_{3} \mathrm{OsP}_{2} \\
& 703.86 \\
& 100(2) \mathrm{K} \\
& 0.71073 \AA
\end{aligned}
$$

Orthorhombic

Pnma

$$
\begin{array}{ll}
\mathrm{a}=14.5071(16) \AA & \alpha=90^{\circ} \\
\mathrm{b}=13.668(2) \AA & \beta=90^{\circ} \\
\mathrm{c}=15.2847(18) \AA & \gamma=90^{\circ}
\end{array}
$$$$
3030.6(7) \AA^{3}
$$$$
4
$$$$
1.543 \mathrm{Mg} / \mathrm{m}^{3}
$$$$
4.336 \mathrm{~mm}^{-1}
$$$$
1424
$$$$
0.470 \times 0.060 \times 0.050 \mathrm{~mm}^{3}
$$$$
\text { Plate, clear dark green }
$$$$
2.443 \text { to } 26.491^{\circ}
$$$$
-18<=\mathrm{h}<=18
$$$$
-17<=\mathrm{k}<=17
$$$$
-19<=\mathrm{l}<=19
$$

38289

$3212[\mathrm{R}(\mathrm{int})=0.1007]$

$98.2 \%$

Full-matrix least-squares on $\mathrm{F}^{2}$

3212 / 16 / 218

0.793

$$
\begin{array}{ll}
\mathrm{R} 1=0.0271, & \mathrm{wR} 2=0.0663 \\
\mathrm{R} 1=0.0392, & \mathrm{wR} 2=0.0765
\end{array}
$$$$
1.843 \text { and }-1.114 \mathrm{e}^{-3}
$$ 


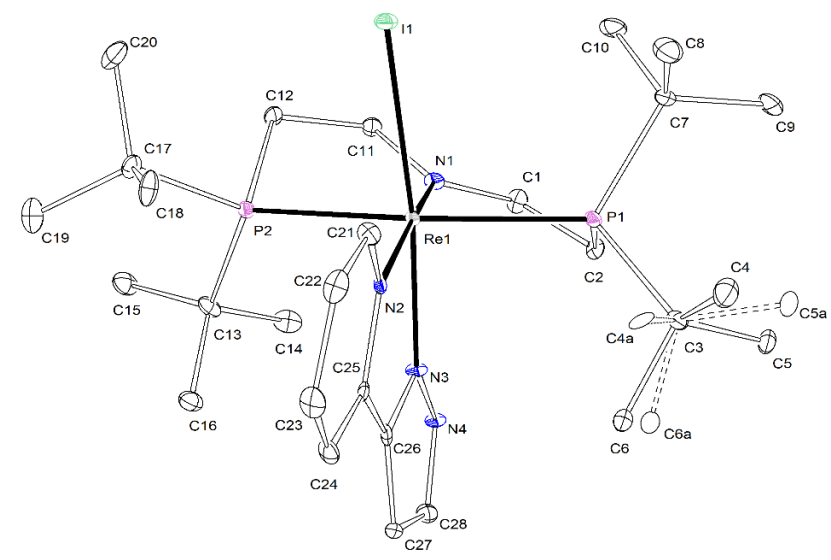

Figure 7.10: Thermal ellipsoid plot of 11 with the anisotropic displacement parameters drawn at the $50 \%$ probability level. The asymmetric unit contains one disordered complex molecule. The disordered complex molecule was refined with population of $0.63(7)$ on the main domain using some restraints and constraints (SADI, EADP).

Empirical formula
Formula weight
Temperature
Wavelength
Crystal system
Space group
Unit cell dimensions

Volume

\section{Z}

Density (calculated)

Absorption coefficient

$\mathrm{F}(000)$

Crystal size

Crystal shape and color

Theta range for data collection

Index ranges

Reflections collected

Independent reflections

Completeness to theta $=25.242^{\circ}$

Refinement method

Data / restraints / parameters

Goodness-of-fit on $\mathrm{F}^{2}$

Final $R$ indices $[\mathrm{I}>2 \operatorname{sigma}(\mathrm{I})]$

$\mathrm{R}$ indices (all data)

Largest diff. peak and hole
$\mathrm{C}_{28} \mathrm{H}_{50} \mathrm{IN}_{4} \mathrm{P}_{2} \mathrm{Re}$

817.76

100(2) K

$0.71073 \AA$

Monoclinic

$\mathrm{P} 21 / \mathrm{c}$

$\mathrm{a}=9.5712(5) \AA$

$\mathrm{b}=15.7700(8) \AA$

$\mathrm{c}=20.5852(10) \AA$

3083.8(3) $\AA^{3}$

4

$1.761 \mathrm{Mg} / \mathrm{m}^{3}$

$5.069 \mathrm{~mm}^{-1}$

1616

$0.242 \times 0.137 \times 0.115 \mathrm{~mm}^{3}$

Block, clear intense red

2.375 to $28.414^{\circ}$

$-12<=\mathrm{h}<=12$

$-21<=\mathrm{k}<=21$

$-26<=\mathrm{l}<=27$

126695

$7724[\mathrm{R}(\mathrm{int})=0.1462]$

$99.9 \%$

Full-matrix least-squares on $\mathrm{F}^{2}$

7724 / 30 / 349

1.070

$\mathrm{R} 1=0.0417$,

$\mathrm{wR} 2=0.0606$

$\mathrm{R} 1=0.0689$,

$\mathrm{wR} 2=0.0659$

$$
\begin{aligned}
& \alpha=90^{\circ} \\
& \beta=97.021(2)^{\circ} \\
& \gamma=90^{\circ}
\end{aligned}
$$

2.042 and $-1.126 \mathrm{eA}^{-3}$ 


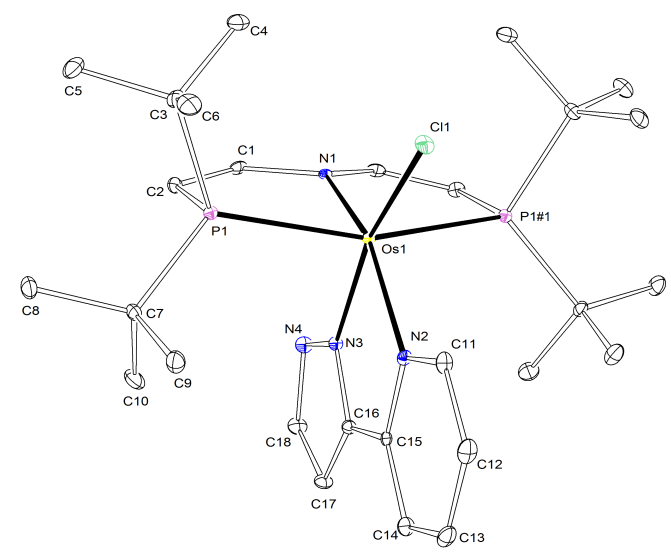

Figure 7.11: Thermal ellipsoid plot of $\mathbf{1 2}$ with the anisotropic displacement parameters drawn at the $50 \%$ probability level. The asymmetric unit contains only a half complex molecule.

Empirical formula

Formula weight

Temperature

Wavelength

Crystal system

Space group

Unit cell dimensions

Volume

Z

Density (calculated)

Absorption coefficient

$\mathrm{F}(000)$

Crystal size

Crystal shape and color

Theta range for data collection

Index ranges

Reflections collected

Independent reflections

Completeness to theta $=25.242^{\circ}$

Refinement method

Data / restraints / parameters

Goodness-of-fit on $\mathrm{F}^{2}$

Final R indices [I $>2 \operatorname{sigma}(\mathrm{I})]$

$\mathrm{R}$ indices (all data)

Largest diff. peak and hole
$\mathrm{C}_{28} \mathrm{H}_{46} \mathrm{ClN}_{4} \mathrm{OsP}_{2}$

726.28

$102(2) \mathrm{K}$

$0.71073 \AA$

Orthorhombic

Pnma

$\mathrm{a}=12.6673(4) \AA$

$\alpha=90^{\circ}$

$\mathrm{b}=13.6362(4) \AA$

$\beta=90^{\circ}$

$\mathrm{c}=17.3873(5) \AA$

$\gamma=90^{\circ}$
$3003.38(16) \AA^{3}$

4

$1.606 \mathrm{Mg} / \mathrm{m}^{3}$

$4.465 \mathrm{~mm}^{-1}$

1460

$0.112 \times 0.062 \times 0.048 \mathrm{~mm}^{3}$

Needle, clear intense red-brown

2.343 to $28.326^{\circ}$

$-16<=\mathrm{h}<=16$

$-16<=\mathrm{k}<=18$

$-23<=\mathrm{l}<=21$

28147

$3876[\mathrm{R}(\mathrm{int})=0.0642]$

$99.9 \%$

Full-matrix least-squares on $\mathrm{F}^{2}$

3876 / 0 / 190

1.039

$\mathrm{R} 1=0.0240$,

$\mathrm{R} 1=0.0369$,

0.573 and $-1.272 \mathrm{e}^{-3}$
$\mathrm{wR} 2=0.0394$

$\mathrm{wR} 2=0.0423$ 


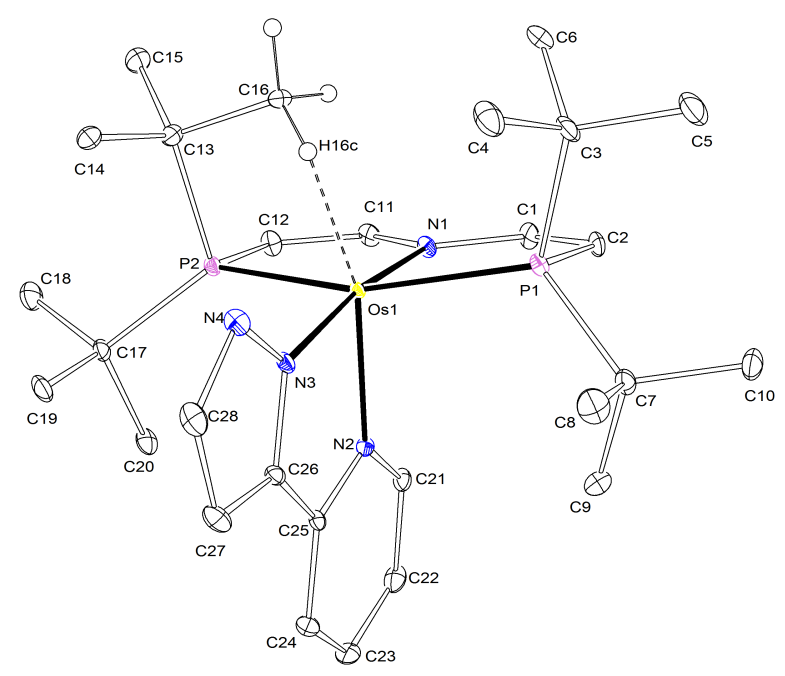

Figure 7.12: Thermal ellipsoid plot of 13 with the anisotropic displacement parameters drawn at the $50 \%$ probability level. The asymmetric unit contains one complex molecule.

Empirical formula

Formula weight

Temperature

Wavelength

Crystal system

Space group

Unit cell dimensions

Volume

Z

Density (calculated)

Absorption coefficient

$\mathrm{F}(000)$

Crystal size

Crystal shape and color

Theta range for data collection

Index ranges

Reflections collected

Independent reflections

Completeness to theta $=25.242^{\circ}$

Refinement method

Data / restraints / parameters

Goodness-of-fit on $\mathrm{F}^{2}$

Final R indices [I $>2 \operatorname{sigma}(\mathrm{I})]$

$\mathrm{R}$ indices (all data)

Largest diff. peak and hole
$\mathrm{C}_{28} \mathrm{H}_{46} \mathrm{~N}_{4} \mathrm{OsP}_{2}$

690.83

102(2) K

$0.71073 \AA$

Monoclinic

$\mathrm{P} 21 / \mathrm{c}$

$\mathrm{a}=16.6529(8) \AA$

$\alpha=90^{\circ}$

$\mathrm{b}=10.2535(4) \AA$

$\beta=91.657(2)^{\circ}$

$\mathrm{c}=17.6988(8) \AA$

$3020.8(2) \AA^{3}$

4

$1.519 \mathrm{Mg} / \mathrm{m}^{3}$

$4.350 \mathrm{~mm}^{-1}$

1392

$0.353 \times 0.191 \times 0.184 \mathrm{~mm}^{3}$

Block, clear intense red

2.296 to $28.396^{\circ}$

$-22<=\mathrm{h}<=22$

$-13<=\mathrm{k}<=13$

$-23<=\mathrm{l}<=23$

102457

$7568[\mathrm{R}(\mathrm{int})=0.1330]$

$100.0 \%$

Full-matrix least-squares on $\mathrm{F}^{2}$

7568 / 0 / 328

1.050

$\mathrm{R} 1=0.0334$,

$\mathrm{wR} 2=0.0554$

$\mathrm{R} 1=0.0573$,

$\mathrm{wR} 2=0.0611$ 


\subsubsection{Syntheses}

\section{Synthesis of LXXV}

$\left[\mathrm{OsCl}_{2}\left(\mathrm{PPh}_{3}\right)_{3}\right]$ (800 mg, $760 \mu \mathrm{mol}, 1.00$ eq.) is dissolved in $\mathrm{C}_{6} \mathrm{H}_{6} /$ pentane $(5: 1,30 \mathrm{~mL})$ and ${ }^{H} \mathrm{PNP}\left(280 \mathrm{mg}, 760 \mu \mathrm{mol}, 1.00\right.$ eq.) in $\mathrm{C}_{6} \mathrm{H}_{6}(5 \mathrm{~mL})$ is added to the solution. The mixture is stirred for $45 \mathrm{~min}$ at $\mathrm{RT}$. The solvent is removed and the solid is washed with pentane at $0^{\circ} \mathrm{C}(4 \times 10 \mathrm{~mL})$. DCM $(15 \mathrm{~mL})$ is added to the solid and the solution is heated to $40^{\circ} \mathrm{C}$ for $16 \mathrm{~h}$. The solvent is removed and the crude product is purified by washing with $\mathrm{Et}_{2} \mathrm{O}(4 \times 10 \mathrm{~mL})$ and $\mathrm{EtOH}(4 \times 5 \mathrm{~mL})$. The solid is extracted with DCM $(5 \times 2 \mathrm{~mL})$ and the solvent is removed. LXXV $(358 \mathrm{mg}, 570 \mu \mathrm{mol}, 75 \%)$ is isolated in form of a green solid. Spectroscopic characterization data was identical with the prior reported values. ${ }^{[83]}$

\section{Synthesis of 1}

LXXV (160 mg, $260 \mu \mathrm{mol}, 1.00$ eq.) and TBP (370 mg, $1.41 \mathrm{mmol}, 7.00$ eq.) are dissolved in $\mathrm{PhCl}(20 \mathrm{~mL})$ and stirred at $50^{\circ} \mathrm{C}$ for $4.5 \mathrm{~h}$. The solvent is removed and the residue is washed with pentanes $(8 \times 10 \mathrm{~mL})$ and extracted with benzene $(5 \times 5 \mathrm{~mL})$. Lyophilization yields 1 as a dark green powder $(149 \mathrm{mg}, 240 \mu \mathrm{mol}, 95 \%)$. Anal. Calcd for $\mathrm{C}_{20} \mathrm{H}_{40} \mathrm{Cl}_{2} \mathrm{~N}_{1} \mathrm{Os}_{1} \mathrm{P}_{2}$ (617.62): C, 38.9; H, 6.53; N, 2.27 Found: C, 39.4; H, 6.36; $\mathrm{N}, 2.15$. NMR $\left(\mathrm{C}_{6} \mathrm{D}_{6}:{ }^{1} \mathrm{H}(300 \mathrm{MHz}, \mathrm{RT}): \delta=43.9(\mathrm{br}, 2 \mathrm{H}, \mathrm{CH}),-0.06(\mathrm{br}, 36 \mathrm{H}\right.$, $\mathrm{P}\left(\mathrm{C}\left(\mathrm{CH}_{3}\right)_{2}\right),-97.7$ (br, 2H, $\left.\mathrm{CH}\right)$. MS (LIFDI, toluene): $m / z=618.0\left(100 \%,\left[\mathrm{M}^{+}\right]\right)$. $\mu_{\text {eff }} 297 \mathrm{~K}=1.65 \mu_{\mathrm{B}}$.

\section{Synthesis of 2}

1 (20.0 mg, $32.4 \mu \mathrm{mol}, 1.00$ eq.) and $\mathrm{CoCp}_{2}(6.1 \mathrm{mg}, 32 \mu \mathrm{mol}, 1.0$ eq.) are dissolved in THF $(2 \mathrm{~mL})$ at $-35^{\circ} \mathrm{C}$ and stirred for $1 \mathrm{~min}$. The solvent is removed and the residue is extracted with pentane. Crystallization at $-35^{\circ} \mathrm{C}$ yields $\mathbf{2}$ in form of dark purple crystals which are suitable for SQUID measurements (10.0 mg, $17.2 \mu \mathrm{mol}, 53 \%)$. Combustion analysis could not be obtained due to decomposition during drying in vacuo, presumably from loss of isobutene. However, SQUID data was reproduced with 3 independent samples. NMR $\left(\mathrm{C}_{6} \mathrm{D}_{6}\right):{ }^{1} \mathrm{H}(300 \mathrm{MHz}, \mathrm{RT}): \delta=18.42\left(\mathrm{ABXX}{ }^{\prime} \mathrm{B}^{\prime} \mathrm{A}^{\prime}, N=\left|{ }^{3} J_{\mathrm{AX}}+{ }^{4} J_{\mathrm{AX}}\right|\right.$ $\left.=17.0 \mathrm{~Hz},{ }^{3} J_{\mathrm{AB}}=6.0 \mathrm{~Hz} .2 \mathrm{H}, \mathrm{NCH}\right),-4.20\left(\mathrm{~A}_{18} \mathrm{XX}^{\prime} \mathrm{A}^{\prime}{ }_{18}, N=\left|{ }^{3} J_{\mathrm{AX}}+{ }^{5} J_{\mathrm{AX}}\right|=\right.$ $6.0 \mathrm{~Hz}, 36 \mathrm{H}, \mathrm{P}\left(\mathrm{C}\left(\mathrm{CH}_{3}\right)_{2}\right),-35.28\left(\mathrm{~d},{ }^{3} J_{\mathrm{AB}}=6.1 \mathrm{~Hz}, 2 \mathrm{H}, \mathrm{PCH}\right) .{ }^{13} \mathrm{C}(125.76 \mathrm{MHz}): \delta$ $=262.4\left(\mathrm{~d},{ }^{1} J_{\mathrm{CH}}=165.1 \mathrm{~Hz}, 2 \mathrm{C}, \mathrm{PCH}\right), 77.1\left(\mathrm{q},{ }^{1} J_{\mathrm{CH}}=125.5,12 \mathrm{C}, \mathrm{P}\left(\mathrm{C}\left(C_{3}\right)_{3}\right)_{2}\right)$, $34.3\left(\mathrm{br}, 4 \mathrm{C}, \mathrm{P}\left(C\left(\mathrm{CH}_{3}\right)_{3}\right)_{2}\right), 31.4\left(\mathrm{~d},{ }^{1} J_{\mathrm{CH}}=162.3 \mathrm{~Hz}, 2 \mathrm{C}, \mathrm{NCH}\right) \cdot{ }^{31} \mathrm{P}\left\{{ }^{1} \mathrm{H}\right\}(161.25$ $\mathrm{MHz}): \delta=-978.2\left(\mathrm{~s}, 2 \mathrm{P}, P\left(\mathrm{C}\left(\mathrm{CH}_{3}\right)_{3}\right)_{2}\right)$. MS (LIFDI, toluene): $m / z=583.1(100 \%$, $\left.\left[\mathrm{M}^{+}\right]\right)$.

\section{Synthesis of 3}

1 (25.0 mg, $40.5 \mu \mathrm{mol}, 1.00$ eq.) and $\mathrm{CoCp}_{2}(7.7 \mathrm{mg}, 42 \mu \mathrm{mol}, 1.0$ eq.) are dissolved in benzene $(5 \mathrm{~mL})$ and stirred for $1 \mathrm{~min}$. $\mathrm{CN}^{t} \mathrm{Bu}(4.6 \mu \mathrm{L}, 41 \mu \mathrm{mol}, 1.0$ eq. $)$ is added and the solution is stirred for an additional minute. After filtration the crude product is purified via column chromatography with silanized silica (benzene). Lyophilization yields $\mathbf{3}$ as a purple powder (18.3 mg, $27.5 \mu \mathrm{mol}, 68 \%$ ). Anal. Calcd for $\mathrm{C}_{25} \mathrm{H}_{49} \mathrm{Cl}_{1} \mathrm{~N}_{2} \mathrm{Os}_{1} \mathrm{P}_{2}$ (665.30): 
C, 45.1; H, 7.42; N, 4.21 Found: C, 45.4; H, 8.02; N, 4.10. NMR $\left(\mathrm{C}_{6} \mathrm{D}_{6}, \mathrm{RT}\right):{ }^{1} \mathrm{H}(400$ MHz): $\delta=7.04\left(\mathrm{ABXX}^{\prime} \mathrm{B}^{\prime} \mathrm{A},, N=\left|{ }^{3} J_{\mathrm{AX}}+{ }^{4} J_{\mathrm{AX}}\right|=17.0 \mathrm{~Hz},{ }^{3} J_{\mathrm{AB}}=5.9 \mathrm{~Hz} .2 \mathrm{H}\right.$, $\mathrm{NCH}$ ) 4.33 (ABXX'B'A', $N=\left|{ }^{2} J_{\mathrm{AX}}+{ }^{4} J_{\mathrm{AX}}{ }^{\prime}\right|=2.6 \mathrm{~Hz},{ }^{3} J_{\mathrm{AB}}=5.9 \mathrm{~Hz}, 2 \mathrm{H}, \mathrm{PC} H$ ), $1.51\left(\mathrm{~A}_{9} \mathrm{XX}^{\prime} \mathrm{A}_{9}{ }_{9}, N=\left|{ }^{3} J_{\mathrm{AX}}+{ }^{5} J_{\mathrm{AX}}\right|=6.6 \mathrm{~Hz}, 18 \mathrm{H}, \mathrm{P}\left(\mathrm{C}\left(\mathrm{CH}_{3}\right)_{3}\right)\right), 1.22\left(\mathrm{~A}_{9} \mathrm{XX}^{\prime}{ }^{\prime}{ }_{9}, N\right.$ $\left.=\left|{ }^{3} J_{\mathrm{AX}}+{ }^{5} J_{\mathrm{AX}}\right|=6.6 \mathrm{~Hz}, 18 \mathrm{H}, \mathrm{P}\left(\mathrm{C}\left(\mathrm{C} H_{3}\right)_{3}\right)\right), 1.19\left(\mathrm{~s}, 9 \mathrm{H}, \mathrm{CN}\left(\mathrm{C}\left(\mathrm{C} H_{3}\right)_{3}\right)\right) .{ }^{13} \mathrm{C}\left\{{ }^{1} \mathrm{H}\right\}$ $(125.76 \mathrm{MHz}): \delta=166.8\left(\mathrm{AXX}^{\prime} \mathrm{A}^{\prime}, N=\left.\right|^{2} J_{\mathrm{AX}}+{ }^{3} J_{\mathrm{AX}} \mid=3.6 \mathrm{~Hz}, 2 \mathrm{C}, \mathrm{N} C \mathrm{H}\right), 89.2$ (AXX'A', $\left.N=\left|{ }^{1} J_{\mathrm{AX}}+{ }^{3} J_{\mathrm{AX}}\right|=23.5 \mathrm{~Hz}, 2 \mathrm{C}, \mathrm{PCH}\right), 58.0\left(\mathrm{~s}, 1 \mathrm{C}, \mathrm{CN}\left(C\left(\mathrm{CH}_{3}\right)_{3}\right), 39.3\right.$ (AXX'A', $N=\left|{ }^{1} J_{\mathrm{AX}}+{ }^{3} J_{\mathrm{AX}}\right|=11.2 \mathrm{~Hz}, 2 \mathrm{C}, \mathrm{P}\left(C\left(\mathrm{CH}_{3}\right)_{3}\right), 36.3$ (AXX'A', $N=\mid{ }^{1} J_{\mathrm{AX}}$ $+{ }^{3} J_{\mathrm{AX}} \mid=11.8 \mathrm{~Hz}, 2 \mathrm{C}, \mathrm{P}\left(C\left(\mathrm{CH}_{3}\right)_{3}\right), 33.3\left(\mathrm{~s}, 3 \mathrm{C}, \mathrm{CN}\left(\mathrm{CH}_{3}\right)_{3}\right), 30.7\left(\mathrm{~A}_{3} \mathrm{XX}^{\prime} \mathrm{A}^{\prime}{ }_{3}, N=\right.$ $\left|{ }^{2} J_{\mathrm{AX}}+{ }^{4} J_{\mathrm{AX}}\right|=2.7 \mathrm{~Hz}, 6 \mathrm{C}, \mathrm{P}\left(\mathrm{C}\left(C \mathrm{H}_{3}\right)_{3}\right), 28.9\left(\mathrm{~A}_{3} \mathrm{XX}^{\prime}{ }^{\prime}{ }_{3}, N=\left|{ }^{2} J_{\mathrm{AX}}+{ }^{4} J_{\mathrm{AX}}\right|=\right.$ $2.7 \mathrm{~Hz}, 6 \mathrm{C}, \mathrm{P}\left(\mathrm{C}\left(\mathrm{CH}_{3}\right)_{3}\right)$. The isonitrile $C N$ resonance was not detected. ${ }^{31} \mathrm{P}\left\{{ }^{1} \mathrm{H}\right\}(161.25$ $\mathrm{MHz}): \delta=55.6\left(\mathrm{~s}, 2 \mathrm{P}, P\left(\mathrm{C}\left(\mathrm{CH}_{3}\right)_{3}\right)_{2}\right)$. MS (LIFDI, toluene): $\mathrm{m} / z=666.2\left(100 \%,\left[\mathrm{M}^{+}\right]\right)$. $\operatorname{IR}(\mathrm{KBr}): \tilde{\nu}=1931(\mathrm{C}=\mathrm{N})$.

\section{Synthesis of 4}

1 (15.0 mg, $24.3 \mu \mathrm{mol}, 1.00$ eq.) and $\mathrm{CoCp}_{2}(4.6 \mathrm{mg}, 24 \mu \mathrm{mol}, 1.0$ eq.) are dissolved in THF $(10 \mathrm{~mL})$ in a $50 \mathrm{~mL}$ J-Young flask and stirred for $1 \mathrm{~min}$ at room temperature. The solution is degassed with three successive freeze-pump-thaw cycles and allowed to warm to room temperature. Upon melting, CO $(600 \mu \mathrm{L}, 1$ bar, $24.3 \mu \mathrm{mol}, 1.00$ eq. $)$ is slowly bubbled through the solution. The solution is stirred for $10 \mathrm{~min}$ at room temperature. After removal of the solvent the crude product is washed with pentanes $(1 \times 1 \mathrm{~mL})$ and extracted with benzene $(3 \times 0.5 \mathrm{~mL})$. The solution is concentrated and the product is further purified via column chromatography with silanized silica (benzene). The solvent is removed and the product is crystallized from $\mathrm{Et}_{2} \mathrm{O}$ at $-35^{\circ} \mathrm{C}$. The crystals are washed with pentanes and dried. Lyophilization yields $4(9.5 \mathrm{mg}, 16 \mu \mathrm{mol}, 64 \%)$ as a purple powder. Anal. Calcd for $\mathrm{C}_{21} \mathrm{H}_{40} \mathrm{~N}_{1} \mathrm{O}_{1} \mathrm{OsP}_{2}$ (610.18): C, 41.3; H, 6.61; N, 2.30; Found: C, 41.0; H, 6.21; N, 2.12. NMR $\left(\mathrm{C}_{6} \mathrm{D}_{6}\right):{ }^{1} \mathrm{H}(400 \mathrm{MHz}): \delta=6.92\left(\right.$ ABXX'B'A' $^{\prime}, N=\left.\right|^{3} J_{\mathrm{AX}}$ $\left.+{ }^{4} J_{\mathrm{AX}} \mid=20.1 \mathrm{~Hz},{ }^{3} J_{\mathrm{AB}}=5.8 \mathrm{~Hz}, 2 \mathrm{H}, \mathrm{NCH}\right), 4.30\left(\mathrm{ABXX}^{\prime} \mathrm{B}^{\prime} \mathrm{A}^{\prime}, N=\left|{ }^{2} J_{\mathrm{AX}}+{ }^{4} J_{\mathrm{AX}}{ }^{\prime}\right|\right.$ $\left.=2.8 \mathrm{~Hz},{ }^{3} J_{\mathrm{AB}}=5.8 \mathrm{~Hz}, 2 \mathrm{H}, \mathrm{PC} H\right), 1.41\left(\mathrm{~A}_{9} \mathrm{XX}^{\prime} \mathrm{A}^{\prime}{ }_{9}, N=\left|{ }^{3} J_{\mathrm{AX}}+{ }^{5} J_{\mathrm{AX}}\right|=7.3 \mathrm{~Hz}\right.$, $\left.18 \mathrm{H}, \mathrm{P}\left(\mathrm{C}\left(\mathrm{CH}_{3}\right)_{3}\right)\right), 1.16\left(\mathrm{~A}_{9} \mathrm{XX}^{\prime} \mathrm{A}_{9}^{\prime}, N=\left|{ }^{3} J_{\mathrm{AX}}+{ }^{5} J_{\mathrm{AX}}\right|=6.8 \mathrm{~Hz}, 18 \mathrm{H}, \mathrm{P}\left(\mathrm{C}\left(\mathrm{CH}_{3}\right)_{3}\right)\right)$. ${ }^{13} \mathrm{C}\left\{{ }^{1} \mathrm{H}\right\}(125.76 \mathrm{MHz}): \delta=168.4\left(\mathrm{t},{ }^{2} J_{\mathrm{CP}}=7.9 \mathrm{~Hz}, 1 \mathrm{C}, C \mathrm{O}\right), 166.8\left(\mathrm{AXX}^{\prime}{ }^{\prime}, N=\right.$ $\left.\left.\right|^{2} J_{\mathrm{AX}}+{ }^{3} J_{\mathrm{AX}} \mid=6.6 \mathrm{~Hz}, 2 \mathrm{C}, \mathrm{NCH}\right), 89.7$ (AXX'A', $N=\left|{ }^{1} J_{\mathrm{AX}}+{ }^{3} J_{\mathrm{AX}}\right|=21.7 \mathrm{~Hz}, 2 \mathrm{C}$, PCH ), 39.2 (AXX'A', $N=\left|{ }^{1} J_{\mathrm{AX}}+{ }^{3} J_{\mathrm{AX}}\right|=11.8 \mathrm{~Hz}, 2 \mathrm{C}, \mathrm{P}\left(C\left(\mathrm{CH}_{3}\right)_{3}\right), 37.0\left(\mathrm{AXX}^{\prime} \mathrm{A}^{\prime}\right.$, $N=\left|{ }^{1} J_{\mathrm{AX}}+{ }^{3} J_{\mathrm{AX}}\right|=12.4 \mathrm{~Hz}, 2 \mathrm{C}, \mathrm{P}\left(C\left(\mathrm{CH}_{3}\right)_{3}\right), 30.0\left(\mathrm{~A}_{3} \mathrm{XXX}^{\prime}{ }^{\prime}{ }_{3}, N=\left.\right|^{2} J_{\mathrm{AX}}+\right.$ ${ }^{4} J_{\mathrm{AX}}{ }^{\prime} \mid=2.3 \mathrm{~Hz}, 6 \mathrm{C}, \mathrm{P}\left(\mathrm{C}\left(\mathrm{CH}_{3}\right)_{3}\right), 28.6\left(\mathrm{~A}_{3} \mathrm{XX}^{\prime} \mathrm{A}^{\prime}{ }_{3}, N=\left|{ }^{2} J_{\mathrm{AX}}+{ }^{4} J_{\mathrm{AX}}\right|=2.7 \mathrm{~Hz}, 6 \mathrm{C}\right.$, $\mathrm{P}\left(\mathrm{C}\left(\mathrm{CH}_{3}\right)_{3}\right) .{ }^{31} \mathrm{P}\left\{{ }^{1} \mathrm{H}\right\}(161.25 \mathrm{MHz}): \delta=65.1\left(\mathrm{~s}, 2 \mathrm{P}, P\left(\mathrm{C}\left(\mathrm{CH}_{3}\right)_{3}\right)_{2}\right) . \mathrm{IR}(\mathrm{Nujol}): \tilde{\nu}=$ $1893(\mathrm{C}=\mathrm{O})$.

\section{Synthesis of 5}

LXXIII (250 mg, $405 \mu \mathrm{mol}, 1.00$ eq.) and $\mathrm{NaI}$ (1.25 g, $8.34 \mathrm{mmol}, 20.6$ eq.) are dissolved in THF and stirred for 90 minutes at room temperature under the exclusion of light. The solvent is evaporated and the residue is extracted with benzene $(6 \times 15 \mathrm{~mL})$. After removal of the solvent the residue is again extracted with benzene $(3 \times 10 \mathrm{~mL})$. Lyophilization yields $\mathbf{5}(295 \mathrm{mg}, 369 \mu \mathrm{mol}, 91 \%)$ in form of a green-brown powder. The product is sufficiently pure for further synthesis. A sample suitable for elemental anal- 
ysis can be obtained by filtration of a benzene solution through a silica plug which is accompanied by decrease of the yield of $30 \%$.

Anal. Calcd for $\mathrm{C}_{20} \mathrm{H}_{44} \mathrm{~N}_{1} \mathrm{I}_{2} \mathrm{P}_{2} \mathrm{Re}_{1}$ (800.5): C, 30.0; H, 5.54; N, 1.75. Found: C, 30.55; $\mathrm{H}, 5.44 ; \mathrm{N}, 1.69$. NMR $\left(\mathrm{C}_{6} \mathrm{D}_{6}, \mathrm{RT}\right):{ }^{1} \mathrm{H}(500 \mathrm{MHz}): \delta=3.31-3.25\left(\mathrm{~m}, 4 \mathrm{H}, \mathrm{PCH} H_{2}\right)$, $2.24\left(36 \mathrm{H}, \mathrm{A}_{18} \mathrm{XX}^{\prime} \mathrm{A}^{\prime}{ }_{18}, N=\left|{ }^{3} J_{\mathrm{AX}}+{ }^{5} J_{\mathrm{AX}}{ }^{\prime}\right|=11.9 \mathrm{~Hz}\right),-0.65--0.71\left(\mathrm{~m}, 4 \mathrm{H}, \mathrm{NCH}_{2}\right)$. ${ }^{13} \mathrm{C}\left\{{ }^{1} \mathrm{H}\right\}(125.8 \mathrm{MHz}): \delta=127.8$ (superimposed with solvent, $2 \mathrm{C}, \mathrm{NCH}_{2}$ ), 69.3 (vt, $N$ $\left.=\left|{ }^{1} J_{\mathrm{CP}}+{ }^{3} J_{\mathrm{CP}}\right|=7.9 \mathrm{~Hz}, 4 \mathrm{C}, \mathrm{P}\left(C\left(\mathrm{CH}_{3}\right)_{3}\right)_{2}\right), 49.1\left(\mathrm{vt},{ }^{1} J_{\mathrm{PC}}=7.0 \mathrm{~Hz}, 2 \mathrm{C}, \mathrm{PCH}_{2}\right)$, $36.0\left(\mathrm{~s} \mathrm{br}, 12 \mathrm{C}, \mathrm{P}\left(\mathrm{C}\left(\mathrm{CH}_{3}\right)_{3}\right)_{2}\right) .{ }^{31} \mathrm{P}\left\{{ }^{1} \mathrm{H}\right\}(202.5 \mathrm{MHz}): \delta=-164.2\left(\mathrm{~s}, 2 \mathrm{P}, P\left(\mathrm{C}\left(\mathrm{CH}_{3}\right)_{3}\right)_{2}\right.$. LIFDI-MS (Toluene): $801.1\left(100 \%,[\mathrm{M}]^{+}\right)$.

\section{Synthesis of 6}

5 (50.0 mg, $62.5 \mu \mathrm{mol}, 100$ eq.) and $\mathrm{Mes}^{*} \mathrm{O}$ (130 mg, $500 \mu \mathrm{mol}, 8.00$ eq.) are dissolved in benzene $(10 \mathrm{~mL})$ and stirred at $50^{\circ} \mathrm{C}$ for $16 \mathrm{~h}$. The solution is concentrated $(3 \mathrm{~mL})$ and after lyophilization the solid is sublimed at $70^{\circ} \mathrm{C}$ under reduced pressure for $12 \mathrm{~h}$. The residue is extracted with pentane $(10 \times 3 \mathrm{~mL})$, the solvent is removed and the crude product is washed with pentane $(2 \times 2 \mathrm{~mL})$ at $-50^{\circ} \mathrm{C}$. The residue is dissolved in benzene and subjected to column chromatography (pentane/benzene 50:50). The solvent is removed and $6(35.0 \mathrm{mg}, 43.9 \mu \mathrm{mol}, 70 \%)$ is obtained as a brown powder.

Anal. Calcd for $\mathrm{C}_{20} \mathrm{H}_{40} \mathrm{~N}_{1} \mathrm{I}_{2} \mathrm{P}_{2} \mathrm{Re}_{1}$ (796.5): C, 30.2; H, 5.06; N, 1.76. Found: C, 30.5; $\mathrm{H}, 5.23 ; \mathrm{N}, 1.73$. NMR $\left(\mathrm{C}_{6} \mathrm{D}_{6}, \mathrm{RT}\right):{ }^{1} \mathrm{H}(500 \mathrm{MHz}): \delta=4.30\left(36 \mathrm{H}, \mathrm{A}_{18} \mathrm{XX}^{\prime} \mathrm{A}_{18}{ }_{18} N\right.$ $\left.=\left|{ }^{3} J_{\mathrm{AX}}+{ }^{5} J_{\mathrm{AX}}\right|=13.4 \mathrm{~Hz}\right), 0.94\left(\mathrm{~A}_{9} \mathrm{BCXX}^{\prime} \mathrm{A}_{9}{ }_{9} \mathrm{~B}^{\prime} \mathrm{C}^{\prime}, N=\left|{ }^{3} J_{\mathrm{CX}}+{ }^{4} J_{\mathrm{CX}}\right|=33.4 \mathrm{~Hz}\right.$, $\left.{ }^{3} J_{\mathrm{BC}}=6.7 \mathrm{~Hz}, 2 \mathrm{H}, \mathrm{NCH}\right),-7.08\left(\mathrm{~d} \mathrm{br},{ }^{3} J_{\mathrm{HH}}=6.7 \mathrm{~Hz}, 2 \mathrm{H}, \mathrm{PCH}\right) \cdot{ }^{13} \mathrm{C}\left\{{ }^{1} \mathrm{H}\right\}(125.8$

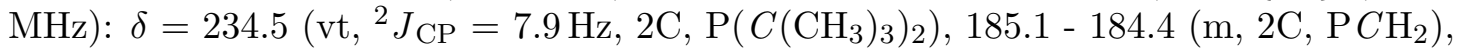
67.5 (s, 2C, $\left.\mathrm{P}\left(\mathrm{C}\left(\mathrm{CH}_{3}\right)_{3}\right)_{2}\right), 42.4$ (s br, $\left.12 \mathrm{C}, \mathrm{P}\left(\mathrm{C}\left(\mathrm{CH}_{3}\right)_{3}\right)_{2}\right)$, $22.4\left(\mathrm{~s}, 2 \mathrm{C}, \mathrm{P}\left(\mathrm{C}\left(\mathrm{CH}_{3}\right)_{3}\right)_{2}\right)$. ${ }^{31} \mathrm{P}\left\{{ }^{1} \mathrm{H}\right\}(202.5 \mathrm{MHz}): \delta=-526.9$ (s, 2P, $\mathrm{P}\left(\mathrm{C}\left(\mathrm{CH}_{3}\right)_{3}\right)_{2}$. LIFDI-MS (Toluene): 797.1 $\left(100 \%,[\mathrm{M}]^{+}\right)$.

\section{Synthesis of 7}

1 (5.0 mg, $8.1 \mu \mathrm{mol}, 1.0$ eq.) and $\mathrm{CoCp}_{2}(1.5 \mu \mathrm{mol}, 8.1 \mu \mathrm{mol}, 1.0$ eq. $)$ are dissolved in benzene $(2 \mathrm{~mL})$ and stirred for 2 minutes at RT and filtered. 2,2'-Bipyridine $(1.3 \mathrm{mg}$, $8.1 \mu \mathrm{mol}, 1.0 \mathrm{eq})$ in benzene $(2 \mathrm{~mL})$ is added and stirring is continued for 5 minutes. The solvent is evaporated and the crude product is washed with pentane $(2 \times 2 \mathrm{~mL})$ and extracted with benzene $(3 \times 1 \mathrm{~mL})$. Lyophilization yields 7 (4.4 mg, $6.0 \mu \mathrm{mol}, 74 \%)$ as a red powder.

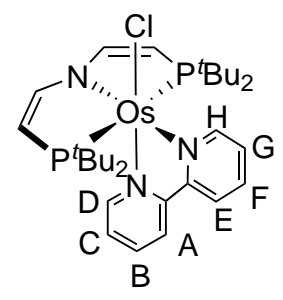

Anal. Calcd. for $\mathrm{C}_{30} \mathrm{H}_{48} \mathrm{ClN}_{3} \mathrm{OsP}_{2}$ (738.36): C, 48.8; H, 6.55; N, 5.69 Found: C, 49.2; $\mathrm{H}, 7.00 ; \mathrm{N}, 5.40$. NMR $\left(\mathrm{CD}_{2} \mathrm{Cl}_{2}, \mathrm{RT}\right):{ }^{1} \mathrm{H} \operatorname{NMR}(400 \mathrm{MHz}): \delta=11.4\left(\mathrm{dd},{ }^{3} J_{\mathrm{HH}}=\right.$ 
$\left.5.3 \mathrm{~Hz},{ }^{4} \mathrm{~J}_{\mathrm{HH}}=2.4 \mathrm{~Hz}, 1 \mathrm{H}, \mathrm{A}-\mathrm{H}\right), 8.41\left(\mathrm{dd},{ }^{3} \mathrm{~J}_{\mathrm{HH}}=6.3 \mathrm{~Hz},{ }^{4} \mathrm{~J}_{\mathrm{HH}}=1.2 \mathrm{~Hz}, 1 \mathrm{H}, \mathrm{E}-\mathrm{H}\right)$, $7.59\left(\mathrm{~A}_{9} \mathrm{BCXX}^{\prime} \mathrm{A}_{9} \mathrm{~B}^{\prime} \mathrm{C}\right.$ ', $\left.N=\left|{ }^{3} J_{\mathrm{CX}}+{ }^{4} J_{\mathrm{CX}},\right|=33.4 \mathrm{~Hz},{ }^{3} J_{\mathrm{BC}}=6.0 \mathrm{~Hz}, 2 \mathrm{H}, \mathrm{NCHCHP}\right)$, $7.20(\mathrm{~m}, 1 \mathrm{H}, \mathrm{D}-\mathrm{H}), 7.03\left(\mathrm{dd},{ }^{3} J_{\mathrm{HH}}=8.3 \mathrm{~Hz},{ }^{4} \mathrm{~J}_{\mathrm{HH}}=1.6 \mathrm{~Hz}, 1 \mathrm{H}, \mathrm{H}-\mathrm{H}\right), 6.56(\mathrm{~m}, 2 \mathrm{H}, \mathrm{B}-\mathrm{H}$ $+\mathrm{C}-\mathrm{H}), 6.31\left(\mathrm{ddd},{ }^{3} J_{\mathrm{HH}}=8.3 \mathrm{~Hz}, 7.2 \mathrm{~Hz},{ }^{4} \mathrm{~J}_{\mathrm{HH}}=1.2 \mathrm{~Hz}, 1 \mathrm{H}, \mathrm{G}-\mathrm{H}\right), 6.15\left(\mathrm{ddd},{ }^{3} J_{\mathrm{HH}}=\right.$ $\left.7.2 \mathrm{~Hz}, 6.3 \mathrm{~Hz},{ }^{4} \mathrm{~J}_{\mathrm{HH}}=1.2 \mathrm{~Hz}, 1 \mathrm{H}, \mathrm{F}-\mathrm{H}\right), 4.06\left(\mathrm{~A}_{9} \mathrm{BCXX}{ }^{\prime}{ }^{\prime}{ }_{9} \mathrm{~B}^{\prime} \mathrm{C}^{\prime}, N=\left|{ }^{2} J_{\mathrm{BX}}+{ }^{4} J_{\mathrm{BX}}\right|=\right.$ $\left.4.1 \mathrm{~Hz},{ }^{3} J_{\mathrm{BC}}=6.0 \mathrm{~Hz}, 2 \mathrm{H}, \mathrm{NCHCHP}\right), 1.65\left(\mathrm{~A}_{9} \mathrm{BCXX}^{\prime} \mathrm{A}_{9}{ }_{9} \mathrm{~B}^{\prime} \mathrm{C}^{\prime},{ }^{3} J_{\mathrm{AX}}=8.5 \mathrm{~Hz}, 18 \mathrm{H}\right.$, $\left.\mathrm{P}\left(\mathrm{C}\left(\mathrm{CH}_{3}\right)_{3}\right)\right), 0.32\left(\mathrm{~A}_{9} \mathrm{BCXX}^{\prime} \mathrm{A}_{9}{ }_{9} \mathrm{~B}^{\prime} \mathrm{C}^{\prime},{ }^{3} J_{\mathrm{AX}}=8.3 \mathrm{~Hz}, 18 \mathrm{H}, \mathrm{P}\left(\mathrm{C}\left(\mathrm{C} H_{3}\right)_{3}\right)\right) .{ }^{13} \mathrm{C}\left\{{ }^{1} \mathrm{H}\right\}-$ $\operatorname{NMR}(101.25 \mathrm{MHz}): \delta=168.9\left(\mathrm{~s}, 1 \mathrm{C}, C-\mathrm{C}_{\mathrm{E}}\right), 165.1\left(\mathrm{~s}, 1 \mathrm{C}, C-\mathrm{C}_{\mathrm{A}}\right), 163.8\left(\mathrm{vt},{ }^{2} J_{\mathrm{CP}}=\right.$ $7.5 \mathrm{~Hz}, 2 \mathrm{C}, \mathrm{NCHCHP}), 154.0\left(\mathrm{~s}, 1 \mathrm{C}, \mathrm{C}_{\mathrm{E}}\right), 153.1\left(\mathrm{~s}, 1 \mathrm{C}, \mathrm{C}_{\mathrm{A}}\right), 130.2\left(\mathrm{~s}, 1 \mathrm{C}, \mathrm{C}_{\mathrm{C}}\right), 126.7(\mathrm{~s}$, $\left.1 \mathrm{C}, \mathrm{C}_{\mathrm{G}}\right), 125.3\left(\mathrm{~s}, 1 \mathrm{C}, \mathrm{C}_{\mathrm{F}}\right), 125.0\left(\mathrm{~s}, 1 \mathrm{C}, \mathrm{C}_{\mathrm{B}}\right), 122.8\left(\mathrm{~s}, 1 \mathrm{C}, \mathrm{C}_{\mathrm{H}}\right), 121.2\left(\mathrm{~s}, 1 \mathrm{C}, \mathrm{C}_{\mathrm{D}}\right), 85.2$ $\left(\right.$ vt, $\left.{ }^{1} J_{\mathrm{CP}}=18.7 \mathrm{~Hz}, 2 \mathrm{C}, \mathrm{NCHCHP}\right), 42.0\left(\mathrm{vt},{ }^{1} J_{\mathrm{CP}}=10.5 \mathrm{~Hz}, 2 \mathrm{C}, \mathrm{P}\left(C\left(\mathrm{CH}_{3}\right)_{3}\right)_{2}\right), 39.1$ (vt, $\left.{ }^{1} J_{\mathrm{CP}}=8.5 \mathrm{~Hz}, 2 \mathrm{C}, \mathrm{P}\left(C\left(\mathrm{CH}_{3}\right)_{3}\right)_{2}\right), 31.8\left(\mathrm{~s} \mathrm{br}, 6 \mathrm{C}, P\left(\mathrm{C}\left(\mathrm{CH}_{3}\right)_{3}\right)_{2}\right) .30 .3\left(\mathrm{vt},{ }^{2} J_{\mathrm{CP}}=\right.$ $\left.\left.2.1 \mathrm{~Hz}, 6 \mathrm{C}, \mathrm{P}\left(\mathrm{C}\left(\mathrm{CH}_{3}\right)_{3}\right)_{2}\right) .{ }^{31} \mathrm{P}\left\{{ }^{1} \mathrm{H}\right\}-\mathrm{NMR}(161.25 \mathrm{MHz}): \delta=19.5\left(\mathrm{~s}, P\left(\mathrm{C}\left(\mathrm{CH}_{3}\right)_{3}\right)_{2}\right)\right)$. All bipy NMR signals could be assigned to two chemically inequivalent ring systems. However, due to a lack of resolution in $2 D$ NMR correlation experiments it might be possible that the position of the ring is not correctly assigned. MS (LIFDI, toluene) $\mathrm{m} / \mathrm{z}$ $=739.1\left(\left[\mathrm{M}^{+}\right]\right), 704.2\left(\left[\mathrm{M}-\mathrm{Cl}^{+}\right]\right)$

\section{Synthesis of 8}

7 is prepared in situ according to the procedure described above and the solvent is removed after addition of 2,2'-bipyridine (1: $35.0 \mathrm{mg}, 56.7 \mu \mathrm{mol}, 1.00$ eq.). An equimolar amount of $\mathrm{NaBPh}_{4}$ in THF $(5 \mathrm{~mL})$ is added and the soution is stirred for 10 minutes at RT. The solvent is removed and the crude product is washed with pentane $(3 \times 5$ $\mathrm{mL})$ and extracted with DCM $(3 \times 3 \mathrm{~mL})$. The solvent is evaporated and the compound is crystallized at $-35^{\circ} \mathrm{C}$ from $\mathrm{THF} /$ pentane. The obtained crystals are washed with pentane $(4 \times 2 \mathrm{~mL})$ and extracted with DCM $(3 \times 1 \mathrm{~mL}) .8(50.6 \mathrm{mg}, 49.5 \mu \mathrm{mol}, 87 \%)$ is obtained as a brown powder.

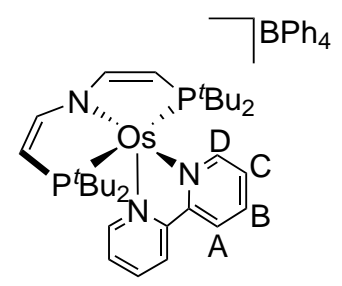

Anal. Calcd. for $\mathrm{C}_{54} \mathrm{H}_{68} \mathrm{BClN}_{3} \mathrm{OsP}_{2}$ (1057.6): C, 61.3; H, 6.48; N, 3.97 Found: C, 61.5; H, 6.53; N, 3.80. NMR ( $\left.d_{8}-\mathrm{THF}, \mathrm{RT}\right):{ }^{1} \mathrm{H}-\mathrm{NMR}:(400 \mathrm{MHz}): \delta=9.05(\mathrm{~s}$ br, $2 \mathrm{H}$, bipy:A-H), 7.82 (d, ${ }^{3} J_{\mathrm{HH}}=8.3 \mathrm{~Hz}, 2 \mathrm{H}$, bipy:B-H), $7.78\left(\mathrm{~A}_{18} \mathrm{BXX}^{\prime} \mathrm{A}^{\prime}{ }_{18} \mathrm{~B}^{\prime} \mathrm{C}^{\prime}, N=\right.$ $\left.\left|{ }^{3} J_{\mathrm{CX}}+{ }^{4} J_{\mathrm{CX}}\right|=35.4 \mathrm{~Hz},{ }^{3} J_{\mathrm{BC}}=5.8 \mathrm{~Hz}, 2 \mathrm{H}, \mathrm{NCHCHP}\right), 7.49\left(\mathrm{t},{ }^{3} J_{\mathrm{HH}}=7.5 \mathrm{~Hz}, 2 \mathrm{H}\right.$, bipy:C-H), 7.38 (t br, $\left.8 \mathrm{H}, \mathrm{BPH}_{4}: \mathrm{o}-\mathrm{H}\right), 7.14$ (t br, ${ }^{3} J_{\mathrm{HH}}=7.1 \mathrm{~Hz}, 2 \mathrm{H}$, bipy:D-H), 7.04 (m br, $\left.8 \mathrm{H}, \mathrm{BPH}_{4},: \mathrm{m}-\mathrm{H}\right), 6.87$ (m br, $\left.4 \mathrm{H}, \mathrm{BPH}_{4}: \mathrm{p}-\mathrm{H}\right), 4.34\left(\mathrm{~A}_{18} \mathrm{BXX}^{\prime} \mathrm{A}_{18} \mathrm{~B}^{\prime} \mathrm{C}\right.$ ', $\left.N=\left|{ }^{2} J_{\mathrm{BX}}+{ }^{4} J_{\mathrm{BX}}\right|=6.2 \mathrm{~Hz},{ }^{3} J_{\mathrm{BC}}=5.8 \mathrm{~Hz}, 2 \mathrm{H}, \mathrm{NCHCHP}\right), 0.49\left(\mathrm{~A}_{18} \mathrm{XX}^{\prime} \mathrm{A}_{18}^{\prime}, N=\right.$ $\left.\left|{ }^{3} J_{\mathrm{AX}}+{ }^{5} J_{\mathrm{AX}}\right|=6.8 \mathrm{~Hz}, 36 \mathrm{H}, \mathrm{P}\left(\mathrm{C}\left(\mathrm{CH}_{3}\right)_{3}\right)_{2}\right) .{ }^{13} \mathrm{C}\left\{{ }^{1} \mathrm{H}\right\}-\mathrm{NMR}(101.25 \mathrm{MHz}): \delta=164.4$ (vt, $\left.{ }^{2} J_{\mathrm{CP}}=7.5 \mathrm{~Hz}, \mathrm{NCHCHP}\right), 164.0\left(\mathrm{q},{ }^{1} J_{\mathrm{C}^{11} \mathrm{~B}}=49 \mathrm{~Hz}, 4 \mathrm{C}, \mathrm{BPh}_{4}: \mathrm{C}_{\mathrm{ipso}}\right), 162.2(\mathrm{~s}, 2 \mathrm{C}$, bipy:NCCN), 156.4 (s, 2C, bipy: $\left.\mathrm{C}_{\mathrm{A}}\right), 135.9\left(\mathrm{q},{ }^{1} J_{\mathrm{CB}}=1.6 \mathrm{~Hz}, 8 \mathrm{C}, \mathrm{BPh}_{4}: \mathrm{C}_{\text {ortho }}\right), 134.1$ 
(s, 2C, bipy: $\mathrm{C}_{\mathrm{C}}$ ), $126.5\left(\mathrm{~s}, 2 \mathrm{C}\right.$, bipy: $\left.\mathrm{C}_{\mathrm{D}}\right), 125.6\left(\mathrm{q},{ }^{1} J_{\mathrm{CB}}=2.7 \mathrm{~Hz}, 8 \mathrm{C}, \mathrm{BPh}_{4}: \mathrm{C}_{\text {meta }}\right), 123.5$ (s, 2C, bipy: $\left.\mathrm{C}_{\mathrm{B}}\right), 121.7\left(\mathrm{~s}, 4 \mathrm{C}, \mathrm{BPh}_{4}: \mathrm{C}_{\mathrm{para}}\right), 85.2$ (vt, $\left.{ }^{1} J_{\mathrm{CP}}=21.0 \mathrm{~Hz}, \mathrm{NCHCHP}\right), 42.6$ (s br, 8C, $\left.\mathrm{P}\left(\mathrm{C}\left(\mathrm{CH}_{3}\right)_{3}\right)_{2}\right), 27.4\left(\mathrm{~s}, 24 \mathrm{C}, \mathrm{P}\left(\mathrm{C}\left(\mathrm{CH}_{3}\right)_{3}\right)_{2}\right)$. ${ }^{31} \mathrm{P}\left\{{ }^{1} \mathrm{H}\right\}-\mathrm{NMR}(161.25 \mathrm{MHz})$ : $\left.\delta=26.9\left(\mathrm{~s}, P\left(\mathrm{C}\left(\mathrm{CH}_{3}\right)_{3}\right)_{2}\right)\right)$.

\section{Reduction of 8}

8 (5.0 mg, 4.9 umol, 1.0 eq.) and $\mathrm{NaHg}(2 \mathrm{M}, 0.33 \mathrm{~g}, 49 \mu \mathrm{mol}, 10 \mathrm{eq})$ are dissolved in THF $(1 \mathrm{~mL})$ and stirred for 1 minute at RT. The solution is filtered and measured by EPR spectroscopy. For UV/vis measurements a stock solution of $\mathbf{8}$ in THF was diluted to appropriate concentrations and a tenfold excess of $\mathrm{NaHg}$ was added.

\section{Synthesis of 10}

8 (12.1 mg, $11.8 \mu \mathrm{mol}, 1.00$ eq.) and $\mathrm{NaBH}_{4}$ (9.0 mg, $0.24 \mathrm{mmol}, 20$ eq.) are dissolved in THF $(3 \mathrm{~mL})$ and stirred for $24 \mathrm{~h}$ at RT. The solvent is removed and the residue is extracted with pentane $(4 \times 1 \mathrm{~mL})$. After evaporation of the solvent and lyophilization $\mathbf{2 1}$ (7.0 mg, $9.9 \mu \mathrm{mol}, 84 \%)$ is obtained as a black powder.

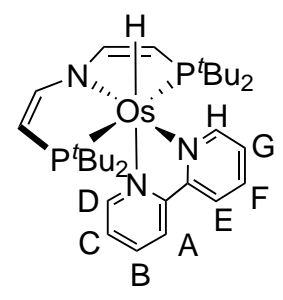

Anal. Calcd. for $\mathrm{C}_{30} \mathrm{H}_{49} \mathrm{ClN}_{3} \mathrm{OsP}_{2}$ (703.92): C, 51.2; H, 7.02; N, 5.97 Found: C, 51.4; H, 7.10; N, 5.90. NMR $\left(\mathrm{C}_{6} \mathrm{D}_{6}, \mathrm{RT}\right):{ }^{1} \mathrm{H}$ NMR $(400 \mathrm{MHz}): \delta=10.27\left(\mathrm{~d},{ }^{3} J_{\mathrm{HH}}\right.$ $=6.2 \mathrm{~Hz}, 1 \mathrm{H}, \mathrm{A}-\mathrm{H}) 8.41\left(\mathrm{~d},{ }^{3} J_{\mathrm{HH}}=5.9 \mathrm{~Hz}, 1 \mathrm{H}, \mathrm{E}-\mathrm{H}\right) 7.59\left(\mathrm{~A}_{9} \mathrm{BCXX}^{\prime} \mathrm{A}_{9}{ }_{9} \mathrm{~B}^{\prime} \mathrm{C}^{\prime}, N=\right.$ $\left.\left|{ }^{3} J_{\mathrm{CX}}+{ }^{4} J_{\mathrm{CX}}\right|=34.0 \mathrm{~Hz},{ }^{3} J_{\mathrm{BC}}=5.5 \mathrm{~Hz}, 2 \mathrm{H}, \mathrm{NCHCHP}\right), 7.36-7.31(\mathrm{~m}, 2 \mathrm{H}, \mathrm{D}-\mathrm{H}, \mathrm{H}-\mathrm{H})$, $6.73-6.68(\mathrm{~m}, 1 \mathrm{H}, \mathrm{G}-\mathrm{H}), 6.65-6.61(\mathrm{~m}, 1 \mathrm{H}, \mathrm{C}-\mathrm{H}), 6.37-6.33(\mathrm{~m}, 1 \mathrm{H}, \mathrm{H}-\mathrm{F}), 6.09$ - $6.04(\mathrm{~m}, 1 \mathrm{H}, \mathrm{B}-\mathrm{H}), 4.26\left(\mathrm{~A}_{9} \mathrm{BCXX}^{\prime} \mathrm{A}_{9} \mathrm{~B}^{\prime} \mathrm{C} ', N=\left|{ }^{2} J_{\mathrm{BX}}+{ }^{4} J_{\mathrm{BX}}\right|=5.5 \mathrm{~Hz},{ }^{3} J_{\mathrm{BC}}=\right.$ $5.5 \mathrm{~Hz}, 2 \mathrm{H}, \mathrm{NCHCHP}), 1.57\left(\mathrm{~A}_{9} \mathrm{BCXX}^{\prime} \mathrm{A}_{9}{ }_{9} \mathrm{~B}^{\prime} \mathrm{C}\right.$ ', $\left.{ }^{3} J_{\mathrm{AX}}=12.5 \mathrm{~Hz}, 18 \mathrm{H}, \mathrm{P}\left(\mathrm{C}\left(\mathrm{CH}_{3}\right)_{3}\right)\right)$, $0.45\left(\mathrm{~A}_{9} \mathrm{BCXX}^{\prime} \mathrm{A}_{9}{ }_{9} \mathrm{~B}^{\prime} \mathrm{C}\right.$ ', $\left.{ }^{3} J_{\mathrm{AX}}=12.2 \mathrm{~Hz}, 18 \mathrm{H}, \mathrm{P}\left(\mathrm{C}\left(\mathrm{CH}_{3}\right)_{3}\right)\right),-11.0\left(\mathrm{t},{ }^{3} J_{\mathrm{HP}}=24.2 \mathrm{~Hz}\right.$, Os- $H$ ). ${ }^{13} \mathrm{C}\left\{{ }^{1} \mathrm{H}\right\}$-NMR $(101.25 \mathrm{MHz}): \delta=161.7$ (vt, ${ }^{2} J_{\mathrm{CP}}=9.0 \mathrm{~Hz}, 2 \mathrm{C}, \mathrm{NCHCHP}$ ), $161.6\left(\mathrm{~s}, 1 \mathrm{C}, C-\mathrm{C}_{\mathrm{A}}\right), 160.2\left(\mathrm{~s}, 1 \mathrm{C}, \mathrm{C}_{\mathrm{A}}\right), 159.4\left(\mathrm{~s}, 1 \mathrm{C}, C-\mathrm{C}_{\mathrm{E}}\right), 149.3\left(\mathrm{~s}, 1 \mathrm{C}, \mathrm{C}_{\mathrm{E}}\right)$, $125.7\left(\mathrm{~s}, 1 \mathrm{C}, \mathrm{C}_{\mathrm{G}}\right), 124.8\left(\mathrm{~s}, 1 \mathrm{C}, \mathrm{C}_{\mathrm{B}}\right), 124.6\left(\mathrm{~s}, 1 \mathrm{C}, \mathrm{C}_{\mathrm{C}}\right), 123.9\left(\mathrm{~s}, 1 \mathrm{C}, \mathrm{C}_{\mathrm{F}}\right), 122.8(\mathrm{~s}$, $1 \mathrm{C}, \mathrm{C}_{\mathrm{H} / \mathrm{D}}$ ), $122.4\left(\mathrm{~s}, 1 \mathrm{C}, \mathrm{C}_{\mathrm{H} / \mathrm{D}}\right), 85.3\left(\mathrm{vt},{ }^{1} J_{\mathrm{CP}}=19.2 \mathrm{~Hz}, 2 \mathrm{C}, \mathrm{NCHCHP}\right), 41.0$ (vt, $\left.{ }^{1} J_{\mathrm{CP}}=8.5 \mathrm{~Hz}, 2 \mathrm{C}, \mathrm{P}\left(C\left(\mathrm{CH}_{3}\right)_{3}\right)_{2}\right), 39.7\left(\mathrm{vt},{ }^{1} J_{\mathrm{CP}}=13.9 \mathrm{~Hz}, 2 \mathrm{C}, \mathrm{P}\left(C\left(\mathrm{CH}_{3}\right)_{3}\right)_{2}\right), 30.1$ (s br, 6C, $\left.P\left(\mathrm{C}\left(\mathrm{CH}_{3}\right)_{3}\right)_{2}\right) .29 .5\left(\right.$ vt, $\left.{ }^{2} J_{\mathrm{CP}}=2.5 \mathrm{~Hz}, 6 \mathrm{C}, \mathrm{P}\left(\mathrm{C}\left(\mathrm{CH}_{3}\right)_{3}\right)_{2}\right) .{ }^{31} \mathrm{P}\left\{{ }^{1} \mathrm{H}\right\}-\mathrm{NMR}$

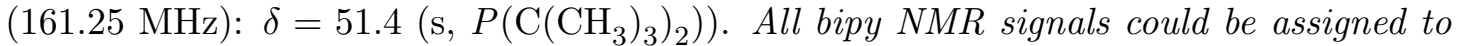
two chemically inequivalent ring systems. However, due to a lack of resolution in $2 D$ NMR correlation experiments it might be possible that the position of the ring is not correctly assigned. IR (ATR, RT) $\tilde{\nu}=2074 \mathrm{~cm}^{-1}(\mathrm{Os}-\mathrm{H})$; MS (LIFDI, toluene) $\frac{m}{z}=$ $705.2\left(\left[\mathrm{M}^{+}\right]\right)$. 


\section{Synthesis of 11}

5 (100 mg, $125 \mu \mathrm{mol}, 1.00$ eq.) and $\mathrm{HPyrPz}(19.0 \mathrm{mg}, 131 \mu \mathrm{mol}, 1.05$ eq.) are dissolved in THF $(5 \mathrm{~mL})$ and stirred for 30s. KHMDS $(23.7 \mathrm{mg}, 118.7 \mu$ molm 0.95 eq.) is added in small portions over the period of 1 minute and stirring is continued for 5 minutes. After addition of $\mathrm{NEt}_{3}(17.4 \mu \mathrm{L}, 124.9 \mu \mathrm{mol}, 1.0 \mathrm{eq}$.) the solution is stirred for $90 \mathrm{~min}$. After evaporation of the solvent the residue is extracted with pentane $(5 \times 5 \mathrm{~mL})$. Lyophilization yields 11 in form of a brown powder $(80.0 \mathrm{mg}, 97.8 \mu \mathrm{mol}, 78 \%)$.

Anal. Calcd for $\mathrm{C}_{28} \mathrm{H}_{50} \mathrm{IN}_{4} \mathrm{P}_{2} \mathrm{Re}$ (817.8): C, 41.1; H, 6.16; N 6.85. Found: C, 41.4; H, 6.06; N, 6.82. NMR $\left(\mathrm{C}_{6} \mathrm{D}_{6}, \mathrm{RT}\right):{ }^{1} \mathrm{H}(500 \mathrm{MHz}): \delta=10.4\left(\mathrm{ddd},{ }^{3} J_{\mathrm{HH}}=6.2 \mathrm{~Hz},{ }^{4} J_{\mathrm{HH}}=\right.$ $\left.1.4 \mathrm{~Hz},{ }^{5} J_{\mathrm{HH}}=0.9 \mathrm{~Hz}, 1 \mathrm{H}, \mathrm{C}_{8} H\right), 7.51\left(\mathrm{~d},{ }^{3} J_{\mathrm{HH}}=2.1 \mathrm{~Hz}, 1 \mathrm{H}, \mathrm{C}_{1} H\right), 7.28\left(\mathrm{ddd},{ }^{3} J_{\mathrm{HH}}\right.$ $\left.=7.9 \mathrm{~Hz},{ }^{4} J_{\mathrm{HH}}=1.6 \mathrm{~Hz},{ }^{5} J_{\mathrm{HH}}=0.9 \mathrm{~Hz}, 1 \mathrm{H}, \mathrm{C}_{5} H\right), 6.45\left(\mathrm{ddd},{ }^{3} J_{\mathrm{HH}}=7.9 \mathrm{~Hz},{ }^{3} J_{\mathrm{HH}}\right.$ $\left.=7.2 \mathrm{~Hz},{ }^{4} J_{\mathrm{HH}}=1.5 \mathrm{~Hz}, 1 \mathrm{H}, \mathrm{C}_{6} H\right), 6.31\left(\mathrm{ddd},{ }^{3} J_{\mathrm{HH}}=7.2 \mathrm{~Hz},{ }^{3} J_{\mathrm{HH}}=6.2 \mathrm{~Hz},{ }^{4} J_{\mathrm{HH}}\right.$ $\left.=1.6 \mathrm{~Hz}, 1 \mathrm{H}, \mathrm{C}_{7} H\right), 6.27\left(\mathrm{~d},{ }^{3} J_{\mathrm{HH}}=2.1 \mathrm{~Hz}, 1 \mathrm{H}, \mathrm{C}_{2} H\right), 5.34-5.23\left(\mathrm{~m}, 2 \mathrm{H}, \mathrm{NCH}_{2}\right)$, $4.65-4.56\left(\mathrm{~m}, 2 \mathrm{H}, \mathrm{NCH}_{2}\right), 2.33-2.24\left(\mathrm{~m}, 2 \mathrm{H}, \mathrm{PCH} H_{2}\right), 2.07-1.99\left(\mathrm{~m}, 2 \mathrm{H}, \mathrm{PCH}_{2}\right)$, $1.49\left(\mathrm{~A}_{9} \mathrm{XX} \mathrm{A}_{9}{ }^{\prime}, N=\left|{ }^{3} J_{\mathrm{AX}}+{ }^{5} J_{\mathrm{AX}}\right|=11.5 \mathrm{~Hz}, 18 \mathrm{H}, \mathrm{P}\left(\mathrm{C}\left(\mathrm{CH}_{3}\right)_{3}\right)_{2}\right), 0.60\left(\mathrm{~A}_{9} \mathrm{XX} \mathrm{A}_{9}\right.$ ', $\left.N=\left|{ }^{3} J_{\mathrm{AX}}+{ }^{5} J_{\mathrm{AX}}\right|=11.8 \mathrm{~Hz}, 18 \mathrm{H}, \mathrm{P}\left(\mathrm{C}\left(\mathrm{C} H_{3}\right)_{3}\right)_{2}\right) \cdot{ }^{13} \mathrm{C}\left\{{ }^{1} \mathrm{H}\right\}(125.8 \mathrm{MHz}): \delta=156.1$ $\left(\mathrm{s}, 1 \mathrm{C}, C_{8}\right), 151.9\left(\mathrm{~s}, 1 \mathrm{C}, C_{4}\right), 147.3\left(\mathrm{~s}, 1 \mathrm{C}, C_{3}\right), 142.2\left(\mathrm{~s}, 1 \mathrm{C}, C_{1}\right), 136.8\left(\mathrm{~s}, 1 \mathrm{C}, C_{5}\right)$, $116.6\left(\mathrm{~s}, 1 \mathrm{C}, C_{7}\right), 115.6\left(\mathrm{~s}, 1 \mathrm{C}, C_{5}\right), 104.0\left(\mathrm{~s}, 1 \mathrm{C}, C_{2}\right), 87.8\left(\mathrm{vt}, N=\left|{ }^{2} J_{\mathrm{PC}}+{ }^{3} J_{\mathrm{PC}}\right|\right.$ $\left.=3.9 \mathrm{~Hz}, 2 \mathrm{C}, \mathrm{NCH} \mathrm{H}_{2}\right), 46.7\left(\mathrm{vt}, N=\left|{ }^{1} J_{\mathrm{CP}}+{ }^{3} J_{\mathrm{CP}}\right|=7.8 \mathrm{~Hz}, 2 \mathrm{C}, \mathrm{P}\left(C\left(\mathrm{CH}_{3}\right)_{3}\right)_{2}\right), 40.6$ (vt, $\left.N=\left|{ }^{1} J_{\mathrm{CP}}+{ }^{3} J_{\mathrm{CP}}\right|=6.4 \mathrm{~Hz}, 2 \mathrm{C}, \mathrm{P}\left(C\left(\mathrm{CH}_{3}\right)_{3}\right)_{2}\right), 30.1\left(\right.$ vt, $N=\left|{ }^{2} J_{\mathrm{CP}}+{ }^{4} J_{\mathrm{CP}}\right|=$ $\left.1.6 \mathrm{~Hz}, 6 \mathrm{C}, \mathrm{P}\left(\mathrm{C}\left(\mathrm{CH}_{3}\right)_{3}\right)_{2}\right), 30.9\left(\mathrm{vt}, N=\mid{ }^{1} J_{\mathrm{PC}}+{ }^{3} J_{\mathrm{PC}}=6.9 \mathrm{~Hz}, 2 \mathrm{C}, \mathrm{PC} \mathrm{H}_{2}\right), 30.1$ (vt, $\left.N=\left|{ }^{2} J_{\mathrm{CP}}+{ }^{4} J_{\mathrm{CP}}\right|=2.4 \mathrm{~Hz}, 6 \mathrm{C}, \mathrm{P}\left(\mathrm{C}\left(\mathrm{CH}_{3}\right)_{3}\right)_{2}\right) .{ }^{31} \mathrm{P}\left\{{ }^{1} \mathrm{H}\right\}(202.5 \mathrm{MHz}): \delta=-27.8(\mathrm{~s}$, 2P, $P\left(\mathrm{C}\left(\mathrm{CH}_{3}\right)_{3}\right)_{2}$. LIFDI-MS (Toluene): $818.2\left(100 \%,[\mathrm{M}]^{+}\right)$.

\section{Synthesis of 12}

$1(80.0 \mathrm{mg}, 120 \mu \mathrm{mol}, 1.00 \mathrm{eq}$.$) and 2-(1H-pyrazol-5-yl)pyridine (19.9 \mathrm{mg}, 137 \mu \mathrm{mol}$, 1.05 eq.) are dissolved in THF $(20 \mathrm{~mL})$. After addition of $\mathrm{NEt}_{3}(80 \mu \mathrm{L}, 574 \mu \mathrm{mol}$, 4.34 eq.) the solution is stirred for $36 \mathrm{~h}$ at room temperature. After removal of the solvent the residue is washed with pentane $(3 \times 5 \mathrm{~mL})$ at $-40^{\circ} \mathrm{C}$. Afterwards the residue is extracted with pentane $(8 \times 10 \mathrm{~mL})$ at room temperature. The solvent is removed and lyophilization yields 12 in form of a yellow-brown powder ( $84.5 \mathrm{mg}, 116 \mu \mathrm{mol}, 90 \%)$.

Anal. Calcd. for $\mathrm{C}_{28} \mathrm{H}_{46} \mathrm{ClN}_{4} \mathrm{OsP}_{2}$ (726.3): C, 46.3; H, 6.38; N, 7.71. Found: C, 46.7; $\mathrm{H}, 6.46 ; \mathrm{N}, 7.79$.

$\operatorname{NMR}\left(\mathrm{THF}-\mathrm{d}_{8}, \mathrm{RT}\right):{ }^{1} \mathrm{H}(300 \mathrm{MHz}) \delta=5.14\left(\mathrm{~s} \mathrm{br}, 18 \mathrm{H}, \mathrm{P}\left(\mathrm{C}\left(\mathrm{CH}_{3}\right)_{3}\right)\right),-1.24(\mathrm{~s} \mathrm{br}, 18 \mathrm{H}$, $\left.\mathrm{P}\left(\mathrm{C}\left(\mathrm{CH}_{3}\right)_{3}\right)\right)$ (other signals cannot be assigned due to paramagnetism).

\section{Synthesis of 13}

Route A:

$12(50.0 \mathrm{mg}, 68.8 \mu \mathrm{mol}, 1.00$ eq.) and $\mathrm{NaHg}(1 \mathrm{w} \%, 1.66 \mathrm{~g}, 72.2 \mu \mathrm{mol}, 1.05$ eq.) are suspended in THF $(10 \mathrm{~mL})$ and stirred for $1 \mathrm{~h}$ at room temperature. After removal of the solvent the residue is extracted with pentane $(4 \times 5 \mathrm{~mL})$, the volume is reduced to ca. $3 \mathrm{~mL}$ and solution is stored at $-40^{\circ} \mathrm{C}$ for 2 days. The precipitate is filtered and washed with cold pentane. Dissolving of the red solid in benzene and lyophilization yields $\mathbf{1 3}$ $(31.0 \mathrm{mg}, 44.9 \mu \mathrm{mol}, 65 \%)$ in form of a red powder. 
Route B:

$1(200 \mathrm{mg}, 323 \mu \mathrm{mol}, 1.00 \mathrm{eq}$.$) and \mathrm{CoCp}_{2}(42.2 \mathrm{mg}, 323 \mu \mathrm{mol}, 1.00$ eq.) are dissolved in THF $(15 \mathrm{~mL})$ and stirred for 2 minutes. 2-(1H-pyrazol-5-yl)pyridine $(47.0 \mathrm{mg}, 323 \mu \mathrm{mol}$, 1.00 eq.) is added in small portions over the course of 3 minutes and stirring is continued for 5 minutes. After addition of KHMDS ( $64.6 \mathrm{mg}, 323 \mu \mathrm{mol}, 1.00$ eq.) the solution is stirred for 90 minutes. The solvent removed and the solid is extracted with pentane $(5$ $\mathrm{x} 10 \mathrm{~mL}$ ). The solvent is evaporated and the product is lyophilized to yield ${ }^{\circ}{ }_{2} \mathrm{pyrpzl}_{3}$ $(210 \mathrm{mg}, 304 \mu \mathrm{mol}, 94 \%)$ in form of a red powder.

Anal. Calcd. for $\mathrm{C}_{28} \mathrm{H}_{46} \mathrm{~N}_{4} \mathrm{OsP}_{2}$ (690.88): C, 48.7; H, 6.71; N, 8.11. Found: C, 48.9; H, 7.05; N, 7.95 .

$\operatorname{NMR}\left(\right.$ THF-d 8, RT): ${ }^{1} \mathrm{H}(500 \mathrm{MHz}) \delta=8.42\left(\mathrm{~d},{ }^{3} J_{\mathrm{HH}}=2.1 \mathrm{~Hz}, 1 \mathrm{H}, \mathrm{C}_{1} \mathrm{H}\right), 7.98(\mathrm{ddd}$, $\left.{ }^{3} J_{\mathrm{HH}}=6.1 \mathrm{~Hz},{ }^{4} J_{\mathrm{HH}}=1.5 \mathrm{~Hz},{ }^{5} J_{\mathrm{HH}}=0.7 \mathrm{~Hz}, 1 \mathrm{H}, \mathrm{C}_{8} H\right), 7.64\left(\mathrm{~A}_{18} \mathrm{BCXX}^{\prime} \mathrm{A}_{18}^{\prime}{ }_{18} \mathrm{C}^{\prime}, N\right.$ $\left.=\left|{ }^{3} J_{\mathrm{CX}}+{ }^{4} J_{\mathrm{CX}}\right|=33.6 \mathrm{~Hz},{ }^{3} J_{\mathrm{BC}}=5.5 \mathrm{~Hz}, 2 \mathrm{H}, \mathrm{NCH}\right), 7.00\left(\mathrm{ddd},{ }^{3} J_{\mathrm{HH}}=8.0 \mathrm{~Hz},{ }^{4} J_{\mathrm{HH}}\right.$ $\left.=1.5 \mathrm{~Hz},{ }^{5} J_{\mathrm{HH}}=0.7 \mathrm{~Hz}, \mathrm{C}_{5} H\right), 6.97\left(\mathrm{~d},{ }^{3} J_{\mathrm{HH}}=2.1 \mathrm{~Hz}, 1 \mathrm{H}, \mathrm{C}_{2} H\right), 6.46\left(\mathrm{~m},{ }^{3} J_{\mathrm{HH}}=\right.$ $\left.8.0 \mathrm{~Hz},{ }^{3} J_{\mathrm{HH}}=7.3 \mathrm{~Hz}, 1 \mathrm{H}, \mathrm{C}_{5} H\right), 5.87\left(\mathrm{~m},{ }^{3} J=7.3 \mathrm{~Hz},{ }^{3} J=6.1 \mathrm{~Hz}, 1 \mathrm{H}, \mathrm{C}_{6} H\right), 4.27$ $\left(\mathrm{A}_{18} \mathrm{BCXX}^{\prime} \mathrm{A}_{18}^{\prime} \mathrm{B}^{\prime} \mathrm{C}^{\prime}, N=\left|{ }^{2} J_{\mathrm{BX}}+{ }^{4} J_{\mathrm{BX}},\right|=5.6 \mathrm{~Hz},{ }^{3} J_{\mathrm{BC}}=5.5 \mathrm{~Hz}, 2 \mathrm{H}, \mathrm{PC} H\right), 0.64(\mathrm{~m}$, $\left.18 \mathrm{H}, \mathrm{P}\left(\mathrm{C}\left(\mathrm{CH}_{3}\right)_{3}\right)\right), 0.57\left(\mathrm{~m}, 18 \mathrm{H}, \mathrm{P}\left(\mathrm{C}\left(\mathrm{CH}_{3}\right)_{3}\right) .{ }^{13} \mathrm{C}\left\{{ }^{1} \mathrm{H}\right\}(128.8 \mathrm{MHz}) \delta=163.7\right.$ (vt, $N$ $\left.=\left|{ }^{2} J_{\mathrm{CP}}+{ }^{3} J_{\mathrm{CP}}\right|=7.8 \mathrm{~Hz}, 2 \mathrm{C}, \mathrm{NCH}\right), 163.5\left(\mathrm{t},{ }^{3} J_{\mathrm{CP}}=1.2 \mathrm{~Hz}, 1 \mathrm{C}, C_{4}\right), 153.9(\mathrm{~s}, 1 \mathrm{C}$, $\left.C_{1}\right), 148.3\left(\mathrm{~s}, 1 \mathrm{C}, C_{3}\right), 139.3\left(\mathrm{~s}, 1 \mathrm{C}, C_{1}\right), 131.2\left(\mathrm{~s}, 1 \mathrm{C}, C_{6}\right), 117.1\left(\mathrm{~s}, 1 \mathrm{C}, C_{7}\right), 116.3($ s, $\left.1 \mathrm{C}, C_{5}\right), 102.9\left(\mathrm{~s}, 1 \mathrm{C}, C_{2}\right), 86.0\left(\mathrm{vt}, N=\left|{ }^{1} J_{\mathrm{CP}}+{ }^{3} J_{\mathrm{CP}}\right|=19.3 \mathrm{~Hz}, 2 \mathrm{C}, \mathrm{P} C \mathrm{H}\right), 47.7$ (vt, $N=\left|{ }^{1} J_{\mathrm{CP}}+{ }^{2} J_{\mathrm{CP}}\right|=11.9 \mathrm{~Hz}, 2 \mathrm{C}, \mathrm{P}\left(C\left(\mathrm{CH}_{3}\right)\right)$ ), 35.4 (vt, $N=\left|{ }^{1} J_{\mathrm{CP}}+{ }^{2} J_{\mathrm{CP}}\right|=$ $\left.10.5 \mathrm{~Hz}, 2 \mathrm{C}, \mathrm{P}\left(C\left(\mathrm{CH}_{3}\right)\right)\right), 28.4\left(\mathrm{vt}, N=\left|{ }^{2} J_{\mathrm{CP}}+{ }^{4} J_{\mathrm{CP}}\right|=2.5 \mathrm{~Hz}, 6 \mathrm{C}, \mathrm{P}\left(\mathrm{C}\left(\mathrm{CH}_{3}\right)_{3}\right)\right.$ ), 25.9 (vt, $N=\left|{ }^{2} J_{\mathrm{CP}}+{ }^{4} J_{\mathrm{CP}}\right|=1.9 \mathrm{~Hz}, 6 \mathrm{C}, \mathrm{P}\left(\mathrm{C}\left(C \mathrm{H}_{3}\right)_{3}\right)$ ). ${ }^{31} \mathrm{P}\left\{{ }^{1} \mathrm{H}\right\}(202.5 \mathrm{MHz}) \delta=$ $24.6\left(\mathrm{~s}, 2 \mathrm{P}, P\left(\mathrm{C}\left(\mathrm{CH}_{3}\right)_{3}\right)\right)$. 


\subsection{Os Nitride and Imide Complexes in Various Coordination Spheres}

Parts of this chapter were adapted from:

J. Abbenseth, S. C. Bete, M. Finger, C. Volkmann, C. Würtele, S. Schneider, Organometallics 2018, 37, 802-811.

Copyright 2018 American Chemical Society.

\subsubsection{Computational Details}

DFT calculations were carried out with the ORCA program package using the PBE and PBE0 functionals. [304], [318], [326] Ahlrichs' basis sets def2-SVP (for geometry optimization and frequency calculation) and def2-TZVP (for single-point energies) were used with a full basis for all elements but Os for which the Stuttgart-Dresden 60 electron core potential was chosen to replace the inner shell 1s-4f orbitals. ${ }^{[307],[308]}$ The RI-J (PBE) approximation in combination with the corresponding auxiliary basis sets of Alrichs was utilized to reduce computational costs in the geometry optimization and frequency calculations. ${ }^{[327]-[329]}$ Grimme's model (D3) with Becke-Johnson damping was used to account for dispersion with the PBE or PBE0 functionals. ${ }^{[306],[330]}$ No symmetry restraints were imposed and the optimized structures were defined as minima (no negative eigenvalues) by vibrational analyses. NBO analysis of $\mathbf{1 8}, \mathbf{1 9}$ and $\mathbf{2 0}$ was carried out with NBO 6.0. ${ }^{3}$ Geometries were analyzed and visualized with Avogadro, molecular orbitals were visualized with Chimera and for NBO/NLMO visualization, Jmol (http://www.jmol.org) was used. ${ }^{[33],[334]}$ The XYZ-Files of all computed species can be obtained free of charge via the article page. TDDFT/EPR calculations were performed with ORCA and the CPCM solvation model was used (pentane, THF). ${ }^{[31]}$ Relativistic treatment was introducted by ZORA. ${ }^{[332]}$

Table 7.4: XYZ-coordinates of computed complex 22.

\begin{tabular}{c|lll|l|lll} 
Atom & $\mathrm{x}$ & $\mathrm{y}$ & $\mathrm{z}$ & Atom & $\mathrm{x}$ & $\mathrm{y}$ & $\mathrm{z}$ \\
\hline $\mathrm{C}$ & 3.66137 & 12.13119 & 17.02107 & $\mathrm{H}$ & 5.92085 & 12.0675 & 12.85937 \\
$\mathrm{H}$ & 4.09274 & 13.07956 & 17.39171 & $\mathrm{C}$ & 4.67158 & 11.93939 & 10.44108 \\
$\mathrm{C}$ & 3.30445 & 11.14571 & 17.89122 & $\mathrm{H}$ & 5.60412 & 11.55532 & 9.97723 \\
$\mathrm{H}$ & 3.44708 & 11.28135 & 18.97274 & $\mathrm{H}$ & 4.84993 & 13.00028 & 10.70357 \\
$\mathrm{C}$ & 4.30489 & 8.44677 & 17.37213 & $\mathrm{H}$ & 3.88201 & 11.88635 & 9.66729 \\
$\mathrm{C}$ & 5.47575 & 9.07747 & 16.59453 & $\mathrm{C}$ & 1.50115 & 12.45277 & 11.58248 \\
$\mathrm{H}$ & 6.38785 & 8.48096 & 16.80258 & $\mathrm{C}$ & 1.94064 & 13.67806 & 10.75936 \\
$\mathrm{H}$ & 5.67343 & 10.11832 & 16.91678 & $\mathrm{H}$ & 2.7122 & 13.4525 & 10.00667 \\
$\mathrm{H}$ & 5.3041 & 9.06472 & 15.50217 & $\mathrm{H}$ & 2.30246 & 14.50336 & 11.40337 \\
$\mathrm{C}$ & 4.74567 & 8.3632 & 18.84732 & $\mathrm{H}$ & 1.05165 & 14.06262 & 10.217 \\
$\mathrm{H}$ & 5.72096 & 7.83539 & 18.87744 & $\mathrm{C}$ & 1.00594 & 11.32302 & 10.66574 \\
$\mathrm{H}$ & 4.05149 & 7.79944 & 19.48974 & $\mathrm{H}$ & 0.09699 & 11.66211 & 10.12623 \\
$\mathrm{H}$ & 4.90808 & 9.36603 & 19.28802 & $\mathrm{H}$ & 0.73298 & 10.41447 & 11.2413 \\
$\mathrm{C}$ & 3.97911 & 7.05576 & 16.80769 & $\mathrm{H}$ & 1.75551 & 11.04301 & 9.9026 \\
$\mathrm{H}$ & 3.65003 & 7.11786 & 15.75168 & $\mathrm{C}$ & 0.35277 & 12.90292 & 12.50032
\end{tabular}

${ }^{3}$ E. D. Glendening, J. K. Badenhoop, A. E. Reed, J. E. Carpenter, J. A. Bohmann, C. M. Morales, C. R. Landis, F. Weinhold, NBO 6.0, Theoretical Chemical Institute, University of Wisconsin, Madison, WI, USA, 2013. 
7.4 Os Nitride and Imide Complexes in Various Coordination Spheres

\begin{tabular}{l|lll|l|lll}
$\mathrm{H}$ & 3.1996 & 6.53523 & 17.39826 & $\mathrm{H}$ & -0.41016 & 13.41946 & 11.88189 \\
$\mathrm{H}$ & 4.89119 & 6.42395 & 16.8395 & $\mathrm{H}$ & 0.69132 & 13.61331 & 13.28025 \\
$\mathrm{C}$ & 1.24442 & 8.95161 & 18.13942 & $\mathrm{H}$ & -0.14072 & 12.04684 & 12.98806 \\
$\mathrm{C}$ & 1.61052 & 8.27902 & 19.47197 & $\mathrm{C}$ & 0.47411 & 12.6519 & 15.94436 \\
$\mathrm{H}$ & 2.14157 & 7.31908 & 19.33018 & $\mathrm{H}$ & 1.41474 & 13.15941 & 16.18926 \\
$\mathrm{H}$ & 0.66738 & 8.05158 & 20.01155 & $\mathrm{C}$ & -0.75048 & 13.21649 & 16.27638 \\
$\mathrm{H}$ & 2.21636 & 8.93211 & 20.12947 & $\mathrm{H}$ & -0.77817 & 14.1887 & 16.78641 \\
$\mathrm{C}$ & 0.34511 & 10.16243 & 18.44393 & $\mathrm{C}$ & -1.93876 & 12.51932 & 15.95422 \\
$\mathrm{H}$ & 0.01661 & 10.68617 & 17.53138 & $\mathrm{H}$ & -2.92352 & 12.92907 & 16.21832 \\
$\mathrm{H}$ & 0.84708 & 10.89758 & 19.10255 & $\mathrm{C}$ & -1.83326 & 11.30086 & 15.29082 \\
$\mathrm{H}$ & -0.56374 & 9.80229 & 18.96924 & $\mathrm{H}$ & -2.73759 & 10.7394 & 15.02276 \\
$\mathrm{C}$ & 0.48952 & 7.94597 & 17.25146 & $\mathrm{C}$ & -0.55972 & 10.78613 & 14.95744 \\
$\mathrm{H}$ & 0.10353 & 8.42019 & 16.33257 & $\mathrm{C}$ & -0.35044 & 9.55364 & 14.21673 \\
$\mathrm{H}$ & -0.38085 & 7.54751 & 17.81307 & $\mathrm{C}$ & -1.39699 & 8.75714 & 13.69427 \\
$\mathrm{H}$ & 1.11791 & 7.08459 & 16.954 & $\mathrm{H}$ & -2.43809 & 9.07472 & 13.83551 \\
$\mathrm{C}$ & 3.8329 & 13.14633 & 14.86797 & $\mathrm{C}$ & -1.11082 & 7.58284 & 13.0056 \\
$\mathrm{H}$ & 4.30321 & 13.98665 & 15.41229 & $\mathrm{H}$ & -1.92247 & 6.96315 & 12.59879 \\
$\mathrm{C}$ & 3.59991 & 13.23834 & 13.52889 & $\mathrm{C}$ & 0.23822 & 7.20612 & 12.84448 \\
$\mathrm{H}$ & 3.89994 & 14.14262 & 12.98138 & $\mathrm{H}$ & 0.52319 & 6.28409 & 12.32038 \\
$\mathrm{C}$ & 4.35975 & 11.06492 & 11.66688 & $\mathrm{C}$ & 1.2323 & 8.02741 & 13.36862 \\
$\mathrm{C}$ & 4.0228 & 9.63429 & 11.21158 & $\mathrm{H}$ & 2.29988 & 7.77991 & 13.27578 \\
$\mathrm{H}$ & 3.09961 & 9.58201 & 10.60458 & $\mathrm{~N}$ & 3.49863 & 12.04125 & 15.642 \\
$\mathrm{H}$ & 3.92426 & 8.95915 & 12.08159 & $\mathrm{~N}$ & 0.5924 & 11.45461 & 15.30958 \\
$\mathrm{H}$ & 4.85584 & 9.25553 & 10.58272 & $\mathrm{~N}$ & 0.96205 & 9.1804 & 14.03374 \\
$\mathrm{C}$ & 5.60363 & 11.04639 & 12.57375 & $\mathrm{~N}$ & 3.66979 & 9.28501 & 14.28417 \\
$\mathrm{H}$ & 6.43608 & 10.57104 & 12.01471 & $\mathrm{P}$ & 2.76165 & 9.59285 & 17.15145 \\
$\mathrm{H}$ & 5.44479 & 10.45631 & 13.49538 & $\mathrm{P}$ & 2.90358 & 11.78878 & 12.71177 \\
$\mathrm{Os}$ & 2.51967 & 10.44596 & 14.80261 & & & & \\
& & & & & & &
\end{tabular}




\subsubsection{Crystallographic Details}

CCDC 1574571 (14), CCDC 1574572 (15), CCDC 1574573 (??), CCDC 1574574 (17), CCDC 1574575 (18), CCDC 1574576 (19) and CCDC 1574577 (20) contain the supplementary crystallographic data for this chapter. This data can be obtained free of charge via http://www.ccdc.cam.ac.uk/ products/csd/request/ (or from Cambridge Crystallographic Data Centre, 12 Union Road, Cambridge, CB2 1EZ, UK. Fax: +44-1223336-033; e-mail: deposit@ccdc.cam.ac.uk). 


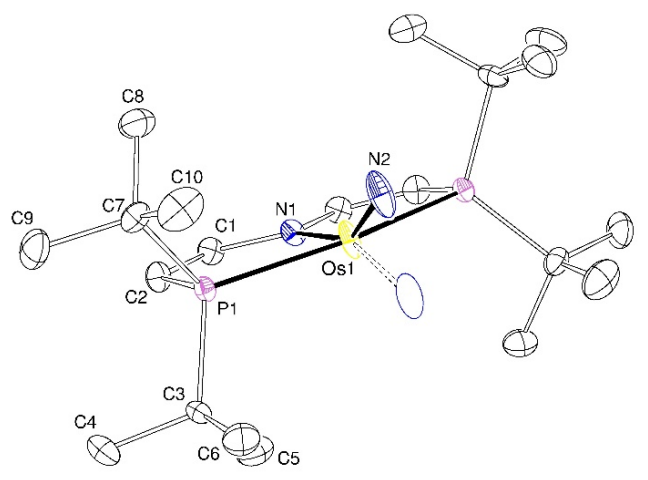

Figure 7.13: Thermal ellipsoid plot of 14 with the anisotropic displacement parameters drawn at the $50 \%$ probability level. The asymmetric unit contains a half complex molecule. The disorder was refined using PART -1 command.

Empirical formula

Formula weight

Temperature

Wavelength

Crystal system

Space group

Unit cell dimensions

Volume

Z

Density (calculated)

Absorption coefficient

$\mathrm{F}(000)$

Crystal size

Crystal shape and color

Theta range for data collection

Index ranges

Reflections collected

Independent reflections

Completeness to theta $=25.242^{\circ}$

Max. and min. transmission

Refinement method

Data / restraints / parameters

Goodness-of-fit on $\mathrm{F}^{2}$

Final R indices $[\mathrm{I}>2 \operatorname{sigma}(\mathrm{I})]$

$\mathrm{R}$ indices (all data)

Largest diff. peak and hole
$\mathrm{C}_{20} \mathrm{H}_{40} \mathrm{~N}_{2} \mathrm{OsP}_{2}$

560.68

106(2) K

$0.71073 \AA$

Monoclinic

$\mathrm{C} 2 / \mathrm{c}$

$$
\begin{array}{ll}
\mathrm{a}=22.3305(19) \AA & \alpha=90^{\circ} \\
\mathrm{b}=7.5982(6) \AA & \beta=104.423(3)^{\circ} \\
\mathrm{c}=14.0141(12) \AA & \gamma=90^{\circ}
\end{array}
$$$$
2302.9(3) \AA^{3}
$$$$
4
$$

$1.617 \mathrm{Mg} / \mathrm{m}^{3}$

$5.682 \mathrm{~mm}^{-1}$

1120

$0.349 \times 0.324 \times 0.122 \mathrm{~mm}^{3}$

Plate, clear intense brown

2.841 to $27.222^{\circ}$

$-28<=\mathrm{h}<=28$

$-9<=\mathrm{k}<=9$

$-18<=\mathrm{l}<=17$

45860

$2565[\mathrm{R}(\mathrm{int})=0.0548]$

$100.0 \%$

0.7455 and 0.4678

Full-matrix least-squares on $\mathrm{F}^{2}$

2565 / 0 / 125

1.116

$\mathrm{R} 1=0.0207$,

$\mathrm{wR} 2=0.0476$

$\mathrm{R} 1=0.0276$,

$\mathrm{wR} 2=0.0527$

0.925 and $-2.005 \mathrm{e}^{-3}$ 


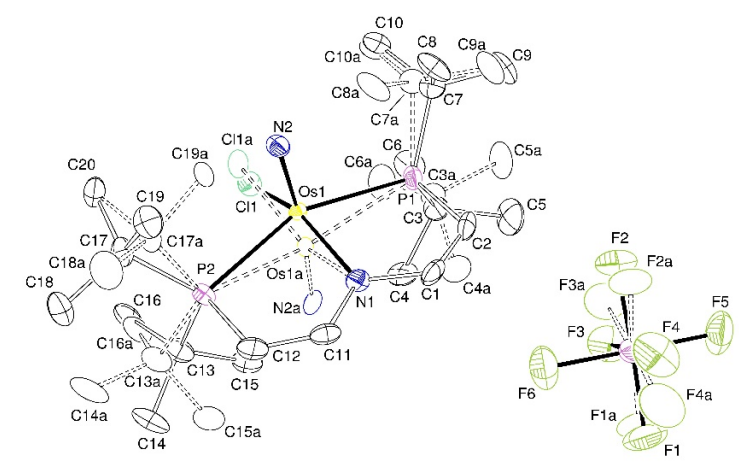

Figure 7.14: Thermal ellipsoid plot of $\mathbf{1 5}$ with the anisotropic displacement parameters drawn at the $50 \%$ probability level. The asymmetric unit contains one disordered complex molecule and one disordered PF6 anion. The disordered complex molecule was refined with population of $0.8345(8)$ on the main domain using some restraints (SADI) and constraints (EADP). The disordered PF6 anion was refined with population of 0.919(5) on the main domain using some restraints (SADI) and constraints (EADP).

Empirical formula

Formula weight

Temperature

Wavelength

Crystal system

Space group

Unit cell dimensions

Volume

Z

Density (calculated)

Absorption coefficient

$\mathrm{F}(000)$

Crystal size

Crystal shape and color

Theta range for data collection

Index ranges

Reflections collected

Independent reflections

Completeness to theta $=25.242^{\circ}$

Max. and min. transmission

Refinement method

Data / restraints / parameters

Goodness-of-fit on $\mathrm{F}^{2}$

Final $R$ indices [I>2sigma(I)]

$\mathrm{R}$ indices (all data)

Largest diff. peak and hole
$\mathrm{C}_{20} \mathrm{H}_{40} \mathrm{ClF}_{6} \mathrm{~N}_{2} \mathrm{OsP}_{3}$

741.10

100(2) K

$0.71073 \AA$

Monoclinic

$\mathrm{P} 21 / \mathrm{c}$

$\mathrm{a}=8.0653(5) \AA$

$\mathrm{b}=12.9580(8) \AA$

$\mathrm{c}=26.7808(17) \AA$

2774.6(3) $\AA^{3}$

4

$1.774 \mathrm{Mg} / \mathrm{m}^{3}$

$4.918 \mathrm{~mm}^{-1}$

1464

$0.156 \times 0.087 \times 0.032 \mathrm{~mm}^{3}$

Block, clear intense yellow

2.196 to $28.351^{\circ}$

$-10<=\mathrm{h}<=10$

$-17<=\mathrm{k}<=17$

$-35<=\mathrm{l}<=35$

119055

$6919[\mathrm{R}(\mathrm{int})=0.0745]$

$100.0 \%$

0.7457 and 0.6217

Full-matrix least-squares on $\mathrm{F}^{2}$

6919 / 71 / 437

1.286

$\mathrm{R} 1=0.0402$,

$\mathrm{wR} 2=0.0681$

$\mathrm{R} 1=0.0506$,

0.956 and $-2.530 \mathrm{e}^{-3}$

$$
\begin{aligned}
& \alpha=90^{\circ} \\
& \beta=97.546(2)^{\circ} \\
& \gamma=90^{\circ}
\end{aligned}
$$




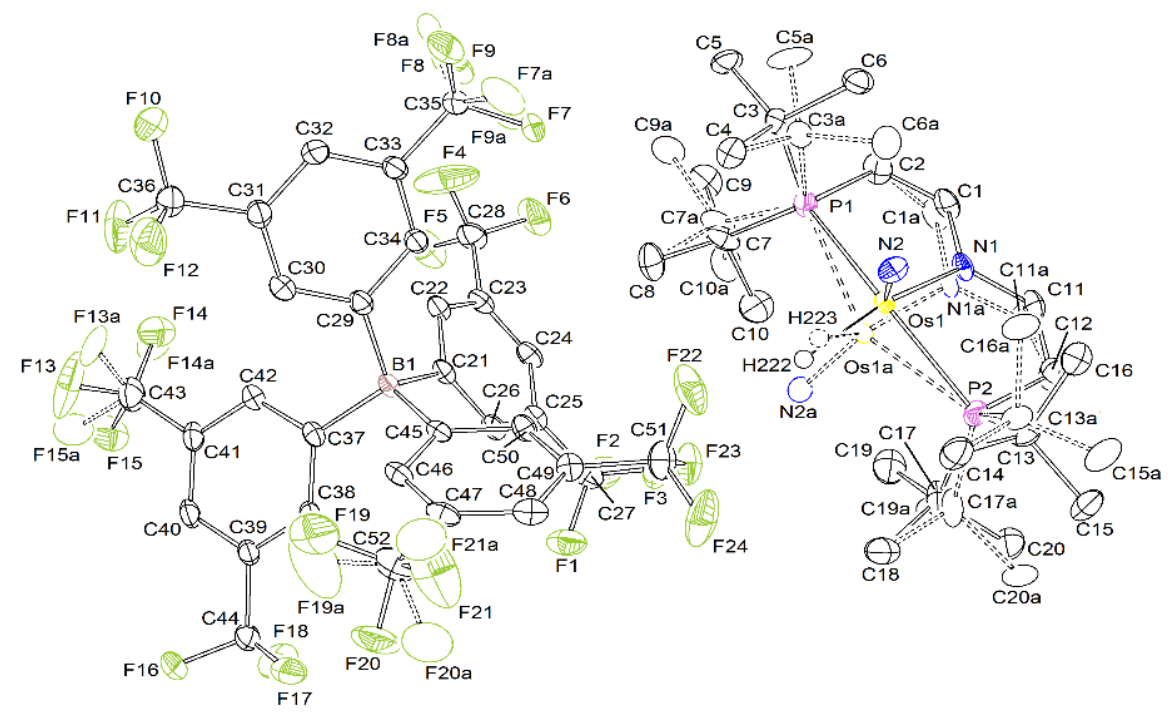

Figure 7.15: Thermal ellipsoid plot of $\mathbf{1 6}$ with the anisotropic displacement parameters drawn at the $50 \%$ probability level. The asymmetric unit contains one disordered complex molecule and one $\mathrm{BArF}_{24}$ anion with three disordered $\mathrm{CF}_{3}$ groups. The disordered complex molecule was refined with population of $0.7826(2)$ on the main domain using some restraints and constrains (SADI, EADP). The Os-H hydrogen atoms were found from the residual density map and isotropically refined. The disordered $\mathrm{CF}_{3}$ groups were refined with populations of $0.735(7)$, $0.68(1)$ and $0.958(2)$ on their main domains using some restraints and constrains (SADI, RIGU, EADP).

\section{Empirical formula \\ Formula weight \\ Temperature \\ Wavelength \\ Crystal system \\ Space group \\ Unit cell dimensions}

Volume

Z

Density (calculated)

Absorption coefficient

$\mathrm{F}(000)$

Crystal size

Crystal shape and color

Theta range for data collection

Index ranges

Reflections collected
$\mathrm{C}_{52} \mathrm{H}_{53} \mathrm{BF}_{24} \mathrm{~N}_{2} \mathrm{OsP}_{2}$

1424.91

$100(2) \mathrm{K}$

$0.71073 \AA$

Triclinic

P-1

$\mathrm{a}=11.9297(5) \AA$

$\alpha=84.800(2)^{\circ}$

$\mathrm{b}=15.5133(6) \AA$

$\beta=84.405(2)^{\circ}$

$\mathrm{c}=15.6315(7) \AA$

2856.7(2) $\AA^{3}$

2

$1.657 \mathrm{Mg} / \mathrm{m}^{3}$

$2.404 \mathrm{~mm}^{-1}$

1412

$0.315 \times 0.239 \times 0.100 \mathrm{~mm}^{3}$

Plate, clear intense yellow

2.257 to $30.508^{\circ}$

$-17<=\mathrm{h}<=17$

$-22<=\mathrm{k}<=22$

$-22<=\mathrm{l}<=22$

164409 
Independent reflections

Completeness to theta $=25.242^{\circ}$

Max. and min. transmission

Refinement method

Data / restraints / parameters

Goodness-of-fit on $\mathrm{F}^{2}$

Final $R$ indices [I $>2 \operatorname{sigma}(\mathrm{I})]$

$\mathrm{R}$ indices (all data)

Largest diff. peak and hole
$17419[\mathrm{R}(\mathrm{int})=0.0447]$

$99.9 \%$

0.7461 and 0.6166

Full-matrix least-squares on $\mathrm{F}^{2}$

17419 / 723 / 939

1.201

$\mathrm{R} 1=0.0307, \quad \quad \mathrm{wR} 2=0.0672$

$\mathrm{R} 1=0.0354$,

$\mathrm{wR} 2=0.0689$ 


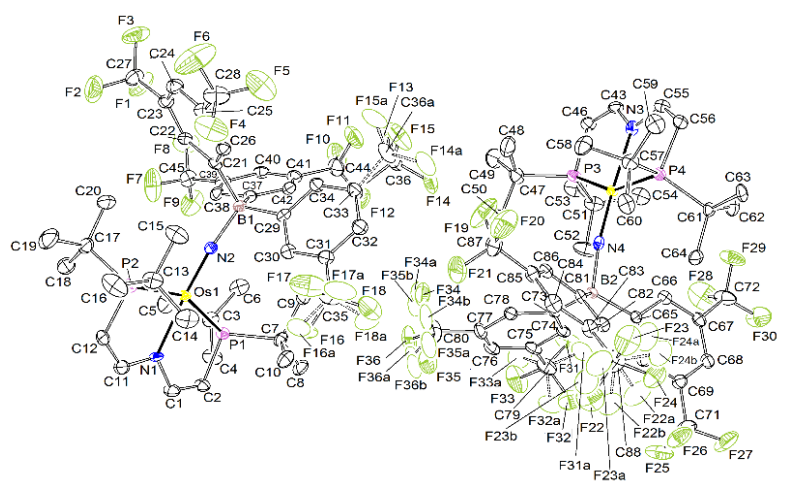

Figure 7.16: Thermal ellipsoid plot of $\mathbf{1 7}$ with the anisotropic displacement parameters drawn at the $50 \%$ probability level. The asymmetric unit contains two disordered complex molecules. Two disordered $\mathrm{CF}_{3}$ groups were refined in three different positions with population of $0.42(2)$, $0.37(2)$ and 0.207(9) using PART/SUMP commands and some restraints (SADI, RIGU). Three other disordered $\mathrm{CF}_{3}$ groups were refined in two different positions with population of $0.55(2)$, 0.64(2) and 0.74(3) on their main domains using PART commands and some restraints and constrains (SADI, RIGU, EADP). The structure was refined as twin using the twin law -100 0-10 101 (BASF: 0.0167(3)).

Empirical formula

Formula weight

Temperature

Wavelength

Crystal system

Space group

Unit cell dimensions

Volume

Z

Density (calculated)

Absorption coefficient

$\mathrm{F}(000)$

Crystal size

Crystal shape and color

Theta range for data collection

Index ranges

Reflections collected

Independent reflections

Completeness to theta $=25.242^{\circ}$

Max. and min. transmission

Refinement method

Data / restraints / parameters

Goodness-of-fit on $\mathrm{F}^{2}$

Final R indices [I $>2 \operatorname{sigma}(\mathrm{I})]$

$\mathrm{R}$ indices (all data)

Largest diff. peak and hole
$\mathrm{C}_{44} \mathrm{H}_{49} \mathrm{BF}_{18} \mathrm{~N}_{2} \mathrm{OsP}_{2}$

1210.80

100(2) K

$0.71073 \AA$

Monoclinic

$\mathrm{P} 21 / \mathrm{c}$

$\mathrm{a}=22.3365(11) \AA$

$\alpha=90^{\circ}$

$\mathrm{b}=20.8614(11) \AA$

$\beta=117.131(2)^{\circ}$

$\mathrm{c}=23.8328(13) \AA$

$9883.4(9) \AA^{3}$

8

$1.627 \mathrm{Mg} / \mathrm{m}^{3}$

$2.746 \mathrm{~mm}^{-1}$

4800

$0.374 \times 0.175 \times 0.080 \mathrm{~mm}^{3}$

Block, clear intense brown

2.154 to $27.485^{\circ}$

$-24<=\mathrm{h}<=29$

$-27<=\mathrm{k}<=27$

$-30<=\mathrm{l}<=30$

370556

$22952[\mathrm{R}(\mathrm{int})=0.1118]$

$99.9 \%$

0.7461 and 0.5790

Full-matrix least-squares on $\mathrm{F}^{2}$

22952 / 998 / 1439

1.135

$\mathrm{R} 1=0.0463$,

$\mathrm{wR} 2=0.1015$

$\mathrm{R} 1=0.0834$,

$\mathrm{wR} 2=0.1195$ 


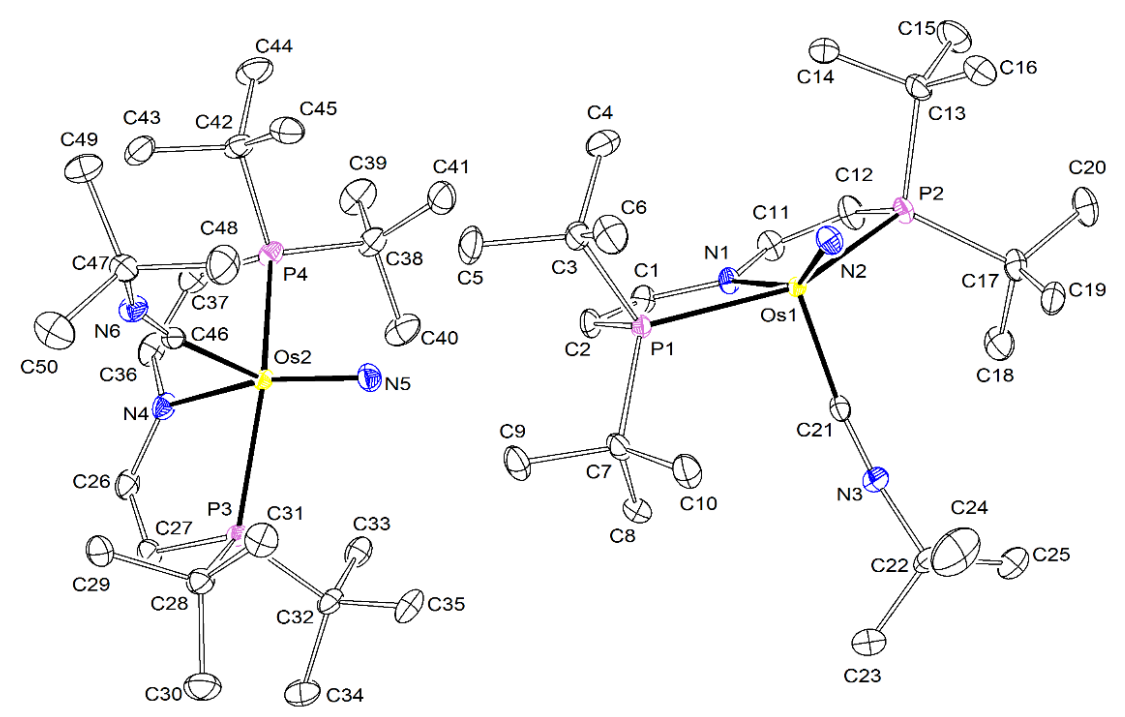

Figure 7.17: Thermal ellipsoid plot of 18 with the anisotropic displacement parameters drawn at the $50 \%$ probability level. The asymmetric unit contains two complex molecules.

Empirical formula

Formula weight

Temperature

Wavelength

Crystal system

Space group

Unit cell dimensions

Volume

Z

Density (calculated)

Absorption coefficient

$\mathrm{F}(000)$

Crystal size

Crystal shape and color

Theta range for data collection

Index ranges

Reflections collected

Independent reflections

Completeness to theta $=25.242^{\circ}$

Max. and min. transmission

Refinement method

Data / restraints / parameters

Goodness-of-fit on $\mathrm{F}^{2}$

Final R indices [I $>2 \operatorname{sigma}(\mathrm{I})]$

$\mathrm{R}$ indices (all data)

Largest diff. peak and hole

159
$\mathrm{C}_{25} \mathrm{H}_{49} \mathrm{~N}_{3} \mathrm{OsP}_{2}$

643.81

100(2) K

$0.71073 \AA$

Monoclinic

$\mathrm{P} 21 / \mathrm{c}$

$\mathrm{a}=11.8920(6) \AA$

$\alpha=90^{\circ}$

$\mathrm{b}=30.3897(15) \AA$

$\mathrm{c}=16.5634(8) \AA$

$5732.5(5) \AA^{3}$

8

$1.492 \mathrm{Mg} / \mathrm{m}^{3}$

$4.577 \mathrm{~mm}^{-1}$

2608

$0.575 \times 0.339 \times 0.262 \mathrm{~mm}^{3}$

Block, clear dark green

2.235 to $28.366^{\circ}$

$-15<=\mathrm{h}<=15$

$-40<=\mathrm{k}<=40$

$-22<=\mathrm{l}<=22$

156514

$14327[\mathrm{R}(\mathrm{int})=0.0431]$

$99.9 \%$

0.7457 and 0.4998

Full-matrix least-squares on $\mathrm{F}^{2}$

14327 / 0 / 589

1.135

$\mathrm{R} 1=0.0190$,

$\mathrm{wR} 2=0.0423$

$\mathrm{R} 1=0.0199$,

$\mathrm{wR} 2=0.0426$ $\beta=106.731(2)^{\circ}$

$\gamma=90^{\circ}$

2.001 and $-1.300 \mathrm{e}^{-3}$ 


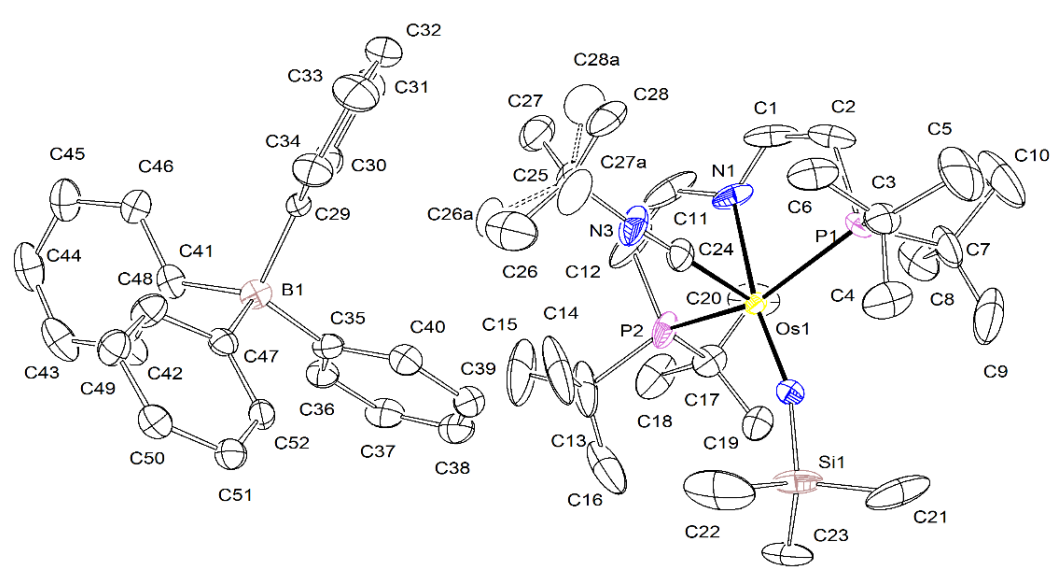

Figure 7.18: Thermal ellipsoid plot of 19 with the anisotropic displacement parameters drawn at the $50 \%$ probability level. The asymmetric unit contains one disordered complex molecule and one $\mathrm{BPh} 4$ anion. The disordered complex molecule was refined with population of $0.709(9)$ on the main domain using some restraints (SADI, RIGU).

Empirical formula

Formula weight

Temperature

Wavelength

Crystal system

Space group

Unit cell dimensions

Volume

Z

Density (calculated)

Absorption coefficient

$\mathrm{F}(000)$

Crystal size

Crystal shape and color

Theta range for data collection

Index ranges

Reflections collected

Independent reflections

Completeness to theta $=67.679^{\circ}$

Max. and min. transmission

Refinement method

Data / restraints / parameters

Goodness-of-fit on $\mathrm{F}^{2}$

Final $R$ indices [I $>2 \operatorname{sigma}(\mathrm{I})]$

$\mathrm{R}$ indices (all data)

Absolute structure parameter

Largest diff. peak and hole
$\mathrm{C}_{52} \mathrm{H}_{78} \mathrm{BN}_{3} \mathrm{OsP}_{2} \mathrm{Si}$

1036.21

129(2) KK

$1.54178 \AA$

Monoclinic

$\mathrm{P} 21$

$\begin{array}{ll}\mathrm{a}=11.5197(4) \AA & \alpha=90^{\circ} \\ \mathrm{b}=16.7973(6) \AA & \beta=90.1691(19)^{\circ} \\ \mathrm{c}=13.4774(5) \AA & \gamma=90^{\circ}\end{array}$

2607.86(16) $\AA^{3}$

2

$1.320 \mathrm{Mg} / \mathrm{m}^{3}$

$5.676 \mathrm{~mm}^{-1}$

1072

$0.258 \times 0.176 \times 0.081 \mathrm{~mm}^{3}$

Plate,

clear dark brown

3.279 to $74.615^{\circ}$

$-14<=\mathrm{h}<=14$

$-18<=\mathrm{k}<=20$

$-16<=\mathrm{l}<=16$

94546

$10499[\mathrm{R}(\mathrm{int})=0.0554]$

$99.9 \%$

0.7538 and 0.5666

Full-matrix least-squares on $\mathrm{F}^{2}$

10499 / 67 / 590

1.088

$\mathrm{R} 1=0.0248$,

$\mathrm{wR} 2=0.0486$

$\mathrm{R} 1=0.0284$,

$\mathrm{wR} 2=0.0496$

0.384 and $-0.675 \mathrm{e}^{-3}$ 


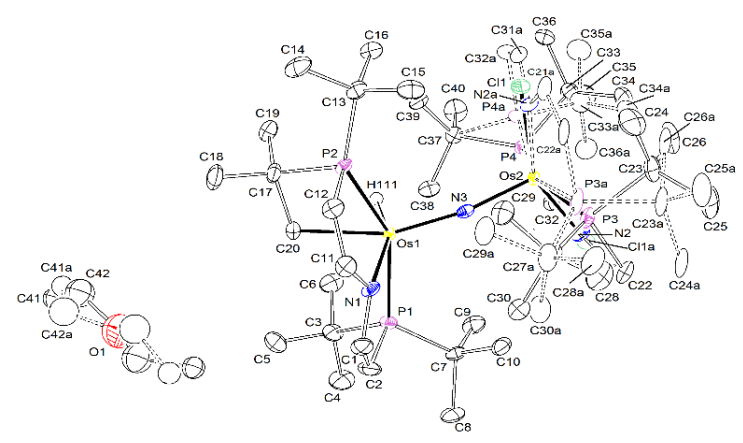

Figure 7.19: Thermal ellipsoid plot of 20 with the anisotropic displacement parameters drawn at the $50 \%$ probability level. The asymmetric unit contains one disordered complex molecule and a quarter of a disordered diethyl ether solvent molecule. The disordered complex molecule was refined with population of $0.933(2)$ on the main domain using some restraints and constraints (SADI, RIGU, EADP). The disordered diethyl ether solvent molecule was refined isotropically with population of $0.58(2)$ on the main domain using some restraints and constraints (SADI, RIGU, EADP). The N-H hydrogen atom was found from the residual density map and isotropically refined. The reflections $-311,510$ and 4489 are removed from the refinement using OMIT commands.

Empirical formula
Formula weight
Temperature
Wavelength
Crystal system
Space group
Unit cell dimensions

Volume

Z

Density (calculated)

Absorption coefficient

$\mathrm{F}(000)$

Crystal size

Crystal shape and color

Theta range for data collection

Index ranges

Reflections collected

Independent reflections

Completeness to theta $=25.242^{\circ}$

Max. and min. transmission

Refinement method

Data / restraints / parameters

Goodness-of-fit on $\mathrm{F}^{2}$

Final R indices [I>2sigma(I)]

$\mathrm{R}$ indices (all data)

Largest diff. peak and hole

$$
\begin{array}{ll}
\mathrm{C}_{40} \mathrm{H}_{80} \mathrm{ClN}_{3} \mathrm{Os}_{2} \mathrm{P}_{4} \times 0.25 \mathrm{C}_{4} \mathrm{H}_{10} \mathrm{O} & \\
1161.33 & \\
100(2) \mathrm{K} & \\
0.71073 \AA & \\
\text { Monoclinic } & \\
\mathrm{C} 2 / \mathrm{c} & \alpha=90^{\circ} \\
\mathrm{a}=40.8402(17) \AA & \beta=92.098(2)^{\circ} \\
\mathrm{b}=19.2523(8) \AA & \gamma=90^{\circ} \\
\mathrm{c}=12.4764(5) \AA &
\end{array}
$$

9803.2(7) $\AA^{3}$

8

$1.574 \mathrm{Mg} / \mathrm{m}^{3}$

$5.395 \mathrm{~mm}^{-1}$

4644

$0.158 \times 0.119 \times 0.057 \mathrm{~mm}^{3}$

Plate, clear dark brown

2.339 to $28.333^{\circ}$

$-54<=\mathrm{h}<=54$

$-25<=\mathrm{k}<=25$

$-16<=\mathrm{l}<=16$

158832

$12204[\mathrm{R}(\mathrm{int})=0.0678]$

$99.9 \%$

0.7457 and 0.6157

Full-matrix least-squares on $\mathrm{F}^{2}$

12204 / 324 / 657

1.051

$\mathrm{R} 1=0.0253$,

$\mathrm{wR} 2=0.0488$

$\mathrm{R} 1=0.0369$,

$\mathrm{wR} 2=0.0519$

2.242 and $-1.627 \mathrm{e}^{-3}$ 


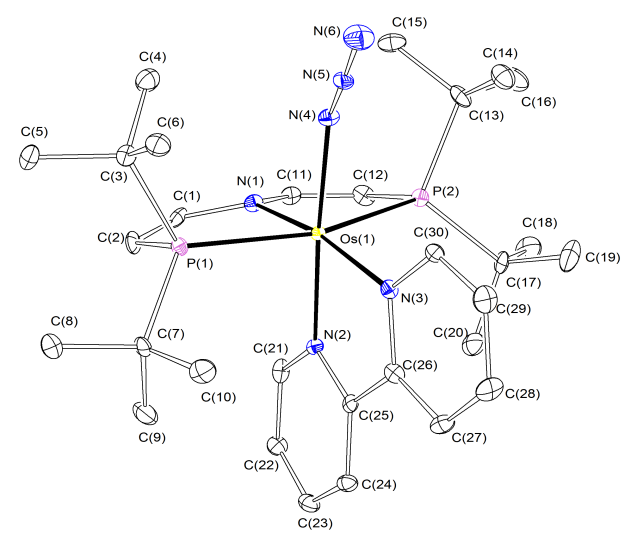

Figure 7.20: Thermal ellipsoid plot of $\mathbf{2 1}$ with the anisotropic displacement parameters drawn at the $50 \%$ probability level.

Empirical formula

Formula weight

Temperature

Wavelength

Crystal system

Space group

Unit cell dimensions

Volume

Z

Density (calculated)

Absorption coefficient

$\mathrm{F}(000)$

Crystal size

Crystal shape and color

Theta range for data collection

Index ranges

Reflections collected

Independent reflections

Completeness to theta $=25.242^{\circ}$

Max. and min. transmission

Refinement method

Data / restraints / parameters

Goodness-of-fit on $\mathrm{F}^{2}$

Final $\mathrm{R}$ indices $\left[\mathrm{I}_{i} 2 \operatorname{sigma}(\mathrm{I})\right]$

$\mathrm{R}$ indices (all data)

Largest diff. peak and hole
$\mathrm{C}_{30} \mathrm{H}_{48} \mathrm{~N}_{6} \mathrm{OsP}_{2}$

744.88

$100(2) \mathrm{K}$

$0.71073 \AA$

Monoclinic

$\mathrm{P} 22_{1} / \mathrm{n}$

$\mathrm{a}=9.1148(3) \AA$

$\alpha=90^{\circ}$

$\mathrm{b}=16.6248(7) \AA$

$\mathrm{c}=21.0265(8) \AA$

$3172.1(2) \AA^{3}$

4

$1.560 \mathrm{Mg} / \mathrm{m}^{3}$

$4.150 \mathrm{~mm}^{-1}$

1504

$0.288 \times 0.169 \times 0.058 \mathrm{~mm}^{3}$

Plate, dark purple

1.946 to $28.368^{\circ}$

$-12<=\mathrm{h}<=12$

$-22<=\mathrm{k}<=22$

$-28<=\mathrm{l}<=28$

124034

$7925[\mathrm{R}(\mathrm{int})=0.1149]$

$100.0 \%$

0.7457 and 0.5574

Full-matrix least-squares on $\mathrm{F}^{2}$

7925 / 0 / 364

1.049

$\mathrm{R} 1=0.0299$,

$\mathrm{wR} 2=0.0439$

$\mathrm{R} 1=0.0490$,

0.665 and $-1.204 \mathrm{e}^{-3}$ $\beta=95.383(2)^{\circ}$

$\gamma=90^{\circ}$ 


\subsubsection{Synthesis}

\section{Synthesis of 14}

Route A: 1 ( $85.5 \mathrm{mg}, 138 \mu \mathrm{mol}, 1.00$ eq.) and $\mathrm{CoCp}_{2}$ (26.2mg, $138 \mu \mathrm{mol}, 1.00$ eq.) are dissolved in THF (10 mL) and stirred for 1 minute under $\mathrm{N}_{2}$ atmosphere. The solution is filtered into a solution of [PPN]N $\mathrm{N}_{3}(400 \mathrm{mg}, 69 \mathrm{mmol}, 5.0$ eq.) and stirred for $10 \mathrm{~min}$. The solvent is removed and the residue is extracted with benzene $(3 \times 10 \mathrm{~mL})$ and pentanes $(10 \times 5 \mathrm{~mL})$. The solution is concentrated and cooled at $-90^{\circ} \mathrm{C}$ for $16 \mathrm{~h}$. The solution is filtered at $-90^{\circ} \mathrm{C}$ and the residue is washed with pentane $(2 \times 5 \mathrm{~mL})$. The product is extracted with benzene $(3 \times 3 \mathrm{~mL})$ and lyophilization yields 14 as a brown powder (48.0 mg, $85.4 \mu \mathrm{mol}, 62 \%)$.

Route B: Complex 16 (10.0 mg, $13.5 \mu \mathrm{mol}, 1.00$ eq.) is dissolved in THF (5 mL). NaHg ( $620 \mathrm{mg}, 0.1 \mathrm{w} \%, 27.0 \mu \mathrm{mol}, 2.00 \mathrm{eq}$.$) is added and the solution is stirred for 5$ minutes. After evaporation of the solvent the crude product is extracted with pentane ( $4 \times 2 \mathrm{~mL})$. [OsN $\left.\left(\mathrm{L}^{3}\right)\right](7.2 \mathrm{mg}, 12.8 \mu \mathrm{mol}, 95 \%)$ is obtained as a brown solid.

Anal. Calcd for $\mathrm{C}_{20} \mathrm{H}_{40} \mathrm{~N}_{2} \mathrm{OsP}_{2}$ (560.73): C, 42.8; H, 7.19; N, 5.00 Found: C, 42.7; H, 7.13; N, 4.90. NMR $\left(\mathrm{C}_{6} \mathrm{D}_{6}, \mathrm{RT}\right):{ }^{1} \mathrm{H}(400 \mathrm{MHz}): \delta=6.99$ (ABXX'B'A', $N=\left.\right|^{3} J_{\mathrm{AX}}$ $\left.+{ }^{4} J_{\mathrm{AX}}, \mid=17.1 \mathrm{~Hz},{ }^{3} J_{\mathrm{AB}}=6.1 \mathrm{~Hz} .2 \mathrm{H}, \mathrm{NCH}\right), 4.56$ (ABXX'B'A', $N=\left|{ }^{2} J_{\mathrm{AX}}+{ }^{4} J_{\mathrm{AX}}{ }^{\prime}\right|$ $\left.=3.7 \mathrm{~Hz},{ }^{3} J_{\mathrm{AB}}=6.1 \mathrm{~Hz}, 2 \mathrm{H}, \mathrm{PC} H\right), 1.54\left(\mathrm{~A}_{18} \mathrm{XX}^{\prime} \mathrm{A}^{\prime}{ }_{18}, N=\left|{ }^{3} J_{\mathrm{AX}}+{ }^{5} J_{\mathrm{AX}}\right|=7.1 \mathrm{~Hz}\right.$, $\left.36 \mathrm{H}, \mathrm{P}\left(\mathrm{C}\left(\mathrm{CH}_{3}\right)_{3}\right)\right) .{ }^{13} \mathrm{C}\left\{{ }^{1} \mathrm{H}\right\}(125.76 \mathrm{MHz}): \delta=166.8$ (AXX'A', $N=\left|{ }^{2} J_{\mathrm{AX}}+{ }^{3} J_{\mathrm{AX}}{ }^{\prime}\right|$ $=9.0 \mathrm{~Hz}, 2 \mathrm{C}, \mathrm{NCH}), 89.2\left(\mathrm{AXX}^{\prime} \mathrm{A}^{\prime},{ }^{1} J_{\mathrm{CP}}=24.3 \mathrm{~Hz} .{ }^{3} J_{\mathrm{CP}}=22.6 \mathrm{~Hz}, 2 \mathrm{C}, \mathrm{PCH}\right), 37.8$ $\left(\mathrm{A}_{2} \mathrm{XX}^{\prime} \mathrm{A}^{\prime}{ }_{2}, N=\left|{ }^{1} J_{\mathrm{AX}}+{ }^{3} J_{\mathrm{AX}}\right|=12.7 \mathrm{~Hz}, 4 \mathrm{C}, \mathrm{P}\left(C\left(\mathrm{CH}_{3}\right)_{3}\right)_{2}\right), 30.8\left(\mathrm{~A}_{6} \mathrm{XX}^{\prime} \mathrm{A}^{\prime}{ }_{6}, N=\right.$ $\left.\left|{ }^{2} J_{\mathrm{AX}}+{ }^{4} J_{\mathrm{AX}},\right|=2.9 \mathrm{~Hz}, 12 \mathrm{C}, \mathrm{P}\left(\mathrm{C}\left(\mathrm{CH}_{3}\right)_{3}\right)_{2}\right) .{ }^{31} \mathrm{P}\left\{{ }^{1} \mathrm{H}\right\}(161.25 \mathrm{MHz}): \delta=81.2(\mathrm{~s}, 2 \mathrm{P}$, $\left.P\left(\mathrm{C}\left(\mathrm{CH}_{3}\right)_{3}\right)_{2}\right)$. MS (LIFDI, toluene): $m / z=562.1\left(100 \%,\left[\mathrm{M}^{+}\right]\right) . \mathrm{IR}(\mathrm{KBr}): \tilde{\nu}=1025$ $(\mathrm{Os} \equiv \mathrm{N})$.

\section{Synthesis of 15}

1 (100 mg, $162 \mu \mathrm{M}, 1.00$ eq.) and $\left[\mathrm{Ph}_{3} \mathrm{C}\right]\left[\mathrm{PF}_{6}\right](62.0 \mathrm{mg}, 160 \mu \mathrm{M}, 0.99$ eq.) are dissolved in THF $(5 \mathrm{~mL}) . \mathrm{TMSN}_{3}(21.2 \mu \mathrm{L}, 160 \mu \mathrm{M}, 0.99$ eq.) in THF $(5 \mathrm{~mL})$ is slowly added and the reaction is stirred for $3 \mathrm{~h}$. After evaporation of the solvent the crude product is washed with $\mathrm{Et}_{2} \mathrm{O}(4 \times 10 \mathrm{~mL})$ and extracted with DCM $(2 \times 5 \mathrm{~mL})$. The solution is layered with $\mathrm{Et}_{2} \mathrm{O}(100 \mathrm{~mL})$ and stored at $-35^{\circ} \mathrm{C}$ for $16 \mathrm{~h}$. After filtration the residue is washed with $\mathrm{Et}_{2} \mathrm{O}(2 \times 5 \mathrm{~mL})$ and extracted with DCM $(2 \times 5 \mathrm{~mL})$. Evaporation of the solvent gives 15 as an orange powder ( $80.0 \mathrm{mg}, 108 \mu \mathrm{M}, 67 \%)$.

Anal. Calcd for $\mathrm{C}_{20} \mathrm{H}_{40} \mathrm{ClF}_{6} \mathrm{~N}_{2} \mathrm{OsP}_{3}$ (741.13): C, 32.4; H, 5.44; N, 3.77 Found: C, 32.6; $\mathrm{H}, 5.52 ; \mathrm{N}, 3.70$. NMR $\left(\mathrm{CD}_{2} \mathrm{Cl}_{2}, \mathrm{RT}\right):{ }^{1} \mathrm{H}(500 \mathrm{MHz}): \delta=7.76$ (ABXX'B'A', $N=\left.\right|^{3} J_{\mathrm{AX}}$ $\left.+{ }^{4} J_{\mathrm{AX}} \mid=19.0 \mathrm{~Hz},{ }^{3} J_{\mathrm{AB}}=6.4 \mathrm{~Hz} .2 \mathrm{H}, \mathrm{NC} H\right), 5.09$ (ABXX'B'A', $N=\left|{ }^{2} J_{\mathrm{AX}}+{ }^{4} J_{\mathrm{AX}}{ }^{\prime}\right|$ $\left.=5.1 \mathrm{~Hz},{ }^{3} J_{\mathrm{AB}}=6.4 \mathrm{~Hz}, 2 \mathrm{H}, \mathrm{PCH}\right), 1.59\left(\mathrm{~A}_{9} \mathrm{XX}^{\prime} \mathrm{A}^{\prime}{ }_{9}, N=\left|{ }^{3} J_{\mathrm{AX}}+{ }^{5} J_{\mathrm{AX}}\right|=8.5 \mathrm{~Hz}\right.$, $\left.18 \mathrm{H}, \mathrm{P}\left(\mathrm{C}\left(\mathrm{CH}_{3}\right)_{3}\right)\right), 1.37\left(\mathrm{~A}_{9} \mathrm{XX}{ }^{\prime}{ }^{\prime}{ }_{9}, N=\left|{ }^{3} J_{\mathrm{AX}}+{ }^{5} J_{\mathrm{AX}}\right|=8.3 \mathrm{~Hz}, 18 \mathrm{H}, \mathrm{P}\left(\mathrm{C}\left(\mathrm{CH}_{3}\right)_{3}\right)\right)$. $\left.{ }^{13} \mathrm{C}^{1}{ }^{1} \mathrm{H}\right\}(125.76 \mathrm{MHz}): \delta=170.0\left(\mathrm{AXX}^{\prime} \mathrm{A}^{\prime}, N=\left|{ }^{2} J_{\mathrm{AX}}+{ }^{3} J_{\mathrm{AX}}\right|=3.6 \mathrm{~Hz}, 2 \mathrm{C}, \mathrm{N} C \mathrm{H}\right.$ ), 93.0 (AXX'A', $N=\left|{ }^{1} J_{\mathrm{AX}}+{ }^{3} J_{\mathrm{AX}}\right|=23.5 \mathrm{~Hz}, 2 \mathrm{C}, \mathrm{PC} H$ ), $40.2\left(\right.$ AXX'A'$^{\prime}, N=\mid{ }^{1} J_{\mathrm{AX}}$ $+{ }^{3} J_{\mathrm{AX}} \mid=12 \mathrm{~Hz}, 6 \mathrm{C}, \mathrm{P}\left(C\left(\mathrm{CH}_{3}\right)_{3}\right), 38.9\left(\mathrm{AXX}^{\prime} \mathrm{A}^{\prime}, N=\left|{ }^{1} J_{\mathrm{AX}}+{ }^{3} J_{\mathrm{AX}}\right|=9.7 \mathrm{~Hz}, 6 \mathrm{C}\right.$, $\mathrm{P}\left(C\left(\mathrm{CH}_{3}\right)_{3}\right), 28.9\left(\mathrm{~A}_{3} \mathrm{XX}^{\prime} \mathrm{A}_{3}^{\prime}, N=\left|{ }^{2} J_{\mathrm{AX}}+{ }^{4} J_{\mathrm{AX}}\right|=1.8 \mathrm{~Hz}, 6 \mathrm{C}, \mathrm{P}\left(\mathrm{C}\left(C \mathrm{CH}_{3}\right)_{3}\right), 28.9(\mathrm{~s}\right.$ br, $6 \mathrm{C}, \mathrm{P}\left(\mathrm{C}\left(\mathrm{CH}_{3}\right)_{3}\right) .{ }^{31} \mathrm{P}\left\{{ }^{1} \mathrm{H}\right\}(161.25 \mathrm{MHz}): \delta=91.5\left(\mathrm{~s}, 2 \mathrm{P}, P\left(\mathrm{C}\left(\mathrm{CH}_{3}\right)_{3}\right)_{2}\right),-145(\mathrm{~h}$, $\left.{ }^{2} J_{\mathrm{PF}}=711 \mathrm{~Hz}, \mathrm{PF}_{6}\right)$. 


\section{Synthesis of 16}

$14(5.0 \mathrm{mg}, 8.9 \mu \mathrm{mol}, 1.0$ eq. $)$ is dissolved in $\mathrm{Et}_{2} \mathrm{O}(2 \mathrm{~mL})$ and cooled to $-35^{\circ} \mathrm{C}$. Brookhart's acid $(9.0 \mathrm{mg}, 8.9 \mu \mathrm{mol}, 1.0 \mathrm{eq}$.) is added and the solution is stirred for $10 \mathrm{~min}$. The solvent is removed and the residue washed with pentanes $(5 \times 1 \mathrm{~mL})$ and extracted with $\mathrm{Et}_{2} \mathrm{O}(2 \times 1 \mathrm{~mL})$. The solvent is removed and $\mathbf{1 6}$ is isolated in form of a yellow solid (10.0 mg, $7.00 \mu \mathrm{mol}, 80 \%)$.

Anal. Calcd for $\mathrm{C}_{52} \mathrm{H}_{53} \mathrm{BF}_{24} \mathrm{~N}_{2} \mathrm{OsP}_{2}$ (1242.96): C, 43.8; H, 3.75; N, 1.97 Found: C, 43.7; $\mathrm{H}, 3.83 ; \mathrm{N}, 1.93$. NMR $\left(\mathrm{CD}_{2} \mathrm{Cl}_{2}, \mathrm{RT}\right):{ }^{1} \mathrm{H}(500 \mathrm{MHz}): \delta=7.74-7.71\left(\mathrm{~m}, 8 \mathrm{H}, \mathrm{C}_{\text {ortho }} H\right)$, 7.59 (ABXX'B'A', $\left.N=\left|{ }^{3} J_{\mathrm{AX}}+{ }^{4} J_{\mathrm{AX}}\right|=19.0 \mathrm{~Hz},{ }^{3} J_{\mathrm{AB}}=6.3 \mathrm{~Hz} .2 \mathrm{H}, \mathrm{NCH}\right), 7.58-$ $7.55\left(\mathrm{~m}, 4 \mathrm{H}, \mathrm{C}_{\mathrm{para}} H\right), 4.99$ (ABXX'B'A', $N=\left|{ }^{2} J_{\mathrm{AX}}+{ }^{4} J_{\mathrm{AX}}{ }^{\prime}\right|=5.3 \mathrm{~Hz},{ }^{3} J_{\mathrm{AB}}=6.3 \mathrm{~Hz}$, $2 \mathrm{H}, \mathrm{PC} H), 1.49\left(\mathrm{~A}_{9} \mathrm{XX}^{\prime} \mathrm{A}^{\prime}{ }_{9}, N=\left|{ }^{3} J_{\mathrm{AX}}+{ }^{5} J_{\mathrm{AX}}\right|=8.2 \mathrm{~Hz}, 18 \mathrm{H}, \mathrm{P}\left(\mathrm{C}\left(\mathrm{CH}_{3}\right)_{3}\right)\right), 1.26$ $\left(\mathrm{A}_{9} \mathrm{XX}^{\prime} \mathrm{A}_{9}^{\prime}, N=\left|{ }^{3} J_{\mathrm{AX}}+{ }^{5} J_{\mathrm{AX}}\right|=8.2 \mathrm{~Hz}, 18 \mathrm{H}, \mathrm{P}\left(\mathrm{C}\left(\mathrm{CH}_{3}\right)_{3}\right)\right), 0.47\left(\mathrm{t},{ }^{2} J_{\mathrm{HP}}=13.5 \mathrm{~Hz}\right.$, Os $H) .{ }^{13} \mathrm{C}\left\{{ }^{1} \mathrm{H}\right\}(125.76 \mathrm{MHz}): \delta=168.3$ (AXX'A', $N=\left|{ }^{2} J_{\mathrm{AX}}+{ }^{3} J_{\mathrm{AX}} \cdot\right|=5.3 \mathrm{~Hz}, 2 \mathrm{C}$, $\mathrm{NCH}), 162.3\left(\mathrm{q},{ }^{1} J_{\mathrm{CB}}=49.8 \mathrm{~Hz}, 4 \mathrm{C}, C_{\mathrm{ipso}}\right), 135.3\left(\mathrm{~s}, 8 \mathrm{C}, C_{\text {ortho }}\right), 129.4$ (qq, ${ }^{2} J_{\mathrm{CF}}=$ $\left.31.5 \mathrm{~Hz},{ }^{3} J_{\mathrm{CB}}=2.8 \mathrm{~Hz}, 8 \mathrm{C}, C_{\mathrm{meta}}\right), 125.1\left(\mathrm{q},{ }^{1} J_{\mathrm{CF}}=272.4 \mathrm{~Hz}, 8 \mathrm{C}, C \mathrm{~F}_{3}\right), 118.0(\mathrm{~h}$, ${ }^{3} J_{\mathrm{CF}}=4.0 \mathrm{~Hz}, 4 \mathrm{C}, C_{\text {para }}$ ), 90.5 (AXX'A', $N=\left|{ }^{1} J_{\mathrm{AX}}+{ }^{3} J_{\mathrm{AX}}\right|=23.9 \mathrm{~Hz}, 2 \mathrm{C}, \mathrm{PC} H$ ), $38.4-38.0\left(\mathrm{~m}, 2 \mathrm{C}, \mathrm{P}\left(C\left(\mathrm{CH}_{3}\right)_{3}\right), 29.1\left(\mathrm{~A}_{3} \mathrm{XX}^{\prime} \mathrm{A}^{\prime}{ }_{3}, N=\left|{ }^{2} J_{\mathrm{AX}}+{ }^{4} J_{\mathrm{AX}}\right|=1.8 \mathrm{~Hz}, 6 \mathrm{C}\right.\right.$, $\mathrm{P}\left(\mathrm{C}\left(\mathrm{CH}_{3}\right)_{3}\right), 28.9\left(\mathrm{~A}_{3} \mathrm{XX}^{\prime} \mathrm{A}_{3}{ }_{3}, N=\left|{ }^{2} J_{\mathrm{AX}}+{ }^{4} J_{\mathrm{AX}},\right|=2.3 \mathrm{~Hz}, 6 \mathrm{C}, \mathrm{P}\left(\mathrm{C}\left(C \mathrm{H}_{3}\right)_{3}\right) .{ }^{31} \mathrm{P}\left\{{ }^{1} \mathrm{H}\right\}\right.$ $(161.25 \mathrm{MHz}): \delta=101.5\left(\mathrm{~s}, 2 \mathrm{P}, P\left(\mathrm{C}\left(\mathrm{CH}_{3}\right)_{3}\right)_{2}\right)$.

\section{Synthesis of 17}

$17(5.0 \mathrm{mg}, 8.9 \mu \mathrm{mol}, 1.0 \mathrm{eq}$.$) and \mathrm{BArF}_{18}(5.8 \mathrm{mg}, 8.9 \mu \mathrm{mol}, 1.0$ eq. $)$ are dissolved in benzene $(2 \mathrm{~mL})$ and stirred for 5 minutes. The crude product is purified via column chromatography over silanized silica (benzene). Lyophilization yields $\mathbf{1 7}$ as a red powder (9.1 mg, $7.5 \mu \mathrm{mol}, 85 \%)$.

Anal. Calcd for $\mathrm{C}_{44} \mathrm{H}_{49} \mathrm{BF}_{18} \mathrm{~N}_{2} \mathrm{OsP}_{2}$ (1210.85): C, 43.7; H, 4.08; N, 2.31 Found: C, 43.4; H, 3.90; N, 2.35. NMR ( $d_{6}$-benzene, RT): ${ }^{1} \mathrm{H}(500 \mathrm{MHz}): \delta=8.31-8.29$ (br, $\left.6 \mathrm{H}, \mathrm{C}_{\text {ortho }} H\right), 7.77-7.75\left(\mathrm{br}, 3 \mathrm{H}, \mathrm{C}_{\text {para }} H\right), 5.69$ (ABXX'B'A', $N=\left|{ }^{3} J_{\mathrm{AX}}+{ }^{4} J_{\mathrm{AX}}\right|=$ $\left.18.0 \mathrm{~Hz},{ }^{3} J_{\mathrm{AB}}=6.3 \mathrm{~Hz} .2 \mathrm{H}, \mathrm{NCH}\right), 4.17$ (ABXX'B'A', $N=\left|{ }^{2} J_{\mathrm{AX}}+{ }^{4} J_{\mathrm{AX}}{ }^{\prime}\right|=4.4 \mathrm{~Hz}$, $\left.{ }^{3} J_{\mathrm{AB}}=6.3 \mathrm{~Hz}, 2 \mathrm{H}, \mathrm{PC} H\right), 1.04\left(\mathrm{~A}_{18} \mathrm{XX}^{\prime} \mathrm{A}^{\prime}{ }_{18}, N=\left|{ }^{3} J_{\mathrm{AX}}+{ }^{5} J_{\mathrm{AX}} \cdot\right|=7.5 \mathrm{~Hz}, 36 \mathrm{H}\right.$, $\left.\mathrm{P}\left(\mathrm{C}\left(\mathrm{CH}_{3}\right)_{3}\right)\right) .{ }^{13} \mathrm{C}\left\{{ }^{1} \mathrm{H}\right\}(125.76 \mathrm{MHz}): \delta=162.7$ (AXX'A', $N=\left|{ }^{2} J_{\mathrm{AX}}+{ }^{3} J_{\mathrm{AX}}{ }^{\prime}\right|=$ $8.6 \mathrm{~Hz}, 2 \mathrm{C}, \mathrm{NCH}), 147.3\left(\mathrm{br}, 1 \mathrm{C}, C_{\text {ipso }}\right), 135.9\left(\mathrm{~s}, 6 \mathrm{C}, C_{\text {ortho }} \mathrm{H}\right), 130.2\left(\mathrm{q},{ }^{2} J_{\mathrm{CF}}=\right.$ $\left.32.14 \mathrm{~Hz}, 6 \mathrm{C}, C_{\text {meta }} \mathrm{H}\right), 124.5\left(\mathrm{q},{ }^{1} J_{\mathrm{CF}}=273.4 \mathrm{~Hz}, 6 \mathrm{C}, \mathrm{CF}_{3}\right), 120.4\left(\mathrm{~h},{ }^{3} J_{\mathrm{CF}}=4.0 \mathrm{~Hz}, 3 \mathrm{C}\right.$, $\left.C_{\text {para }} \mathrm{H}\right), 94.2\left(\right.$ AXX'A' $\left., N=\left|{ }^{1} J_{\mathrm{AX}}+{ }^{3} J_{\mathrm{AX}}\right|=24.2 \mathrm{~Hz}, 2 \mathrm{C}, \mathrm{PC} H\right), 38.3\left(\mathrm{~A}_{2} \mathrm{XX}^{\prime} \mathrm{A}^{\prime}{ }_{2}, N\right.$ $\left.=\left|{ }^{1} J_{\mathrm{AX}}+{ }^{3} J_{\mathrm{AX}}\right|=12.0 \mathrm{~Hz}, 4 \mathrm{C}, \mathrm{P}\left(C\left(\mathrm{CH}_{3}\right)_{3}\right)_{2}\right), 30.4\left(\mathrm{~A}_{6} \mathrm{XX}^{\prime} \mathrm{A}_{6}^{\prime}, N=\left|{ }^{2} J_{\mathrm{AX}}+{ }^{4} J_{\mathrm{AX}}\right|\right.$ $\left.=2.4 \mathrm{~Hz}, 12 \mathrm{C}, \mathrm{P}\left(\mathrm{C}\left(\mathrm{CH}_{3}\right)_{3}\right)_{2}\right) .{ }^{31} \mathrm{P}\left\{{ }^{1} \mathrm{H}\right\}(161.25 \mathrm{MHz}): \delta=82.7\left(\mathrm{~s}, 2 \mathrm{P}, \mathrm{P}\left(\mathrm{C}\left(\mathrm{CH}_{3}\right)_{3}\right)_{2}\right.$.

\section{Hydrogenolysis of 14}

$14(9.8 \mathrm{mg}, 17.5 \mathrm{mmol}, 1.0$ eq. $)$ is dissolved in toluene- $\mathrm{d}_{8}(0.5 \mathrm{~mL})$ and heated to $100^{\circ} \mathrm{C}$ for $48 \mathrm{~h}$ under a dihydrogen atmosphere $(1 \mathrm{bar})$. The reaction is followed by ${ }^{31} \mathrm{P}$ NMR spectroscopy with $\mathrm{PPh}_{3} \mathrm{O}$ as internal standard (capillary). Ammonia was quantified by indophenolic titration. 


\section{Synthesis of 18}

$14\left(20.4 \mathrm{mg}, 36.1 \mu \mathrm{mol}, 1.00\right.$ eq.) and $\mathrm{CN}^{t} \mathrm{Bu}(4.3 \mathrm{mg}, 38 \mu \mathrm{mol}, 1.1$ eq.) are dissolved in thawing benzene $(5 \mathrm{~mL})$ and stirred for $10 \mathrm{~s}$. The solvent is evacuated and $\mathbf{1 8}$ is isolated in form of a green solid $(23.1 \mathrm{mg}, 36.1 \mu \mathrm{mol}$, quant.). 18 is only stable at low temperatures $\left(<-50^{\circ} \mathrm{C}\right)$ and cannot be stored as a solid without decomposition over the course of a few hours which prevented reproducible characterization by combustion analysis. Small amounts of benzene are still visible in the NMR spectra.

$\operatorname{NMR}\left(d_{8}-\mathrm{THF},-50^{\circ} \mathrm{C}\right):{ }^{1} \mathrm{H}(400 \mathrm{MHz}): \delta=6.94$ (ABXX'B'A', $N=\left|{ }^{3} J_{\mathrm{AX}}+{ }^{4} J_{\mathrm{AX}}\right|$ $\left.=16.3 \mathrm{~Hz},{ }^{3} J_{\mathrm{AB}}=5.7 \mathrm{~Hz} .2 \mathrm{H}, \mathrm{NCH}\right), 5.74\left(\mathrm{ABXX}{ }^{\prime} \mathrm{B}^{\prime} \mathrm{A}^{\prime}, N=\left|{ }^{2} J_{\mathrm{AX}}+{ }^{4} J_{\mathrm{AX}}{ }^{\prime}\right|=4.2 \mathrm{~Hz}\right.$, $\left.{ }^{3} J_{\mathrm{AB}}=5.7 \mathrm{~Hz}, 2 \mathrm{H}, \mathrm{PCH}\right), 1.51\left(\mathrm{~s}, 9 \mathrm{H}, \mathrm{CN}\left(\mathrm{C}\left(\mathrm{CH}_{3}\right)_{3}\right)\right), 1.40\left(\mathrm{~A}_{9} \mathrm{XX}^{\prime} \mathrm{A}_{9}^{\prime}, N={ }^{3} J_{\mathrm{AX}}+\right.$ $\left.{ }^{5} J_{\mathrm{AX}} \mid=6.5 \mathrm{~Hz}, 18 \mathrm{H}, \mathrm{P}\left(\mathrm{C}\left(\mathrm{CH}_{3}\right)_{3}\right)\right), 1.34\left(\mathrm{~A}_{9} \mathrm{XX}^{\prime} \mathrm{A}_{9}^{\prime}, N=\left|{ }^{3} J_{\mathrm{AX}}+{ }^{5} J_{\mathrm{AX}}\right|=7.2 \mathrm{~Hz}\right.$, $\left.18 \mathrm{H}, \mathrm{P}\left(\mathrm{C}\left(\mathrm{CH}_{3}\right)_{3}\right)\right) .{ }^{13} \mathrm{C}\left\{{ }^{1} \mathrm{H}\right\}(125.76 \mathrm{MHz}): \delta=161.0$ (AXX'A', $N=\left|{ }^{2} J_{\mathrm{AX}}+{ }^{3} J_{\mathrm{AX}}{ }^{\prime}\right|$ $=7.3 \mathrm{~Hz}, 2 \mathrm{C}, \mathrm{N} C \mathrm{H}), 155.9\left(\mathrm{t},{ }^{2} J_{\mathrm{CP}}=3.2 \mathrm{~Hz}, 1 \mathrm{C}, \mathrm{Os} C\right), 82.2\left(\mathrm{AXX}^{\prime} \mathrm{A}^{\prime}, N=\left.\right|^{1} J_{\mathrm{AX}}+\right.$ $\left.{ }^{3} J_{\mathrm{AX}} \mid=26.2 \mathrm{~Hz}, 2 \mathrm{C}, \mathrm{PC} H\right), 57.4\left(\mathrm{~s}, 1 \mathrm{C}, \mathrm{CN}\left(C\left(\mathrm{CH}_{3}\right)_{3}\right)_{2}\right), 38.1\left(\mathrm{AXX}^{\prime} \mathrm{A}^{\prime}, N=\left.\right|^{1} J_{\mathrm{AX}}+\right.$ ${ }^{3} J_{\mathrm{AX}}, \mid=13.6 \mathrm{~Hz}, 2 \mathrm{C}, \mathrm{P}\left(C\left(\mathrm{CH}_{3}\right)_{3}\right), 37.9\left(\mathrm{AXX}^{\prime} \mathrm{A}^{\prime}, N=\left|{ }^{1} J_{\mathrm{AX}}+{ }^{3} J_{\mathrm{AX}}\right|=11.3 \mathrm{~Hz}, 2 \mathrm{C}\right.$, $\left.\mathrm{P}\left(C\left(\mathrm{CH}_{3}\right)_{3}\right), 32.3\left(\mathrm{~s}, 3 \mathrm{C}, \mathrm{CC}\left(C \mathrm{H}_{3}\right)_{3}\right)_{2}\right), 31.6-31.4\left(\mathrm{br}, 12 \mathrm{C}, \mathrm{P}\left(\mathrm{C}\left(C \mathrm{H}_{3}\right)_{3}\right)_{2}\right) .{ }^{31} \mathrm{P}\left\{{ }^{1} \mathrm{H}\right\}$ $(161.25 \mathrm{MHz}): \delta=73.3\left(\mathrm{~s}, 2 \mathrm{P}, P\left(\mathrm{C}\left(\mathrm{CH}_{3}\right)_{3}\right)_{2}\right)$. IR (nujol): $\tilde{\nu}=2007(\mathrm{C}=\mathrm{N})$.

\section{Synthesis of 19}

$14(21.3 \mathrm{mg}, 38.0 \mu \mathrm{mol}, 1.00 \mathrm{eq}$.$) and \mathrm{NaBPh}_{4}(13.0 \mathrm{mg}, 38.0 \mu \mathrm{mol}, 1.00$ eq. $)$ are dissolved in $\mathrm{THF}$ and $\mathrm{CN} t \mathrm{Bu}(4.30 \mu \mathrm{L}, 38.0 \mu \mathrm{mol}, 1.00 \mathrm{eq}$.$) is added. The solution is stirred for$ 30 seconds and $\mathrm{SiMe}_{3} \mathrm{I}(4.92 \mu \mathrm{L}, 38.0 \mu \mathrm{mol}, 1.00$ eq. $)$ is added. The solution is stirred for 2 minutes and the solvent is removed. The residue is washed with $\mathrm{Et}_{2} \mathrm{O}$ and extracted with DCM. The extract is layered with pentanes and stored at $-35^{\circ} \mathrm{C}$ for $16 \mathrm{~h}$. After filtration the residue is washed with pentane $(4 \times 2 \mathrm{~mL})$ and extracted with DCM $(2 \mathrm{x}$ $2 \mathrm{~mL}) .19$ is isolated in form of an orange solid (20.1 mg, $19.4 \mu \mathrm{mol}, 51 \%)$.

Anal. Calcd for $\mathrm{C}_{52} \mathrm{H}_{78} \mathrm{~B}_{1} \mathrm{~N}_{3} \mathrm{Os}_{1} \mathrm{P}_{2} \mathrm{Si}$ (1036.29): C, 60.27; H, 7.59; N, 4.05 Found: C, 59.97; H, 7.25; N, 4.01. NMR $\left(d_{8}-\mathrm{THF},-50{ }^{\circ} \mathrm{C}\right):{ }^{1} \mathrm{H}(500 \mathrm{MHz}): \delta=7.34-7.29(\mathrm{~m}$, $\left.8 \mathrm{H}, \mathrm{C}_{\text {meta }} H\right), 7.15$ (ABXX'B'A', $N=\left|{ }^{3} J_{\mathrm{AX}}+{ }^{4} J_{\mathrm{AX}}\right|=18.3 \mathrm{~Hz},{ }^{3} J_{\mathrm{AB}}=5.8 \mathrm{~Hz} .2 \mathrm{H}$, $\mathrm{NCH}), 7.05-7.00\left(\mathrm{~m}, 8 \mathrm{H}, \mathrm{C}_{\text {ortho }} H\right), 6.90-6.85\left(\mathrm{~m}, 4 \mathrm{H}, \mathrm{C}_{\text {para }} H\right), 4.33$ (ABXX'B' A', $N$ $\left.=\left|{ }^{2} J_{\mathrm{AX}}+{ }^{4} J_{\mathrm{AX}}\right|=5.9 \mathrm{~Hz},{ }^{3} J_{\mathrm{AB}}=5.8 \mathrm{~Hz}, 2 \mathrm{H}, \mathrm{PCH}\right), 1.43\left(\mathrm{~s}, 9 \mathrm{H}, \mathrm{CN}\left(\mathrm{C}\left(\mathrm{CH}_{3}\right)_{3}\right)\right), 1.41$ $\left(\mathrm{A}_{9} \mathrm{XX}^{\prime} \mathrm{A}^{\prime}{ }_{9}, N=\left|{ }^{3} J_{\mathrm{AX}}+{ }^{5} J_{\mathrm{AX}}\right|=7.7 \mathrm{~Hz}, 18 \mathrm{H}, \mathrm{P}\left(\mathrm{C}\left(\mathrm{CH}_{3}\right)_{3}\right)\right), 1.24\left(\mathrm{~A}_{9} \mathrm{XX}^{\prime}{ }^{\prime}{ }_{9}, N=\right.$ $\left.\left|{ }^{3} J_{\mathrm{AX}}+{ }^{5} J_{\mathrm{AX}}\right|=7.8 \mathrm{~Hz}, 18 \mathrm{H}, \mathrm{P}\left(\mathrm{C}\left(\mathrm{CH}_{3}\right)_{3}\right)\right), 0.46\left(\mathrm{~s}, 9 \mathrm{H}, \mathrm{Si}\left(\mathrm{C} H_{3}\right)_{3}\right) \cdot{ }^{13} \mathrm{C}\left\{{ }^{1} \mathrm{H}\right\}(125.76$ $\mathrm{MHz}): \delta=164.6\left(\mathrm{q}^{1}{ }^{1} J_{\mathrm{CB}}=49.0 \mathrm{~Hz}, 4 \mathrm{C}, C_{\text {ipso }}\right), 161.1\left(\mathrm{AXX}^{\prime} \mathrm{A}^{\prime}, N=\left.\right|^{2} J_{\mathrm{AX}}+{ }^{3} J_{\mathrm{AX}} \mid\right.$ $=5.5 \mathrm{~Hz}, 2 \mathrm{C}, \mathrm{NCH}), 136.4\left(\mathrm{~s}, 8 \mathrm{C}, C_{\text {meta }}\right), 132.3(\mathrm{br}, 1 \mathrm{C}, \mathrm{Os} C), 126.2\left(\mathrm{q},{ }^{2} J_{\mathrm{CB}}=2.9 \mathrm{~Hz}\right.$, $4 \mathrm{C}, C_{\text {ortho }}$ ), $122.3\left(\mathrm{~s}, 8 \mathrm{C}, C_{\text {para }}\right.$ ), 83.1 (AXX'A', $N=\left|{ }^{1} J_{\mathrm{AX}}+{ }^{3} J_{\mathrm{AX}}{ }^{\prime}\right|=27.6 \mathrm{~Hz}, 2 \mathrm{C}$, $\mathrm{PCH}), 59.8\left(\mathrm{~s}, 1 \mathrm{C}, \mathrm{CN}\left(C\left(\mathrm{CH}_{3}\right)_{3}\right)_{2}\right), 41.6$ (AXX'A', $N=\left|{ }^{1} J_{\mathrm{AX}}+{ }^{3} J_{\mathrm{AX}}\right|=12.8 \mathrm{~Hz}, 2 \mathrm{C}$, $\mathrm{P}\left(C\left(\mathrm{CH}_{3}\right)_{3}\right), 41.2\left(\mathrm{AXX}^{\prime} \mathrm{A}^{\prime}, N=\left|{ }^{1} J_{\mathrm{AX}}+{ }^{3} J_{\mathrm{AX}}\right|=12.1 \mathrm{~Hz}, 2 \mathrm{C}, \mathrm{P}\left(C\left(\mathrm{CH}_{3}\right)_{3}\right), 32.0(\mathrm{~s}\right.$, $\left.\left.3 \mathrm{C}, \mathrm{CC}\left(\mathrm{CH}_{3}\right)_{3}\right)_{2}\right), 31.5\left(\mathrm{~A}_{3} \mathrm{XX}^{\prime} \mathrm{A}^{\prime}{ }_{3}, N=\left|{ }^{2} J_{\mathrm{AX}}+{ }^{4} J_{\mathrm{AX}}\right|=1.7 \mathrm{~Hz}, 6 \mathrm{C}, \mathrm{P}\left(\mathrm{C}\left(C_{3} \mathrm{H}_{3}\right)_{3}\right)\right.$, $29.5\left(\mathrm{~A}_{3} \mathrm{XX}^{\prime} \mathrm{A}_{3}{ }_{3}, N=\left.\right|^{2} J_{\mathrm{AX}}+{ }^{4} J_{\mathrm{AX}} \mid=2.0 \mathrm{~Hz}, 6 \mathrm{C}, \mathrm{P}\left(\mathrm{C}\left(C \mathrm{H}_{3}\right)_{3}\right), 2.21\left(\mathrm{~s}, 3 \mathrm{C}, \mathrm{Si}\left(C \mathrm{H}_{3}\right)_{3}\right)\right.$. ${ }^{31} \mathrm{P}\left\{{ }^{1} \mathrm{H}\right\}(161.25 \mathrm{MHz}): \delta=91.3\left(\mathrm{~s}, 2 \mathrm{P}, \mathrm{P}\left(\mathrm{C}\left(\mathrm{CH}_{3}\right)_{3}\right)_{2}\right)$. IR (nujol): $\tilde{\nu}=2128(\mathrm{C}=\mathrm{N})$. 


\section{Synthesis of 20}

Route A: 2 (11.0 mg, $17.83 \mu \mathrm{mol}, 1.00$ eq.) and $\mathrm{CoCp}_{2}(3.4 \mathrm{mg}, 18 \mu \mathrm{mol}, 1.0$ eq.) are dissolved in benzene $(5 \mathrm{~mL})$ and stirred for 2 minutes at RT. A solution of 14 (10.0 mg, $17.83 \mu \mathrm{mol}, 1.0 \mathrm{eq})$ in pentane $(5 \mathrm{~mL})$ is slowly added and the solution is stirred for 20 minutes at RT The solvent is removed and the residue extracted with pentanes $(4 \mathrm{x}$ $5 \mathrm{~mL}$ ). The filtrate is concentrated and the crude product is purified via column chromatography with silanized silica (pentane). The solvent is removed and lyophilization yields 20 as a green powder (17.0 mg, $14.9 \mu \mathrm{mol}, 83 \%$ ).

Route B: 2 (20.0 mg, $32.4 \mu \mathrm{mol}, 1.00$ eq.) and $\mathrm{CoCp}_{2}(6.2 \mathrm{mg}, 32 \mu \mathrm{mol}, 1.0$ eq.) are dissolved in benzene $(5 \mathrm{~mL})$ and stirred for 2 minutes. [TMS]N $3(2.2 \mu \mathrm{L}, 17 \mu \mathrm{mol}, 0.50$ eq. $)$ in pentane $(5 \mathrm{~mL})$ is slowly added and the solution is stirred for additional 20 minutes. The solvent is removed and the residue extracted with pentanes $(4 \times 5 \mathrm{~mL})$. The filtrate is concentrated and the crude product is purified via column chromatography with silanized silica (pentane). The solvent is removed and lyophilization yields $\mathbf{2 0}$ as a green powder (13.2 mg, $11.5 \mu \mathrm{mol}, 71 \%)$.

Anal. Calcd for $\mathrm{C}_{40} \mathrm{H}_{80} \mathrm{Cl}_{1} \mathrm{~N}_{3} \mathrm{Os}_{2} \mathrm{P}_{4}$ (1142.9): C, 42.0; H, 7.06; N, 3.67 Found: C, 41.9; $\mathrm{H}, 6.94 ; \mathrm{N}, 3.48$. NMR (toluene- $\left.d_{8}, \mathrm{RT}\right):{ }^{1} \mathrm{H}(500 \mathrm{MHz}): \delta=6.99\left(\mathrm{~m},{ }^{3} J_{\mathrm{HH}}=6.1 \mathrm{~Hz}\right.$, $1 \mathrm{H}, \mathrm{NCH}), 6.89\left(\mathrm{~m},{ }^{3} J_{\mathrm{HH}}=6.0 \mathrm{~Hz}, 1 \mathrm{H}, \mathrm{NCH}\right), 6.67\left(\mathrm{~m},{ }^{3} J_{\mathrm{HH}}=6.3 \mathrm{~Hz}, 1 \mathrm{H}, \mathrm{NCH}\right)$, $6.61\left(\mathrm{~m},{ }^{3} J_{\mathrm{HH}}=6.3 \mathrm{~Hz}, 1 \mathrm{H}, \mathrm{NCH}\right), 4.44\left(\mathrm{~m},{ }^{3} J_{\mathrm{HH}}=6.0 \mathrm{~Hz}, 1 \mathrm{H}, \mathrm{PCH}\right), 4.34\left(\mathrm{~m},{ }^{3} J_{\mathrm{HH}}\right.$ $=6.3 \mathrm{~Hz}, 1 \mathrm{H}, \mathrm{PCH}), 4.31\left(\mathrm{~m},{ }^{3} J_{\mathrm{HH}}=6.1 \mathrm{~Hz}, 1 \mathrm{H}, \mathrm{PCH}\right), 4.18\left(\mathrm{~m},{ }^{3} J_{\mathrm{HH}}=6.3 \mathrm{~Hz}, 1 \mathrm{H}\right.$, $\mathrm{PCH}), 1.93-1.07(\mathrm{~m}, 69 \mathrm{H}), 0.81\left(\mathrm{~m}, 1 \mathrm{H}, \mathrm{OsCH}_{2}\right),-0.56\left(\mathrm{~m}, 1 \mathrm{H}, \mathrm{OsCH}_{2}\right),-4.18(\mathrm{~m}, 1 \mathrm{H}$, OsH $) .{ }^{13} \mathrm{C}\left\{{ }^{1} \mathrm{H}\right\}(125.76 \mathrm{MHz}) \delta=168.5\left(\mathrm{dd},{ }^{2} J_{\mathrm{CP}}=10.3 \mathrm{~Hz},{ }^{3} J_{\mathrm{CP}}=2.7 \mathrm{~Hz}, 1 \mathrm{C}, \mathrm{NCH}\right)$, $168.4\left(\mathrm{dd},{ }^{2} J_{\mathrm{CP}}=10.6 \mathrm{~Hz},{ }^{3} J_{\mathrm{CP}}=2.9 \mathrm{~Hz}, 1 \mathrm{C}, \mathrm{NCH}\right), 162.4\left(\mathrm{dd},{ }^{2} J_{\mathrm{CP}}=12.4 \mathrm{~Hz},{ }^{3} J_{\mathrm{CP}}=\right.$ $2.0 \mathrm{~Hz}, 1 \mathrm{C}, \mathrm{N} C \mathrm{H}), 161.2\left(\mathrm{dd},{ }^{2} J_{\mathrm{CP}}=10.1 \mathrm{~Hz},{ }^{3} J_{\mathrm{CP}}=2.4 \mathrm{~Hz}, 1 \mathrm{C}, \mathrm{NCH}\right), 93.9\left(\mathrm{dd},{ }^{1} J_{\mathrm{CP}}\right.$ $\left.=35.0 \mathrm{~Hz},{ }^{3} J_{\mathrm{CP}}=2.5 \mathrm{~Hz}, 1 \mathrm{C}, \mathrm{PCH}\right), 93.6\left(\mathrm{~d},{ }^{1} J_{\mathrm{CP}}=39.3 \mathrm{~Hz}, 1 \mathrm{C}, \mathrm{PCH}\right), 93.2\left(\mathrm{dd},{ }^{1} J_{\mathrm{CP}}\right.$ $\left.=35.2 \mathrm{~Hz},{ }^{3} J_{\mathrm{CP}}=1.7 \mathrm{~Hz}, 1 \mathrm{C}, \mathrm{PCH}\right), 90.6\left(\mathrm{~d},{ }^{1} J_{\mathrm{CP}}=38.8 \mathrm{~Hz}, 1 \mathrm{C}, \mathrm{PCH}\right), 58.1\left(\mathrm{dd},{ }^{1} J_{\mathrm{CP}}\right.$ $\left.=35.2 \mathrm{~Hz},{ }^{3} J_{\mathrm{CP}}=5.4 \mathrm{~Hz}, 1 \mathrm{C}, \mathrm{P}\left(C\left(\mathrm{CH}_{3}\right)_{2} \mathrm{CH}_{2} \mathrm{Os}\right)\right), 39.1\left(\mathrm{dd},{ }^{1} J_{\mathrm{CP}}=18.7 \mathrm{~Hz},{ }^{3} J_{\mathrm{CP}}=\right.$ $\left.2.7 \mathrm{~Hz}, 1 \mathrm{C}, \mathrm{P}\left(C\left(\mathrm{CH}_{3}\right)_{3}\right)\right), 38.1\left(\mathrm{~m}, 2 \mathrm{C}, \mathrm{P}\left(C\left(\mathrm{CH}_{3}\right)_{3}\right)\right), 37.5\left(\mathrm{dd},{ }^{1} J_{\mathrm{CP}}=13.9 \mathrm{~Hz},{ }^{3} J_{\mathrm{CP}}=\right.$ $\left.4.0 \mathrm{~Hz}, 1 \mathrm{C}, \mathrm{P}\left(C\left(\mathrm{CH}_{3}\right)_{3}\right)\right), 37.3\left(\mathrm{dd},{ }^{1} J_{\mathrm{CP}}=13.2 \mathrm{~Hz},{ }^{3} J_{\mathrm{CP}}=4.0 \mathrm{~Hz}, 1 \mathrm{C}, \mathrm{P}\left(C\left(\mathrm{CH}_{3}\right)_{3}\right)\right)$, $36.0\left(\mathrm{dd},{ }^{1} J_{\mathrm{CP}}=13.9 \mathrm{~Hz},{ }^{3} J_{\mathrm{CP}}=5.1 \mathrm{~Hz}, 1 \mathrm{C}, \mathrm{P}\left(C\left(\mathrm{CH}_{3}\right)_{3}\right)\right), 34.5\left(\mathrm{dd},{ }^{1} J_{\mathrm{CP}}=6.6 \mathrm{~Hz}\right.$, $\left.{ }^{3} J_{\mathrm{CP}}=2.7 \mathrm{~Hz}, 1 \mathrm{C}, \mathrm{P}\left(C\left(\mathrm{CH}_{3}\right)_{3}\right)\right), 33.2-29.8(21 \mathrm{C}), 32.3\left(\mathrm{~d},{ }^{2} J_{\mathrm{CP}}=3.6 \mathrm{~Hz}, 1 \mathrm{C}\right.$, $\left.\mathrm{P}\left(\mathrm{C}\left(\mathrm{CH}_{3}\right)_{2} \mathrm{CH}_{2} \mathrm{Os}\right)\right), 9.13\left(\mathrm{dd},{ }^{2} J_{\mathrm{CP}}=30.4 \mathrm{~Hz},{ }^{2} J_{\mathrm{CP}}=5.4 \mathrm{~Hz}, \mathrm{P}\left(\mathrm{C}\left(\mathrm{CH}_{3}\right)_{2} C \mathrm{H}_{2} \mathrm{Os}\right)\right)$. ${ }^{31} \mathrm{P}\left\{{ }^{1} \mathrm{H}\right\}(202.46 \mathrm{MHz}): \delta=38.0\left(\mathrm{~d},{ }^{2} J_{\mathrm{PP}}=250.2 \mathrm{~Hz}, 1 \mathrm{P}, P\left(\mathrm{C}\left(\mathrm{CH}_{3}\right)_{3}\right)\right), 19.7\left(\mathrm{~m},{ }^{2} J_{\mathrm{PP}}\right.$ $\left.=291.6 \mathrm{~Hz}, N=\left|{ }^{4} J_{\mathrm{PP}}+{ }^{4} J_{\mathrm{PP}}\right|=2.5 \mathrm{~Hz}, 1 \mathrm{P}, P\left(\mathrm{C}\left(\mathrm{CH}_{3}\right)_{3}\right)_{2}\right), 16.6\left(\mathrm{~m},{ }^{2} J_{\mathrm{PP}}=291.6 \mathrm{~Hz}\right.$, $\left.N=\left|{ }^{4} J_{\mathrm{PP}}+{ }^{4} J_{\mathrm{PP}}\right|=2.5 \mathrm{~Hz}, 1 \mathrm{P}, P\left(\mathrm{C}\left(\mathrm{CH}_{3}\right)_{3}\right)_{2}\right),-15.5\left(\mathrm{~m},{ }^{2} J_{\mathrm{PP}}=250.1 \mathrm{~Hz}, N=\mid{ }^{4} J_{\mathrm{PP}}\right.$ $\left.+{ }^{4} J_{\mathrm{PP}} \mid=2.5 \mathrm{~Hz}, 1 \mathrm{P}, P\left(\mathrm{C}\left(\mathrm{CH}_{3}\right)_{2} \mathrm{CH}_{2} \mathrm{Os}\right)\right)$. MS (LIFDI, toluene): $m / z=1143.5(100 \%$, $\left.\left[\mathrm{M}^{+}\right]\right)$.

\section{Synthesis of 21}

Route A: 1 (30.0 mg, $48.6 \mu \mathrm{mol}, 1.00$ eq.) and $\mathrm{CoCp}_{2}(9.2 \mathrm{mg}, 49 \mu \mathrm{mol}, 1.0$ eq.) are dissolved in benzene $(2 \mathrm{~mL})$ and stirred for 2 minutes at RT. 2,2'-Bipyridine (7.6 mg, $48.6 \mu \mathrm{mol}, 1.0$ eq.) is added and stirring is continued for 5 minutes. $\operatorname{TMSN}_{3}(64 \mu \mathrm{L}$, $486 \mu \mathrm{mol}, 10 \mathrm{eq}$.$) is added and the solution is filtered after 1$ hour of stirring. The solvent is evaporated and the residue is washed with pentane $(5 \times 1 \mathrm{~mL})$ at $-20^{\circ} \mathrm{C}$. The product is extracted with benzene $(5 \times 0.5 \mathrm{~mL})$ and $\mathbf{2 1}(22.4 \mathrm{mg}, 30.1 \mu \mathrm{mol}, 62 \%)$ is 
obtained in form of a red powder after lyophilization.

Route B: 7 (10.0 mg, $13.5 \mu \mathrm{mol}, 1.00$ eq.) is dissolved in benzene $(2 \mathrm{~mL}), \mathrm{TMSN}_{3}(17.8 \mu \mathrm{L}$, $135 \mu \mathrm{mol}, 10.0$ eq.) is added and the solution is stirred for 1 hour at RT. The purification is identical with Route A.

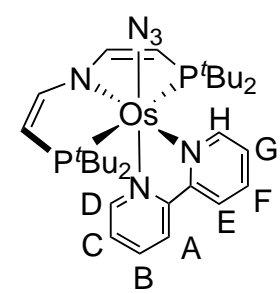

Anal. Calcd. for $\mathrm{C}_{30} \mathrm{H}_{48} \mathrm{ClN}_{6} \mathrm{OsP}_{2}$ (744.93): Route A: C, 48.4; H, 6.50; N, 11.3 Found: C, 48.8; H, 6.70; N, 10.7. Route B: C, 48.4; H, 6.50; N, 11.3 Found: C, 48.6; H, 6.38; N, 10.9. NMR $\left(\mathrm{C}_{6} \mathrm{D}_{6}, \mathrm{RT}\right):{ }^{1} \mathrm{H}$ NMR $(400 \mathrm{MHz}): \delta=10.40-10.36(\mathrm{~m}, 1 \mathrm{H}, \mathrm{A}-$ $\mathrm{H}), 8.29\left(\mathrm{dd},{ }^{3} J_{\mathrm{HH}}=6.2 \mathrm{~Hz},{ }^{4} \mathrm{~J}_{\mathrm{HH}}=1.4 \mathrm{~Hz}, 1 \mathrm{H}, \mathrm{E}-\mathrm{H}\right), 7.48\left(\mathrm{~A}_{9} \mathrm{BCXX}^{\prime}{ }^{\prime}{ }_{9} \mathrm{~B}^{\prime} \mathrm{C}^{\prime}, N=\right.$ $\left.\left|{ }^{3} J_{\mathrm{CX}}+{ }^{4} J_{\mathrm{CX}}\right|=34.0 \mathrm{~Hz},{ }^{3} J_{\mathrm{BC}}=5.9 \mathrm{~Hz}, 2 \mathrm{H}, \mathrm{NCHCHP}\right), 7.14-7.10(\mathrm{~m}, 1 \mathrm{H}, \mathrm{D}-\mathrm{H}), 6.94$ $\left(\mathrm{dd},{ }^{3} J_{\mathrm{HH}}=8.3 \mathrm{~Hz},{ }^{4} \mathrm{~J}_{\mathrm{HH}}=1.6 \mathrm{~Hz}, 1 \mathrm{H}, \mathrm{H}-\mathrm{H}\right), 6.59-6.52(\mathrm{~m}, 2 \mathrm{H}, \mathrm{B}-\mathrm{H}, \mathrm{C}-\mathrm{H}) 6.24(\mathrm{ddd}$, $\left.{ }^{3} J_{\mathrm{HH}}=8.3 \mathrm{~Hz}, 7.2 \mathrm{~Hz},{ }^{4} \mathrm{~J}_{\mathrm{HH}}=1.4 \mathrm{~Hz}, 1 \mathrm{H}, \mathrm{G}-\mathrm{H}\right), 6.03\left(\mathrm{ddd},{ }^{3} J_{\mathrm{HH}}=7.2 \mathrm{~Hz}, 6.2 \mathrm{~Hz}\right.$, $\left.{ }^{4} \mathrm{~J}_{\mathrm{HH}}=1.6 \mathrm{~Hz}, 1 \mathrm{H}, \mathrm{F}-\mathrm{H}\right), 4.00\left(\mathrm{~A}_{9} \mathrm{BCXX}{ }^{\prime}{ }^{\prime}{ }_{9} \mathrm{~B}^{\prime} \mathrm{C} ', N=\left|{ }^{2} J_{\mathrm{BX}}+{ }^{4} J_{\mathrm{BX}}\right|=6.0 \mathrm{~Hz},{ }^{3} J_{\mathrm{BC}}=\right.$ $5.9 \mathrm{~Hz}, 2 \mathrm{H}, \mathrm{NCHCHP}), 1.60\left(\mathrm{~A}_{9} \mathrm{BCXX}^{\prime} \mathrm{A}_{9}{ }_{9} \mathrm{~B}^{\prime} \mathrm{C}^{\prime},{ }^{3} J_{\mathrm{AX}}=12.4 \mathrm{~Hz}, 18 \mathrm{H}, \mathrm{P}\left(\mathrm{C}\left(\mathrm{C} H_{3}\right)_{3}\right)\right)$, $0.31\left(\mathrm{~A}_{9} \mathrm{BCXX}{ }^{\prime}{ }^{\prime}{ }_{9} \mathrm{~B}{ }^{\prime} \mathrm{C}^{\prime},{ }^{3} J_{\mathrm{AX}}=12.4 \mathrm{~Hz}, 18 \mathrm{H}, \mathrm{P}\left(\mathrm{C}\left(\mathrm{CH}_{3}\right)_{3}\right)\right) .{ }^{13} \mathrm{C}\left\{{ }^{1} \mathrm{H}\right\}-\mathrm{NMR}(101.25$ $\mathrm{MHz}): \delta=163.7\left(\mathrm{vt},{ }^{2} J_{\mathrm{CP}}=7.5 \mathrm{~Hz}, 2 \mathrm{C}, \mathrm{NCHCHP}\right), 168.1\left(\mathrm{~s}, 1 \mathrm{C}, \mathrm{C}_{\mathrm{A}}\right), 163.6(\mathrm{~s}, 1 \mathrm{C}$,

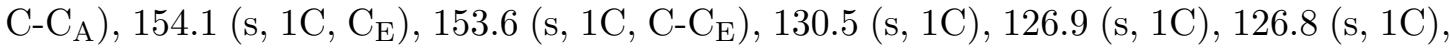
$125.3(\mathrm{~s}, 1 \mathrm{C}), 122.7(\mathrm{~s}, 1 \mathrm{C}), 121.2(\mathrm{~s}, 1 \mathrm{C}), 84.2\left(\mathrm{vt},{ }^{1} J_{\mathrm{CP}}=19.2 \mathrm{~Hz}, 2 \mathrm{C}, \mathrm{NCH} C \mathrm{HP}\right), 38.3$ $\left(\right.$ vt, $\left.{ }^{1} J_{\mathrm{CP}}=10.7 \mathrm{~Hz}, 2 \mathrm{C}, \mathrm{P}\left(C\left(\mathrm{CH}_{3}\right)_{3}\right)_{2}\right), 38.2\left(\mathrm{vt},{ }^{1} J_{\mathrm{CP}}=9.5 \mathrm{~Hz}, 2 \mathrm{C}, \mathrm{P}\left(C\left(\mathrm{CH}_{3}\right)_{3}\right)_{2}\right)$, $30.5\left(\mathrm{vt},{ }^{2} J_{\mathrm{CP}}=2.2 \mathrm{~Hz}, 6 \mathrm{C}, \mathrm{P}\left(\mathrm{C}\left(\mathrm{CH}_{3}\right)_{3}\right)_{2}\right), 29.6\left(\mathrm{vt},{ }^{2} J_{\mathrm{CP}}=2.3 \mathrm{~Hz}, 6 \mathrm{C}, \mathrm{P}\left(\mathrm{C}\left(\mathrm{CH}_{3}\right)_{3}\right)_{2}\right)$. ${ }^{31} \mathrm{P}\left\{{ }^{1} \mathrm{H}\right\}$-NMR $\left.(161.25 \mathrm{MHz}): \delta=21.6\left(\mathrm{~s}, P\left(\mathrm{C}\left(\mathrm{CH}_{3}\right)_{3}\right)_{2}\right)\right)$. All bipy ${ }^{1} \mathrm{H}$ NMR signals could be assigned to two chemically inequivalent ring systems. However, due to a lack of resolution in 2D NMR correlatiokn experiments not all carbon signals could be assigned and it might be possible that the position of the ring is not correctly assigned.. IR (Nujol, $\mathrm{RT}) \tilde{\nu}=2072\left(\mathrm{Os}-N_{3}\right)$.

\section{One-electron oxidation of 21}

21 (5.0 mg, $6.7 \mu \mathrm{mol}, 1.0$ eq.) is dissolved in THF ( $1 \mathrm{~mL})$ and $\mathrm{AgSbF}_{6}(2.3 \mathrm{mg}, 6.7 \mu \mathrm{mol}$, 2.0 eq.) is added. The solution is stirred for 1 minute at RT and filtered. The solvent evaporated, redisolved in THF- $\mathrm{d}_{8}$ and measured by NMR which showed the formation of $\mathbf{8}$ as sole reaction product.

\section{Two-electron oxidation of 21 and successive reduction}

21 (5.0 mg, $6.7 \mu \mathrm{mol}, 1.0$ eq.) is dissolved in THF $(1 \mathrm{~mL})$ and $\mathrm{AgSbF}_{6}(4.6 \mathrm{mg}, 13 \mu \mathrm{mol}$, 2.0 eq.) is added in one portion under heavy stirring. The solution is stirred for 1 minute at $\mathrm{RT}$ and filtered. The solvent evaporated, redisolved in $\mathrm{CD}_{2} \mathrm{Cl}_{2}$ and measured by NMR which showed the formation of $\mathbf{2 3}$ accompanied by a minor asymmetric impurity 
$\left({ }^{31} \mathrm{P}\left\{{ }^{1} \mathrm{H}\right\}-\mathrm{NMR}(161.25 \mathrm{MHz}): \delta=34.9\left(\mathrm{~d},{ }^{2} J_{\mathrm{PP}}=223.4 \mathrm{~Hz}, P\left(\mathrm{C}_{\left(\mathrm{CH}_{3}\right.}\right)_{3}\right)_{2}\right), 7.10(\mathrm{~d}$, $\left.\left.{ }^{2} J_{\mathrm{PP}}=223.4 \mathrm{~Hz}, \mathrm{P}\left(\mathrm{C}\left(\mathrm{CH}_{3}\right)_{3}\right)_{2}\right)\right)$ and organic impurities.

$\operatorname{NMR}\left(\mathrm{CD}_{2} \mathrm{Cl}_{2}, \mathrm{RT}\right):{ }^{1} \mathrm{H}$ NMR $(300 \mathrm{MHz}): \delta=9.75-9.70(\mathrm{~m}, 1 \mathrm{H}), 8.92-8.87(\mathrm{~m}, 1 \mathrm{H})$, $8.70-8.60(\mathrm{~m}, 1 \mathrm{H}), 8.43-8.35(\mathrm{~m}, 1 \mathrm{H}), 8.16-7.87(\mathrm{~m}, 2 \mathrm{H}), 8.05\left(\mathrm{~A}_{9} \mathrm{BCXX}^{\prime} \mathrm{A}_{9}{ }_{9} \mathrm{~B}^{\prime} \mathrm{C}^{\prime}\right.$, $\left.N=\left|{ }^{3} J_{\mathrm{CX}}+{ }^{4} J_{\mathrm{CX}}\right|=38.6 \mathrm{~Hz},{ }^{3} J_{\mathrm{BC}}=6.0 \mathrm{~Hz}, 2 \mathrm{H}, \mathrm{NCHCHP}\right), 7.39-7.23(\mathrm{~m}, 2 \mathrm{H})$, $4.96\left(\mathrm{~A}_{9} \mathrm{BCXX}{ }^{\prime}{ }_{9}{ }_{9} \mathrm{~B}^{\prime} \mathrm{C}^{\prime}, N=\left.\right|^{2} J_{\mathrm{BX}}+{ }^{4} J_{\mathrm{BX}} \mid=10.2 \mathrm{~Hz},{ }^{3} J_{\mathrm{BC}}=6.0 \mathrm{~Hz}, 2 \mathrm{H}, \mathrm{NCHC} H \mathrm{P}\right)$, $1.50\left(\mathrm{~A}_{9} \mathrm{BCXX} \mathrm{A}^{\prime}{ }_{9} \mathrm{~B} \mathrm{C}^{\prime},{ }^{3} J_{\mathrm{AX}}=16.4 \mathrm{~Hz}, 18 \mathrm{H}, \mathrm{P}\left(\mathrm{C}\left(\mathrm{CH}_{3}\right)_{3}\right)\right), 0.67\left(\mathrm{~A}_{9} \mathrm{BCXX}^{\prime}{ }^{\prime}{ }_{9} \mathrm{~B}^{\prime} \mathrm{C}\right.$ ', $\left.{ }^{3} J_{\mathrm{AX}}=16.4 \mathrm{~Hz}, 18 \mathrm{H}, \mathrm{P}\left(\mathrm{C}\left(\mathrm{CH}_{3}\right)_{3}\right)\right) .{ }^{31} \mathrm{P}\left\{{ }^{1} \mathrm{H}\right\}-\mathrm{NMR}(121.49 \mathrm{MHz}): \delta=78.7(\mathrm{~s}$,

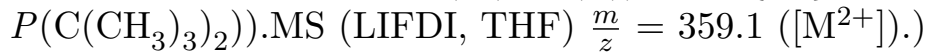

This solvent is evaporated and the residue is redissolved in THF. To this solution $\mathrm{CoCp}_{2}$ $(1.2 \mathrm{mg}, 6.4 \mu \mathrm{mol}, 1.0 \mathrm{eq}$.) is added at RT. NMR spectroscopy shows exclusive formation of 8 . 


\subsection{Transition Metal Complexes with Metal-Pnictogen Multiple Bonds}

Parts of this chapter were adapted from:

J. Abbenseth, M. Diefenbach, A. Hinz, L. Alig, C. Würtele, J. M. Goicoechea*, M. C. Holthausen*, S. Schneider*, 2019, 58, 10966-10970.

J. Abbenseth, D. Delony, M. C. Neben, C. Würtele, B. de Bruin, S. Schneider, Angew. Chem. Int. Ed. 2019, 58, 6338-6341.

with permission from WILEY-VCH Verlag GmbH \& Co. KGaA, Weinheim

\subsection{1 $\mathrm{P}_{2}$ and $\mathrm{As}_{2}$ Complexes from Coupling of Terminal Re Pnictides - Computational Details}

Based on a detailed evaluation study of Minenkov et al. the $\omega$ B97XD meta-hybrid density functional as implemented in the Gaussian program along with the StuttgartDresden effective core potentials (SDD ECP) to replace the innermost core electrons of all non-hydrogen atoms (C, N: 2 electrons; P: 10 electrons; As: 28 electrons, Re: 60 electrons) was employed. ${ }^{[308],[335]-[338]}$ The corresponding standard [2s2p] SDD valence basis sets for $\mathrm{C}, \mathrm{N}, \mathrm{P}$, As were augmented by a single d-polarization function, i.e., the 'HONDO 7 polarization' functions for $\mathrm{C}(0.72), \mathrm{N}(0.98)$, and $\mathrm{P}(0.465)$ as well as the 'Huzinaga polarization' for As (0.293) as provided by the EMSL basis set library. [339], [340] The standard [6s5p3d] SDD valence basis set for Re and the D95 double- $\zeta$ basis set for $\mathrm{H}$ were used. This level of density functional theory, dubbed $\omega \mathrm{B} 97 \mathrm{XD} / \mathrm{BS} 1$ was used for geometry optimizations and Hessian evaluations. ${ }^{[308],[335],[336],[341]}$ Based on minimum structures optimized at this level subsequent single-point energy calculations were performed employing the 6-311++G(2d,2p) basis set for $\mathrm{H}, \mathrm{C}, \mathrm{N}, \mathrm{P}$, and As in combination with the SDD ECP/basis set representation of Re, all as implemented in the basis set library of the Gaussian program. [308], [335], [336], [342]-[345] Wave functions obtained at this level of theory, dubbed $\omega \mathrm{B} 97 \mathrm{XD} / \mathrm{BS} 2$, were used for bonding analysis. Natural bond orbital (NBO) analysis was performed with the NBO 6.0 program interfaced with Gaussian 09. The programs AIMAll and Multiwfn were used for QTAIM analysis. Molecular graphics were rendered with the programs CylView and Chemcraft.

A refined molecular structure of $\mathbf{2 7}$ was optimized at the $\omega$ B97XD3/def2-TZVP level, applying the rijcosx approximation as implemented in the ORCA program. ${ }^{[318]}$ Based on this structure, ${ }^{31} \mathrm{P}$ NMR chemical shift calculations were performed with the NMR module ${ }^{[346],[347]}$ of the ADF program. ${ }^{[348]}$ Relativistic spin-orbit contributions to nuclear magnetic shielding constants were accounted for by means of the two-component ZORA formalism implemented in ADF. ${ }^{[349]-[351]}$ The PBE0 hybrid functional ${ }^{\text {?, }[326],[352]}$ was employed in combination with the TZ2P Slater-orbital basis set. ${ }^{[353]} 31 \mathrm{P}$ NMR chemical shifts were computed relative to the (bare) $\left[\mathrm{P}\left(\mathrm{CH}_{3}\right)_{4}\right]^{+}$cation as standard and incremented for its experimentally determined 31P NMR chemical shift relative to $85 \%$ aqueous phosphoric acid $\left(\delta^{31} \mathrm{P}\left(\left[\mathrm{PMe}_{4}\right] \mathrm{Br}\right)=25.1,{ }^{[354]}\right.$ thus $\delta^{\text {calc }}(\mathrm{X})=\sigma^{\text {calc }}\left(\left[\mathrm{PMe}_{4}\right]^{+}\right)-$ $\left.\sigma^{\text {calc }}(\mathrm{X})+25.1\right)$. Solvent effects were taken into account with the COSMO model implemented in ADF (solvent tetrahydrofuran). ${ }^{[355],[356]}$ The appendix lists several relevant characteristics derived from the electron density at the bcp, located at $\mathbf{r}_{\mathrm{b}}$ along the bond path connecting two interacting atoms, which are customarily used for the classification of bonds. In homopolar bonding situations, a covalent interaction is characterized by a 
negative Laplacian at the corresponding bcp. The magnitudes of the electron density $\rho \mathbf{r}_{\mathrm{b}}$ and its Laplacian $\nabla^{2} \mathbf{r}_{\mathrm{b}}$, and the relative total energy density $H \mathbf{r}_{\mathrm{b}} / \rho \mathbf{r}_{\mathrm{b}}$ relate to the strength of a covalent bond. The total energy density $H=V+G$ is the sum of the potential energy density $V$ (always negative) and the kinetic energy density $G$ (always positive). Covalent, electron-sharing bonding situations are indicated by the ratios $H\left(\mathbf{r}_{\mathrm{b}}\right) / \rho\left(\mathbf{r}_{\mathrm{b}}\right)<0$ and $G\left(\mathbf{r}_{\mathrm{b}}\right) / \rho\left(\mathbf{r}_{\mathrm{b}}\right)<1$. Further, the ellipticity $\epsilon$ of the density at the bcp serves as indicator for $\pi$-double bond character, and the delocalization index $\delta(\mathrm{A}, \mathrm{B})$ is a measure for the number of electron pairs shared between two nuclei A and B. Compared to homonuclear bonds, the degree of covalency is generally reduced with increasing polarity of the interaction. As a consequence, in polar bonds the delocalization index is smaller than the formal bond order. Also, the bcp usually resides close to a nodal surface in the Laplacian distribution, an effect which further amplifies with diffuseness of valence electron density in heavy atoms. Thus, the sign of $\nabla^{2} \rho\left(\mathbf{r}_{\mathrm{b}}\right)$ is unsuitable for a bond classification.

Table 7.6: XYZ-coordinates of computed complex 27.

\begin{tabular}{c|lll|l|lll} 
Atom & $\mathrm{x}$ & $\mathrm{y}$ & $\mathrm{z}$ & Atom & $\mathrm{x}$ & $\mathrm{y}$ & $\mathrm{z}$ \\
\hline $\mathrm{C}$ & -4.08824 & -1.96221 & 0.84562 & $\mathrm{H}$ & 2.40229 & 3.10119 & 2.26253 \\
$\mathrm{C}$ & -3.71229 & -2.2656 & -0.47151 & $\mathrm{H}$ & -6.19948 & -4.10404 & -1.47485 \\
$\mathrm{~N}$ & -4.46868 & -3.02268 & -1.27316 & $\mathrm{H}$ & -7.02538 & -3.65781 & 0.83226 \\
$\mathrm{C}$ & -5.61383 & -3.49171 & -0.79082 & $\mathrm{H}$ & -3.43796 & -1.34987 & 1.46123 \\
$\mathrm{C}$ & -6.07889 & -3.24526 & 0.49598 & $\mathrm{H}$ & -5.60133 & -2.23982 & 2.34646 \\
$\mathrm{C}$ & -5.28715 & -2.45894 & 1.32924 & $\mathrm{H}$ & -2.29583 & -2.60329 & -3.08673 \\
$\mathrm{C}$ & -2.45491 & -1.75769 & -1.0274 & $\mathrm{H}$ & 0.0609 & -1.15235 & -3.00839 \\
$\mathrm{~N}$ & -1.66805 & -0.93912 & -0.31263 & $\mathrm{H}$ & 2.31506 & -1.58352 & -2.64559 \\
$\mathrm{~N}$ & -0.60392 & -0.65735 & -1.06251 & $\mathrm{H}$ & 1.83378 & -3.13993 & -1.96464 \\
$\mathrm{C}$ & -0.70677 & -1.28056 & -2.25891 & $\mathrm{H}$ & 3.34438 & -3.01009 & -2.8798 \\
$\mathrm{C}$ & -1.88959 & -2.00092 & -2.28748 & $\mathrm{H}$ & 5.35511 & -1.03928 & -0.51545 \\
$\mathrm{Re}$ & 0.95484 & 0.58056 & -0.2498 & $\mathrm{H}$ & 4.21305 & -0.16445 & -1.55918 \\
$\mathrm{~N}$ & 1.71126 & 1.2794 & 1.47635 & $\mathrm{H}$ & 5.11592 & -1.54479 & -2.19005 \\
$\mathrm{C}$ & 2.68135 & 0.58655 & 2.32372 & $\mathrm{H}$ & 4.92348 & -3.74629 & -1.11587 \\
$\mathrm{C}$ & 3.45792 & -0.49007 & 1.56861 & $\mathrm{H}$ & 3.50364 & -4.23005 & -0.19081 \\
$\mathrm{P}$ & 2.33508 & -1.32993 & 0.36383 & $\mathrm{H}$ & 4.74708 & -3.20133 & 0.55541 \\
$\mathrm{C}$ & 3.49679 & -2.14661 & -0.90588 & $\mathrm{H}$ & 3.16515 & -3.8949 & 1.9245 \\
$\mathrm{C}$ & 4.60272 & -1.15502 & -1.30246 & $\mathrm{H}$ & 1.69995 & -4.17334 & 2.8648 \\
$\mathrm{P}$ & 2.04586 & 1.31811 & -1.88568 & $\mathrm{H}$ & 2.6581 & -2.72132 & 3.1521 \\
$\mathrm{P}$ & -0.56826 & 2.45751 & 0.18418 & $\mathrm{H}$ & -0.1759 & -2.71515 & 2.81447 \\
$\mathrm{C}$ & -1.19968 & 3.51107 & -1.26418 & $\mathrm{H}$ & -0.49411 & -1.60526 & 1.4812 \\
$\mathrm{C}$ & -0.01377 & 4.17526 & -1.97912 & $\mathrm{H}$ & 0.64291 & -1.15086 & 2.77523 \\
$\mathrm{C}$ & 1.40976 & -2.66598 & 1.35678 & $\mathrm{H}$ & 1.44135 & -4.31728 & -0.10736 \\
$\mathrm{C}$ & 0.28696 & -1.97392 & 2.14883 & $\mathrm{H}$ & 0.11569 & -3.15365 & -0.34449 \\
$\mathrm{C}$ & 0.73015 & -3.66335 & 0.40538 & $\mathrm{H}$ & 0.06205 & -4.3067 & 0.9921 \\
$\mathrm{C}$ & 2.30102 & -3.39988 & 2.36967 & $\mathrm{H}$ & -1.65404 & 5.32771 & -0.1254 \\
$\mathrm{C}$ & -1.98406 & 2.17654 & 1.4413 & $\mathrm{H}$ & -3.07333 & 4.29659 & -0.39754 \\
$\mathrm{C}$ & -3.28925 & 1.75513 & 0.74725 & $\mathrm{H}$ & -2.40109 & 5.23922 & -1.72392 \\
$\mathrm{C}$ & 0.65078 & 3.50257 & 1.09399 & $\mathrm{H}$ & -0.38605 & 4.66097 & -2.8896
\end{tabular}




\begin{tabular}{l|lll|l|lll}
$\mathrm{C}$ & 1.44501 & 2.60354 & 2.03985 & $\mathrm{H}$ & 0.74982 & 3.45503 & -2.28606 \\
$\mathrm{C}$ & -1.5888 & 1.01449 & 2.36815 & $\mathrm{H}$ & 0.46218 & 4.9482 & -1.36642 \\
$\mathrm{C}$ & -2.22888 & 3.41594 & 2.31659 & $\mathrm{H}$ & -1.21397 & 1.78633 & -2.60464 \\
$\mathrm{C}$ & 4.19559 & -3.40382 & -0.3693 & $\mathrm{H}$ & -2.2319 & 3.14573 & -3.1297 \\
$\mathrm{C}$ & 2.69009 & -2.49213 & -2.16645 & $\mathrm{H}$ & -2.77336 & 2.07408 & -1.83045 \\
$\mathrm{C}$ & -1.89761 & 2.56779 & -2.25803 & $\mathrm{H}$ & -1.38088 & 3.61707 & 2.9803 \\
$\mathrm{C}$ & -2.1387 & 4.64904 & -0.83661 & $\mathrm{H}$ & -3.09881 & 3.22079 & 2.95691 \\
$\mathrm{H}$ & 4.21062 & 0.01237 & 0.95546 & $\mathrm{H}$ & -2.44077 & 4.32317 & 1.74633 \\
$\mathrm{H}$ & 3.97665 & -1.17011 & 2.2515 & $\mathrm{H}$ & -3.75182 & 2.55918 & 0.16979 \\
$\mathrm{H}$ & 3.40952 & 1.31194 & 2.7181 & $\mathrm{H}$ & -4.01101 & 1.45916 & 1.52012 \\
$\mathrm{H}$ & 2.18641 & 0.15281 & 3.20883 & $\mathrm{H}$ & -3.12915 & 0.88953 & 0.09972 \\
$\mathrm{H}$ & 0.21453 & 4.35627 & 1.6213 & $\mathrm{H}$ & -1.56576 & 0.08075 & 1.8063 \\
$\mathrm{H}$ & 1.33173 & 3.88316 & 0.32767 & $\mathrm{H}$ & -2.35078 & 0.92351 & 3.15459 \\
$\mathrm{H}$ & 0.92104 & 2.5183 & 3.00772 & $\mathrm{H}$ & -0.62196 & 1.15786 & 2.85713
\end{tabular}

Table 7.7: XYZ-coordinates of computed complex 28.

\begin{tabular}{c|lll|l|lll} 
Atom & $\mathrm{x}$ & $\mathrm{y}$ & $\mathrm{z}$ & Atom & $\mathrm{x}$ & $\mathrm{y}$ & $\mathrm{z}$ \\
\hline $\mathrm{C}$ & -4.3902 & -1.72852 & 0.68012 & $\mathrm{H}$ & 2.39011 & 2.83389 & 2.50015 \\
$\mathrm{C}$ & -3.95146 & -2.04633 & -0.61382 & $\mathrm{H}$ & -6.49985 & -3.67487 & -1.80819 \\
$\mathrm{~N}$ & -4.70853 & -2.73484 & -1.47469 & $\mathrm{H}$ & -7.44592 & -3.18167 & 0.44293 \\
$\mathrm{C}$ & -5.91689 & -3.1163 & -1.0774 & $\mathrm{H}$ & -3.73695 & -1.17622 & 1.34716 \\
$\mathrm{C}$ & -6.44815 & -2.84464 & 0.17836 & $\mathrm{H}$ & -6.02048 & -1.89806 & 2.0709 \\
$\mathrm{C}$ & -5.6552 & -2.13214 & 1.0743 & $\mathrm{H}$ & -2.38985 & -2.50984 & -3.1207 \\
$\mathrm{C}$ & -2.62529 & -1.63287 & -1.08165 & $\mathrm{H}$ & 0.05693 & -1.23939 & -2.88861 \\
$\mathrm{~N}$ & -1.83228 & -0.86508 & -0.32011 & $\mathrm{H}$ & 2.29748 & -1.70771 & -2.52845 \\
$\mathrm{~N}$ & -0.69998 & -0.6715 & -0.99561 & $\mathrm{H}$ & 1.66446 & -3.23322 & -1.90491 \\
$\mathrm{C}$ & -0.7669 & -1.30099 & -2.19254 & $\mathrm{H}$ & 3.23023 & -3.20384 & -2.73303 \\
$\mathrm{C}$ & -1.99478 & -1.93122 & -2.29867 & $\mathrm{H}$ & 5.23673 & -1.41681 & -0.21991 \\
$\mathrm{Re}$ & 0.89401 & 0.46156 & -0.1079 & $\mathrm{H}$ & 4.20504 & -0.42766 & -1.2733 \\
$\mathrm{~N}$ & 1.61822 & 1.07197 & 1.65709 & $\mathrm{H}$ & 5.04482 & -1.83847 & -1.92385 \\
$\mathrm{C}$ & 2.50831 & 0.3024 & 2.5275 & $\mathrm{H}$ & 4.67193 & -4.05966 & -0.92459 \\
$\mathrm{C}$ & 3.25955 & -0.79584 & 1.77829 & $\mathrm{H}$ & 3.18403 & -4.48351 & -0.08114 \\
$\mathrm{P}$ & 2.15224 & -1.53135 & 0.49368 & $\mathrm{H}$ & 4.447 & -3.5486 & 0.75115 \\
$\mathrm{C}$ & 3.33277 & -2.38412 & -0.73646 & $\mathrm{H}$ & 2.78466 & -4.16551 & 2.02875 \\
$\mathrm{C}$ & 4.51637 & -1.45246 & -1.0437 & $\mathrm{H}$ & 1.25972 & -4.40553 & 2.88136 \\
$\mathrm{As}$ & 2.14395 & 1.22159 & -1.7567 & $\mathrm{H}$ & 2.26309 & -3.00691 & 3.26421 \\
$\mathrm{P}$ & -0.54732 & 2.40109 & 0.32784 & $\mathrm{H}$ & -0.53146 & -2.84781 & 2.79689 \\
$\mathrm{C}$ & -1.10576 & 3.52212 & -1.10099 & $\mathrm{H}$ & -0.7449 & -1.7034 & 1.47284 \\
$\mathrm{C}$ & 0.11867 & 4.13441 & -1.795 & $\mathrm{H}$ & 0.35616 & -1.32065 & 2.81863 \\
$\mathrm{C}$ & 1.11574 & -2.84509 & 1.40713 & $\mathrm{H}$ & 1.13532 & -4.46415 & -0.0935 \\
$\mathrm{C}$ & -0.00867 & -2.11773 & 2.16424 & $\mathrm{H}$ & -0.12366 & -3.23501 & -0.36003 \\
$\mathrm{C}$ & 0.43348 & -3.78839 & 0.40358 & $\mathrm{H}$ & -0.28832 & -4.4124 & 0.94606 \\
$\mathrm{C}$ & 1.91969 & -3.6459 & 2.44304 & $\mathrm{H}$ & -1.45494 & 5.3428 & 0.06675 \\
$\mathrm{C}$ & -2.00175 & 2.16687 & 1.5551 & $\mathrm{H}$ & -2.9313 & 4.39927 & -0.21597 \\
$\mathrm{C}$ & -3.32377 & 1.85679 & 0.83469 & $\mathrm{H}$ & -2.2104 & 5.32211 & -1.53006
\end{tabular}


7.5 Transition Metal Complexes with Metal-Pnictogen Multiple Bonds

\begin{tabular}{l|lll|l|lll}
$\mathrm{C}$ & 0.69884 & 3.36212 & 1.29282 & $\mathrm{H}$ & -0.22116 & 4.67797 & -2.68518 \\
$\mathrm{C}$ & 1.41184 & 2.39927 & 2.24031 & $\mathrm{H}$ & 0.83124 & 3.37733 & -2.13456 \\
$\mathrm{C}$ & -1.69657 & 0.95086 & 2.44578 & $\mathrm{H}$ & 0.64849 & 4.84856 & -1.15602 \\
$\mathrm{C}$ & -2.17728 & 3.38989 & 2.46997 & $\mathrm{H}$ & -1.21154 & 1.81961 & -2.46575 \\
$\mathrm{C}$ & 3.92958 & -3.69469 & -0.20323 & $\mathrm{H}$ & -2.12952 & 3.25038 & -2.98787 \\
$\mathrm{C}$ & 2.57698 & -2.64924 & -2.04685 & $\mathrm{H}$ & -2.76042 & 2.20043 & -1.71151 \\
$\mathrm{C}$ & -1.84786 & 2.64073 & -2.11916 & $\mathrm{H}$ & -1.33723 & 3.49983 & 3.16429 \\
$\mathrm{C}$ & -1.97941 & 4.70397 & -0.65317 & $\mathrm{H}$ & -3.07923 & 3.24122 & 3.0776 \\
$\mathrm{H}$ & 4.07485 & -0.32512 & 1.2227 & $\mathrm{H}$ & -2.30034 & 4.33115 & 1.92911 \\
$\mathrm{H}$ & 3.69369 & -1.52927 & 2.46465 & $\mathrm{H}$ & -3.73018 & 2.7105 & 0.28743 \\
$\mathrm{H}$ & 3.25376 & 0.97619 & 2.97643 & $\mathrm{H}$ & -4.06969 & 1.57709 & 1.59055 \\
$\mathrm{H}$ & 1.94681 & -0.12614 & 3.37453 & $\mathrm{H}$ & -3.21245 & 1.00988 & 0.15345 \\
$\mathrm{H}$ & 0.29382 & 4.22544 & 1.82917 & $\mathrm{H}$ & -1.72984 & 0.03722 & 1.85228 \\
$\mathrm{H}$ & 1.42206 & 3.72236 & 0.55606 & $\mathrm{H}$ & -2.47056 & 0.88587 & 3.22305 \\
$\mathrm{H}$ & 0.85627 & 2.32388 & 3.19101 & $\mathrm{H}$ & -0.72691 & 1.0117 & 2.94595
\end{tabular}

Table 7.8: XYZ-coordinates of computed complex 29.

\begin{tabular}{c|lll|l|lll} 
Atom & $\mathrm{x}$ & $\mathrm{y}$ & $\mathrm{z}$ & Atom & $\mathrm{x}$ & $\mathrm{y}$ & $\mathrm{z}$ \\
\hline $\mathrm{Re}$ & -0.19434 & -0.11397 & 0 & $\mathrm{H}$ & -4.34273 & -0.1177 & 2.51529 \\
$\mathrm{P}$ & -0.53782 & -0.40157 & 2.42575 & $\mathrm{H}$ & -3.32217 & -1.19921 & 1.56945 \\
$\mathrm{P}$ & -0.53782 & -0.40157 & -2.42575 & $\mathrm{H}$ & -3.32399 & 0.53884 & 1.23971 \\
$\mathrm{~N}$ & -1.01178 & -1.91397 & 0 & $\mathrm{C}$ & -2.6669 & -0.90421 & 4.29185 \\
$\mathrm{C}$ & -0.515 & -2.24688 & 2.43237 & $\mathrm{H}$ & -3.58328 & -0.50893 & 4.74759 \\
$\mathrm{H}$ & -0.92173 & -2.6992 & 3.34153 & $\mathrm{H}$ & -1.9146 & -0.9645 & 5.07975 \\
$\mathrm{H}$ & 0.53502 & -2.55019 & 2.35436 & $\mathrm{H}$ & -2.89557 & -1.9224 & 3.95662 \\
$\mathrm{C}$ & -1.26065 & -2.74142 & 1.19784 & $\mathrm{C}$ & 0.42023 & -0.09792 & 5.15183 \\
$\mathrm{H}$ & -2.34233 & -2.79445 & 1.38364 & $\mathrm{H}$ & -0.43128 & 0.49435 & 5.49598 \\
$\mathrm{H}$ & -0.95178 & -3.77605 & 0.99162 & $\mathrm{H}$ & 1.2798 & 0.18888 & 5.77154 \\
$\mathrm{C}$ & -0.515 & -2.24688 & -2.43237 & $\mathrm{H}$ & 0.21687 & -1.15605 & 5.35442 \\
$\mathrm{H}$ & 0.53502 & -2.55019 & -2.35436 & $\mathrm{C}$ & 2.07284 & -0.62756 & 3.39443 \\
$\mathrm{H}$ & -0.92173 & -2.6992 & -3.34153 & $\mathrm{H}$ & 2.87605 & -0.20277 & 4.0095 \\
$\mathrm{C}$ & -1.26065 & -2.74142 & -1.19784 & $\mathrm{H}$ & 2.3851 & -0.54941 & 2.35143 \\
$\mathrm{H}$ & -0.95178 & -3.77605 & -0.99162 & $\mathrm{H}$ & 1.99386 & -1.68779 & 3.65816 \\
$\mathrm{H}$ & -2.34233 & -2.79445 & -1.38364 & $\mathrm{C}$ & 1.06504 & 1.643 & 3.45551 \\
$\mathrm{C}$ & -2.28327 & 0.00882 & 3.11229 & $\mathrm{H}$ & 0.18749 & 2.27663 & 3.61189 \\
$\mathrm{C}$ & 0.77945 & 0.15096 & 3.68113 & $\mathrm{H}$ & 1.44241 & 1.82918 & 2.44516 \\
$\mathrm{C}$ & -2.28327 & 0.00882 & -3.11229 & $\mathrm{H}$ & 1.83626 & 1.96877 & 4.16519 \\
$\mathrm{C}$ & 0.77945 & 0.15096 & -3.68113 & $\mathrm{C}$ & -2.6669 & -0.90421 & -4.29185 \\
$\mathrm{~N}$ & 1.78402 & -1.00735 & 0 & $\mathrm{H}$ & -2.89557 & -1.9224 & -3.95662 \\
$\mathrm{C}$ & 4.34541 & -2.16298 & 0 & $\mathrm{H}$ & -1.9146 & -0.9645 & -5.07975 \\
$\mathrm{C}$ & 2.91143 & -0.24172 & 0 & $\mathrm{H}$ & -3.58328 & -0.50893 & -4.74759 \\
$\mathrm{C}$ & 1.95641 & -2.34846 & 0 & $\mathrm{C}$ & -3.36098 & -0.20987 & -2.03319 \\
$\mathrm{C}$ & 3.19687 & -2.9553 & 0 & $\mathrm{H}$ & -3.32399 & 0.53884 & -1.23971 \\
$\mathrm{C}$ & 4.19379 & -0.78921 & 0 & $\mathrm{H}$ & -3.32217 & -1.19921 & -1.56945 \\
$\mathrm{H}$ & 1.05335 & -2.94561 & 0 & $\mathrm{H}$ & -4.34273 & -0.1177 & -2.51529
\end{tabular}




\begin{tabular}{l|lll|l|lll}
$\mathrm{H}$ & 3.25731 & -4.03899 & 0 & $\mathrm{C}$ & -2.3634 & 1.47919 & -3.54572 \\
$\mathrm{H}$ & 5.05184 & -0.12388 & 0 & $\mathrm{H}$ & -2.03876 & 2.16406 & -2.75356 \\
$\mathrm{H}$ & 5.3335 & -2.61383 & 0 & $\mathrm{H}$ & -3.4096 & 1.71994 & -3.77339 \\
$\mathrm{C}$ & 2.62916 & 1.16407 & 0 & $\mathrm{H}$ & -1.78035 & 1.69033 & -4.44687 \\
$\mathrm{C}$ & 3.37995 & 2.35359 & 0 & $\mathrm{C}$ & 0.42023 & -0.09792 & -5.15183 \\
$\mathrm{C}$ & 2.43361 & 3.35403 & 0 & $\mathrm{H}$ & 0.21687 & -1.15605 & -5.35442 \\
$\mathrm{~N}$ & 1.19865 & 2.77801 & 0 & $\mathrm{H}$ & 1.2798 & 0.18888 & -5.77154 \\
$\mathrm{~N}$ & 1.31505 & 1.44436 & 0 & $\mathrm{H}$ & -0.43128 & 0.49435 & -5.49598 \\
$\mathrm{H}$ & 4.45464 & 2.47122 & 0 & $\mathrm{C}$ & 1.06504 & 1.643 & -3.45551 \\
$\mathrm{H}$ & 2.53231 & 4.43087 & 0 & $\mathrm{H}$ & 1.44241 & 1.82918 & -2.44516 \\
$\mathrm{P}$ & -1.55495 & 1.85284 & 0 & $\mathrm{H}$ & 0.18749 & 2.27663 & -3.61189 \\
$\mathrm{P}$ & -0.43787 & 3.55582 & 0 & $\mathrm{H}$ & 1.83626 & 1.96877 & -4.16519 \\
$\mathrm{C}$ & -2.3634 & 1.47919 & 3.54572 & $\mathrm{C}$ & 2.07284 & -0.62756 & -3.39443 \\
$\mathrm{H}$ & -3.4096 & 1.71994 & 3.77339 & $\mathrm{H}$ & 2.3851 & -0.54941 & -2.35143 \\
$\mathrm{H}$ & -2.03876 & 2.16406 & 2.75356 & $\mathrm{H}$ & 2.87605 & -0.20277 & -4.0095 \\
$\mathrm{H}$ & -1.78035 & 1.69033 & 4.44687 & $\mathrm{H}$ & 1.99386 & -1.68779 & -3.65816 \\
$\mathrm{C}$ & -3.36098 & -0.20987 & 2.03319 & & & &
\end{tabular}

Table 7.9: XYZ-coordinates of computed complex 29 for NMR calculations.

\begin{tabular}{c|lll|l|lll} 
Atom & $\mathrm{x}$ & $\mathrm{y}$ & $\mathrm{z}$ & Atom & $\mathrm{x}$ & $\mathrm{y}$ & $\mathrm{z}$ \\
\hline $\mathrm{Re}$ & -0.27778 & -0.07896 & 0.00014 & $\mathrm{H}$ & -4.31155 & 0.10372 & 2.79774 \\
$\mathrm{P}$ & -0.54519 & -0.41368 & 2.45822 & $\mathrm{H}$ & -3.45761 & -1.01454 & 1.73701 \\
$\mathrm{P}$ & -0.54504 & -0.41415 & -2.45782 & $\mathrm{H}$ & -3.32275 & 0.72691 & 1.49773 \\
$\mathrm{~N}$ & -1.06454 & -1.86069 & 0.00032 & $\mathrm{C}$ & -2.61044 & -1.00114 & 4.36631 \\
$\mathrm{C}$ & -0.55891 & -2.23865 & 2.42030 & $\mathrm{H}$ & -3.47004 & -0.5996 & 4.90830 \\
$\mathrm{H}$ & -0.96742 & -2.68664 & 3.32432 & $\mathrm{H}$ & -1.8319 & -1.20048 & 5.09663 \\
$\mathrm{H}$ & 0.47859 & -2.56577 & 2.34019 & $\mathrm{H}$ & -2.9277 & -1.95404 & 3.93833 \\
$\mathrm{C}$ & -1.32241 & -2.69681 & 1.19103 & $\mathrm{C}$ & 0.50606 & -0.3207 & 5.15853 \\
$\mathrm{H}$ & -2.39889 & -2.72983 & 1.38958 & $\mathrm{H}$ & -0.34938 & 0.21058 & 5.56596 \\
$\mathrm{H}$ & -1.03015 & -3.72753 & 0.96622 & $\mathrm{H}$ & 1.36953 & -0.04848 & 5.76960 \\
$\mathrm{C}$ & -0.55891 & -2.23912 & -2.41968 & $\mathrm{H}$ & 0.34591 & -1.39237 & 5.29496 \\
$\mathrm{H}$ & 0.47862 & -2.5662 & -2.33973 & $\mathrm{C}$ & 2.1116 & -0.71713 & 3.34683 \\
$\mathrm{H}$ & -0.96754 & -2.68728 & -3.32357 & $\mathrm{H}$ & 2.89702 & -0.37235 & 4.02185 \\
$\mathrm{C}$ & -1.32217 & -2.69716 & -1.19020 & $\mathrm{H}$ & 2.45114 & -0.51861 & 2.33461 \\
$\mathrm{H}$ & -1.02966 & -3.72776 & -0.96517 & $\mathrm{H}$ & 2.02725 & -1.79522 & 3.48839 \\
$\mathrm{H}$ & -2.39864 & -2.73055 & -1.38870 & $\mathrm{C}$ & 1.10961 & 1.53748 & 3.58328 \\
$\mathrm{C}$ & -2.22994 & 0.00451 & 3.26554 & $\mathrm{H}$ & 0.24676 & 2.15243 & 3.82700 \\
$\mathrm{C}$ & 0.81996 & 0.03548 & 3.69857 & $\mathrm{H}$ & 1.44897 & 1.80794 & 2.58236 \\
$\mathrm{C}$ & -2.22957 & 0.00448 & -3.26527 & $\mathrm{H}$ & 1.90552 & 1.80268 & 4.28297 \\
$\mathrm{C}$ & 0.81986 & 0.03486 & -3.69843 & $\mathrm{C}$ & -2.60957 & -1.00054 & -4.36675 \\
$\mathrm{~N}$ & 1.76168 & -0.89195 & 0.00013 & $\mathrm{H}$ & -2.92703 & -1.95366 & -3.93940 \\
$\mathrm{C}$ & 4.35749 & -1.91028 & -0.00032 & $\mathrm{H}$ & -1.83063 & -1.19954 & -5.09679 \\
$\mathrm{C}$ & 2.83483 & -0.06746 & -0.00023 & $\mathrm{H}$ & -3.46888 & -0.59872 & -4.90898 \\
$\mathrm{C}$ & 1.9961 & -2.21712 & 0.00038 & $\mathrm{C}$ & -3.37982 & -0.05607 & -2.24915 \\
$\mathrm{C}$ & 3.25811 & -2.75945 & 0.00010 & $\mathrm{H}$ & -3.32292 & 0.72624 & -1.49759
\end{tabular}


7.5 Transition Metal Complexes with Metal-Pnictogen Multiple Bonds

\begin{tabular}{l|lll|l|lll}
$\mathrm{C}$ & 4.13651 & -0.55075 & -0.00043 & $\mathrm{H}$ & -3.4578 & -1.0151 & -1.73749 \\
$\mathrm{H}$ & 1.12704 & -2.8549 & 0.00082 & $\mathrm{H}$ & -4.31131 & 0.10354 & -2.79815 \\
$\mathrm{H}$ & 3.37298 & -3.83463 & 0.00023 & $\mathrm{C}$ & -2.23225 & 1.42957 & -3.83161 \\
$\mathrm{H}$ & 4.95727 & 0.15369 & -0.00067 & $\mathrm{H}$ & -1.95877 & 2.16552 & -3.07371 \\
$\mathrm{H}$ & 5.36468 & -2.30574 & -0.00049 & $\mathrm{H}$ & -3.24507 & 1.66779 & -4.16670 \\
$\mathrm{C}$ & 2.47737 & 1.32804 & -0.00041 & $\mathrm{H}$ & -1.57745 & 1.55584 & -4.69089 \\
$\mathrm{C}$ & 3.1593 & 2.55583 & -0.00098 & $\mathrm{C}$ & 0.50528 & -0.32134 & -5.15824 \\
$\mathrm{C}$ & 2.1632 & 3.4989 & -0.00100 & $\mathrm{H}$ & 0.34526 & -1.39304 & -5.29458 \\
$\mathrm{~N}$ & 0.96866 & 2.85809 & -0.00054 & $\mathrm{H}$ & 1.36839 & -0.049 & -5.76976 \\
$\mathrm{~N}$ & 1.16008 & 1.53884 & -0.00018 & $\mathrm{H}$ & -0.3505 & 0.20978 & -5.56523 \\
$\mathrm{H}$ & 4.21985 & 2.74056 & -0.00133 & $\mathrm{C}$ & 1.10945 & 1.53685 & -3.58323 \\
$\mathrm{H}$ & 2.20719 & 4.57579 & -0.00136 & $\mathrm{H}$ & 1.44907 & 1.80734 & -2.58239 \\
$\mathrm{P}$ & -1.69907 & 1.81838 & -0.00034 & $\mathrm{H}$ & 0.24648 & 2.15178 & -3.82670 \\
$\mathrm{P}$ & -0.67936 & 3.53012 & -0.00055 & $\mathrm{H}$ & 1.90516 & 1.80212 & -4.28311 \\
$\mathrm{C}$ & -2.23298 & 1.42929 & 3.83261 & $\mathrm{C}$ & 2.11164 & -0.71775 & -3.34712 \\
$\mathrm{H}$ & -3.24596 & 1.66723 & 4.16742 & $\mathrm{H}$ & 2.45153 & -0.51919 & -2.33502 \\
$\mathrm{H}$ & -1.95927 & 2.16568 & 3.07523 & $\mathrm{H}$ & 2.89681 & -0.37294 & -4.02241 \\
$\mathrm{H}$ & -1.57859 & 1.55516 & 4.69223 & $\mathrm{H}$ & 2.02729 & -1.79584 & -3.48863 \\
$\mathrm{C}$ & -3.37986 & -0.05565 & 2.24901 & & & &
\end{tabular}

Table 7.10: XYZ-coordinates of computed complex 30.

\begin{tabular}{c|lll|l|lll} 
Atom & $\mathrm{x}$ & $\mathrm{y}$ & $\mathrm{z}$ & Atom & $\mathrm{x}$ & $\mathrm{y}$ & $\mathrm{z}$ \\
\hline $\mathrm{Re}$ & 0.03197 & 0.36729 & 0 & $\mathrm{C}$ & 0.71418 & 2.40038 & -2.42855 \\
$\mathrm{As}$ & -2.16449 & -0.81827 & 0 & $\mathrm{H}$ & 1.78587 & 2.20033 & -2.33896 \\
$\mathrm{As}$ & -1.78426 & -3.01211 & 0 & $\mathrm{H}$ & 0.56716 & 2.98194 & -3.34166 \\
$\mathrm{P}$ & -0.13183 & 0.76235 & 2.42344 & $\mathrm{C}$ & -1.86163 & 1.19072 & -3.14576 \\
$\mathrm{P}$ & -0.13183 & 0.76235 & -2.42344 & $\mathrm{C}$ & -2.77014 & 1.8275 & -2.07487 \\
$\mathrm{~N}$ & 0.09386 & 2.32838 & 0 & $\mathrm{H}$ & -3.65212 & 2.24156 & -2.57827 \\
$\mathrm{~N}$ & 2.26294 & 0.38003 & 0 & $\mathrm{H}$ & -2.29718 & 2.64429 & -1.52595 \\
$\mathrm{~N}$ & 0.77983 & -1.69716 & 0 & $\mathrm{H}$ & -3.13539 & 1.10227 & -1.34703 \\
$\mathrm{~N}$ & 0.15831 & -2.8757 & 0 & $\mathrm{C}$ & -1.76182 & 2.22107 & -4.28718 \\
$\mathrm{C}$ & 0.25517 & 3.18094 & 1.20194 & $\mathrm{H}$ & -2.74447 & 2.30265 & -4.7672 \\
$\mathrm{H}$ & -0.68495 & 3.71072 & 1.39831 & $\mathrm{H}$ & -1.04354 & 1.96064 & -5.06437 \\
$\mathrm{H}$ & 0.99398 & 3.96505 & 0.99056 & $\mathrm{H}$ & -1.51365 & 3.21843 & -3.90863 \\
$\mathrm{C}$ & 0.71418 & 2.40038 & 2.42855 & $\mathrm{C}$ & -2.57417 & -0.07319 & -3.65148 \\
$\mathrm{H}$ & 0.56716 & 2.98194 & 3.34166 & $\mathrm{H}$ & -2.60564 & -0.86311 & -2.89299 \\
$\mathrm{H}$ & 1.78587 & 2.20033 & 2.33896 & $\mathrm{H}$ & -2.12039 & -0.4846 & -4.5562 \\
$\mathrm{C}$ & -1.86163 & 1.19072 & 3.14576 & $\mathrm{H}$ & -3.61266 & 0.18053 & -3.89678 \\
$\mathrm{C}$ & -2.77014 & 1.8275 & 2.07487 & $\mathrm{C}$ & 0.81163 & -0.31838 & -3.67216 \\
$\mathrm{H}$ & -3.13539 & 1.10227 & 1.34703 & $\mathrm{C}$ & 0.64488 & 0.08482 & -5.14308 \\
$\mathrm{H}$ & -2.29718 & 2.64429 & 1.52595 & $\mathrm{H}$ & -0.37054 & -0.05657 & -5.51864 \\
$\mathrm{H}$ & -3.65212 & 2.24156 & 2.57827 & $\mathrm{H}$ & 1.30134 & -0.54783 & -5.75347 \\
$\mathrm{C}$ & -1.76182 & 2.22107 & 4.28718 & $\mathrm{H}$ & 0.9433 & 1.12395 & -5.31948 \\
$\mathrm{H}$ & -2.74447 & 2.30265 & 4.7672 & $\mathrm{C}$ & 0.37529 & -1.77973 & -3.47465 \\
$\mathrm{H}$ & -1.51365 & 3.21843 & 3.90863 & $\mathrm{H}$ & -0.68681 & -1.93941 & -3.6725
\end{tabular}




\begin{tabular}{l|lll|l|lll}
$\mathrm{H}$ & -1.04354 & 1.96064 & 5.06437 & $\mathrm{H}$ & 0.5838 & -2.12568 & -2.45895 \\
$\mathrm{C}$ & -2.57417 & -0.07319 & 3.65148 & $\mathrm{H}$ & 0.93895 & -2.41721 & -4.16713 \\
$\mathrm{H}$ & -3.61266 & 0.18053 & 3.89678 & $\mathrm{C}$ & 2.31219 & -0.22512 & -3.35184 \\
$\mathrm{H}$ & -2.12039 & -0.4846 & 4.5562 & $\mathrm{H}$ & 2.5276 & -0.45462 & -2.30899 \\
$\mathrm{H}$ & -2.60564 & -0.86311 & 2.89299 & $\mathrm{H}$ & 2.73251 & 0.75744 & -3.58924 \\
$\mathrm{C}$ & 0.81163 & -0.31838 & 3.67216 & $\mathrm{H}$ & 2.84655 & -0.96 & -3.96571 \\
$\mathrm{C}$ & 2.31219 & -0.22512 & 3.35184 & $\mathrm{C}$ & 2.99498 & 1.5117 & 0 \\
$\mathrm{H}$ & 2.84655 & -0.96 & 3.96571 & $\mathrm{H}$ & 2.44368 & 2.43968 & 0 \\
$\mathrm{H}$ & 2.73251 & 0.75744 & 3.58924 & $\mathrm{C}$ & 4.3747 & 1.52655 & 0 \\
$\mathrm{H}$ & 2.5276 & -0.45462 & 2.30899 & $\mathrm{H}$ & 4.89506 & 2.47767 & 0 \\
$\mathrm{C}$ & 0.37529 & -1.77973 & 3.47465 & $\mathrm{C}$ & 5.06614 & 0.31711 & 0 \\
$\mathrm{H}$ & -0.68681 & -1.93941 & 3.6725 & $\mathrm{H}$ & 6.15039 & 0.29451 & 0 \\
$\mathrm{H}$ & 0.93895 & -2.41721 & 4.16713 & $\mathrm{C}$ & 4.33463 & -0.85408 & 0 \\
$\mathrm{H}$ & 0.5838 & -2.12568 & 2.45895 & $\mathrm{H}$ & 4.82041 & -1.82353 & 0 \\
$\mathrm{C}$ & 0.64488 & 0.08482 & 5.14308 & $\mathrm{C}$ & 2.94292 & -0.7915 & 0 \\
$\mathrm{H}$ & -0.37054 & -0.05657 & 5.51864 & $\mathrm{C}$ & 2.09701 & -1.95278 & 0 \\
$\mathrm{H}$ & 0.9433 & 1.12395 & 5.31948 & $\mathrm{C}$ & 2.32893 & -3.33629 & 0 \\
$\mathrm{H}$ & 1.30134 & -0.54783 & 5.75347 & $\mathrm{H}$ & 3.27291 & -3.86128 & 0 \\
$\mathrm{C}$ & 0.25517 & 3.18094 & -1.20194 & $\mathrm{C}$ & 1.06528 & -3.88225 & 0 \\
$\mathrm{H}$ & 0.99398 & 3.96505 & -0.99056 & $\mathrm{H}$ & 0.73654 & -4.91122 & 0 \\
$\mathrm{H}$ & -0.68495 & 3.71072 & -1.39831 & & & &
\end{tabular}

Table 7.11: XYZ-coordinates of computed compound $\mathrm{P}_{2}$.

\begin{tabular}{c|lll|l|lll} 
Atom & $\mathrm{x}$ & $\mathrm{y}$ & $\mathrm{z}$ & Atom & $\mathrm{x}$ & $\mathrm{y}$ & $\mathrm{z}$ \\
\hline $\mathrm{P}$ & 0 & 0 & 0.9478 & $\mathrm{P}$ & 0 & 0 & -0.9478
\end{tabular}

Table 7.12: XYZ-coordinates of computed compound $\mathrm{P}_{2} \mathrm{H}_{2}$.

\begin{tabular}{c|lll|c|lll} 
Atom & $\mathrm{x}$ & $\mathrm{y}$ & $\mathrm{z}$ & Atom & $\mathrm{x}$ & $\mathrm{y}$ & $\mathrm{z}$ \\
\hline $\mathrm{P}$ & 0 & 1.02017 & 0 & $\mathrm{P}$ & 0 & -1.02017 & 0 \\
$\mathrm{H}$ & 1.42934 & 1.1292 & 0 & $\mathrm{H}$ & -1.42934 & -1.1292 & 0
\end{tabular}

Table 7.13: XYZ-coordinates of computed compound $\mathrm{P}_{2} \mathrm{H}_{4}$.

\begin{tabular}{c|lll|l|lll} 
Atom & $\mathrm{x}$ & $\mathrm{y}$ & $\mathrm{z}$ & Atom & $\mathrm{x}$ & $\mathrm{y}$ & $\mathrm{z}$ \\
\hline $\mathrm{P}$ & 0 & 1.3155 & 0 & $\mathrm{P}$ & 0 & -1.13155 & 0 \\
$\mathrm{H}$ & 0.98088 & 1.23025 & 1.03186 & $\mathrm{H}$ & 0.98088 & 1.23025 & -1.03186 \\
$\mathrm{H}$ & -0.98088 & -1.23025 & 1.03186 & $\mathrm{H}$ & -0.9888 & -1.23025 & -1.03186
\end{tabular}

\subsubsection{Oxygenation of a Terminal Re Phosphide Complex - Computational Details}

DFT calculations were carried out with the ORCA program package using the PBE and PBE0 functionals. [304], [318], [326] Ahlrichs' basis sets def2-SVP (for geometry optimization 
and frequency calculation) and def2-TZVP (for single-point energies) were used with a full basis for all elements but Os for which the Stuttgart-Dresden 60 electron core potential was chosen to replace the inner shell 1s-4f orbitals. ${ }^{[307],[308]}$ The RI-J (PBE) approximation in combination with the corresponding auxiliary basis sets of Alrichs was utilized to reduce computational costs in the geometry optimization and frequency calculations. ${ }^{[327]-[329]}$ Grimme's model (D3) with Becke-Johnson damping was used to account for dispersion with the PBE or PBE0 functionals. ${ }^{[306],[330]}$ No symmetry restraints were imposed and the optimized structures were defined as minima (no negative eigenvalues) by vibrational analyses. NBO analysis of $\mathbf{1 8}, \mathbf{1 9}$ and $\mathbf{2 0}$ was carried out with NBO 6.0. Geometries were analyzed and visualized with Avogadro, molecular orbitals were visualized with Chimera and for NBO/NLMO visualization, Jmol (http://www.jmol.org) was used. 34 was computed with and without the $\left[\mathrm{K}\left(\mathrm{C}_{12} \mathrm{H}_{24} \mathrm{O}_{6}\right)\right]$ cation. No significant structural changes were observed. Therefore, the smaller model was utlized to reduce computational costs.

Table 7.14: XYZ-coordinates of computed complex $\left[\operatorname{Re}\left(\mathrm{L}^{1}\right)\left(\kappa N^{2}-\mathrm{PyrPz}\right)(\mathrm{PO})\right]$.

\begin{tabular}{c|lll|l|lll} 
Atom & $\mathrm{x}$ & $\mathrm{y}$ & $\mathrm{z}$ & Atom & $\mathrm{x}$ & $\mathrm{y}$ & $\mathrm{z}$ \\
\hline $\mathrm{C}$ & -4.02244 & -2.23688 & 1.06678 & $\mathrm{H}$ & -6.10793 & -4.46189 & -1.27152 \\
$\mathrm{C}$ & -3.68536 & -2.45207 & -0.29462 & $\mathrm{H}$ & -6.84313 & -4.17901 & 1.119 \\
$\mathrm{~N}$ & -4.43525 & -3.24214 & -1.10074 & $\mathrm{H}$ & -3.38526 & -1.58379 & 1.68 \\
$\mathrm{C}$ & -5.52112 & -3.82701 & -0.5813 & $\mathrm{H}$ & -5.4427 & -2.70159 & 2.64284 \\
$\mathrm{C}$ & -5.93692 & -3.67474 & 0.75232 & $\mathrm{H}$ & -2.49915 & -2.47226 & -3.0411 \\
$\mathrm{C}$ & -5.15987 & -2.85626 & 1.58967 & $\mathrm{H}$ & -0.1893 & -0.87845 & -3.04419 \\
$\mathrm{C}$ & -2.50537 & -1.82333 & -0.88872 & $\mathrm{H}$ & 2.12375 & -1.5301 & -2.63196 \\
$\mathrm{~N}$ & -1.6796 & -1.02105 & -0.16059 & $\mathrm{H}$ & 1.75343 & -3.15808 & -2.00539 \\
$\mathrm{~N}$ & -0.70005 & -0.60349 & -0.97677 & $\mathrm{H}$ & 3.23011 & -2.8966 & -2.98909 \\
$\mathrm{C}$ & -0.88589 & -1.1207 & -2.23446 & $\mathrm{H}$ & 5.24612 & -0.9476 & -0.55195 \\
$\mathrm{C}$ & -2.03775 & -1.91214 & -2.22282 & $\mathrm{H}$ & 4.07642 & -0.10579 & -1.62442 \\
$\mathrm{Re}$ & 0.88808 & 0.58375 & -0.16496 & $\mathrm{H}$ & 5.03683 & -1.47036 & -2.24438 \\
$\mathrm{~N}$ & 1.74369 & 1.29855 & 1.46305 & $\mathrm{H}$ & 4.84754 & -3.7314 & -1.25126 \\
$\mathrm{C}$ & 2.67852 & 0.55254 & 2.33237 & $\mathrm{H}$ & 3.43293 & -4.23258 & -0.28689 \\
$\mathrm{C}$ & 3.41907 & -0.55352 & 1.58844 & $\mathrm{H}$ & 4.71714 & -3.21595 & 0.4551 \\
$\mathrm{P}$ & 2.26199 & -1.3487 & 0.36024 & $\mathrm{H}$ & 3.09342 & -4.04589 & 1.78788 \\
$\mathrm{C}$ & 3.41878 & -2.12824 & -0.96502 & $\mathrm{H}$ & 1.66288 & -4.27166 & 2.83077 \\
$\mathrm{C}$ & 4.4939 & -1.09445 & -1.3518 & $\mathrm{H}$ & 2.72079 & -2.86134 & 3.08171 \\
$\mathrm{P}$ & 1.86296 & 1.19457 & -1.98774 & $\mathrm{H}$ & -0.23026 & -2.75961 & 2.83301 \\
$\mathrm{P}$ & -0.53195 & 2.53204 & 0.1667 & $\mathrm{H}$ & -0.48099 & -1.53466 & 1.55496 \\
$\mathrm{C}$ & -1.01878 & 3.64499 & -1.32194 & $\mathrm{H}$ & 0.71519 & -1.24597 & 2.88185 \\
$\mathrm{C}$ & 0.25724 & 4.29301 & -1.8912 & $\mathrm{H}$ & 1.33983 & -4.32629 & -0.17423 \\
$\mathrm{C}$ & 1.35683 & -2.72343 & 1.35363 & $\mathrm{H}$ & 0.01532 & -3.11995 & -0.3533 \\
$\mathrm{C}$ & 0.28683 & -2.00612 & 2.20012 & $\mathrm{H}$ & -0.04223 & -4.33348 & 0.95589 \\
$\mathrm{C}$ & 0.6376 & -3.67181 & 0.37796 & $\mathrm{H}$ & -1.58201 & 5.44413 & -0.16546 \\
$\mathrm{C}$ & 2.27575 & -3.51097 & 2.30167 & $\mathrm{H}$ & -2.98082 & 4.41844 & -0.63919
\end{tabular}

${ }^{4}$ E. D. Glendening, J. K. Badenhoop, A. E. Reed, J. E. Carpenter, J. A. Bohmann, C. M. Morales, C. R. Landis, F. Weinhold, NBO 6.0, Theoretical Chemical Institute, University of Wisconsin, Madison, WI, USA, 2013. 


\begin{tabular}{l|lll|l|lll}
$\mathrm{C}$ & -2.05075 & 2.28779 & 1.33099 & $\mathrm{H}$ & -2.14713 & 5.41268 & -1.85945 \\
$\mathrm{C}$ & -3.27423 & 1.84293 & 0.51013 & $\mathrm{H}$ & 0.00509 & 4.7782 & -2.85722 \\
$\mathrm{C}$ & 0.6607 & 3.53859 & 1.17944 & $\mathrm{H}$ & 1.0697 & 3.5698 & -2.09791 \\
$\mathrm{C}$ & 1.42612 & 2.59465 & 2.10137 & $\mathrm{H}$ & 0.65952 & 5.08126 & -1.22449 \\
$\mathrm{C}$ & -1.70338 & 1.13769 & 2.29787 & $\mathrm{H}$ & -0.94957 & 1.91315 & -2.67767 \\
$\mathrm{C}$ & -2.37588 & 3.5451 & 2.15541 & $\mathrm{H}$ & -1.86807 & 3.32762 & -3.29992 \\
$\mathrm{C}$ & 4.13322 & -3.39673 & -0.46946 & $\mathrm{H}$ & -2.58494 & 2.26134 & -2.0528 \\
$\mathrm{C}$ & 2.57542 & -2.44931 & -2.21166 & $\mathrm{H}$ & -1.56137 & 3.8006 & 2.86191 \\
$\mathrm{C}$ & -1.64252 & 2.72897 & -2.39234 & $\mathrm{H}$ & -3.28038 & 3.3403 & 2.76703 \\
$\mathrm{C}$ & -1.98813 & 4.78319 & -0.95796 & $\mathrm{H}$ & -2.59106 & 4.43563 & 1.53807 \\
$\mathrm{H}$ & 4.22059 & -0.09699 & 0.97695 & $\mathrm{H}$ & -3.66376 & 2.64283 & -0.146 \\
$\mathrm{H}$ & 3.89342 & -1.26973 & 2.28805 & $\mathrm{H}$ & -4.08967 & 1.56685 & 1.21215 \\
$\mathrm{H}$ & 3.41562 & 1.27018 & 2.75882 & $\mathrm{H}$ & -3.04388 & 0.94386 & -0.09451 \\
$\mathrm{H}$ & 2.12224 & 0.14223 & 3.2095 & $\mathrm{H}$ & -1.55698 & 0.1975 & 1.73412 \\
$\mathrm{H}$ & 0.19169 & 4.36676 & 1.74566 & $\mathrm{H}$ & -2.56086 & 0.98856 & 2.98968 \\
$\mathrm{H}$ & 1.36621 & 3.97776 & 0.44751 & $\mathrm{H}$ & -0.8121 & 1.34412 & 2.92018 \\
$\mathrm{H}$ & 0.86367 & 2.42478 & 3.05058 & $\mathrm{O}$ & 3.00486 & 1.98731 & -2.61105 \\
$\mathrm{H}$ & 2.3863 & 3.06893 & 2.40889 & & & &
\end{tabular}

Table 7.15: XYZ-coordinates of computed complex $34+\mathrm{K}\left(\mathrm{C}_{12} \mathrm{H}_{24} \mathrm{O}_{6}\right)$.

\begin{tabular}{c|lll|l|lll} 
Atom & $\mathrm{x}$ & $\mathrm{y}$ & $\mathrm{z}$ & $\mathrm{Atom}$ & $\mathrm{x}$ & $\mathrm{y}$ & $\mathrm{z}$ \\
\hline $\mathrm{C}$ & -1.75406 & 0.10323 & -1.49309 & $\mathrm{H}$ & 4.92202 & -0.36076 & 1.24084 \\
$\mathrm{C}$ & -1.46407 & -1.29578 & -0.93455 & $\mathrm{Re}$ & 1.47914 & -0.22992 & -1.02839 \\
$\mathrm{~N}$ & -0.04567 & -1.57465 & -0.77371 & $\mathrm{~N}$ & 3.19076 & 0.94199 & -1.67028 \\
$\mathrm{H}$ & -1.9287 & -2.07588 & -1.58644 & $\mathrm{C}$ & 3.68959 & 2.05589 & -1.05682 \\
$\mathrm{H}$ & -1.97773 & -1.39965 & 0.05665 & $\mathrm{C}$ & 4.75604 & 2.79779 & -1.55968 \\
$\mathrm{C}$ & 0.16138 & -2.9853 & -0.4783 & $\mathrm{H}$ & 3.1654 & 2.34606 & -0.13186 \\
$\mathrm{C}$ & 1.59826 & -3.32783 & -0.06574 & $\mathrm{C}$ & 5.37075 & 2.40951 & -2.76664 \\
$\mathrm{H}$ & -0.10539 & -3.59688 & -1.37682 & $\mathrm{H}$ & 5.09296 & 3.68553 & -1.00471 \\
$\mathrm{H}$ & -0.55287 & -3.30369 & 0.32129 & $\mathrm{C}$ & 4.90106 & 1.25343 & -3.39123 \\
$\mathrm{P}$ & 2.48244 & -1.81581 & 0.55877 & $\mathrm{H}$ & 6.20205 & 2.98667 & -3.19753 \\
$\mathrm{H}$ & 2.166 & -3.60454 & -0.97826 & $\mathrm{C}$ & 3.83878 & 0.52054 & -2.82565 \\
$\mathrm{H}$ & 1.63168 & -4.17678 & 0.64626 & $\mathrm{H}$ & 5.3542 & 0.88041 & -4.32035 \\
$\mathrm{P}$ & -0.28709 & 0.71027 & -2.47127 & $\mathrm{~N}$ & 2.1619 & -2.59954 & -3.09446 \\
$\mathrm{H}$ & -1.84361 & 0.80541 & -0.64462 & $\mathrm{~N}$ & 2.38527 & -1.36279 & -2.61895 \\
$\mathrm{H}$ & -2.69949 & 0.12259 & -2.07255 & $\mathrm{C}$ & 3.02084 & -2.7772 & -4.13504 \\
$\mathrm{C}$ & -0.48814 & 2.65229 & -2.57109 & $\mathrm{C}$ & 3.82016 & -1.63897 & -4.33877 \\
$\mathrm{C}$ & -1.3023 & 3.16556 & -1.37055 & $\mathrm{H}$ & 3.01896 & -3.72555 & -4.68892 \\
$\mathrm{H}$ & -0.93454 & 2.80228 & -0.39396 & $\mathrm{C}$ & 3.38398 & -0.74785 & -3.3324 \\
$\mathrm{H}$ & -1.23234 & 4.27452 & -1.3447 & $\mathrm{H}$ & 4.60822 & -1.48312 & -5.08333 \\
$\mathrm{H}$ & -2.37421 & 2.89325 & -1.45182 & $\mathrm{P}$ & 0.76526 & 1.22596 & 0.58605 \\
$\mathrm{C}$ & 0.93821 & 3.22482 & -2.49212 & $\mathrm{O}$ & 1.58712 & 2.45605 & 1.10362 \\
$\mathrm{H}$ & 1.60191 & 2.81387 & -3.27956 & $\mathrm{O}$ & -0.60879 & 1.15126 & 1.32543 \\
$\mathrm{H}$ & 0.90192 & 4.32985 & -2.6136 & $\mathrm{~K}$ & -0.18661 & 3.14797 & 3.07879 \\
$\mathrm{H}$ & 1.39862 & 3.00091 & -1.51346 & $\mathrm{O}$ & -0.22834 & 5.21579 & 1.2163
\end{tabular}


7.5 Transition Metal Complexes with Metal-Pnictogen Multiple Bonds

\begin{tabular}{|c|c|c|c|c|c|c|}
\hline-1.16575 & 3.20667 & -3.83385 & $\mathrm{O}$ & -2.54271 & 4.35526 & 2.36299 \\
\hline-2.22211 & 2.89114 & -3.92271 & $\mathrm{O}$ & -0.20087 & 1.26469 & 5.18798 \\
\hline-1.15869 & 4.31652 & -3.76512 & $\mathrm{O}$ & 1.85348 & 5.42319 & 3.34419 \\
\hline-0.63309 & 2.93507 & -4.76449 & $\mathrm{O}$ & 1.79894 & 3.1169 & 4.93656 \\
\hline-0.60142 & -0.02829 & -4.23201 & $\mathrm{O}$ & -2.71568 & 2.00843 & 3.89614 \\
\hline-0.68 & -1.55963 & -4.06441 & $\mathrm{C}$ & 1.09131 & 5.69199 & 0.99036 \\
\hline-1.65131 & -1.85999 & -3.62477 & $\mathrm{C}$ & 1.76579 & 6.29231 & 2.22851 \\
\hline-0.62259 & -2.02594 & -5.0714 & $\mathrm{H}$ & 1.0988 & 6.4633 & 0.17969 \\
\hline 0.13901 & -1.98909 & -3.46002 & $\mathrm{H}$ & 1.64173 & 4.79822 & 0.63085 \\
\hline-1.92159 & 0.39616 & -4.90735 & $\mathrm{H}$ & 1.20184 & 7.18093 & 2.58389 \\
\hline-2.14063 & -0.32653 & -5.7219 & $\mathrm{H}$ & 2.77902 & 6.65432 & 1.92525 \\
\hline-1.88738 & 1.3977 & -5.36592 & C & 2.88427 & 4.44162 & 3.25491 \\
\hline-2.78333 & 0.35997 & -4.20917 & $\mathrm{C}$ & 3.02424 & 3.7484 & 4.59722 \\
\hline 0.59849 & 0.33983 & -5.12003 & $\mathrm{H}$ & 3.86121 & 4.92875 & 3.01305 \\
\hline 0.46805 & -0.10463 & -6.12991 & $\mathrm{H}$ & 2.66044 & 3.68845 & 2.46319 \\
\hline 1.54421 & -0.05055 & -4.70179 & $\mathrm{H}$ & 3.30567 & 4.48131 & 5.39173 \\
\hline 0.70824 & 1.43563 & -5.24999 & $\mathrm{H}$ & 3.84576 & 2.99731 & 4.511 \\
\hline 1.95864 & -1.6933 & 2.42574 & $\mathrm{C}$ & -1.20972 & 6.16578 & 1.57868 \\
\hline 0.41867 & -1.74789 & 2.47967 & $\mathrm{C}$ & -2.56612 & 5.48214 & 1.50961 \\
\hline 0.03923 & -2.7567 & 2.22861 & $\mathrm{H}$ & -1.04476 & 6.55355 & 2.61316 \\
\hline-0.06457 & -1.00484 & 1.82014 & $\mathrm{H}$ & -1.21032 & 7.04284 & 0.88572 \\
\hline 0.10184 & -1.52927 & 3.52241 & $\mathrm{H}$ & -2.77333 & 5.16621 & 0.45874 \\
\hline 2.43895 & -0.35894 & 3.02994 & $\mathrm{H}$ & -3.35583 & 6.21287 & 1.81254 \\
\hline 3.50564 & -0.3895 & 3.31898 & $\mathrm{C}$ & -3.72656 & 3.57088 & 2.37816 \\
\hline 2.30885 & 0.50109 & 2.34478 & $\mathrm{C}$ & -3.3761 & 2.11783 & 2.64073 \\
\hline 1.84082 & -0.13724 & 3.93856 & $\mathrm{H}$ & -4.24623 & 3.62189 & 1.39284 \\
\hline 2.47785 & -2.852 & 3.29386 & $\mathrm{H}$ & -4.42994 & 3.95619 & 3.15575 \\
\hline 3.5758 & -2.83467 & 3.42868 & $\mathrm{H}$ & -2.71286 & 1.73028 & 1.83152 \\
\hline 2.02461 & -2.76789 & 4.30501 & $\mathrm{H}$ & -4.32198 & 1.52132 & 2.64126 \\
\hline 2.1867 & -3.84175 & 2.8894 & $\mathrm{C}$ & -2.301 & 0.67881 & 4.18513 \\
\hline 4.33822 & -2.35785 & 0.53277 & $\mathrm{C}$ & -1.47452 & 0.69225 & 5.45544 \\
\hline 4.55702 & -3.83336 & 0.9124 & $\mathrm{H}$ & -1.69893 & 0.26921 & 3.341 \\
\hline 4.05032 & -4.51272 & 0.19998 & $\mathrm{H}$ & -3.18763 & 0.01507 & 4.33777 \\
\hline 4.21729 & -4.08658 & 1.93109 & $\mathrm{H}$ & -1.35977 & -0.3501 & 5.83879 \\
\hline 5.64427 & -4.05896 & 0.8591 & $\mathrm{H}$ & -2.01271 & 1.28707 & 6.23171 \\
\hline 4.85595 & -2.1608 & -0.90015 & $\mathrm{C}$ & 1.89801 & 2.13566 & 5.94745 \\
\hline 5.91829 & -2.48527 & -0.94185 & $\mathrm{C}$ & 0.50176 & 1.68512 & 6.34186 \\
\hline 4.8156 & -1.10002 & -1.2001 & $\mathrm{H}$ & 2.40553 & 2.53805 & 6.85849 \\
\hline 4.2953 & -2.75424 & -1.64598 & $\mathrm{H}$ & 2.49732 & 1.26683 & 5.58322 \\
\hline 5.14998 & -1.42643 & 1.44874 & $\mathrm{H}$ & -0.04356 & 2.52546 & 6.83898 \\
\hline 6.23426 & -1.58308 & 1.2593 & $\mathrm{H}$ & 0.59695 & 0.85775 & 7.08744 \\
\hline 4.97092 & -1.61852 & 2.52374 & & & & \\
\hline
\end{tabular}


Table 7.16: XYZ-coordinates of computed complex 34.

\begin{tabular}{|c|c|c|c|c|c|c|c|}
\hline Atom & $\mathrm{x}$ & $\mathrm{y}$ & $\mathrm{z}$ & Atom & $\mathrm{x}$ & $\mathrm{y}$ & $\mathrm{Z}$ \\
\hline $\mathrm{C}$ & -0.9394 & 1.51121 & -2.05382 & $\mathrm{H}$ & -0.67582 & -0.13011 & 1.48768 \\
\hline $\mathrm{C}$ & -1.40362 & 0.11101 & -1.63422 & $\mathrm{H}$ & -1.19297 & -0.79297 & 3.06399 \\
\hline $\mathrm{N}$ & -0.3199 & -0.74823 & -1.18994 & $\mathrm{C}$ & 1.48625 & -0.74943 & 3.19649 \\
\hline $\mathrm{H}$ & -1.95957 & -0.38336 & -2.47116 & $\mathrm{H}$ & 2.37391 & -1.24604 & 3.62978 \\
\hline $\mathrm{H}$ & -2.15357 & 0.22018 & -0.80709 & $\mathrm{H}$ & 1.84305 & 0.13114 & 2.62665 \\
\hline $\mathrm{C}$ & -0.80779 & -2.1011 & -0.98776 & $\mathrm{H}$ & 0.87353 & -0.36688 & 4.04118 \\
\hline $\mathrm{C}$ & 0.21221 & -3.02867 & -0.31623 & $\mathrm{C}$ & 0.37251 & -2.99495 & 3.16146 \\
\hline $\mathrm{H}$ & -1.10219 & -2.54752 & -1.97355 & $\mathrm{H}$ & 1.30347 & -3.47678 & 3.51627 \\
\hline $\mathrm{H}$ & -1.75111 & -2.07336 & -0.38376 & $\mathrm{H}$ & -0.22085 & -2.72958 & 4.06404 \\
\hline $\mathrm{P}$ & 1.47204 & -2.03116 & 0.6288 & $\mathrm{H}$ & -0.21752 & -3.74261 & 2.59302 \\
\hline $\mathrm{H}$ & 0.80957 & -3.52559 & -1.10925 & $\mathrm{C}$ & 2.87352 & -3.34442 & 0.93543 \\
\hline $\mathrm{H}$ & -0.28569 & -3.80716 & 0.29805 & $\mathrm{C}$ & 2.34727 & -4.7703 & 1.17936 \\
\hline $\mathrm{P}$ & 0.84237 & 1.45079 & -2.59438 & $\mathrm{H}$ & 1.78822 & -5.14924 & 0.30156 \\
\hline $\mathrm{H}$ & -0.93322 & 2.14506 & -1.14663 & $\mathrm{H}$ & 1.69662 & -4.85924 & 2.06676 \\
\hline $\mathrm{H}$ & -1.61277 & 1.95936 & -2.81394 & $\mathrm{H}$ & 3.21441 & -5.45147 & 1.33135 \\
\hline $\mathrm{C}$ & 1.47788 & 3.30483 & -2.50028 & $\mathrm{C}$ & 3.74618 & -3.38243 & -0.32772 \\
\hline $\mathrm{C}$ & 0.61869 & 4.11191 & -1.50851 & $\mathrm{H}$ & 4.54146 & -4.14805 & -0.189 \\
\hline $\mathrm{H}$ & 0.4983 & 3.62925 & -0.51957 & $\mathrm{H}$ & 4.23817 & -2.41059 & -0.50471 \\
\hline $\mathrm{H}$ & 1.10651 & 5.09777 & -1.34385 & $\mathrm{H}$ & 3.17469 & -3.65006 & -1.23602 \\
\hline $\mathrm{H}$ & -0.39531 & 4.30506 & -1.9143 & $\mathrm{C}$ & 3.77238 & -2.89081 & 2.09746 \\
\hline $\mathrm{C}$ & 2.9245 & 3.23066 & -1.98001 & $\mathrm{H}$ & 4.68719 & -3.52337 & 2.11792 \\
\hline $\mathrm{H}$ & 3.56189 & 2.57156 & -2.60309 & $\mathrm{H}$ & 3.28421 & -2.98658 & 3.0858 \\
\hline $\mathrm{H}$ & 3.37051 & 4.24967 & -1.98615 & $\mathrm{H}$ & 4.09568 & -1.83797 & 1.96801 \\
\hline $\mathrm{H}$ & 2.95686 & 2.85422 & -0.94176 & Re & 1.64418 & -0.18896 & -0.96111 \\
\hline $\mathrm{C}$ & 1.48212 & 4.09197 & -3.82054 & $\mathrm{~N}$ & 3.77719 & 0.139 & -1.08667 \\
\hline $\mathrm{H}$ & 0.46357 & 4.26023 & -4.21737 & $\mathrm{C}$ & 4.52258 & 0.93048 & -0.25428 \\
\hline $\mathrm{H}$ & 1.92397 & 5.09294 & -3.61981 & $\mathrm{C}$ & 5.88893 & 1.14395 & -0.42338 \\
\hline $\mathrm{H}$ & 2.09946 & 3.61765 & -4.60696 & $\mathrm{H}$ & 3.94549 & 1.41647 & 0.55624 \\
\hline $\mathrm{C}$ & 0.70501 & 0.95059 & -4.46772 & $\mathrm{C}$ & 6.57156 & 0.53309 & -1.49585 \\
\hline $\mathrm{C}$ & -0.03927 & -0.39942 & -4.50967 & $\mathrm{H}$ & 6.40956 & 1.80172 & 0.28854 \\
\hline $\mathrm{H}$ & -1.12376 & -0.26068 & -4.33064 & $\mathrm{C}$ & 5.83655 & -0.30806 & -2.33388 \\
\hline $\mathrm{H}$ & 0.07074 & -0.82752 & -5.53078 & $\mathrm{H}$ & 7.64678 & 0.69979 & -1.66209 \\
\hline $\mathrm{H}$ & 0.34814 & -1.14752 & -3.79483 & $\mathrm{C}$ & 4.46147 & -0.51484 & -2.10825 \\
\hline $\mathrm{C}$ & -0.10085 & 1.91158 & -5.36516 & $\mathrm{H}$ & 6.31099 & -0.83926 & -3.17217 \\
\hline $\mathrm{H}$ & -0.39385 & 1.36468 & -6.28853 & $\mathrm{~N}$ & 1.76758 & -2.6134 & -3.09113 \\
\hline $\mathrm{H}$ & 0.46718 & 2.80091 & -5.68425 & $\mathrm{~N}$ & 2.3573 & -1.59119 & -2.44475 \\
\hline $\mathrm{H}$ & -1.03972 & 2.25152 & -4.88124 & $\mathrm{C}$ & 2.70601 & -3.13801 & -3.92687 \\
\hline $\mathrm{C}$ & 2.12781 & 0.77204 & -5.02202 & $\mathrm{C}$ & 3.92827 & -2.45249 & -3.82068 \\
\hline $\mathrm{H}$ & 2.07861 & 0.44083 & -6.08303 & $\mathrm{H}$ & 2.44838 & -3.99113 & -4.57001 \\
\hline $\mathrm{H}$ & 2.68859 & 0.0053 & -4.45578 & $\mathrm{C}$ & 3.66402 & -1.46273 & -2.84388 \\
\hline $\mathrm{H}$ & 2.7126 & 1.71378 & -4.99366 & $\mathrm{H}$ & 4.86856 & -2.64641 & -4.34838 \\
\hline $\mathrm{C}$ & 0.62985 & -1.71276 & 2.35117 & $\mathrm{P}$ & 1.2292 & 1.4653 & 0.63862 \\
\hline $\mathrm{C}$ & -0.74198 & -1.06089 & 2.0833 & $\mathrm{O}$ & 2.36905 & 2.16053 & 1.41552 \\
\hline
\end{tabular}




$$
\begin{array}{l|lll|l|lll}
\mathrm{H} & -1.43735 & -1.76379 & 1.58461 & \mathrm{O} & -0.19227 & 1.9805 & 0.946
\end{array}
$$

Table 7.17: XYZ-coordinates of computed compound $\mathrm{PO}_{2}^{-}$.

\begin{tabular}{c|lll|c|lll} 
Atom & $\mathrm{x}$ & $\mathrm{y}$ & $\mathrm{z}$ & Atom & $\mathrm{x}$ & $\mathrm{y}$ & $\mathrm{z}$ \\
\hline $\mathrm{P}$ & 1.0000 & 0.96031 & 0.96031 & $\mathrm{O}$ & 1.00000 & 2.45798 & 0.58170 \\
$\mathrm{O}$ & 1.00000 & 0.58170 & 2.457982 & & & &
\end{tabular}

\subsubsection{Interconversion of Os PHMes* and PMes* Complexes by PCET - Computational Details}

DFT calculations were carried out with the ORCA program package using the PBE and PBE0 functionals. [304], [318], [326] Ahlrichs' basis sets def2-SVP (for geometry optimization and frequency calculation) and def2-TZVP (for single-point energies) were used with a full basis for all elements but Os for which the Stuttgart-Dresden 60 electron core potential was chosen to replace the inner shell 1s-4f orbitals. ${ }^{[307],[308]}$ The RI-J (PBE) approximation in combination with the corresponding auxiliary basis sets of Alrichs was utilized to reduce computational costs in the geometry optimization and frequency calculations. ${ }^{[327]-[329]}$ Grimme's model (D3) with Becke-Johnson damping was used to account for dispersion with the PBE or PBE0 functionals. ${ }^{[306],[330]}$ No symmetry restraints were imposed and the optimized structures were defined as minima (no negative eigenvalues) by vibrational analyses. Geometries were analyzed and visualized with Avogadro, molecular orbitals were visualized with Chimera. ${ }^{[333],[334]}$

\section{BDE Calculations}

Geometry optimizations were carried out with the Turbomole program package coupled to the PQS Baker optimizer via the BOpt package, at the spin unrestricted riDFT level using the BP86 functional and the resolution-of-identity (ri) method. ${ }^{[357]-[362]}$ The def2-TZVP basis set (small-core pseudopotentials on Os ) and Grimme's version 3 dispersion corrections (disp3, "zero damping") for all geometry optimizations was

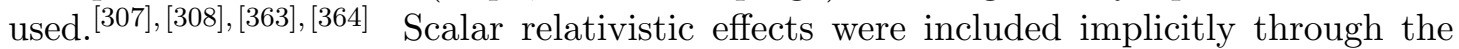
use of the Os ECPs. All minima (no imaginary frequencies) were characterized by calculating the Hessian matrix. ZPE and gas-phase thermal corrections (entropy and enthalpy, $298 \mathrm{~K}, 1$ bar) from these analyses were calculated. Spectroscopic properties and BDEs were evaluated using the full atom model of the complexes. The calculated BDEs were calibrated (taking advantage of internal error cancelation) against the know experimental BDE of propene, using the Hess cycle shown in Scheme 7.1.

\section{EPR Property Calculations}

EPR parameters of $\mathbf{3 8}$ were calculated with the ADF program system at the B3LYP / ZORA / QZ4P level, using the coordinates from the structure optimized in Turbomole as input. ${ }^{[365]-[370]}$ Unrestricted SPINORBIT ZORA COLLINEAR calculations were used to compute the SOC corrected HFI-tensors and Zeeman corrected g-tensors. 


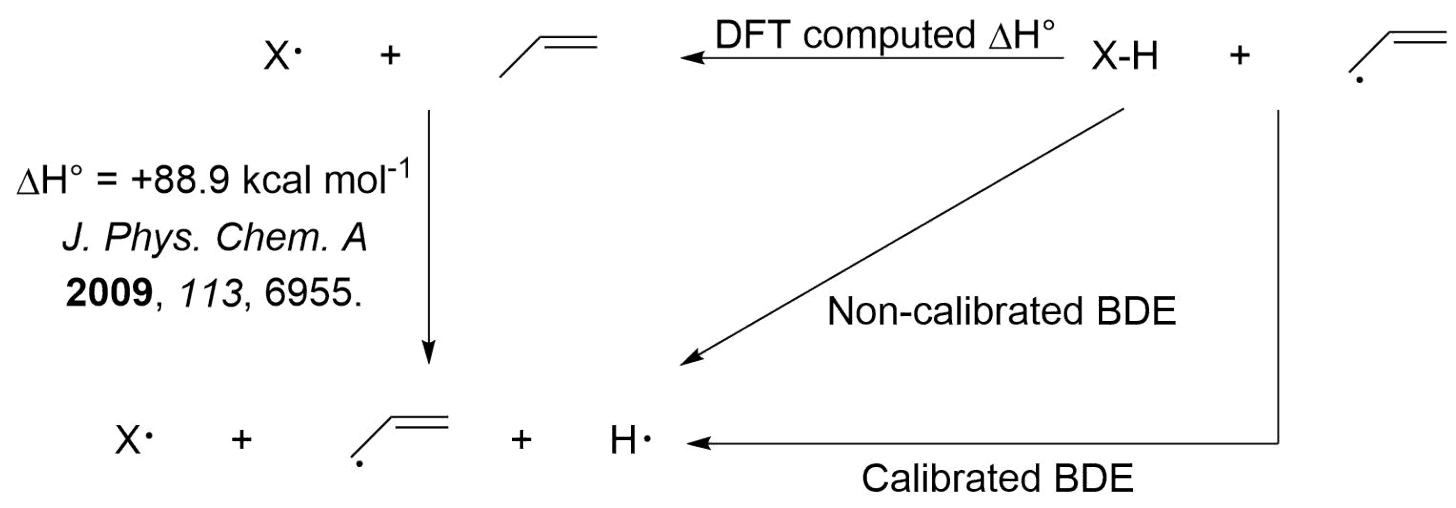

Scheme 7.1: Calibration of the calculated BDE values.

\section{An Isolable Terminal Chlorophosphinidene Complex - Computational Details}

DFT calculations were carried out with the ORCA program package using the PBE and PBE0 functionals. [304], [318], [326] Ahlrichs' basis sets def2-SVP (for geometry optimization and frequency calculation) and def2-TZVP (for single-point energies) were used with a full basis for all elements but Os for which the Stuttgart-Dresden 60 electron core potential was chosen to replace the inner shell 1s-4f orbitals. ${ }^{[307],[308]}$ The RI-J (PBE) approximation in combination with the corresponding auxiliary basis sets of Alrichs was utilized to reduce computational costs in the geometry optimization and frequency calculations. ${ }^{[327]-[329]}$ Grimme's model (D3) with Becke-Johnson damping was used to account for dispersion with the PBE or PBE0 functionals. ${ }^{[306],[330]}$ No symmetry restraints were imposed and the optimized structures were defined as minima (no negative eigenvalues, one negative eigenvalue for transition states) by vibrational analyses. Geometries were analyzed and visualized with Cylview ${ }^{5}$ and molecular orbitals, spindensities and difference densities were visualized with Chimera. ${ }^{[333],[334]}$

Table 7.18: XYZ-coordinates of computed complex $44^{\mathrm{tBu}}$.

\begin{tabular}{c|lll|l|lll} 
Atom & $\mathrm{x}$ & $\mathrm{y}$ & $\mathrm{z}$ & Atom & $\mathrm{x}$ & $\mathrm{y}$ & $\mathrm{z}$ \\
\hline $\mathrm{Os}$ & 3.12227 & 8.55294 & 10.92059 & $\mathrm{C}$ & 1.75915 & 9.69305 & 7.88058 \\
$\mathrm{Cl}$ & 3.73634 & 10.85463 & 11.32611 & $\mathrm{H}$ & 1.69428 & 10.27049 & 6.93455 \\
$\mathrm{Cl}$ & 2.11686 & 5.62267 & 12.96935 & $\mathrm{H}$ & 1.70109 & 8.61494 & 7.63061 \\
$\mathrm{P}$ & 0.81745 & 8.97699 & 10.34799 & $\mathrm{H}$ & 2.74925 & 9.89711 & 8.32569 \\
$\mathrm{P}$ & 5.38653 & 7.76907 & 10.61123 & $\mathrm{C}$ & 3.70984 & 5.89521 & 9.63594 \\
$\mathrm{P}$ & 2.77429 & 7.73151 & 12.82215 & $\mathrm{H}$ & 3.40991 & 4.93885 & 9.16651 \\
$\mathrm{~N}$ & 2.69586 & 6.76738 & 9.93482 & $\mathrm{C}$ & 5.03423 & 6.16606 & 9.87697 \\
$\mathrm{C}$ & 1.40069 & 6.46141 & 9.60048 & $\mathrm{H}$ & 5.80751 & 5.42397 & 9.63316 \\
$\mathrm{H}$ & 1.23019 & 5.45651 & 9.17095 & $\mathrm{C}$ & 6.46714 & 7.46089 & 12.1678 \\
$\mathrm{C}$ & 0.35875 & 7.33878 & 9.76412 & $\mathrm{C}$ & 6.4132 & 8.70222 & 13.07373 \\
$\mathrm{H}$ & -0.66523 & 7.04976 & 9.48996 & $\mathrm{H}$ & 6.81552 & 9.60715 & 12.58424
\end{tabular}

${ }^{5}$ Cylview, V 1.0b (build 562), C. Y. Legault, Université de Sherbrooke, Canada, 2009 (http://www.cylview.org). 
7.5 Transition Metal Complexes with Metal-Pnictogen Multiple Bonds

\begin{tabular}{l|lll|l|lll}
$\mathrm{C}$ & -0.38267 & 9.48592 & 11.75557 & $\mathrm{H}$ & 7.01127 & 8.51299 & 13.98984 \\
$\mathrm{C}$ & -1.73478 & 9.98795 & 11.2195 & $\mathrm{H}$ & 5.37335 & 8.9294 & 13.37826 \\
$\mathrm{H}$ & -1.64172 & 10.93511 & 10.65537 & $\mathrm{C}$ & 7.92006 & 7.11803 & 11.793 \\
$\mathrm{H}$ & -2.40588 & 10.1838 & 12.08261 & $\mathrm{H}$ & 8.44899 & 7.9582 & 11.30593 \\
$\mathrm{H}$ & -2.23565 & 9.23633 & 10.57653 & $\mathrm{H}$ & 7.97884 & 6.22864 & 11.13349 \\
$\mathrm{C}$ & 0.29439 & 10.57626 & 12.60364 & $\mathrm{H}$ & 8.4762 & 6.87453 & 12.72281 \\
$\mathrm{H}$ & 0.57395 & 11.46663 & 12.01097 & $\mathrm{C}$ & 5.87733 & 6.25369 & 12.91053 \\
$\mathrm{H}$ & 1.22279 & 10.19958 & 13.07279 & $\mathrm{H}$ & 4.81217 & 6.39275 & 13.18292 \\
$\mathrm{H}$ & -0.39936 & 10.8983 & 13.4093 & $\mathrm{H}$ & 6.43734 & 6.10357 & 13.85728 \\
$\mathrm{C}$ & -0.64316 & 8.24046 & 12.61348 & $\mathrm{H}$ & 5.93751 & 5.32439 & 12.31178 \\
$\mathrm{H}$ & -1.2062 & 7.46406 & 12.06074 & $\mathrm{C}$ & 6.37309 & 8.76242 & 9.29861 \\
$\mathrm{H}$ & -1.23259 & 8.5345 & 13.50732 & $\mathrm{C}$ & 5.3139 & 9.22418 & 8.28301 \\
$\mathrm{H}$ & 0.28923 & 7.76673 & 12.98006 & $\mathrm{H}$ & 5.81595 & 9.70179 & 7.41468 \\
$\mathrm{C}$ & 0.6232 & 10.12536 & 8.82345 & $\mathrm{H}$ & 4.64554 & 9.9792 & 8.74107 \\
$\mathrm{C}$ & -0.71074 & 9.91265 & 8.08895 & $\mathrm{H}$ & 4.70705 & 8.37818 & 7.90092 \\
$\mathrm{H}$ & -0.67983 & 10.47448 & 7.13161 & $\mathrm{C}$ & 7.02655 & 10.00752 & 9.91879 \\
$\mathrm{H}$ & -1.58547 & 10.27745 & 8.65407 & $\mathrm{H}$ & 7.43191 & 10.64114 & 9.10048 \\
$\mathrm{H}$ & -0.87071 & 8.84477 & 7.84044 & $\mathrm{H}$ & 7.8703 & 9.75928 & 10.59015 \\
$\mathrm{C}$ & 0.82689 & 11.59239 & 9.22816 & $\mathrm{H}$ & 6.28153 & 10.60918 & 10.4788 \\
$\mathrm{H}$ & 0.88306 & 12.21957 & 8.3132 & $\mathrm{C}$ & 7.39979 & 7.88269 & 8.56805 \\
$\mathrm{H}$ & 1.77179 & 11.72207 & 9.79601 & $\mathrm{H}$ & 6.91349 & 7.01564 & 8.07906 \\
$\mathrm{H}$ & -0.01084 & 11.98054 & 9.84088 & $\mathrm{H}$ & 8.20092 & 7.505 & 9.22765 \\
$\mathrm{H}$ & 7.88317 & 8.48782 & 7.7712 & & & &
\end{tabular}

Table 7.19: XYZ-coordinates of computed complex $44^{\mathrm{iPr}}$.

\begin{tabular}{c|lll|c|lll} 
Atom & $\mathrm{x}$ & $\mathrm{y}$ & $\mathrm{z}$ & Atom & $\mathrm{x}$ & $\mathrm{y}$ & $\mathrm{z}$ \\
\hline $\mathrm{Os}$ & 3.14555 & 8.47126 & 10.82874 & $\mathrm{C}$ & 3.70073 & 5.84781 & 9.49445 \\
$\mathrm{Cl}$ & 3.83829 & 10.74839 & 10.9717 & $\mathrm{H}$ & 3.36691 & 4.92239 & 8.98864 \\
$\mathrm{Cl}$ & 3.77012 & 6.21721 & 13.70386 & $\mathrm{C}$ & 5.00479 & 6.02942 & 9.85072 \\
$\mathrm{P}$ & 0.86735 & 8.991 & 10.30698 & $\mathrm{H}$ & 5.75248 & 5.24604 & 9.66161 \\
$\mathrm{P}$ & 5.37418 & 7.64054 & 10.58959 & $\mathrm{C}$ & 6.54009 & 7.32758 & 12.01785 \\
$\mathrm{P}$ & 2.57548 & 7.78329 & 12.75991 & $\mathrm{C}$ & 6.59778 & 8.52235 & 12.97695 \\
$\mathrm{~N}$ & 2.71608 & 6.79302 & 9.74135 & $\mathrm{C}$ & 7.92344 & 6.80728 & 11.6127 \\
$\mathrm{C}$ & 1.40857 & 6.52907 & 9.37497 & $\mathrm{H}$ & 8.53562 & 7.59002 & 11.12166 \\
$\mathrm{H}$ & 1.23467 & 5.55861 & 8.87364 & $\mathrm{H}$ & 7.86245 & 5.93535 & 10.93109 \\
$\mathrm{C}$ & 0.37569 & 7.39423 & 9.61026 & $\mathrm{H}$ & 8.47609 & 6.48098 & 12.51868 \\
$\mathrm{H}$ & -0.64519 & 7.14334 & 9.29123 & $\mathrm{H}$ & 5.99007 & 6.51344 & 12.53598 \\
$\mathrm{C}$ & -0.34945 & 9.39478 & 11.67186 & $\mathrm{C}$ & 6.27032 & 8.60483 & 9.22522 \\
$\mathrm{C}$ & -1.79814 & 9.56677 & 11.20766 & $\mathrm{H}$ & 5.40554 & 8.9622 & 8.62188 \\
$\mathrm{H}$ & -1.93157 & 10.5079 & 10.63726 & $\mathrm{C}$ & 7.00629 & 9.83908 & 9.75855 \\
$\mathrm{H}$ & -2.47099 & 9.61997 & 12.08894 & $\mathrm{H}$ & 7.34085 & 10.4673 & 8.90654 \\
$\mathrm{H}$ & -2.14479 & 8.72563 & 10.57428 & $\mathrm{H}$ & 7.91261 & 9.55847 & 10.33196 \\
$\mathrm{C}$ & 0.13357 & 10.55351 & 12.55468 & $\mathrm{H}$ & 6.34693 & 10.45821 & 10.39844 \\
$\mathrm{H}$ & -0.27177 & 8.45608 & 12.26679 & $\mathrm{C}$ & 7.15063 & 7.74102 & 8.31422 \\
$\mathrm{C}$ & 0.70042 & 10.215 & 8.87585 & $\mathrm{H}$ & 6.58955 & 6.88709 & 7.88821
\end{tabular}




\begin{tabular}{l|lll|l|lll}
$\mathrm{C}$ & -0.49376 & 9.95679 & 7.94987 & $\mathrm{H}$ & 8.03448 & 7.34113 & 8.84773 \\
$\mathrm{H}$ & -0.4292 & 10.62436 & 7.06537 & $\mathrm{H}$ & 7.52615 & 8.35663 & 7.4699 \\
$\mathrm{H}$ & -1.45969 & 10.1646 & 8.45074 & $\mathrm{H}$ & 0.1355 & 11.5175 & 12.01169 \\
$\mathrm{H}$ & -0.5142 & 8.9127 & 7.58318 & $\mathrm{H}$ & 1.1616 & 10.37862 & 12.92669 \\
$\mathrm{C}$ & 0.78733 & 11.67397 & 9.33612 & $\mathrm{H}$ & -0.53856 & 10.65868 & 13.43085 \\
$\mathrm{H}$ & 0.90531 & 12.33572 & 8.45318 & $\mathrm{H}$ & 7.12389 & 9.39085 & 12.53713 \\
$\mathrm{H}$ & 1.65513 & 11.83839 & 10.00639 & $\mathrm{H}$ & 7.13611 & 8.23267 & 13.90285 \\
$\mathrm{H}$ & -0.13893 & 11.98846 & 9.85864 & $\mathrm{H}$ & 5.58111 & 8.85332 & 13.26284 \\
$\mathrm{H}$ & 1.63003 & 9.98146 & 8.30918 & & & &
\end{tabular}


Table 7.20: XYZ-coordinates of computed tolane.

\begin{tabular}{c|lll|l|lll} 
Atom & $\mathrm{x}$ & $\mathrm{y}$ & $\mathrm{z}$ & Atom & $\mathrm{x}$ & $\mathrm{y}$ & $\mathrm{z}$ \\
\hline $\mathrm{C}$ & -6.05987 & 2.49433 & 0.03129 & $\mathrm{C}$ & -1.22817 & 1.50396 & 0.05189 \\
$\mathrm{C}$ & -4.66256 & 2.56505 & 0.03318 & $\mathrm{C}$ & 0.19432 & 1.57197 & 0.05535 \\
$\mathrm{C}$ & -6.70788 & 1.24771 & 0.0437 & $\mathrm{C}$ & 0.85903 & 2.82621 & 0.04121 \\
$\mathrm{H}$ & -6.65099 & 3.42265 & 0.01981 & $\mathrm{C}$ & 2.25663 & 2.88744 & 0.04522 \\
$\mathrm{C}$ & -5.94634 & 0.06701 & 0.05819 & $\mathrm{H}$ & 0.25925 & 3.7477 & 0.02695 \\
$\mathrm{H}$ & -7.8075 & 1.1957 & 0.04199 & $\mathrm{C}$ & 3.02001 & 1.70791 & 0.06322 \\
$\mathrm{C}$ & -4.54857 & 0.12612 & 0.06028 & $\mathrm{H}$ & 2.75789 & 3.86732 & 0.03408 \\
$\mathrm{H}$ & -6.44926 & -0.91208 & 0.06812 & $\mathrm{C}$ & 2.37386 & 0.46044 & 0.07721 \\
$\mathrm{C}$ & -3.88197 & 1.37934 & 0.04769 & $\mathrm{H}$ & 4.11955 & 1.76158 & 0.06648 \\
$\mathrm{H}$ & -3.94984 & -0.79612 & 0.07171 & $\mathrm{C}$ & 0.97665 & 0.38754 & 0.07341 \\
$\mathrm{H}$ & -4.15176 & 3.53887 & 0.02327 & $\mathrm{H}$ & 2.96642 & -0.46695 & 0.09141 \\
$\mathrm{C}$ & -2.45943 & 1.44573 & 0.04953 & $\mathrm{H}$ & 0.46693 & -0.58683 & 0.0846
\end{tabular}

Table 7.21: XYZ-coordinates of computed complex $46^{\mathrm{tBu}}$.

\begin{tabular}{c|lll|l|lll} 
Atom & $\mathrm{x}$ & $\mathrm{y}$ & $\mathrm{z}$ & Atom & $\mathrm{x}$ & $\mathrm{y}$ & $\mathrm{z}$ \\
\hline $\mathrm{C}$ & -3.28673 & 3.00663 & 1.21724 & $\mathrm{H}$ & -0.08081 & 5.68081 & -1.79696 \\
$\mathrm{C}$ & -3.91743 & 1.79105 & 1.29631 & $\mathrm{H}$ & 0.25102 & 3.92341 & -1.61885 \\
$\mathrm{P}$ & -1.71479 & 2.98676 & 0.33577 & $\mathrm{H}$ & 0.59731 & 5.05863 & -0.26491 \\
$\mathrm{H}$ & -3.67586 & 3.87621 & 1.76425 & $\mathrm{C}$ & -0.37036 & 2.90069 & 1.72827 \\
$\mathrm{~N}$ & -3.46161 & 0.67251 & 0.64016 & $\mathrm{C}$ & -0.18523 & 4.23198 & 2.47145 \\
$\mathrm{H}$ & -4.82894 & 1.66204 & 1.91045 & $\mathrm{H}$ & -1.14063 & 4.61795 & 2.87882 \\
$\mathrm{C}$ & -4.07602 & -0.53871 & 0.82794 & $\mathrm{H}$ & 0.2766 & 5.01484 & 1.84097 \\
$\mathrm{C}$ & -3.67528 & -1.69069 & 0.19554 & $\mathrm{H}$ & 0.49604 & 4.06295 & 3.3335 \\
$\mathrm{P}$ & -2.25005 & -1.53552 & -0.8958 & $\mathrm{C}$ & 0.97251 & 2.44905 & 1.12873 \\
$\mathrm{H}$ & -4.2125 & -2.63335 & 0.37183 & $\mathrm{H}$ & 1.69317 & 2.24885 & 1.95051 \\
$\mathrm{C}$ & -0.77583 & -2.3026 & 0.06982 & $\mathrm{H}$ & 0.87796 & 1.53292 & 0.51448 \\
$\mathrm{C}$ & -2.62919 & -2.57559 & -2.45258 & $\mathrm{H}$ & 1.41497 & 3.22104 & 0.47182 \\
$\mathrm{C}$ & -0.48439 & -1.23425 & 1.13539 & $\mathrm{C}$ & -0.87279 & 1.86498 & 2.75057 \\
$\mathrm{H}$ & 0.36745 & -1.54327 & 1.77911 & $\mathrm{H}$ & -1.7438 & 2.24756 & 3.31568 \\
$\mathrm{H}$ & -0.17895 & -0.26917 & 0.66849 & $\mathrm{H}$ & -1.18242 & 0.91837 & 2.27527 \\
$\mathrm{H}$ & -1.36287 & -1.05981 & 1.78698 & $\mathrm{H}$ & -0.0589 & 1.63796 & 3.47152 \\
$\mathrm{C}$ & -1.13142 & -3.61139 & 0.78937 & $\mathrm{Os}$ & -1.94793 & 0.85634 & -0.79148 \\
$\mathrm{H}$ & -0.30376 & -3.88095 & 1.48063 & $\mathrm{Cl}$ & 0.09973 & 1.02313 & -2.08926 \\
$\mathrm{H}$ & -2.05202 & -3.49782 & 1.39609 & $\mathrm{P}$ & -3.40765 & 1.09573 & -2.35923 \\
$\mathrm{H}$ & -1.2723 & -4.46069 & 0.09696 & $\mathrm{Cl}$ & -5.49215 & 1.28229 & -1.83877 \\
$\mathrm{C}$ & 0.45486 & -2.46339 & -0.8321 & $\mathrm{C}$ & -0.9152 & -0.10723 & -6.56181 \\
$\mathrm{H}$ & 1.34117 & -2.69042 & -0.20137 & $\mathrm{C}$ & -1.41153 & 0.70924 & -5.5358 \\
$\mathrm{H}$ & 0.66743 & -1.5331 & -1.39863 & $\mathrm{C}$ & -1.78262 & -0.94483 & -7.28215 \\
$\mathrm{H}$ & 0.34008 & -3.30096 & -1.54686 & $\mathrm{H}$ & 0.15982 & -0.09221 & -6.7977 \\
$\mathrm{C}$ & -1.61584 & -2.19857 & -3.54431 & $\mathrm{C}$ & -3.15281 & -0.97077 & -6.9721 \\
$\mathrm{H}$ & -1.55022 & -1.10412 & -3.67851 & $\mathrm{H}$ & -1.38861 & -1.58201 & -8.08879 \\
$\mathrm{H}$ & -1.92377 & -2.64152 & -4.51423 & $\mathrm{C}$ & -3.65607 & -0.16026 & -5.94485
\end{tabular}




\begin{tabular}{l|lll|l|lll}
$\mathrm{H}$ & -0.59875 & -2.56398 & -3.31278 & $\mathrm{H}$ & -3.83419 & -1.62575 & -7.53665 \\
$\mathrm{C}$ & -4.0533 & -2.20929 & -2.90262 & $\mathrm{C}$ & -2.78912 & 0.69016 & -5.22758 \\
$\mathrm{H}$ & -4.81475 & -2.51067 & -2.15788 & $\mathrm{H}$ & -4.72708 & -0.17498 & -5.69181 \\
$\mathrm{H}$ & -4.27212 & -2.71641 & -3.86569 & $\mathrm{H}$ & -0.73717 & 1.34221 & -4.93995 \\
$\mathrm{H}$ & -4.17336 & -1.11952 & -3.06937 & $\mathrm{C}$ & -3.31178 & 1.55756 & -4.15433 \\
$\mathrm{C}$ & -2.58539 & -4.08659 & -2.17557 & $\mathrm{C}$ & -3.84006 & 2.73313 & -3.97719 \\
$\mathrm{H}$ & -2.92353 & -4.62641 & -3.08613 & $\mathrm{C}$ & -4.32781 & 4.0169 & -4.31683 \\
$\mathrm{H}$ & -3.25831 & -4.38551 & -1.34663 & $\mathrm{C}$ & -3.47913 & 5.00344 & -4.89897 \\
$\mathrm{H}$ & -1.56426 & -4.44313 & -1.94672 & $\mathrm{C}$ & -3.97512 & 6.2755 & -5.19629 \\
$\mathrm{C}$ & -1.55222 & 4.64119 & -0.58704 & $\mathrm{H}$ & -2.42994 & 4.74855 & -5.10521 \\
$\mathrm{C}$ & -2.47618 & 4.48697 & -1.79733 & $\mathrm{C}$ & -5.32232 & 6.59493 & -4.94485 \\
$\mathrm{H}$ & -2.52171 & 5.44232 & -2.35877 & $\mathrm{H}$ & -3.30547 & 7.02759 & -5.64153 \\
$\mathrm{H}$ & -2.10021 & 3.70076 & -2.47833 & $\mathrm{C}$ & -6.17192 & 5.62684 & -4.37986 \\
$\mathrm{H}$ & -3.51055 & 4.21851 & -1.50266 & $\mathrm{H}$ & -5.70783 & 7.59563 & -5.19024 \\
$\mathrm{C}$ & -2.02925 & 5.85095 & 0.23326 & $\mathrm{C}$ & -5.68643 & 4.35705 & -4.05339 \\
$\mathrm{H}$ & -1.98659 & 6.75342 & -0.41432 & $\mathrm{H}$ & -7.22577 & 5.87167 & -4.17713 \\
$\mathrm{H}$ & -1.41057 & 6.05332 & 1.12386 & $\mathrm{H}$ & -6.33663 & 3.60368 & -3.58618 \\
$\mathrm{H}$ & -3.08326 & 5.73444 & 0.55379 & $\mathrm{H}$ & -4.93015 & -0.55098 & 1.53181 \\
$\mathrm{C}$ & -0.10957 & 4.82735 & -1.08561 & & & &
\end{tabular}

Table 7.22: XYZ-coordinates of computed complex $46^{\mathrm{iPr}}$.

\begin{tabular}{c|lll|l|lll} 
Atom & $\mathrm{x}$ & $\mathrm{y}$ & $\mathrm{z}$ & Atom & $\mathrm{x}$ & $\mathrm{y}$ & $\mathrm{z}$ \\
\hline $\mathrm{C}$ & -3.51603 & 2.33248 & 0.5367 & $\mathrm{C}$ & -0.59328 & 2.64652 & 0.72523 \\
$\mathrm{C}$ & -3.98879 & 1.04675 & 0.4438 & $\mathrm{C}$ & -0.71536 & 3.60109 & 1.91216 \\
$\mathrm{P}$ & -2.05293 & 2.66912 & -0.46187 & $\mathrm{H}$ & -1.6164 & 3.36661 & 2.51642 \\
$\mathrm{H}$ & -4.00447 & 3.06288 & 1.19573 & $\mathrm{H}$ & -0.78181 & 4.65983 & 1.59312 \\
$\mathrm{~N}$ & -3.43098 & 0.10517 & -0.38993 & $\mathrm{H}$ & 0.16936 & 3.5089 & 2.57864 \\
$\mathrm{H}$ & -4.85572 & 0.7149 & 1.04729 & $\mathrm{H}$ & 0.27006 & 2.9294 & 0.08502 \\
$\mathrm{C}$ & -3.95406 & -1.16518 & -0.46486 & $\mathrm{C}$ & -0.39988 & 1.18858 & 1.16896 \\
$\mathrm{C}$ & -3.42871 & -2.14086 & -1.27605 & $\mathrm{H}$ & -1.29782 & 0.79763 & 1.69003 \\
$\mathrm{P}$ & -1.91038 & -1.67928 & -2.14145 & $\mathrm{H}$ & -0.17631 & 0.52567 & 0.29911 \\
$\mathrm{H}$ & -3.88808 & -3.13949 & -1.31293 & $\mathrm{H}$ & 0.46575 & 1.09686 & 1.85915 \\
$\mathrm{C}$ & -0.52227 & -2.58377 & -1.23718 & $\mathrm{Os}$ & -1.87205 & 0.64844 & -1.66357 \\
$\mathrm{C}$ & -2.06942 & -2.28358 & -3.90547 & $\mathrm{Cl}$ & 0.27284 & 1.17438 & -2.65112 \\
$\mathrm{H}$ & -0.50595 & -2.0057 & -0.28431 & $\mathrm{P}$ & -3.18107 & 1.13746 & -3.30463 \\
$\mathrm{C}$ & -0.8204 & -4.04469 & -0.88579 & $\mathrm{Cl}$ & -4.76091 & 2.5624 & -2.98811 \\
$\mathrm{H}$ & -0.02292 & -4.44353 & -0.22287 & $\mathrm{C}$ & -3.05572 & -0.43932 & -8.45475 \\
$\mathrm{H}$ & -1.78654 & -4.14999 & -0.35413 & $\mathrm{C}$ & -2.64293 & 0.42292 & -7.43157 \\
$\mathrm{H}$ & -0.85001 & -4.68789 & -1.78741 & $\mathrm{C}$ & -4.11882 & -1.335 & -8.24198 \\
$\mathrm{C}$ & 0.83094 & -2.39883 & -1.93225 & $\mathrm{H}$ & -2.54006 & -0.41786 & -9.42682 \\
$\mathrm{H}$ & 1.00222 & -1.34369 & -2.22848 & $\mathrm{C}$ & -4.77445 & -1.3601 & -6.99975 \\
$\mathrm{H}$ & 0.90312 & -3.03164 & -2.83914 & $\mathrm{H}$ & -4.4379 & -2.01222 & -9.04864 \\
$\mathrm{H}$ & 1.65172 & -2.70767 & -1.25098 & $\mathrm{C}$ & -4.3635 & -0.50041 & -5.97184 \\
$\mathrm{C}$ & -0.9493 & -1.74862 & -4.80566 & $\mathrm{H}$ & -5.61464 & -2.05071 & -6.83171 \\
$\mathrm{H}$ & -0.70283 & -0.69239 & -4.57562 & $\mathrm{C}$ & -3.29421 & 0.39393 & -6.17867
\end{tabular}


7.5 Transition Metal Complexes with Metal-Pnictogen Multiple Bonds

\begin{tabular}{l|lll|l|lll}
$\mathrm{H}$ & -0.01854 & -2.33738 & -4.68711 & $\mathrm{H}$ & -4.8739 & -0.50137 & -4.99464 \\
$\mathrm{H}$ & -1.2585 & -1.81339 & -5.86926 & $\mathrm{H}$ & -1.80541 & 1.12043 & -7.5833 \\
$\mathrm{H}$ & -3.00633 & -1.75185 & -4.18309 & $\mathrm{C}$ & -2.85215 & 1.29577 & -5.11192 \\
$\mathrm{C}$ & -2.31065 & -3.78441 & -4.08467 & $\mathrm{C}$ & -2.21708 & 2.40929 & -4.90265 \\
$\mathrm{H}$ & -2.61882 & -3.99007 & -5.13169 & $\mathrm{C}$ & -1.6078 & 3.67817 & -4.9677 \\
$\mathrm{H}$ & -3.11068 & -4.16766 & -3.42051 & $\mathrm{C}$ & -0.19186 & 3.8315 & -5.00069 \\
$\mathrm{H}$ & -1.3912 & -4.37316 & -3.89371 & $\mathrm{C}$ & 0.36922 & 5.10586 & -5.10634 \\
$\mathrm{C}$ & -2.15504 & 4.37416 & -1.22329 & $\mathrm{H}$ & 0.43855 & 2.93646 & -4.90961 \\
$\mathrm{H}$ & -2.71307 & 4.16255 & -2.1597 & $\mathrm{C}$ & -0.4461 & 6.25183 & -5.15616 \\
$\mathrm{C}$ & -2.97114 & 5.38418 & -0.41149 & $\mathrm{H}$ & 1.46496 & 5.21016 & -5.13807 \\
$\mathrm{H}$ & -3.02934 & 6.34497 & -0.96465 & $\mathrm{C}$ & -1.84393 & 6.11135 & -5.09313 \\
$\mathrm{H}$ & -2.51486 & 5.59928 & 0.57633 & $\mathrm{H}$ & 0.00719 & 7.25119 & -5.23562 \\
$\mathrm{H}$ & -4.0071 & 5.02994 & -0.24489 & $\mathrm{C}$ & -2.42913 & 4.84521 & -4.99858 \\
$\mathrm{C}$ & -0.75611 & 4.87379 & -1.59797 & $\mathrm{H}$ & -2.48937 & 7.00271 & -5.12162 \\
$\mathrm{H}$ & -0.83883 & 5.74534 & -2.27909 & $\mathrm{H}$ & -3.52133 & 4.72709 & -4.94886 \\
$\mathrm{H}$ & -0.17441 & 4.09067 & -2.12488 & $\mathrm{H}$ & -4.83665 & -1.3694 & 0.17197 \\
$\mathrm{H}$ & -0.18294 & 5.19701 & -0.70383 & & & &
\end{tabular}

Table 7.23: XYZ-coordinates of computed complex $45^{\mathrm{tBu}}$.

\begin{tabular}{c|lll|l|lll} 
Atom & $\mathrm{x}$ & $\mathrm{y}$ & $\mathrm{z}$ & Atom & $\mathrm{x}$ & $\mathrm{y}$ & $\mathrm{z}$ \\
\hline $\mathrm{C}$ & -3.33994 & 2.98349 & 1.31192 & $\mathrm{H}$ & -0.06498 & 5.79189 & -1.53245 \\
$\mathrm{C}$ & -3.95223 & 1.75297 & 1.37551 & $\mathrm{H}$ & 0.28376 & 4.03466 & -1.39727 \\
$\mathrm{P}$ & -1.75547 & 2.99943 & 0.44636 & $\mathrm{H}$ & 0.55643 & 5.13097 & 0.00571 \\
$\mathrm{H}$ & -3.73276 & 3.8278 & 1.89594 & $\mathrm{C}$ & -0.42683 & 2.91369 & 1.86088 \\
$\mathrm{~N}$ & -3.48933 & 0.65223 & 0.69802 & $\mathrm{C}$ & -0.26583 & 4.23085 & 2.63602 \\
$\mathrm{H}$ & -4.85605 & 1.60271 & 1.99712 & $\mathrm{H}$ & -1.22943 & 4.58836 & 3.05027 \\
$\mathrm{C}$ & -4.09068 & -0.57093 & 0.84881 & $\mathrm{H}$ & 0.18308 & 5.03857 & 2.02863 \\
$\mathrm{C}$ & -3.68424 & -1.69338 & 0.16675 & $\mathrm{H}$ & 0.41572 & 4.05331 & 3.49619 \\
$\mathrm{P}$ & -2.28791 & -1.46489 & -0.94978 & $\mathrm{C}$ & 0.93094 & 2.49437 & 1.26738 \\
$\mathrm{H}$ & -4.20981 & -2.6482 & 0.30992 & $\mathrm{H}$ & 1.63395 & 2.24431 & 2.09099 \\
$\mathrm{C}$ & -0.76516 & -2.15875 & -0.00214 & $\mathrm{H}$ & 0.85475 & 1.61907 & 0.59403 \\
$\mathrm{C}$ & -2.61755 & -2.52445 & -2.49851 & $\mathrm{H}$ & 1.38864 & 3.30601 & 0.67253 \\
$\mathrm{C}$ & -0.52506 & -1.04124 & 1.02457 & $\mathrm{C}$ & -0.9257 & 1.86006 & 2.86664 \\
$\mathrm{H}$ & 0.37739 & -1.24453 & 1.6412 & $\mathrm{H}$ & -1.80501 & 2.22883 & 3.42813 \\
$\mathrm{H}$ & -0.29827 & -0.06347 & 0.51886 & $\mathrm{H}$ & -1.22378 & 0.91438 & 2.38432 \\
$\mathrm{H}$ & -1.39072 & -0.91847 & 1.70174 & $\mathrm{H}$ & -0.11572 & 1.63619 & 3.59303 \\
$\mathrm{C}$ & -1.04281 & -3.46132 & 0.75788 & $\mathrm{Os}$ & -1.97959 & 0.89006 & -0.72594 \\
$\mathrm{H}$ & -0.1943 & -3.67562 & 1.44401 & $\mathrm{Cl}$ & -0.00842 & 1.15923 & -2.15711 \\
$\mathrm{H}$ & -1.96234 & -3.37899 & 1.37134 & $\mathrm{P}$ & -3.43119 & 1.35871 & -2.27907 \\
$\mathrm{H}$ & -1.15015 & -4.33226 & 0.08536 & $\mathrm{Cl}$ & -5.48215 & 0.83169 & -1.84376 \\
$\mathrm{C}$ & 0.46343 & -2.28291 & -0.91159 & $\mathrm{C}$ & -1.04363 & -0.11322 & -6.79511 \\
$\mathrm{H}$ & 1.36738 & -2.43992 & -0.28407 & $\mathrm{C}$ & -1.44026 & 0.57005 & -5.63804 \\
$\mathrm{H}$ & 0.62043 & -1.36402 & -1.51391 & $\mathrm{C}$ & -1.99377 & -0.77271 & -7.59312 \\
$\mathrm{H}$ & 0.3855 & -3.15193 & -1.59256 & $\mathrm{H}$ & 0.02156 & -0.13799 & -7.07194 \\
$\mathrm{C}$ & -1.55856 & -2.18257 & -3.56357 & $\mathrm{C}$ & -3.35086 & -0.74459 & -7.23202
\end{tabular}




\begin{tabular}{l|lll|l|lll}
$\mathrm{H}$ & -1.33394 & -1.10008 & -3.58669 & $\mathrm{H}$ & -1.67515 & -1.30951 & -8.49941 \\
$\mathrm{H}$ & -1.92404 & -2.47731 & -4.56943 & $\mathrm{C}$ & -3.76051 & -0.05813 & -6.08034 \\
$\mathrm{H}$ & -0.60652 & -2.71444 & -3.38589 & $\mathrm{H}$ & -4.09883 & -1.25839 & -7.8548 \\
$\mathrm{C}$ & -4.01648 & -2.1388 & -3.00736 & $\mathrm{C}$ & -2.80672 & 0.6037 & -5.27617 \\
$\mathrm{H}$ & -4.80209 & -2.32636 & -2.25002 & $\mathrm{H}$ & -4.82173 & -0.03183 & -5.79083 \\
$\mathrm{H}$ & -4.24835 & -2.734 & -3.91563 & $\mathrm{H}$ & -0.70412 & 1.0535 & -4.97754 \\
$\mathrm{H}$ & -4.08014 & -1.07379 & -3.28931 & $\mathrm{C}$ & -3.20746 & 1.2988 & -4.05771 \\
$\mathrm{C}$ & -2.61015 & -4.02876 & -2.1863 & $\mathrm{C}$ & -3.69447 & 2.49328 & -3.63665 \\
$\mathrm{H}$ & -2.91471 & -4.58757 & -3.09759 & $\mathrm{C}$ & -4.1671 & 3.75466 & -4.18125 \\
$\mathrm{H}$ & -3.32477 & -4.29567 & -1.38147 & $\mathrm{C}$ & -3.61763 & 4.27677 & -5.37512 \\
$\mathrm{H}$ & -1.60606 & -4.39406 & -1.90117 & $\mathrm{C}$ & -4.09457 & 5.48646 & -5.89506 \\
$\mathrm{C}$ & -1.57182 & 4.69241 & -0.42516 & $\mathrm{H}$ & -2.80854 & 3.72815 & -5.87991 \\
$\mathrm{C}$ & -2.42555 & 4.57895 & -1.68918 & $\mathrm{C}$ & -5.12862 & 6.17793 & -5.24047 \\
$\mathrm{H}$ & -2.46098 & 5.55593 & -2.21514 & $\mathrm{H}$ & -3.65871 & 5.89361 & -6.81942 \\
$\mathrm{H}$ & -2.00017 & 3.82969 & -2.38207 & $\mathrm{C}$ & -5.68955 & 5.65505 & -4.06255 \\
$\mathrm{H}$ & -3.46565 & 4.28792 & -1.44545 & $\mathrm{H}$ & -5.49996 & 7.12859 & -5.65115 \\
$\mathrm{C}$ & -2.11037 & 5.86392 & 0.4124 & $\mathrm{C}$ & -5.21025 & 4.45232 & -3.53018 \\
$\mathrm{H}$ & -2.03651 & 6.79564 & -0.18983 & $\mathrm{H}$ & -6.50571 & 6.19118 & -3.55538 \\
$\mathrm{H}$ & -1.55246 & 6.03156 & 1.34901 & $\mathrm{H}$ & -5.64307 & 4.02336 & -2.61476 \\
$\mathrm{H}$ & -3.18075 & 5.723 & 0.66207 & $\mathrm{H}$ & -4.93827 & -0.61917 & 1.55922 \\
$\mathrm{C}$ & -0.11099 & 4.9156 & -0.85027 & & & &
\end{tabular}

Table 7.24: XYZ-coordinates of computed complex $45^{\mathrm{iPr}}$.

\begin{tabular}{c|lll|l|lll} 
Atom & $\mathrm{x}$ & $\mathrm{y}$ & $\mathrm{z}$ & Atom & $\mathrm{x}$ & $\mathrm{y}$ & $\mathrm{z}$ \\
\hline $\mathrm{C}$ & -3.4394 & 2.33839 & 0.64607 & $\mathrm{H}$ & -0.00696 & 5.02836 & -0.9378 \\
$\mathrm{C}$ & -3.98467 & 1.08471 & 0.49507 & $\mathrm{C}$ & -0.49374 & 2.63929 & 0.83824 \\
$\mathrm{P}$ & -1.96117 & 2.63717 & -0.33577 & $\mathrm{C}$ & -0.60663 & 3.60343 & 2.0185 \\
$\mathrm{H}$ & -3.89545 & 3.06732 & 1.33035 & $\mathrm{H}$ & -1.51198 & 3.38384 & 2.62213 \\
$\mathrm{~N}$ & -3.46772 & 0.14229 & -0.35787 & $\mathrm{H}$ & -0.65841 & 4.66118 & 1.69399 \\
$\mathrm{H}$ & -4.88356 & 0.78642 & 1.06813 & $\mathrm{H}$ & 0.27457 & 3.50387 & 2.68852 \\
$\mathrm{C}$ & -4.06256 & -1.08895 & -0.48924 & $\mathrm{H}$ & 0.36411 & 2.91565 & 0.18758 \\
$\mathrm{C}$ & -3.56234 & -2.07154 & -1.30941 & $\mathrm{C}$ & -0.30099 & 1.1848 & 1.29346 \\
$\mathrm{P}$ & -1.99053 & -1.66785 & -2.1043 & $\mathrm{H}$ & -1.20154 & 0.80071 & 1.81606 \\
$\mathrm{H}$ & -4.07263 & -3.04259 & -1.38908 & $\mathrm{H}$ & -0.08013 & 0.51836 & 0.42758 \\
$\mathrm{C}$ & -0.67803 & -2.64714 & -1.16127 & $\mathrm{H}$ & 0.56277 & 1.09797 & 1.9865 \\
$\mathrm{C}$ & -2.09836 & -2.25025 & -3.87847 & $\mathrm{Os}$ & -1.8376 & 0.63135 & -1.56302 \\
$\mathrm{H}$ & -0.66355 & -2.0689 & -0.20799 & $\mathrm{Cl}$ & 0.35174 & 1.08828 & -2.51299 \\
$\mathrm{C}$ & -1.06243 & -4.08921 & -0.81655 & $\mathrm{P}$ & -3.06349 & 1.38618 & -3.16215 \\
$\mathrm{H}$ & -0.31696 & -4.52335 & -0.11618 & $\mathrm{Cl}$ & -5.10081 & 1.89492 & -2.73986 \\
$\mathrm{H}$ & -2.05576 & -4.14135 & -0.32879 & $\mathrm{C}$ & -2.51664 & -0.11371 & -8.43129 \\
$\mathrm{H}$ & -1.08523 & -4.73649 & -1.71556 & $\mathrm{C}$ & -2.34591 & 0.6758 & -7.28786 \\
$\mathrm{C}$ & 0.70569 & -2.53145 & -1.80988 & $\mathrm{C}$ & -3.48546 & -1.1322 & -8.4495 \\
$\mathrm{H}$ & 0.93711 & -1.48718 & -2.10431 & $\mathrm{H}$ & -1.88719 & 0.06289 & -9.31676 \\
$\mathrm{H}$ & 0.77918 & -3.17162 & -2.71187 & $\mathrm{C}$ & -4.29352 & -1.35485 & -7.32125 \\
$\mathrm{H}$ & 1.48775 & -2.87501 & -1.10021 & $\mathrm{H}$ & -3.61348 & -1.75283 & -9.34949
\end{tabular}


7.5 Transition Metal Complexes with Metal-Pnictogen Multiple Bonds

\begin{tabular}{l|lll|l|lll}
$\mathrm{C}$ & -0.92486 & -1.74627 & -4.72687 & $\mathrm{C}$ & -4.1246 & -0.57226 & -6.17255 \\
$\mathrm{H}$ & -0.65078 & -0.70266 & -4.47152 & $\mathrm{H}$ & -5.06083 & -2.14393 & -7.33895 \\
$\mathrm{H}$ & -0.02131 & -2.36918 & -4.57684 & $\mathrm{C}$ & -3.14318 & 0.44556 & -6.14195 \\
$\mathrm{H}$ & -1.19065 & -1.78979 & -5.80361 & $\mathrm{H}$ & -4.75848 & -0.72403 & -5.28519 \\
$\mathrm{H}$ & -3.00458 & -1.68839 & -4.19159 & $\mathrm{H}$ & -1.58446 & 1.46962 & -7.26217 \\
$\mathrm{C}$ & -2.38031 & -3.7414 & -4.08036 & $\mathrm{C}$ & -2.96837 & 1.2342 & -4.93778 \\
$\mathrm{H}$ & -2.64201 & -3.93334 & -5.14286 & $\mathrm{C}$ & -2.55325 & 2.45371 & -4.49916 \\
$\mathrm{H}$ & -3.2247 & -4.10069 & -3.45891 & $\mathrm{C}$ & -1.97754 & 3.72363 & -4.91253 \\
$\mathrm{H}$ & -1.49151 & -4.36125 & -3.84636 & $\mathrm{C}$ & -0.644 & 3.78192 & -5.37821 \\
$\mathrm{C}$ & -2.06866 & 4.31351 & -1.15911 & $\mathrm{C}$ & -0.07637 & 5.01641 & -5.72137 \\
$\mathrm{H}$ & -2.75554 & 4.08803 & -2.00526 & $\mathrm{H}$ & -0.05158 & 2.85571 & -5.41777 \\
$\mathrm{C}$ & -2.71507 & 5.42322 & -0.32616 & $\mathrm{C}$ & -0.82709 & 6.19814 & -5.60671 \\
$\mathrm{H}$ & -2.83766 & 6.33448 & -0.94893 & $\mathrm{H}$ & 0.96492 & 5.05666 & -6.07408 \\
$\mathrm{H}$ & -2.09736 & 5.7063 & 0.55013 & $\mathrm{C}$ & -2.15101 & 6.14471 & -5.13722 \\
$\mathrm{H}$ & -3.71884 & 5.13048 & 0.03916 & $\mathrm{H}$ & -0.37783 & 7.16513 & -5.87816 \\
$\mathrm{C}$ & -0.70118 & 4.69631 & -1.73776 & $\mathrm{C}$ & -2.72508 & 4.91696 & -4.78343 \\
$\mathrm{H}$ & -0.80939 & 5.53325 & -2.45847 & $\mathrm{H}$ & -2.74 & 7.06968 & -5.04282 \\
$\mathrm{H}$ & -0.23137 & 3.84229 & -2.26726 & $\mathrm{H}$ & -3.75736 & 4.86386 & -4.40729 \\
$\mathrm{H}$ & -4.97947 & -1.25759 & 0.10787 & & & &
\end{tabular}

Table 7.25: XYZ-coordinates of computed complex 44-rotamere.

\begin{tabular}{c|lll|l|lll} 
Atom & $\mathrm{x}$ & $\mathrm{y}$ & $\mathrm{z}$ & Atom & $\mathrm{x}$ & $\mathrm{y}$ & $\mathrm{z}$ \\
\hline $\mathrm{Os}$ & 3.14508 & 8.4993 & 10.89374 & $\mathrm{C}$ & 1.97158 & 10.19876 & 8.11838 \\
$\mathrm{Cl}$ & 3.73986 & 10.77975 & 11.41308 & $\mathrm{H}$ & 1.90915 & 10.82616 & 7.20406 \\
$\mathrm{Cl}$ & 2.61078 & 9.0572 & 14.52932 & $\mathrm{H}$ & 2.14852 & 9.15231 & 7.79557 \\
$\mathrm{P}$ & 0.84258 & 9.02113 & 10.32055 & $\mathrm{H}$ & 2.83662 & 10.55366 & 8.71188 \\
$\mathrm{P}$ & 5.38295 & 7.63493 & 10.62408 & $\mathrm{C}$ & 3.67773 & 5.84189 & 9.55659 \\
$\mathrm{P}$ & 2.92834 & 7.68049 & 12.82295 & $\mathrm{H}$ & 3.35917 & 4.92149 & 9.02977 \\
$\mathrm{~N}$ & 2.69327 & 6.76749 & 9.81754 & $\mathrm{C}$ & 4.98986 & 6.02506 & 9.91023 \\
$\mathrm{C}$ & 1.41064 & 6.55352 & 9.36624 & $\mathrm{H}$ & 5.738 & 5.24726 & 9.70094 \\
$\mathrm{H}$ & 1.22991 & 5.59438 & 8.84371 & $\mathrm{C}$ & 6.50949 & 7.35187 & 12.14765 \\
$\mathrm{C}$ & 0.39652 & 7.46128 & 9.52552 & $\mathrm{C}$ & 6.44611 & 8.59822 & 13.04848 \\
$\mathrm{H}$ & -0.61201 & 7.23769 & 9.15079 & $\mathrm{H}$ & 6.78214 & 9.5145 & 12.52959 \\
$\mathrm{C}$ & -0.44874 & 9.31643 & 11.70038 & $\mathrm{H}$ & 7.10311 & 8.44168 & 13.92983 \\
$\mathrm{C}$ & -1.83976 & 9.57729 & 11.09756 & $\mathrm{H}$ & 5.4212 & 8.7946 & 13.41755 \\
$\mathrm{H}$ & -1.87845 & 10.52847 & 10.53392 & $\mathrm{C}$ & 7.96625 & 7.05475 & 11.75307 \\
$\mathrm{H}$ & -2.5763 & 9.65518 & 11.92518 & $\mathrm{H}$ & 8.46292 & 7.92171 & 11.27811 \\
$\mathrm{H}$ & -2.17501 & 8.7545 & 10.43378 & $\mathrm{H}$ & 8.04894 & 6.18022 & 11.07673 \\
$\mathrm{C}$ & -0.00833 & 10.49887 & 12.58094 & $\mathrm{H}$ & 8.53813 & 6.81437 & 12.67429 \\
$\mathrm{H}$ & -0.07234 & 11.46601 & 12.05113 & $\mathrm{C}$ & 5.96161 & 6.13068 & 12.90275 \\
$\mathrm{H}$ & 1.02643 & 10.37559 & 12.94998 & $\mathrm{H}$ & 4.89092 & 6.25006 & 13.17242 \\
$\mathrm{H}$ & -0.6771 & 10.55459 & 13.46549 & $\mathrm{H}$ & 6.52529 & 6.01015 & 13.85148 \\
$\mathrm{C}$ & -0.51913 & 8.03603 & 12.54575 & $\mathrm{H}$ & 6.05877 & 5.19788 & 12.31418 \\
$\mathrm{H}$ & -0.81042 & 7.15262 & 11.94508 & $\mathrm{C}$ & 6.32541 & 8.56993 & 9.23938 \\
$\mathrm{H}$ & -1.26749 & 8.18258 & 13.35259 & $\mathrm{C}$ & 5.23792 & 8.88416 & 8.19849
\end{tabular}




\begin{tabular}{l|lll|l|lll}
$\mathrm{H}$ & 0.44257 & 7.81329 & 13.04839 & $\mathrm{H}$ & 5.69649 & 9.38637 & 7.32047 \\
$\mathrm{C}$ & 0.67655 & 10.33994 & 8.93549 & $\mathrm{H}$ & 4.48533 & 9.57726 & 8.61795 \\
$\mathrm{C}$ & -0.5214 & 10.06672 & 8.01137 & $\mathrm{H}$ & 4.7267 & 7.96784 & 7.84108 \\
$\mathrm{H}$ & -0.52527 & 10.82699 & 7.20035 & $\mathrm{C}$ & 6.89641 & 9.89034 & 9.77885 \\
$\mathrm{H}$ & -1.4964 & 10.13102 & 8.52738 & $\mathrm{H}$ & 7.2723 & 10.49482 & 8.92531 \\
$\mathrm{H}$ & -0.44455 & 9.07014 & 7.53425 & $\mathrm{H}$ & 7.74912 & 9.73094 & 10.46689 \\
$\mathrm{C}$ & 0.61984 & 11.75756 & 9.52328 & $\mathrm{H}$ & 6.11445 & 10.48015 & 10.30168 \\
$\mathrm{H}$ & 0.7363 & 12.49148 & 8.69709 & $\mathrm{C}$ & 7.4072 & 7.71423 & 8.56116 \\
$\mathrm{H}$ & 1.44338 & 11.92542 & 10.24693 & $\mathrm{H}$ & 6.99559 & 6.75414 & 8.19228 \\
$\mathrm{H}$ & -0.34895 & 11.97072 & 10.01463 & $\mathrm{H}$ & 8.2692 & 7.50091 & 9.21571 \\
$\mathrm{H}$ & 7.79205 & 8.26977 & 7.67922 & & & &
\end{tabular}

Table 7.26: XYZ-coordinates of computed complex 44-rotamere-transition state.

\begin{tabular}{c|lll|l|lll} 
Atom & $\mathrm{x}$ & $\mathrm{y}$ & $\mathrm{z}$ & Atom & $\mathrm{x}$ & $\mathrm{y}$ & $\mathrm{z}$ \\
\hline $\mathrm{Os}$ & 3.1267 & 8.55419 & 10.98028 & $\mathrm{C}$ & 1.85274 & 9.8046 & 8.01917 \\
$\mathrm{Cl}$ & 3.72874 & 10.87068 & 11.07946 & $\mathrm{H}$ & 1.79197 & 10.37582 & 7.06896 \\
$\mathrm{Cl}$ & 1.54851 & 7.49272 & 14.36916 & $\mathrm{H}$ & 1.88473 & 8.72648 & 7.76328 \\
$\mathrm{P}$ & 0.78414 & 8.98043 & 10.43107 & $\mathrm{H}$ & 2.79759 & 10.0887 & 8.51261 \\
$\mathrm{P}$ & 5.43597 & 7.7719 & 10.67256 & $\mathrm{C}$ & 3.73707 & 5.93883 & 9.65363 \\
$\mathrm{P}$ & 3.21747 & 7.93176 & 13.01012 & $\mathrm{H}$ & 3.4227 & 5.00563 & 9.14884 \\
$\mathrm{~N}$ & 2.72077 & 6.82894 & 9.95365 & $\mathrm{C}$ & 5.05372 & 6.16659 & 9.93479 \\
$\mathrm{C}$ & 1.42787 & 6.51656 & 9.56056 & $\mathrm{H}$ & 5.81414 & 5.41812 & 9.6712 \\
$\mathrm{H}$ & 1.29631 & 5.5295 & 9.07986 & $\mathrm{C}$ & 6.6389 & 7.40878 & 12.13176 \\
$\mathrm{C}$ & 0.37434 & 7.36367 & 9.73008 & $\mathrm{C}$ & 6.68036 & 8.64273 & 13.05081 \\
$\mathrm{H}$ & -0.63616 & 7.06812 & 9.41696 & $\mathrm{H}$ & 7.06417 & 9.54068 & 12.53302 \\
$\mathrm{C}$ & -0.58638 & 9.39901 & 11.70528 & $\mathrm{H}$ & 7.34733 & 8.4355 & 13.914 \\
$\mathrm{C}$ & -1.84479 & 10.05385 & 11.10922 & $\mathrm{H}$ & 5.67614 & 8.88794 & 13.44812 \\
$\mathrm{H}$ & -1.65285 & 11.04869 & 10.66812 & $\mathrm{C}$ & 8.05943 & 7.05877 & 11.65658 \\
$\mathrm{H}$ & -2.57417 & 10.19858 & 11.9349 & $\mathrm{H}$ & 8.56611 & 7.90328 & 11.15378 \\
$\mathrm{H}$ & -2.33351 & 9.41278 & 10.34983 & $\mathrm{H}$ & 8.07173 & 6.17914 & 10.98188 \\
$\mathrm{C}$ & 0.04603 & 10.35158 & 12.73682 & $\mathrm{H}$ & 8.66885 & 6.79572 & 12.54736 \\
$\mathrm{H}$ & 0.43223 & 11.27435 & 12.26194 & $\mathrm{C}$ & 6.10032 & 6.19102 & 12.90415 \\
$\mathrm{H}$ & 0.88137 & 9.87069 & 13.27636 & $\mathrm{H}$ & 5.07767 & 6.35928 & 13.29569 \\
$\mathrm{H}$ & -0.72162 & 10.64363 & 13.48438 & $\mathrm{H}$ & 6.76303 & 5.99983 & 13.77455 \\
$\mathrm{C}$ & -1.03264 & 8.07157 & 12.34717 & $\mathrm{H}$ & 6.0746 & 5.28033 & 12.27444 \\
$\mathrm{H}$ & -1.67 & 7.4897 & 11.65277 & $\mathrm{C}$ & 6.38101 & 8.70817 & 9.27917 \\
$\mathrm{H}$ & -1.6332 & 8.29489 & 13.25444 & $\mathrm{C}$ & 5.29511 & 9.15117 & 8.28411 \\
$\mathrm{H}$ & -0.18684 & 7.43674 & 12.66141 & $\mathrm{H}$ & 5.77739 & 9.54711 & 7.36537 \\
$\mathrm{C}$ & 0.64605 & 10.15542 & 8.90437 & $\mathrm{H}$ & 4.68224 & 9.96241 & 8.71714 \\
$\mathrm{C}$ & -0.62847 & 9.90622 & 8.08018 & $\mathrm{H}$ & 4.63726 & 8.31041 & 7.9826 \\
$\mathrm{H}$ & -0.5696 & 10.51494 & 7.15295 & $\mathrm{C}$ & 7.07235 & 9.9617 & 9.83891 \\
$\mathrm{H}$ & -1.55411 & 10.19948 & 8.60286 & $\mathrm{H}$ & 7.44417 & 10.57378 & 8.98891 \\
$\mathrm{H}$ & -0.71602 & 8.84586 & 7.77275 & $\mathrm{H}$ & 7.9453 & 9.7223 & 10.47592 \\
$\mathrm{C}$ & 0.75214 & 11.62704 & 9.33612 & $\mathrm{H}$ & 6.35898 & 10.58349 & 10.41707 \\
$\mathrm{H}$ & 0.84154 & 12.26258 & 8.42919 & $\mathrm{C}$ & 7.37101 & 7.80346 & 8.52575
\end{tabular}


7.5 Transition Metal Complexes with Metal-Pnictogen Multiple Bonds

\begin{tabular}{l|lll|l|lll}
$\mathrm{H}$ & 1.65096 & 11.79808 & 9.96184 & $\mathrm{H}$ & 6.85809 & 6.93499 & 8.06784 \\
$\mathrm{H}$ & -0.14116 & 11.97422 & 9.88971 & $\mathrm{H}$ & 8.19759 & 7.42853 & 9.1527 \\
$\mathrm{H}$ & 7.82354 & 8.39309 & 7.7 & & & &
\end{tabular}

Table 7.27: XYZ-coordinates of computed complex 44-oxidative addition.

\begin{tabular}{|c|c|c|c|c|c|c|c|}
\hline Atom & $\mathrm{x}$ & $\mathrm{y}$ & $\mathrm{z}$ & Atom & $\mathrm{x}$ & $\mathrm{y}$ & $\mathrm{z}$ \\
\hline Os & 3.13635 & 8.6355 & 10.70109 & $\mathrm{C}$ & 0.68874 & 10.17801 & 7.59878 \\
\hline $\mathrm{Cl}$ & 3.69332 & 10.09514 & 8.73895 & $\mathrm{H}$ & 0.51991 & 11.07409 & 6.96393 \\
\hline $\mathrm{Cl}$ & 3.66169 & 10.5851 & 11.99842 & $\mathrm{H}$ & 0.1018 & 9.34296 & 7.16878 \\
\hline $\mathrm{P}$ & 0.73834 & 9.05628 & 10.18376 & $\mathrm{H}$ & 1.76035 & 9.9236 & 7.55647 \\
\hline $\mathrm{P}$ & 5.47491 & 7.81353 & 10.60769 & $\mathrm{C}$ & 3.84571 & 6.1494 & 9.20368 \\
\hline $\mathrm{P}$ & 2.64253 & 7.40395 & 12.33133 & $\mathrm{H}$ & 3.6012 & 5.27135 & 8.57473 \\
\hline $\mathrm{N}$ & 2.8487 & 7.08147 & 9.35068 & $\mathrm{C}$ & 5.07739 & 6.24173 & 9.80511 \\
\hline $\mathrm{C}$ & 1.58333 & 6.8185 & 8.87798 & $\mathrm{H}$ & 5.83403 & 5.46714 & 9.60971 \\
\hline $\mathrm{H}$ & 1.48402 & 5.92583 & 8.23053 & $\mathrm{C}$ & 6.3502 & 7.44097 & 12.29307 \\
\hline $\mathrm{C}$ & 0.479 & 7.56613 & 9.19271 & $\mathrm{C}$ & 7.05312 & 8.7188 & 12.78535 \\
\hline $\mathrm{H}$ & -0.5012 & 7.2929 & 8.77421 & $\mathrm{H}$ & 7.93709 & 8.9865 & 12.17983 \\
\hline $\mathrm{C}$ & -0.42434 & 8.92183 & 11.74491 & $\mathrm{H}$ & 7.40446 & 8.55667 & 13.82618 \\
\hline $\mathrm{C}$ & -1.88111 & 9.38367 & 11.56321 & $\mathrm{H}$ & 6.34948 & 9.57688 & 12.78902 \\
\hline $\mathrm{H}$ & -1.96819 & 10.48024 & 11.47604 & $\mathrm{C}$ & 7.31122 & 6.24214 & 12.18499 \\
\hline $\mathrm{H}$ & -2.44533 & 9.08728 & 12.47323 & $\mathrm{H}$ & 8.1555 & 6.4131 & 11.49818 \\
\hline $\mathrm{H}$ & -2.38315 & 8.91354 & 10.69713 & $\mathrm{H}$ & 6.77758 & 5.32681 & 11.86036 \\
\hline $\mathrm{C}$ & 0.21213 & 9.76276 & 12.86783 & $\mathrm{H}$ & 7.73932 & 6.03531 & 13.18894 \\
\hline $\mathrm{H}$ & 0.34216 & 10.82159 & 12.5683 & $\mathrm{C}$ & 5.30157 & 7.0708 & 13.34538 \\
\hline $\mathrm{H}$ & 1.21851 & 9.40008 & 13.15386 & $\mathrm{H}$ & 4.66296 & 7.94228 & 13.60228 \\
\hline $\mathrm{H}$ & -0.44151 & 9.73526 & 13.76553 & $\mathrm{H}$ & 5.80777 & 6.7529 & 14.28129 \\
\hline $\mathrm{C}$ & -0.4989 & 7.43834 & 12.13151 & $\mathrm{H}$ & 4.67173 & 6.21271 & 13.02117 \\
\hline $\mathrm{H}$ & -1.08543 & 6.8493 & 11.40058 & $\mathrm{C}$ & 6.8003 & 8.61227 & 9.4285 \\
\hline $\mathrm{H}$ & -0.96995 & 7.3423 & 13.13254 & $\mathrm{C}$ & 6.34551 & 8.35364 & 7.97798 \\
\hline $\mathrm{H}$ & 0.49762 & 6.9443 & 12.20419 & $\mathrm{H}$ & 7.04484 & 8.8898 & 7.3023 \\
\hline $\mathrm{C}$ & 0.20036 & 10.51645 & 9.02343 & $\mathrm{H}$ & 5.32759 & 8.73318 & 7.78285 \\
\hline $\mathrm{C}$ & -1.32153 & 10.71736 & 8.89607 & $\mathrm{H}$ & 6.38403 & 7.27602 & 7.72569 \\
\hline $\mathrm{H}$ & -1.48979 & 11.38593 & 8.02488 & $\mathrm{C}$ & 6.89465 & 10.12327 & 9.70791 \\
\hline $\mathrm{H}$ & -1.77578 & 11.21329 & 9.76958 & $\mathrm{H}$ & 7.56945 & 10.5799 & 8.95246 \\
\hline $\mathrm{H}$ & -1.86838 & 9.776 & 8.68933 & $\mathrm{H}$ & 7.31588 & 10.34145 & 10.70567 \\
\hline $\mathrm{C}$ & 0.84441 & 11.80018 & 9.57512 & $\mathrm{H}$ & 5.90714 & 10.61348 & 9.63044 \\
\hline $\mathrm{H}$ & 0.60464 & 12.64773 & 8.89782 & $\mathrm{C}$ & 8.19087 & 7.96579 & 9.56498 \\
\hline $\mathrm{H}$ & 1.9443 & 11.7089 & 9.65115 & $\mathrm{H}$ & 8.16571 & 6.8712 & 9.39387 \\
\hline $\mathrm{H}$ & 0.44121 & 12.05269 & 10.57721 & $\mathrm{H}$ & 8.68619 & 8.16641 & 10.5331 \\
\hline $\mathrm{H}$ & 8.84078 & 8.40285 & 8.77668 & & & & \\
\hline
\end{tabular}


Table 7.28: XYZ-coordinates of computed complex 44-oxidative addition-transition state.

\begin{tabular}{|c|c|c|c|c|c|c|c|}
\hline Atom & $\mathrm{x}$ & $\mathrm{y}$ & $\mathrm{Z}$ & Atom & $\mathrm{x}$ & $\mathrm{y}$ & Z \\
\hline Os & 3.1085 & 8.50577 & 10.97573 & $\mathrm{H}$ & 1.33815 & 10.67102 & 6.9961 \\
\hline $\mathrm{Cl}$ & 3.72209 & 10.81229 & 10.26271 & $\mathrm{H}$ & 1.55269 & 8.97022 & 7.51182 \\
\hline $\mathrm{Cl}$ & 3.49304 & 9.73267 & 13.2883 & $\mathrm{H}$ & 2.55062 & 10.27622 & 8.25676 \\
\hline $\mathrm{P}$ & 0.78133 & 9.0015 & 10.36564 & $\mathrm{C}$ & 3.80819 & 6.16051 & 9.24635 \\
\hline $\mathrm{P}$ & 5.44782 & 7.77277 & 10.66046 & $\mathrm{H}$ & 3.52601 & 5.30975 & 8.5974 \\
\hline $\mathrm{P}$ & 2.62603 & 7.27461 & 12.60065 & $\mathrm{C}$ & 5.07188 & 6.25322 & 9.75943 \\
\hline $\mathrm{N}$ & 2.80572 & 7.06952 & 9.50706 & $\mathrm{H}$ & 5.8298 & 5.50405 & 9.49073 \\
\hline $\mathrm{C}$ & 1.51725 & 6.72351 & 9.15742 & $\mathrm{C}$ & 6.46239 & 7.26658 & 12.21516 \\
\hline $\mathrm{H}$ & 1.41315 & 5.80698 & 8.5474 & $\mathrm{C}$ & 6.89572 & 8.5239 & 12.98725 \\
\hline $\mathrm{C}$ & 0.41699 & 7.42777 & 9.56113 & $\mathrm{H}$ & 7.67032 & 9.09915 & 12.44906 \\
\hline $\mathrm{H}$ & -0.58987 & 7.11785 & 9.24861 & $\mathrm{H}$ & 7.33005 & 8.21323 & 13.96112 \\
\hline $\mathrm{C}$ & -0.47707 & 9.26806 & 11.79 & $\mathrm{H}$ & 6.03089 & 9.18539 & 13.1926 \\
\hline $\mathrm{C}$ & -1.89898 & 9.58748 & 11.29513 & $\mathrm{C}$ & 7.68301 & 6.40327 & 11.84605 \\
\hline $\mathrm{H}$ & -1.95432 & 10.57331 & 10.79827 & $\mathrm{H}$ & 8.46709 & 6.98561 & 11.33283 \\
\hline $\mathrm{H}$ & -2.57455 & 9.62716 & 12.17559 & $\mathrm{H}$ & 7.41686 & 5.53446 & 11.21209 \\
\hline $\mathrm{H}$ & -2.29708 & 8.81578 & 10.60597 & $\mathrm{H}$ & 8.13147 & 6.00702 & 12.78134 \\
\hline $\mathrm{C}$ & 0.04133 & 10.40842 & 12.68484 & $\mathrm{C}$ & 5.54639 & 6.42555 & 13.11546 \\
\hline $\mathrm{H}$ & 0.09192 & 11.37242 & 12.14507 & $\mathrm{H}$ & 4.76754 & 7.05884 & 13.59292 \\
\hline $\mathrm{H}$ & 1.05347 & 10.19496 & 13.08164 & $\mathrm{H}$ & 6.1456 & 5.99533 & 13.94511 \\
\hline $\mathrm{H}$ & -0.65343 & 10.5366 & 13.54181 & $\mathrm{H}$ & 5.06726 & 5.58724 & 12.56934 \\
\hline $\mathrm{C}$ & -0.55258 & 7.96857 & 12.59977 & $\mathrm{C}$ & 6.54345 & 8.73442 & 9.3863 \\
\hline $\mathrm{H}$ & -0.921 & 7.11296 & 12.00098 & $\mathrm{C}$ & 5.63549 & 8.96193 & 8.16019 \\
\hline $\mathrm{H}$ & -1.22955 & 8.11791 & 13.4668 & $\mathrm{H}$ & 6.22325 & 9.50606 & 7.39081 \\
\hline $\mathrm{H}$ & 0.4345 & 7.68472 & 13.03221 & $\mathrm{H}$ & 4.75595 & 9.57911 & 8.41436 \\
\hline $\mathrm{C}$ & 0.45207 & 10.25348 & 8.9243 & $\mathrm{H}$ & 5.29963 & 8.0045 & 7.71682 \\
\hline $\mathrm{C}$ & -0.89433 & 9.96756 & 8.22978 & $\mathrm{C}$ & 7.02192 & 10.08183 & 9.95046 \\
\hline $\mathrm{H}$ & -0.97021 & 10.64636 & 7.3547 & $\mathrm{H}$ & 7.45022 & 10.67964 & 9.11755 \\
\hline $\mathrm{H}$ & -1.77589 & 10.15358 & 8.86459 & $\mathrm{H}$ & 7.82133 & 9.95517 & 10.70474 \\
\hline $\mathrm{H}$ & -0.94555 & 8.93138 & 7.84349 & $\mathrm{H}$ & 6.18754 & 10.66162 & 10.3884 \\
\hline $\mathrm{C}$ & 0.49394 & 11.70018 & 9.43853 & $\mathrm{C}$ & 7.75138 & 7.90212 & 8.91933 \\
\hline $\mathrm{H}$ & 0.42453 & 12.39176 & 8.57182 & $\mathrm{H}$ & 7.46968 & 6.87572 & 8.61523 \\
\hline $\mathrm{H}$ & 1.44295 & 11.91026 & 9.96898 & $\mathrm{H}$ & 8.55659 & 7.84057 & 9.67375 \\
\hline $\mathrm{H}$ & -0.35899 & 11.92652 & 10.10816 & $\mathrm{H}$ & 8.1849 & 8.40043 & 8.02651 \\
\hline $\mathrm{C}$ & 1.55055 & 10.01843 & 7.86956 & & & & \\
\hline
\end{tabular}




\subsubsection{Crystallographic Details}

CCDC-1894136 (37), CCDC-1894134 (38), CCDC-1894133 (41) and CCDC-1894135 (43) contain the supplementary crystallographic data for this chapter. This data can be obtained free of charge via http://www.ccdc.cam.ac.uk/ products/csd/request/ (or

from Cambridge Crystallographic Data Centre, 12 Union Road, Cambridge, CB2 1EZ, UK. Fax: +44-1223- 336-033; e-mail: deposit@ccdc.cam.ac.uk). 


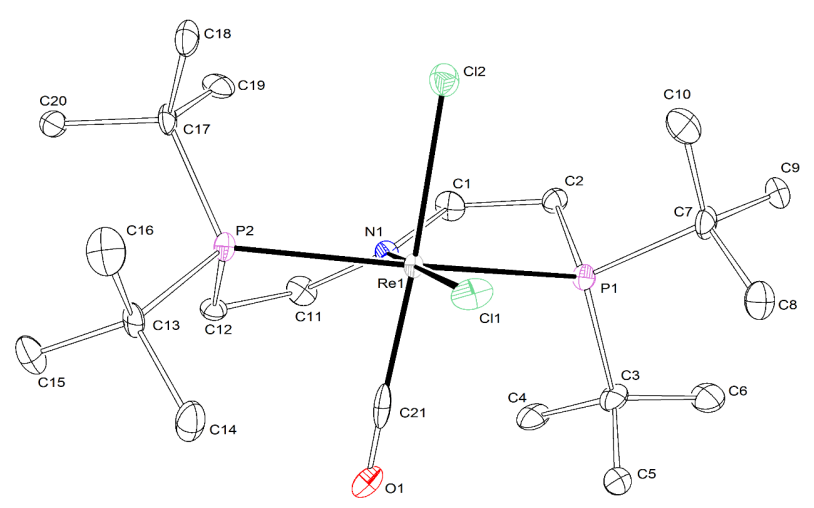

Figure 7.21: Thermal ellipsoid plot of $\mathbf{2 4}$ with the anisotropic displacement parameters drawn at the $50 \%$ probability level. The asymmetric unit contains one complex molecule.

Empirical formula

Formula weight

Temperature

Wavelength

Crystal system

Space group

Unit cell dimensions

Volume

Z

Density (calculated)

Absorption coefficient

$\mathrm{F}(000)$

Crystal size

Crystal shape and color

Theta range for data collection

Index ranges

Reflections collected

Independent reflections

Completeness to theta $=25.242^{\circ}$

Refinement method

Data / restraints / parameters

Goodness-of-fit on $\mathrm{F}^{2}$

Final $\mathrm{R}$ indices $[\mathrm{I}>2 \operatorname{sigma}(\mathrm{I})]$

$\mathrm{R}$ indices (all data)

Largest diff. peak and hole
$\mathrm{C}_{21} \mathrm{H}_{44} \mathrm{Cl}_{2} \mathrm{NOP}_{2} \mathrm{Re}$

645.61

100(2) K

$0.71073 \AA$

Monoclinic

$\mathrm{C} 2 / \mathrm{c}$

$\mathrm{a}=30.7099(14) \AA$

$\mathrm{b}=12.8064(6) \AA$

$\mathrm{c}=14.2870(6) \AA$

$5229.2(4) \AA^{3}$

8

$1.640 \mathrm{Mg} / \mathrm{m}^{3}$

$4.987 \mathrm{~mm}^{-1}$

2592

$0.180 \times 0.120 \times 0.052 \mathrm{~mm}^{3}$

Plate, clear intense green

2.486 to $26.450^{\circ}$

$-38<=\mathrm{h}<=38$

$-16<=\mathrm{k}<=16$

$-17<=\mathrm{l}<=17$

88105

$5382[\mathrm{R}(\mathrm{int})=0.1671]$

$99.9 \%$

Full-matrix least-squares on $\mathrm{F}^{2}$

5382 / 0 / 265

1.037

$\mathrm{R} 1=0.0371$,

$\mathrm{wR} 2=0.0626$

$\mathrm{R} 1=0.0607$,

$\mathrm{wR} 2=0.0681$$$
\begin{aligned}
& \beta=111.463(2)^{\circ} \\
& \gamma=90^{\circ}
\end{aligned}
$$$$
\gamma=90^{\circ}
$$

1.436 and $-1.305 \mathrm{e}^{-3}$ 


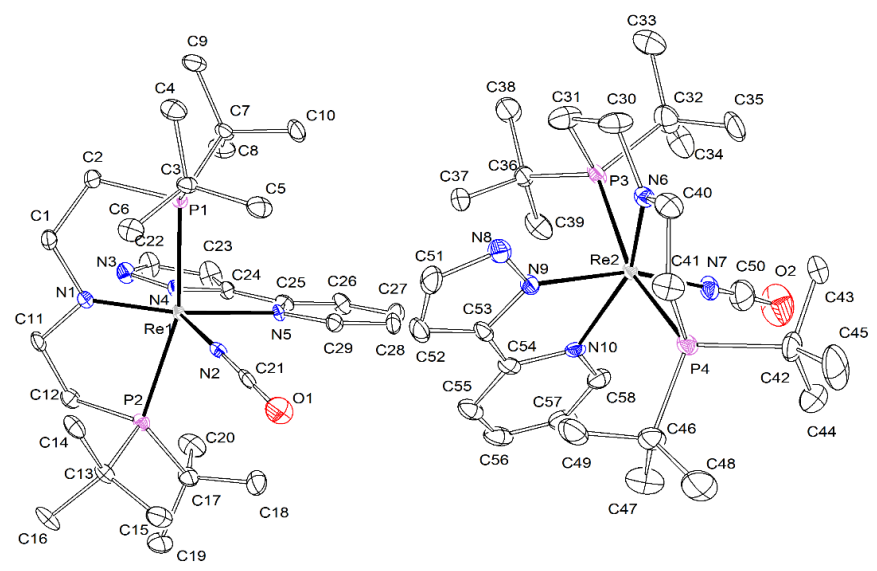

Figure 7.22: Thermal ellipsoid plot of $\mathbf{2 5}$ with the anisotropic displacement parameters drawn at the $50 \%$ probability level. The asymmetric unit contains two complex molecules.

Empirical formula

Formula weight

Temperature

Wavelength

Crystal system

Space group

Unit cell dimensions

Volume

Z

Density (calculated)

Absorption coefficient

$\mathrm{F}(000)$

Crystal size

Crystal shape and color

Theta range for data collection

Index ranges

Reflections collected

Independent reflections

Completeness to theta $=25.242^{\circ}$

Refinement method

Data / restraints / parameters

Goodness-of-fit on $\mathrm{F}^{2}$

Final $R$ indices $[\mathrm{I}>2 \operatorname{sigma}(\mathrm{I})]$

$\mathrm{R}$ indices (all data)

Largest diff. peak and hole
$\mathrm{C}_{29} \mathrm{H}_{50} \mathrm{~N}_{5} \mathrm{OP}_{2} \mathrm{Re}$

732.88

$100(2) \mathrm{K}$

$0.71073 \AA$

Monoclinic

$\mathrm{P} 22_{1} / \mathrm{n}$

$\mathrm{a}=18.0402(6) \AA$

$\mathrm{b}=10.1427(4) \AA$

$\mathrm{c}=34.6876(12) \AA$

$6239.6(4) \AA^{3}$

8

$1.560 \mathrm{Mg} / \mathrm{m}^{3}$

$4.028 \mathrm{~mm}^{-1}$

2976

$0.206 \times 0.120 \times 0.114 \mathrm{~mm}^{3}$

Block, clear intense brown

2.297 to $28.282^{\circ}$

$-24<=\mathrm{h}<=24$

$-13<=\mathrm{k}<=13$

$-46<=\mathrm{l}<=46$

194220

$15482[\mathrm{R}(\mathrm{int})=0.0752]$

$100.0 \%$

Full-matrix least-squares on $\mathrm{F}^{2}$

15482 / 0 / 709

1.117

$\mathrm{R} 1=0.0370$,

$\mathrm{R} 1=0.0533$,

$\mathrm{wR} 2=0.0588$

$\mathrm{wR} 2=0.0621$$$
\gamma=90^{\circ}
$$

1.539 and $-1.729 \mathrm{e} \AA^{-3}$ 


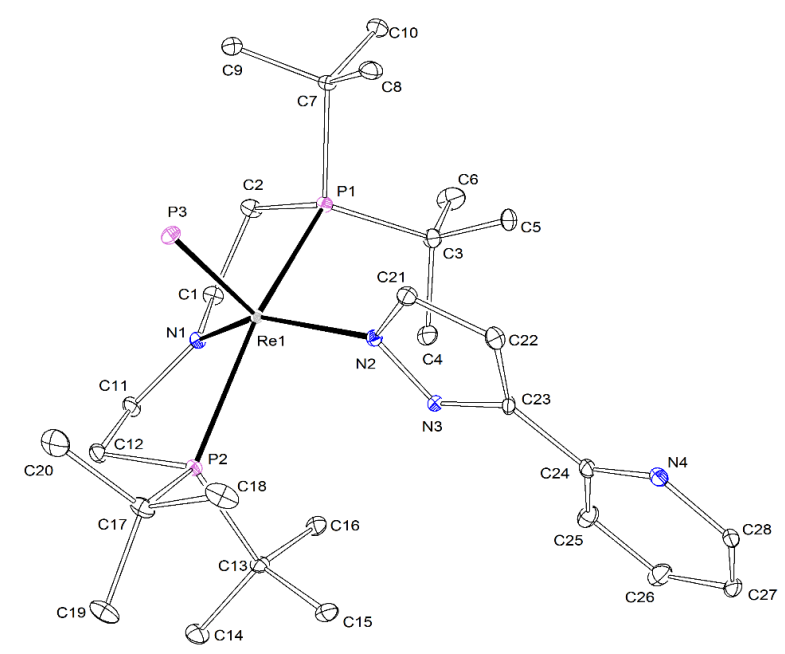

Figure 7.23: Thermal ellipsoid plot of $\mathbf{2 7}$ with the anisotropic displacement parameters drawn at the $50 \%$ probability level. The asymmetric unit contains one complex molecule.

Empirical formula

Formula weight

Temperature

Wavelength

Crystal system

Space group

Unit cell dimensions

Volume

Z

Density (calculated)

Absorption coefficient

$\mathrm{F}(000)$

Crystal size

Crystal shape and color

Theta range for data collection

Index ranges

Reflections collected

Independent reflections

Completeness to theta $=25.242^{\circ}$

Refinement method

Data / restraints / parameters

Goodness-of-fit on $\mathrm{F}^{2}$

Final $\mathrm{R}$ indices [I $>2$ sigma(I)]

$\mathrm{R}$ indices (all data)

Largest diff. peak and hole
$\mathrm{C}_{28} \mathrm{H}_{50} \mathrm{~N}_{4} \mathrm{P}_{3} \mathrm{Re}$

721.83

101(2) K

$0.71073 \AA$

Monoclinic

$\mathrm{P} 21 / \mathrm{n}$

$\mathrm{a}=10.8199(4) \AA$

$\mathrm{b}=20.3333(7) \AA$

$\mathrm{c}=14.7109(5) \AA$

$3130.77(19) \AA^{3}$

4

$1.531 \mathrm{Mg} / \mathrm{m}^{3}$

$4.058 \mathrm{~mm}^{-1}$

1464

$0.247 \times 0.137 \times 0.128 \mathrm{~mm}^{3}$

Plate, clear intense yellow-brown

2.188 to $30.625^{\circ}$

$-14<=\mathrm{h}<=15$

$-29<=\mathrm{k}<=29$

$-21<=\mathrm{l}<=21$

98300

$9626[\mathrm{R}(\mathrm{int})=0.0611]$

$99.9 \%$

Full-matrix least-squares on $\mathrm{F}^{2}$

9626 / 0 / 337

1.018

$\mathrm{R} 1=0.0248$,

$\mathrm{R} 1=0.0380$,

0.733 and $-0.742 \mathrm{e} \AA^{-3}$

$$
\begin{aligned}
& \alpha=90^{\circ} \\
& \beta=104.6830(10)^{\circ} \\
& \gamma=90^{\circ}
\end{aligned}
$$

$\mathrm{wR} 2=0.0382$

${ }_{\mathrm{wR} 2}=0.0406$ 


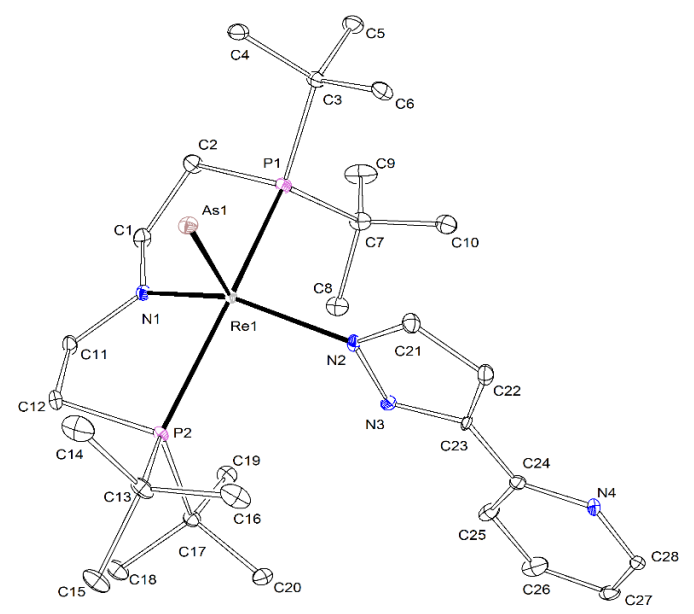

Figure 7.24: Thermal ellipsoid plot of $\mathbf{2 8}$ with the anisotropic displacement parameters drawn at the $50 \%$ probability level. The asymmetric unit contains one complex molecule.

Empirical formula

Formula weight

Temperature

Wavelength

Crystal system

Space group

Unit cell dimensions

Volume

Z

Density (calculated)

Absorption coefficient

$\mathrm{F}(000)$

Crystal size

Crystal shape and color

Theta range for data collection

Index ranges

Reflections collected

Independent reflections

Completeness to theta $=25.242^{\circ}$

Refinement method

Data / restraints / parameters

Goodness-of-fit on $\mathrm{F}^{2}$

Final R indices $[\mathrm{I}>2 \operatorname{sigma}(\mathrm{I})]$

$\mathrm{R}$ indices (all data)

Largest diff. peak and hole
$\mathrm{C}_{28} \mathrm{H}_{50} \mathrm{AsN}_{4} \mathrm{P}_{2} \mathrm{Re}$

765.78

100(2) K

$0.71073 \AA$

Monoclinic

$\mathrm{P} 22_{1} / \mathrm{n}$

$\mathrm{a}=10.7835(4) \AA$

$\mathrm{b}=20.3684(6) \AA$

$\mathrm{c}=14.7721(5) \AA$

$3141.73(18) \AA^{3}$

4

$1.619 \mathrm{Mg} / \mathrm{m}^{3}$

$5.038 \mathrm{~mm}^{-1}$

1536

$0.279 \times 0.085 \times 0.042 \mathrm{~mm}^{3}$

Plate, clear intense yellow

2.192 to $30.572^{\circ}$

$-15<=\mathrm{h}<=15$

$-29<=\mathrm{k}<=29$

$-21<=\mathrm{l}<=21$

136063

$9617[\mathrm{R}(\mathrm{int})=0.1195]$

$99.9 \%$

Full-matrix least-squares on $\mathrm{F}^{2}$

9617 / 0 / 337

1.064

$\mathrm{R} 1=0.0370$,

$\mathrm{R} 1=0.0630$,

$\mathrm{wR} 2=0.0435$

0.816 and $-1.145 \mathrm{e}^{-3}$

$$
\begin{aligned}
& \alpha=90^{\circ} \\
& \beta=104.465(2)^{\circ} \\
& \gamma=90^{\circ}
\end{aligned}
$$




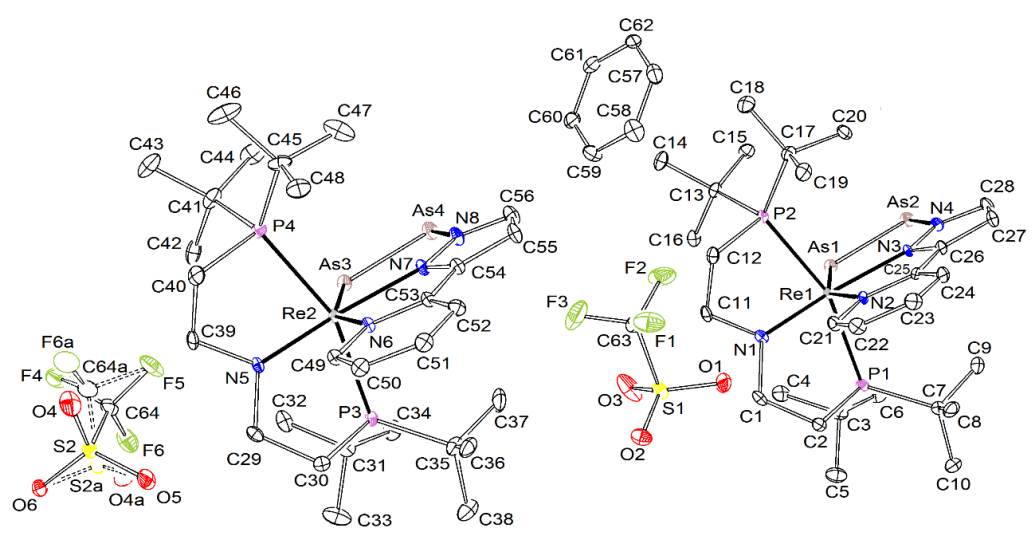

Figure 7.25: Thermal ellipsoid plot of $\mathbf{3 0}$ with the anisotropic displacement parameters drawn at the $50 \%$ probability level. The asymmetric unit contains two complex molecules, two $\mathrm{CF}_{3} \mathrm{SO}_{3}-$ anions and one benzene solvent molecule. One disordered $\mathrm{CF}_{3} \mathrm{SO}_{3}-$ anions was refined with population of $0.858(6)$ on the main domain using some restraints (RIGU).

Empirical formula

Formula weight

Temperature

Wavelength

Crystal system

Space group

Unit cell dimensions

Volume

Z

Density (calculated)

Absorption coefficient

$\mathrm{F}(000)$

Crystal size

Crystal shape and color

Theta range for data collection

Index ranges

Reflections collected

Independent reflections

Completeness to theta $=25.242^{\circ}$

Refinement method

Data / restraints / parameters

Goodness-of-fit on $\mathrm{F}^{2}$

Final $R$ indices [I>2sigma(I)]

$\mathrm{R}$ indices (all data)

Largest diff. peak and hole
$\mathrm{C}_{64} \mathrm{H}_{106} \mathrm{As}_{4} \mathrm{~F}_{6} \mathrm{~N}_{8} \mathrm{O}_{6} \mathrm{P}_{4} \mathrm{Re}_{2} \mathrm{~S}_{2}$

2057.64

$105(2) \mathrm{K}$

$0.71073 \AA$

Monoclinic

$\mathrm{P} 21 / \mathrm{n}$

$\mathrm{a}=10.8124(5) \AA$

$\mathrm{b}=21.0452(10) \AA$

$\mathrm{c}=34.0720(15) \AA$

$7710.8(6) \AA^{3}$

4

$1.772 \mathrm{Mg} / \mathrm{m}^{3}$

$5.045 \mathrm{~mm}^{-1}$

4088

$0.246 \times 0.142 \times 0.078 \mathrm{~mm}^{3}$

Block,

$\alpha=90^{\circ}$

$\beta=95.9810(10)^{\circ}$

$\gamma=90^{\circ}$

dark red-brown

2.263 to $28.383^{\circ}$

$-14<=\mathrm{h}<=14$

$-28<=\mathrm{k}<=28$

$-45<=\mathrm{l}<=45$

181199

$19313[\mathrm{R}(\mathrm{int})=0.0758]$

$99.9 \%$

Full-matrix least-squares on $\mathrm{F}^{2}$

19313 / 108 / 926

1.059

$\mathrm{R} 1=0.0393$,

$\mathrm{wR} 2=0.0703$

$\mathrm{R} 1=0.0601$,

$\mathrm{wR} 2=0.0755$ 


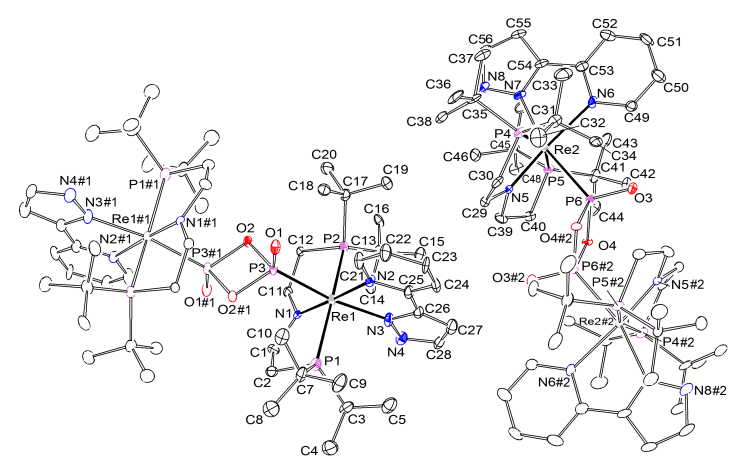

Figure 7.26: Thermal ellipsoid plot of $\mathbf{3 1}$ with the anisotropic displacement parameters drawn at the $50 \%$ probability level. The asymmetric unit contains two half complex molecules and was refined using some restraints and constraints (RIGU, EADP). The SQUEEZE algorithm was used to omit three disordered $\mathrm{Et}_{2} \mathrm{O}$ solvent molecules. ${ }^{[371]}$

\section{Empirical formula \\ Formula weight \\ Temperature \\ Wavelength \\ Crystal system \\ Space group \\ Unit cell dimensions}

Volume

Z

Density (calculated)

Absorption coefficient

$\mathrm{F}(000)$

Crystal size

Crystal shape and color

Theta range for data collection

Index ranges

Reflections collected

Independent reflections

Completeness to theta $=25.242^{\circ}$

Refinement method

Data / restraints / parameters

Goodness-of-fit on $\mathrm{F}^{2}$

Final R indices [I $>2 \operatorname{sigma}(\mathrm{I})]$

$\mathrm{R}$ indices (all data)

Largest diff. peak and hole

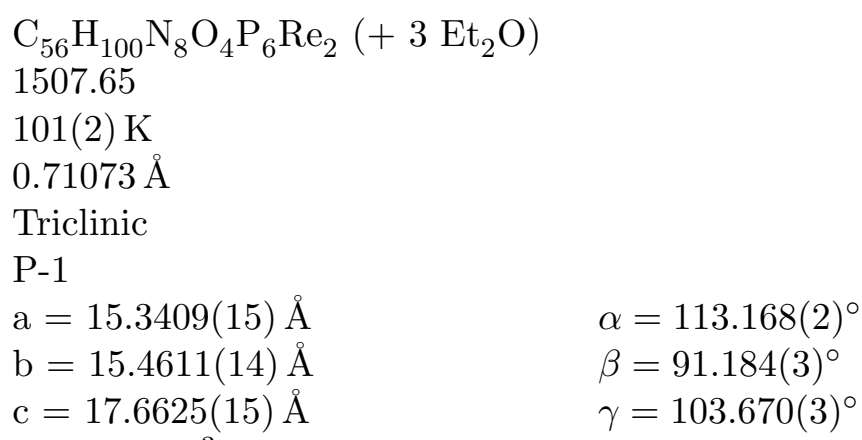

Full-matrix least-squares on $\mathrm{F}^{2}$ 13625 / 732 / 697

1.026

$\mathrm{R} 1=0.0432$,

$\mathrm{R} 1=0.0816$,

$\mathrm{wR} 2=0.0907$

1.852 and $-1.154 \mathrm{e}^{-3}$ 


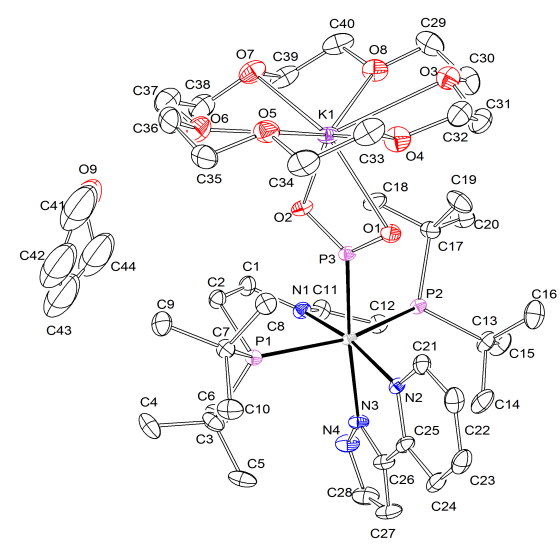

Figure 7.27: Thermal ellipsoid plot of $\mathbf{3 4}$ with the anisotropic displacement parameters drawn at the $50 \%$ probability level. The asymmetric unit contains one complex a disordered THF molecule. THF was set to a population of 0.5 and refined using some restraints and constraints (SADI, EADP).

Empirical formula

Formula weight

Temperature

Wavelength

Crystal system

Space group

Unit cell dimensions

Volume

Z

Density (calculated)

Absorption coefficient

$\mathrm{F}(000)$

Crystal size

Crystal shape and color

Theta range for data collection

Index ranges

Reflections collected

Independent reflections

Completeness to theta $=25.242^{\circ}$

Refinement method

Data / restraints / parameters

Goodness-of-fit on $\mathrm{F}^{2}$

Final R indices [I $>2 \operatorname{sigma}(\mathrm{I})]$

$\mathrm{R}$ indices (all data)

Largest diff. peak and hole
$\mathrm{C}_{40} \mathrm{H}_{73} \mathrm{KN}_{4} \mathrm{O}_{8} \mathrm{P}_{3} \operatorname{Rex0} \cdot 5\left(\mathrm{C}_{4} \mathrm{H}_{8} \mathrm{O}\right)$

1092.28

$100(2) \mathrm{K}$

$0.71073 \AA$

Monoclinic

$\mathrm{P} 21 / \mathrm{c}$

$\mathrm{a}=12.8339(4) \AA$

$\alpha=90^{\circ}$

$\mathrm{b}=23.5174(8) \AA$

$\beta=100.086(2)^{\circ}$

$\mathrm{c}=16.6688(5) \AA$

4953.2(3) $\AA^{3}$

4

$1.465 \mathrm{Mg} / \mathrm{m}^{3}$

$2.686 \mathrm{~mm}^{-1}$

2256

$0.454 \times 0.162 \times 0.100 \mathrm{~mm}^{3}$

Plate, clear intense purple

2.364 to $28.369^{\circ}$

$-17<=\mathrm{h}<=17$

$-31<=\mathrm{k}<=31$

$-22<=\mathrm{l}<=22$

215272

$12383[\mathrm{R}(\mathrm{int})=0.0992]$

$99.9 \%$

Full-matrix least-squares on $\mathrm{F}^{2}$

12383 / 4 / 558

1.048

$\mathrm{R} 1=0.0291$,

$\mathrm{wR} 2=0.0519$

$\mathrm{R} 1=0.0454$,

$\mathrm{wR} 2=0.0566$ 


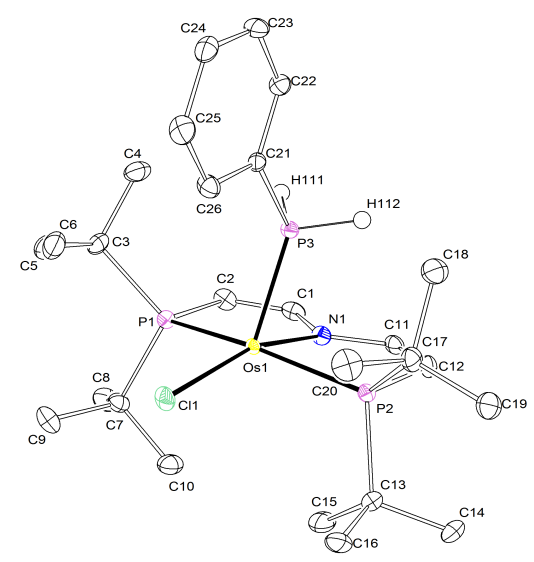

Figure 7.28: Thermal ellipsoid plot of $\mathbf{3 6}$ with the anisotropic displacement parameters drawn at the $50 \%$ probability level. The asymmetric unit contains one complex molecule. The P-H hydrogen atoms were found from the residual density map and isotropically refined.

Empirical formula

Formula weight

Temperature

Wavelength

Crystal system

Space group

Unit cell dimensions

Volume

Z

Density (calculated)

Absorption coefficient

$\mathrm{F}(000)$

Crystal size

Crystal shape and color

Theta range for data collection

Index ranges

Reflections collected

Independent reflections

Completeness to theta $=25.242^{\circ}$

Max. and min. transmission

Refinement method

Data / restraints / parameters

Goodness-of-fit on $\mathrm{F}^{2}$

Final $\mathrm{R}$ indices $[\mathrm{I}>2 \operatorname{sigma}(\mathrm{I})]$

$\mathrm{R}$ indices (all data)

Largest diff. peak and hole
$\mathrm{C}_{26} \mathrm{H}_{47} \mathrm{ClNOsP}_{3}$

692.20

100(2) K

$0.71073 \AA$

Monoclinic

$\mathrm{P} 21 / \mathrm{c}$

$\mathrm{a}=18.2079(6) \AA$

$\alpha=90^{\circ}$

$\mathrm{b}=8.1419(3) \AA$

$\mathrm{c}=21.6350(8) \AA$

2950.40(18) $\AA^{3}$

4

$1.558 \mathrm{Mg} / \mathrm{m}^{3}$

$4.590 \mathrm{~mm}^{-1}$

1392

$0.193 \times 0.103 \times 0.065 \mathrm{~mm}^{3}$

Block, clear intense blue

2.432 to $30.594 \circ$

$-26<=\mathrm{h}<=26$

$-11<=\mathrm{k}<=11$

$-28<=\mathrm{l}<=30$

106188

$9079[\mathrm{R}(\mathrm{int})=0.0735]$

$99.9 \%$

0.7461 and 0.5601

Full-matrix least-squares on $\mathrm{F}^{2}$

9079 / 0 / 309

1.056

$\mathrm{R} 1=0.0273$,

$\mathrm{wR} 2=0.0423$

$\mathrm{R} 1=0.0407$,

$\mathrm{wR} 2=0.0448$ $\beta=113.0890(10)^{\circ}$

$\gamma=90^{\circ}$

0.704 and $-0.997 \mathrm{e} \AA^{-3}$ 


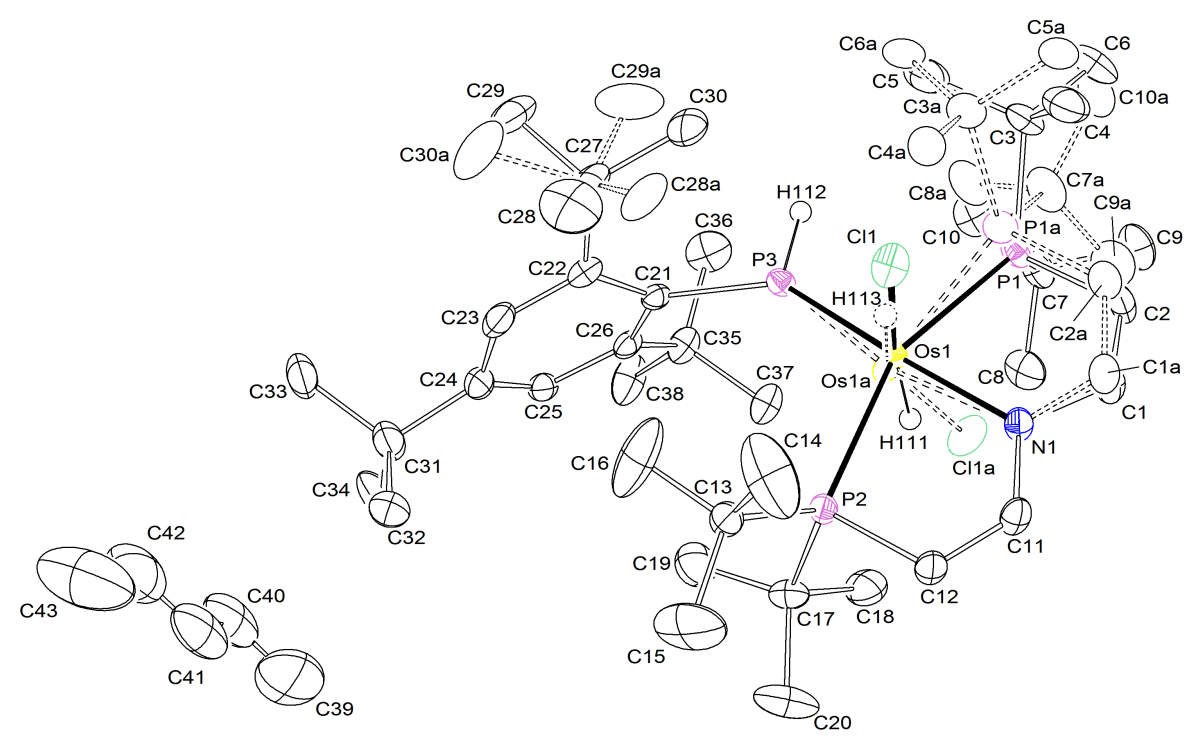

Figure 7.29: Thermal ellipsoid plot of $\mathbf{3 7}$ with the anisotropic displacement parameters drawn at the $50 \%$ probability level. The asymmetric unit contains one disordered complex molecule and one disordered pentane solvent molecule. The disordered Os(PNP)-part of the complex molecule was refined with population of $0.915(2)$ on the main domain using some restraints and constraints (SADI, RIGU, EADP). The disordered $-\mathrm{C}\left(\mathrm{CH}_{3}\right)_{3}$-part of the complex molecule was refined with population of $0.737(7)$ on the main domain using some restraints (SADI, RIGU). The disordered solvent molecule was refined using PART-1 command and some restraints (SADI, RIGU) and constraints (EADP). The Os-H and P-H hydrogen atoms were found from the residual density map and isotropically refined.

Empirical formula

Formula weight

Temperature

Wavelength

Crystal system

Space group

Unit cell dimensions

Volume

Z

Density (calculated)

Absorption coefficient

$\mathrm{F}(000)$

Crystal size

Crystal shape and color

Theta range for data collection

Index ranges

$$
\mathrm{C}_{39} \mathrm{H}_{71} \mathrm{ClNOsP}_{3} \cdot 0.5 \mathrm{C}_{5} \mathrm{H}_{12}
$$

$$
896.59
$$

100(2) K

$0.71073 \AA$

Monoclinic

$\mathrm{C} 2 / \mathrm{c}$

$$
\begin{array}{ll}
\mathrm{a}=28.7206(11) \AA & \alpha=90^{\circ} \\
\mathrm{b}=12.8908(5) \AA & \beta=99.050(2)^{\circ} \\
\mathrm{c}=23.8342(9) \AA & \gamma=90^{\circ}
\end{array}
$$$$
8714.3(6) \AA^{3}
$$

8

$1.367 \mathrm{Mg} / \mathrm{m}^{3}$

$3.125 \mathrm{~mm}^{-1}$

3720

$0.163 \times 0.105 \times 0.054 \mathrm{~mm}^{3}$

Plate, clear intense blue-green

2.370 to $28.352^{\circ}$

$$
\begin{aligned}
& -38<=\mathrm{h}<=38 \\
& -17<=\mathrm{k}<=17 \\
& -31<=\mathrm{l}<=31
\end{aligned}
$$


Reflections collected

Independent reflections

Completeness to theta $=25.242^{\circ}$

Refinement method

Data / restraints / parameters

Goodness-of-fit on $\mathrm{F}^{2}$

Final $\mathrm{R}$ indices $[\mathrm{I}>2 \operatorname{sigma}(\mathrm{I})]$

$\mathrm{R}$ indices (all data)

Largest diff. peak and hole
152470

$10862[\mathrm{R}($ int $)=0.0597]$

$99.9 \%$

Full-matrix least-squares on $\mathrm{F}^{2}$

10862 / 619 / 612

1.201

$\mathrm{R} 1=0.0332$,

$\mathrm{wR} 2=0.0546$

$\mathrm{R} 1=0.0420$,

$\mathrm{wR} 2=0.0564$

1.000 and $-1.522 \mathrm{e}^{-3}$

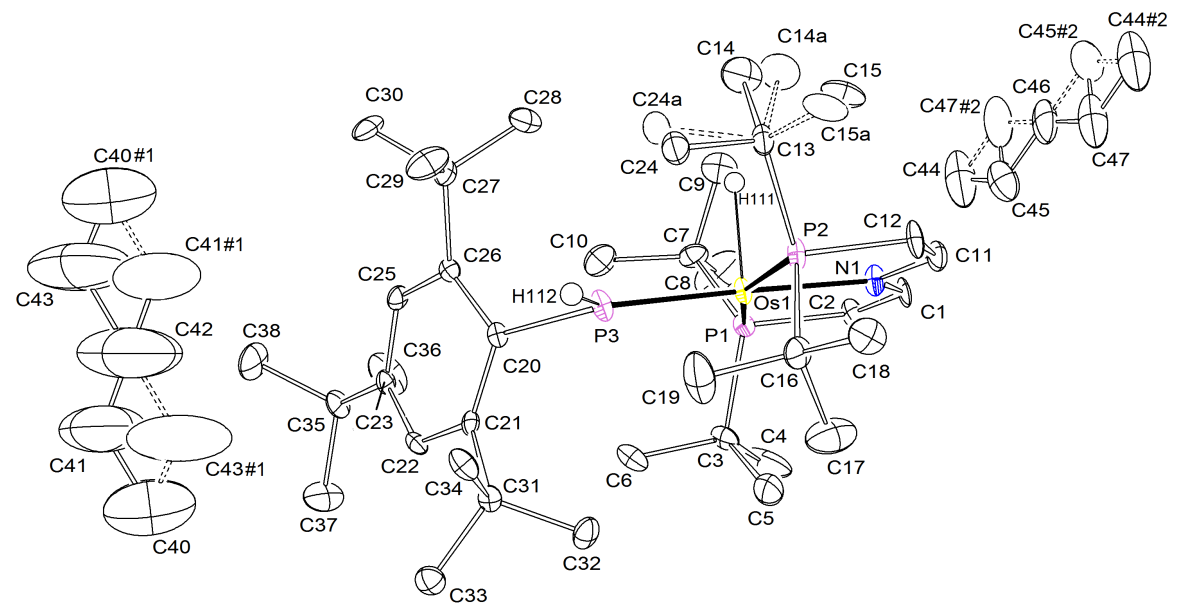

Figure 7.30: Thermal ellipsoid plot of $\mathbf{3 8}$ with the anisotropic displacement parameters drawn at the $50 \%$ probability level. The asymmetric unit contains one disordered complex molecule and two half pentane solvent molecules located at special positions. The disordered complex molecule was refined with population of $0.71(1)$ on the main domain using some restraints (SADI) and constraints (EADP). The Os-H and P-H hydrogen atoms were found from the residual density map and isotropically refined.

Empirical formula

Formula weight

Temperature

Wavelength

Crystal system

Space group

Unit cell dimensions

Volume

Z

Density (calculated)

Absorption coefficient

$\mathrm{F}(000)$

Crystal size
$\mathrm{C}_{43} \mathrm{H}_{83} \mathrm{NOsP}_{3}$

897.21

100(2) K

$0.71073 \AA$

Monoclinic

$\mathrm{P} 2{ }_{1} / \mathrm{n}$

$\mathrm{a}=14.2026(6) \AA$

$\alpha=90^{\circ}$

$\mathrm{b}=15.4095(7) \AA$

$\beta=97.2097(18)^{\circ}$

$\mathrm{c}=21.0809(10) \AA$

$4577.2(4) \AA^{3}$

4

$1.302 \mathrm{Mg} / \mathrm{m}^{3}$

$2.918 \mathrm{~mm}^{-1}$

1876

$0.238 \times 0.211 \times 0.162 \mathrm{~mm}^{3}$ 
Crystal shape and color

Theta range for data collection

Index ranges

Reflections collected

Independent reflections

Completeness to theta $=25.242^{\circ}$

Refinement method

Data / restraints / parameters

Goodness-of-fit on $\mathrm{F}^{2}$

Final R indices [I $>2 \operatorname{sigma}(\mathrm{I})]$

$\mathrm{R}$ indices (all data)

Largest diff. peak and hole
Block, dark green

2.267 to $27.976^{\circ}$

$-18<=\mathrm{h}<=18$

$-20<=\mathrm{k}<=20$

$-27<=\mathrm{l}<=27$

124611

$11010[\mathrm{R}(\mathrm{int})=0.1202]$

$99.9 \%$

Full-matrix least-squares on $\mathrm{F}^{2}$

11010 / 45 / 498

1.053

$\mathrm{R} 1=0.0416, \quad \mathrm{wR} 2=0.0531$

$\mathrm{R} 1=0.1021$,

0.695 and $-0.830 \mathrm{e}^{-3}$
$\mathrm{wR} 2=0.0636$ 


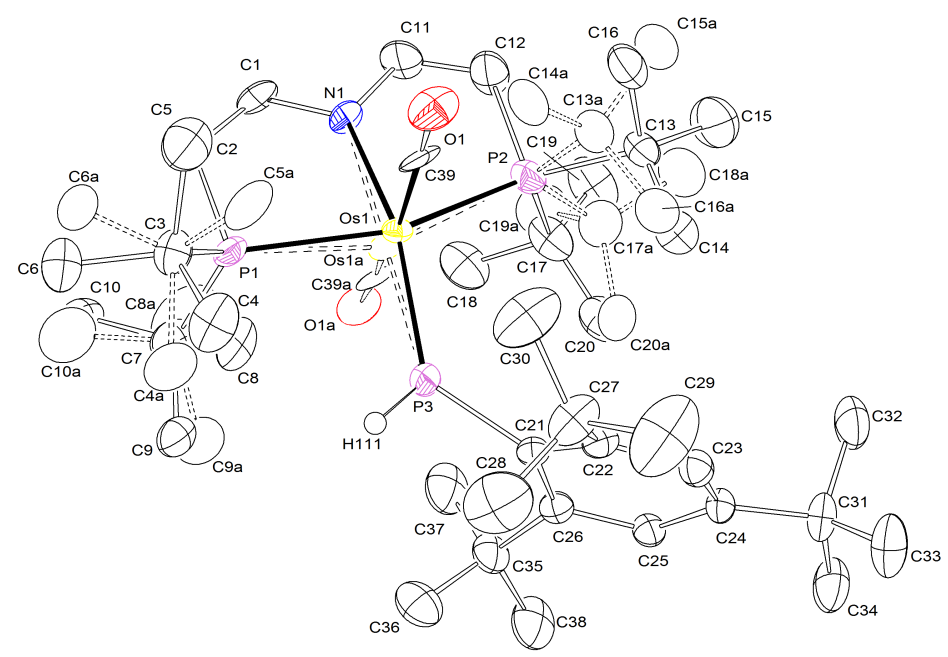

Figure 7.31: Thermal ellipsoid plot of $\mathbf{4 1}$ with the anisotropic displacement parameters drawn at the $50 \%$ probability level. The asymmetric unit contains one disordered complex molecule. The disordered complex molecule was refined with population of 0.682(7) on the main domain using some restraints and constraints (RIGU, SADI, EADP). The P-H hydrogen atom was found from the residual density map and isotropically refined. The structure was refined as an inversion twin using the twin law -100 0-10 00-1 (BASF: 0.061(16)). The reflections -2 2 4, 144,019 and -2110 are removed from the refinement using OMIT commands.

Empirical formula

Formula weight

Temperature

Wavelength

Crystal system

Space group

Unit cell dimensions

Volume

Z

Density (calculated)

Absorption coefficient

$\mathrm{F}(000)$

Crystal size

Crystal shape and color

Theta range for data collection

Index ranges

Reflections collected

Independent reflections

Completeness to theta $=25.242^{\circ}$

Refinement method
$\mathrm{C}_{39} \mathrm{H}_{70} \mathrm{NOOsP}_{3}$

852.07

100(2) K

$0.71073 \AA$

Orthorhombic

$\mathrm{P} 2{ }_{1} 2_{1} 2_{1}$

$\mathrm{a}=20.1894(14) \AA$

$\alpha=90^{\circ}$

$\mathrm{b}=11.4930(8) \AA$

$\beta=90^{\circ}$

$\mathrm{c}=17.4542(14) \AA$

$\gamma=90^{\circ}$

4050.0(5) $\AA^{3}$

4

$1.397 \mathrm{Mg} / \mathrm{m}^{3}$

$3.296 \mathrm{~mm}^{-1}$

1760

$0.265 \times 0.178 \times 0.120 \mathrm{~mm}^{3}$

Block, clear intense red

2.331 to $28.424^{\circ}$

$-26<=\mathrm{h}<=26$

$-15<=\mathrm{k}<=15$

$-23<=\mathrm{l}<=23$

79045

$10098[\mathrm{R}(\mathrm{int})=0.1626]$

$99.9 \%$

Full-matrix least-squares on $\mathrm{F}^{2}$ 
Data / restraints / parameters 10098 / 396 / 559

Goodness-of-fit on $\mathrm{F}^{2} \quad 1.155$

Final R indices $[\mathrm{I}>2 \operatorname{sigma}(\mathrm{I})] \quad \mathrm{R} 1=0.0588, \quad \mathrm{wR} 2=0.1161$

$\mathrm{R}$ indices (all data)

$\mathrm{R} 1=0.0762$

$\mathrm{wR} 2=0.1245$

Absolute structure parameter

$0.061(16)$

Largest diff. peak and hole

1.550 and $-2.048 \mathrm{e}^{-3}$ 


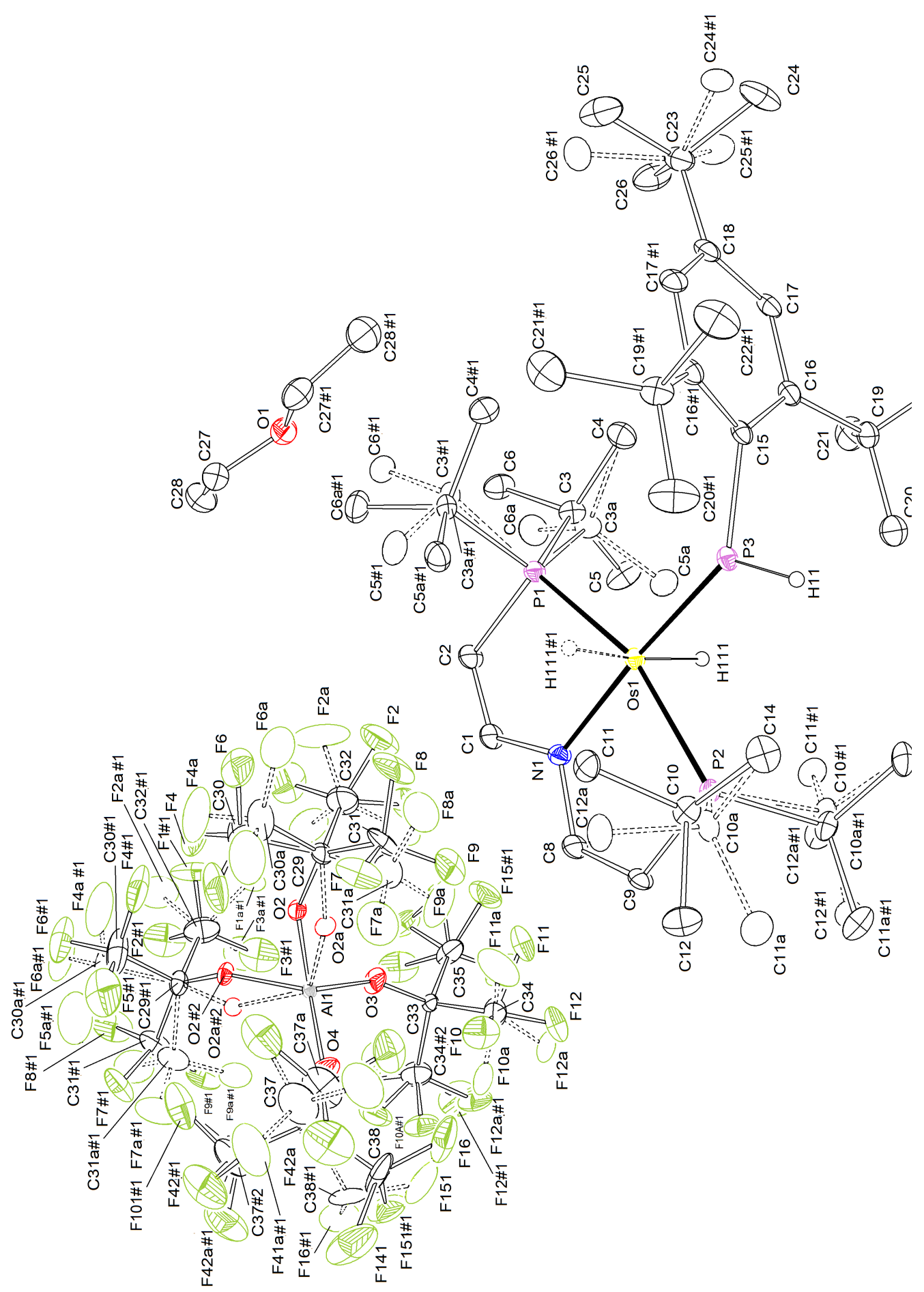

Figure 7.32: Thermal ellipsoid plot of $\mathbf{4 3}$ with the anisotropic displacement parameters drawn at the $50 \%$ probability level. The asymmetric unit contains a half disordered complex molecule, a half disordered $\left[\mathrm{Al}\left\{\mathrm{OC}\left(\mathrm{CF}_{3}\right)_{3}\right\}_{4}\right]^{-}$anion and a half diethyl ether solvent molecule. The disorder was refined with site occupation factors of 0.5 for both sites using PART commands and some restraints and constraints (SADI, RIGU, EADP). The Os-H hydrogen atom was found from the residual density map and isotropically refined using DFIX. 
Empirical formula

Formula weight

Temperature

Wavelength

Crystal system

Space group

Unit cell dimensions

Volume

\section{Z}

Density (calculated)

Absorption coefficient

$\mathrm{F}(000)$

Crystal size

Crystal shape and color

Theta range for data collection

Index ranges

Reflections collected

Independent reflections

Completeness to theta $=25.242^{\circ}$

Refinement method

Data / restraints / parameters

Goodness-of-fit on $\mathrm{F}^{2}$

Final $\mathrm{R}$ indices $[\mathrm{I}>2 \operatorname{sigma}(\mathrm{I})]$

$\mathrm{R}$ indices (all data)

Largest diff. peak and hole
$\mathrm{C}_{58} \mathrm{H}_{81} \mathrm{AlF}_{36} \mathrm{NO}_{5} \mathrm{OsP}_{3}$

1866.32

100(2) K

$0.71073 \AA$

Monoclinic

$\mathrm{P} 22_{1} / \mathrm{m}$

$\mathrm{a}=14.9152(8) \AA$

$\alpha=90^{\circ}$

$\mathrm{b}=15.4377(8) \AA$

$\mathrm{c}=16.7109(9) \AA$

3663.1(3) $\AA^{3}$

2

$1.692 \mathrm{Mg} / \mathrm{m}^{3}$

$1.955 \mathrm{~mm}^{-1}$

1868

$0.513 \times 0.242 \times 0.128 \mathrm{~mm}^{3}$

Plate, clear intense green-blue

2.195 to $28.443^{\circ}$

$-19<=\mathrm{h}<=19$

$-20<=\mathrm{k}<=20$

$-22<=\mathrm{l}<=22$

99819

$9531[\mathrm{R}(\mathrm{int})=0.0648]$

$99.9 \%$

Full-matrix least-squares on $\mathrm{F}^{2}$

9531 / $301 / 741$

1.086

$\mathrm{R} 1=0.0508, \quad \mathrm{wR} 2=0.1312$

$\mathrm{R} 1=0.0573$,

$\mathrm{wR} 2=0.1378$ 


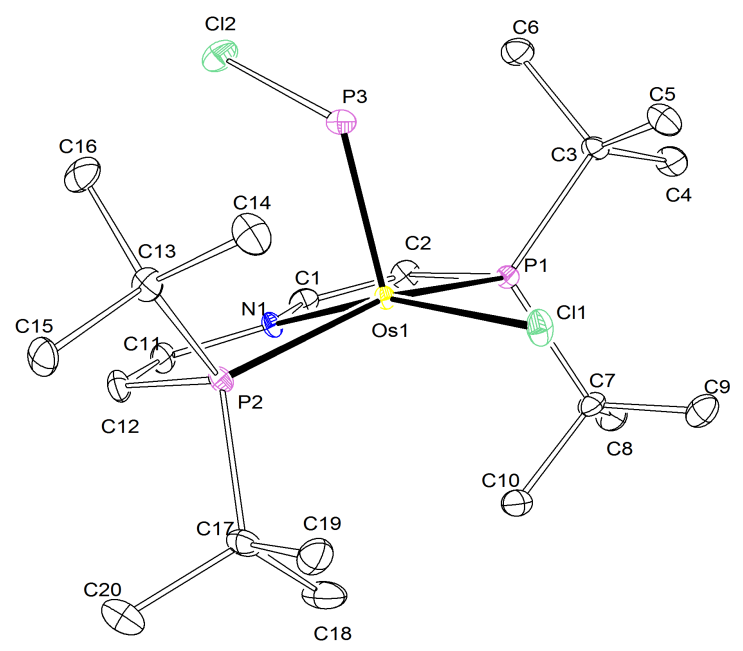

Figure 7.33: Thermal ellipsoid plot of $\mathbf{4 4}$ with the anisotropic displacement parameters drawn at the $50 \%$ probability level. The asymmetric unit contains one complex molecule.

Empirical formula

Formula weight

Temperature

Wavelength

Crystal system

Space group

Unit cell dimensions

Volume

Z

Density (calculated)

Absorption coefficient

$\mathrm{F}(000)$

Crystal size

Crystal shape and color

Theta range for data collection

Index ranges

Reflections collected

Independent reflections

Completeness to theta $=25.242^{\circ}$

Refinement method

Data / restraints / parameters

Goodness-of-fit on $\mathrm{F}^{2}$

Final $R$ indices [I $>2 \operatorname{sigma}(\mathrm{I})]$

$\mathrm{R}$ indices (all data)

Largest diff. peak and hole

$$
\mathrm{C}_{20} \mathrm{H}_{40} \mathrm{Cl}_{2} \mathrm{NOsP}_{3}
$$

$$
648.54
$$

293(2) K

$0.71073 \AA$

Monoclinic

$\mathrm{P} 2{ }_{1} / \mathrm{n}$

$\mathrm{a}=12.2910(7) \AA$

$\alpha=90^{\circ}$

$\mathrm{b}=14.2611(7) \AA$

$\mathrm{c}=15.1564(8) \AA$

2529.8(2) $\AA^{3}$

4

$1.703 \mathrm{Mg} / \mathrm{m}^{3}$

$5.449 \mathrm{~mm}^{-1}$

1288

$0.541 \times 0.370 \times 0.215 \mathrm{~mm}^{3}$

Block, clear intense purple

2.251 to $28.362^{\circ}$.

$-16<=\mathrm{h}<=16$

$-18<=\mathrm{k}<=19$

$-20<=\mathrm{l}<=20$

99312

$6305[\mathrm{R}(\mathrm{int})=0.1252]$

$100.0 \%$

Full-matrix least-squares on $\mathrm{F}^{2}$

6305 / 0 / 256

1.019

$\mathrm{R} 1=0.0268$,

$\mathrm{wR} 2=0.0458$

$\mathrm{R} 1=0.0454$,

$\mathrm{wR} 2=0.0504$ $\beta=107.780(2)^{\circ}$

$\gamma=90^{\circ}$

1.081 and $-1.328 \mathrm{e}^{-3}$ 


\subsubsection{Isothermal Titration Calorimetry}

The bond dissociation energy (BDE) of $\mathbf{3 8}$ was determined by ITC using a TA INSTRUMENTS NanoITC equipped with a $24 \mathrm{~K}$ gold cell ( $1 \mathrm{~mL}$ sampe volume) and a $250 \mu \mathrm{L}$ titration syringe operated in overfill mode. The instrument was run under an indert atmosphere inside an argon filled glove box. The syringe concentration was chosen to be 6.4 times higher than the cell concentration to obtain a titration range from 0 - 2 eq.. Mes*O was used as a titrant. Both reactant solutions were prepared with $\mathrm{Na} / \mathrm{K}$ dried benzene with a sample mass of more than $10.0 \mathrm{mg}$ for sufficient accuracy. Titrations were carried out at $10^{\circ} \mathrm{C}$ to suppress decay of $\mathbf{3 9}$ to $\mathbf{4 0}$ and corrected vs. addition of Mes* ${ }^{*}$ to pure benzene in the sampe cell. Further experimental conditions are detailed in Table 7.29. The data were evaluated using the implemented NanoAnalyze software. ${ }^{6}$

Table 7.29: Experimental details of ITC in benzene.

\begin{tabular}{l|cc}
\hline Concentration / volume of 38 & $1.02 \mathrm{mM}$ & $950 \mu \mathrm{L}$ \\
Concentration / volume of Mes*O & $9.18 \mathrm{mM}$ & $250 \mu \mathrm{L}$ \\
Injection Steps / Injected Volume & 33 & $7.5 \mu \mathrm{L}$ \\
Waiting time & $350-650 \mathrm{~s}$ & \\
Stirring rate & $350 \mathrm{rpm}$ & \\
Equilibration time before start of the experiment & $1514 \mathrm{~s}$ & \\
\hline
\end{tabular}
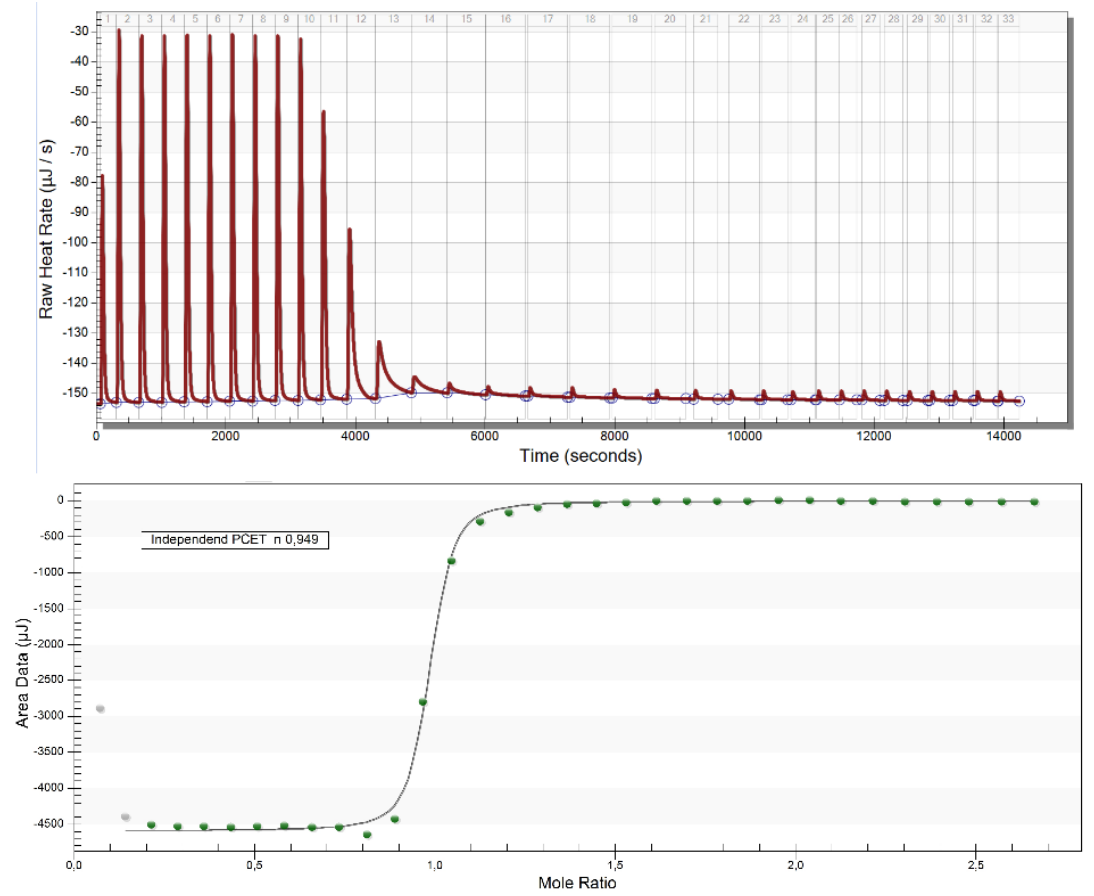

Figure 7.34: Top: Representative thermogram of calorimetric titration of $\mathbf{3 8}$ with $\mathrm{Mes}^{*} \mathrm{O}$ in benzene; Bottom: Integrated titration curve.

${ }^{6}$ a) Measurment by ITCRun Version 3.4.6.0, TA Instruments, 2017; b) Evaluation by NanoAnalyze Version 3.7.5 TA Instruments, 2015. 
A representative thermogram is shown in Figure 7.34. The first two peaks of each titration were generally neglected due to diffusion of reactant solution into the cell during equilibration of the instrument. Around the addition of one equivalent of $\mathrm{Mes}^{*} \mathrm{O}$, the peaks show increased tailing, which is tentatively attributed to follow-up reactivity of 39. The $\mathrm{P}-\mathrm{H} \mathrm{BDE}$ was therefore determined at an early titration stage from the average of injections 3-6 (Tables 7.30, 7.31) while reliable BDFE determination was not possible. A cumulative error of $5 \%$ for stock solution concentrations was estimated from the fitted $\mathrm{n}$-value $(0.95)$ which gives rise to an error in $\Delta \mathrm{H}$ of $\pm 0.8 \mathrm{kcal} \cdot \mathrm{mol}^{-1}$.

Table 7.30: Integrated heats of the injections 3-6 for the BDE determination of $\mathbf{3 8}$ in benzene.

\begin{tabular}{c|ccc}
\hline Injection & Heats $/ \mu \mathrm{J} 1^{\text {st }}$ run & Heats $/ \mu \mathrm{J} 2^{\text {nd }}$ run & Added moles $/ \mathrm{nmol}$ \\
\hline 3 & 4682 & 4678 & 68.2 \\
4 & 4708 & 4678 & 68.2 \\
5 & 4703 & 4711 & 68.2 \\
6 & 4716 & 4711 & 68.2 \\
Mean & 4702 & 4694 & \\
\hline
\end{tabular}

Table 7.31: Results of the calorimetric BDE determination of 38 in benzene.

\begin{tabular}{|c|c|c|}
\hline & $\Delta \mathrm{H} / \mathrm{kJ} \cdot \mathrm{mol}^{-1}$ & $\Delta \mathrm{H} / \mathrm{kcal} \cdot \mathrm{mol}^{-1}$ \\
\hline Mean $1^{\text {st }}$ run & -68.9 & -16.5 \\
\hline Mean $2^{\text {nd }}$ run & -68.8 & -16.4 \\
\hline Mean & -68.9 & -16.5 \\
\hline $\mathrm{Mes}^{*} \mathrm{O}$ & & 81.6 \\
\hline $\mathrm{BDE}(\mathrm{P}-\mathrm{H})$ of $\mathbf{3 8}$ & & $65.1 \pm 0.8$ \\
\hline
\end{tabular}

The BDE measurement was also performed in THF to allow to construct a thermochemical square-scheme of $\mathbf{3 8}$ ( Table 7.32).

Table 7.32: Experimental details of ITC in THF.

\begin{tabular}{l|cc}
\hline Concentration / volume of $\mathbf{3 8}$ & $1.40 \mathrm{mM}$ & $950 \mu \mathrm{L}$ \\
Concentration / volume of Mes*O & $11.17 \mathrm{mM}$ & $250 \mu \mathrm{L}$ \\
Injection Steps / Injected Volume & 49 & $5 \mu \mathrm{L}$ \\
Waiting time & $300 \mathrm{~s}$ & \\
Stirring rate & $350 \mathrm{rpm}$ & \\
Equilibration time before start of the experiment & $1671 \mathrm{~s}$ & \\
\hline
\end{tabular}

The $\mathrm{P}-\mathrm{H} \mathrm{BDE}$ of $\mathbf{3 8}$ was calculated from the mean heat evolved at the peaks 2-5 (Figure 7.35). The $\mathrm{O}-\mathrm{H}$ BDE of Mes* $\mathrm{OH}$ in THF was determined by a square-scheme using the oxidation potential of the $\mathrm{Mes}^{*} \mathrm{O} / \mathrm{Mes}^{*} \mathrm{O}^{-}$redox couple $\left(E^{\circ}=-0.955 \mathrm{~V}\right.$ vs. $\mathrm{Fc}^{0 /+}$, Figure 7.35). The $\mathrm{p} K_{\mathrm{a}}$ of $\mathrm{Mes}^{*} \mathrm{OH}$ was calculated from the reported value in DMSO according to Ding $\left(\mathrm{p} K_{\mathrm{a}}^{\mathrm{THF}}\left(\mathrm{Mes}^{*} \mathrm{OH}\right)=1.03 \cdot 17.8+11.3=29.6\right)$. This results in a BDE of $84.5 \mathrm{kcal} \cdot \mathrm{mol}^{-1}$. 

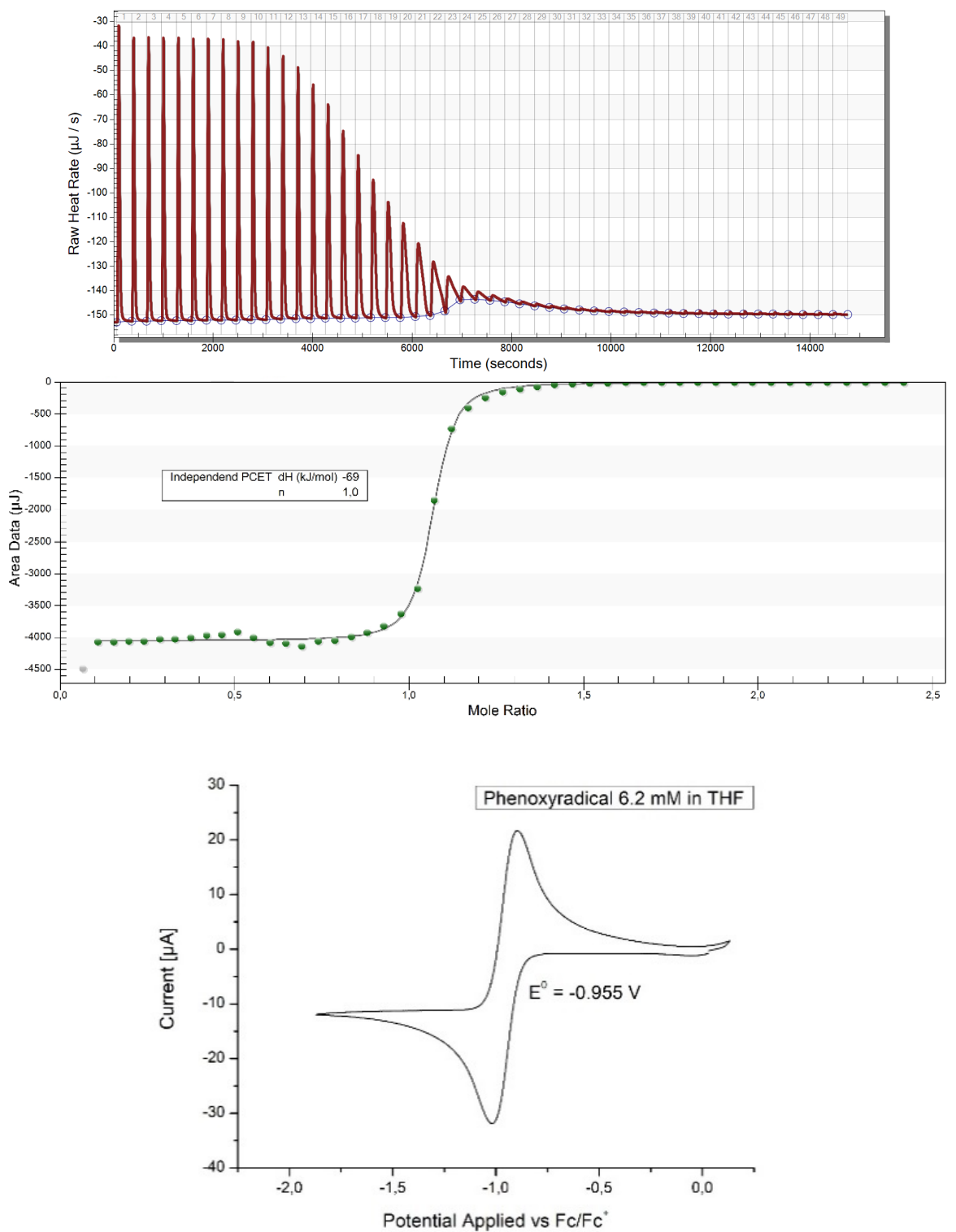

Figure 7.35: Top: Thermogram of calorimetric titration of $\mathbf{3 8}$ with Mes*O in THF; Middle: Integrated titration curve; Bottom: Cyclic voltammogram of Mes* $\mathrm{O}, 100 \mathrm{Vm} \cdot \mathrm{s}^{-1}, 0.1 \mathrm{M} \mathrm{NBu}_{4} \mathrm{PF}_{6}$, THF, RT.

Table 7.33: Integrated heats of the injections 2-5 for the BDE determination of 38 in THF.

\begin{tabular}{c|cc}
\hline Injection & Heats $/ \mu \mathrm{J} 1^{\text {st }}$ run & Added moles / nmol \\
\hline 2 & 4069 & 56.9 \\
3 & 4066 & 56.9 \\
4 & 4050 & 56.9 \\
5 & 4049 & 56.9 \\
Mean & 4059 & \\
\hline
\end{tabular}


Table 7.34: Results of the calorimetric BDE determination of 38 in THF.

\begin{tabular}{c|cc}
\hline & $\Delta H / \mathrm{kJ} \cdot \mathrm{mol}^{-1}$ & $\Delta H / \mathrm{kcal} \cdot \mathrm{mol}^{-1}$ \\
\hline Mean & -71 & -17 \\
Mes*O & & 84.5 \\
BDE $(\mathrm{P}-\mathrm{H})$ of $\mathbf{3 8}$ & & $67 \pm 1$ \\
\hline
\end{tabular}

\subsubsection{Syntheses}

\section{Reaction of LXXIII with $\left[\mathrm{Na}(\operatorname{diox})_{1.39}\right][\mathrm{PCO}]$}

LXXIII (5.0 mg, $8.1 \mu \mathrm{mol}, 1.0$ eq.) and [Na(diox $\left.)_{1.39}\right][\mathrm{PCO}](1.6 \mathrm{mg}, 8.1 \mu \mathrm{mol}, 1.0$ eq.) are dissolved in $\mathrm{C}_{6} \mathrm{D}_{6}(0.5 \mathrm{~mL})$ and stirred for $1 \mathrm{~h}$ at room temperature. Longer reaction times lead to no further consumption of the starting material and precipitation of a black solid.

NMR $\left(\mathrm{C}_{6} \mathrm{D}_{6}, \mathrm{RT}\right):{ }^{31} \mathrm{P}\left\{{ }^{1} \mathrm{H}\right\}(162.1 \mathrm{MHz})=1063.6$ (br, 1P, Re $\left.\equiv \mathrm{P}\right), 87.2$ (br, 2P,

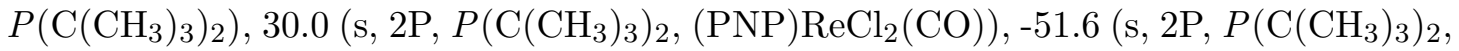
1a).

\section{Synthesis of 24}

LXXIII (123 mg, $198 \mu \mathrm{mol}, 1.00$ eq.) is dissolved in benzene and degassed via two freezepump-thaw cycles. CO (1.2 bar) is applied and the solution stirred for 1 minute. The green solution is degassed via two freeze-pump-thaw cycles and lyophilized overnight. The residue is washed with pentane $(3 \times 5 \mathrm{~mL})$ and $\mathrm{Et}_{2} \mathrm{O}(3 \times 5 \mathrm{~mL})$ and extracted with benzene. Lyophilization yields $\mathbf{2 4}(104 \mathrm{mg}, 161 \mu \mathrm{mol}, 81 \%)$ as a green powder.

Anal. Calcd for $\mathrm{C}_{21} \mathrm{H}_{44} \mathrm{~N}_{1} \mathrm{I}_{2} \mathrm{OP}_{2} \mathrm{Re}_{1}$ (645.6): C, 39.1; H, 6.87; N, 2.17. Found: C, 39.4; $\mathrm{H}, 6.27 ; \mathrm{N}, 2.22$. NMR $\left(\mathrm{CD}_{2} \mathrm{Cl}_{2}, \mathrm{RT}\right):{ }^{1} \mathrm{H}(300 \mathrm{MHz}): \delta=4.11-3.85\left(\mathrm{~m}, 4 \mathrm{H}, \mathrm{NCH}_{2}\right)$, $2.74-2.60\left(\mathrm{~m}, 2 \mathrm{H}, \mathrm{PCH} H_{2}\right), 2.34-2.30\left(\mathrm{~m}, 2 \mathrm{H}, \mathrm{PC} H_{2}\right), 1.43\left(\mathrm{~A}_{9} \mathrm{XX}^{\prime} \mathrm{A}_{9}{ }^{\prime}, N={ }^{3} J_{\mathrm{AX}}\right.$ $\left.+{ }^{5} J_{\mathrm{AX}} \mid=12.7 \mathrm{~Hz}, \mathrm{P}\left(\mathrm{C}\left(\mathrm{CH}_{3}\right)_{3}\right)_{2}\right), 1.31\left(\mathrm{~A}_{9} \mathrm{XX}^{\prime} \mathrm{A}_{9}{ }^{\prime}, N=\left|{ }^{3} J_{\mathrm{AX}}+{ }^{5} J_{\mathrm{AX}}\right|=12.9 \mathrm{~Hz}\right.$, $\left.\mathrm{P}\left(\mathrm{C}(\mathrm{CH})_{3}\right)_{2}\right) .{ }^{13} \mathrm{C}\left\{{ }^{1} \mathrm{H}\right\}(75.5 \mathrm{MHz}) \delta=195.8\left(\mathrm{t},{ }^{3} J_{\mathrm{PC}}=7.6 \mathrm{~Hz}, 1 \mathrm{C}, C \mathrm{O}\right), 93.4$ (vt, $\left.N=\left|{ }^{2} J_{\mathrm{PC}}+{ }^{3} J_{\mathrm{PC}}\right|=3.0 \mathrm{~Hz}, 2 \mathrm{C}, \mathrm{NCH}_{2}\right), 42.2\left(\mathrm{vt}, N=\left|{ }^{1} J_{\mathrm{CP}}+{ }^{3} J_{\mathrm{CP}}\right|=7.7 \mathrm{~Hz}, 2 \mathrm{C}\right.$, $\left.\mathrm{P}\left(C\left(\mathrm{CH}_{3}\right)_{3}\right)_{2}\right), 41.2\left(\right.$ vt, $\left.N=\left|{ }^{1} J_{\mathrm{CP}}+{ }^{3} J_{\mathrm{CP}}\right|=8.5 \mathrm{~Hz}, 2 \mathrm{C}, \mathrm{P}\left(C\left(\mathrm{CH}_{3}\right)_{3}\right)_{2}\right), 31.6$ (vt, $\left.N=\left|{ }^{1} J_{\mathrm{PC}}+{ }^{3} J_{\mathrm{PC}}\right|=9.3 \mathrm{~Hz}, 2 \mathrm{C}, \mathrm{PC} \mathrm{H}_{2}\right), 30.9\left(\mathrm{vt}, N=\left|{ }^{2} J_{\mathrm{CP}}+{ }^{4} J_{\mathrm{CP}}\right|=1.5 \mathrm{~Hz}\right.$, 6C, $\left.\mathrm{P}\left(\mathrm{C}\left(\mathrm{CH}_{3}\right)_{3}\right)_{2}\right), 30.4\left(\mathrm{vt}, N=\left|{ }^{2} J_{\mathrm{CP}}+{ }^{4} J_{\mathrm{CP}}\right|=2.0 \mathrm{~Hz}, 6 \mathrm{C}, \mathrm{P}\left(\mathrm{C}\left(\mathrm{CH}_{3}\right)_{3}\right)_{2}\right) \cdot{ }^{31} \mathrm{P}\left\{{ }^{1} \mathrm{H}\right\}$ $(121.5 \mathrm{MHz}) \delta=31.5\left(\mathrm{~s}, 2 \mathrm{P}, \mathrm{P}\left(\mathrm{C}\left(\mathrm{CH}_{3}\right)_{3}\right)_{2}\right)$. LIFDI-MS (THF): $645.1\left(100 \%,[\mathrm{M}]^{+}\right)$.

\section{Reaction of 5 with $\left[\mathrm{Na}(\operatorname{diox})_{1.39}\right][\mathrm{PCO}]$}

5 (5.0 mg, $8.1 \mu \mathrm{mol}, 1.0$ eq.) and $\left[\mathrm{Na}(\text { diox })_{1.39}\right][\mathrm{PCO}](1.6 \mathrm{mg}, 8.1 \mu \mathrm{mol}, 1.0$ eq. $)$ are dissolved in THF- $\mathrm{d}_{8}(0.5 \mathrm{~mL})$ and stirred for $1 \mathrm{~h}$ at room temperature.

NMR (THF, RT): ${ }^{31} \mathrm{P}\left\{{ }^{1} \mathrm{H}\right\}(162.1 \mathrm{MHz})=1092.4$ (br, 1P, Re $\left.\equiv \mathrm{P}\right), 84.7$ (br, 2P, $\left.\mathrm{P}\left(\mathrm{C}\left(\mathrm{CH}_{3}\right)_{3}\right)_{2}\right)$.

\section{Synthesis of 25}

11 (25.0 mg, $30.6 \mu \mathrm{mol}, 1.00$ eq.) and [ $\left.\mathrm{NBu}_{4}\right][\mathrm{NCO}](49.5 \mathrm{mg}, 153 \mu \mathrm{mol}, 5.00$ eq.) are dissolved in THF $(10 \mathrm{~mL})$ and stirred for $90 \mathrm{~min}$ at room temperature. After evaporation 
of the solvent the residue is extracted with benzene $(3 \times 5 \mathrm{~mL})$. After removal of the solvent the residue is extracted with pentane $(15 \times 5 \mathrm{~mL})$. The volume of the pentane solution is reduced until product starts to precipitate (ca. $5 \mathrm{~mL}$ ). The solution is stored at $-35^{\circ} \mathrm{C}$ for 2 days. After filtration the precipitate is washed with cold pentane $(2 \mathrm{~mL})$. Lyophilization yields 25 in form of a brown powder (18.0 mg, $24.6 \mu \mathrm{mol}, 80 \%$ ).

Anal. Calcd for $\mathrm{C}_{29} \mathrm{H}_{50} \mathrm{~N}_{5} \mathrm{OP}_{2} \mathrm{Re}(732.9)$ : C, 47.5; H, 6.88; N 9.56. Found: C, 48.0; H, 6.90; N, 9.40. NMR $\left(\mathrm{C}_{6} \mathrm{D}_{6}, \mathrm{RT}\right):{ }^{1} \mathrm{H}(500 \mathrm{MHz}): \delta=9.82\left(\mathrm{ddd},{ }^{3} J_{\mathrm{HH}}=6.1 \mathrm{~Hz},{ }^{4} J_{\mathrm{HH}}=\right.$ $\left.1.4 \mathrm{~Hz},{ }^{5} J_{\mathrm{HH}}=0.9 \mathrm{~Hz}, 1 \mathrm{H}, \mathrm{C}_{8} H\right), 7.68\left(\mathrm{~d},{ }^{3} J_{\mathrm{HH}}=2.1 \mathrm{~Hz}, 1 \mathrm{H}, \mathrm{C}_{1} H\right), 7.27\left(\mathrm{ddd},{ }^{3} J_{\mathrm{HH}}\right.$ $\left.=8.0 \mathrm{~Hz},{ }^{4} J_{\mathrm{HH}}=1.5 \mathrm{~Hz},{ }^{5} J_{\mathrm{HH}}=0.9 \mathrm{~Hz}, 1 \mathrm{H}, \mathrm{C}_{5} H\right), 6.56\left(\mathrm{ddd},{ }^{3} J_{\mathrm{HH}}=8.0 \mathrm{~Hz},{ }^{3} J_{\mathrm{HH}}=\right.$ $\left.7.2 \mathrm{~Hz},{ }^{5} J_{\mathrm{HH}}=1.5 \mathrm{~Hz}, 1 \mathrm{H}, \mathrm{C}_{6} H\right), 6.34\left(\mathrm{~d},{ }^{3} J_{\mathrm{HH}}=2.1 \mathrm{~Hz}, 1 \mathrm{H}, \mathrm{C}_{2} H\right), 6.32\left(\mathrm{ddd},{ }^{3} J_{\mathrm{HH}}\right.$ $\left.=7.2 \mathrm{~Hz},{ }^{3} J_{\mathrm{HH}}=6.1 \mathrm{~Hz},{ }^{4} J_{\mathrm{HH}}=1.5 \mathrm{~Hz}, 1 \mathrm{H}, \mathrm{C}_{7} H\right), 4.39-4.25\left(\mathrm{~m}, 2 \mathrm{H}, \mathrm{NCH}_{2}\right), 3.46$ - $3.37\left(\mathrm{~m}, 2 \mathrm{H}, \mathrm{NCH}_{2}\right), 2.07-1.96\left(\mathrm{~m}, 2 \mathrm{H}, \mathrm{PCH}_{2}\right), 1.81-1.71\left(\mathrm{~m}, 2 \mathrm{H}, \mathrm{PCH}_{2}\right), 1.28$ $\left(\mathrm{A}_{9} \mathrm{XX}^{\prime} \mathrm{A}_{9}{ }^{\prime}, N=\left|{ }^{3} J_{\mathrm{AX}}+{ }^{5} J_{\mathrm{AX}}{ }^{\prime}\right|=11.4 \mathrm{~Hz}, 18 \mathrm{H}, \mathrm{P}\left(\mathrm{C}\left(\mathrm{CH}_{3}\right)_{3}\right)_{2}\right), 0.39\left(\mathrm{~A}_{9} \mathrm{XX}^{\prime} \mathrm{A}_{9}{ }^{\prime}, N=\right.$ $\left.\left|{ }^{3} J_{\mathrm{AX}}+{ }^{5} J_{\mathrm{AX}}\right|=12.1 \mathrm{~Hz}, 18 \mathrm{H}, \mathrm{P}\left(\mathrm{C}\left(\mathrm{CH}_{3}\right)_{3}\right)_{2}\right) \cdot{ }^{13} \mathrm{C}\left\{{ }^{1} \mathrm{H}\right\}(125.8 \mathrm{MHz}): \delta=159.6(\mathrm{br}$, $1 \mathrm{C}, \mathrm{NCO}), 150.9\left(\mathrm{~s}, 1 \mathrm{C}, C_{4}\right), 147.9\left(\mathrm{~s}, 1 \mathrm{C}, C_{8}\right), 145.4\left(\mathrm{~s}, 1 \mathrm{C}, C_{3}\right), 142.8\left(\mathrm{~s}, 1 \mathrm{C}, C_{1}\right)$, $135.2\left(\mathrm{~s}, 1 \mathrm{C}, C_{6}\right), 117.4\left(\mathrm{~s}, 1 \mathrm{C}, C_{5}\right), 117.4\left(\mathrm{~s}, 1 \mathrm{C}, C_{7}\right), 104.3\left(\mathrm{~s}, 1 \mathrm{C}, C_{2}\right), 83.6(\mathrm{vt}, N$ $\left.=\left|{ }^{2} J_{\mathrm{PC}}+{ }^{3} J_{\mathrm{PC}}\right|=4.3 \mathrm{~Hz}, 2 \mathrm{C}, \mathrm{NCH}_{2}\right), 41.8\left(\mathrm{vt}, N=\left|{ }^{1} J_{\mathrm{CP}}+{ }^{3} J_{\mathrm{CP}}\right|=8.0 \mathrm{~Hz}, 2 \mathrm{C}\right.$, $\left.\mathrm{P}\left(C\left(\mathrm{CH}_{3}\right)_{3}\right)_{2}\right), 38.3\left(\mathrm{vt}, N=\left|{ }^{1} J_{\mathrm{CP}}+{ }^{3} J_{\mathrm{CP}}\right|=7.0 \mathrm{~Hz}, 2 \mathrm{C}, \mathrm{P}\left(C\left(\mathrm{CH}_{3}\right)_{3}\right)_{2}\right), 30.9$ (vt, $N$ $\left.=\left|{ }^{2} J_{\mathrm{CP}}+{ }^{4} J_{\mathrm{CP}}\right|=1.8 \mathrm{~Hz}, 6 \mathrm{C}, \mathrm{P}\left(\mathrm{C}\left(\mathrm{CH}_{3}\right)_{3}\right)_{2}\right), 29.3\left(\mathrm{vt}, N=\left|{ }^{1} J_{\mathrm{PC}}+{ }^{3} J_{\mathrm{PC}}\right|=2.5 \mathrm{~Hz}\right.$, 2C, $\left.\mathrm{PC} \mathrm{H}_{2}\right), 28.7$ (vt, $\left.N=\left|{ }^{2} J_{\mathrm{CP}}+{ }^{4} J_{\mathrm{CP}}\right|=7.3 \mathrm{~Hz}, 6 \mathrm{C}, \mathrm{P}\left(\mathrm{C}\left(\mathrm{CH}_{3}\right)_{3}\right)_{2}\right) . .{ }^{31} \mathrm{P}\left\{{ }^{1} \mathrm{H}\right\}(202.5$ $\mathrm{MHz}) \delta=8.0\left(\mathrm{~s}, 2 \mathrm{P}, P\left(\mathrm{C}\left(\mathrm{CH}_{3}\right)_{3}\right)_{2}\right)$. LIFDI-MS (Toluene): $733.2\left(100 \%,[\mathrm{M}]^{+}\right)$. IR $\left(\mathrm{ATR}, \mathrm{cm}^{-1}\right): \tilde{\nu}=2234(\mathrm{NCO})$.

\section{Irradiation and heating of 25}

Heating: $4 \mathbf{a}\left(5.0 \mathrm{mg}, 6.8 \mu \mathrm{mol}, 1.0\right.$ eq.) are dissolved in $\mathrm{C}_{6} \mathrm{D}_{6}(0.5 \mathrm{~mL})$ in a Y-Joung NMR tube and heated to $80^{\circ} \mathrm{C}$ for 4 hours. The ${ }^{31} \mathrm{P}\left\{{ }^{1} \mathrm{H}\right\}$ and ${ }^{1} \mathrm{H}$ NMR spectra indicated no conversion of the starting material.

Irradiation: $4 \mathbf{a}(5 \mathrm{mg}, 6.82 \mu \mathrm{mol}, 1.0$ eq. $)$ are dissolved in $\mathrm{C}_{6} \mathrm{D}_{6}(0.5 \mathrm{~mL})$ in a Y-Joung NMR tube and irradiated at room temperature overnight. The ${ }^{31} \mathrm{P}\left\{{ }^{1} \mathrm{H}\right\}$ and ${ }^{1} \mathrm{H}$ NMR spectra indicated no conversion of the starting material.

\section{Synthesis of 27}

11 (58.0 mg, $70.9 \mu \mathrm{mol}, 1.00$ eq.) and [Na(diox) 1.39$][\mathrm{PCO}](15.3 \mathrm{mg}, 74.5 \mu \mathrm{mol}, 1.05$ eq.) are dissolved in THF at $-80^{\circ} \mathrm{C}$. The solution is warmed to $-10^{\circ} \mathrm{C}$ and stirred for $1 \mathrm{~h}$. After warming to room temperature the solvent is evaporated. The residue is washed with pentane $(4 \times 1 \mathrm{~mL})$ and extracted with benzene $(3 \times 1 \mathrm{~mL})$. Lyophilization yields $\mathbf{2 7}$ in form of a brown powder $(32.5 \mathrm{mg}, 42.0 \mu \mathrm{mol}, 63 \%)$.

Anal. Calcd for $\mathrm{C}_{28} \mathrm{H}_{50} \mathrm{IN}_{4} \mathrm{P}_{2} \mathrm{Re}$ (817.8): C, 46.6; H, 6.98; N 7.76. Found: C, 46.6; H, 6.88; N, 7.55. NMR $\left(\right.$ THF-d $\left.8,0{ }^{\circ} \mathrm{C}\right):{ }^{1} \mathrm{H}(500 \mathrm{MHz}): \delta=8.37\left(\mathrm{ddd},{ }^{3} J_{\mathrm{HH}}=4.8 \mathrm{~Hz}\right.$, $\left.{ }^{4} J_{\mathrm{HH}}=1.8 \mathrm{~Hz},{ }^{5} J_{\mathrm{HH}}=0.9 \mathrm{~Hz}, 1 \mathrm{H}, \mathrm{C}_{8} H\right), 8.31\left(\mathrm{~d},{ }^{3} J_{\mathrm{HH}}=1.8 \mathrm{~Hz}, 1 \mathrm{H}, \mathrm{C}_{1} H\right), 7.60$ $\left(\mathrm{m}, 1 \mathrm{H}, \mathrm{C}_{5} H\right), 7.42\left(\mathrm{ddd},{ }^{3} J_{\mathrm{HH}}=8.0 \mathrm{~Hz},{ }^{3} J_{\mathrm{HH}}=7.3 \mathrm{~Hz},{ }^{4} J_{\mathrm{HH}}=1.8 \mathrm{~Hz}, 1 \mathrm{H}, \mathrm{C}_{6} H\right)$, $6.83\left(\mathrm{~m}, 1 \mathrm{H}, \mathrm{C}_{2} H\right), 6.81\left(\mathrm{~m}, 1 \mathrm{H}, \mathrm{C}_{7} H\right), 3.63-3.55\left(\mathrm{~m}, 2 \mathrm{H}, \mathrm{NCH}_{2}\right.$, superimposed with solvent), $3.40-3.28\left(\mathrm{~m}, 2 \mathrm{H}, \mathrm{NCH}_{2}\right), 2.51-2.43\left(\mathrm{~m}, 2 \mathrm{H}, \mathrm{PCH}_{2}\right), 1.94-1.86(\mathrm{~m}, 2 \mathrm{H}$, $\left.\mathrm{PCH}_{2}\right), 1.42\left(\mathrm{~A}_{9} \mathrm{XX}^{\prime} \mathrm{A}_{9}{ }^{\prime}, N=\left|{ }^{3} J_{\mathrm{AX}}+{ }^{5} J_{\mathrm{AX}}\right|=13.0 \mathrm{~Hz}, 18 \mathrm{H}, \mathrm{P}\left(\mathrm{C}\left(\mathrm{CH}_{3}\right)_{3}\right)_{2}\right), 1.10$ $\left(\mathrm{A}_{9} \mathrm{XX}^{\prime} \mathrm{A}_{9}{ }^{\prime}, N=\left|{ }^{3} J_{\mathrm{AX}}+{ }^{5} J_{\mathrm{AX}}{ }^{\prime}\right|=13.0 \mathrm{~Hz}, 18 \mathrm{H}, \mathrm{P}\left(\mathrm{C}\left(\mathrm{CH}_{3}\right)_{3}\right)_{2}\right) .{ }^{13} \mathrm{C}\left\{{ }^{1} \mathrm{H}\right\}(125.8 \mathrm{MHz})$ : 
$\delta=155.7\left(\mathrm{~s}, 1 \mathrm{C}, C_{4}\right), 152.2\left(\mathrm{~s}, 1 \mathrm{C}, C_{3}\right), 150.5\left(\mathrm{~s}, 1 \mathrm{C}, C_{1} \mathrm{H}\right), 149.6\left(\mathrm{~s}, 1 \mathrm{C}, C_{8} \mathrm{H}\right), 135.6$ $\left(\mathrm{s}, 1 \mathrm{C}, C_{6} \mathrm{H}\right), 120.2\left(\mathrm{~s}, 1 \mathrm{C}, C_{7} \mathrm{H}\right), 119.3\left(\mathrm{~s}, 1 \mathrm{C}, C_{5} \mathrm{H}\right), 105.8\left(\mathrm{~s}, 1 \mathrm{C}, C_{2} \mathrm{H}\right), 75.0(\mathrm{vt}$, $\left.N=\left|{ }^{2} J_{\mathrm{PC}}+{ }^{3} J_{\mathrm{PC}}\right|=3.5 \mathrm{~Hz}, 2 \mathrm{C}, \mathrm{N} C \mathrm{H}_{2}\right), 38.4\left(\mathrm{vt}, N=\left|{ }^{1} J_{\mathrm{CP}}+{ }^{3} J_{\mathrm{CP}}\right|=9.0 \mathrm{~Hz}\right.$, $\left.2 \mathrm{C}, \mathrm{P}\left(C\left(\mathrm{CH}_{3}\right)_{3}\right)_{2}\right), 34.3$ (vt, $\left.N=\left|{ }^{1} J_{\mathrm{CP}}+{ }^{3} J_{\mathrm{CP}}\right|=8.8 \mathrm{~Hz}, 2 \mathrm{C}, \mathrm{P}\left(C\left(\mathrm{CH}_{3}\right)_{3}\right)_{2}\right), 31.7$ (vt, $\left.N=\left|{ }^{1} J_{\mathrm{PC}}+{ }^{3} J_{\mathrm{PC}}\right|=10.4 \mathrm{~Hz}, 2 \mathrm{C}, \mathrm{PC} \mathrm{H}_{2}\right), 30.4$ (br, 6C, $\left.\mathrm{P}\left(\mathrm{C}\left(\mathrm{CH}_{3}\right)_{3}\right)_{2}\right), 29.0$ (br, $\left.6 \mathrm{C}, \mathrm{P}\left(\mathrm{C}\left(\mathrm{CH}_{3}\right)_{3}\right)_{2}\right) .{ }^{31} \mathrm{P}\left\{{ }^{1} \mathrm{H}\right\}(204.5 \mathrm{MHz}) \delta=1069.4$ (s br, 1P; Re $\left.\equiv P\right), 91.0$ (s, 2P, $\left.P\left(\mathrm{C}\left(\mathrm{CH}_{3}\right)_{3}\right)_{2}\right)$. LIFDI-MS (Toluene): $722.2\left(100 \%,[\mathrm{M}]^{+}\right)$.

\section{Synthesis of 28}

11 (20.0 mg, $24.5 \mu \mathrm{mol}, 1.00$ eq.) and [Na(diox) $\left.{ }_{3.31}\right][\mathrm{AsCO}](10.7 \mathrm{mg}, 25.7 \mu \mathrm{mol}, 1.05$ eq.) are dissolved in $\mathrm{THF}$ at $-80^{\circ} \mathrm{C}$ and the solution is allowed to warm to room temperature over the course of 90 minutes. The solvent is evaporated and the residue is washed with pentane $(3 \times 1 \mathrm{~mL})$ and extracted with benzene $(3 \times 2 \mathrm{~mL})$. Lyophilization yields 28 in form of a brown powder $(13.2 \mathrm{mg}, 17.2 \mu \mathrm{mol}, 70 \%)$.

Anal. Calcd for $\mathrm{C}_{28} \mathrm{H}_{50} \mathrm{AsN}_{4} \mathrm{P}_{2} \mathrm{Re}$ (765.8): C, 43.9; H, 6.58; N 7.32. Found: C, 43.7; $\mathrm{H}, 6.17 ; \mathrm{N}, 6.91$. NMR $\left(\mathrm{THF}-\mathrm{d}_{8}, 0{ }^{\circ} \mathrm{C}\right):{ }^{1} \mathrm{H}(500 \mathrm{MHz}): \delta=8.41\left(\mathrm{~d},{ }^{3} J_{\mathrm{HH}}=1.8 \mathrm{~Hz}\right.$, $\left.1 \mathrm{H}, \mathrm{C}_{1} H\right), 8.37\left(\mathrm{ddd},{ }^{3} J_{\mathrm{HH}}=4.8 \mathrm{~Hz},{ }^{4} J_{\mathrm{HH}}=1.8 \mathrm{~Hz},{ }^{5} J_{\mathrm{HH}}=0.9 \mathrm{~Hz}, 1 \mathrm{H}, \mathrm{C}_{8} H\right), 7.58$ $\left(\mathrm{m}, 1 \mathrm{H}, \mathrm{C}_{5} H\right), 7.41\left(\mathrm{ddd},{ }^{3} J_{\mathrm{HH}}=8.0 \mathrm{~Hz},{ }^{3} J_{\mathrm{HH}}=7.4 \mathrm{~Hz},{ }^{4} J_{\mathrm{HH}}=1.9 \mathrm{~Hz}, 1 \mathrm{H}, \mathrm{C}_{6} H\right)$, $6.82\left(\mathrm{~m}, 2 \mathrm{H}, \mathrm{C}_{2} H, \mathrm{C}_{7} H\right.$, superimposed), $3.55-3.47\left(\mathrm{~m}, 2 \mathrm{H}, \mathrm{NCH}_{2}\right), 3.41-3.31(\mathrm{~m}, 2 \mathrm{H}$, $\left.\mathrm{NCH}_{2}\right), 2.53-2.46\left(\mathrm{~m}, 2 \mathrm{H}, \mathrm{PCH}_{2}\right), 1.94-1.84\left(\mathrm{~m}, 2 \mathrm{H}, \mathrm{PCH}_{2}\right), 1.40\left(\mathrm{~A}_{9} \mathrm{XX}^{\prime} \mathrm{A}_{9},, N=\right.$ $\left.\left|{ }^{3} J_{\mathrm{AX}}+5 J_{\mathrm{AX}}\right|=13.0 \mathrm{~Hz}, 18 \mathrm{H}, \mathrm{P}\left(\mathrm{C}\left(\mathrm{CH}_{3}\right)_{3}\right)_{2}\right), 1.08\left(\mathrm{~A}_{9} \mathrm{XX}^{\prime} \mathrm{A}_{9}{ }^{\prime}, N=\left|{ }^{3} J_{\mathrm{AX}}+{ }^{5} J_{\mathrm{AX}}\right|\right.$ $\left.=12.9 \mathrm{~Hz}, 18 \mathrm{H}, \mathrm{P}\left(\mathrm{C}\left(\mathrm{CH}_{3}\right)_{3}\right)_{2}\right) \cdot{ }^{13} \mathrm{C}\left\{{ }^{1} \mathrm{H}\right\}(125.8 \mathrm{MHz}): \delta=155.6\left(\mathrm{~s}, 1 \mathrm{C}, C_{4}\right), 152.1(\mathrm{~s}$, $\left.1 \mathrm{C}, C_{3}\right), 151.0\left(\mathrm{~s}, 1 \mathrm{C}, C_{1} \mathrm{H}\right), 149.5\left(\mathrm{~s}, 1 \mathrm{C}, C_{8} \mathrm{H}\right), 135.5\left(\mathrm{~s}, 1 \mathrm{C}, C_{6} \mathrm{H}\right), 120.1(\mathrm{~s}, 1 \mathrm{C}$, $\left.C_{7} \mathrm{H}\right), 119.2\left(\mathrm{~s}, 1 \mathrm{C}, C_{5} \mathrm{H}\right), 105.6\left(\mathrm{~s}, 1 \mathrm{C}, C_{2} \mathrm{H}\right), 75.6\left(\mathrm{vt}, N=\mid{ }^{2} J_{\mathrm{PC}}+{ }^{3} J_{\mathrm{PC}}=3.5 \mathrm{~Hz}\right.$, $2 \mathrm{C}, \mathrm{NCH}_{2}$ ), 38.6 (vt, $\left.N=\left|{ }^{1} J_{\mathrm{CP}}+{ }^{3} J_{\mathrm{CP}}\right|=8.8 \mathrm{~Hz}, 2 \mathrm{C}, \mathrm{P}\left(C\left(\mathrm{CH}_{3}\right)_{3}\right)_{2}\right), 33.5$ (vt, $N=$ $\left.\left|{ }^{1} J_{\mathrm{CP}}+{ }^{3} J_{\mathrm{CP}}\right|=8.7 \mathrm{~Hz}, 2 \mathrm{C}, \mathrm{P}\left(C\left(\mathrm{CH}_{3}\right)_{3}\right)_{2}\right), 32.3\left(\mathrm{vt}, N=\left|{ }^{1} J_{\mathrm{PC}}+{ }^{3} J_{\mathrm{PC}}\right|=10.2 \mathrm{~Hz}\right.$, 2C, $\left.\mathrm{PC} \mathrm{H}_{2}\right), 30.5$ (br, 6C, $\left.\mathrm{P}\left(\mathrm{C}\left(\mathrm{CH}_{3}\right)_{3}\right)_{2}\right), 28.6$ (br, 6C, $\left.\mathrm{P}\left(\mathrm{C}\left(\mathrm{CH}_{3}\right)_{3}\right)_{2}\right) .{ }^{31} \mathrm{P}\left\{{ }^{1} \mathrm{H}\right\}(204.5$ $\mathrm{MHz}) \delta=89.9\left(\mathrm{~s}, 2 \mathrm{P}, P\left(\mathrm{C}\left(\mathrm{CH}_{3}\right)_{3}\right)_{2}\right)$. LIFDI-MS (Toluene): $766.2\left(100 \%,[\mathrm{M}]^{+}\right)$.

\section{Synthesis of 26}

When followed by variable-temperature NMR, the transient species $\mathbf{2 6}$ can be observed by ${ }^{31} \mathrm{P}\left\{{ }^{1} \mathrm{H}\right\}$ and ${ }^{1} \mathrm{H}$ NMR. NMR $\left(\mathrm{THF}-\mathrm{d}_{8},-20{ }^{\circ} \mathrm{C}\right):{ }^{1} \mathrm{H}(400 \mathrm{MHz}) \delta=9.81\left(\mathrm{~d}\right.$ br, ${ }^{3} J_{\mathrm{HH}}$ $\left.=5.9 \mathrm{~Hz}, 1 \mathrm{H}, \mathrm{C}_{8} H\right), 7.92-7.90(\mathrm{~m}, 1 \mathrm{H}, \mathrm{PyrPz}), 7.46-7.37$ (m, 1H, PyrPz, superimposed with 5), 7.22 - 7.18 (m, 1H, PyrPz), 6.96 (br, 1H, PyrPz), 6.29 (br, 1H, PyrPz), 4.87 - $4.74\left(\mathrm{~m}, 2 \mathrm{H}, \mathrm{NCH}_{2}\right), 4.40-4.29\left(\mathrm{~m}, 2 \mathrm{H}, \mathrm{NCH}_{2}\right), 2.32-2.28\left(\mathrm{~m}, 2 \mathrm{H}, \mathrm{PCH}_{2}\right), 1.99$ - $1.89\left(\mathrm{~m}, 2 \mathrm{H}, \mathrm{PC} H_{2}\right.$, superimposed with 5), $1.33\left(\mathrm{~A}_{9} \mathrm{XXA}_{9}, N=\left|{ }^{3} J_{\mathrm{AX}}+{ }^{5} J_{\mathrm{AX}}\right|=\right.$ $\left.10.6 \mathrm{~Hz}, 18 \mathrm{H}, \mathrm{P}\left(\mathrm{C}\left(\mathrm{CH}_{3}\right)_{3}\right)_{2}\right), 0.38\left(\mathrm{~A}_{9} \mathrm{XXA}_{9}, N=\left|{ }^{3} J_{\mathrm{AX}}+{ }^{5} J_{\mathrm{AX}}\right|=11.6 \mathrm{~Hz}, 18 \mathrm{H}\right.$, $\left.\mathrm{P}\left(\mathrm{C}\left(\mathrm{CH}_{3}\right)_{3}\right)_{2}\right) .{ }^{31} \mathrm{P}\left\{{ }^{1} \mathrm{H}\right\}(204.5 \mathrm{MHz}) \delta=1.92$ (br, 2P, $P\left(\mathrm{C}_{\left.\left.\left(\mathrm{CH}_{3}\right)_{3}\right)_{2}\right)}\right),-268.8$ (br, 1P, $\mathrm{PCO})$.

\section{Oxidation of 27 to 29}

27 (16.0 mg, $22.2 \mu \mathrm{mol}, 1.00$ eq.) and [FeCp 2 ] OTf (7.5 mg, $22 \mu \mathrm{mol}, 1.0$ eq.) are dissolved in THF- $\mathrm{d}_{8}(0.5 \mathrm{~mL})$ in a J-Young NMR tube and shaken for 1 minute at room temperature. The solution is directly transferred to the NMR machine and measured at $-38^{\circ} \mathrm{C}$. 
The spectroscopic yield was determined to be $44 \%$.

$\operatorname{NMR}\left(\right.$ THF-d $\left._{8},-38^{\circ} \mathrm{C}\right):{ }^{1} \mathrm{H}(500 \mathrm{MHz}): \delta=8.62\left(\mathrm{~d},{ }^{3} J_{\mathrm{HH}}=5.6 \mathrm{~Hz}, 1 \mathrm{H}, \mathrm{C}_{8} H\right), 7.96$ $\left(\mathrm{d},{ }^{3} J_{\mathrm{HH}}=7.7 \mathrm{~Hz}, 1 \mathrm{H}, \mathrm{C}_{5} H\right), 7.84\left(\mathrm{~d},{ }^{3} J_{\mathrm{HH}}=2.7 \mathrm{~Hz}, 1 \mathrm{H}, \mathrm{C}_{1} H\right), 7.28-7.24(\mathrm{~m}, 1 \mathrm{H}$, $\left.\mathrm{C}_{6} H\right), 7.24-7.19\left(\mathrm{~m}, 1 \mathrm{H}, \mathrm{C}_{7} H\right), 6.91\left(\mathrm{~d},{ }^{3} J_{\mathrm{HH}}=2.7 \mathrm{~Hz}, 1 \mathrm{H}, \mathrm{C}_{2} H\right), 4.86-4.68(\mathrm{~m}$, $\left.2 \mathrm{H}, \mathrm{NCH}_{2}\right), 3.08-2.97\left(\mathrm{~m}, 2 \mathrm{H}, \mathrm{NCH}_{2}\right), 2.61-2.52\left(\mathrm{~m}, 2 \mathrm{H}, \mathrm{PCH}_{2}\right), 2.20-2.09(\mathrm{~m}$, $\left.2 \mathrm{H}, \mathrm{PCH} \mathrm{H}_{2}\right), 1.39-1.26$ (br, $\left.18 \mathrm{H}, \mathrm{P}\left(\mathrm{C}\left(\mathrm{CH}_{3}\right)_{3}\right)_{2}\right), 0.59-0.50$ (br, $\left.18 \mathrm{H}, \mathrm{P}\left(\mathrm{C}(\mathrm{CH})_{3}\right)_{2}\right)$. ${ }^{13} \mathrm{C}\left\{{ }^{1} \mathrm{H}\right\}(125.8 \mathrm{MHz}): \delta=157.9\left(\mathrm{~s}, 1 \mathrm{C}, C_{4}\right), 156.2\left(\mathrm{~s}, 1 \mathrm{C}, C_{8}\right), 147.1\left(\mathrm{~s}, 1 \mathrm{C}, C_{3}\right), 140.6$ $\left(\mathrm{s}, 1 \mathrm{C}, C_{7}\right), 135.2\left(\mathrm{~s}, 1 \mathrm{C}, C_{1}\right), 123.8\left(\mathrm{~s}, 1 \mathrm{C}, C_{5}\right), 123.6\left(\mathrm{~s}, 1 \mathrm{C}, C_{6}\right), 106.6\left(\mathrm{~s}, 1 \mathrm{C} . C_{2}\right)$, $82.4\left(\right.$ vt $\left., N=\left|{ }^{2} J_{\mathrm{PC}}+{ }^{3} J_{\mathrm{PC}}\right|=5.0 \mathrm{~Hz}, 2 \mathrm{C}, \mathrm{NCH}_{2}\right), 42.8\left(\right.$ vt, $N=\left|{ }^{1} J_{\mathrm{CP}}+{ }^{3} J_{\mathrm{CP}}\right|=$ $\left.7.6 \mathrm{~Hz}, 2 \mathrm{C}, \mathrm{P}\left(C\left(\mathrm{CH}_{3}\right)_{3}\right)_{2}\right), 41.5\left(\mathrm{vt}, N=\left|{ }^{1} J_{\mathrm{CP}}+{ }^{3} J_{\mathrm{CP}}\right|=11.3 \mathrm{~Hz}, 2 \mathrm{C}, \mathrm{P}\left(C\left(\mathrm{CH}_{3}\right)_{3}\right)_{2}\right)$, 29.7 (br, 6C, P $\left.\left(\mathrm{C}\left(\mathrm{CH}_{3}\right)_{3}\right)_{2}\right), 29.3\left(\mathrm{br}, 6 \mathrm{C}, \mathrm{P}\left(\mathrm{C}\left(\mathrm{CH}_{3}\right)_{3}\right)_{2}\right), 26.5$ (vt, $N=\left|{ }^{1} J_{\mathrm{PC}}+{ }^{3} J_{\mathrm{PC}}\right|$ $\left.=8.1 \mathrm{~Hz}, 2 \mathrm{C}, \mathrm{PC} \mathrm{H}_{2}\right),{ }^{31} \mathrm{P}\left\{{ }^{1} \mathrm{H}\right\}(204.5 \mathrm{MHz}) \delta=656.7\left(\mathrm{~d},{ }^{1} J_{\mathrm{PP}}=560 \mathrm{~Hz}, 1 \mathrm{P}, P_{1}\right)$, $231.6\left(\mathrm{~d},{ }^{1} J_{\mathrm{PP}}=560 \mathrm{~Hz}, 1 \mathrm{P}, P_{2}\right), 30.3\left(\mathrm{br}, 2 \mathrm{P}, P\left(\mathrm{C}\left(\mathrm{CH}_{3}\right)_{3}\right)_{2}\right) .{ }^{31} \mathrm{P}\left\{{ }^{1} \mathrm{H}\right\}(162.0 \mathrm{MHz}) \delta$ $=659.4\left(\mathrm{dt},{ }^{1} J_{\mathrm{PP}}=562 \mathrm{~Hz},{ }^{2} J_{\mathrm{PP}}=11.8 \mathrm{~Hz}, 1 \mathrm{P}, P_{1}\right), 234.7\left(\mathrm{dt},{ }^{1} J_{\mathrm{PP}}=562 \mathrm{~Hz},{ }^{3} J_{\mathrm{PP}}\right.$ $\left.=3.9 \mathrm{~Hz}, 1 \mathrm{P}, P_{2}\right), 31.0\left(\mathrm{dd},{ }^{2} J_{\mathrm{PP}}=11.8 \mathrm{~Hz},{ }^{3} J_{\mathrm{PP}}=3.9 \mathrm{~Hz}, 1 \mathrm{P}, P\left(\mathrm{C}\left(\mathrm{CH}_{3}\right)_{3}\right)_{2}\right)$.

\section{Oxidation of 28 to 30}

3b (20.0 mg, $26.1 \mu \mathrm{mol}, 1.00$ eq.) and [FeCp 2 ] OTf ( $8.7 \mathrm{mg}, 26 \mu \mathrm{mol}, 1.0$ eq.) are dissolved in THF- $\mathrm{d}_{8}(0.5 \mathrm{~mL})$ in a J-Young NMR tube and shaken for 1 minute at room temperature. The solution is directly transferred to the NMR machine and measured at $-38^{\circ} \mathrm{C}$. The spectroscopic yield was determined to be $33 \%$.

$\operatorname{NMR}\left(\right.$ THF- $\left.\mathrm{d}_{8},-38^{\circ} \mathrm{C}\right):{ }^{1} \mathrm{H}(500 \mathrm{MHz}): \delta=8.44\left(\mathrm{~d},{ }^{3} J_{\mathrm{HH}}=6.03 \mathrm{~Hz}, 1 \mathrm{H}, \mathrm{C}_{8} H\right), 7.86$ $\left(\mathrm{d},{ }^{3} J_{\mathrm{HH}}=2.7 \mathrm{~Hz}, 1 \mathrm{H}, \mathrm{C}_{1} H\right), 7.85-7.83\left(\mathrm{~m}, 1 \mathrm{H}, \mathrm{C}_{5} H\right), 7.31-7.25\left(\mathrm{~m}, 1 \mathrm{H}, \mathrm{C}_{7} H\right), 7.21$ - $7.16\left(\mathrm{~m}, 1 \mathrm{H}, \mathrm{C}_{6} H\right), 6.83\left(\mathrm{~d},{ }^{3} J_{\mathrm{HH}}=2.7 \mathrm{~Hz}, 1 \mathrm{H}, \mathrm{C}_{2} H\right), 4.92-4.81\left(\mathrm{~m}, 2 \mathrm{H}, \mathrm{NCH}_{2}\right)$, $3.13-3.08\left(\mathrm{~m}, 2 \mathrm{H}, \mathrm{NCH}_{2}\right), 2.61-2.52\left(\mathrm{~m}, 2 \mathrm{H}, \mathrm{PCH}_{2}\right), 2.15-2.08\left(\mathrm{~m}, 2 \mathrm{H}, \mathrm{PCH}_{2}\right)$, 1.30 - $1.28\left(\mathrm{~m}, 18 \mathrm{H}, \mathrm{P}\left(\mathrm{C}\left(\mathrm{CH}_{3}\right)_{3}\right)_{2}\right), 0.62-0.61\left(\mathrm{~m}, 18 \mathrm{H}, \mathrm{P}\left(\mathrm{C}(\mathrm{CH})_{3}\right)_{3}\right){ }_{2}{ }^{13} \mathrm{C}\left\{{ }^{1} \mathrm{H}\right\}(125.8$ $\mathrm{MHz}): \delta=158.7\left(\mathrm{~s}, 1 \mathrm{C}, C_{4}\right), 155.7\left(\mathrm{~s}, 1 \mathrm{C}, C_{8}\right), 148.0\left(\mathrm{~s}, 1 \mathrm{C}, C_{3}\right), 140.8\left(\mathrm{~s}, 1 \mathrm{C}, C_{6}\right)$, $139.6\left(\mathrm{~s}, 1 \mathrm{C}, C_{1}\right), 123.4\left(\mathrm{~s}, 1 \mathrm{C}, C_{5}\right), 123.3\left(\mathrm{~s}, 1 \mathrm{C}, C_{7}\right), 106.9\left(\mathrm{~s}, 1 \mathrm{C}, C_{2}\right), 84.8$ (vt, $N$ $\left.=\left|{ }^{2} J_{\mathrm{PC}}+{ }^{3} J_{\mathrm{PC}}\right|=4.6 \mathrm{~Hz}, 2 \mathrm{C}, \mathrm{NCH}_{2}\right), 44.1$ (vt, $N=\left|{ }^{1} J_{\mathrm{CP}}+{ }^{3} J_{\mathrm{CP}}\right|=7.3 \mathrm{~Hz}, 2 \mathrm{C}$, $\left.\mathrm{P}\left(C\left(\mathrm{CH}_{3}\right)_{3}\right)_{2}\right), 41.5\left(\mathrm{vt}, N=\left|{ }^{1} J_{\mathrm{CP}}+{ }^{3} J_{\mathrm{CP}}\right|=11.2 \mathrm{~Hz}, 2 \mathrm{C}, \mathrm{P}\left(C\left(\mathrm{CH}_{3}\right)_{3}\right)_{2}\right), 30.3(\mathrm{br}$, $\left.6 \mathrm{C}, \mathrm{P}\left(\mathrm{C}\left(\mathrm{CH}_{3}\right)_{3}\right)_{2}\right), 29.5\left(\mathrm{br}, 6 \mathrm{C}, \mathrm{P}\left(\mathrm{C}\left(\mathrm{CH}_{3}\right)_{3}\right)_{2}\right), 26.7$ (vt, $N=\left|{ }^{1} J_{\mathrm{PC}}+{ }^{3} J_{\mathrm{PC}}\right|=8.1 \mathrm{~Hz}$, $\left.2 \mathrm{C}, \mathrm{PC} \mathrm{H}_{2}\right) .{ }^{31} \mathrm{P}\left\{{ }^{1} \mathrm{H}\right\}(162.0 \mathrm{MHz}) \delta=26.5\left(\mathrm{~s}, 2 \mathrm{P}, P\left(\mathrm{C}\left(\mathrm{CH}_{3}\right)_{3}\right)_{2}\right)$.

\section{Synthesis of 31}

27 (10.0 mg, $13.9 \mu \mathrm{mol}, 1.00$ eq.) and pyridine-N-oxide ( $2.6 \mathrm{mg}, 28 \mu \mathrm{mol}, 2.0$ eq.) are dissolved in benzene $(0.5 \mathrm{~mL})$ and heated to $70^{\circ} \mathrm{C}$ for $2 \mathrm{~h}$. After cooling to room temperature the solution is filtered and the residue is extracted with benzene $(2 \times 0.5 \mathrm{~mL})$. The volume is reduced to $0.5 \mathrm{~mL}$ and the crude product is purified by column chromatography over silanized silica $\left(\mathrm{C}_{6} \mathrm{H}_{6} / \mathrm{THF}\right)$. The solvent is removed, the residue is extracted with benzene and subsequent lyophilization yields 31 in form of a red powder $(8.1 \mathrm{mg}$, $5.4 \mu \mathrm{mol}, 77 \%)$.

Anal. Calcd for $\mathrm{C}_{56} \mathrm{H}_{100} \mathrm{~N}_{8} \mathrm{O}_{4} \mathrm{P}_{6} \mathrm{Re}_{2}$ (1507.7): C, 44.6; H, 6.69; N, 7.43. Found: C, 44.8; $\mathrm{H}, 6.80 ; \mathrm{N}, 7.33$. NMR $\left(\mathrm{C}_{6} \mathrm{D}_{6}, 25^{\circ} \mathrm{C}\right):{ }^{1} \mathrm{H}(500 \mathrm{MHz}): \delta=11.3\left(\mathrm{~d},{ }^{3} J_{\mathrm{HH}}=5.9 \mathrm{~Hz}, 2 \mathrm{H}\right.$, $\left.\mathrm{C}_{8} H\right), 7.77\left(\mathrm{~d},{ }^{3} J_{\mathrm{HH}}=1.6 \mathrm{~Hz}, 2 \mathrm{H}, \mathrm{C}_{1} H\right), 7.33\left(\mathrm{ddd},{ }^{3} J_{\mathrm{HH}}=8.0 \mathrm{~Hz},{ }^{4} J_{\mathrm{HH}}=1.5 \mathrm{~Hz}\right.$, $\left.{ }^{5} J_{\mathrm{HH}}=0.8 \mathrm{~Hz}, 2 \mathrm{H}, \mathrm{C}_{5} H\right), 6.81\left(\mathrm{ddd},{ }^{3} J_{\mathrm{HH}}=7.5 \mathrm{~Hz},{ }^{3} J_{\mathrm{HH}}=6.0 \mathrm{~Hz},{ }^{4} J_{\mathrm{HH}}=1.5 \mathrm{~Hz}\right.$, 


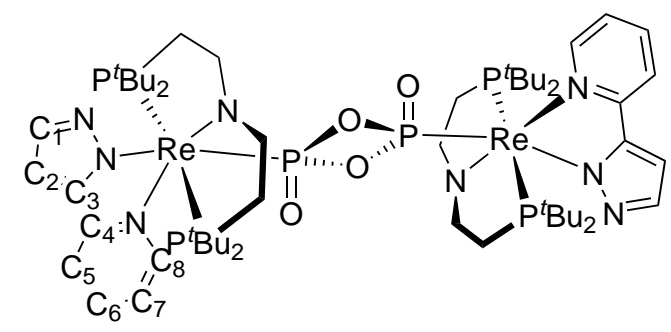

$\left.2 \mathrm{H}, \mathrm{C}_{7} H\right), 6.42\left(\mathrm{ddd},{ }^{3} J_{\mathrm{HH}}=7.5 \mathrm{~Hz},{ }^{3} J_{\mathrm{HH}}=6.0 \mathrm{~Hz},{ }^{4} J_{\mathrm{HH}}=1.5 \mathrm{~Hz}, 2 \mathrm{H}, \mathrm{C}_{6} H\right), 6.36$ $\left(\mathrm{m},{ }^{3} J_{\mathrm{HH}}=1.8 \mathrm{~Hz}, 2 \mathrm{H}, \mathrm{C}_{2} H\right), 3.74-3.38\left(\mathrm{~m}, 8 \mathrm{H}, \mathrm{NCH}_{2}\right), 2.28-2.08\left(\mathrm{~m}, 8 \mathrm{H}, \mathrm{PCH}_{2}\right)$, $1.23\left(\mathrm{~A}_{9} \mathrm{XX}^{\prime} \mathrm{A}_{9}{ }^{\prime}, N=\left|{ }^{2} J_{\mathrm{AX}}+{ }^{4} J_{\mathrm{AX}}\right|=12.6 \mathrm{~Hz}, 18 \mathrm{H}, \mathrm{P}\left(\mathrm{C}\left(\mathrm{CH}_{3}\right)_{3}\right)_{2}\right), 0.51\left(\mathrm{~A}_{9} \mathrm{XX}^{\prime} \mathrm{A}_{9}\right.$ ', $\left.N=\left|{ }^{2} J_{\mathrm{AX}}+{ }^{4} J_{\mathrm{AX}}\right|=12.1 \mathrm{~Hz}, 18 \mathrm{H}, \mathrm{P}\left(\mathrm{C}\left(\mathrm{CH}_{3}\right)_{3}\right)_{2}\right) \cdot{ }^{13} \mathrm{C}\left\{{ }^{1} \mathrm{H}\right\}(125.8 \mathrm{MHz}): \delta=155.8$ $\left(\mathrm{s}, 2 \mathrm{C}, C_{8}\right), 150.7\left(\mathrm{~d},{ }^{3} J_{\mathrm{CP}}=2.8 \mathrm{~Hz}, 2 \mathrm{C}, C_{4}\right), 149.1\left(\mathrm{~d},{ }^{3} J_{\mathrm{CP}}=4.3 \mathrm{~Hz}, 2 \mathrm{C}, C_{3}\right), 143.1$ $\left(\mathrm{d},{ }^{4} J_{\mathrm{CP}}=4.1 \mathrm{~Hz}, 2 \mathrm{C}, C_{1}\right), 138.6\left(\mathrm{~s}, 2 \mathrm{C}, C_{6}\right), 117.9\left(\mathrm{~s}, 2 \mathrm{C}, C_{7}\right), 115.9\left(\mathrm{~s}, 2 \mathrm{C}, C_{5}\right), 104.4$ $\left(\mathrm{d},{ }^{4} J_{\mathrm{CP}}=1.2 \mathrm{~Hz}, 2 \mathrm{C}, C_{2}\right), 96.6\left(\mathrm{vt}, N=\left|{ }^{2} J_{\mathrm{PC}}+{ }^{3} J_{\mathrm{PC}}\right|=7.6 \mathrm{~Hz},{ }^{3} J_{\mathrm{CP}}=1.0 \mathrm{~Hz}\right.$, $\left.4 \mathrm{C}, \mathrm{NCH}_{2}\right), 41.5\left(\mathrm{vt}, N=\left|{ }^{1} J_{\mathrm{CP}}+{ }^{3} J_{\mathrm{CP}}\right|=18.9 \mathrm{~Hz}, 4 \mathrm{C}, \mathrm{P}\left(C\left(\mathrm{CH}_{3}\right)_{3}\right)_{2}\right), 40.7$ (vt, $N=$ $\left.\left|{ }^{1} J_{\mathrm{CP}}+{ }^{3} J_{\mathrm{CP}}\right|=14.2 \mathrm{~Hz}, 4 \mathrm{C}, \mathrm{P}\left(C\left(\mathrm{CH}_{3}\right)_{3}\right)_{2}\right), 31.1\left(\mathrm{vt}, N=\left|{ }^{2} J_{\mathrm{CP}}+{ }^{4} J_{\mathrm{CP}}\right|=3.9 \mathrm{~Hz}\right.$, $\left.12 \mathrm{C}, \mathrm{P}\left(\mathrm{C}\left(\mathrm{CH}_{3}\right)_{3}\right)_{2}\right), 29.6$ (vt, $\left.N=\left|{ }^{2} J_{\mathrm{CP}}+{ }^{4} J_{\mathrm{CP}}\right|=4.5 \mathrm{~Hz}, 12 \mathrm{C}, \mathrm{P}\left(\mathrm{C}\left(\mathrm{CH}_{3}\right)_{3}\right)_{2}\right), 28.2$ (vt, $\left.N=\left|{ }^{1} J_{\mathrm{PC}}+{ }^{3} J_{\mathrm{PC}}\right|=15.3 \mathrm{~Hz}, 4 \mathrm{C}, \mathrm{PC} \mathrm{H}_{2}\right) .{ }^{31} \mathrm{P}\left\{{ }^{1} \mathrm{H}\right\}(204.5 \mathrm{MHz}) \delta=245.7(\mathrm{t}$, $\left.{ }^{2} J_{\mathrm{PP}}=20.7 \mathrm{~Hz}, 2 \mathrm{P} ; \mathrm{P}_{2} \mathrm{O}_{4}\right), 13.2\left(\mathrm{~d},{ }^{2} J_{\mathrm{PP}}=20.7 \mathrm{~Hz}, 4 \mathrm{P}, \mathrm{P}\left(\mathrm{C}\left(\mathrm{CH}_{3}\right)_{3}\right)_{2}\right)$. 


\section{One-electron reduction of 31}

31 (5.0 mg, $3.3 \mu \mathrm{mol}, 1.0$ eq.) and $\mathrm{C}_{10} \mathrm{H}_{20} \mathrm{O}_{5}(790 \mu \mathrm{L}, 4.0 \mu \mathrm{mol}, 1.0$ eq. $)$ are dissolved in THF $(1 \mathrm{~mL})$ and cooled to $-35^{\circ} \mathrm{C} . \mathrm{NaHg}(0.1 \mathrm{w} \%, 76 \mathrm{mg}, 3.3 \mu \mathrm{mol}, 1.0$ eq. $)$ is added and the reaction is stirred for 10 minutes while warming to room temperature. The obtained dark red solution is filtered and analyzed.

\section{Synthesis of 34}

31 (10.0 mg, $6.63 \mu \mathrm{mol}, 1.00$ eq.) and $\mathrm{C}_{12} \mathrm{H}_{24} \mathrm{O}_{6}$ (3.5 mg, $13 \mu \mathrm{mol}, 2.0$ eq.) are dissolved in $\mathrm{THF}$ and cooled to $-80^{\circ} \mathrm{C}$. $\mathrm{KC}_{8}(2.0 \mathrm{mg}, 15 \mu \mathrm{mol}, 2.2 \mathrm{eq}$.$) is added in one portion$ and the solution is slowly allowed to warm to room temperature. After stirring for 30 minutes at room temperature the formed carbon is removed by filtration. The solvent is removed in vacuo and the residue is extracted with benzene $(3 \times 1 \mathrm{~mL})$. The solvent is removed in vacuo and the product is crystallized from $\mathrm{THF} /$ pentane at $-80^{\circ} \mathrm{C}$ for 3 days. The supernatant is decanted off and the residue is extracted with benzene. Lyophilization affords $\mathbf{3 4}$ in form of a purple powder $(8.3 \mathrm{mg}, 7.9 \mu \mathrm{mol}, 59 \%)$.

Anal. Calcd for $\mathrm{C}_{40} \mathrm{H}_{74} \mathrm{KN}_{4} \mathrm{O}_{8} \mathrm{P}_{3} \mathrm{Re}$ (1057.3): C, 45.4; H, 7.06; N, 5.30. Found: C, 45.8; $\mathrm{H}, 7.23 ; \mathrm{N}, 5.28$.

\section{Synthesis of $\left[\mathrm{Fe}\left(\mathrm{C}_{5} \mathrm{Me}_{5}\right)_{2}\right]\left[\mathrm{Al}\left(\mathrm{O}\left(\mathrm{C}\left(\mathrm{CF}_{3}\right)_{3}\right)_{4}\right]\right.$}

$\left[\mathrm{Fe}\left(\mathrm{C}_{5} \mathrm{Me}_{5}\right)_{2}\right](25.0 \mathrm{mg}, 76.9 \mu \mathrm{mol}, 1.00$ eq. $)$ and $\mathrm{Ag}\left[\mathrm{Al}\left(\mathrm{O}\left(\mathrm{C}\left(\mathrm{CF}_{3}\right)_{3}\right)_{4}\right](80.0 \mathrm{mg}, 74.2 \mu \mathrm{mol}\right.$, 0.97 eq. $)$ are dissolved in $\mathrm{DCM}(3 \mathrm{~mL})$ at $0^{\circ} \mathrm{C}$ and stirred for 30 minutes. The solution is filtered, concentrated and layered with pentane $(10 \mathrm{~mL})$. The solution is kept at $-35^{\circ} \mathrm{C}$ for 1 day. The precipitate is filtered off, washed with pentane $(3 \times 1 \mathrm{~mL})$ and extracted with THF $(3 \times 1 \mathrm{~mL})$. The product is dried in vacuo. $\left[\mathrm{Fe}\left(\mathrm{C}_{5} \mathrm{Me}_{5}\right)_{2}\right]\left[\mathrm{Al}\left(\mathrm{O}\left(\mathrm{C}\left(\mathrm{CF}_{3}\right)_{3}\right)_{4}\right]\right.$ is obtained in form of a turquoise powder $(78 \mathrm{mg}, 59.7 \mu \mathrm{mol}, 78 \%)$.

Anal. Calcd for $\mathrm{C}_{36} \mathrm{H}_{30} \mathrm{AlF}_{36} \mathrm{FeO}_{4}$ (1293.4): C, 33.4; H, 2.34. Found: C, 33.6; H, 2.16.

\section{Oxidation of 34 with $\left[\mathrm{Fe}\left(\mathrm{C}_{5} \mathrm{Me}_{5}\right)_{2}\right]\left[\mathrm{Al}\left(\mathrm{O}\left(\mathrm{C}\left(\mathrm{CF}_{3}\right)_{3}\right)_{4}\right]\right.$}

34 (5.0 mg, $4.7 \mu \mathrm{mol}, 1.0$ eq.) and $\left[\mathrm{Fe}\left(\mathrm{C}_{5} \mathrm{Me}_{5}\right)_{2}\right]\left[\mathrm{Al}\left(\mathrm{O}\left(\mathrm{C}\left(\mathrm{CF}_{3}\right)_{3}\right)_{4}\right](6.1 \mathrm{mg}, 4.7 \mu \mathrm{mol}, 1.0\right.$ eq. $)$ are placed in a J-Young NMR tube. THF- $\mathrm{d}_{8}$ is added via vacuum condensation and the solvent is thawed at $-90^{\circ} \mathrm{C}$. The sample is gradually warmed to room temperature in the NMR machine.

\section{Synthesis of 36}

1 (30.0 mg, $48.6 \mu \mathrm{mol}, 1.00$ eq.) and $\mathrm{CoCp}_{2}(9.6 \mathrm{mg}, 51 \mu \mathrm{mol}, 1.0$ eq.) are dissolved in $\mathrm{Et}_{2} \mathrm{O}(5 \mathrm{~mL})$ and stirred for one minute at room temperature. $\mathrm{PH}_{2} \mathrm{Ph}(77.3 \mu \mathrm{L}, 47.1 \mu \mathrm{mol}$, 0.97 eq., $10 \mathrm{w} \%$ in hexane) is added and stirring is continued for 30 minutes. The solvent is evaporated and the residue is extracted with pentane $(3 \times 2 \mathrm{~mL})$. The solution is concentrated and purified by column chromatography (pentane). 36 (26.1 mg, $37.8 \mu \mathrm{mol}$, $80 \%)$ is obtained as a green solid.

Anal. Calcd for $\mathrm{C}_{26} \mathrm{H}_{47} \mathrm{ClNOsP}_{3}$ (692.27): C, 45.1; H, 6.84; N, 2.02 Found: C, 45.6; H, 6.39; N, 1.84. NMR (THF- $\left.\mathrm{d}_{8},-30^{\circ}\right):{ }^{1} \mathrm{H}(300 \mathrm{MHz}): \delta=7.83-7.71\left(\mathrm{~m}, 2 \mathrm{H}, \mathrm{C}_{\text {ortho }} H\right)$, $7.36\left(\mathrm{~A}_{18} \mathrm{BCXX}^{\prime} \mathrm{A}^{\prime}{ }_{18} \mathrm{~B}^{\prime} \mathrm{C}^{\prime}, N=\left|{ }^{3} J_{\mathrm{CX}}+{ }^{4} J_{\mathrm{CX}}{ }^{\prime}\right|=33.6 \mathrm{~Hz},{ }^{3} J_{\mathrm{BC}}=5.7 \mathrm{~Hz}, 2 \mathrm{H}, \mathrm{NCH}\right), 7.30$ 
- $7.21\left(\mathrm{~m}, 3 \mathrm{H}, \mathrm{C}_{\text {meta }} H, \mathrm{C}_{\mathrm{para}} H\right), 4.96\left(\mathrm{dt},{ }^{1} J_{\mathrm{HP}}=337 \mathrm{~Hz},{ }^{2} J_{\mathrm{HP}}=8.9 \mathrm{~Hz}, 2 \mathrm{H}, \mathrm{PH} \mathrm{H}_{2} \mathrm{Ph}\right)$, $4.49\left(\mathrm{~A}_{18} \mathrm{BCXX}^{\prime}{ }^{\prime}{ }_{18} \mathrm{~B}^{\prime} \mathrm{C}^{\prime}, N=\left|{ }^{2} J_{\mathrm{BX}}+{ }^{4} J_{\mathrm{BX}}\right|=8.0 \mathrm{~Hz},{ }^{3} J_{\mathrm{BC}}=5.7 \mathrm{~Hz}, 2 \mathrm{H}, \mathrm{PCH}\right)$, $1.21\left(\mathrm{~A}_{18} \mathrm{BCXX}^{\prime} \mathrm{A}_{18} \mathrm{~B}^{\prime} \mathrm{C}^{\prime},{ }^{3} J_{\mathrm{AX}}=13.0 \mathrm{~Hz}, 18 \mathrm{H}, \mathrm{PC}\left(\mathrm{CH}_{3}\right)_{3}\right), 1.17\left(\mathrm{~A}_{18} \mathrm{BCXX}^{\prime} \mathrm{A}_{18} \mathrm{~B}^{\prime} \mathrm{C}^{\prime}\right.$, $\left.{ }^{3} J_{\mathrm{AX}}=12.6 \mathrm{~Hz}, 18 \mathrm{H}, \mathrm{PC}\left(\mathrm{CH}_{3}\right)_{3}\right) \cdot{ }^{13} \mathrm{C}\left\{{ }^{1} \mathrm{H}\right\}(125.76 \mathrm{MHz}): \delta=164.7\left(\mathrm{vt},{ }^{2} J_{\mathrm{CP}}=6.6 \mathrm{~Hz}\right.$, $2 \mathrm{C}, \mathrm{NCH}), 135.8\left(\mathrm{~d},{ }^{2} J_{\mathrm{CP}}=10.4 \mathrm{~Hz}, 2 \mathrm{C}, C_{\text {ortho }} \mathrm{H}\right), 132.8\left(\mathrm{~d},{ }^{2} J_{\mathrm{CP}}=61.6 \mathrm{~Hz}, 1 \mathrm{C}\right.$, $\left.C_{\text {ipso }} \mathrm{H}\right), 130.7\left(\mathrm{~d},{ }^{4} J_{\mathrm{CP}}=2.7 \mathrm{~Hz}, 1 \mathrm{C}, C_{\text {para }} \mathrm{H}\right), 129.0\left(\mathrm{~d},{ }^{3} J_{\mathrm{CP}}=11.2 \mathrm{~Hz}, 2 \mathrm{C}, C_{\text {meta }} \mathrm{H}\right)$, $90.0\left(\mathrm{vt},{ }^{1} J_{\mathrm{CP}}=19.5 \mathrm{~Hz}, 2 \mathrm{C}, \mathrm{PCH}\right), 42.5\left(\mathrm{vt},{ }^{1} J_{\mathrm{CP}}=10.3 \mathrm{~Hz}, 2 \mathrm{C}, \mathrm{PC}\left(\mathrm{CH}_{3}\right)_{3}\right), 37.7$ $\left(\mathrm{vt},{ }^{1} J_{\mathrm{CP}}=11.4 \mathrm{~Hz}, 2 \mathrm{C}, \mathrm{PC}\left(\mathrm{CH}_{3}\right)_{3}\right), 31.0\left(\mathrm{vt},{ }^{1} J_{\mathrm{CP}}=2.8 \mathrm{~Hz}, 6 \mathrm{C}, \mathrm{PC}\left(\mathrm{CH}_{3}\right)_{3}\right), 30.3$ (vt, $\left.{ }^{1} J_{\mathrm{CP}}=2.6 \mathrm{~Hz}, 6 \mathrm{C}, \mathrm{PC}\left(\mathrm{CH}_{3}\right)_{3}\right) .{ }^{31} \mathrm{P}\left\{{ }^{1} \mathrm{H}\right\}(161.25 \mathrm{MHz}): \delta=51.0\left(\mathrm{~d},{ }^{2} J_{\mathrm{PP}}=18.7 \mathrm{~Hz}\right.$, $\left.2 \mathrm{P}, P C\left(\mathrm{CH}_{3}\right)_{3}\right),-62.2\left(\mathrm{t},{ }^{2} J_{\mathrm{PP}}=18.7 \mathrm{~Hz}, 1 \mathrm{P}, \mathrm{PH}_{2} \mathrm{Ph}\right)$. IR $\left(\right.$ Nujol, $\left.\mathrm{cm}^{-1}\right): 2320,2292$ $\left(\tilde{\nu}_{\mathrm{PH}_{2}}\right)$. LIFDI-MS (Toluene): $693.1\left(100 \%, \mathrm{M}^{+}\right)$.

\section{Synthesis of 37}

1 (42.5 mg, $68.8 \mu \mathrm{mol}, 1.00$ eq.) and $\mathrm{CoCp}_{2}(13.1 \mathrm{mg}, 69.3 \mu \mathrm{mol}, 1.00 \mathrm{eq}$.$) are dissolved in$ $\mathrm{Et}_{2} \mathrm{O}(5 \mathrm{~mL})$ and stirred for $1 \mathrm{~min}$ at room temperature. $\mathrm{PH}_{2} \mathrm{Mes}^{*}(19.2 \mathrm{mg}, 68.8 \mu \mathrm{mol}$, 1.00 eq.) is added and stirring is continued for $2 \mathrm{~h}$. After filtration the solvent is evaporated and the crude product is extracted with pentane $(10 \times 3 \mathrm{~mL})$. The solution is concentrated and stored at $-40^{\circ}$ for 3 days. The solvent is decanted off and the solid is washed with cold pentane. After extraction with benzene $(3 \times 5 \mathrm{~mL})$ Lyophilization yields $37 \mathrm{in}$ form of a blue powder $(42.8 \mathrm{mg}, 49.7 \mu \mathrm{mol}, 72 \%)$.

Anal. Calcd for $\mathrm{C}_{38} \mathrm{H}_{71} \mathrm{ClNOsP}_{3}$ (860.58): C, 53.04; H, 8.32; N, 1.63 Found: C, 53.54; H, 7.82; N, 1.45. NMR $\left(\right.$ THF- $\left.\mathrm{d}_{8},-30^{\circ}\right):{ }^{1} \mathrm{H}(500 \mathrm{MHz}): \delta=18.5\left(\mathrm{~d},{ }^{1} J_{\mathrm{HP}}=337.2 \mathrm{~Hz}, 1 \mathrm{H}\right.$, PHMes $\left.{ }^{*}\right), 7.34\left(\mathrm{~d},{ }^{4} J_{\mathrm{HH}}=1.93 \mathrm{~Hz}, 1 \mathrm{H}, \mathrm{C}_{\text {meta }} H, \mathrm{Mes}^{*}\right), 7.26\left(\mathrm{~d},{ }^{4} J_{\mathrm{HH}}=1.93 \mathrm{~Hz}, 1 \mathrm{H}\right.$, $\mathrm{C}_{\text {meta }} H$, Mes $\left.^{*}\right), 6.82-6.70(\mathrm{~m}, 2 \mathrm{H}, \mathrm{NCHCHP}), 4.35\left(\mathrm{~m},{ }^{3} J_{\mathrm{HH}}=6.0 \mathrm{~Hz}, 1 \mathrm{H}, \mathrm{NCHC} H \mathrm{P}\right)$, $4.14\left(\mathrm{~m},{ }^{3} J_{\mathrm{HH}}=6.0 \mathrm{~Hz}, 1 \mathrm{H}, \mathrm{NCHCHP}\right), 1.64\left(\mathrm{~d},{ }^{3} J_{\mathrm{HP}}=10.5 \mathrm{~Hz}, 9 \mathrm{H}, \mathrm{PC}(\mathrm{CH})_{3}\right), 1.45$ $\left(\mathrm{d},{ }^{3} J_{\mathrm{HP}}=9.8 \mathrm{~Hz}, 9 \mathrm{H}, \mathrm{PC}\left(\mathrm{CH}_{3}\right)_{3}\right), 1.36\left(\mathrm{~s}, 9 \mathrm{H}, \mathrm{C}_{\text {ortho }} \mathrm{C}\left(\mathrm{CH}_{3}\right)_{3}, \mathrm{Mes}^{*}\right), 1.34(\mathrm{~s}, 9 \mathrm{H}$, $\left.\mathrm{C}_{\text {ortho }} \mathrm{C}\left(\mathrm{CH}_{3}\right)_{3}, \mathrm{Mes}^{*}\right), 1.30\left(\mathrm{~s}, 9 \mathrm{H}, \mathrm{C}_{\mathrm{para}} \mathrm{C}\left(\mathrm{CH}_{3}\right)_{3}, \mathrm{Mes}^{*}\right), 1.17\left(\mathrm{~d},{ }^{3} J_{\mathrm{HP}}=10.7 \mathrm{~Hz}\right.$, $\left.9 \mathrm{H}, \mathrm{PC}\left(\mathrm{CH}_{3}\right)_{3}\right), 1.00\left(\mathrm{~d},{ }^{3} J_{\mathrm{HP}}=9.7 \mathrm{~Hz}, 9 \mathrm{H}, \mathrm{PC}\left(\mathrm{CH}_{3}\right)_{3}\right),-5.37(\mathrm{~m}, 1 \mathrm{H}, \mathrm{Os} H) .{ }^{13} \mathrm{C}\left\{{ }^{1} \mathrm{H}\right\}$ $(125.76 \mathrm{MHz}): \delta=162.8\left(\mathrm{dd},{ }^{2} J_{\mathrm{CP}}=11.5 \mathrm{~Hz},{ }^{3} J_{\mathrm{CP}}=4.7 \mathrm{~Hz}, 1 \mathrm{C}, \mathrm{NCHCHP}\right), 162.1(\mathrm{dd}$, $\left.{ }^{2} J_{\mathrm{CP}}=10.4 \mathrm{~Hz},{ }^{3} J_{\mathrm{CP}}=3.8 \mathrm{~Hz}, 1 \mathrm{C}, \mathrm{NCHCHP}\right), 153.9\left(\mathrm{~s}, 1 \mathrm{C}, C_{\text {ortho }}, \mathrm{Mes}^{*}\right), 152.6(\mathrm{~s}$, $1 \mathrm{C}, C_{\text {ortho }}$, Mes*), 148.9 (s, 1C, $C_{\text {para }}$, Mes*), 146.4 (br, $1 \mathrm{C}, C_{\text {ipso }}$, Mes*), 125.5 (s, 1C, $C_{\text {meta }}$, Mes*), 123.1 (s, $1 \mathrm{C}, C_{\text {meta }}$, Mes $\left.{ }^{*}\right), 93.9\left(\mathrm{~m},{ }^{1} J_{\mathrm{CP}}=31.5 \mathrm{~Hz},{ }^{3} J_{\mathrm{CP}}=7.3 \mathrm{~Hz}, 1 \mathrm{C}\right.$, $\mathrm{NCH} C \mathrm{HP}), 92.6\left(\mathrm{~m},{ }^{1} J_{\mathrm{CP}}=34.5 \mathrm{~Hz},{ }^{3} J_{\mathrm{CP}}=4.9 \mathrm{~Hz}, 1 \mathrm{C}, \mathrm{NCHCHP}\right), 42.8\left(\mathrm{dd},{ }^{1} J_{\mathrm{CP}}=\right.$ $\left.14.3 \mathrm{~Hz},{ }^{3} J_{\mathrm{CP}}=7.7 \mathrm{~Hz} 1 \mathrm{C}, \mathrm{PC}\left(\mathrm{CH}_{3}\right)_{3}\right), 40.7\left(\mathrm{~s}, 1 \mathrm{C}, \mathrm{C}_{\mathrm{para}} C\left(\mathrm{CH}_{3}\right)_{3}, \mathrm{Mes}^{*}\right) 40.0(\mathrm{~s}, 1 \mathrm{C}$, $\mathrm{C}_{\text {ortho }} C\left(\mathrm{CH}_{3}\right)_{3}$, Mes*), $39.6\left(\mathrm{dd},{ }^{1} J_{\mathrm{CP}}=13.6 \mathrm{~Hz},{ }^{3} J_{\mathrm{CP}}=6.5 \mathrm{~Hz} 1 \mathrm{C}, \mathrm{P} C\left(\mathrm{CH}_{3}\right)_{3}\right), 38.8$ $\left(\mathrm{dd},{ }^{1} J_{\mathrm{CP}}=21.4 \mathrm{~Hz},{ }^{3} J_{\mathrm{CP}}=6.1 \mathrm{~Hz}, 1 \mathrm{C}, \mathrm{PC}\left(\mathrm{CH}_{3}\right)_{3}\right), 35.9\left(\mathrm{dd},{ }^{1} J_{\mathrm{CP}}=19.0 \mathrm{~Hz},{ }^{3} J_{\mathrm{CP}}=\right.$ $\left.6.0 \mathrm{~Hz}, 1 \mathrm{C}, \mathrm{PC}\left(\mathrm{CH}_{3}\right)_{3}\right), 35.6$ (s, $\left.1 \mathrm{C}, \mathrm{C}_{\text {ortho }} C\left(\mathrm{CH}_{3}\right)_{3}, \mathrm{Mes}^{*}\right), 33.7$ (br, 3C, $\left.\mathrm{PC}\left(\mathrm{CH}_{3}\right)_{3}\right)$, 33.5 (br, 3C, $\left.\mathrm{PC}\left(\mathrm{CH}_{3}\right)_{3}\right), 33.2\left(\mathrm{~s}, 3 \mathrm{C}, \mathrm{C}_{\text {ortho }} \mathrm{C}\left(\mathrm{CH}_{3}\right)_{3}\right), 32.8\left(\mathrm{~s}, 3 \mathrm{C}, \mathrm{C}_{\mathrm{para}} \mathrm{C}\left(\mathrm{CH}_{3}\right)_{3}\right)$, 31.6 (s, 3C C $\left.\mathrm{C}_{\text {ortho }} \mathrm{C}\left(\mathrm{CH}_{3}\right)_{3}\right)$, 31.5-30.8 (br, 6C, $\left.\mathrm{PC}\left(\mathrm{CH}_{3}\right)_{3}\right) .{ }^{31} \mathrm{P}\left\{{ }^{1} \mathrm{H}\right\}(161.25 \mathrm{MHz}): \delta$ $=103.9(\mathrm{~m}, 1 \mathrm{P}$, PHMes$*), 31.6\left(\mathrm{dd},{ }^{2} J_{\mathrm{PP}}=259.8 \mathrm{~Hz},{ }^{2} J_{\mathrm{PP}}=20.1 \mathrm{~Hz}, 1 \mathrm{P}, P \mathrm{C}\left(\mathrm{CH}_{3}\right)_{3}\right)$, $30.0\left(\mathrm{dd},{ }^{2} J_{\mathrm{PP}}=259.8 \mathrm{~Hz},{ }^{2} J_{\mathrm{PP}}=18.3 \mathrm{~Hz}, 1 \mathrm{P}, P \mathrm{C}\left(\mathrm{CH}_{3}\right)_{3}\right) . \quad$ IR $\left(\right.$ Nujol, $\left.\mathrm{cm}^{-1}\right): 2349$ $\left(\tilde{\nu}_{\mathrm{PH}}\right.$, partially superimposed with the absorbance of $\mathrm{CO}_{2}$ in the atmosphere). LIFDI-MS (Toluene): $861.5\left(39 \%,[\mathrm{M}]^{+}, 825.5\left(100 \%,[\mathrm{M}-\mathrm{HCl}]^{+}\right)\right.$. 


\section{Synthesis of 38}

Route A: 37 (30 mg, $34.9 \mu \mathrm{mol}, 1.00$ eq.) and $\mathrm{CoCp}_{2}(6.6 \mathrm{mg}, 35 \mu \mathrm{mol}, 1.0$ eq.) are dissolved in pentane $(3 \mathrm{~mL})$ and stirred for 5 minutes at room temperature. After filtration and extraction of the residue with pentane $(3 \times 1 \mathrm{~mL})$ the solvent is evaporated. After column chromatography (silanized silica, pentane), the solvent is evaporated. $\mathbf{3 8}$ is obtained after lyophilization as a dark green powder (23.0 mg, $27.9 \mu \mathrm{mol}, 80 \%)$.

Anal. Calcd for $\mathrm{C}_{38} \mathrm{H}_{71} \mathrm{NOsP}_{3}$ (825.1): C, 55.1; H, 8.53; N, 1.68 Found: C, 54.9; H, 8.65; N, 1.65. IR (Nujol, $\left.\mathrm{cm}^{-1}\right): 2345\left(\tilde{\nu}_{\mathrm{PH}}\right) 2180\left(\tilde{\nu}_{\mathrm{OsH}}\right)$. LIFDI-MS (Toluene): 826.4 $\left(100 \%,[\mathrm{M}]^{+}\right)$.

Route B: 1 (35.0 mg, $56.7 \mu \mathrm{mol}, 1.00 \mathrm{eq})$ and $\mathrm{CoCp}_{2}(21.4 \mathrm{mg}, 113 \mu \mathrm{mol}, 2.00 \mathrm{eq}$.$) are$ dissolved in $\mathrm{C}_{6} \mathrm{H}_{6}(3 \mathrm{~mL})$ and stirred for 2 minutes at room temperature. $\mathrm{PH}_{2} \mathrm{Mes}^{*}$ $(15.8 \mathrm{mg}, 56.7 \mathrm{\mu mol}, 1.00$ eq.) is added and stirring is continued for additional 4 hours. The solvent is removed and the crude product is extracted with pentane $(5 \times 1 \mathrm{~mL})$ and the solvent is evaporated. After column chromatography (silanized silica, pentane) the solvent is evaporated. Lyophilization yields $\mathbf{3 8}$ as a dark green powder (41.8 $\mathrm{mg}$, $50.7 \mu \mathrm{mol}, 89 \%$ )

Anal. Calcd for $\mathrm{C}_{38} \mathrm{H}_{71} \mathrm{NOsP}_{3}$ (825.1): C, 55.3; H, 8.67; N, 1.70 Found: C, 54.9; H, 8.65; N, 1.65. IR (Nujol, $\left.\mathrm{cm}^{-1}\right): 2345\left(\tilde{\nu}_{\mathrm{PH}}\right) 2180\left(\tilde{\nu}_{\mathrm{OsH}}\right)$. LIFDI-MS (Toluene): 826.4 $\left(100 \%,[\mathrm{M}]^{+}\right) \cdot \mu_{\mathrm{eff}}{ }^{298 \mathrm{~K}}=1.51 \mu_{\mathrm{B}}$

\section{Synthesis of 39}

Route A: 38 (10.0 mg, $12.1 \mu \mathrm{mol}, 1.00$ eq.) and TEMPO (5.7 mg, 38 pmol, 3.0 eq.) are dissolved in pentane and stirred for 10 minutes at room temperature. After column chromatography (silanized silica, pentane) and removal of the solvent $\mathbf{3 9}$ is obtained in form of a violet powder $(9.0 \mathrm{mg}, 11 \mu \mathrm{mol}, 90 \%)$.

$\operatorname{NMR}\left(\mathrm{THF}_{-} \mathrm{d}_{8},-30^{\circ}\right):{ }^{1} \mathrm{H}(500 \mathrm{MHz}): \delta=7.36\left(\mathrm{~d},{ }^{4} J_{\mathrm{HH}}=1.8 \mathrm{~Hz}, 1 \mathrm{H}, \mathrm{C}_{\text {meta }} H\right), 7.23$ $\left(\mathrm{m},{ }^{3} J_{\mathrm{HH}}=6.1 \mathrm{~Hz}, 2 \mathrm{H}, \mathrm{NCH}\right), 7.03\left(\mathrm{~d},{ }^{4} J_{\mathrm{HH}}=1.8 \mathrm{~Hz}, 1 \mathrm{H}, \mathrm{C}_{\text {meta }} H\right), 4.63\left(\mathrm{~m},{ }^{3} J_{\mathrm{HH}}\right.$ $=5.0 \mathrm{~Hz}, 2 \mathrm{H}, \mathrm{PCH}), 1.55\left(\mathrm{~s}, 9 \mathrm{H}, \mathrm{C}_{\text {ortho }} \mathrm{C}\left(\mathrm{CH}_{3}\right)_{3}\right), 1.35\left(\mathrm{~s}, 9 \mathrm{H}, \mathrm{C}_{\text {ortho }} \mathrm{C}\left(\mathrm{CH}_{3}\right)_{3}\right), 1.34$ $\left(\mathrm{s}, 9 \mathrm{H}, \mathrm{C}_{\mathrm{para}} \mathrm{C}\left(\mathrm{CH}_{3}\right)_{3}\right), 1.22$ (br, $\left.18 \mathrm{H}, \mathrm{PC}\left(\mathrm{CH}_{3}\right)_{3}\right), 1.20$ (br, $\left.18 \mathrm{H}, \mathrm{PC}\left(\mathrm{CH}_{3}\right)_{3}\right),-15.91$ $\left(\mathrm{t},{ }^{2} J_{\mathrm{HP}}=14.5 \mathrm{~Hz}, 1 \mathrm{H}, \mathrm{Os} H\right) \cdot{ }^{31} \mathrm{P}\left\{{ }^{1} \mathrm{H}\right\}(161.25 \mathrm{MHz}): \delta=825.1\left(\mathrm{t},{ }^{2} J_{\mathrm{PP}}=36.6 \mathrm{~Hz}\right.$, $1 \mathrm{P}, \mathrm{PH}), 43.2\left(\mathrm{~d},{ }^{2} J_{\mathrm{PP}}=36.6 \mathrm{~Hz}, 2 \mathrm{P}, \mathrm{PC}\left(\mathrm{CH}_{3}\right)_{3}\right)$. LIFDI-MS (Toluene): 825.5 (100\%, $\left.[\mathrm{M}]^{+}\right)$.

Route B: 38 (10 mg, $12.1 \mu \mathrm{mol}, 1.0$ eq. $)$ is dissolved in THF $(1 \mathrm{~mL})$ and cooled to $-80^{\circ}$. $\mathrm{Ag}\left[\mathrm{Al}\left(\mathrm{O}_{-}{ }^{t} \mathrm{Bu}-\mathrm{F}_{9}\right)_{4}\right](13.0 \mathrm{mg}, 12.1 \mu \mathrm{mol}, 1.00$ eq. $)$ is added and the solution is stirred for 30 seconds. $\mathrm{KOtBu}(1.4 \mathrm{mg}, 1.0 \mu \mathrm{mol}, 1.0 \mathrm{eq}$.) is added and stirring is continued for 3 minutes. After evaporation of the solvent the crude product is extracted with pentane and purified by chromatography (silanized silica, pentane). NMR spectra $\left(-30^{\circ}\right)$ were identical with spectra of the product obtained by route A.

\section{Synthesis of 40}

Complex 39 is prepared by route A and dissolved in benzene- $\mathrm{d}_{6}(0.5 \mathrm{~mL})$, transferred to a J-Young NMR tube and stirred at room temperature until complete conversion of 6 is detected by NMR. Lyophilization yields a brown powder as a mixture of the two diastereomers of $\mathbf{7}$ in a ratio about $60(\mathbf{A}): 40(\mathbf{B})$ according to NMR spectroscopy. 
Anal. Calcd for $\mathrm{C}_{39} \mathrm{H}_{70} \mathrm{NOOsP}_{3}$ (825.1):C, 55.4; H, 8.56; N, 1.70 Found: C, 55.5; H, 8.63, N, 1.68. LIFDI-MS (Toluene): $825.5\left(100 \%,[\mathrm{M}]^{+}\right)$.

$\operatorname{NMR}\left(\mathbf{A}, \mathrm{C}_{6} \mathrm{D}_{6}, \mathrm{RT}\right):{ }^{1} \mathrm{H}(500 \mathrm{MHz}): \delta=8.34\left(\mathrm{~m},{ }^{1} J_{\mathrm{HP}}=308.7 \mathrm{~Hz},{ }^{3} J_{\mathrm{HP}}=9.14,{ }^{3} J_{\mathrm{HP}}\right.$ $=4.67, \mathrm{P} H), 7.55-7.40\left(\mathrm{~m}, 3 \mathrm{H}, \mathrm{NCH}, \mathrm{C}_{\text {meta }} H\right), 7.18-7.15\left(\mathrm{~m}, 1 \mathrm{H}, \mathrm{C}_{\text {meta }} H\right), 4.52-4.44$ (m, 2H, $\mathrm{PCH}$ ), 2.92-2.85 (m, $\left.1 \mathrm{H}, \mathrm{PCH}_{2} \mathrm{C}\left(\mathrm{CH}_{3}\right)_{2}\right), 2.69-2.63\left(\mathrm{~m}, 1 \mathrm{H}, \mathrm{PCH}{ }_{2} \mathrm{C}\left(\mathrm{CH}_{3}\right)_{2}\right)$, $1.71\left(\mathrm{~s}, 9 \mathrm{H}, \mathrm{C}_{\text {ortho }} \mathrm{C}\left(\mathrm{CH}_{3}\right)_{3}\right), 1.64\left(\mathrm{~m}, 3 \mathrm{H}, \mathrm{PCH}_{2} \mathrm{C}\left(\mathrm{CH}_{3}\right)_{2}\right), 1.49\left(\mathrm{~d},{ }^{2} J_{\mathrm{HP}}=12.4 \mathrm{~Hz}\right.$, $\left.9 \mathrm{H}, \mathrm{PC}\left(\mathrm{CH}_{3}\right)_{3}\right), 1.32\left(\mathrm{~s}, 9 \mathrm{H}, \mathrm{C}_{\mathrm{para}} \mathrm{C}\left(\mathrm{CH}_{3}\right)_{3}\right), 1.25\left(\mathrm{~d},{ }^{2} J_{\mathrm{HP}}=12.1 \mathrm{~Hz}, 9 \mathrm{H}, \mathrm{PC}\left(\mathrm{CH}_{3}\right)_{3}\right)$, $1.14\left(\mathrm{~m}, 3 \mathrm{H}, \mathrm{PCH}_{2} \mathrm{C}\left(\mathrm{CH}_{3}\right)_{2}\right), 0.96\left(\mathrm{~d},{ }^{2} J_{\mathrm{HP}}=12.7 \mathrm{~Hz}, 9 \mathrm{H}, \mathrm{PC}\left(\mathrm{CH}_{3}\right)_{3}\right), 0.93\left(\mathrm{~d},{ }^{2} J_{\mathrm{HP}}=\right.$ $\left.11.0 \mathrm{~Hz}, 9 \mathrm{H}, \mathrm{PC}\left(\mathrm{CH}_{3}\right)_{3}\right),-44.7\left(\mathrm{ddd},{ }^{2} J_{\mathrm{HP}}=18.4 \mathrm{~Hz},{ }^{2} J_{\mathrm{HP}}=13.1 \mathrm{~Hz},{ }^{2} J_{\mathrm{HP}}=13.0 \mathrm{~Hz}\right.$, OsH $) .{ }^{31} \mathrm{P}\left\{{ }^{1} \mathrm{H}\right\}(161.25 \mathrm{MHz}): \delta=64.9\left(\mathrm{dd},{ }^{2} J_{\mathrm{PP}}=234.4 \mathrm{~Hz},{ }^{2} J_{\mathrm{PP}}=11.5 \mathrm{~Hz}\right), 53.0$ $\left(\mathrm{dd},{ }^{2} J_{\mathrm{PP}}=234.4 \mathrm{~Hz},{ }^{2} J_{\mathrm{PP}}=10.9 \mathrm{~Hz}\right),-54.6\left(\mathrm{dd},{ }^{2} J_{\mathrm{PP}}=11.5 \mathrm{~Hz},{ }^{2} J_{\mathrm{PP}}=10.9 \mathrm{~Hz}\right)$ $\operatorname{NMR}\left(\mathbf{B}, \mathrm{C}_{6} \mathrm{D}_{6}, \mathrm{RT}\right):{ }^{1} \mathrm{H}(500 \mathrm{MHz}): \delta=8.43\left(\mathrm{~m},{ }^{1} J_{\mathrm{HP}}=300.7 \mathrm{~Hz},{ }^{3} J_{\mathrm{HP}}=8.7,{ }^{3} J_{\mathrm{HP}}\right.$ $=4.8, \mathrm{P} H), 7.55-7.40\left(\mathrm{~m}, 3 \mathrm{H}, \mathrm{NCH}, \mathrm{C}_{\text {meta }} H\right), 7.18-7.15\left(\mathrm{~m}, 1 \mathrm{H}, \mathrm{C}_{\text {meta }} H\right), 4.57-4.52$ $(\mathrm{m}, 2 \mathrm{H}, \mathrm{PCH}), 2.53-2.41\left(\mathrm{~m}, 2 \mathrm{H}, \mathrm{PC} \mathrm{H}_{2} \mathrm{C}\left(\mathrm{CH}_{3}\right)_{2}\right), 1.51\left(\mathrm{~s}, 9 \mathrm{H}, \mathrm{C}_{\text {ortho }} \mathrm{C}\left(\mathrm{CH}_{3}\right)_{3}\right), 1.50$ $\left(\mathrm{m}, 3 \mathrm{H}, \mathrm{PCH}_{2} \mathrm{C}\left(\mathrm{CH}_{3}\right)_{2}\right), 1.43\left(\mathrm{~d},{ }^{2} J_{\mathrm{HP}}=11.4 \mathrm{~Hz}, 9 \mathrm{H}, \mathrm{PC}\left(\mathrm{CH}_{3}\right)_{3}\right), 1.41\left(\mathrm{~d},{ }^{2} J_{\mathrm{HP}}=\right.$ $\left.12.43 \mathrm{~Hz}, 9 \mathrm{H}, \mathrm{PC}\left(\mathrm{CH}_{3}\right)_{3}\right), 1.33\left(\mathrm{~s}, 9 \mathrm{H}, \mathrm{C}_{\mathrm{para}} \mathrm{C}\left(\mathrm{CH}_{3}\right)_{3}\right), 1.11\left(\mathrm{~d},{ }^{2} J_{\mathrm{HP}}=12.6 \mathrm{~Hz}, 9 \mathrm{H}\right.$, $\left.\mathrm{PC}\left(\mathrm{CH}_{3}\right)_{3}\right), 1.11\left(\mathrm{~m}, 3 \mathrm{H}, \mathrm{PCH}_{2} \mathrm{C}\left(\mathrm{CH}_{3}\right)_{2}\right), 0.91\left(\mathrm{~d},{ }^{2} J_{\mathrm{HP}}=12.5 \mathrm{~Hz}, 9 \mathrm{H}, \mathrm{PC}\left(\mathrm{CH}_{3}\right)_{3}\right)$, $-45.7\left(\mathrm{ddd},{ }^{2} J_{\mathrm{HP}}=21.4 \mathrm{~Hz},{ }^{2} J_{\mathrm{HP}}=14.4 \mathrm{~Hz},{ }^{2} J_{\mathrm{HP}}=14.4 \mathrm{~Hz}, \mathrm{Os} H\right) .{ }^{31} \mathrm{P}\left\{{ }^{1} \mathrm{H}\right\}(161.25$ $\mathrm{MHz}): \delta=68.0\left(\mathrm{dd},{ }^{2} J_{\mathrm{PP}}=234.8 \mathrm{~Hz},{ }^{2} J_{\mathrm{PP}}=10.5 \mathrm{~Hz}\right), 58.0\left(\mathrm{dd},{ }^{2} J_{\mathrm{PP}}=234.8 \mathrm{~Hz}\right.$, $\left.{ }^{2} J_{\mathrm{PP}}=10.8 \mathrm{~Hz}\right),-55.4\left(\mathrm{dd},{ }^{2} J_{\mathrm{PP}}=10.8 \mathrm{~Hz},{ }^{2} J_{\mathrm{PP}}=10.5 \mathrm{~Hz}\right)$.

\section{Synthesis of 41}

38 (20.0 mg, $24.2 \mu \mathrm{mol}, 1.00$ eq.) and TEMPO (11.4 mg, $72.7 \mu \mathrm{mol}, 3.00 \mathrm{eq})$ are dissolved in pentane and stirred for $10 \mathrm{~min}$ at room temperature. The solution is degassed by three freeze-pump-thaw cycles and one atmosphere of $\mathrm{CO}$ is added. The solution is stirred at $-80^{\circ}$ for 5 minutes and the solvent is removed $i$. vac. The crude product is purified via column chromatography (silanized silica, pentane) and crystalized at $-80^{\circ}$ to give $\mathbf{4 1}$ (16.5 mg, $19.4 \mu \mathrm{mol}, 80 \%)$ as dark red crystals.

Anal. Calcd for $\mathrm{C}_{39} \mathrm{H}_{70} \mathrm{NOOsP}_{3}$ (825.1): C, 54.9; H, 8.28; N, 1.64 Found: C, 54.6; H, 8.34; N, 1.55. NMR $($ THF-d 8$):{ }^{1} \mathrm{H}(500 \mathrm{MHz}): \delta=9.79\left(\mathrm{ddd},{ }^{1} J_{\mathrm{PH}}=308.9 \mathrm{~Hz},{ }^{3} J_{\mathrm{PH}}\right.$ $\left.=13.3 \mathrm{~Hz},{ }^{3} J_{\mathrm{PH}}=4.9 \mathrm{~Hz}, 1 \mathrm{H}, \mathrm{P} H \mathrm{Mes}^{*}\right), 7.44\left(\mathrm{ddd},{ }^{3} J_{\mathrm{PH}}=36.6 \mathrm{~Hz},{ }^{3} J_{\mathrm{HH}}=6.0 \mathrm{~Hz}\right.$, $\left.{ }^{4} J_{\mathrm{PH}}=1.4 \mathrm{~Hz}, 1 \mathrm{H}, \mathrm{NCH}\right), 7.33\left(\mathrm{ddd},{ }^{3} J_{\mathrm{PH}}=34.6 \mathrm{~Hz},{ }^{3} J_{\mathrm{HH}}=5.9 \mathrm{~Hz},{ }^{4} J_{\mathrm{PH}}=2.2 \mathrm{~Hz}\right.$, $1 \mathrm{H}, \mathrm{NCH}), 7.24(\mathrm{~m}, 2 \mathrm{H}$, Cmeta $H), 4.44\left(\mathrm{~m},{ }^{2} J_{\mathrm{PH}}=5.1 \mathrm{~Hz},{ }^{3} J_{\mathrm{HH}}=6.0 \mathrm{~Hz},{ }^{4} J_{\mathrm{PH}}=\right.$ $1.7 \mathrm{~Hz}, 1 \mathrm{H}, \mathrm{PCH}), 4.33\left(\mathrm{~m},{ }^{2} J_{\mathrm{PH}}=5.0 \mathrm{~Hz},{ }^{3} J_{\mathrm{HH}}=5.8 \mathrm{~Hz},{ }^{4} J_{\mathrm{PH}}=1.8 \mathrm{~Hz}, 1 \mathrm{H}, \mathrm{PCH}\right)$, $\left.\left.1.62\left(\mathrm{~s}, 9 \mathrm{H}, \mathrm{C}_{\text {ortho }}\left(\mathrm{CH}_{3}\right)_{3}\right)\right), 1.61\left(\mathrm{~s}, 9 \mathrm{H}, \mathrm{C}_{\text {ortho }}\left(\mathrm{CH}_{3}\right)_{3}\right)\right), 1.51\left(\mathrm{~d},{ }^{3} J_{\mathrm{PH}}=13.5 \mathrm{~Hz}, 9 \mathrm{H}\right.$, $\left.\left.\left.\left.\mathrm{PC}\left(\mathrm{CH}_{3}\right)_{3}\right)\right), 1.31\left(\mathrm{~d},{ }^{3} J_{\mathrm{PH}}=13.0 \mathrm{~Hz}, 9 \mathrm{H}, \mathrm{PC}\left(\mathrm{C} H_{3}\right)_{3}\right)\right), 1.31\left(\mathrm{~s}, 9 \mathrm{H}, \mathrm{C}_{\mathrm{para}}\left(\mathrm{CH}_{3}\right)_{3}\right)\right)$, $\left.\left.1.03\left(\mathrm{~d},{ }^{3} J_{\mathrm{PH}}=13.8 \mathrm{~Hz}, 9 \mathrm{H}, \mathrm{PC}\left(\mathrm{CH}_{3}\right)_{3}\right)\right), 0.81\left(\mathrm{~d},{ }^{3} J_{\mathrm{PH}}=13.4 \mathrm{~Hz}, 9 \mathrm{H}, \mathrm{PC}\left(\mathrm{CH}_{3}\right)_{3}\right)\right)$. ${ }^{13} \mathrm{C}\left\{{ }^{1} \mathrm{H}\right\}(125.76 \mathrm{MHz}): \delta=181.7\left(\mathrm{ddd},{ }^{2} J_{\mathrm{CP}}=48.3 \mathrm{~Hz},{ }^{2} J_{\mathrm{CP}}=6.0,{ }^{2} J_{\mathrm{CP}}=5.6,1 \mathrm{C}\right.$, Os- $C \mathrm{O}), 164.9\left(\mathrm{~d},{ }^{2} J_{\mathrm{CP}}=12.0 \mathrm{~Hz}, 1 \mathrm{C}, \mathrm{NCH}\right), 163.7\left(\mathrm{~d},{ }^{2} J_{\mathrm{CP}}=11.0 \mathrm{~Hz}, 1 \mathrm{C}, \mathrm{NCH}\right)$, 155.7 (br, $\left.1 \mathrm{C}, \mathrm{C}_{\text {ortho }}\right), 155.6\left(\mathrm{~d},{ }^{2} J_{\mathrm{CP}}=4.1 \mathrm{~Hz}, 1 \mathrm{C}, C^{\text {ortho }}\right), 148.8\left(\mathrm{~d},{ }^{4} J_{\mathrm{CP}}=2.2 \mathrm{~Hz}, 1 \mathrm{C}\right.$, $\left.\mathrm{C}^{\text {para }}\right), 140.2\left(\mathrm{~d},{ }^{1} J_{\mathrm{CP}}=6.1 \mathrm{~Hz}, 1 \mathrm{C}, \mathrm{C}_{\mathrm{ipso}}\right), 123.0\left(\mathrm{~d},{ }^{3} J_{\mathrm{CP}}=5.1 \mathrm{~Hz}, 1 \mathrm{C}, \mathrm{C}_{\text {meta }}\right), 122.2$ $\left(\mathrm{d},{ }^{3} J_{\mathrm{CP}}=10.2 \mathrm{~Hz}, 1 \mathrm{C}, \mathrm{C}_{\text {meta }}\right), 88.4\left(\mathrm{dd},{ }^{1} J_{\mathrm{CP}}=45.5 \mathrm{~Hz},{ }^{3} J_{\mathrm{CP}}=3.7 \mathrm{~Hz}, 1 \mathrm{C}, \mathrm{PCH}\right)$, $86.7\left(\mathrm{dd},{ }^{1} J_{\mathrm{CP}}=46.8 \mathrm{~Hz},{ }^{3} J_{\mathrm{CP}}=5.0 \mathrm{~Hz}, 1 \mathrm{C}, \mathrm{PCH}\right), 41.9\left(\mathrm{ddd},{ }^{1} J_{\mathrm{CP}}=22.0 \mathrm{~Hz},{ }^{3} J_{\mathrm{CP}}\right.$ $\left.=7.4,{ }^{3} J_{\mathrm{CP}}=3.1 \mathrm{~Hz}, 1 \mathrm{C}, \mathrm{PC}\left(\mathrm{CH}_{3}\right)_{3}\right), 40.8\left(\mathrm{ddd},{ }^{1} J_{\mathrm{CP}}=21.1 \mathrm{~Hz},{ }^{3} J_{\mathrm{CP}}=7.5,{ }^{3} J_{\mathrm{CP}}\right.$ $\left.=2.9 \mathrm{~Hz}, 1 \mathrm{C}, \mathrm{PC}\left(\mathrm{CH}_{3}\right)_{3}\right), 40.7\left(\mathrm{~s}, 1 \mathrm{C}, \mathrm{C}_{\text {ortho }} C\left(\mathrm{CH}_{3}\right)_{3}\right), 40.0\left(\mathrm{~s}, 1 \mathrm{C}, \mathrm{C}_{\text {ortho }} C\left(\mathrm{CH}_{3}\right)_{3}\right)$, 
$39.9\left(\mathrm{dd},{ }^{1} J_{\mathrm{CP}}=23.5 \mathrm{~Hz},{ }^{3} J_{\mathrm{CP}}=2.2,1 \mathrm{C}, \mathrm{PC}\left(\mathrm{CH}_{3}\right)_{3}\right), 37.5\left(\mathrm{dd},{ }^{1} J_{\mathrm{CP}}=24.2 \mathrm{~Hz}\right.$, $\left.{ }^{3} J_{\mathrm{CP}}=2.0,1 \mathrm{C}, \mathrm{P} C\left(\mathrm{CH}_{3}\right)_{3}\right), 35.3\left(\mathrm{~s}, 1 \mathrm{C}, \mathrm{C}_{\mathrm{para}} C\left(\mathrm{CH}_{3}\right)_{3}\right), 35.1\left(\mathrm{~s}, 3 \mathrm{C}, \mathrm{C}_{\text {ortho }} \mathrm{C}\left(\mathrm{CH}_{3}\right)_{3}\right)$, $33.6\left(\mathrm{~s}, 3 \mathrm{C}, \mathrm{C}_{\text {ortho }} \mathrm{C}\left(\mathrm{CH}_{3}\right)_{3}\right), 31.9\left(\mathrm{~s}, 3 \mathrm{C}, \mathrm{C}_{\text {para }} \mathrm{C}\left(\mathrm{CH}_{3}\right)_{3}\right), 31.4\left(\mathrm{~d},{ }^{2} J_{\mathrm{CP}}=4.9 \mathrm{~Hz}, 3 \mathrm{C}\right.$, $\left.\mathrm{PC}\left(\mathrm{CH}_{3}\right)_{3}\right), 31.3\left(\mathrm{~d},{ }^{2} J_{\mathrm{CP}}=3.8 \mathrm{~Hz}, 3 \mathrm{C}, \mathrm{PC}\left(\mathrm{CH}_{3}\right)_{3}\right), 30.5\left(\mathrm{br}, 3 \mathrm{C}, \mathrm{PC}\left(\mathrm{CH}_{3}\right)_{3}\right), 30.2$ (br, 3C, $\left.\mathrm{PC}\left(C \mathrm{H}_{3}\right)_{3}\right) .{ }^{31} \mathrm{P}\left\{{ }^{1} \mathrm{H}\right\}(161.25 \mathrm{MHz}): \delta=66.9\left(\mathrm{dd},{ }^{2} J_{\mathrm{PP}}=197,1 \mathrm{~Hz},{ }^{2} J_{\mathrm{PP}}=\right.$ 35.4, $\left.1 \mathrm{P}, P \mathrm{C}\left(\mathrm{CH}_{3}\right)_{3}\right), 53.5\left(\mathrm{dd},{ }^{2} J_{\mathrm{PP}}=197,1 \mathrm{~Hz},{ }^{2} J_{\mathrm{PP}}=35.3,1 \mathrm{P}, P \mathrm{C}\left(\mathrm{CH}_{3}\right)_{3}\right), 17.7$ $\left(\mathrm{dd},{ }^{2} J_{\mathrm{PP}}=35.4 \mathrm{~Hz},{ }^{2} J_{\mathrm{PP}}=35.3,1 \mathrm{P}\right.$, PHMes$\left.{ }^{*}\right) . \quad \mathrm{IR}\left(\mathrm{KBr}, \mathrm{cm}^{-1}\right): 2333\left(\tilde{\nu}_{\mathrm{PH}}\right), 1888$ $\left(\tilde{\nu}_{\mathrm{CO}}\right)$. LIFDI-MS (Toluene): $853.4\left(100 \%,[\mathrm{M}]^{+}\right)$.

\section{Reaction of 39 with $\mathrm{PhCCH}$}

38 (20.0 mg, $24.2 \mu \mathrm{mol}, 1.00$ eq.) and TEMPO (11.4 mg, $72.7 \mu \mathrm{mol}, 3.00 \mathrm{eq})$ are dissolved in pentane and stirred for $10 \mathrm{~min}$ at room temperature. TEMPOH is removed by column chromatography (pentane, silanized silica), $\mathrm{PhCCH}(2.7 \mu \mathrm{L}, 24 \mu \mathrm{mol}, 1.0$ eq.) is added and stirring is continued for $16 \mathrm{~h}$.

$\operatorname{NMR}\left(\mathrm{C}_{6} \mathrm{D}_{6}, \mathrm{RT}\right):{ }^{1} \mathrm{H}(500 \mathrm{MHz}): \delta=9.39\left(\mathrm{dddd},{ }^{1} J_{\mathrm{HP}}=308.7 \mathrm{~Hz},{ }^{3} J_{\mathrm{HP}}=12.3 \mathrm{~Hz}\right.$, $\left.{ }^{3} J_{\mathrm{HP}}=5.3 \mathrm{~Hz},{ }^{4} J_{\mathrm{HH}}=1.6 \mathrm{~Hz}, 1 \mathrm{H}, H \mathrm{PMes}^{*}\right), 7.41\left(\mathrm{~d},{ }^{4} J_{\mathrm{HH}}=2.3 \mathrm{~Hz}, \mathrm{C}_{\text {meta }} \mathrm{H}, \mathrm{HPMes}{ }^{*}\right)$, $7.40\left(\mathrm{~d},{ }^{4} J_{\mathrm{HH}}=2.3 \mathrm{~Hz}, \mathrm{C}_{\text {meta }} \mathrm{H}, \mathrm{HPMes}{ }^{*}\right), 7.29\left(\mathrm{~m},{ }^{3} J_{\mathrm{HH}}=6.2 \mathrm{~Hz}, 1 \mathrm{H}, \mathrm{NCHCHP}\right), 7.21$ $\left(\mathrm{m}, 2 \mathrm{H}, \mathrm{C}_{\text {meta }} \mathrm{H}, \mathrm{Ph}\right), 7.20\left(\mathrm{~m},{ }^{3} J_{\mathrm{HH}}=6.5 \mathrm{~Hz}, 1 \mathrm{H}, \mathrm{NCHCHP}\right), 6.87\left(\mathrm{~d},{ }^{3} J_{\mathrm{HH}}=7.4 \mathrm{~Hz}\right.$, $\left.2 \mathrm{H}, \mathrm{C}_{\text {ortho }} \mathrm{H}, \mathrm{Ph}\right), 6.76\left(\mathrm{tt},{ }^{3} J_{\mathrm{HH}}=7.3 \mathrm{~Hz},{ }^{4} J_{\mathrm{HH}}=1.2 \mathrm{~Hz}, 1 \mathrm{H}, \mathrm{C}_{\mathrm{para}} \mathrm{H}, \mathrm{Ph}\right), 4.37$ $\left(\mathrm{m},{ }^{3} J_{\mathrm{HH}}=6.2 \mathrm{~Hz}, 1 \mathrm{H}, \mathrm{NCHCHP}\right), 4.36\left(\mathrm{~m}, 1 \mathrm{H},{ }^{3} J_{\mathrm{HH}}=6.5 \mathrm{~Hz} \mathrm{NCHCHP}\right), 2.68$ $\left(\mathrm{m},{ }^{4} J_{\mathrm{HH}}=1.6 \mathrm{~Hz}, \mathrm{Os}=\mathrm{C}=\mathrm{CHPh}\right), 1.81\left(\mathrm{~s}, 9 \mathrm{H}, \mathrm{C}_{\text {ortho }} \mathrm{C}\left(\mathrm{CH}_{3}\right)_{3}, \mathrm{HPMes}^{*}\right), 1.79(\mathrm{~s}$, $\left.9 \mathrm{H}, \mathrm{C}_{\text {ortho }} \mathrm{C}\left(\mathrm{CH}_{3}\right)_{3}, \mathrm{HPMes}^{*}\right), 1.46\left(\mathrm{~d},{ }^{3} J_{\mathrm{HP}}=13.8 \mathrm{~Hz}, 9 \mathrm{H}, \mathrm{PC}\left(\mathrm{CH}_{3}\right)_{3}\right), 1.36(\mathrm{~s}, 9 \mathrm{H}$, $\mathrm{C}_{\text {para }} \mathrm{C}\left(\mathrm{CH}_{3}\right)_{3}$, HPMes $\left.{ }^{*}\right), 1.34\left(\mathrm{br}, 9 \mathrm{H}, \mathrm{PC}\left(\mathrm{CH}_{3}\right)_{3}\right), 1.12\left(\mathrm{~d},{ }^{3} J_{\mathrm{HP}}=14.0 \mathrm{~Hz}, \mathrm{PC}\left(\mathrm{CH}_{3}\right)_{3}\right)$, $0.84\left(\mathrm{~d},{ }^{3} J_{\mathrm{HP}}=13.2 \mathrm{~Hz}, 9 \mathrm{H}, \mathrm{PC}\left(\mathrm{CH}_{3}\right)_{3}\right) .{ }^{13} \mathrm{C}\left\{{ }^{1} \mathrm{H}\right\}(125.76 \mathrm{MHz}): \delta=274.8(\mathrm{ddd}$, $\left.{ }^{2} J_{\mathrm{CP}}=37.0 \mathrm{~Hz},{ }^{2} J_{\mathrm{CP}}=8.7 \mathrm{~Hz},{ }^{2} J_{\mathrm{CP}}=8.2 \mathrm{~Hz}, 1 \mathrm{C}, \mathrm{Os}=\mathrm{C}=\mathrm{CHPh}\right), 164.9\left(\mathrm{~d},{ }^{2} J_{\mathrm{CP}}=\right.$ $12.0 \mathrm{~Hz}, 1 \mathrm{C}, \mathrm{NCHCHP}), 163.7\left(\mathrm{~d},{ }^{2} J_{\mathrm{CP}}=11.2 \mathrm{~Hz}, 1 \mathrm{C}, \mathrm{NCHCHP}\right), 156.7\left(\mathrm{~d},{ }^{2} J_{\mathrm{CP}}=\right.$ $\left.15.9 \mathrm{~Hz}, 1 \mathrm{C}, C_{\text {ortho }} \mathrm{C}\left(\mathrm{CH}_{3}\right)_{3}, \mathrm{HPMes}^{*}\right), 156.0\left(\mathrm{~d},{ }^{2} J_{\mathrm{CP}}=6.4 \mathrm{~Hz}, 1 \mathrm{C}, C_{\text {ortho }} \mathrm{C}\left(\mathrm{CH}_{3}\right)_{3}\right.$, HPMes*), 148.3 (s, 1C, $C_{\text {para }} \mathrm{C}\left(\mathrm{CH}_{3}\right)_{3}$, HPMes*), 140.0 (s, $1 \mathrm{C}, C_{i p s o} \mathrm{C}\left(\mathrm{CH}_{3}\right)_{3}$, HPMes$\left.^{*}\right)$, 133.7 (s, 1C, $\left.C_{\text {ipso }}, \mathrm{Ph}\right), 124.4$ (s, 2C, $\left.C_{\text {ortho }}, \mathrm{Ph}\right), 122.7$ (s, 1C, $\left.C_{\text {para }}, \mathrm{Ph}\right), 122.3$ $\left(\mathrm{d},{ }^{3} J_{\mathrm{CP}}=4.8 \mathrm{~Hz}, 1 \mathrm{C}, C_{\text {meta }} C(C H 3) 3\right.$, HPMes $\left.^{*}\right), 121.6\left(\mathrm{~d},{ }^{3} J_{\mathrm{CP}}=11.3 \mathrm{~Hz}, 1 \mathrm{C}\right.$, $C_{\text {meta }} C(C H 3) 3$, HPMes*), $113.2(\mathrm{~s}, 1 \mathrm{C}, \mathrm{Os}=\mathrm{C}=\mathrm{CHPh}), 92.2\left(\mathrm{~d},{ }^{1} J_{\mathrm{CP}}=42.6 \mathrm{~Hz}, 1 \mathrm{C}\right.$, $\mathrm{NCH} C \mathrm{HP}), 90.3\left(\mathrm{dd},{ }^{1} J_{\mathrm{CP}}=43.5 \mathrm{~Hz},{ }^{3} J_{\mathrm{CP}}=4.5 \mathrm{~Hz}, 1 \mathrm{C}, \mathrm{NCHCHP}\right), 40.8\left(\mathrm{~d},{ }^{3} J_{\mathrm{CP}}=\right.$ $\left.21.16 \mathrm{~Hz}, 1 \mathrm{C}, \mathrm{PC}\left(\mathrm{CH}_{3}\right)_{3}\right), 40.6\left(\mathrm{~s}, 1 \mathrm{C}, \mathrm{C}_{\text {ortho }} \mathrm{C}\left(\mathrm{CH}_{3}\right)_{3}, \mathrm{HPMes}^{*}\right), 40.0\left(\mathrm{~d},{ }^{3} J_{\mathrm{CP}}=20.8 \mathrm{~Hz}\right.$, $\left.1 \mathrm{C}, \mathrm{PC}\left(\mathrm{CH}_{3}\right)_{3}\right), 39.4\left(\mathrm{~s}, 1 \mathrm{C}, \mathrm{C}_{\text {ortho }} \mathrm{C}\left(\mathrm{CH}_{3}\right)_{3}\right.$, HPMes $\left.^{*}\right), 37.8\left(\mathrm{dd},{ }^{3} J_{\mathrm{CP}}=19.0 \mathrm{~Hz}\right.$, $\left.{ }^{5} J_{\mathrm{CP}}=3.2 \mathrm{~Hz}, 1 \mathrm{C}, \mathrm{PC}\left(\mathrm{CH}_{3}\right)_{3}\right), 35.9\left(\mathrm{dd},{ }^{3} J_{\mathrm{CP}}=20.3 \mathrm{~Hz},{ }^{5} J_{\mathrm{CP}}=3.1 \mathrm{~Hz}, 1 \mathrm{C}, \mathrm{PC}\left(\mathrm{CH}_{3}\right)_{3}\right)$, 35.0 (s, 3C, $\mathrm{C}_{\text {ortho }} \mathrm{C}\left(\mathrm{CH}_{3}\right)_{3}$, HPMes*), 33.5 (s, 3C, $\mathrm{C}_{\text {ortho }} \mathrm{C}\left(\mathrm{CH}_{3}\right)_{3}$, HPMes*), 31.7 (s, 3C, $\mathrm{C}_{\text {para }} \mathrm{C}\left(\mathrm{CH}_{3}\right)_{3}$, HPMes*), $31.2\left(\mathrm{~s}, 3 \mathrm{C}, \mathrm{PC}\left(\mathrm{CH}_{3}\right)_{3}\right), 31.0$ (s, 3C, $\left.\mathrm{PC}\left(\mathrm{CH}_{3}\right)_{3}\right), 30.0$ (br, 6C, $\left.\mathrm{PC}\left(\mathrm{CH}_{3}\right)_{3}\right) .{ }^{31} \mathrm{P}\left\{{ }^{1} \mathrm{H}\right\}(161.25 \mathrm{MHz}): \delta=50.0\left(\mathrm{dd},{ }^{2} J_{\mathrm{PP}}=242.3 \mathrm{~Hz},{ }^{2} J_{\mathrm{PP}}=24.6 \mathrm{~Hz}\right.$ $1 \mathrm{P}, P C(C H 3) 3), 36.5\left(\mathrm{dd},{ }^{2} J_{\mathrm{PP}}=242.3 \mathrm{~Hz},{ }^{2} J_{\mathrm{PP}}=18.2 \mathrm{~Hz} 1 \mathrm{P}, P C(C H 3) 3\right), 3.52(\mathrm{dd}$, ${ }^{2} J_{\mathrm{PP}}=24.6 \mathrm{~Hz},{ }^{2} J_{\mathrm{PP}}=18.2 \mathrm{~Hz}, 1 \mathrm{P}$, PMes$\left.*\right)$.

\section{Synthesis of 43}

38 (10.0 mg, $12.1 \mu \mathrm{mol}, 1.00$ eq.) is dissolved in $\mathrm{Et}_{2} \mathrm{O}(1 \mathrm{~mL})$ and cooled to $-35^{\circ} . \mathrm{Ag}[\mathrm{Al}(\mathrm{O}-$ $\left.\left.{ }^{t} \mathrm{Bu}-\mathrm{F}_{9}\right)_{4}\right](13.0 \mathrm{mg}, 12.1 \mu \mathrm{mol}, 1.00 \mathrm{eq}$.$) is added and the solution is stirred for 30 \mathrm{sec}-$ onds. After filtration the solvent is removed and the residue is extracted with $\mathrm{Et}_{2} \mathrm{O}$ 
$(5 \times 0.5 \mathrm{~mL})$ at $-35^{\circ}$. After concentration the solution is layered with pentane $(5 \mathrm{~mL})$ and stored at $-85^{\circ}$ for $24 \mathrm{~h}$. The solution is decanted off and the solid is washed with pentane at $-30^{\circ}(5 \times 1 \mathrm{~mL})$. After drying in vacuo at $-30^{\circ} 43$ is obtained as a blue solid (18.0 mg, $10.0 \mu \mathrm{mol}, 83 \%)$ NMR spectroscopic characterization shows minor decomposition products and some residual solvent $\left(\mathrm{Et}_{2} \mathrm{O} /\right.$ pentane). Elemental analysis did not give reproducible data due to high reactivity.

NMR $\left(\right.$ THF $\left.-\mathrm{d}_{8},-30^{\circ} \mathrm{C}\right):{ }^{1} \mathrm{H}(500 \mathrm{MHz}): \delta=18.3\left(\mathrm{~d},{ }^{1} J_{\mathrm{HP}}=345.5 \mathrm{~Hz}, 1 \mathrm{H}, \mathrm{P} H\right.$ Mes $\left.^{*}\right)$, $7.86-7.68(\mathrm{~m}, 2 \mathrm{H}, \mathrm{NCHCHP}), 7.50\left(\mathrm{~d},{ }^{4} J_{\mathrm{HH}}=1.83 \mathrm{~Hz}, 1 \mathrm{H}, \mathrm{C}_{\text {meta }} H, \mathrm{Mes}^{*}\right), 7.42(\mathrm{~d}$, $\left.{ }^{4} J_{\mathrm{HH}}=1.893 \mathrm{~Hz}, 1 \mathrm{H}, \mathrm{C}_{\text {meta }} H, \mathrm{Mes}^{*}\right), 5.79(\mathrm{~m}, 1 \mathrm{H}, \mathrm{NCHC} H \mathrm{P}), 5.55(\mathrm{~m}, 1 \mathrm{H}, \mathrm{NCHC} H \mathrm{P})$, $1.58\left(\mathrm{~d},{ }^{3} J_{\mathrm{HP}}=14.0 \mathrm{~Hz}, 9 \mathrm{H}, \mathrm{PC}\left(\mathrm{CH}_{3}\right)_{3}\right), 1.52\left(\mathrm{~d},{ }^{3} J_{\mathrm{HP}}=13.9 \mathrm{~Hz}, 9 \mathrm{H}, \mathrm{PC}\left(\mathrm{CH}_{3}\right)_{3}\right)$, $1.46\left(\mathrm{~s}, 9 \mathrm{H}, \mathrm{C}_{\text {ortho }} \mathrm{C}\left(\mathrm{CH}_{3}\right)_{3}, \mathrm{Mes}^{*}\right), 1.40$ (s, $\left.9 \mathrm{H}, \mathrm{C}_{\text {ortho }} \mathrm{C}\left(\mathrm{CH}_{3}\right)_{3}, \mathrm{Mes}^{*}\right), 1.36$ (s, 9H, $\mathrm{C}_{\text {para }} \mathrm{C}\left(\mathrm{CH}_{3}\right)_{3}$, Mes*), 1.10-1.00 (m, 18H, PC $\left.\left.(\mathrm{CH})_{3}\right)_{3}\right),-21.7$ (m, $1 \mathrm{H}$, Os $\left.H\right) .{ }^{13} \mathrm{C}\left\{{ }^{1} \mathrm{H}\right\}$ (125.76 MHz): $\delta=168.2(\mathrm{~m}, 1 \mathrm{C}, \mathrm{NCHCHP}), 167.4(\mathrm{~m}, 1 \mathrm{C}, \mathrm{NCHCHP}), 154.5$ (s, 1C, $C_{\text {para }}, M^{*}$ ), 151.6 (s, 1C, $C_{\text {ortho }}$, Mes*), 151.9 (s, 1C, $C_{\text {ortho }}$, Mes*), 143.7 (br, 1C, $\left.C_{\text {ipso }}, M^{*}\right), 125.16$ (br, 2C, $\left.C_{\text {meta }}, M^{*}\right), 122.3\left(\mathrm{q},{ }^{1} J_{\mathrm{CF}}=291.8 \mathrm{~Hz}, 12 \mathrm{C}, C \mathrm{~F}_{3}\right.$ ), 110.0-108.9 (m, 2C, NCHCHP), 79.9 (br, $\left.4 \mathrm{C}, C \mathrm{CF}_{3}\right), 40.8$ (s, $\left.1 \mathrm{C}, \mathrm{C}_{\text {ortho }} C\left(\mathrm{CH}_{3}\right)_{3}\right)$, $40.3\left(\mathrm{~d},{ }^{1} J_{\mathrm{CP}}=21.6 \mathrm{~Hz}, 1 \mathrm{C}, \mathrm{P} C\left(\mathrm{CH}_{3}\right)_{3}\right), 40.1\left(\mathrm{~s}, 1 \mathrm{C}, \mathrm{C}_{\text {ortho }} C\left(\mathrm{CH}_{3}\right)_{3}\right), 38.2\left(\mathrm{~d},{ }^{1} J_{\mathrm{CP}}\right.$ $\left.=21.3 \mathrm{~Hz}, 1 \mathrm{C}, \mathrm{PC}\left(\mathrm{CH}_{3}\right)_{3}\right), 37.73\left(\mathrm{~d},{ }^{1} J_{\mathrm{CP}}=22.7 \mathrm{~Hz}, 1 \mathrm{C}, \mathrm{PC}\left(\mathrm{CH}_{3}\right)_{3}\right), 35.8(\mathrm{~s}, 1 \mathrm{C}$, $\left.\mathrm{C}_{\text {para }} C\left(\mathrm{CH}_{3}\right)_{3}\right), 34.7\left(\mathrm{~d},{ }^{1} J_{\mathrm{CP}}=22.4 \mathrm{~Hz}, 1 \mathrm{C}, \mathrm{PC}\left(\mathrm{CH}_{3}\right)_{3}\right), 33.8\left(\mathrm{~s}, 3 \mathrm{C}, \mathrm{C}_{\text {ortho }} \mathrm{C}\left(C_{3} \mathrm{H}_{3}\right)_{3}\right)$, 33.6 (s, 3C, $\left.\mathrm{C}_{\text {ortho }} \mathrm{C}\left(\mathrm{CH}_{3}\right)_{3}\right), 31.4\left(\mathrm{~s}, 3 \mathrm{C}, \mathrm{C}_{\text {para }} \mathrm{C}\left(\mathrm{CH}_{3}\right)_{3}\right), 30.9$ (br, 3C, $\left.\mathrm{PC}\left(\mathrm{CH}_{3}\right)_{3}\right)$, 30.7 (br, 3C, $\left.\mathrm{PC}\left(\mathrm{CH}_{3}\right)_{3}\right), 30.0$ (br, 3C, $\left.\mathrm{PC}\left(\mathrm{CH}_{3}\right)_{3}\right), 29.3$ (br, $\left.3 \mathrm{C}, \mathrm{PC}\left(C \mathrm{H}_{3}\right)_{3}\right) .{ }^{31} \mathrm{P}\left\{{ }^{1} \mathrm{H}\right\}$ $(161.25 \mathrm{MHz}): \delta=169.2(\mathrm{~m}, 1 \mathrm{P}, P \mathrm{H} \mathrm{Mes} *), 61.5\left(\mathrm{dd},{ }^{2} J_{\mathrm{PP}}=224.0 \mathrm{~Hz},{ }^{2} J_{\mathrm{PP}}=18.0 \mathrm{~Hz}\right.$, $\left.1 \mathrm{P}, P \mathrm{C}\left(\mathrm{CH}_{3}\right)_{3}\right), 52.28\left(\mathrm{dd},{ }^{2} J_{\mathrm{PP}}=224.0 \mathrm{~Hz},{ }^{2} J_{\mathrm{PP}}=14.2 \mathrm{~Hz}, 1 \mathrm{P}, P \mathrm{C}\left(\mathrm{CH}_{3}\right)_{3}\right)$. IR (Nujol, $\left.\mathrm{cm}^{-1}\right): 2350\left(\tilde{\nu}_{\mathrm{PH}}\right)$. LIFDI-MS (THF): $826.4\left(100 \%,[\mathrm{M}]^{+}\right)$.

\section{Synthesis of 44}

1 (15.0 mg, $24.3 \mu \mathrm{mol}, 1.00$ eq.) and $\mathrm{CoCp}_{2}(4.6 \mathrm{mg}, 24 \mu \mathrm{mol}, 1.0 \mathrm{eq}$.$) are dissolved in$ toluene $(1 \mathrm{~mL})$ stirred for 1 minutes and filtered. The residue is extracted with toluene $(0.5 \mathrm{~mL})$ and the combined filtrates are cooled to $-80^{\circ} \mathrm{C}$. ClPA (A/ClPA: $75 / 25,18.4$ $\mathrm{mg}, 94.8 \mu \mathrm{mol}, 3.9$ eq.) is added and the solution is warmed to room temperature over $1 \mathrm{~h}$. The solution is filtered and the solvent is removed. DCM $(1 \mathrm{~mL})$ is added and the solution is cooled to $-80^{\circ} \mathrm{C}$ for $4 \mathrm{~h}$. The anthracene is decanted and more anthracene is crystallized from $\mathrm{DCM} /$ pentane $(0.5 \mathrm{~mL} / 5 \mathrm{~mL})$ at $-80^{\circ} \mathrm{C}$. The anthracene is decanted and the obtained solution is subjected to filtration through an activated charcoal column. The solvent is removed the crude product is layered with pentane $(3 \mathrm{~mL})$ and stored at $-30^{\circ} \mathrm{C}$ for 2 days. The solution is decanted and the solid is dried in vacuo. $44(8.0 \mathrm{mg}$, $13 \mu \mathrm{mol}, 50 \%)$ is obtained as a purple solid.

Anal. Calcd. for $\mathrm{C}_{20} \mathrm{H}_{40} \mathrm{Cl}_{2} \mathrm{NOsP}_{3}(648,6)$ : C, 37.0; H, 6.22; N, 2.16. Found: C, 37.4; $\mathrm{H}, 6.53 ; \mathrm{N}, 2.03$.

$\operatorname{NMR}\left(\mathrm{CD}_{2} \mathrm{Cl}_{2}, \mathrm{RT}\right):{ }^{1} \mathrm{H}(400 \mathrm{MHz}) \delta=7.36\left(\mathrm{~A}_{18} \mathrm{BCXX}^{\prime} \mathrm{A}_{18}{ }_{18} \mathrm{~B}^{\prime} \mathrm{C}^{\prime}, N=\left|{ }^{3} J_{\mathrm{CX}}+{ }^{4} J_{\mathrm{CX}}\right|\right.$ $\left.=35.7 \mathrm{~Hz},{ }^{3} J_{\mathrm{BC}}=6.1 \mathrm{~Hz}, 2 \mathrm{H}, \mathrm{NCH}\right), 4.32\left(\mathrm{~A}_{18} \mathrm{BCXX}^{\prime} \mathrm{A}_{18}^{\prime} \mathrm{B}^{\prime} \mathrm{C}^{\prime}, N=\left|{ }^{2} J_{\mathrm{BX}}+{ }^{4} J_{\mathrm{BX}}\right|\right.$ $\left.=6.6 \mathrm{~Hz},{ }^{3} J_{\mathrm{BC}}=6.1 \mathrm{~Hz}, 2 \mathrm{H}, \mathrm{PCH}\right), 1.37\left(\mathrm{~A}_{18} \mathrm{BCXX}^{\prime} \mathrm{A}_{18}{ }_{18} \mathrm{~B}^{\prime} \mathrm{C}^{\prime},{ }^{3} J_{\mathrm{AX}}=14.7 \mathrm{~Hz}, 18 \mathrm{H}\right.$, $\left.\mathrm{P}\left(\mathrm{C}\left(\mathrm{CH}_{3}\right)_{3}\right)\right), 1.24\left(\mathrm{~A}_{18} \mathrm{BCXX}^{\prime} \mathrm{A}_{18}{ }_{18} \mathrm{~B}^{\prime} \mathrm{C}^{\prime},{ }^{3} J_{\mathrm{AX}}=14.3 \mathrm{~Hz}, 18 \mathrm{H}, \mathrm{P}\left(\mathrm{C}\left(C \mathrm{H}_{3}\right)_{3}\right)\right) .{ }^{13} \mathrm{C}\left\{{ }^{1} \mathrm{H}\right\}$ $(128.8 \mathrm{MHz}) \delta=168.1\left(\mathrm{vt}, N=\left.\right|^{2} J_{\mathrm{CP}}+{ }^{3} J_{\mathrm{CP}} \mid=5.6 \mathrm{~Hz}, 2 \mathrm{C}, \mathrm{NCH}\right), 87.2(\mathrm{vt}, N$ $\left.=\left|{ }^{1} J_{\mathrm{CP}}+{ }^{3} J_{\mathrm{CP}}\right|=23.4 \mathrm{~Hz}, 2 \mathrm{C}, \mathrm{PCH}\right), 40.1$ (vt, $N=\left|{ }^{1} J_{\mathrm{CP}}+{ }^{2} J_{\mathrm{CP}}\right|=12.5 \mathrm{~Hz}, 2 \mathrm{C}$, 
$\left.\mathrm{P}\left(C\left(\mathrm{CH}_{3}\right)\right)\right), 35.9\left(\mathrm{vt}, N=\left|{ }^{1} J_{\mathrm{CP}}+{ }^{2} J_{\mathrm{CP}}\right|=11.0 \mathrm{~Hz}, 2 \mathrm{C}, \mathrm{P}\left(C\left(\mathrm{CH}_{3}\right)\right)\right), 29.8(\mathrm{vt}, N=$ $\left.\left.\right|^{2} J_{\mathrm{CP}}+{ }^{4} J_{\mathrm{CP}} \mid=2.3 \mathrm{~Hz}, 6 \mathrm{C}, \mathrm{P}\left(\mathrm{C}\left(C_{3}\right)_{3}\right)\right), 29.4\left(\mathrm{br}, 6 \mathrm{C}, \mathrm{P}\left(\mathrm{C}\left(C \mathrm{H}_{3}\right)_{3}\right)\right) .{ }^{31} \mathrm{P}\left\{{ }^{1} \mathrm{H}\right\}(202.5$ $\mathrm{MHz}) \delta=858.4$ (s br, 1P, $P$ Cl), 70.4 (s br, 2P, $P\left(\mathrm{C}\left(\mathrm{CH}_{3}\right)_{3}\right)$ ). LIFDI-MS (Toluol): 649.2 $\left(\left[\mathrm{M}^{+}\right]\right)$.

\section{Reaction of 1,7 and 12 with $\mathrm{PCO}^{-}$}

$7 \mu \mathrm{mol}$ of the chosen complex and $14 \mu \mathrm{mol}$ of $\left[\mathrm{Na}(\text { diox })_{1.33}\right][\mathrm{PCO}]$ are dissolved in THF $(0.5 \mathrm{~mL})$ and stirred for 5 minutes at room temperature. NMR spectroscopy reveals quantiative reduction without any detectable follow-up reactions in all cases.

\section{Reaction of 44 with $\mathrm{Na}^{+}$and $\mathrm{Tl}^{+}$}

$7 \mu \mathrm{mol}$ of 44 and $7 \mu \mathrm{mol}$ of $\mathrm{NaBPh}_{4}$ or TlOTf are dissolved in THF $(0.5 \mathrm{~mL})$ and stirred for one hour at room temperature. No conversion was detected by NMR spectroscopy.

\section{Reaction of 44 with unsaturated substrates}

44 (5.0 mg, $7.72 \mu \mathrm{mol}, 1.0$ eq.) and cyclohexane/tolane/dimethyl butadiene (5.0 eq.) are dissolved in $\mathrm{C}_{6} \mathrm{D}_{6}$ and heated to $80^{\circ} \mathrm{C}$ for $3 \mathrm{~h}$. No conversion was observed by ${ }^{31} \mathrm{P}$ NMR spectroscopy.

\section{Reaction of 44 with [TBA] [WN(ODipp) $\left.{ }_{4}\right]$}

$44\left(5.0 \mathrm{mg}, 7.7 \mu \mathrm{mol}, 1.0\right.$ eq., prepared in situ) and [TBA] [WN(ODipp) $\left.{ }_{4}\right](9.3 \mathrm{mg}, 7.7 \mu \mathrm{mol}$, 1.0 eq.) are dissolved in THF $(0.5 \mathrm{~mL})$ and heated to $70^{\circ} \mathrm{C}$ for $24 \mathrm{~h}$. The solvent is removed, the residue extracted with benzene and characterized by NMR spectroscopy.

NMR $\left(\mathrm{C}_{6} \mathrm{D}_{6}, \mathrm{RT}\right):{ }^{1} \mathrm{H}(400 \mathrm{MHz}) \delta=7.10-6.86\left(\mathrm{~m}, 5 \mathrm{H}, \mathrm{NH} \mathrm{H}, \mathrm{C}_{\text {ortho/meta }} H\right)$, $4.22\left(\mathrm{~A}_{18} \mathrm{BCXX}^{\prime} \mathrm{A}_{18}{ }_{18} \mathrm{~B}^{\prime} \mathrm{C}^{\prime}, N=\left.\right|^{2} J_{\mathrm{BX}}+{ }^{4} J_{\mathrm{BX}} \mid=5.9 \mathrm{~Hz},{ }^{3} J_{\mathrm{BC}}=5.7 \mathrm{~Hz}, 2 \mathrm{H}, \mathrm{PC} H\right), 3.30$ $\left(\mathrm{h},{ }^{3} J_{\mathrm{HH}}=6.8 \mathrm{~Hz}, 2 \mathrm{H}, \mathrm{CH}\left(\mathrm{CH}_{3}\right)_{2}\right) 1.46\left(\mathrm{~A}_{18} \mathrm{BCXX}^{\prime} \mathrm{A}^{\prime}{ }_{18} \mathrm{~B}^{\prime} \mathrm{C}{ }^{\prime},{ }^{3} J_{\mathrm{AX}}=14.0 \mathrm{~Hz}, 18 \mathrm{H}\right.$, $\left.\mathrm{P}\left(\mathrm{C}\left(\mathrm{CH}_{3}\right)_{3}\right)\right), 1.30\left(\mathrm{~A}_{18} \mathrm{BCXX}^{\prime} \mathrm{A}_{18} \mathrm{~B}^{\prime} \mathrm{C}^{\prime},{ }^{3} J_{\mathrm{AX}}=13.6 \mathrm{~Hz}, 18 \mathrm{H}, \mathrm{P}\left(\mathrm{C}\left(C \mathrm{H}_{3}\right)_{3}\right)\right), 1.15(\mathrm{~d}$, $\left.{ }^{3} J_{\mathrm{HH}}=6.8 \mathrm{~Hz}, 12 \mathrm{H}, \mathrm{CH}\left(\mathrm{CH}_{3}\right)_{3}\right) .{ }^{31} \mathrm{P}\left\{{ }^{1} \mathrm{H}\right\}(202.5 \mathrm{MHz}) \delta=791.2\left(\mathrm{t},{ }^{2} J_{\mathrm{PP}}=16.8 \mathrm{~Hz}\right.$, 1P, PODipp), $56.7\left(\mathrm{~d},{ }^{2} J_{\mathrm{PP}}=16.8 \mathrm{~Hz}, 2 \mathrm{P}, P\left(\mathrm{C}\left(\mathrm{CH}_{3}\right)_{3}\right)\right)$. 


\subsection{Proton-Induced Splitting of Dinitrogen by Dimeric Mo PNP Pincer Complexes}

\subsubsection{Computational Details}

DFT calculations were carried out with the ORCA program package using the PBE0 functional. ${ }^{[304],[318],[326]}$ Ahlrichs' basis sets def2-SVP (for geometry optimization and frequency calculation) and def2-TZVPP (for single-point energies) were used with a full basis for all elements but Mo for which the Stuttgart-Dresden electron core potential was chosen to replace the inner shell orbitals. ${ }^{[307],[308]}$ The RI-J (PBE) approximation in combination with the corresponding auxiliary basis sets of Alrichs was utilized to reduce computational costs in the geometry optimization and frequency calculations. ${ }^{[327]-[329]}$ Grimme's model (D3) with Becke-Johnson damping was used to account for dispersion with the PBE0 functional. ${ }^{[306],[330]}$ No symmetry restraints were imposed and the optimized structures were defined as minima (no negative eigenvalues) by vibrational analyses. The CPCM solvation model was used (THF). ${ }^{[331]}$ Relativistic treatment was introducted by ZORA. ${ }^{[32]}$ Geometries were analyzed and visualized with Avogadro and molecular orbitals, spin-densities and difference densities were visualized with Chimera. ${ }^{[333],[334]}$

Table 7.35: XYZ-coordinates of computed complex XCVII.

\begin{tabular}{c|lll|l|lll} 
Atom & $\mathrm{x}$ & $\mathrm{y}$ & $\mathrm{z}$ & Atom & $\mathrm{x}$ & $\mathrm{y}$ & $\mathrm{z}$ \\
\hline $\mathrm{Mo}$ & -0.00633 & 2.40204 & -0.03111 & $\mathrm{Mo}$ & -0.05531 & -2.39797 & 0.03397 \\
$\mathrm{C}$ & -2.15749 & 4.67282 & 1.96298 & $\mathrm{C}$ & -2.09258 & -4.60745 & -2.14174 \\
$\mathrm{P}$ & -1.57741 & 2.86697 & 1.81326 & $\mathrm{P}$ & -1.50367 & -2.81035 & -1.91947 \\
$\mathrm{~N}$ & 1.27879 & 3.02406 & 1.35695 & $\mathrm{~N}$ & 1.30474 & -3.04065 & -1.27255 \\
$\mathrm{P}$ & 1.96682 & 2.76214 & -1.45507 & $\mathrm{P}$ & 1.81131 & -2.80799 & 1.58292 \\
$\mathrm{C}$ & 2.14217 & 4.47674 & -2.25717 & $\mathrm{C}$ & 1.88464 & -4.51989 & 2.40402 \\
$\mathrm{Cl}$ & -1.52543 & 3.46674 & -1.65147 & $\mathrm{Cl}$ & -1.70938 & -3.4405 & 1.53141 \\
$\mathrm{C}$ & -3.00928 & 1.67908 & 2.15373 & $\mathrm{C}$ & -2.88918 & -1.59927 & -2.34986 \\
$\mathrm{C}$ & 2.45788 & 1.40481 & -2.68281 & $\mathrm{C}$ & 2.25097 & -1.45425 & 2.83523 \\
$\mathrm{~N}$ & -0.08572 & 0.6191 & -0.04511 & $\mathrm{~N}$ & -0.10246 & -0.6138 & 0.06309 \\
$\mathrm{C}$ & 3.25505 & 2.73353 & -0.13315 & $\mathrm{C}$ & 3.18453 & -2.8218 & 0.34868 \\
$\mathrm{H}$ & 4.21089 & 3.1972 & -0.41813 & $\mathrm{H}$ & 4.10288 & -3.31889 & 0.69475 \\
$\mathrm{H}$ & 3.44405 & 1.66853 & 0.05664 & $\mathrm{H}$ & 3.42254 & -1.76388 & 0.17521 \\
$\mathrm{C}$ & 2.68586 & 3.35105 & 1.13602 & $\mathrm{C}$ & 2.68145 & -3.41654 & -0.959 \\
$\mathrm{H}$ & 2.83532 & 4.45138 & 1.12911 & $\mathrm{H}$ & 2.79272 & -4.52123 & -0.94851 \\
$\mathrm{H}$ & 3.28512 & 2.9894 & 1.99509 & $\mathrm{H}$ & 3.34903 & -3.07274 & -1.77379 \\
$\mathrm{C}$ & 0.96724 & 3.23579 & 2.7687 & $\mathrm{C}$ & 1.08434 & -3.22845 & -2.70427 \\
$\mathrm{H}$ & 1.7614 & 2.77132 & 3.38775 & $\mathrm{H}$ & 1.93502 & -2.78767 & -3.26254 \\
$\mathrm{H}$ & 1.00768 & 4.3163 & 3.02373 & $\mathrm{H}$ & 1.10258 & -4.30718 & -2.96957 \\
$\mathrm{C}$ & -0.3716 & 2.64564 & 3.18769 & $\mathrm{C}$ & -0.20153 & -2.58658 & -3.2016 \\
$\mathrm{H}$ & -0.25841 & 1.55696 & 3.28741 & $\mathrm{H}$ & -0.0515 & -1.49953 & -3.26157 \\
$\mathrm{H}$ & -0.7029 & 3.04134 & 4.1597 & $\mathrm{H}$ & -0.47421 & -2.94678 & -4.20497 \\
$\mathrm{C}$ & 1.42497 & 4.50395 & -3.60858 & $\mathrm{C}$ & 1.0515 & -4.51713 & 3.68765 \\
$\mathrm{H}$ & 1.38247 & 5.54669 & -3.96506 & $\mathrm{H}$ & 0.95843 & -5.55362 & 4.05288 \\
$\mathrm{H}$ & 0.39043 & 4.14137 & -3.51027 & $\mathrm{H}$ & 0.03668 & -4.13739 & 3.49359
\end{tabular}




\begin{tabular}{|c|c|c|c|c|c|c|c|}
\hline $\mathrm{H}$ & 1.94491 & 3.91813 & -4.37927 & $\mathrm{H}$ & 1.51207 & -3.92817 & 4.49289 \\
\hline $\mathrm{C}$ & 3.59605 & 4.92607 & -2.40593 & $\mathrm{C}$ & 3.30901 & -5.00232 & 2.67937 \\
\hline $\mathrm{H}$ & 3.61607 & 5.89984 & -2.92354 & $\mathrm{H}$ & 3.26028 & -5.97971 & 3.1883 \\
\hline $\mathrm{H}$ & 4.20904 & 4.22901 & -2.99093 & $\mathrm{H}$ & 3.88219 & -4.32398 & 3.32326 \\
\hline $\mathrm{H}$ & 4.07965 & 5.0723 & -1.42892 & $\mathrm{H}$ & 3.87607 & -5.15382 & 1.7491 \\
\hline $\mathrm{C}$ & 1.41688 & 5.46658 & -1.33775 & $\mathrm{C}$ & 1.22042 & -5.49964 & 1.42939 \\
\hline $\mathrm{H}$ & 1.57715 & 6.49058 & -1.71494 & $\mathrm{H}$ & 1.29677 & -6.52013 & 1.84074 \\
\hline $\mathrm{H}$ & 1.77926 & 5.43453 & -0.30078 & $\mathrm{H}$ & 1.69625 & -5.50541 & 0.43889 \\
\hline $\mathrm{H}$ & 0.33687 & 5.27172 & -1.34035 & $\mathrm{H}$ & 0.1552 & -5.264 & 1.31123 \\
\hline C & -1.08768 & 5.55304 & 1.30629 & $\mathrm{C}$ & -1.10863 & -5.51089 & -1.38951 \\
\hline $\mathrm{H}$ & -1.37015 & 6.61189 & 1.43172 & $\mathrm{H}$ & -1.38026 & -6.56469 & -1.57007 \\
\hline $\mathrm{H}$ & -1.02812 & 5.34861 & 0.22993 & $\mathrm{H}$ & -1.17049 & -5.32854 & -0.30903 \\
\hline $\mathrm{H}$ & -0.0903 & 5.42004 & 1.74557 & $\mathrm{H}$ & -0.06653 & -5.37511 & -1.70769 \\
\hline $\mathrm{C}$ & -3.4532 & 4.88384 & 1.17675 & $\mathrm{C}$ & -3.46419 & -4.80984 & -1.49363 \\
\hline $\mathrm{H}$ & -3.67522 & 5.96376 & 1.14702 & $\mathrm{H}$ & -3.70036 & -5.88712 & -1.5016 \\
\hline $\mathrm{H}$ & -4.31617 & 4.38605 & 1.63917 & $\mathrm{H}$ & -4.27136 & -4.29574 & -2.03272 \\
\hline $\mathrm{H}$ & -3.34515 & 4.53368 & 0.13924 & $\mathrm{H}$ & -3.45717 & -4.47479 & -0.44563 \\
\hline C & -2.33415 & 5.11402 & 3.41613 & $\mathrm{C}$ & -2.12619 & -5.02846 & -3.61107 \\
\hline $\mathrm{H}$ & -2.71676 & 6.14853 & 3.43112 & $\mathrm{H}$ & -2.52795 & -6.05336 & -3.68082 \\
\hline $\mathrm{H}$ & -1.37948 & 5.1157 & 3.96242 & $\mathrm{H}$ & -1.11977 & -5.04574 & -4.05427 \\
\hline $\mathrm{H}$ & -3.04601 & 4.49044 & 3.97209 & $\mathrm{H}$ & -2.76147 & -4.38108 & -4.22944 \\
\hline $\mathrm{C}$ & 3.68811 & 1.73382 & -3.5254 & $\mathrm{C}$ & 3.3961 & -1.81661 & 3.77823 \\
\hline $\mathrm{H}$ & 3.93904 & 0.85887 & -4.14865 & $\mathrm{H}$ & 3.62394 & -0.94456 & 4.41421 \\
\hline $\mathrm{H}$ & 4.56662 & 1.95056 & -2.89813 & $\mathrm{H}$ & 4.31574 & -2.06942 & 3.22839 \\
\hline $\mathrm{H}$ & 3.52712 & 2.58155 & -4.2042 & $\mathrm{H}$ & 3.15014 & -2.65158 & 4.44734 \\
\hline C & 1.23888 & 1.1352 & -3.56856 & $\mathrm{C}$ & 0.97667 & -1.13393 & 3.62079 \\
\hline $\mathrm{H}$ & 1.44284 & 0.26757 & -4.21827 & $\mathrm{H}$ & 1.16529 & -0.27614 & 4.28811 \\
\hline $\mathrm{H}$ & 0.98462 & 1.98521 & -4.21325 & $\mathrm{H}$ & 0.63551 & -1.97293 & 4.23935 \\
\hline $\mathrm{H}$ & 0.35732 & 0.91478 & -2.95124 & $\mathrm{H}$ & 0.15826 & -0.87534 & 2.93566 \\
\hline 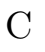 & 2.75119 & 0.1313 & -1.88651 & $\mathrm{C}$ & 2.65163 & -0.19799 & 2.05806 \\
\hline $\mathrm{H}$ & 2.87544 & -0.70237 & -2.59652 & $\mathrm{H}$ & 2.76117 & 0.63287 & 2.77406 \\
\hline $\mathrm{H}$ & 1.93454 & -0.1431 & -1.20811 & $\mathrm{H}$ & 1.89459 & 0.10637 & 1.3248 \\
\hline $\mathrm{H}$ & 3.68569 & 0.20489 & -1.31105 & $\mathrm{H}$ & 3.62051 & -0.31285 & 1.5504 \\
\hline $\mathrm{C}$ & -2.40655 & 0.30179 & 2.44429 & $\mathrm{C}$ & -2.24806 & -0.22453 & -2.55681 \\
\hline $\mathrm{H}$ & -3.21113 & -0.45043 & 2.45745 & $\mathrm{H}$ & -3.03923 & 0.5394 & -2.61604 \\
\hline $\mathrm{H}$ & -1.91431 & 0.26143 & 3.42663 & $\mathrm{H}$ & -1.67752 & -0.16794 & -3.49496 \\
\hline $\mathrm{H}$ & -1.68806 & -0.01536 & 1.67914 & $\mathrm{H}$ & -1.58909 & 0.06418 & -1.72968 \\
\hline C & -3.89105 & 2.07603 & 3.33615 & $\mathrm{C}$ & -3.66723 & -1.96057 & -3.61366 \\
\hline $\mathrm{H}$ & -4.64381 & 1.28683 & 3.49987 & $\mathrm{H}$ & -4.39782 & -1.16161 & -3.82366 \\
\hline $\mathrm{H}$ & -4.4349 & 3.01543 & 3.16913 & $\mathrm{H}$ & -4.22912 & -2.89929 & -3.51837 \\
\hline $\mathrm{H}$ & -3.3096 & 2.16991 & 4.26643 & $\mathrm{H}$ & -3.00678 & -2.03674 & -4.49152 \\
\hline $\mathrm{C}$ & -3.83104 & 1.5791 & 0.86499 & $\mathrm{C}$ & -3.81758 & -1.515 & -1.13454 \\
\hline $\mathrm{H}$ & -4.58068 & 0.77873 & 0.97364 & $\mathrm{H}$ & -4.5364 & -0.69332 & -1.28431 \\
\hline $\mathrm{H}$ & -3.18641 & 1.33787 & 0.00831 & $\mathrm{H}$ & -3.24562 & -1.31374 & -0.21748 \\
\hline 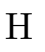 & -4.36205 & 2.5085 & 0.62722 & $\mathrm{H}$ & -4.38867 & -2.4369 & -0.97234 \\
\hline
\end{tabular}


Table 7.36: XYZ-coordinates of computed complex 48.

\begin{tabular}{|c|c|c|c|c|c|c|c|}
\hline Atom & $\mathrm{x}$ & $\mathrm{y}$ & $\mathrm{z}$ & Atom & $\mathrm{x}$ & $\mathrm{y}$ & $\mathrm{z}$ \\
\hline Mo & 0.00426 & 2.45656 & -0.03567 & Mo & -0.03754 & -2.34801 & 0.0077 \\
\hline $\mathrm{C}$ & -2.16825 & 4.68443 & 1.99836 & $\mathrm{C}$ & -2.08498 & -4.63382 & -2.12369 \\
\hline $\mathrm{P}$ & -1.60786 & 2.87930 & 1.86028 & $\mathrm{P}$ & -1.51066 & -2.8327 & -1.93329 \\
\hline $\mathrm{N}$ & 1.28752 & 3.06338 & 1.35297 & $\mathrm{~N}$ & 1.30142 & -3.00495 & -1.28115 \\
\hline $\mathrm{P}$ & 2.03423 & 2.80816 & -1.49581 & $\mathrm{P}$ & 1.81465 & -2.83953 & 1.58399 \\
\hline $\mathrm{C}$ & 2.15611 & 4.52932 & -2.27672 & $\mathrm{C}$ & 1.8447 & -4.54159 & 2.41878 \\
\hline $\mathrm{Cl}$ & -1.4979 & 3.3327 & -1.64247 & $\mathrm{Cl}$ & -1.68268 & -3.34417 & 1.49426 \\
\hline $\mathrm{C}$ & -3.02171 & 1.66987 & 2.16058 & $\mathrm{C}$ & -2.90317 & -1.62624 & -2.35186 \\
\hline $\mathrm{C}$ & 2.504 & 1.44257 & -2.71069 & $\mathrm{C}$ & 2.25324 & -1.47277 & 2.82389 \\
\hline $\mathrm{N}$ & -0.0717 & 0.68578 & -0.09122 & $\mathrm{~N}$ & -0.05322 & -0.5349 & $5.67 \mathrm{E}-04$ \\
\hline $\mathrm{C}$ & 3.26581 & 2.76664 & -0.13372 & $\mathrm{C}$ & 3.18392 & -2.87371 & 0.35032 \\
\hline $\mathrm{H}$ & 4.23063 & 3.21574 & -0.40998 & $\mathrm{H}$ & 4.08175 & -3.4075 & 0.69326 \\
\hline $\mathrm{H}$ & 3.43967 & 1.70104 & 0.06751 & $\mathrm{H}$ & 3.46319 & -1.8237 & 0.18933 \\
\hline $\mathrm{C}$ & 2.68835 & 3.40636 & 1.12005 & $\mathrm{C}$ & 2.66352 & -3.43977 & -0.96058 \\
\hline $\mathrm{H}$ & 2.81359 & 4.50639 & 1.0897 & $\mathrm{H}$ & 2.71898 & -4.54634 & -0.95692 \\
\hline $\mathrm{H}$ & 3.29019 & 3.07054 & 1.98474 & $\mathrm{H}$ & 3.34284 & -3.12243 & -1.7742 \\
\hline $\mathrm{C}$ & 0.97007 & 3.23579 & 2.76883 & $\mathrm{C}$ & 1.07723 & -3.21924 & -2.71271 \\
\hline $\mathrm{H}$ & 1.7703 & 2.7699 & 3.37481 & $\mathrm{H}$ & 1.92622 & -2.7824 & -3.27342 \\
\hline $\mathrm{H}$ & 0.99581 & 4.31102 & 3.03485 & $\mathrm{H}$ & 1.10672 & -4.30155 & -2.95106 \\
\hline $\mathrm{C}$ & -0.35845 & 2.61846 & 3.17907 & $\mathrm{C}$ & -0.21405 & -2.59944 & -3.21594 \\
\hline $\mathrm{H}$ & -0.23821 & 1.52807 & 3.24828 & $\mathrm{H}$ & -0.07999 & -1.51145 & -3.29543 \\
\hline $\mathrm{H}$ & -0.68252 & 2.98207 & 4.16533 & $\mathrm{H}$ & -0.48539 & -2.98025 & -4.21129 \\
\hline $\mathrm{C}$ & 1.46205 & 4.54889 & -3.6408 & $\mathrm{C}$ & 1.00388 & -4.5115 & 3.69749 \\
\hline $\mathrm{H}$ & 1.41253 & 5.59213 & -3.99121 & $\mathrm{H}$ & 0.89472 & -5.54253 & 4.07102 \\
\hline $\mathrm{H}$ & 0.43035 & 4.17377 & -3.57403 & $\mathrm{H}$ & -0.00509 & -4.12037 & 3.49872 \\
\hline $\mathrm{H}$ & 2.0042 & 3.97667 & -4.40523 & $\mathrm{H}$ & 1.46971 & -3.92451 & 4.50068 \\
\hline $\mathrm{C}$ & 3.60797 & 4.99438 & -2.40091 & $\mathrm{C}$ & 3.26256 & -5.03636 & 2.70866 \\
\hline $\mathrm{H}$ & 3.62288 & 5.97111 & -2.91073 & $\mathrm{H}$ & 3.19491 & -6.00316 & 3.23351 \\
\hline $\mathrm{H}$ & 4.23357 & 4.30899 & -2.98633 & $\mathrm{H}$ & 3.84226 & -4.35797 & 3.34593 \\
\hline $\mathrm{H}$ & 4.07888 & 5.13984 & -1.41782 & $\mathrm{H}$ & 3.83316 & -5.21533 & 1.7857 \\
\hline $\mathrm{C}$ & 1.39998 & 5.49706 & -1.35889 & $\mathrm{C}$ & 1.17737 & -5.5222 & 1.44802 \\
\hline $\mathrm{H}$ & 1.54287 & 6.52418 & -1.73114 & $\mathrm{H}$ & 1.23832 & -6.53748 & 1.87212 \\
\hline $\mathrm{H}$ & 1.75432 & 5.47476 & -0.31917 & $\mathrm{H}$ & 1.66509 & -5.54993 & 0.46356 \\
\hline $\mathrm{H}$ & 0.32205 & 5.29034 & -1.37112 & $\mathrm{H}$ & 0.11626 & -5.27947 & 1.31539 \\
\hline C & -1.10156 & 5.55854 & 1.32922 & $\mathrm{C}$ & -1.09281 & -5.52134 & -1.36455 \\
\hline $\mathrm{H}$ & -1.38546 & 6.61657 & 1.4483 & $\mathrm{H}$ & -1.35547 & -6.57769 & -1.53778 \\
\hline $\mathrm{H}$ & -1.04424 & 5.35559 & 0.25177 & $\mathrm{H}$ & -1.1555 & -5.33554 & -0.28547 \\
\hline $\mathrm{H}$ & -0.10223 & 5.43586 & 1.76662 & $\mathrm{H}$ & -0.05173 & -5.38397 & -1.68596 \\
\hline $\mathrm{C}$ & -3.47704 & 4.90017 & 1.23479 & $\mathrm{C}$ & -3.45594 & -4.8376 & -1.47395 \\
\hline $\mathrm{H}$ & -3.69956 & 5.97915 & 1.22447 & $\mathrm{H}$ & -3.68354 & -5.91587 & -1.47442 \\
\hline $\mathrm{H}$ & -4.33211 & 4.39698 & 1.70435 & $\mathrm{H}$ & -4.26653 & -4.33596 & -2.01884 \\
\hline $\mathrm{H}$ & -3.39564 & 4.56861 & 0.18938 & $\mathrm{H}$ & -3.45637 & -4.49677 & -0.42822 \\
\hline $\mathrm{C}$ & -2.32346 & 5.11029 & 3.45895 & $\mathrm{C}$ & -2.11591 & -5.06533 & -3.59049 \\
\hline
\end{tabular}




\begin{tabular}{l|lll|l|lll}
$\mathrm{H}$ & -2.69667 & 6.1468 & 3.48781 & $\mathrm{H}$ & -2.51509 & -6.091 & -3.64898 \\
$\mathrm{H}$ & -1.36438 & 5.09973 & 3.99695 & $\mathrm{H}$ & -1.1101 & -5.0872 & -4.03456 \\
$\mathrm{H}$ & -3.03762 & 4.48856 & 4.01379 & $\mathrm{H}$ & -2.75449 & -4.42787 & -4.21528 \\
$\mathrm{C}$ & 3.72302 & 1.78746 & -3.56496 & $\mathrm{C}$ & 3.3808 & -1.84708 & 3.78395 \\
$\mathrm{H}$ & 3.97066 & 0.91715 & -4.19413 & $\mathrm{H}$ & 3.61354 & -0.97458 & 4.41674 \\
$\mathrm{H}$ & 4.60784 & 2.00524 & -2.94778 & $\mathrm{H}$ & 4.30376 & -2.11856 & 3.24975 \\
$\mathrm{H}$ & 3.55017 & 2.6357 & -4.23932 & $\mathrm{H}$ & 3.11189 & -2.6715 & 4.45609 \\
$\mathrm{C}$ & 1.27628 & 1.16148 & -3.5822 & $\mathrm{C}$ & 0.97463 & -1.12754 & 3.59161 \\
$\mathrm{H}$ & 1.49569 & 0.3127 & -4.24985 & $\mathrm{H}$ & 1.17521 & -0.28268 & 4.27156 \\
$\mathrm{H}$ & 0.99409 & 2.01383 & -4.21188 & $\mathrm{H}$ & 0.60261 & -1.96209 & 4.19762 \\
$\mathrm{H}$ & 0.40617 & 0.89867 & -2.96461 & $\mathrm{H}$ & 0.17208 & -0.84355 & 2.89769 \\
$\mathrm{C}$ & 2.82078 & 0.17825 & -1.90741 & $\mathrm{C}$ & 2.68664 & -0.22964 & 2.04287 \\
$\mathrm{H}$ & 2.93941 & -0.65628 & -2.61652 & $\mathrm{H}$ & 2.79344 & 0.60552 & 2.75422 \\
$\mathrm{H}$ & 2.01847 & -0.10411 & -1.21507 & $\mathrm{H}$ & 1.94736 & 0.06958 & 1.28984 \\
$\mathrm{H}$ & 3.76566 & 0.26131 & -1.35113 & $\mathrm{H}$ & 3.66422 & -0.36134 & 1.55685 \\
$\mathrm{C}$ & -2.4065 & 0.29474 & 2.43154 & $\mathrm{C}$ & -2.27548 & -0.2485 & -2.57725 \\
$\mathrm{H}$ & -3.20789 & -0.45999 & 2.43653 & $\mathrm{H}$ & -3.07766 & 0.50321 & -2.64248 \\
$\mathrm{H}$ & -1.91293 & 0.24139 & 3.41211 & $\mathrm{H}$ & -1.70984 & -0.19475 & -3.51863 \\
$\mathrm{H}$ & -1.69149 & -0.01123 & 1.65863 & $\mathrm{H}$ & -1.61675 & 0.05212 & -1.75483 \\
$\mathrm{C}$ & -3.90052 & 2.05254 & 3.3509 & $\mathrm{C}$ & -3.6835 & -2.00683 & -3.60939 \\
$\mathrm{H}$ & -4.65074 & 1.25975 & 3.50202 & $\mathrm{H}$ & -4.42438 & -1.21833 & -3.82025 \\
$\mathrm{H}$ & -4.44797 & 2.99178 & 3.19837 & $\mathrm{H}$ & -4.23498 & -2.94973 & -3.50328 \\
$\mathrm{H}$ & -3.31984 & 2.13216 & 4.28252 & $\mathrm{H}$ & -3.0288 & -2.08227 & -4.49116 \\
$\mathrm{C}$ & -3.84331 & 1.58664 & 0.87019 & $\mathrm{C}$ & -3.82619 & -1.53992 & -1.13263 \\
$\mathrm{H}$ & -4.60743 & 0.80188 & 0.98375 & $\mathrm{H}$ & -4.56046 & -0.7342 & -1.2925 \\
$\mathrm{H}$ & -3.21074 & 1.32242 & 0.01129 & $\mathrm{H}$ & -3.25602 & -1.31714 & -0.21942 \\
$\mathrm{H}$ & -4.36007 & 2.5236 & 0.63162 & $\mathrm{H}$ & -4.38217 & -2.46777 & -0.95555
\end{tabular}

Table 7.37: XYZ-coordinates of computed complex 49.

\begin{tabular}{c|lll|l|lll} 
Atom & $\mathrm{x}$ & $\mathrm{y}$ & $\mathrm{z}$ & Atom & $\mathrm{x}$ & $\mathrm{y}$ & $\mathrm{z}$ \\
\hline $\mathrm{Mo}$ & 0.01909 & 2.40383 & -0.00396 & $\mathrm{H}$ & -4.31468 & 2.55294 & 0.60752 \\
$\mathrm{C}$ & -2.14320 & 4.72541 & 1.97812 & $\mathrm{Mo}$ & -0.03942 & -2.39508 & 0.01086 \\
$\mathrm{P}$ & -1.60184 & 2.91341 & 1.88275 & $\mathrm{C}$ & -2.0923 & -4.65216 & -2.15898 \\
$\mathrm{~N}$ & 1.29330 & 3.01893 & 1.36081 & $\mathrm{P}$ & -1.54086 & -2.84688 & -1.98542 \\
$\mathrm{P}$ & 2.03531 & 2.82714 & -1.49605 & $\mathrm{~N}$ & 1.30366 & -3.03695 & -1.27495 \\
$\mathrm{C}$ & 2.12693 & 4.54634 & -2.28426 & $\mathrm{P}$ & 1.86478 & -2.88358 & 1.62639 \\
$\mathrm{Cl}$ & -1.46550 & 3.25865 & -1.59593 & $\mathrm{C}$ & 1.84275 & -4.596 & 2.43104 \\
$\mathrm{C}$ & -3.02380 & 1.71231 & 2.18472 & $\mathrm{Cl}$ & -1.65461 & -3.21618 & 1.48833 \\
$\mathrm{C}$ & 2.49267 & 1.45327 & -2.70839 & $\mathrm{C}$ & -2.91882 & -1.62302 & -2.37516 \\
$\mathrm{~N}$ & -0.04737 & 0.61427 & -0.03284 & $\mathrm{C}$ & 2.27975 & -1.51199 & 2.85844 \\
$\mathrm{C}$ & 3.27242 & 2.79115 & -0.14114 & $\mathrm{~N}$ & -0.06603 & -0.60458 & 0.05207 \\
$\mathrm{H}$ & 4.22285 & 3.2692 & -0.41742 & $\mathrm{C}$ & 3.18826 & -2.90053 & 0.35465 \\
$\mathrm{H}$ & 3.47818 & 1.72914 & 0.04989 & $\mathrm{H}$ & 4.09583 & -3.42245 & 0.68988 \\
$\mathrm{C}$ & 2.68472 & 3.40851 & 1.11582 & $\mathrm{H}$ & 3.455 & -1.84888 & 0.18344 \\
$\mathrm{H}$ & 2.76382 & 4.51116 & 1.08865 & $\mathrm{C}$ & 2.65841 & -3.4863 & -0.9427
\end{tabular}


7.6 Proton-Induced Splitting of Dinitrogen by Dimeric Mo PNP Pincer COMPLEXes

\begin{tabular}{|c|c|c|c|c|c|c|c|}
\hline $\mathrm{H}$ & 3.29642 & 3.09421 & 1.98004 & $\mathrm{H}$ & 2.68921 & -4.59123 & -0.92007 \\
\hline $\mathrm{C}$ & 0.97856 & 3.23362 & 2.77630 & $\mathrm{H}$ & 3.33792 & -3.19301 & -1.76242 \\
\hline $\mathrm{H}$ & 1.77710 & 2.77161 & 3.38517 & $\mathrm{C}$ & 1.07651 & -3.2218 & -2.7107 \\
\hline $\mathrm{H}$ & 1.02754 & 4.31452 & 3.00591 & $\mathrm{H}$ & 1.93206 & -2.78702 & -3.259 \\
\hline $\mathrm{C}$ & -0.35745 & 2.65238 & 3.20422 & $\mathrm{H}$ & 1.09412 & -4.30102 & -2.9535 \\
\hline $\mathrm{H}$ & -0.25997 & 1.56241 & 3.30802 & $\mathrm{C}$ & -0.20407 & -2.5801 & -3.21122 \\
\hline $\mathrm{H}$ & -0.67326 & 3.05257 & 4.17865 & $\mathrm{H}$ & -0.06642 & -1.49113 & -3.26882 \\
\hline $\mathrm{C}$ & 1.42949 & 4.55316 & -3.64678 & $\mathrm{H}$ & -0.46395 & -2.93729 & -4.21811 \\
\hline $\mathrm{H}$ & 1.37535 & 5.59462 & -4.00029 & $\mathrm{C}$ & 1.01708 & -4.56454 & 3.71964 \\
\hline $\mathrm{H}$ & 0.39848 & 4.17667 & -3.58030 & $\mathrm{H}$ & 0.9096 & -5.59683 & 4.08768 \\
\hline $\mathrm{H}$ & 1.97347 & 3.98358 & -4.41147 & $\mathrm{H}$ & 0.00504 & -4.17141 & 3.54569 \\
\hline $\mathrm{C}$ & 3.57770 & 5.01591 & -2.41377 & $\mathrm{H}$ & 1.49466 & -3.98604 & 4.52112 \\
\hline $\mathrm{H}$ & 3.58362 & 5.98585 & -2.93562 & $\mathrm{C}$ & 3.26084 & -5.10281 & 2.70291 \\
\hline $\mathrm{H}$ & 4.20814 & 4.33048 & -2.99352 & $\mathrm{H}$ & 3.18831 & -6.07596 & 3.21373 \\
\hline $\mathrm{H}$ & 4.04906 & 5.18013 & -1.43406 & $\mathrm{H}$ & 3.84623 & -4.43881 & 3.35012 \\
\hline $\mathrm{C}$ & 1.36955 & 5.51251 & -1.36721 & $\mathrm{H}$ & 3.826 & -5.27394 & 1.77534 \\
\hline $\mathrm{H}$ & 1.51346 & 6.53856 & -1.74050 & $\mathrm{C}$ & 1.15432 & -5.55322 & 1.45269 \\
\hline $\mathrm{H}$ & 1.72490 & 5.49592 & -0.32761 & $\mathrm{H}$ & 1.19459 & -6.57102 & 1.8712 \\
\hline $\mathrm{H}$ & 0.29151 & 5.30975 & -1.38055 & $\mathrm{H}$ & 1.64189 & -5.59169 & 0.46901 \\
\hline $\mathrm{C}$ & -1.06040 & 5.57689 & 1.30769 & $\mathrm{H}$ & 0.09559 & -5.29858 & 1.32075 \\
\hline $\mathrm{H}$ & -1.33791 & 6.63835 & 1.40426 & $\mathrm{C}$ & -1.10796 & -5.52732 & -1.37697 \\
\hline $\mathrm{H}$ & -0.98944 & 5.3585 & 0.23482 & $\mathrm{H}$ & -1.36819 & -6.58489 & -1.54158 \\
\hline $\mathrm{H}$ & -0.06856 & 5.46041 & 1.76385 & $\mathrm{H}$ & -1.18348 & -5.33944 & -0.29837 \\
\hline $\mathrm{C}$ & -3.44428 & 4.93909 & 1.20061 & $\mathrm{H}$ & -0.06286 & -5.39901 & -1.68885 \\
\hline $\mathrm{H}$ & -3.65781 & 6.01916 & 1.17738 & $\mathrm{C}$ & -3.47786 & -4.86005 & -1.54188 \\
\hline $\mathrm{H}$ & -4.30793 & 4.45051 & 1.66937 & $\mathrm{H}$ & -3.70349 & -5.93775 & -1.56169 \\
\hline $\mathrm{H}$ & -3.36023 & 4.59853 & 0.15848 & $\mathrm{H}$ & -4.27701 & -4.35453 & -2.09871 \\
\hline $\mathrm{C}$ & -2.30575 & 5.17089 & 3.43273 & $\mathrm{H}$ & -3.51154 & -4.53724 & -0.49121 \\
\hline $\mathrm{H}$ & -2.66960 & 6.21062 & 3.44048 & $\mathrm{C}$ & -2.08882 & -5.07483 & -3.62925 \\
\hline $\mathrm{H}$ & -1.35193 & 5.16351 & 3.97989 & $\mathrm{H}$ & -2.47151 & -6.10541 & -3.69793 \\
\hline $\mathrm{H}$ & -3.03224 & 4.56768 & 3.99135 & $\mathrm{H}$ & -1.07631 & -5.08153 & -4.05795 \\
\hline $\mathrm{C}$ & 3.70403 & 1.80754 & -3.57113 & $\mathrm{H}$ & -2.72976 & -4.44622 & -4.2605 \\
\hline $\mathrm{H}$ & 3.95209 & 0.93983 & -4.20328 & $\mathrm{C}$ & 3.3933 & -1.90287 & 3.83026 \\
\hline $\mathrm{H}$ & 4.59266 & 2.03037 & -2.96188 & $\mathrm{H}$ & 3.62289 & -1.03466 & 4.46862 \\
\hline $\mathrm{H}$ & 3.52034 & 2.65373 & -4.24426 & $\mathrm{H}$ & 4.3219 & -2.17751 & 3.3084 \\
\hline $\mathrm{C}$ & 1.26061 & 1.16607 & -3.57114 & $\mathrm{H}$ & 3.10986 & -2.72619 & 4.4972 \\
\hline $\mathrm{H}$ & 1.48619 & 0.32993 & -4.25245 & $\mathrm{C}$ & 0.99329 & -1.15942 & 3.61117 \\
\hline $\mathrm{H}$ & 0.96182 & 2.02045 & -4.18982 & $\mathrm{H}$ & 1.20326 & -0.33577 & 4.31253 \\
\hline $\mathrm{H}$ & 0.39810 & 0.88636 & -2.94984 & $\mathrm{H}$ & 0.59614 & -1.9968 & 4.19734 \\
\hline $\mathrm{C}$ & 2.82693 & 0.19201 & -1.90792 & $\mathrm{H}$ & 0.20529 & -0.83395 & 2.91807 \\
\hline $\mathrm{H}$ & 2.94316 & -0.64092 & -2.61931 & $\mathrm{C}$ & 2.73508 & -0.27848 & 2.07405 \\
\hline $\mathrm{H}$ & 2.03450 & -0.08852 & -1.20331 & $\mathrm{H}$ & 2.82343 & 0.56126 & 2.78159 \\
\hline $\mathrm{H}$ & 3.77862 & 0.27984 & -1.36451 & $\mathrm{H}$ & 2.01812 & 0.02036 & 1.29894 \\
\hline $\mathrm{C}$ & -2.42452 & 0.33876 & 2.49443 & $\mathrm{H}$ & 3.72617 & -0.41465 & 1.6182 \\
\hline $\mathrm{H}$ & -3.23700 & -0.40404 & 2.50738 & $\mathrm{C}$ & -2.27996 & -0.24869 & -2.58551 \\
\hline $\mathrm{H}$ & -1.94416 & 0.30109 & 3.48237 & $\mathrm{H}$ & -3.07977 & 0.50541 & -2.64337 \\
\hline
\end{tabular}




\begin{tabular}{c|lll|l|lll}
$\mathrm{H}$ & -1.70363 & 0.0121 & 1.73526 & $\mathrm{H}$ & -1.71523 & -0.1857 & -3.52647 \\
$\mathrm{C}$ & -3.90763 & 2.13285 & 3.35963 & $\mathrm{H}$ & -1.623 & 0.04301 & -1.75766 \\
$\mathrm{H}$ & -4.66698 & 1.35147 & 3.52247 & $\mathrm{C}$ & -3.68874 & -1.99927 & -3.64179 \\
$\mathrm{H}$ & -4.44574 & 3.07188 & 3.17953 & $\mathrm{H}$ & -4.4273 & -1.20887 & -3.8501 \\
$\mathrm{H}$ & -3.33561 & 2.2288 & 4.29464 & $\mathrm{H}$ & -4.24351 & -2.94085 & -3.54584 \\
$\mathrm{C}$ & -3.83638 & 1.6081 & 0.89039 & $\mathrm{H}$ & -3.0303 & -2.06726 & -4.52094 \\
$\mathrm{H}$ & -4.63342 & 0.86131 & 1.03052 & $\mathrm{C}$ & -3.84849 & -1.54665 & -1.16016 \\
$\mathrm{H}$ & -3.20939 & 1.28237 & 0.04840 & $\mathrm{H}$ & -4.6077 & -0.77049 & -1.34364 \\
$\mathrm{H}$ & -4.31468 & 2.55294 & 0.60752 & $\mathrm{H}$ & -3.29799 & -1.27552 & -0.24782 \\
$\mathrm{Mo}$ & -0.03942 & -2.39508 & 0.01086 & $\mathrm{H}$ & -4.3781 & -2.48663 & -0.96709
\end{tabular}




\subsubsection{Crystallographic Details}

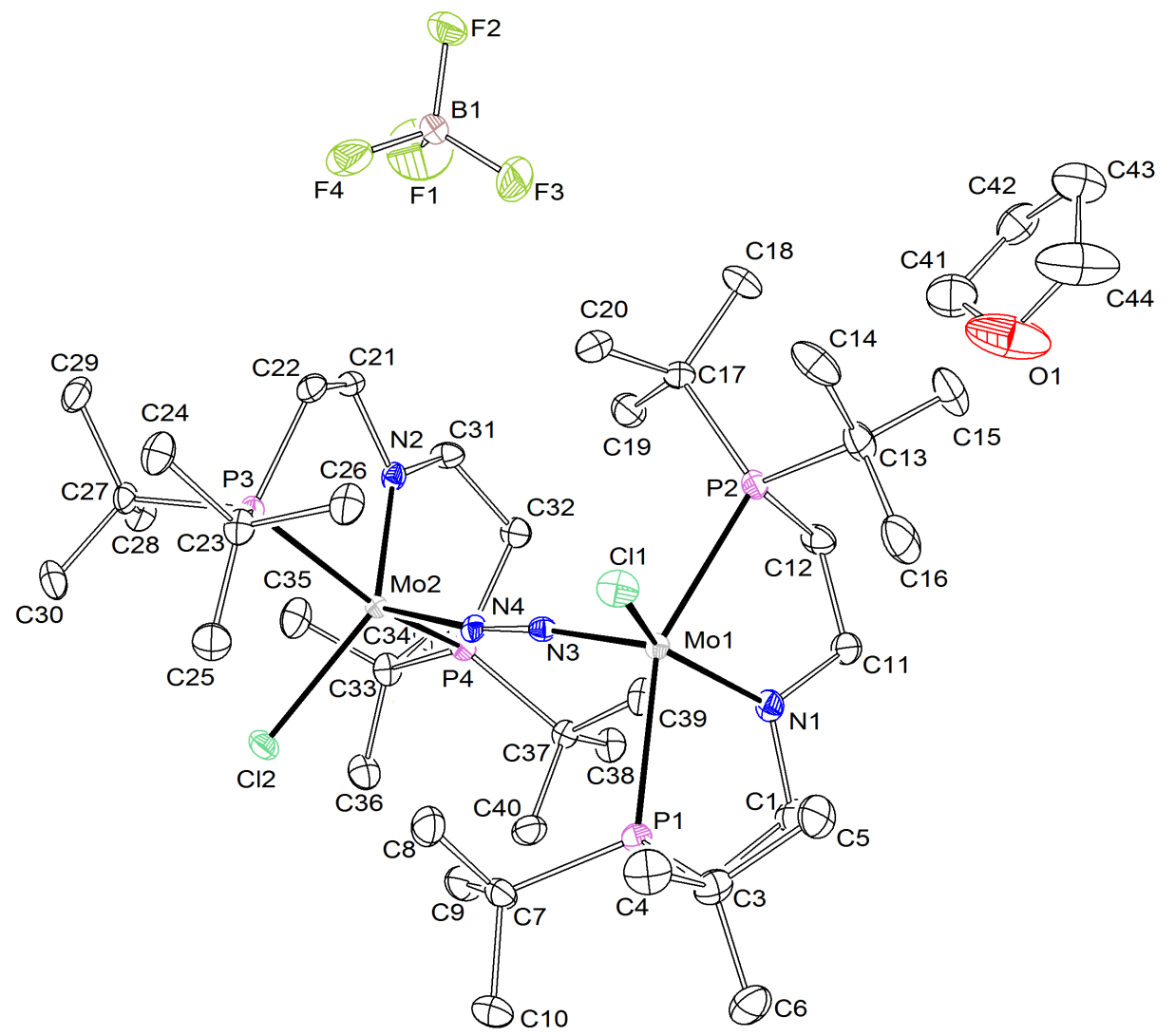

Figure 7.36: Thermal ellipsoid plot of $\mathbf{4 8}$ with the anisotropic displacement parameters drawn at the $50 \%$ probability level. The asymmetric unit contains one complex molecule, one $\mathrm{BF}_{4}^{-}$ anion and one THF solvent molecule. The structure was refined as an inversion twin using the twin law -100 0-10 00-1 (BASF: 0.49(3)). The reflections 1053,1042 , -8 3 5, 631 , -10 21,64 $3,552,541,510,1036$ and 1004 are removed from the refinement using OMIT commands.

Empirical formula

Formula weight

Temperature

Wavelength

Crystal system

Space group

Unit cell dimensions

Volume

$\mathrm{Z}$

Density (calculated)

Absorption coefficient

$\mathrm{F}(000)$
$\mathrm{C}_{44} \mathrm{H}_{96} \mathrm{BCl}_{2} \mathrm{~F}_{4} \mathrm{Mo}_{2} \mathrm{~N}_{4} \mathrm{OP}_{4}$

1170.71

$102(2) \mathrm{K}$

$0.71073 \AA$

Monoclinic

$\mathrm{P} 21$

$\mathrm{a}=12.3704(6) \AA$

$\alpha=90^{\circ}$

$\mathrm{b}=13.7799(7) \AA$

$\beta=95.233(2)^{\circ}$

$\mathrm{c}=16.8847(8) \AA$

$2866.2(2) \AA^{3}$

2

$1.357 \mathrm{Mg} / \mathrm{m}^{3}$

$0.690 \mathrm{~mm}^{-1}$

1230 
Crystal size

Crystal shape and color

Theta range for data collection

Index ranges

Reflections collected

Independent reflections

Completeness to theta $=25.242^{\circ}$

Refinement method

Data / restraints / parameters

Goodness-of-fit on $\mathrm{F}^{2}$

Final R indices [I $>2 \operatorname{sigma}(\mathrm{I})]$

$\mathrm{R}$ indices (all data)

Absolute structure parameter

Largest diff. peak and hole
$0.373 \times 0.165 \times 0.118 \mathrm{~mm}^{3}$

Plate, clear intense brown

2.218 to $28.389^{\circ}$

$-16<=\mathrm{h}<=16$

$-18<=\mathrm{k}<=18$

$-22<=\mathrm{l}<=22$

109664

$14315[\mathrm{R}(\mathrm{int})=0.0528]$

$99.9 \%$

Full-matrix least-squares on $\mathrm{F}^{2}$

14315 / 1 / 584

1.088

$\mathrm{R} 1=0.0334, \quad \mathrm{wR} 2=0.0737$

$\mathrm{R} 1=0.0390$,

$\mathrm{wR} 2=0.0775$

1.034 and $-0.787 \mathrm{e}^{-3}$ 


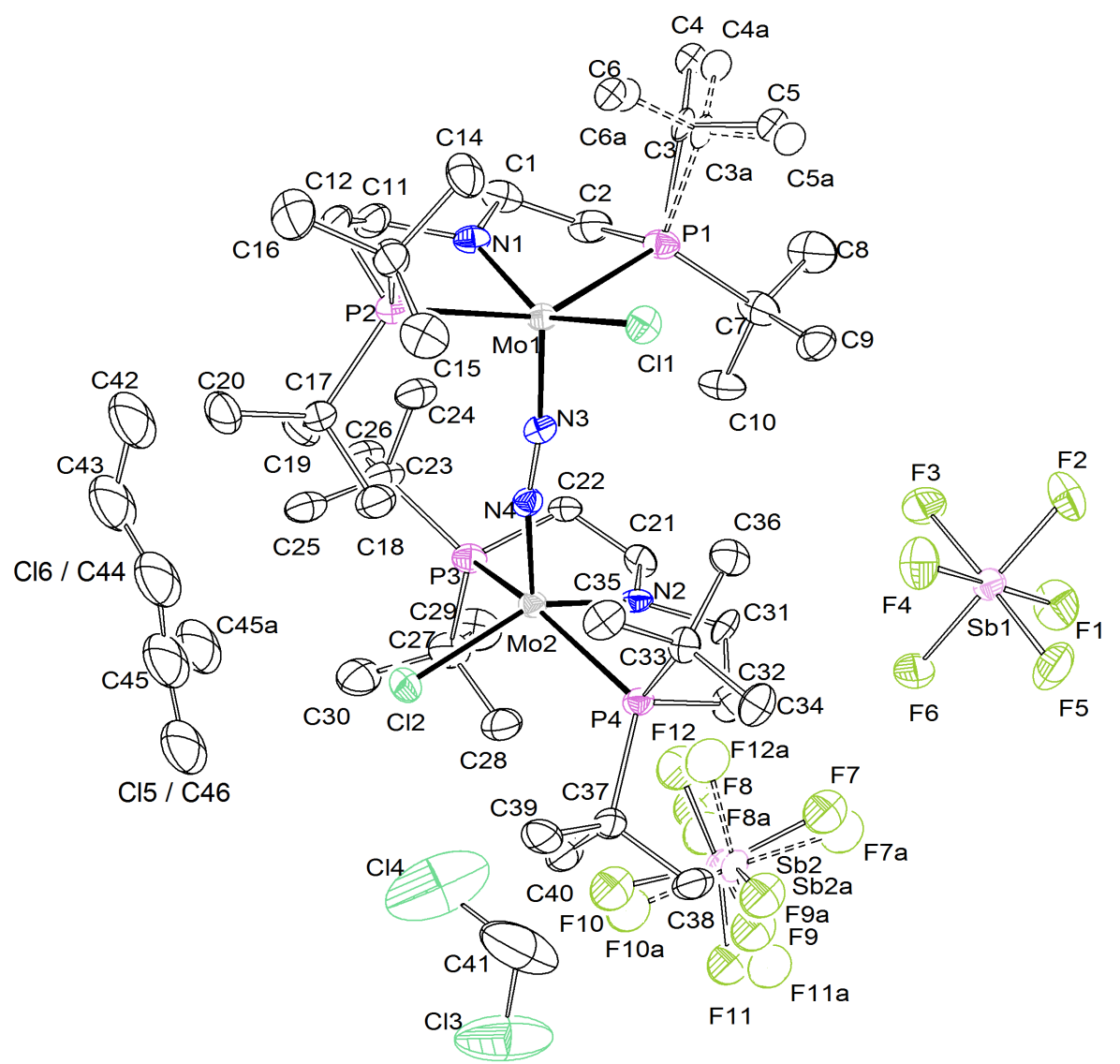

Figure 7.37: Thermal ellipsoid plot of $\mathbf{4 9}$ with the anisotropic displacement parameters drawn at the $50 \%$ probability level. The asymmetric unit contains one disordered complex molecule, two $\mathrm{SbF}_{6}^{-}$anions, one and a half dichloromethane and half a pentane molecule. The disordered complex molecule was set to a population of 0.5 using some restraints and constraints (SADI, EADP). The solvent molecules were set to a population of 0.5 for one of the dichloromethane and pentane molecules using some restraints and constrains (SADI, EADP, EXYZ). One of the $\mathrm{SbF}_{6}^{-}$anions was disordered and refined with a population of $0.73(1)$ for the main domain using some restraints and constraints (SADI, EADP).

Empirical formula

Formula weight

Temperature

Wavelength

Crystal system

Space group

Unit cell dimensions

Volume

Z

Density (calculated)
$\mathrm{C}_{44} \mathrm{H}_{97} \mathrm{Cl}_{5} \mathrm{~F}_{12} \mathrm{Mo}_{2} \mathrm{~N}_{4} \mathrm{P}_{4} \mathrm{Sb}_{2}$

1646.76

100(2) K

$0.71073 \AA$

Triclinic

P-1

$\mathrm{a}=13.9158(14) \AA$

$\alpha=108.380(3)^{\circ}$

$\mathrm{b}=15.9147(17) \AA$

$\mathrm{c}=17.1828(19) \AA$

$3277.1(6) \AA^{3}$

2

$1.669 \mathrm{Mg} / \mathrm{m}^{3}$ $\beta=95.891(3)^{\circ}$

$\gamma=110.746(3)^{\circ}$ 


$\begin{array}{lll}\text { Absorption coefficient } & 1.558 \mathrm{~mm}^{-1} \\ \mathrm{~F}(000) & 1656 \\ \text { Crystal size } & 0.248 \times 0.240 \times 0.216 \mathrm{~mm}^{3} & \\ \text { Crystal shape and color } & \text { Block, clear intense brown } & \\ \text { Theta range for data collection } & 2.219 \text { to } 25.815^{\circ} \\ \text { Index ranges } & -16<=\mathrm{h}<=17 \\ & -19<=\mathrm{k}<=19 \\ & -21<=\mathrm{l}<=21 \\ \text { Reflections collected } & 92374 \\ \text { Independent reflections } & 12553[\mathrm{R}(\text { int })=0.1037] & \\ \text { Completeness to theta }=25.242^{\circ} & 99.9 \% & \\ \text { Refinement method } & \text { Full-matrix least-squares on } \mathrm{F}^{2} & \\ \text { Data / restraints / parameters } & 12553 / 58 / 650 & \mathrm{wR} 2=0.1659 \\ \text { Goodness-of-fit on } \mathrm{F}^{2} & 1.095 & \mathrm{wR} 2=0.1863 \\ \text { Final R indices [I }>2 \text { sigma(I) }] & \mathrm{R} 1=0.0744 \\ \text { R indices (all data) } & \mathrm{R} 1=0.1104 \\ \text { Largest diff. peak and hole } & 1.985 \text { and }-1.643 \mathrm{eA}^{-3} & \end{array}$




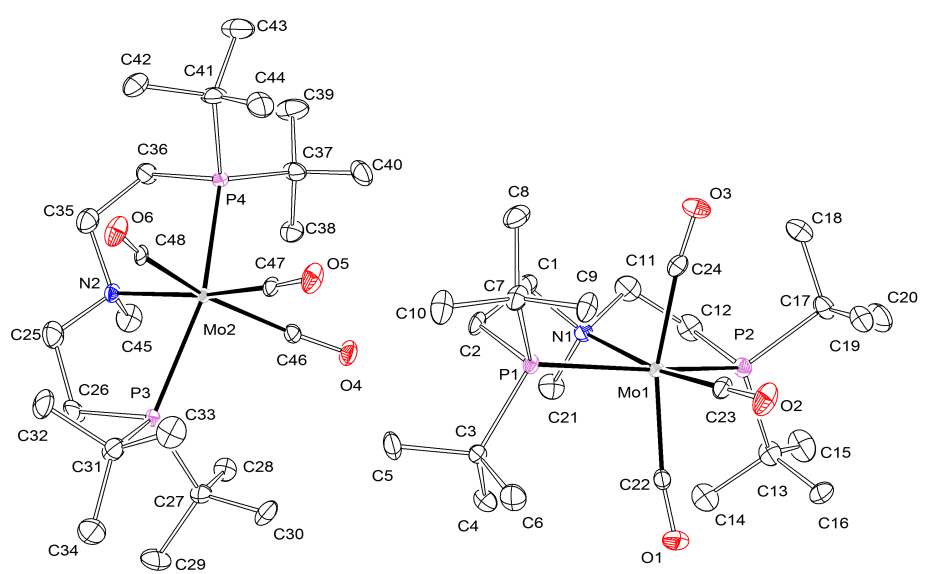

Figure 7.38: Thermal ellipsoid plot of $\mathbf{5 1}$ with the anisotropic displacement parameters drawn at the $50 \%$ probability level. The asymmetric unit contains two complex molecules. The reflections 0137,1157 and $730-3$ are removed from the refinement using OMIT commands.

Empirical formula

Formula weight

Temperature

Wavelength

Crystal system

Space group

Unit cell dimensions

Volume

Z

Density (calculated)

Absorption coefficient

$\mathrm{F}(000)$

Crystal size

Crystal shape and color

Theta range for data collection

Index ranges

Reflections collected

Independent reflections

Completeness to theta $=25.242^{\circ}$

Refinement method

Data / restraints / parameters

Goodness-of-fit on $\mathrm{F}^{2}$

Final R indices [I $>2 \operatorname{sigma}(\mathrm{I})]$

$\mathrm{R}$ indices (all data)

Largest diff. peak and hole

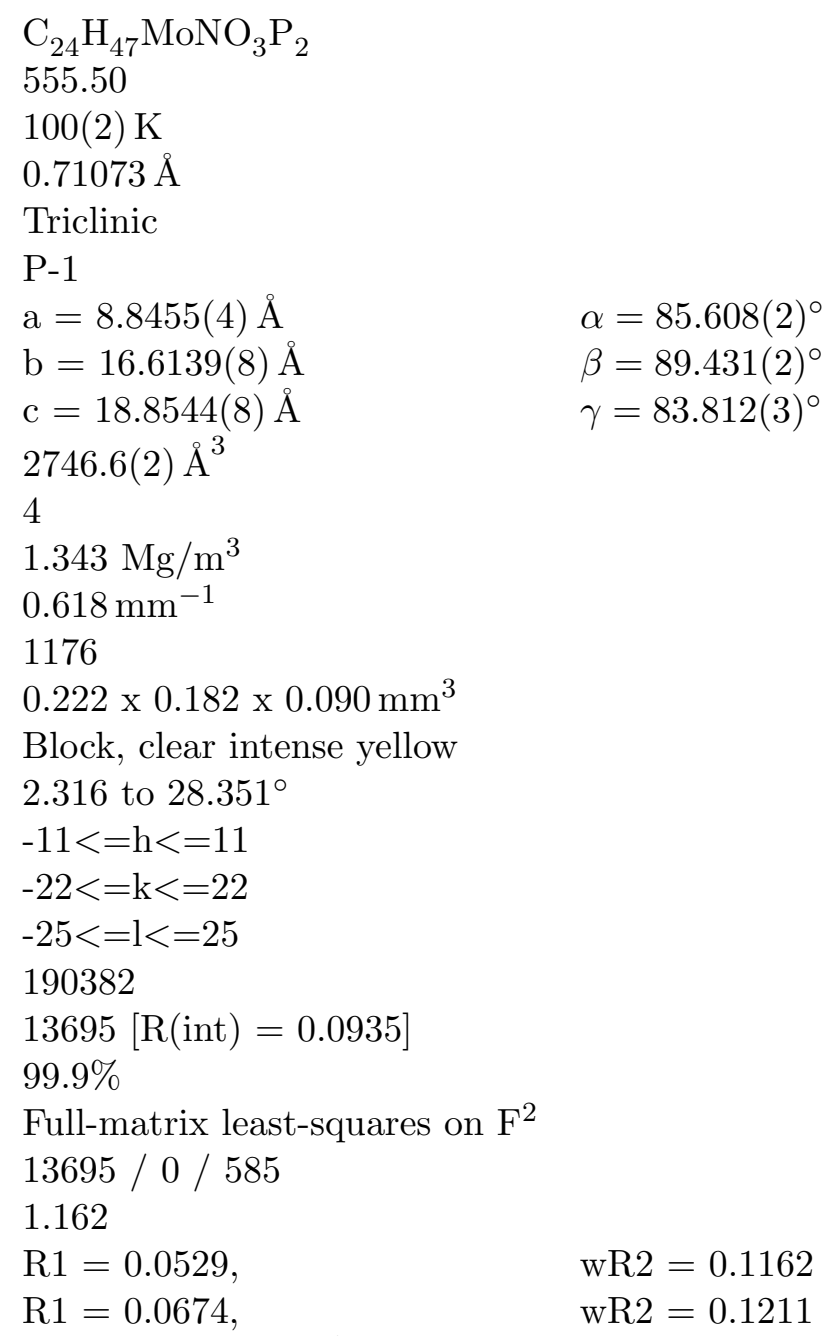

Triclinic

P-1

$\mathrm{a}=8.8455(4) \AA$

$\alpha=85.608(2)^{\circ}$

$\mathrm{b}=16.6139(8) \AA$

$\beta=89.431(2)^{\circ}$

$\mathrm{c}=18.8544(8) \AA$

$2746.6(2) \AA^{3}$

4

$1.343 \mathrm{Mg} / \mathrm{m}^{3}$

$0.618 \mathrm{~mm}^{-1}$

1176

$0.222 \times 0.182 \times 0.090 \mathrm{~mm}^{3}$

Block, clear intense yellow

2.316 to $28.351^{\circ}$

$-11<=\mathrm{h}<=11$

$-22<=\mathrm{k}<=22$

$-25<=\mathrm{l}<=25$

190382

$13695[\mathrm{R}(\mathrm{int})=0.0935]$

$99.9 \%$

Full-matrix least-squares on $\mathrm{F}^{2}$

13695 / 0 / 585

1.162

$\mathrm{R} 1=0.0529$,

$\mathrm{wR} 2=0.1162$

$\mathrm{R} 1=0.0674$,

$\mathrm{wR} 2=0.1211$ 


\subsubsection{Syntheses}

\section{Synthesis of 48}

XCVII (10.2 mg, $10.1 \mu \mathrm{mol}, 1.00$ eq.) and $\mathrm{Ag}\left[\mathrm{Al}(\mathrm{OR})_{4}\right](10.8,10.1 \mu \mathrm{mol}, 1.00$ eq.) are dissolved in benzene and stirred for 16 at room temperature. The solution is filtered and the residue is extracted with benzene $(15 \times 2 \mathrm{~mL})$. The solvent is removed and the residue is dissolved in chlorobenzene $(2 \mathrm{~mL})$, layered with pentane and stored at $-35^{\circ} \mathrm{C}$ for 2 days. The precipitate is washed with benzene/pentane (1:1) and extracted with chlorobenzene $(4 \times 2 \mathrm{~mL})$. After removal of the solvent $\mathrm{P}$ is obtained as a dark brown powder $(17.5 \mathrm{mg}, 8.84 \mu \mathrm{mol}, 88 \%)$

Anal. Cald for $\mathrm{C}_{56} \mathrm{H}_{88} \mathrm{AlCl}_{2} \mathrm{~F}_{36} \mathrm{Mo}_{2} \mathrm{~N}_{4} \mathrm{O}_{4} \mathrm{P}_{4}$ (1978.98): C, 40.0; H, 4.48; N, 2.83. Found: $\mathrm{C}, 34.0 ; \mathrm{H}, 4.68 ; \mathrm{N}, 1.44$.

Like parent XCVII, 48 most likely loses dinitrogen during combustion leading to: ${ }^{280]}$

Anal. Cald for $\mathrm{C}_{56} \mathrm{H}_{88} \mathrm{AlCl}_{2} \mathrm{~F}_{36} \mathrm{Mo}_{2} \mathrm{~N}_{2} \mathrm{O}_{4} \mathrm{P}_{4}$ (1950.97): C, 34.5; H, 4.55; N, 1.44. Found: $\mathrm{C}, 34.0 ; \mathrm{H}, 4.68 ; \mathrm{N}, 1.44$.

NMR $\left(\right.$ THF-d 8, RT): ${ }^{1} \mathrm{H}(300 \mathrm{MHz}) \delta=2.66\left(\mathrm{br}, 18 \mathrm{H}, \mathrm{PC}\left(\mathrm{CH}_{3}\right)_{3}\right), 2.41-1.98(\mathrm{br}, 54 \mathrm{H}$, $\left.\mathrm{PC}\left(\mathrm{CH}_{3}\right)_{3}\right),-2.59(\mathrm{br}, 2 \mathrm{H}),-4.02(\mathrm{br}, 4 \mathrm{H}),-4.71(\mathrm{br}, 2 \mathrm{H}),-24.08(\mathrm{br}, 2 \mathrm{H}),-30.97$ (br, 2H), -32.32 (br, 2H), -35.37 (br, $2 \mathrm{H})$.

Single-crystals suitable for X-ray diffraction were obtained upon reacting XCVII with one equivalent of $\mathrm{AgBF}_{4}$ in $\mathrm{THF}$ and successive vapor diffusion of pentane at $-35^{\circ} \mathrm{C}$.

\section{Synthesis of 49}

XCVII (12.5 mg, $12.4 \mu \mathrm{mol}, 1.00$ eq.) and $\mathrm{Ag}\left[\mathrm{Al}(\mathrm{OR})_{4}\right](25.2 \mathrm{mg}, 23.5 \mu \mathrm{mol}, 1.90$ eq.) are dissolved in benzene and stirred for $16 \mathrm{~h}$ at room temperature. The solution is filtered, washed with benzene $(5 \times 2 \mathrm{~mL})$ and the residue is extracted with DCM $(3 \times 2 \mathrm{~mL})$. The solution is concentrated, layered with toluene and stored at $-80^{\circ} \mathrm{C}$ for 2 days. The crude product is filtered, washed with benzene $(2 \times 2 \mathrm{~mL})$ and extracted with DCM. The solution Is pushed through a celite pad and dried. $\mathrm{P}$ is obtained as a brown powder (25.0 mg, $8.48 \mu \mathrm{mol}, 69 \%)$.

Anal. Cald for $\mathrm{C}_{72} \mathrm{H}_{88} \mathrm{Al}_{2} \mathrm{Cl}_{2} \mathrm{~F}_{72} \mathrm{Mo}_{2} \mathrm{~N}_{4} \mathrm{O}_{8} \mathrm{P}_{4}$ (2946.08): C, 29.4; H, 3.01; N, 1.90. Found: $\mathrm{C}, 29.2 ; \mathrm{H}, 3.30 ; \mathrm{N}, 1.66$.

$\operatorname{NMR}\left(\mathrm{CD}_{2} \mathrm{Cl}_{2}, \mathrm{RT}\right):{ }^{1} \mathrm{H}(300 \mathrm{MHz}) \delta=3.81\left(\mathrm{br}, 18 \mathrm{H}, \mathrm{PC}\left(\mathrm{CH}_{3}\right)_{3}\right), 3.58-2.88(\mathrm{br}, 54 \mathrm{H}$, $\left.\mathrm{PC}\left(\mathrm{CH}_{3}\right)_{3}\right),-5.46(\mathrm{br}, 4 \mathrm{H}),-13.08(\mathrm{br}, 2 \mathrm{H}),-15.8(\mathrm{br}, 2 \mathrm{H}),-58.93(\mathrm{br}, 2 \mathrm{H}),-71.26$ (br, 2H), -83.05 (br, 2H), -88.56 (br, 2H).

Single-crystals suitable for X-ray diffraction were obtained upon reacting XCVII with two equivalents of $\mathrm{AgSbF}_{6}$ in $\mathrm{DCM}$ and successive vapor diffusion of pentane at $-35^{\circ} \mathrm{C}$.

\section{Protonation of XCVII to XCVIII}

A J-Young NMR tube is filled with XCVII (11.1 mg, $10.9 \mu \mathrm{mol}, 1.00$ eq.) and THF $(0.3 \mathrm{~mL})$ is added carfully. HOTf $(2.0 \mu \mathrm{L}, 23 \mu \mathrm{mol}, 2.1 \mathrm{eq}$.$) is placed on top of the$ tube. After cooling to $-40^{\circ} \mathrm{C}$ the tube is shaken for 2 minutes at $-40^{\circ} \mathrm{C}$. The tube is transferred to the spectrometer and emerged in liquid nitrogen. 


\section{Synthesis of ${ }^{\mathrm{Me}} \mathbf{L}$}

${ }^{t} \mathrm{Bu}_{2} \mathrm{PCl}$ (3.80 g, $21.0 \mathrm{mmol}$, 1.0 eq.) is dissolved in THF $(20 \mathrm{~mL})$ and added slowly to a suspension of $\mathrm{Li}(439 \mathrm{mg}, 63.1 \mathrm{mmol}, 3.0 \mathrm{eq}$.$) in \mathrm{THF}(20 \mathrm{~mL})$ at $0^{\circ} \mathrm{C}$. The suspension is allowed to warm to room temperature and stirred for $4 \mathrm{~d}$. In a separate flask $\mathrm{N}\left(\mathrm{CH}_{2} \mathrm{CH}_{2} \mathrm{Cl}\right)_{2} \mathrm{Me} \cdot \mathrm{HCl}(1.94 \mathrm{~g}, 10.1 \mathrm{mmol}, 0.48$ eq. $)$ is deprotonated in THF $(10 \mathrm{~mL})$ by slow addition of n-butyllithium (2.5 M in n-hexane, $4.21 \mathrm{~mL}, 10.5 \mathrm{mmol}, 0.5$ eq.) at $-70^{\circ} \mathrm{C}$ and subsequent warming to room temperature. To this flask the greenish solution of ${ }^{t} \mathrm{Bu}_{2} \mathrm{PLi}$ is added to slowly at $-70^{\circ} \mathrm{C}$. The solution is heated to $70^{\circ} \mathrm{C}$ for $16 \mathrm{~h}$ and after cooling to room temperature, the solution is filtered, and the volume is reduced to $10 \mathrm{~mL}$. Impurities are extracted upon addition of degassed water $(3 \times 20 \mathrm{~mL})$ and stirring for 30 minutes before removing the aqueous phase. After evaporation of the solvent ${ }^{\mathrm{Me}} \mathrm{L}(3.8 \mathrm{~g}, 9.60 \mathrm{mmol}, 92 \%)$ is obtained in form of a colorless oil. NMR $\left(\mathrm{C}_{6} \mathrm{D}_{6}\right.$, $\mathrm{RT}):{ }^{1} \mathrm{H}(500 \mathrm{MHz}): \delta=2.81-2.74\left(\mathrm{~m}, 4 \mathrm{H}, \mathrm{NCH} \mathrm{H}_{2}\right), 2.30(\mathrm{~s}, 3 \mathrm{H}, \mathrm{Me}), 1.71-1.64$ $\left(\mathrm{m}, 4 \mathrm{H}, \mathrm{PC} \mathrm{H}_{2}\right), 1.11\left(\mathrm{~d},{ }^{3} J_{\mathrm{HP}}=10.7 \mathrm{~Hz}, 36 \mathrm{H}, \mathrm{PC}\left(\mathrm{CH}_{3}\right){ }_{3} \cdot{ }^{13} \mathrm{C}\left\{{ }^{1} \mathrm{H}\right\}(125.8 \mathrm{MHz}): \delta\right.$ $=58.8\left(\mathrm{~d},{ }^{2} J_{\mathrm{CP}}=37.2 \mathrm{~Hz}, 2 \mathrm{C}, \mathrm{NCH}_{2}\right), 41.9(\mathrm{~s}, 1 \mathrm{C}, \mathrm{Me}), 31.1\left(\mathrm{~d},{ }^{1} J_{\mathrm{CP}}=22.6 \mathrm{~Hz}, 4 \mathrm{C}\right.$, $\left.\mathrm{PC}\left(\mathrm{CH}_{3}\right)_{3}\right), 29.7\left(\mathrm{~d},{ }^{2} J_{\mathrm{CP}}=14.1 \mathrm{~Hz}, 12 \mathrm{C}, \mathrm{PC}\left(\mathrm{CH}_{3}\right)_{3}\right), 19.9\left(\mathrm{~d},{ }^{1} J_{\mathrm{CP}}=22.5 \mathrm{~Hz}, 2 \mathrm{C}\right.$, $\left.\mathrm{PCH}_{2}\right) .{ }^{31} \mathrm{P}\left\{{ }^{1} \mathrm{H}\right\}(202.5 \mathrm{MHz}): \delta=24.5\left(\mathrm{~s}, 2 \mathrm{P}, \mathrm{PC}\left(\mathrm{CH}_{3}\right)_{3}\right)$. HR-ESI $\left(\frac{m}{z}\right): 376.3268$ $\left([\mathrm{M}+\mathrm{H}]^{+}\right.$, calcd.: 376.3256$)$.

\section{Attempted complexation of $\left[\mathrm{MoCl}_{3}(\text { thf })_{3}\right]$ by ${ }^{\mathrm{Me}} \mathrm{L}$}

PhCl, elevated temperatures: $\left[\mathrm{MoCl}_{3}(\mathrm{thf})_{3}\right](64.0 \mathrm{mg}, 154 \mu \mathrm{mol}, 1.00$ eq. $)$ and ${ }^{\mathrm{Me}} \mathrm{L}(80.0 \mathrm{mg}$, $213 \mu \mathrm{mol}, 1.38$ eq.) are dissolved in $\mathrm{PhCl}(10 \mathrm{~mL})$ and heated to $50^{\circ} \mathrm{C}$ for $16 \mathrm{~h}$. The solvent is removed, the residue is washed with pentane $(5 \times 5 \mathrm{~mL})$ and extracted with benzene $(3 \times 5 \mathrm{~mL})$. Lyophilization yields and orange powder.

$\operatorname{NMR}\left(\mathrm{C}_{6} \mathrm{D}_{6}, \mathrm{RT}\right):{ }^{1} \mathrm{H}(300 \mathrm{MHz}): \delta=25.39,16.76,10.9,5.99,1.12,-7.69,-20.66,-63.26$. DCM, THF (1:2): $\left[\mathrm{MoCl}_{3}(\mathrm{thf})_{3}\right]\left(10.0 \mathrm{mg}, 24.1 \mu \mathrm{mol}, 1.00\right.$ eq.) and ${ }^{\mathrm{Me}} \mathrm{L}(8.9 \mathrm{mg}, 24 \mu \mathrm{mol}$, 1.0 eq.) are dissolved in $\mathrm{DMC} / \mathrm{THF}(10 \mathrm{~mL}, 1: 2)$ and heated stirred at room temperature for $16 \mathrm{~h}$. The solvent is removed, the residue is washed with pentane $(5 \times 5 \mathrm{~mL})$ and extracted with benzene $(3 \times 5 \mathrm{~mL})$. Lyophilization yields and orange powder.

$\operatorname{NMR}\left(\mathrm{C}_{6} \mathrm{D}_{6}, \mathrm{RT}\right):{ }^{1} \mathrm{H}(300 \mathrm{MHz}): \delta=25.57,16.84,10.14,9.50,6.01,1.12,-8.00,-63.73$.

\section{Synthesis of 51}

$\left[\mathrm{Mo}(\mathrm{CO})_{3}\right.$ (toluene)] (121 mg, $470 \mu \mathrm{mol}, 1.00$ eq.) and ${ }^{\mathrm{Me}} \mathrm{L}(180 \mathrm{mg}, 480 \mu \mathrm{mol}, 1.02 \mathrm{eq})$. are dissolved in benzene $(5 \mathrm{~mL})$ in the dark and stirred for $16 \mathrm{~h}$ at room temperature. The solvent is removed and the product is crystallized from pentane at $-80^{\circ} \mathrm{C} . \quad \mathbf{5 1}$ ( $210 \mathrm{mg}, 378 \mu \mathrm{mol}, 80 \%$ ) is obtained in form of a yellow powder.

Anal. Cald for $\mathrm{C}_{24} \mathrm{H}_{47} \mathrm{MoNO}_{3} \mathrm{P}_{2}$ (555.55): C, 51.9; H, 8.53; N, 2.52. Found: C, 51.8; $\mathrm{H}, 8.17 ; \mathrm{N}, 2.40$. NMR $\left(\mathrm{C}_{6} \mathrm{D}_{6}, \mathrm{RT}\right):{ }^{1} \mathrm{H}(500 \mathrm{MHz}): \delta=2.34-2.29\left(\mathrm{~m}, 2 \mathrm{H}, \mathrm{NCH}_{2}\right)$, $2.01-1.61\left(\mathrm{~m}, 2 \mathrm{H}, \mathrm{NCH}_{2}\right), 1.86(\mathrm{~s}, 3 \mathrm{H}, \mathrm{Me}), 1.57-1.50\left(\mathrm{~m}, 2 \mathrm{H}, \mathrm{PCH}_{2}\right), 1.36(18 \mathrm{H}$, $\left.\mathrm{A}_{18} \mathrm{XX}^{\prime} \mathrm{A}^{\prime}{ }_{18}, N=\left|{ }^{3} J_{\mathrm{AX}}+{ }^{5} J_{\mathrm{AX}}\right|=12.5 \mathrm{~Hz}\right), 1.31\left(18 \mathrm{H}, \mathrm{A}_{18} \mathrm{XX}^{\prime} \mathrm{A}^{\prime}{ }_{18}, N=\left.\right|^{3} J_{\mathrm{AX}}+\right.$ $\left.{ }^{5} J_{\mathrm{AX}} \mid=12.3 \mathrm{~Hz}\right), 1.31-1.27\left(\mathrm{~m}, 2 \mathrm{H}, \mathrm{PCH} \mathrm{H}_{2}\right) \cdot{ }^{13} \mathrm{C}\left\{{ }^{1} \mathrm{H}\right\}(125.8 \mathrm{MHz}): \delta=232.1(\mathrm{t}$, $\left.{ }^{2} J_{\mathrm{PP}}=5.8 \mathrm{~Hz}, 1 \mathrm{C}, C \mathrm{O}\right), 226.9\left(\mathrm{t},{ }^{2} J_{\mathrm{PP}}=9.5 \mathrm{~Hz}, 1 \mathrm{C}, C \mathrm{O}\right), 225.1\left(\mathrm{t},{ }^{2} J_{\mathrm{PP}}=8.7 \mathrm{~Hz}\right.$, $1 \mathrm{C}, C \mathrm{O}$ ), $65.2\left(\mathrm{vt}, N=\left|{ }^{1} J_{\mathrm{CP}}+{ }^{3} J_{\mathrm{CP}}\right|=5.3 \mathrm{~Hz}, 2 \mathrm{C}, \mathrm{NCH}_{2}\right.$ ), 47.3 (s, $1 \mathrm{C}, \mathrm{Me}$ ), 37.8 
$\left(\right.$ vt, $\left.{ }^{1} J_{\mathrm{PC}}=4.4 \mathrm{~Hz}, 2 \mathrm{C}, \mathrm{PC}\left(\mathrm{CH}_{3}\right)_{3}\right), 36.3\left(\mathrm{vt},{ }^{1} J_{\mathrm{PC}}=4.4 \mathrm{~Hz}, 2 \mathrm{C}, \mathrm{PC}\left(\mathrm{CH}_{3}\right)_{3}\right), 30.8$ (vt, $\left.{ }^{2} J_{\mathrm{PC}}=3.0 \mathrm{~Hz}, 6 \mathrm{C}, \mathrm{PC}\left(\mathrm{CH}_{3}\right)_{3}\right), 30.3\left(\mathrm{br}, 6 \mathrm{C}, \mathrm{PC}\left(\mathrm{CH}_{3}\right)_{3}\right), 22.3\left(\mathrm{vt}, N=\left.\right|^{2} J_{\mathrm{CP}}+\right.$ ${ }^{3} J_{\mathrm{CP}} \mid=3.6 \mathrm{~Hz}, 2 \mathrm{C}, \mathrm{NCH}_{2} .{ }^{31} \mathrm{P}\left\{{ }^{1} \mathrm{H}\right\}(202.5 \mathrm{MHz}): \delta=99.3\left(\mathrm{~s}, 2 \mathrm{P}, P\left(\mathrm{C}\left(\mathrm{CH}_{3}\right)_{3}\right)_{2}\right.$. IR $\left(\mathrm{ATR}, \mathrm{cm}^{-1}\right): \tilde{\nu}_{\mathrm{CO}}=1911,1805,1784$. 
Appendix 


\section{Appendix}

\subsection{Os(II) and Re(III) PNP Halide Complexes}

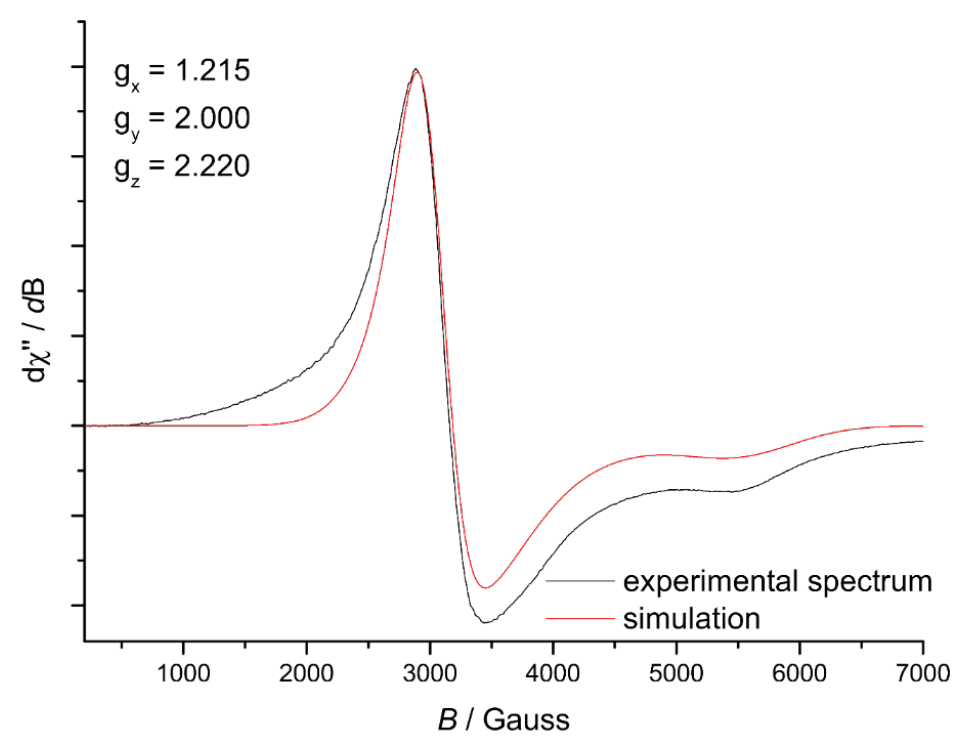

Appendix 8.1.1: EPR powder spectrum of 1, $140 \mathrm{~K}$, Freq.: $9.448 \mathrm{GHz}$.

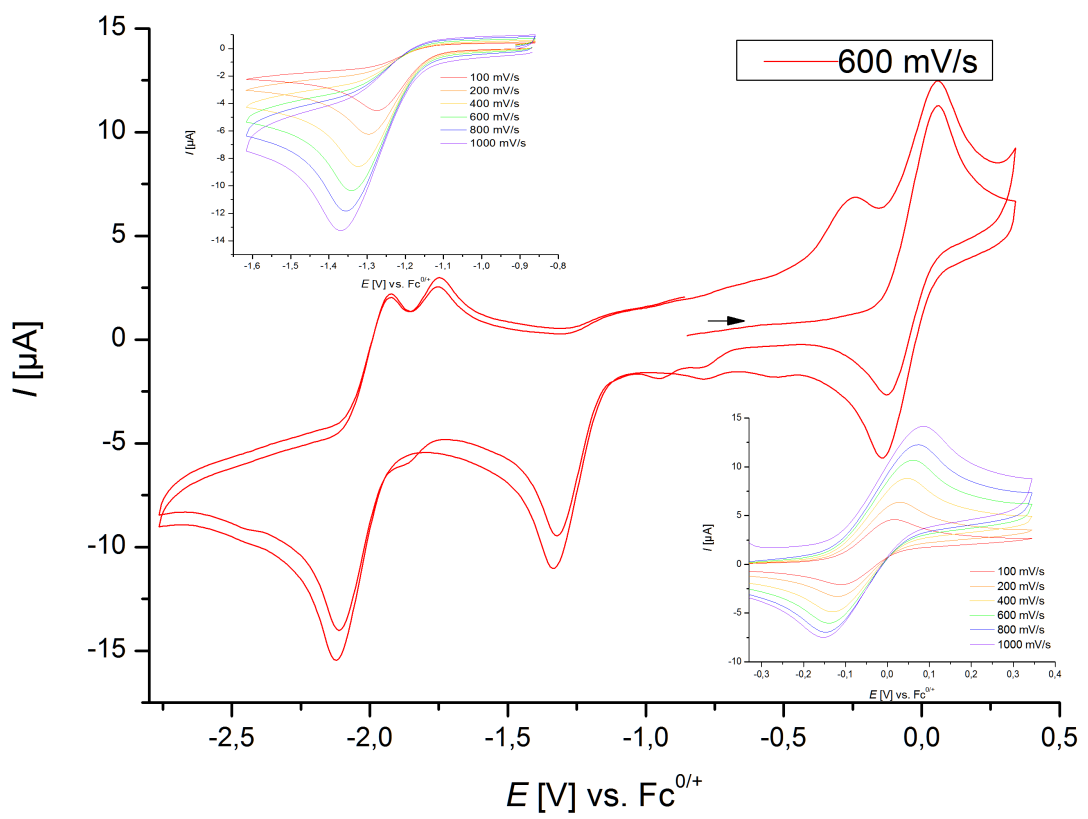

Appendix 8.1.2: Cyclovoltammogramm of 1, 0.1 mM, 0.1 M NBu $\mathrm{PF}_{6}$, THF, RT; Inset: First reduction and oxidation event. 

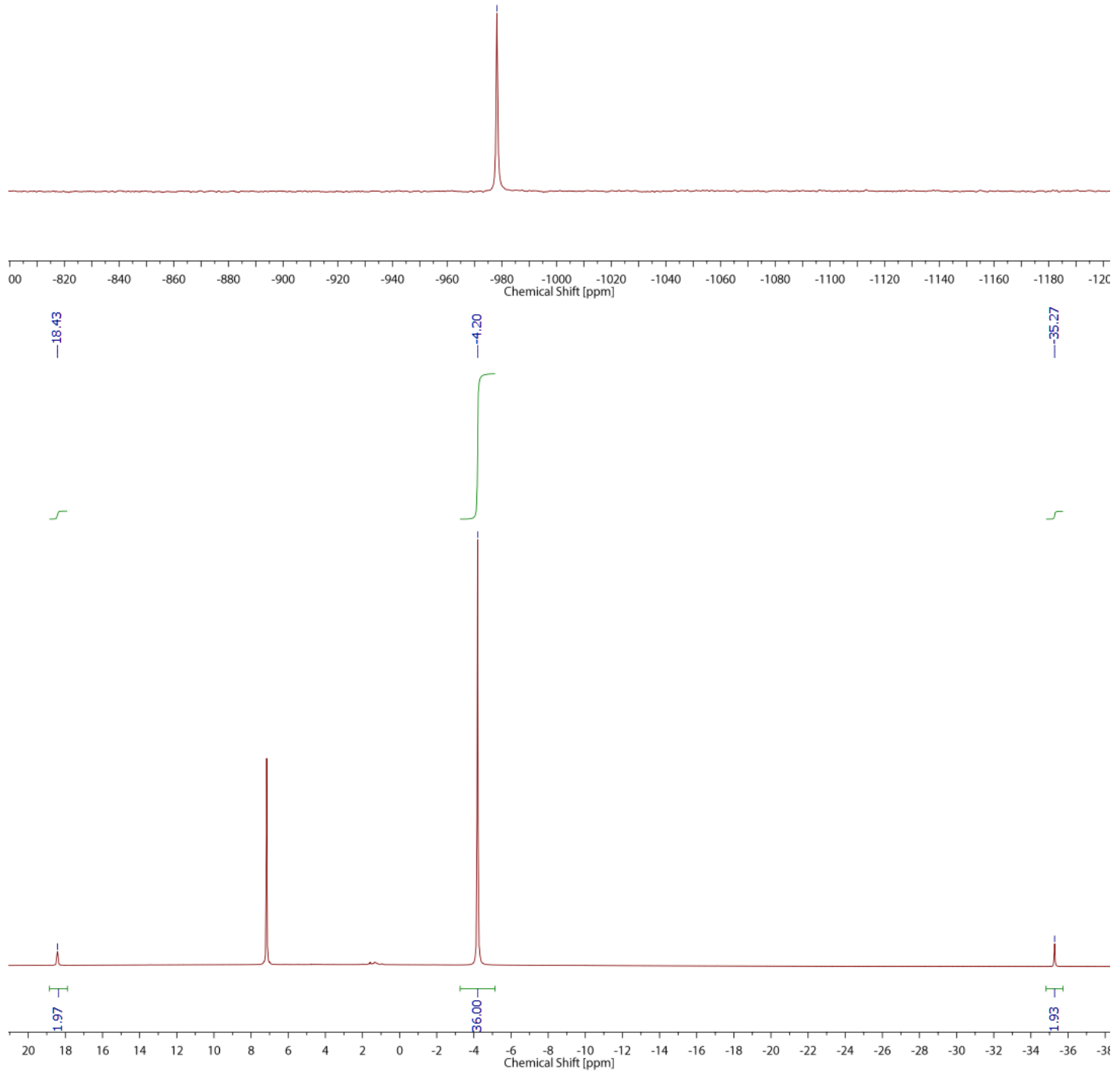

Appendix 8.1.3: Top: ${ }^{31} \mathrm{P}\left\{{ }^{1} \mathrm{H}\right\}$ NMR spectrum of 2, $\mathrm{C}_{6} \mathrm{D}_{6}$, RT; Bottom: ${ }^{1} \mathrm{H}$ NMR spectrum of $2, \mathrm{C}_{6} \mathrm{D}_{6}, \mathrm{RT}$. 


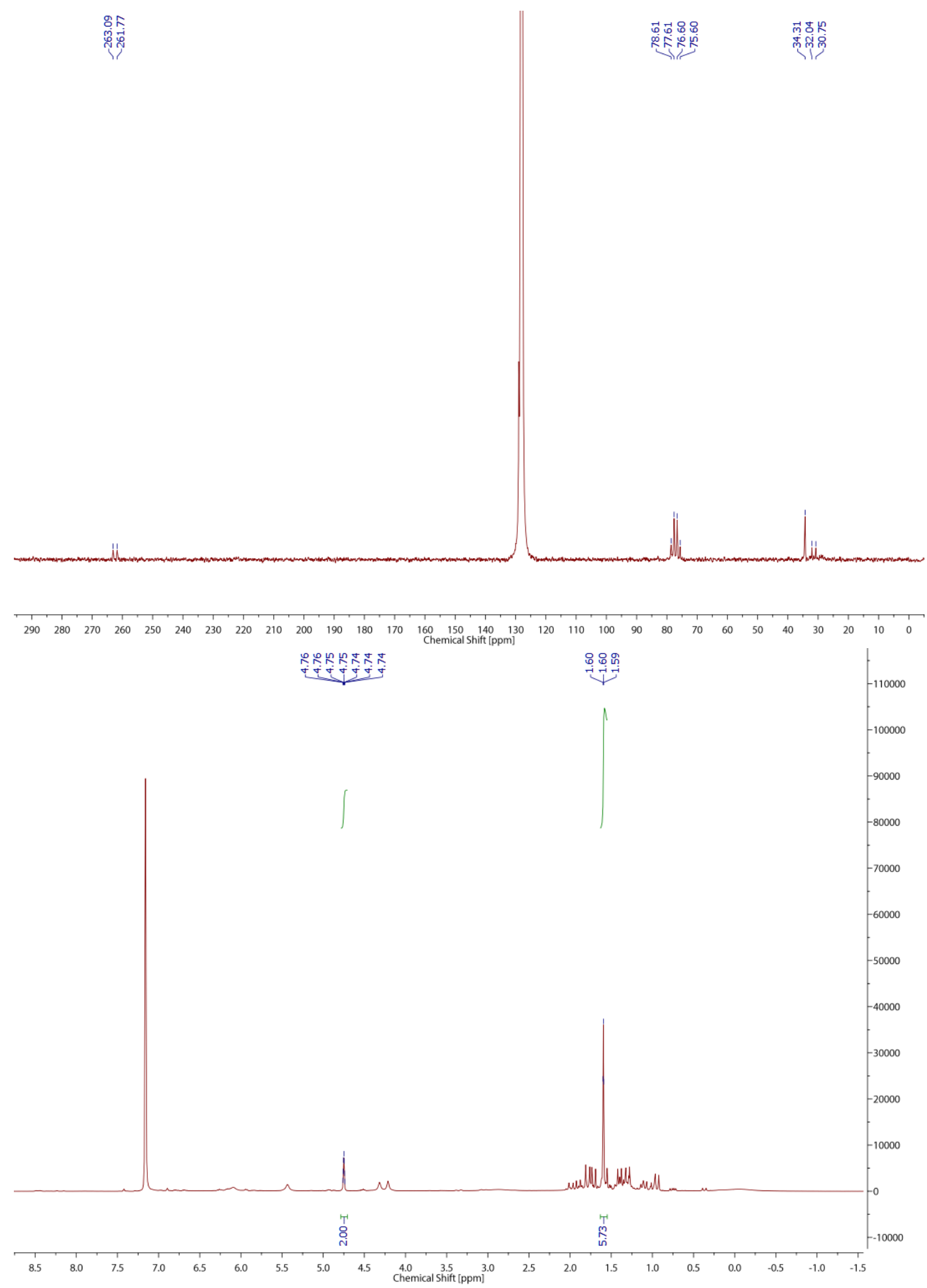

Appendix 8.1.4: Top: ${ }^{13} \mathrm{C}\left\{{ }^{1} \mathrm{H}\right\}$ NMR spectrum of 2, $\mathrm{C}_{6} \mathrm{D}_{6}$, RT; Bottom: ${ }^{1} \mathrm{H}$ NMR spectrum of 2 after $24 \mathrm{~h}$ at room temperature showing formation of isobutene, $\mathrm{C}_{6} \mathrm{D}_{6}, \mathrm{RT}$. 


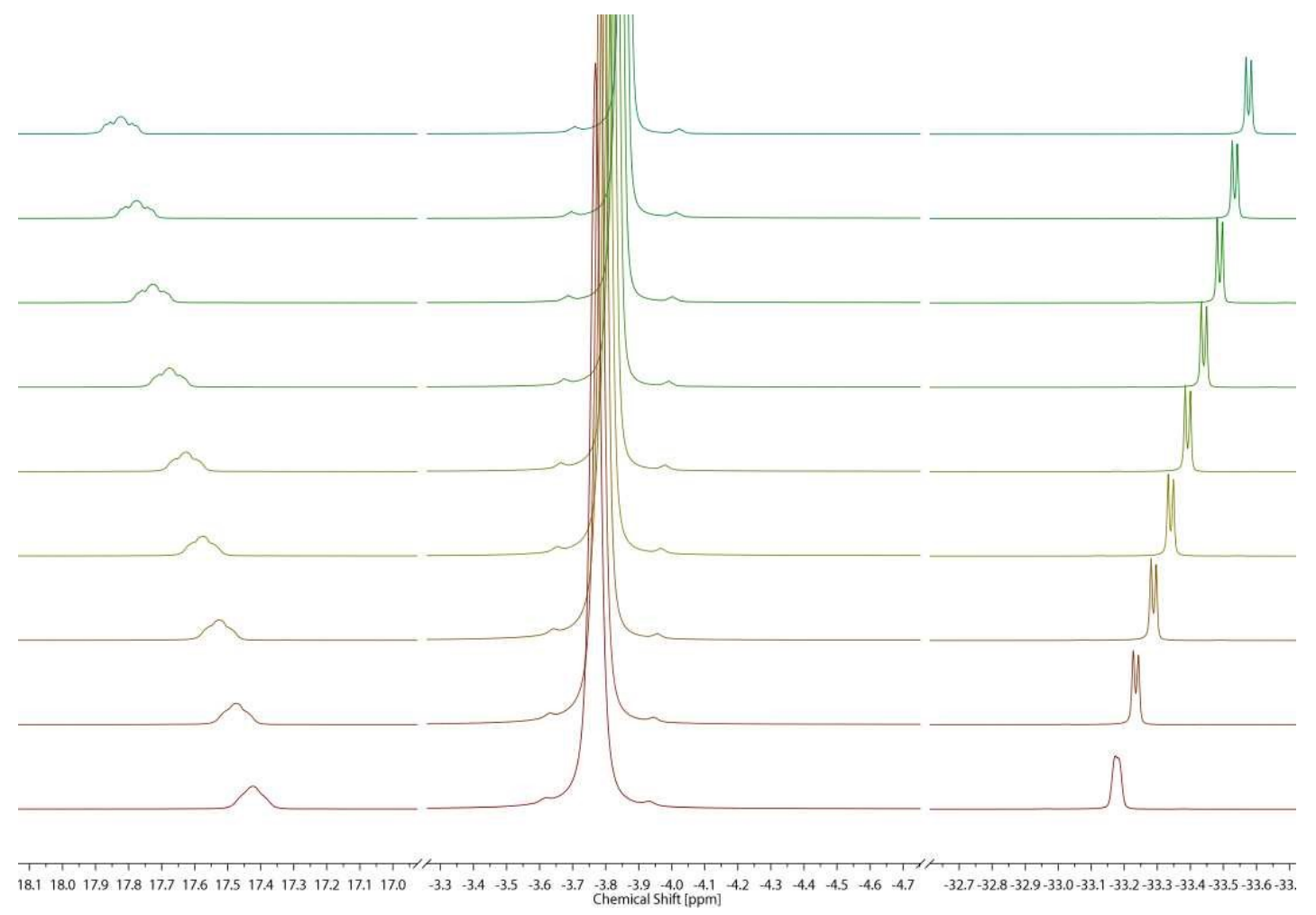

Appendix 8.1.5: $\mathrm{VT}{ }^{1} \mathrm{H}$ NMR spectrum of 2, Toluene- $d_{8},-55^{\circ} \mathrm{C}$ (red) to $-15^{\circ} \mathrm{C}$ (green), $5^{\circ} \mathrm{C}$ steps.

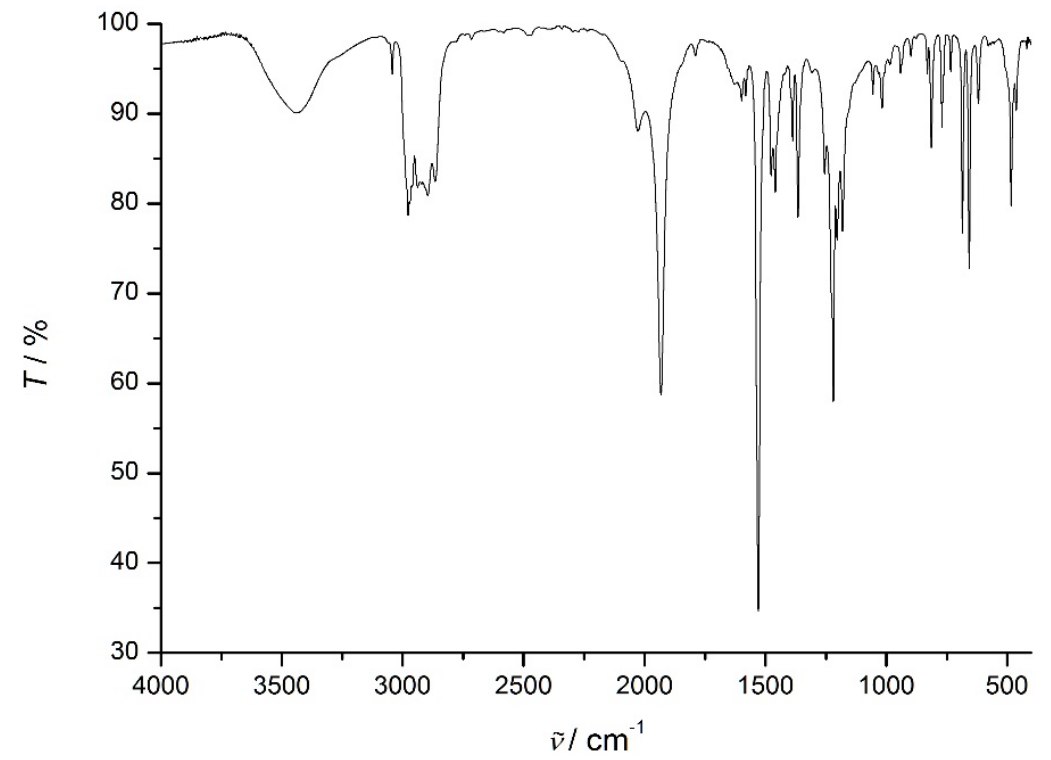

Appendix 8.1.6: $\mathrm{VT}{ }^{1} \mathrm{H}$ NMR spectrum of 2, Toluene- $d_{8},-55^{\circ} \mathrm{C}$ (red) to $-15^{\circ} \mathrm{C}$ (green), $5^{\circ} \mathrm{C}$ steps. 


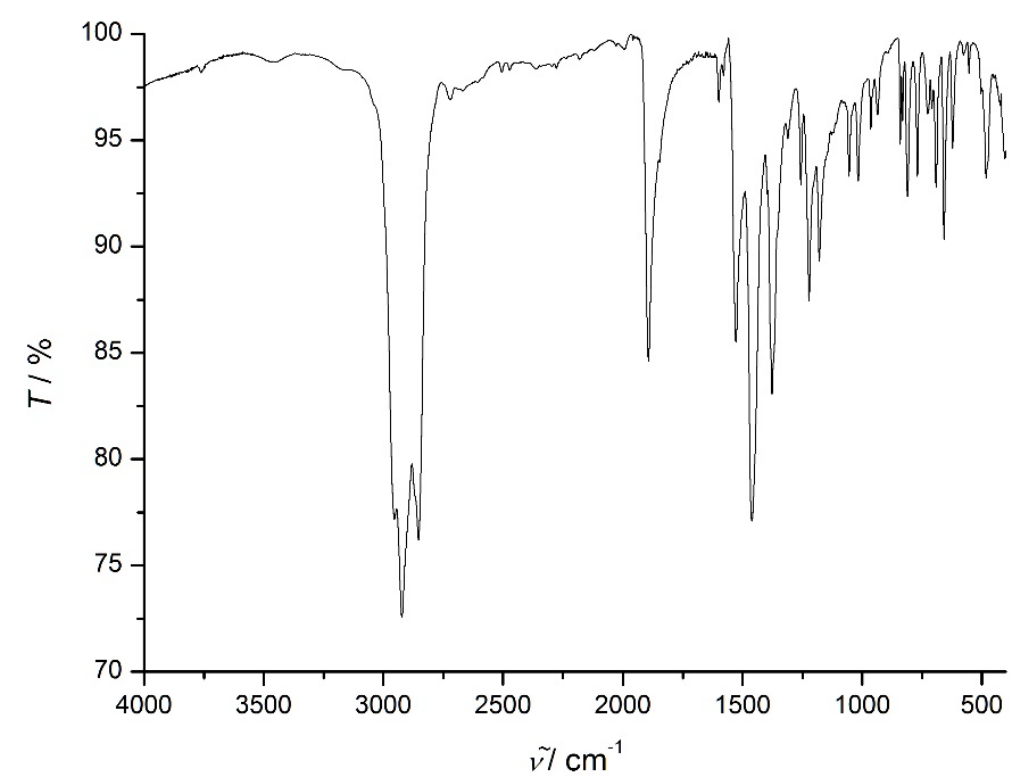

Appendix 8.1.7: Top: ${ }^{13} \mathrm{C}\left\{{ }^{1} \mathrm{H}\right\}$ NMR spectrum of 2, $\mathrm{C}_{6} \mathrm{D}_{6}$, RT; Bottom: ${ }^{1} \mathrm{H}$ NMR spectrum of 2 after $24 \mathrm{~h}$ at room temperature showing formation of isobutene, $\mathrm{C}_{6} \mathrm{D}_{6}, \mathrm{RT}$.

Table 8.1: Experimental and computed EPR parameters (Hyperfine coupling constants are given in $\mathrm{MHz})$.

\begin{tabular}{c|lll}
\hline Orientation & Parameter & Exp. & DFT \\
\hline \multirow{3}{*}{$\mathrm{x}$} & $\mathrm{g}$ & 1.8980 & 1.9118 \\
& $\mathrm{~A}_{1}\left({ }^{14} \mathrm{~N}\right)$ & & 2.06 \\
& $\mathrm{~A}_{2}\left({ }^{14} \mathrm{~N}\right)$ & & 1.53 \\
$\mathrm{y}$ & $\mathrm{g}$ & 1.9756 & 1.9723 \\
& $\mathrm{~A}_{1}\left({ }^{14} \mathrm{~N}\right)$ & & 1.82 \\
& $\mathrm{~A}_{2}\left({ }^{14} \mathrm{~N}\right)$ & & 1.70 \\
$\mathrm{z}$ & $\mathrm{g}$ & 2.015 & 2.008 \\
& $\mathrm{~A}_{1}\left({ }^{14} \mathrm{~N}\right)$ & 40 & 30.0 \\
& $\mathrm{~A}_{2}\left({ }^{14} \mathrm{~N}\right)$ & 40 & 27.0 \\
\hline
\end{tabular}




\subsection{Os Nitride and Imide Complexes in Various Coordination Spheres}
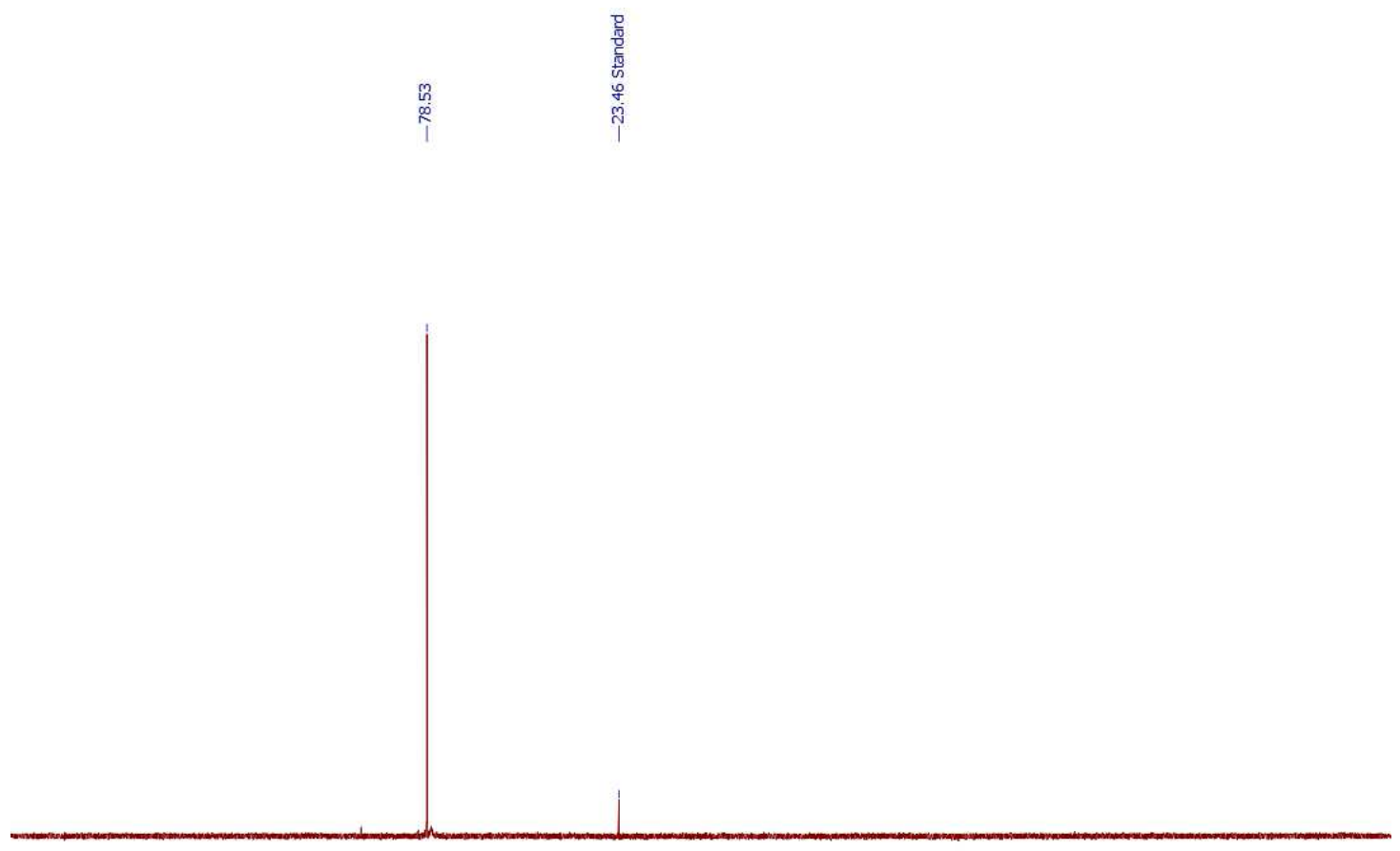

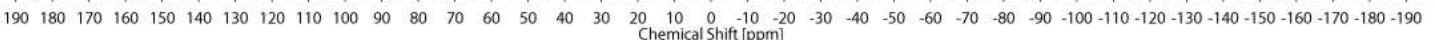

Appendix 8.2.1: ${ }^{31} \mathrm{P}\left\{{ }^{1} \mathrm{H}\right\}$ NMR spectrum of the reaction mixture after hydrogenolysis of 14, toluene-d 8 , RT.

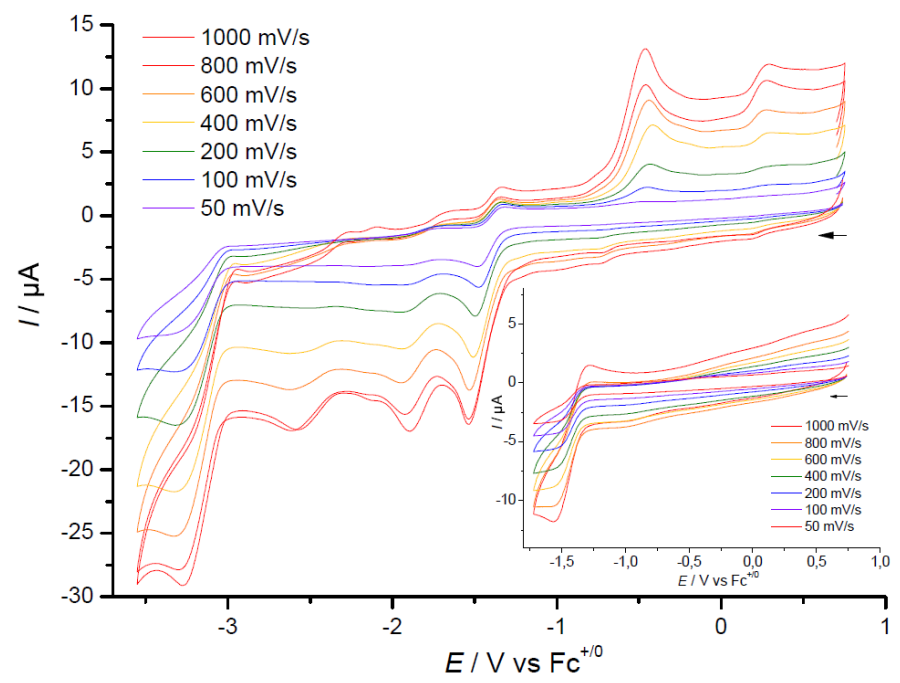

Appendix 8.2.2: Cyclic voltammogram of 15, $1 \mathrm{mM}, 0.1 \mathrm{M} \mathrm{NBu}_{4} \mathrm{PF}_{6}$, THF, RT. 


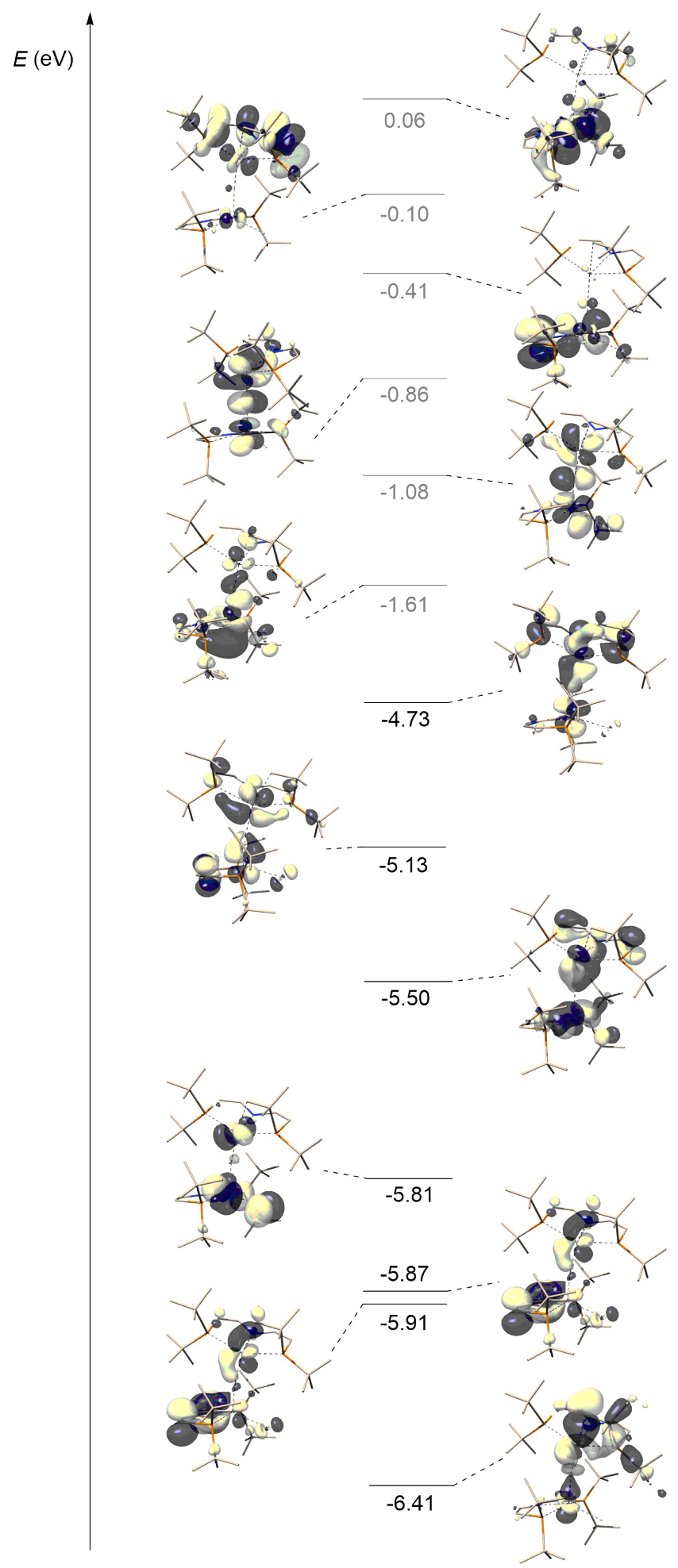

Appendix 8.2.3: Computed molecular orbital scheme of 20. Unoccupied molecular orbitals are drawn in grey and energies are given in $\mathrm{eV}$. 


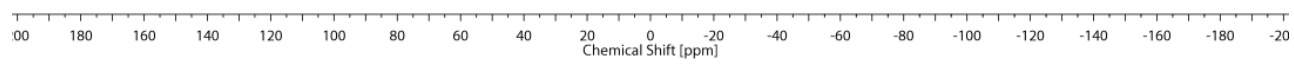

Appendix 8.2.4: ${ }^{31} \mathrm{P}\left\{{ }^{1} \mathrm{H}\right\}$ NMR spectrum of 21 after irradiation, THF- $\mathrm{d}_{8}$, RT, after 1 h of irradiation.

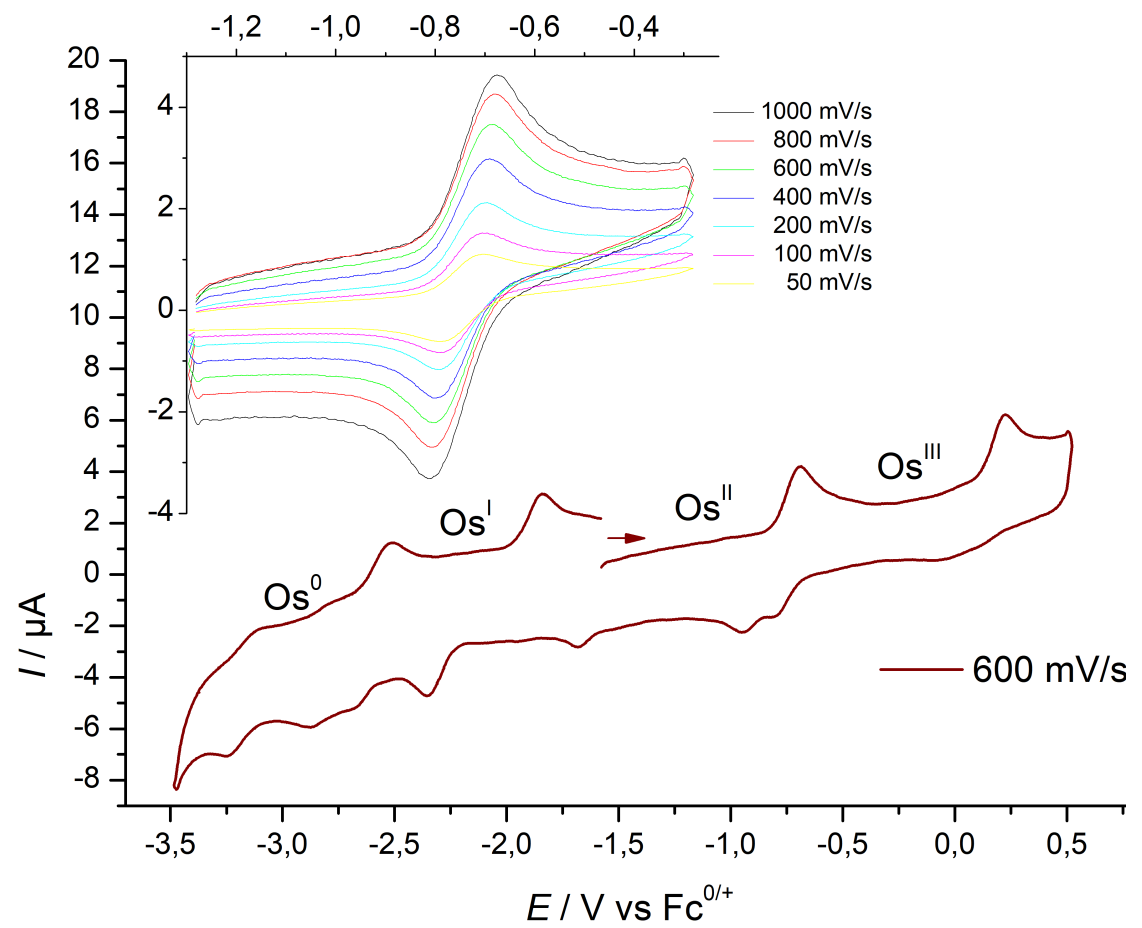

Appendix 8.2.5: Cyclic voltammogram of 21, $200 \mathrm{mV} \cdot \mathrm{s}^{-1}, 1 \mathrm{mM}, 0.1 \mathrm{M} \mathrm{NBu}_{4} \mathrm{PF}_{6}$, THF, RT. Inset: Isolated $1^{\text {st }}$ oxidative wave. 

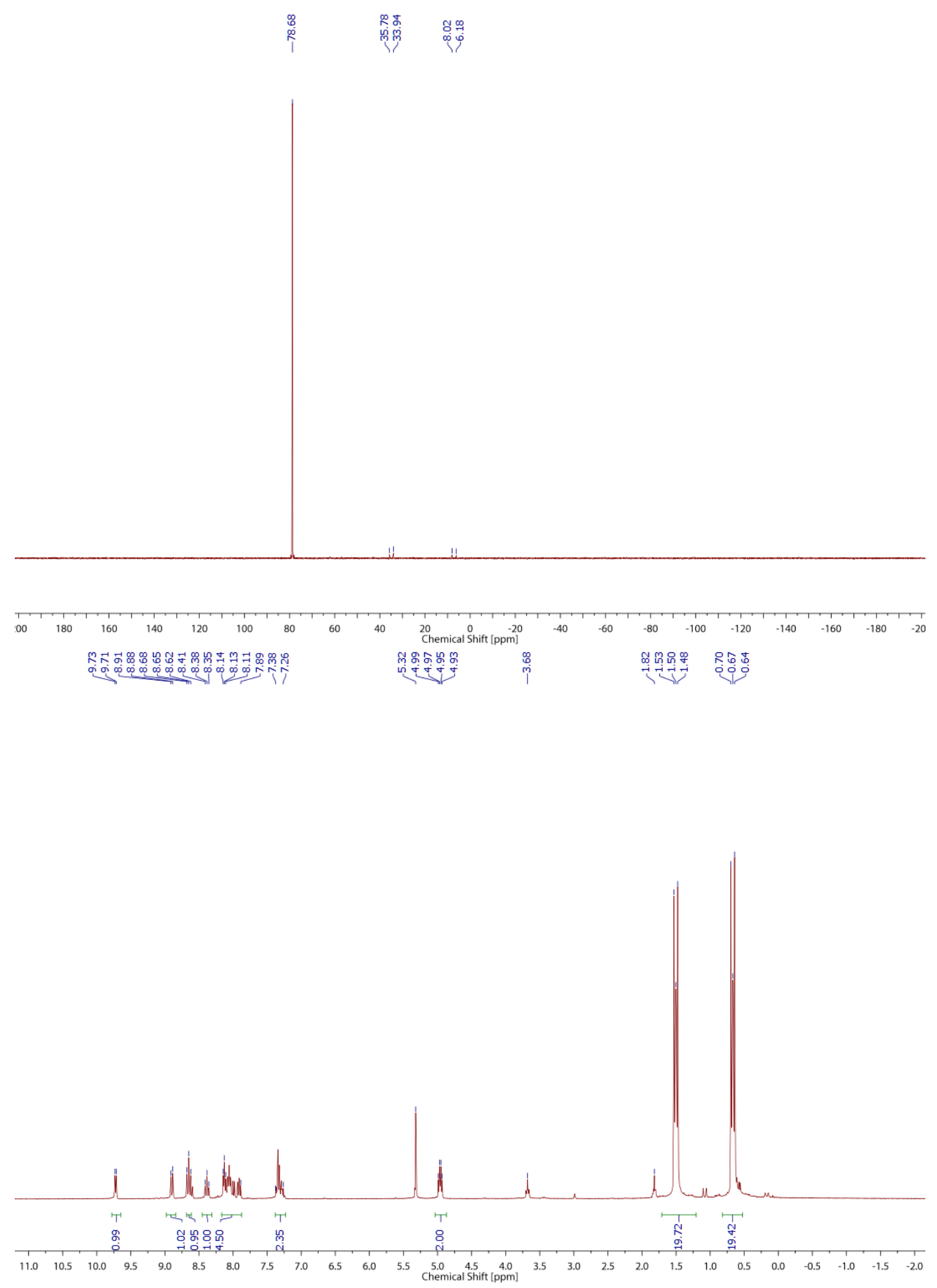

Appendix 8.2.6: Top: ${ }^{31} \mathrm{P}\left\{{ }^{1} \mathrm{H}\right\}$ NMR spectrum of the two-electron oxidation of $\mathbf{2 1}, \mathrm{CD}_{2} \mathrm{Cl}_{2}$, RT; Bottom: ${ }^{1} \mathrm{H}$ NMR spectrum of the two-electron oxidation of $\mathbf{2 1}, \mathrm{CD}_{2} \mathrm{Cl}_{2}, \mathrm{RT}$. 

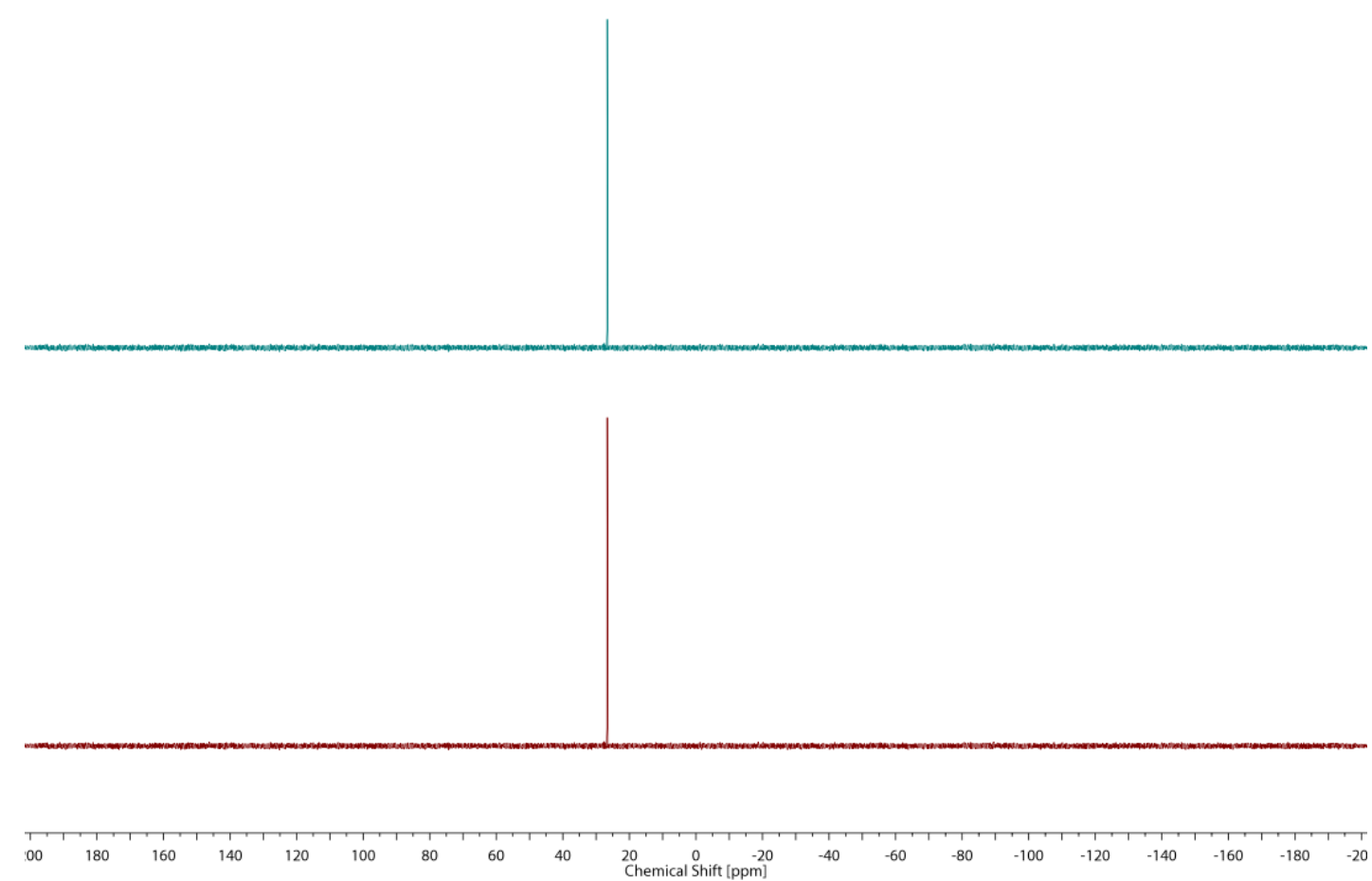

Appendix 8.2.7: Top: ${ }^{31} \mathrm{P}\left\{{ }^{1} \mathrm{H}\right\}$ NMR spectrum of the two-electron oxidation of 21 and successive reduction, $\mathrm{CD}_{2} \mathrm{Cl}_{2}$, RT; Bottom: ${ }^{31} \mathrm{P}\left\{{ }^{1} \mathrm{H}\right\}$ NMR spectrum of the one-electron oxidation of $\mathbf{2 1}, \mathrm{CD}_{2} \mathrm{Cl}_{2}$, RT. 


\subsection{Transition Metal Complexes with Metal-Pnictogen Multiple Bonds}
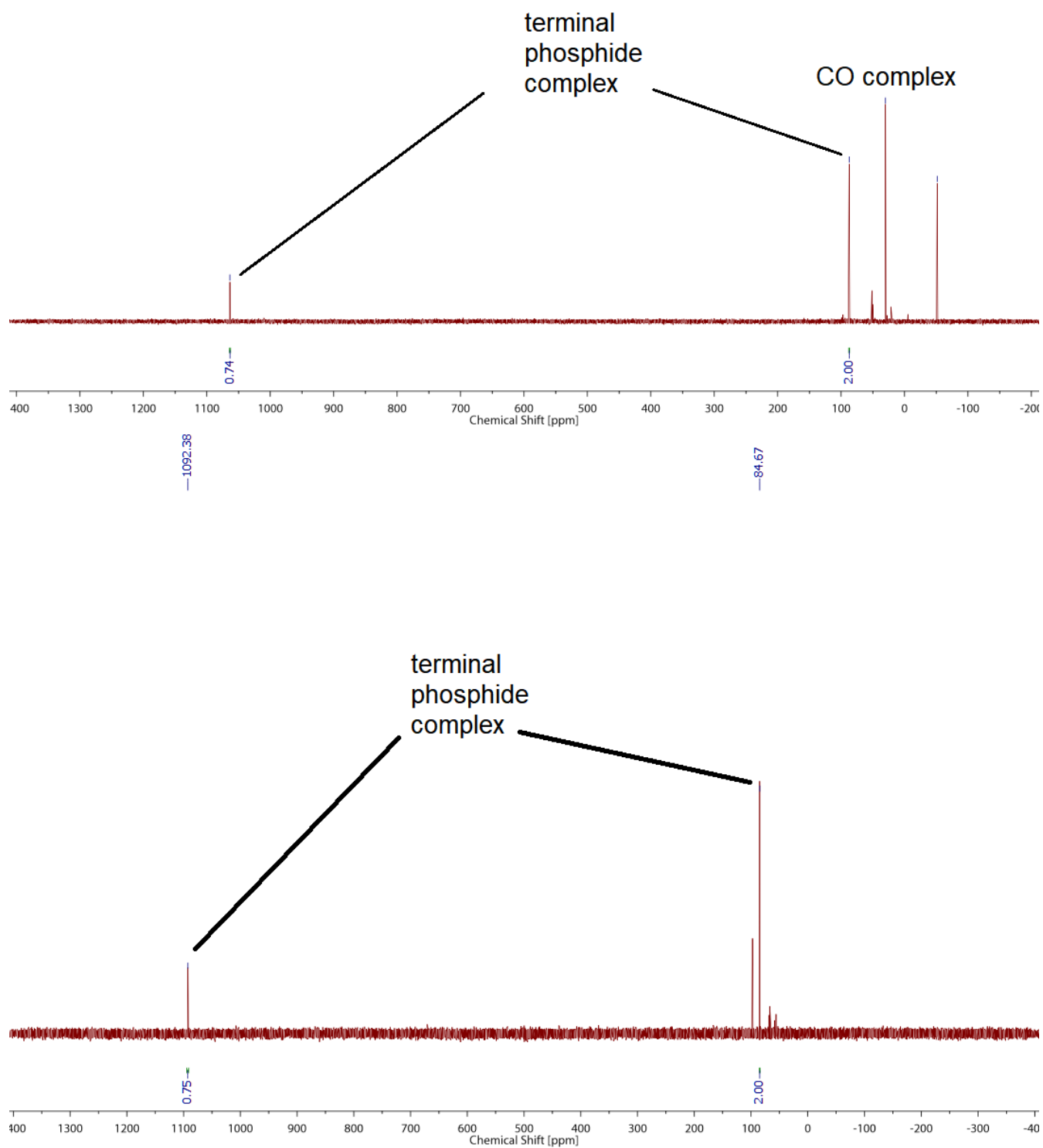

Appendix 8.3.1: Top: ${ }^{31} \mathrm{P}\left\{{ }^{1} \mathrm{H}\right\}$ NMR spectrum of the reaction of LXXIII with $\mathrm{Na}$ (diox) ${ }_{1.39} \mathrm{PCO}$; Bottom: ${ }^{31} \mathrm{P}\left\{{ }^{1} \mathrm{H}\right\}$ NMR spectrum of the reaction of $\mathbf{5}$ with $\mathrm{Na}($ diox $){ }_{1.39} \mathrm{PCO}$, $\mathrm{C}_{6} \mathrm{D}_{6}, \mathrm{RT}$ 

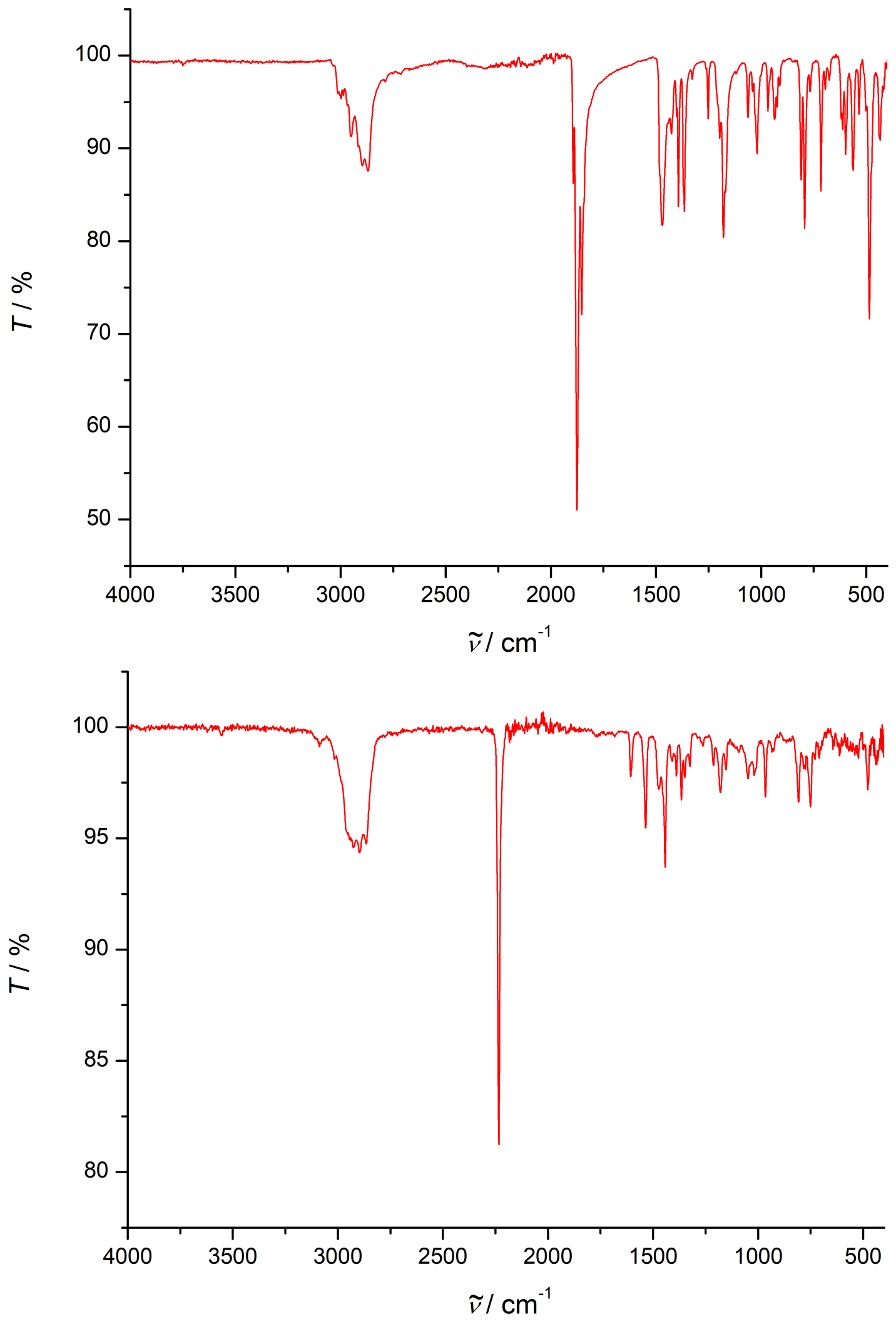

Appendix 8.3.2: Top: IR spectrum of 24, RT; Bottom: IR spectrum of 25, RT.. 

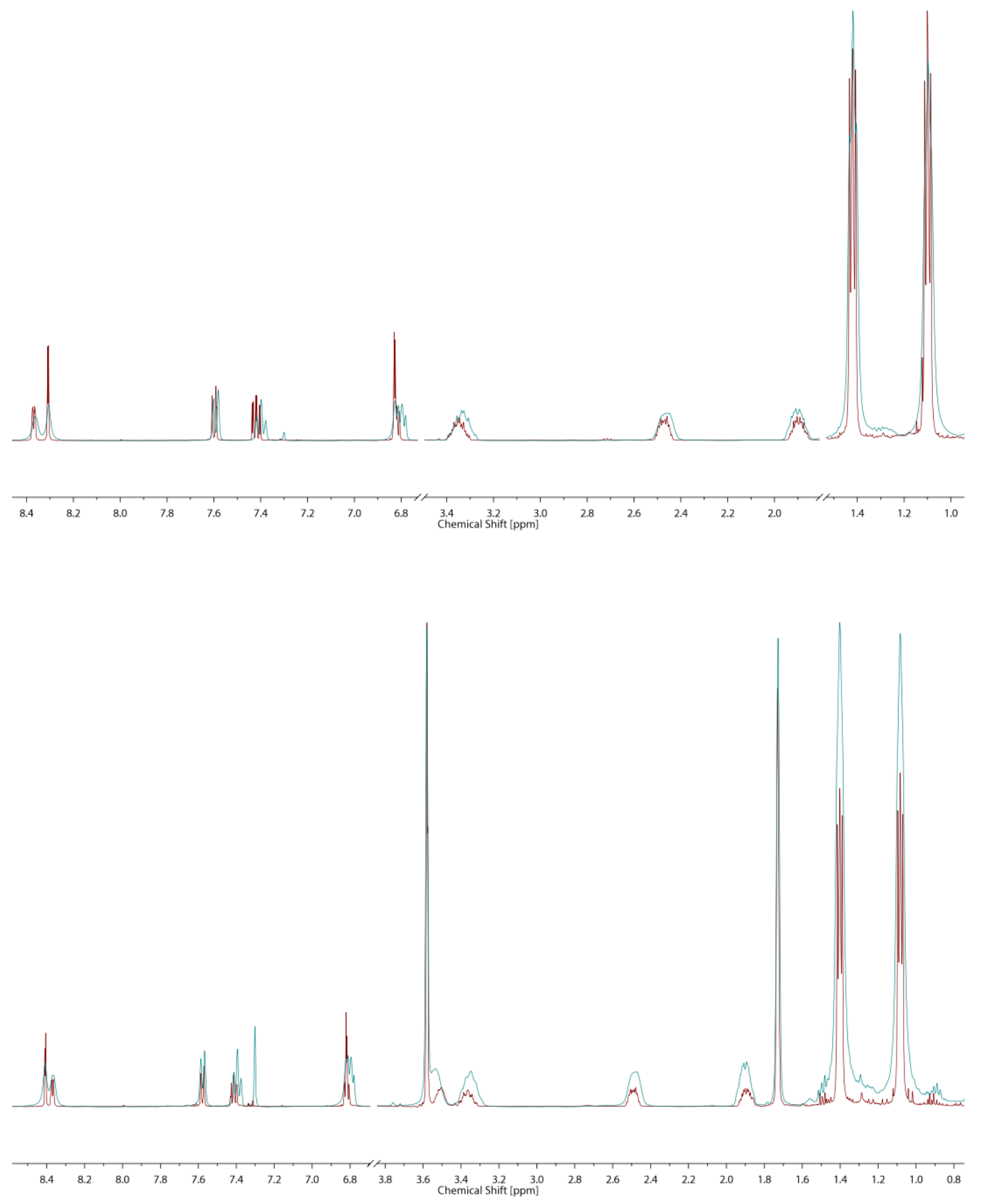

Appendix 8.3.3: Top: ${ }^{1} \mathrm{H}$ NMR spectrum of 27; Bottom: ${ }^{1} \mathrm{H}$ NMR spectrum of ??, THF- $\mathrm{d}_{8}$, $\mathrm{RT}$ (turquoise), $0^{\circ} \mathrm{C}$ (red). 


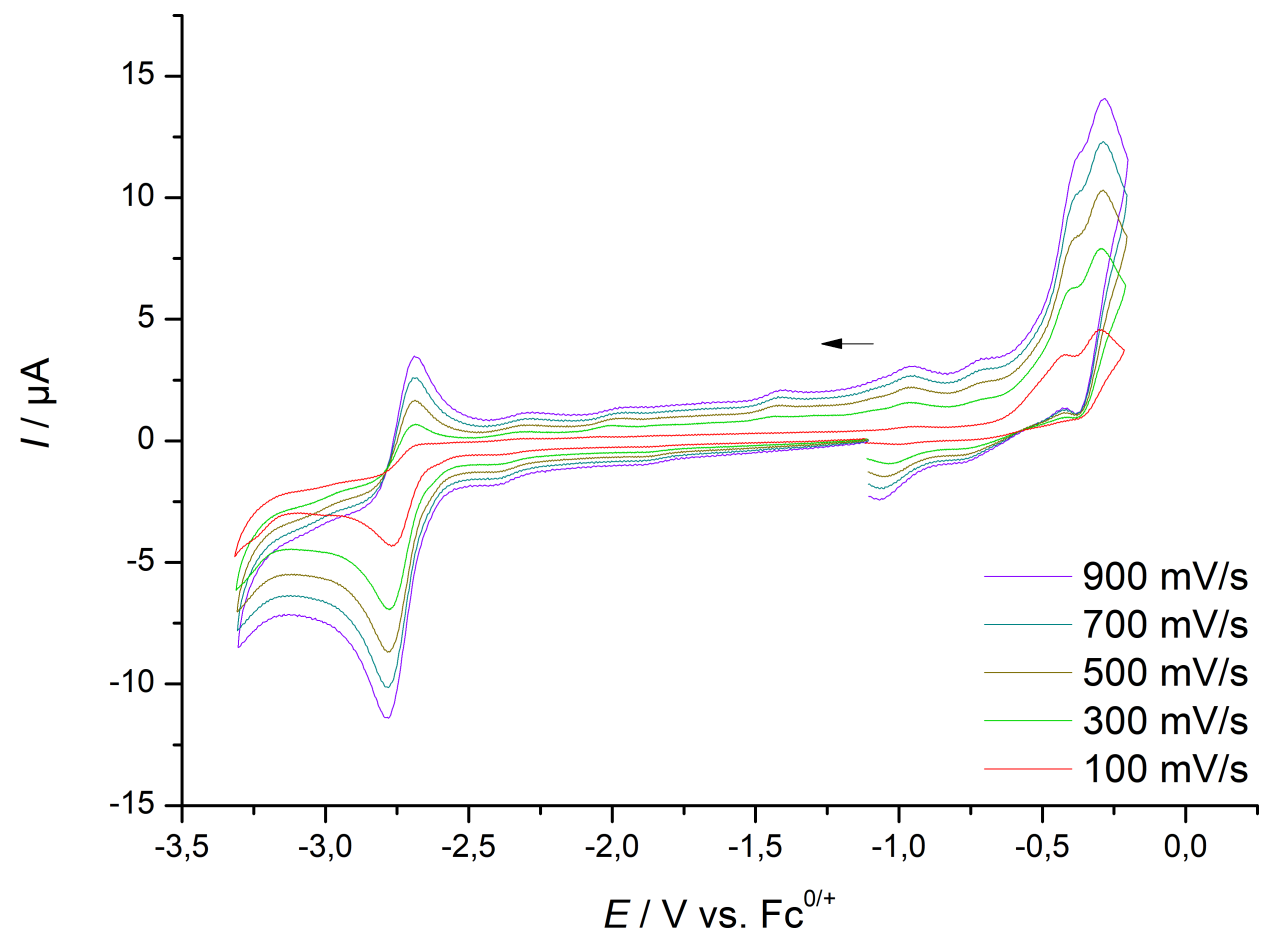

Appendix 8.3.4: Cyclic voltammogram of $\mathbf{2 7}, 1 \mathrm{mM}, 0.1 \mathrm{M} \mathrm{NBu}_{4} \mathrm{PF}_{6}$, THF, RT.

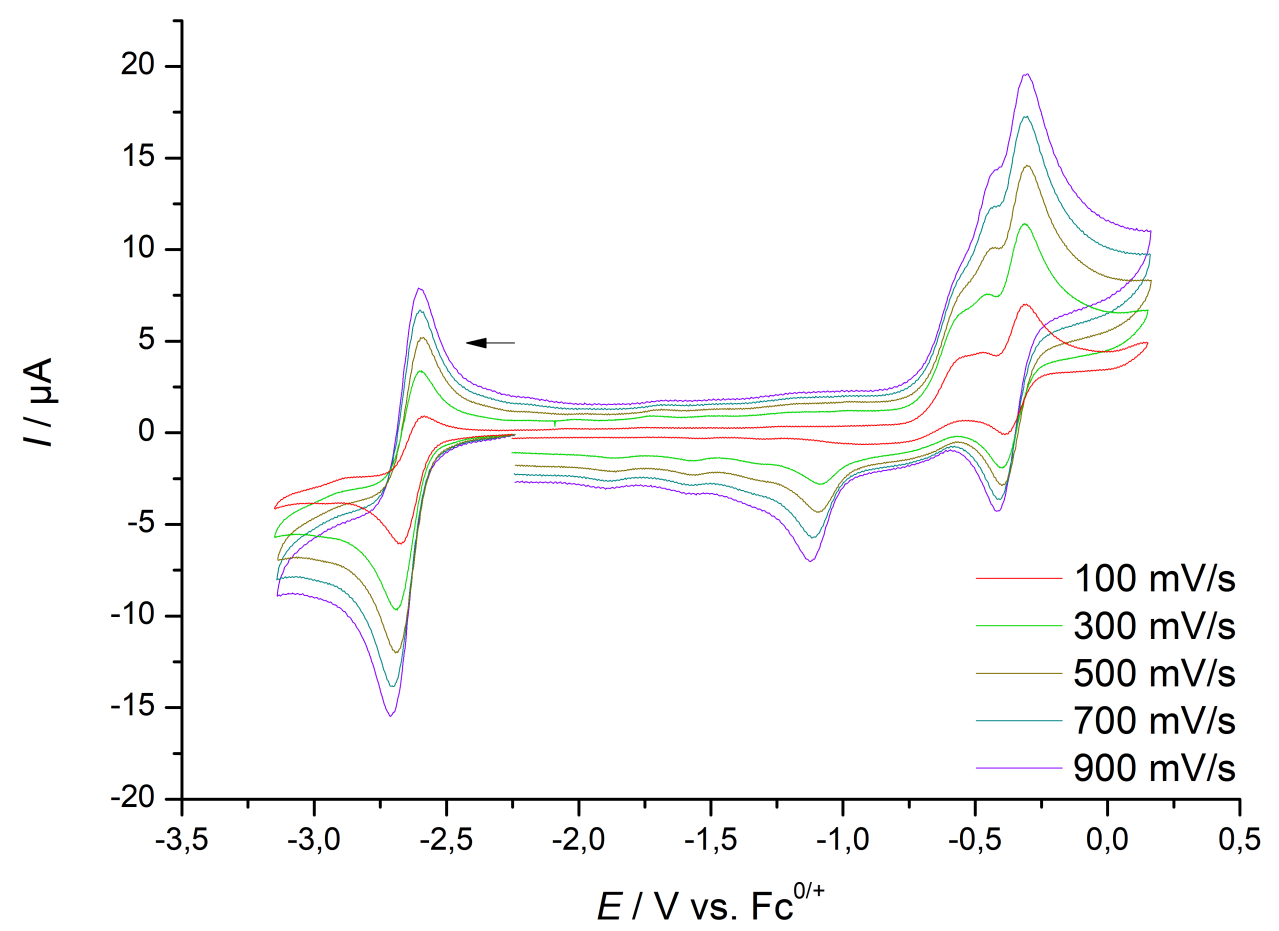

Appendix 8.3.5: Cyclic voltammogram of 28, $1 \mathrm{mM}, 0.1 \mathrm{M} \mathrm{NBu}_{4} \mathrm{PF}_{6}$, THF, RT. 

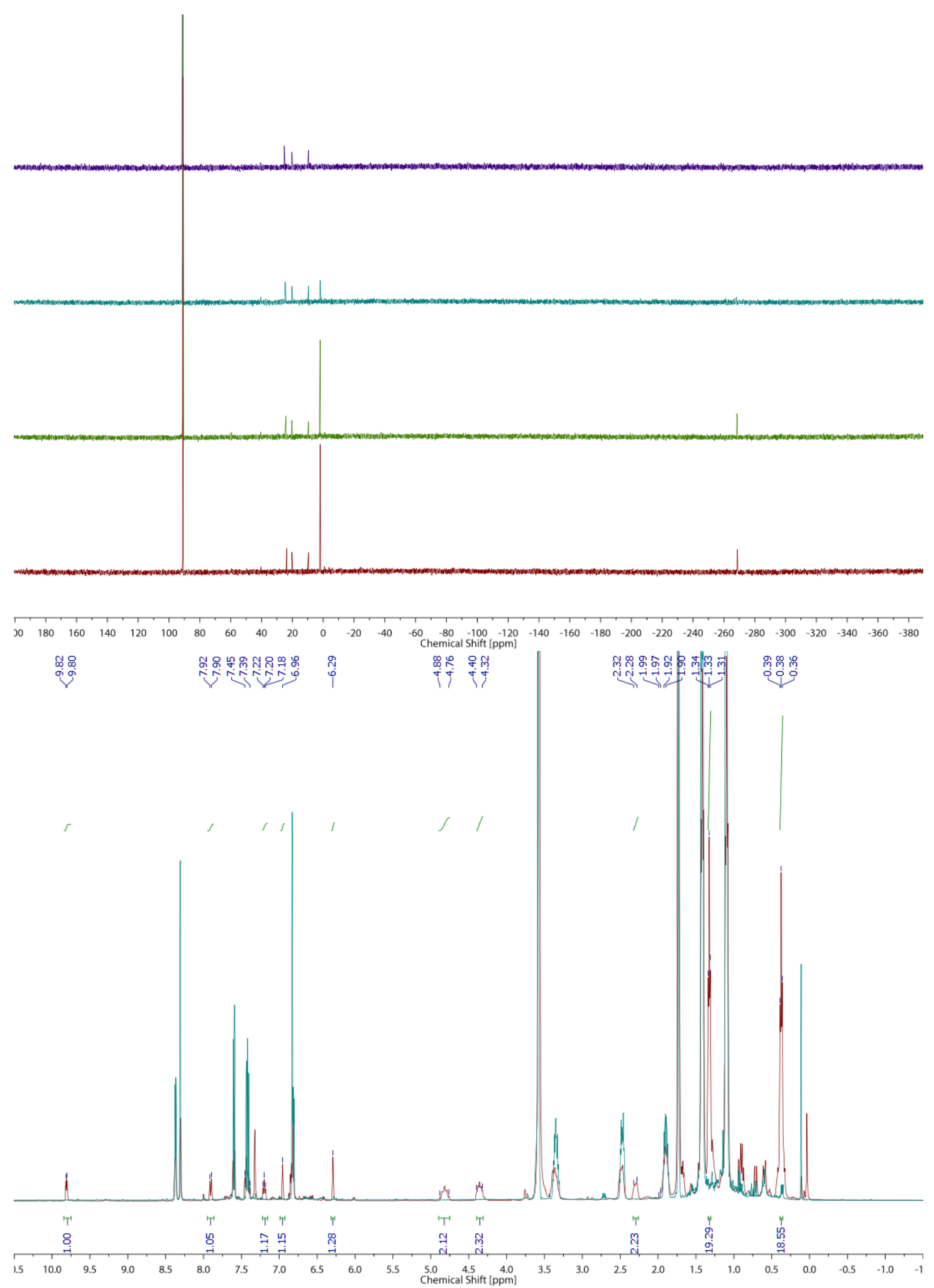

Appendix 8.3.6: Top: VT ${ }^{31} \mathrm{P}\left\{{ }^{1} \mathrm{H}\right\}$ NMR spectra of the reaction of $\mathbf{1 1}$ with $\mathrm{Na}(\text { diox })_{1.39} \mathrm{PCO}$, THF- $\mathrm{d}_{8},-20^{\circ} \mathrm{C}$ (red), $-10^{\circ} \mathrm{C}$ (green), $0^{\circ} \mathrm{C}$ (teal), $10^{\circ} \mathrm{C}$ (purple); Bottom: ${ }^{1} \mathrm{H}$ NMR spectra of the reaction of $\mathbf{1 1}$ with $\mathrm{Na}(\text { diox })_{1.39} \mathrm{PCO}$, THF- $\mathrm{d}_{8},-20^{\circ} \mathrm{C}$ (red), pure $\mathbf{2 7}$, THF- $\mathrm{d}_{8}, 0^{\circ} \mathrm{C}$ (teal), peaks belonging to $\mathbf{2 6}$ are marked in blue. 

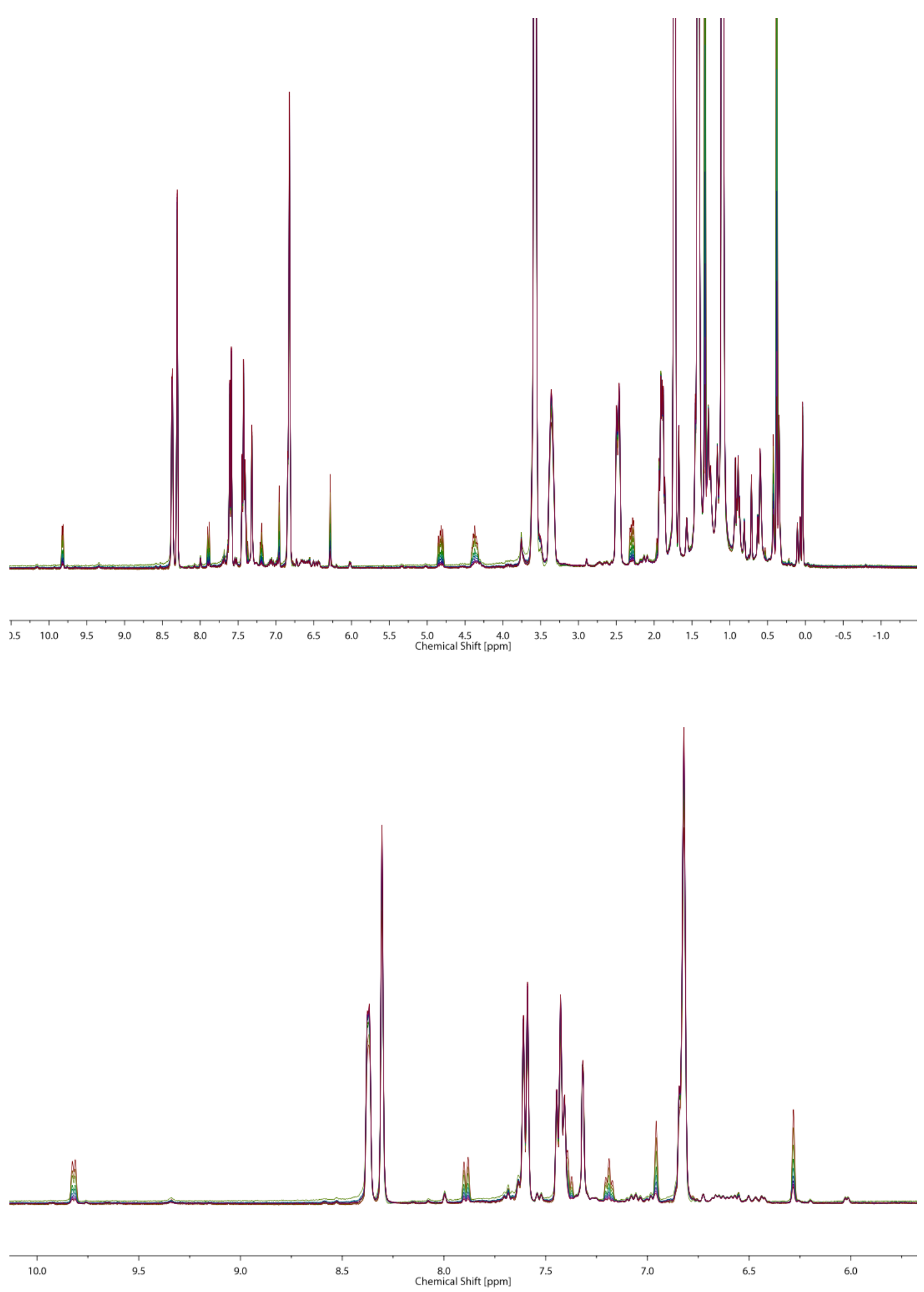

Appendix 8.3.7: Top: ${ }^{1} \mathrm{H}\left\{{ }^{31} \mathrm{P}\right\}$ NMR spectra of the reaction of 11 with $\mathrm{Na}(\operatorname{diox})_{1.39} \mathrm{PCO}$, THF- $\mathrm{d}_{8},-10^{\circ} \mathrm{C}$, the reaction was followed for $50 \mathrm{~min}$; Bottom: ${ }^{1} \mathrm{H}\left\{{ }^{31} \mathrm{P}\right\}$ NMR spectra of the aromatic region of the reaction of $\mathbf{1 1}$ with $\mathrm{Na}(\text { diox })_{1.39} \mathrm{PCO}, \mathrm{THF}-\mathrm{d}_{8},-10^{\circ} \mathrm{C}$, the reaction was followed for $50 \mathrm{~min}$. 
8 APPENDIX
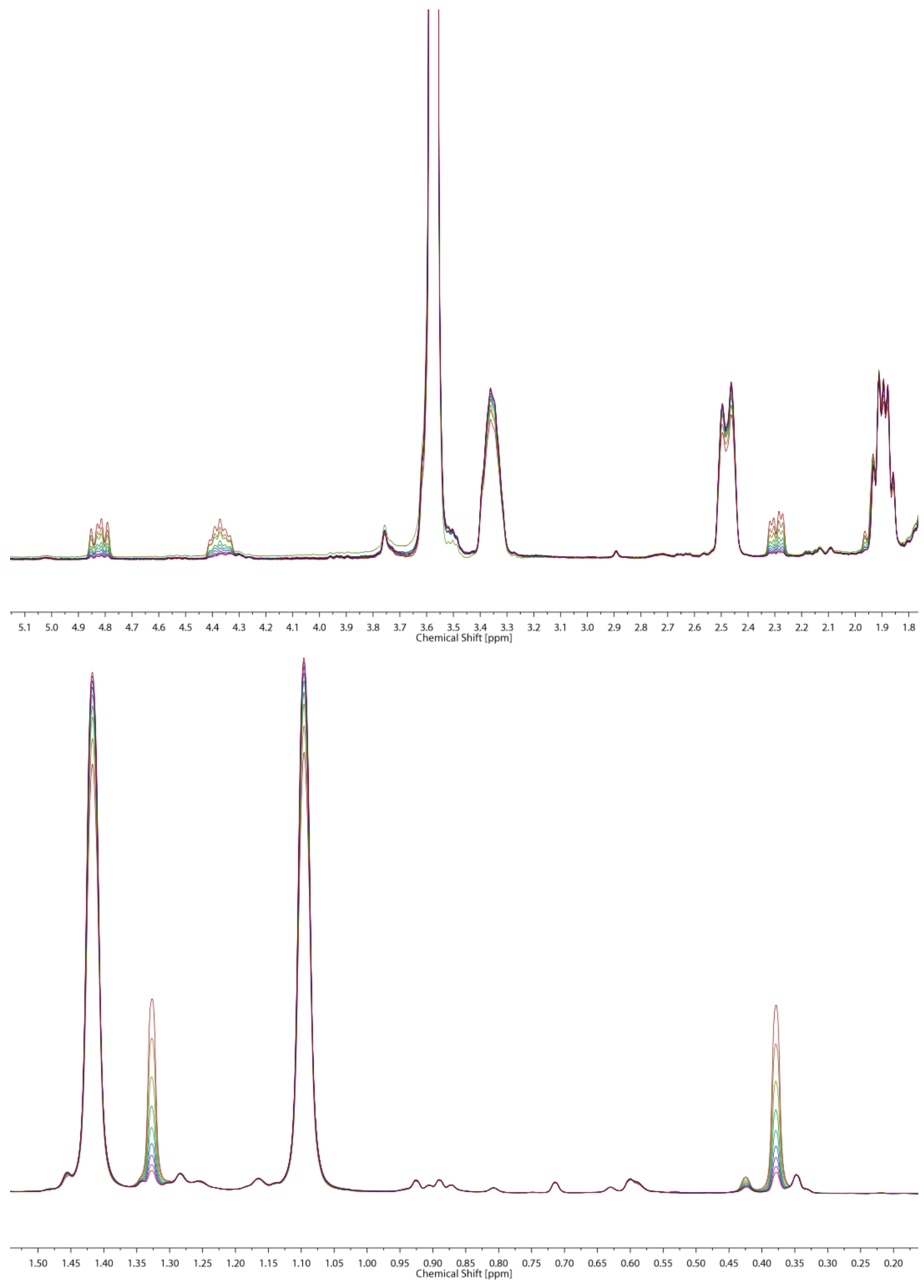

Appendix 8.3.8: Top: ${ }^{1} \mathrm{H}\left\{{ }^{31} \mathrm{P}\right\}$ NMR spectra of the PNP-aliphatic region of the reaction of 11 with $\mathrm{Na}(\text { dion })_{1.39} \mathrm{PCO}, \mathrm{THF}-\mathrm{d}_{8},-10^{\circ} \mathrm{C}$, the reaction was followed for $50 \mathrm{~min}$; Bottom: ${ }^{1} \mathrm{H}\left\{{ }^{31} \mathrm{P}\right\}$ $\mathrm{NMR}$ spectra of the $\mathrm{PNP}_{-}{ }^{t} \mathrm{Bu}$ region of the reaction of $\mathbf{1 1}$ with $\mathrm{Na}(\text { dion })_{1.39} \mathrm{PCO}, \mathrm{THF}_{-} \mathrm{d}_{8}$, $-10^{\circ} \mathrm{C}$, the reaction was followed for $50 \mathrm{~min}$.

255 

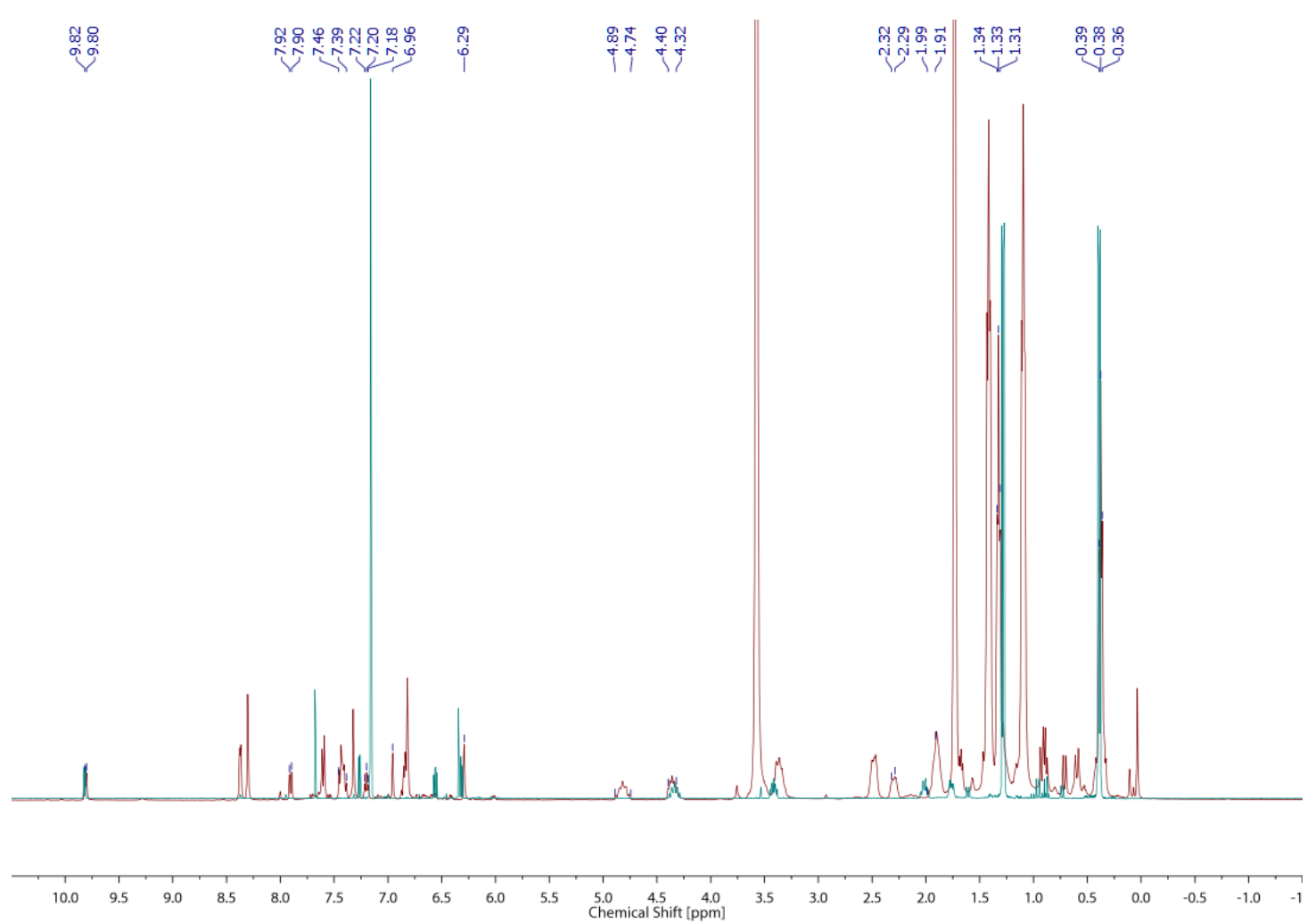

Appendix 8.3.9: Top: ${ }^{1} \mathrm{H}\left\{{ }^{31} \mathrm{P}\right\}$ NMR spectra of the reaction of 11 with $\mathrm{Na}(\operatorname{diox})_{1.39} \mathrm{PCO}$, THF- $\mathrm{d}_{8},-20^{\circ} \mathrm{C}$ (red), pure 25 (teal), $\mathrm{C}_{6} \mathrm{D}_{6}, \mathrm{RT}$. 

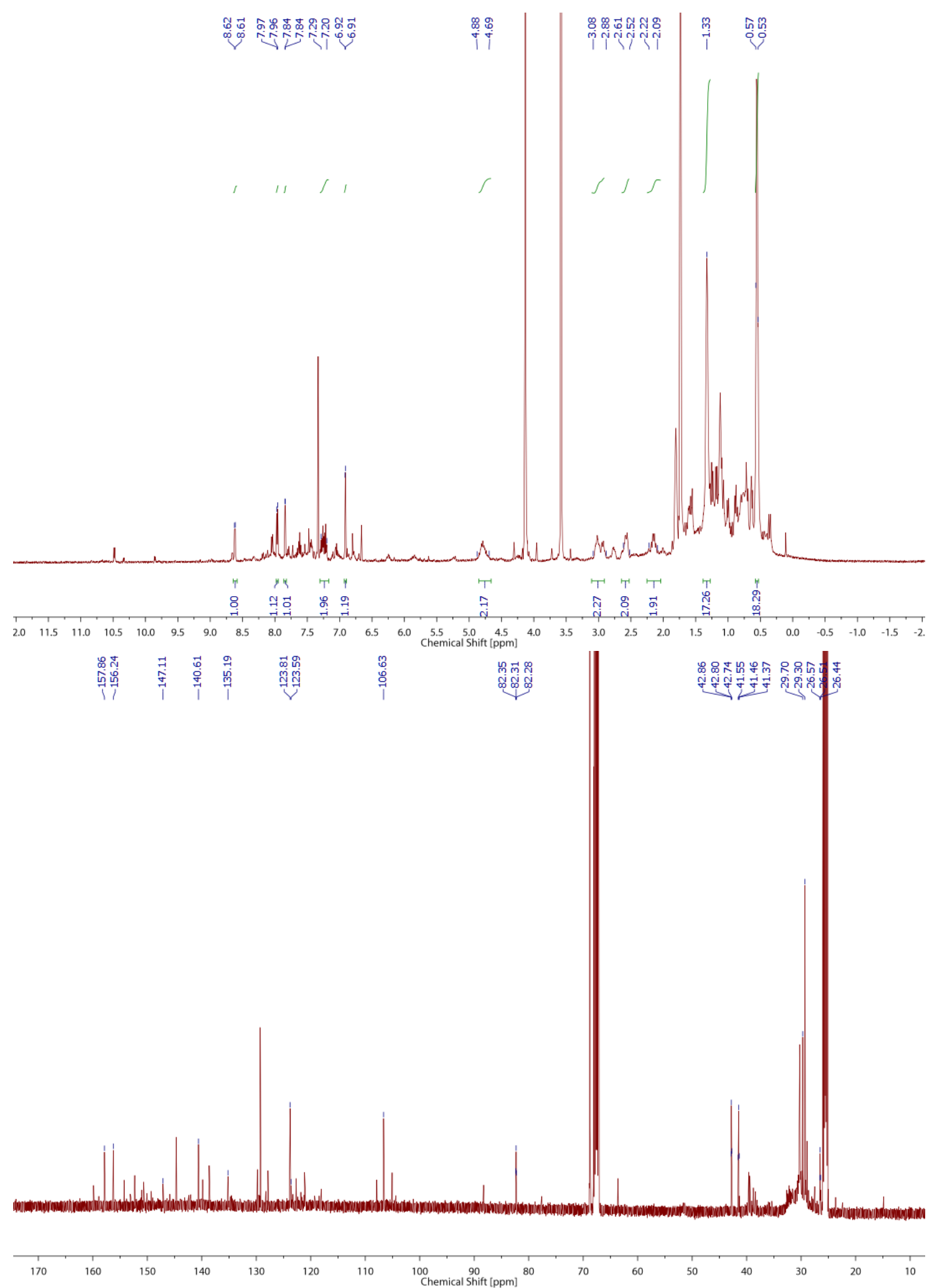

Appendix 8.3.10: Top: ${ }^{1} \mathrm{H}$ NMR spectrum of the oxidation of 27 with [Fc][OTf], THF- $\mathrm{d}_{8}$, $-38^{\circ} \mathrm{C}$; Bottom: ${ }^{13} \mathrm{C}\left\{{ }^{1} \mathrm{H}\right\}$ NMR spectrum of the oxidation of $\mathbf{2 7}$ with $[\mathrm{Fc}][\mathrm{OTf}]$, THF- $\mathrm{d}_{8},-38^{\circ} \mathrm{C}$. 

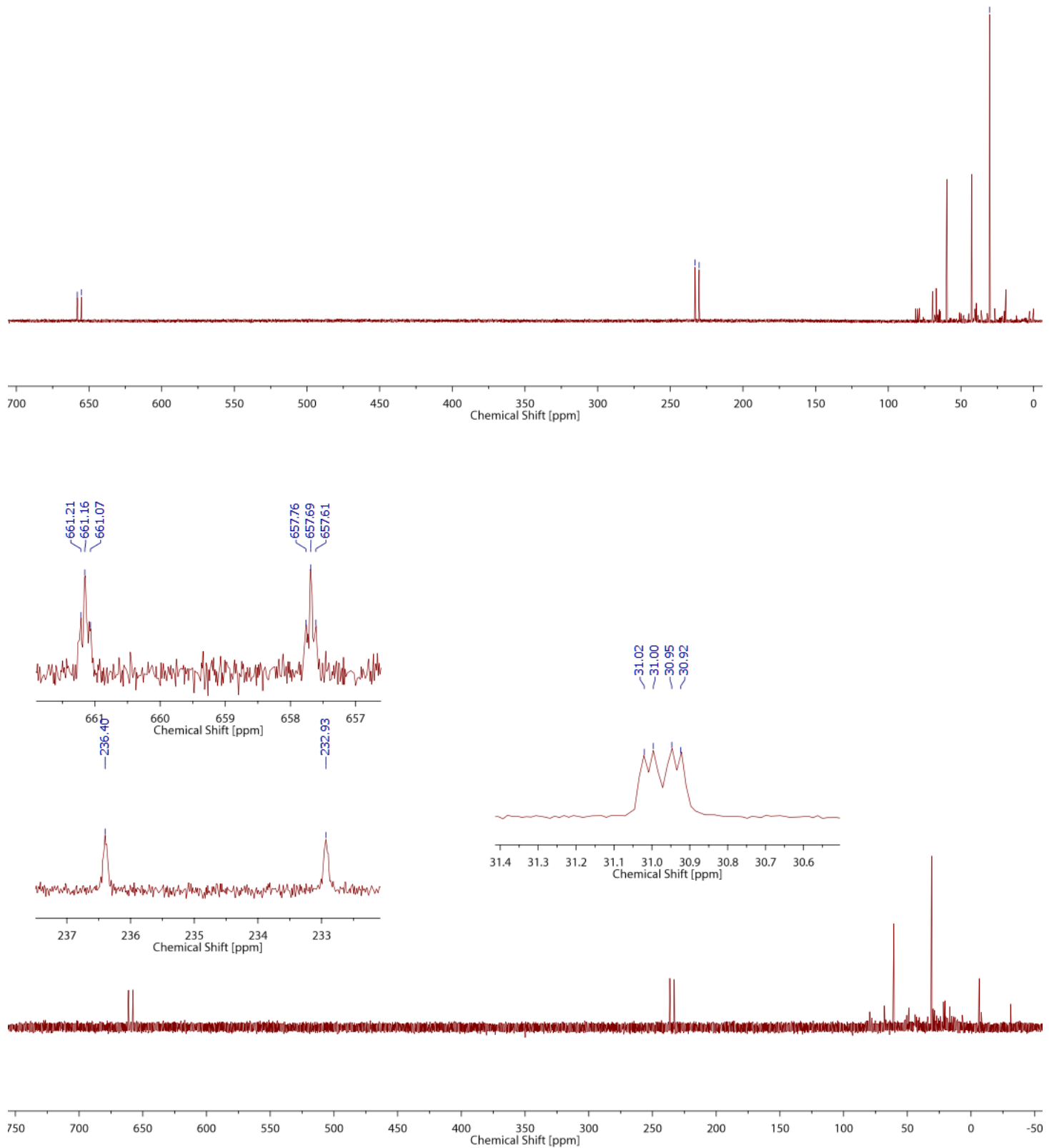

Appendix 8.3.11: Top: ${ }^{31} \mathrm{P}\left\{{ }^{1} \mathrm{H}\right\}$ NMR spectrum of the oxidation of $\mathbf{2 7}$ with [Fc][OTf], THF-8, $-38^{\circ} \mathrm{C}$; Bottom: ${ }^{31} \mathrm{P}\left\{{ }^{1}\right\}$ NMR spectrum of the oxidation of 27 with $[\mathrm{Fc}][\mathrm{OTf}]$, THF-8, RT. 

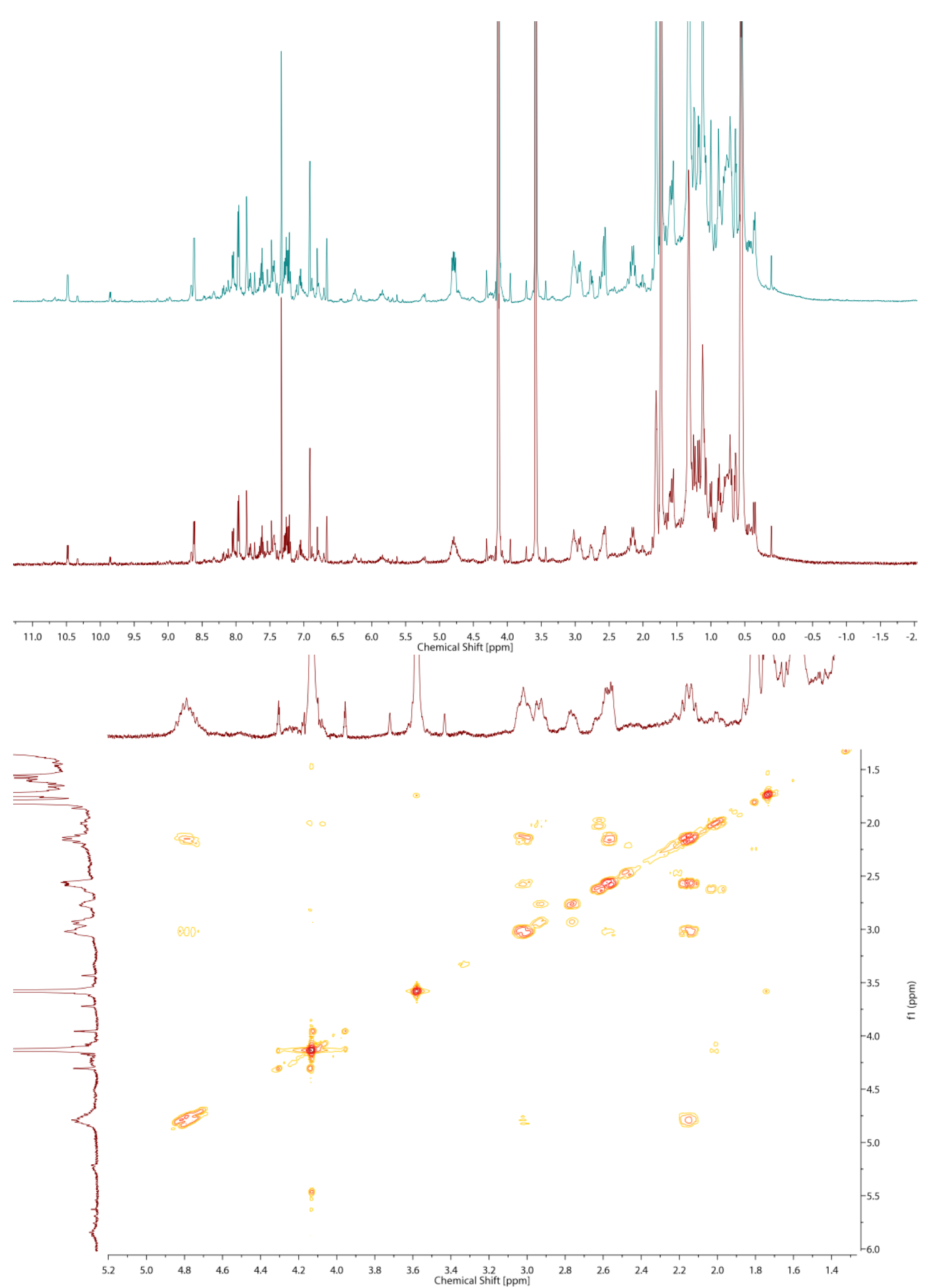

Appendix 8.3.12: Top: ${ }^{1} \mathrm{H}\left\{{ }^{31} \mathrm{P}\right\}$ NMR spectrum of the oxidation of $\mathbf{2 7}$ with [Fc][OTf], THF$\mathrm{d}_{8},-38^{\circ} \mathrm{C}$, decoupled at $31 \mathrm{ppm}$ (top) and without decoupling (bottom); Bottom: COSY NMR spectrum of the PNP pincer region of the oxidation of $\mathbf{2 7}$ with $[\mathrm{Fc}][\mathrm{OTf}]$, THF- $\mathrm{d}_{8},-38^{\circ} \mathrm{C}$. 


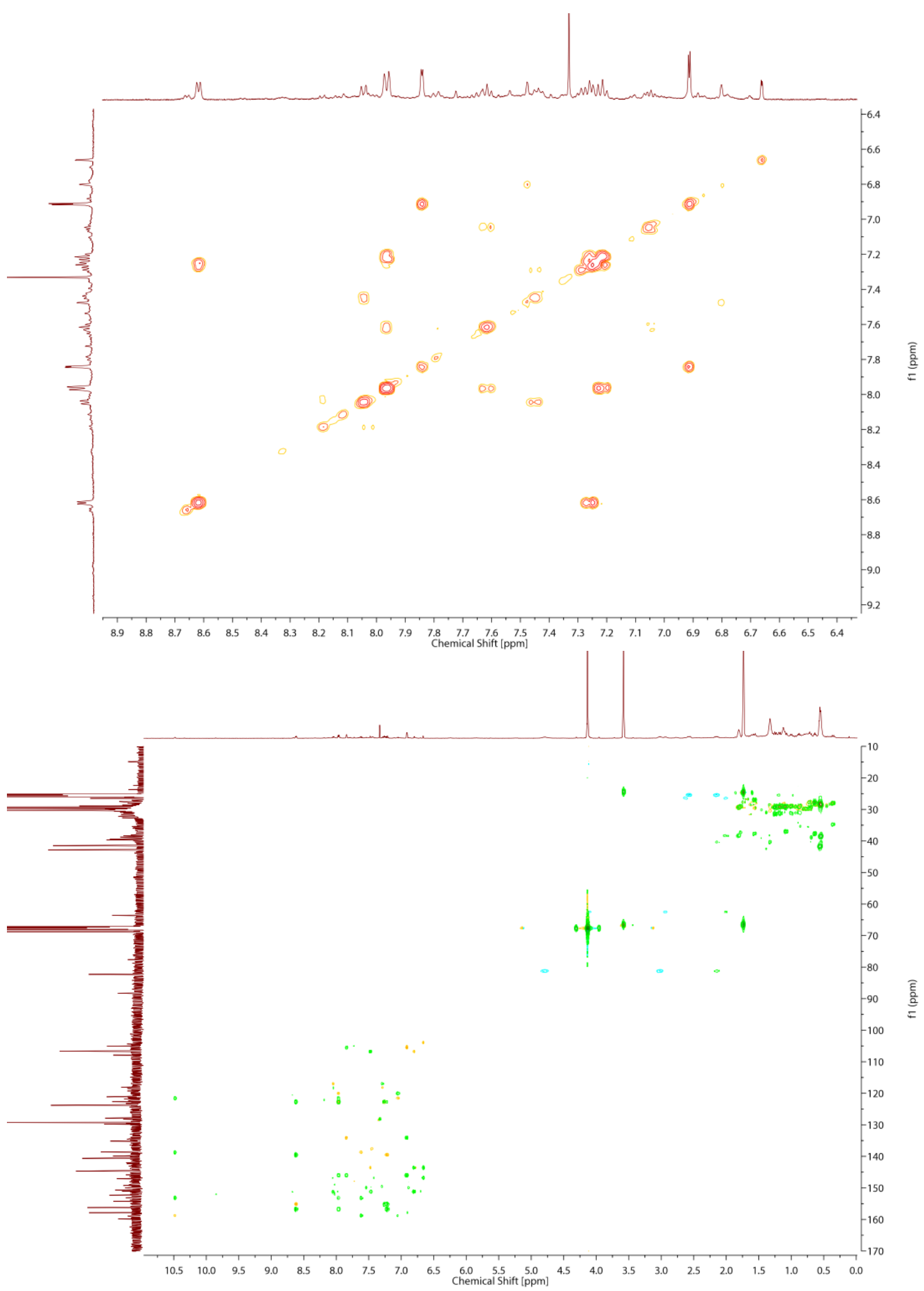

Appendix 8.3.13: Top: COSY NMR spectrum of the PNP pincer region of the oxidation of 27 with [Fc][OTf], THF- $\mathrm{d}_{8},-38^{\circ} \mathrm{C}$; Bottom: HSQC (green/yellow) and HMBC (blue) spectra of the oxidation of 27 with [Fc][OTf], THF- $\mathrm{d}_{8},-38^{\circ} \mathrm{C}$. 

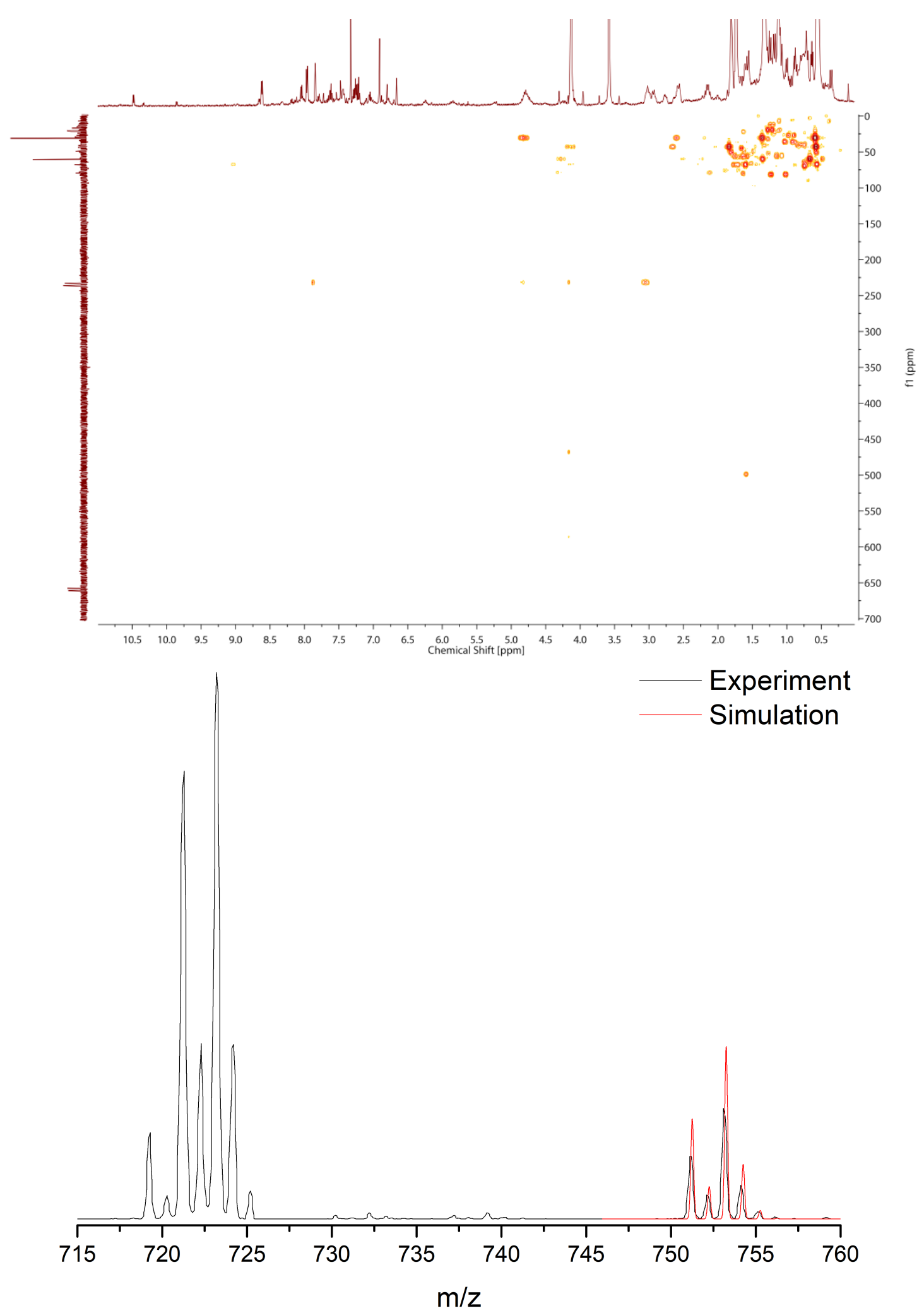

Appendix 8.3.14: Top: ${ }^{31} \mathrm{P}\left\{{ }^{1} \mathrm{H}\right\}$ NMR spectrum of the oxidation of $\mathbf{2 7}$ with [Fc][OTf], THF$\mathrm{d}_{8},-38^{\circ} \mathrm{C}$; Bottom: ESI spectrum of the oxidation of $\mathbf{2 7}$ with [Fc][OTf], THF, RT. 

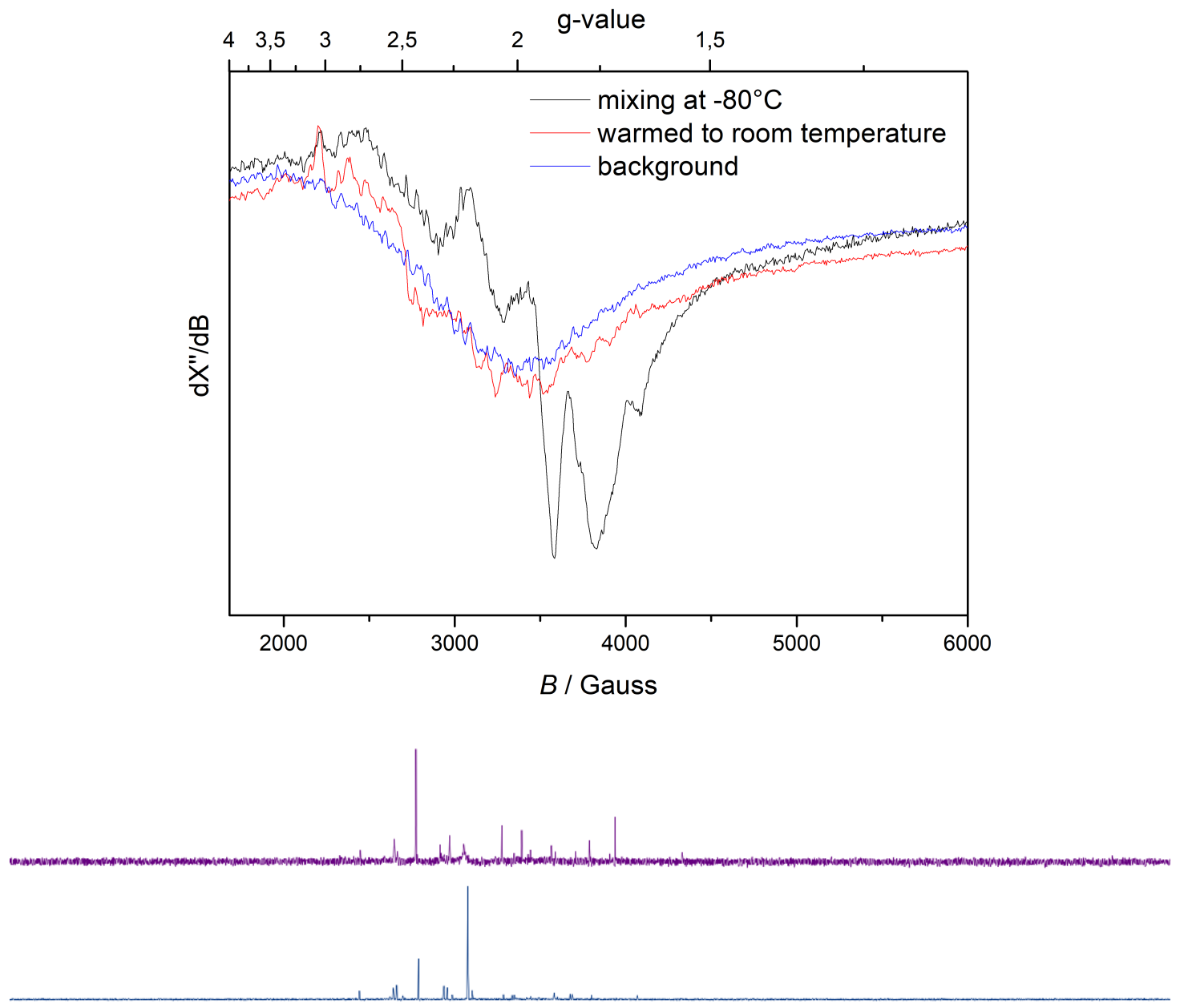

J0 $190 \begin{array}{lllllllllllllllllllllllllllll}180 & 170 & 160 & 150 & 140 & 130 & 120 & 110 & 100 & 90 & 80 & 70 & 60 & 50 & 40 & 30 & 20 & 10 & 0 & -10 & -20 & -30 & -40 & -50 & -60 & -70 & -80 & -90 & -100-110-120-130-140-150-160-170-180-190\end{array}$

Appendix 8.3.15: Top: EPR spectra of the oxidation of 27 with [Fc][OTf], THF; Bottom: ${ }^{31} \mathrm{P}\left\{{ }^{1} \mathrm{H}\right\}$ NMR spectra of the oxidation of 27 with [Fc][OTf], THF-d $8,-80^{\circ} \mathrm{C}$ (red), $-70^{\circ} \mathrm{C}$ (yellow), $-60^{\circ} \mathrm{C}$ (green), $-50^{\circ} \mathrm{C}$ (blue), $\mathrm{RT}$ (purple). 

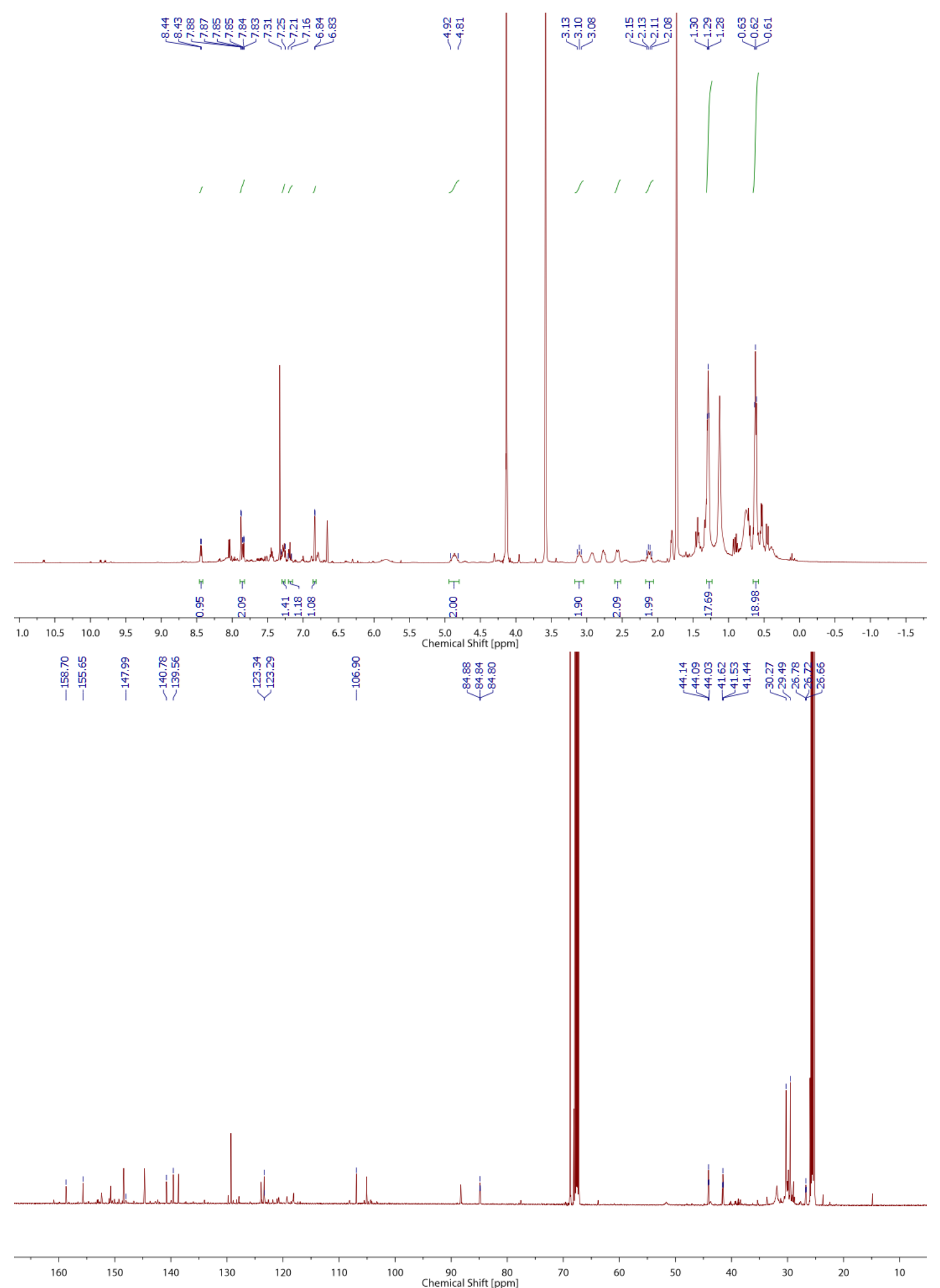

Appendix 8.3.16: Top: ${ }^{1} \mathrm{H}$ NMR spectrum of the oxidation of $\mathbf{2 8}$ with [Fc][OTf], THF- $\mathrm{d}_{8}$, $-38^{\circ} \mathrm{C}$; Bottom: ${ }^{13} \mathrm{C}\left\{{ }^{1} \mathrm{H}\right\}$ NMR spectrum of the oxidation of $\mathbf{2 8}$ with [Fc][OTf], THF-d $8,-38^{\circ} \mathrm{C}$. 

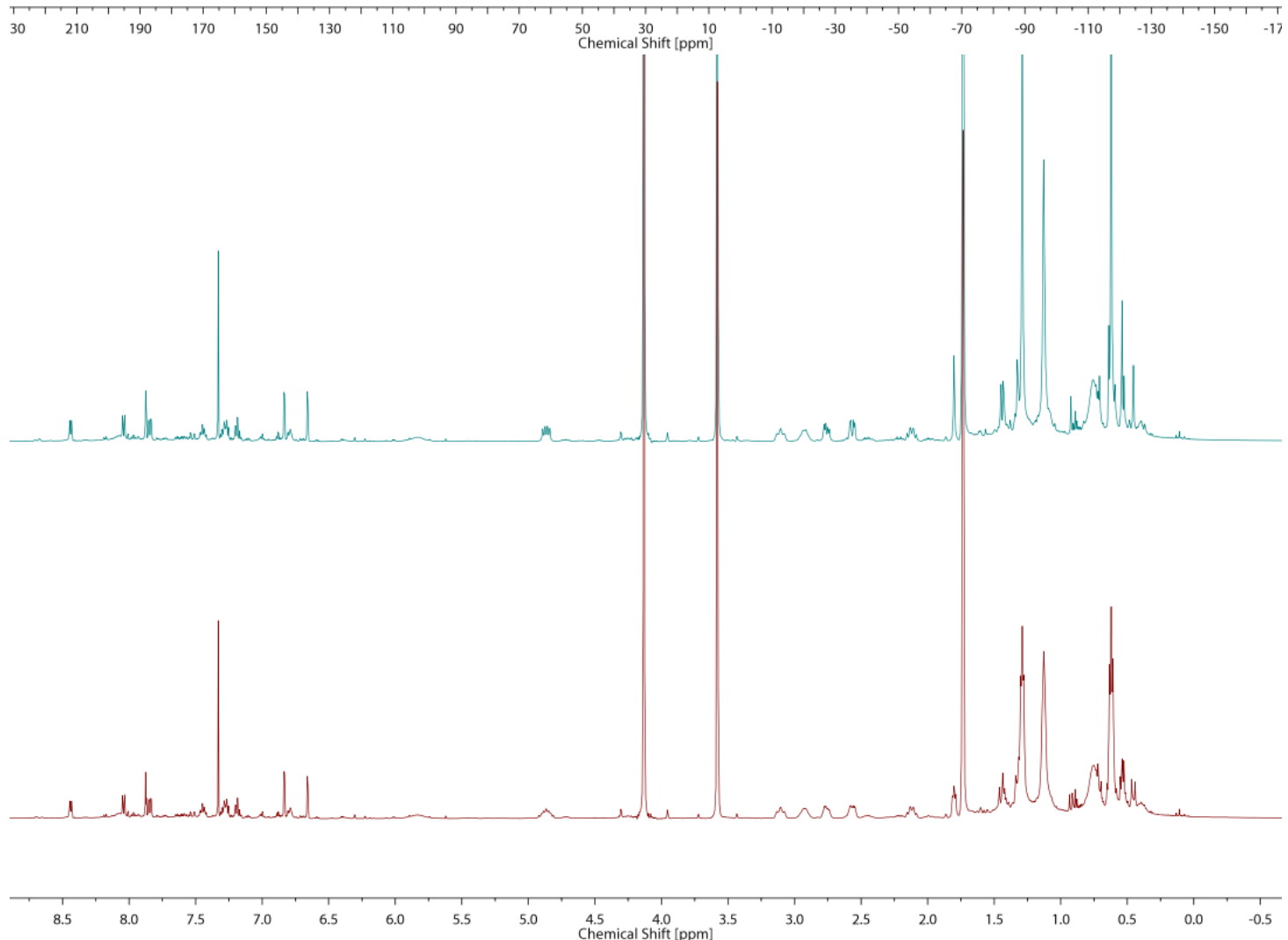

Appendix 8.3.17: Top: ${ }^{31} \mathrm{P}\left\{{ }^{1} \mathrm{H}\right\}$ NMR spectrum of the oxidation of $\mathbf{2 8}$ with [Fc][OTf], THF-8, $-38^{\circ} \mathrm{C}$; Bottom: ${ }^{31} \mathrm{P}\left\{{ }^{1} \mathrm{H}\right\}$ NMR spectrum of the oxidation of $\mathbf{2 8}$ with [Fc][OTf], THF-8, RT. 

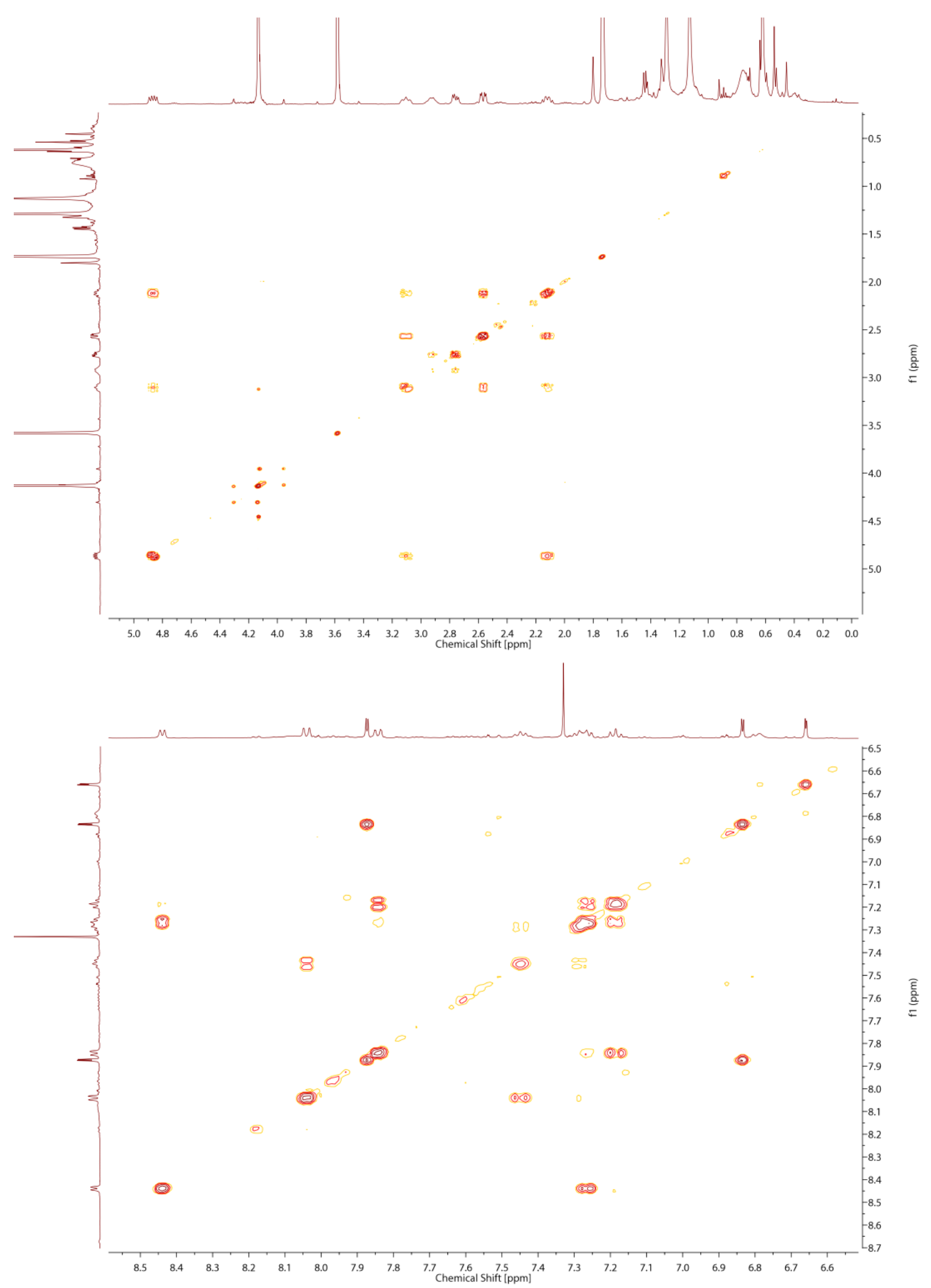

Appendix 8.3.18: Top: COSY NMR spectrum of the PNP pincer region of the oxidation of 28 with $[\mathrm{Fc}][\mathrm{OTf}]$, THF- $\mathrm{d}_{8},-38^{\circ} \mathrm{C}$ : Bottom: COSY NMR spectrum of the aromatic region of the oxidation of 28 with $[\mathrm{Fc}][\mathrm{OTf}], \mathrm{THF}-\mathrm{d}_{8},-38^{\circ} \mathrm{C}$. 


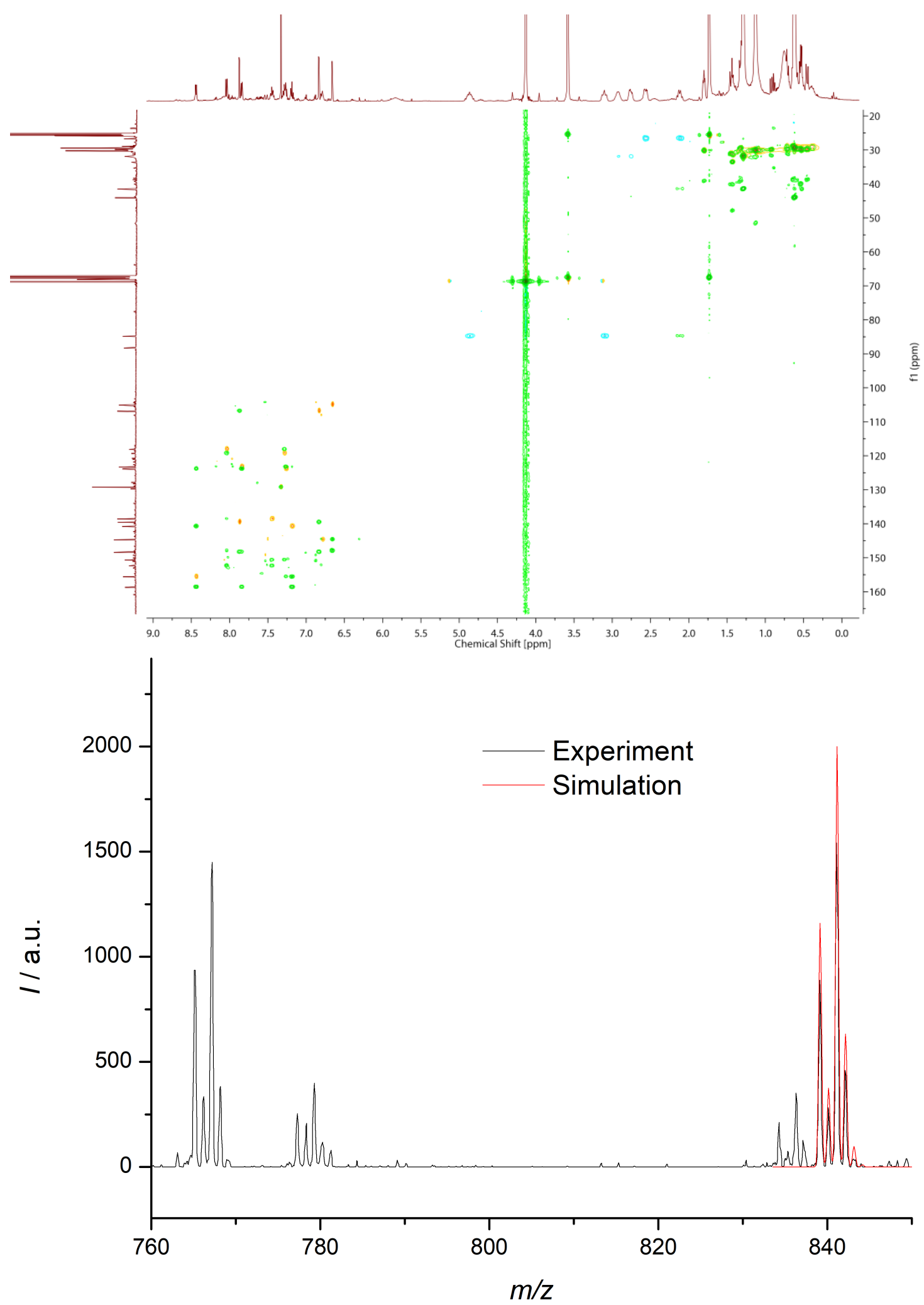

Appendix 8.3.19: Top: HSQC (green/yellow) and HMBC (blue) spectra of the oxidation of 28 with [Fc][OTf], THF-d $8,-38^{\circ} \mathrm{C}$; Bottom: ESI spectrum of the oxidation of 28 with [Fc][OTf], THF, RT. 

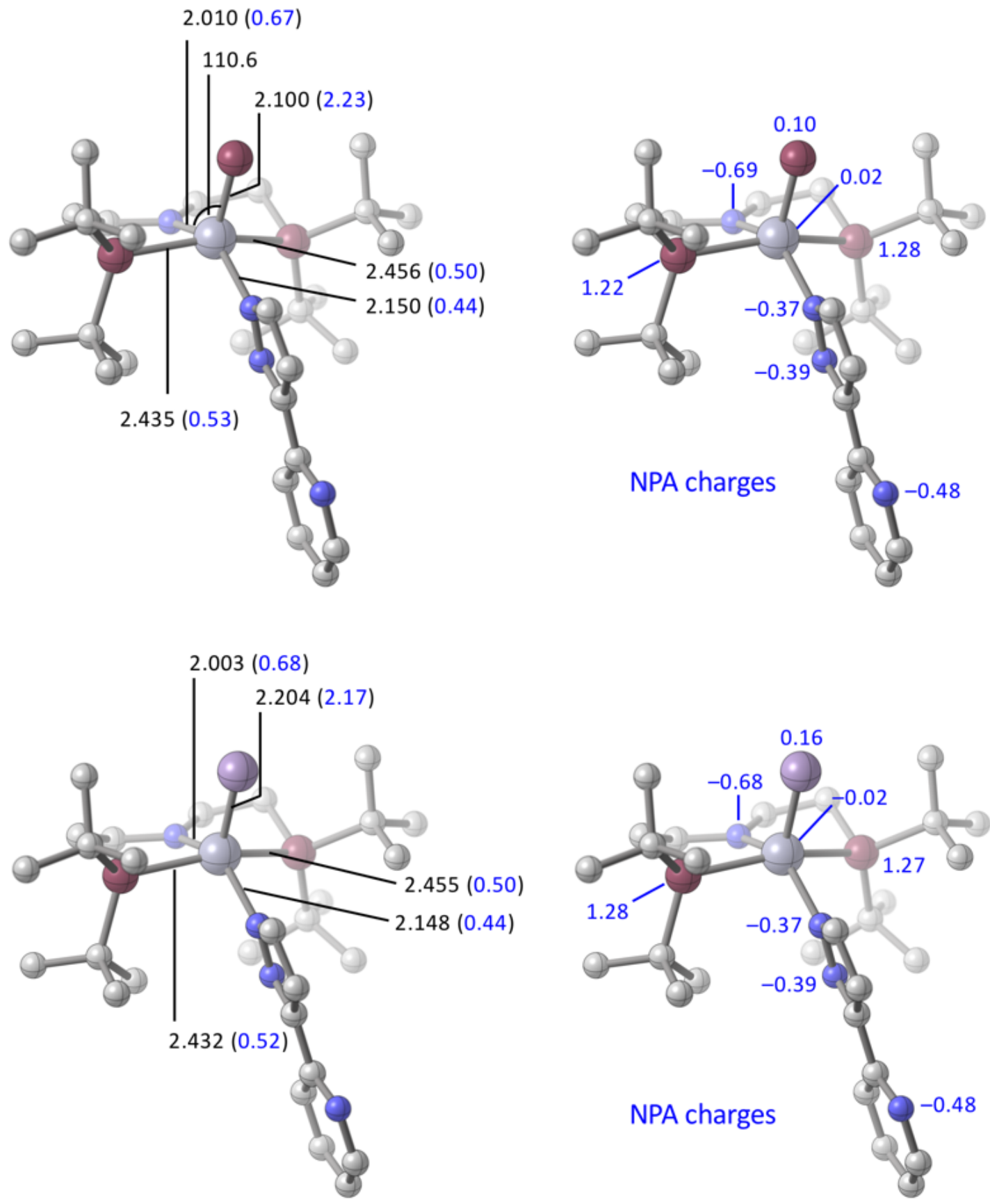

Appendix 8.3.20: Computed structures and properties of $\mathbf{2 7}$ (top) and $\mathbf{2 8}$ (bottom, $\omega \mathrm{B} 97 \mathrm{XD} / \mathrm{BS} 2 / / \omega \mathrm{B} 97 \mathrm{XD} / \mathrm{BS} 1$. Left: Selected bond lengths in $\AA$ (Wiberg bond indices in blue); Right: NPA charges. 

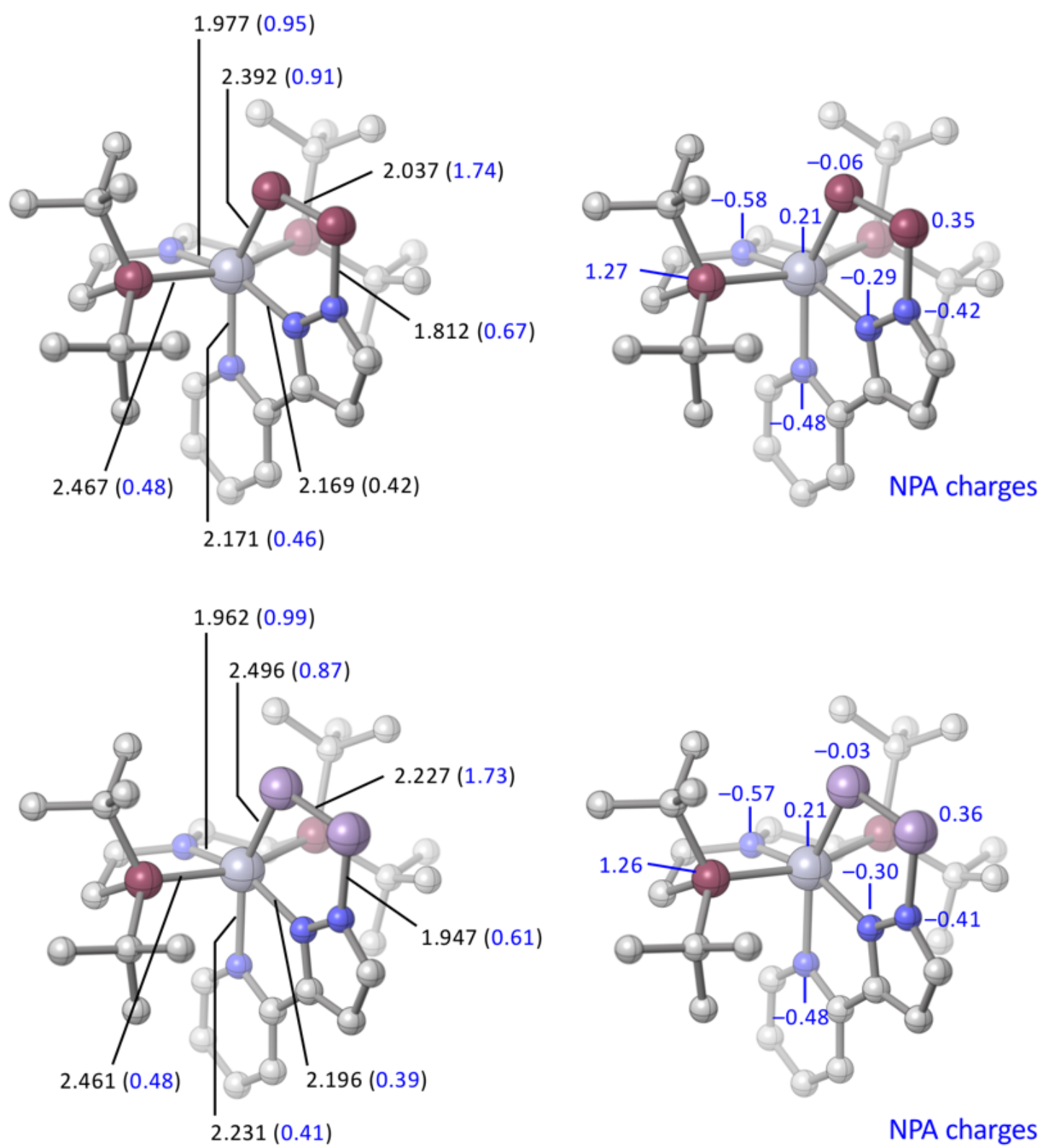

Appendix 8.3.21: Computed structures and properties of 29 (top) and 30 (bottom, $\omega B 97 X D / B S 2 / / \omega B 97 X D / B S 1$. Left: Selected bond lengths in $\AA$ (Wiberg bond indices in blue); Right: NPA charges. 


\begin{tabular}{|c|c|c|c|c|c|c|c|}
\hline Species & Bond & $\begin{array}{c}\rho\left(\mathbf{r}_{\mathrm{b}}\right) \\
/ \\
\mathrm{e} \AA^{-3}\end{array}$ & $\begin{array}{c}\nabla^{2} \rho\left(\mathbf{r}_{\mathrm{b}}\right) \\
/ / \\
\mathrm{e} \AA^{-5}\end{array}$ & $\begin{array}{c}H\left(\mathbf{r}_{\mathrm{b}}\right) / \rho\left(\mathbf{r}_{\mathrm{b}}\right) \\
\text { Hartree/e }\end{array}$ & $\begin{array}{c}G\left(\mathbf{r}_{\mathrm{b}}\right) / \rho\left(\mathbf{r}_{\mathrm{b}}\right) \\
/ \\
\text { Hartree } / \mathrm{e}\end{array}$ & $\epsilon$ & $\delta(\mathrm{A}, \mathrm{B})$ \\
\hline $\mathrm{P}_{2}$ & $\mathrm{P}-\mathrm{P}$ & 1.15 & -5.74 & -0.74 & 0.39 & 0.00 & 3.04 \\
\hline $\mathrm{P}_{2} \mathrm{H}_{2}$ & $\mathrm{P}-\mathrm{P}$ & 0.95 & -4.07 & -0.59 & 0.29 & 0.40 & 1.96 \\
\hline $\mathrm{P}_{2} \mathrm{H}_{4}$ & $\mathrm{P}-\mathrm{P}$ & 0.71 & -2.48 & -0.44 & 0.19 & 0.07 & 0.99 \\
\hline \multirow[t]{6}{*}{27} & Re1 - P3 & 1.07 & -1.34 & -0.68 & 0.56 & 0.04 & 2.47 \\
\hline & Re1 - P1 & 0.58 & 2.71 & -0.35 & 0.63 & 0.57 & 0.78 \\
\hline & Re1 - P2 & 0.60 & 2.76 & -0.36 & 0.63 & 0.57 & 0.81 \\
\hline & Re1 - N1 & 0.88 & 10.94 & -0.36 & 1.14 & 0.37 & 0.95 \\
\hline & Re1 - N2 & 0.65 & 8.49 & -0.25 & 1.07 & 0.90 & 0.63 \\
\hline & N2 - N3 & 2.54 & -14.66 & -0.93 & 0.53 & 0.13 & 1.41 \\
\hline \multirow[t]{6}{*}{28} & Re1 - As1 & 0.95 & 0.96 & -0.61 & 0.65 & 0.06 & 2.40 \\
\hline & Re1 - P1 & 0.58 & 2.76 & -0.35 & 0.64 & 0.56 & 0.79 \\
\hline & Re1 - P2 & 0.60 & 2.80 & -0.36 & 0.64 & 0.54 & 0.83 \\
\hline & Re1 - N1 & 0.90 & 11.09 & -0.37 & 1.15 & 0.37 & 0.97 \\
\hline & Re1 - N2 & 0.65 & 8.54 & -0.25 & 1.07 & 0.88 & 0.64 \\
\hline & N2 - N3 & 2.53 & -14.61 & -0.93 & 0.53 & 0.13 & 1.41 \\
\hline \multirow[t]{8}{*}{29} & Re1 - P3 & 0.66 & 1.35 & -0.41 & 0.51 & 0.80 & 1.11 \\
\hline & Re1 - P1 & 0.57 & 2.78 & -0.35 & 0.65 & 0.23 & 0.81 \\
\hline & Re1 - P2 & 0.57 & 2.78 & -0.35 & 0.65 & 0.23 & 0.81 \\
\hline & Re1 - N1 & 0.93 & 12.45 & -0.38 & 1.22 & 0.21 & 1.10 \\
\hline & Re1 - N3 & 0.58 & 8.95 & -0.18 & 1.17 & 1.38 & 0.57 \\
\hline & N3 - N4 & 2.49 & -14.28 & -0.91 & 0.51 & 0.16 & 1.32 \\
\hline & P3 - P4 & 0.94 & -3.84 & -0.58 & 0.30 & 0.36 & 1.71 \\
\hline & $\mathrm{P} 4-\mathrm{N} 4$ & 0.87 & 2.64 & -0.79 & 1.00 & 0.11 & 0.79 \\
\hline \multirow[t]{8}{*}{30} & Re1 - As1 & 0.59 & 1.14 & -0.38 & 0.48 & 0.76 & 1.07 \\
\hline & Re1 - P1 & 0.57 & 2.91 & -0.35 & 0.66 & 0.23 & 0.83 \\
\hline & Re1 - P2 & 0.57 & 2.91 & -0.35 & 0.66 & 0.23 & 0.83 \\
\hline & Re1 - N1 & 0.97 & 12.75 & -0.39 & 1.22 & 0.25 & 1.14 \\
\hline & Re1 - N3 & 0.54 & 8.42 & -0.17 & 1.15 & 1.56 & 0.55 \\
\hline & N3 - N4 & 2.53 & -14.78 & -0.93 & 0.52 & 0.16 & 1.34 \\
\hline & As1 - As2 & 0.77 & -1.46 & -0.53 & 0.40 & 0.27 & 1.70 \\
\hline & As2 - N4 & 0.79 & 4.03 & -0.55 & 0.91 & 0.09 & 0.77 \\
\hline
\end{tabular}

Appendix 8.3.22: Topological characteristics computed at the bond critical points (Atom numbering as in the XRD structures). 
a)

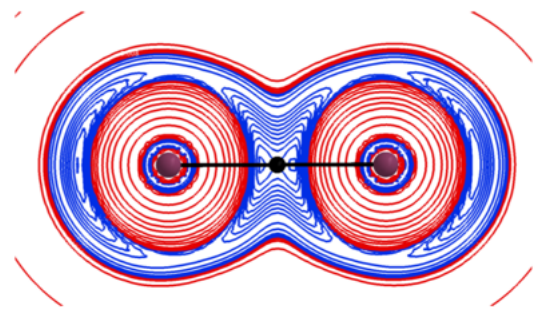

b)

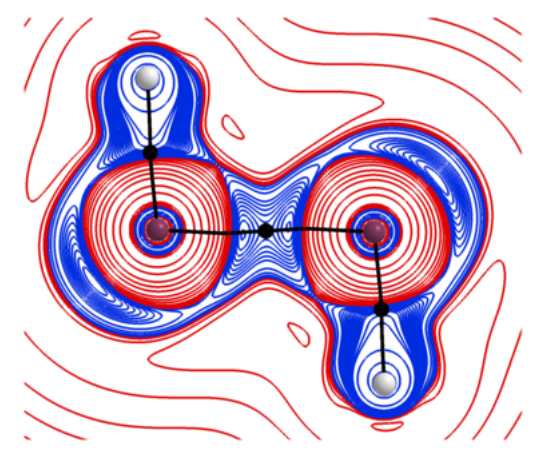

c)

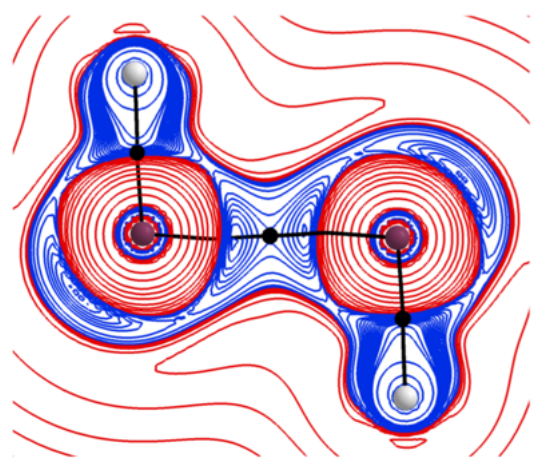

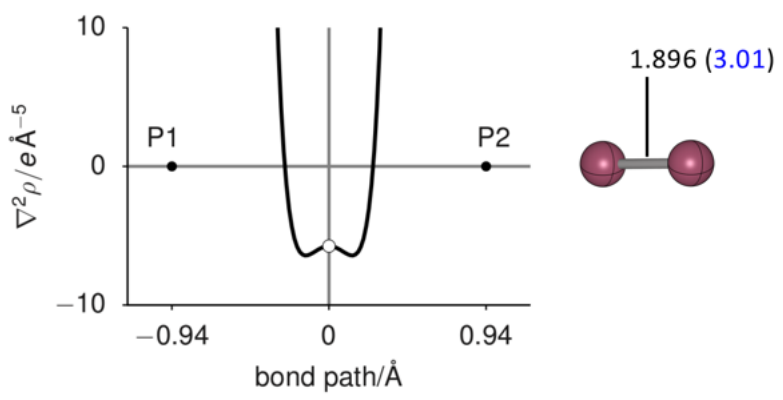
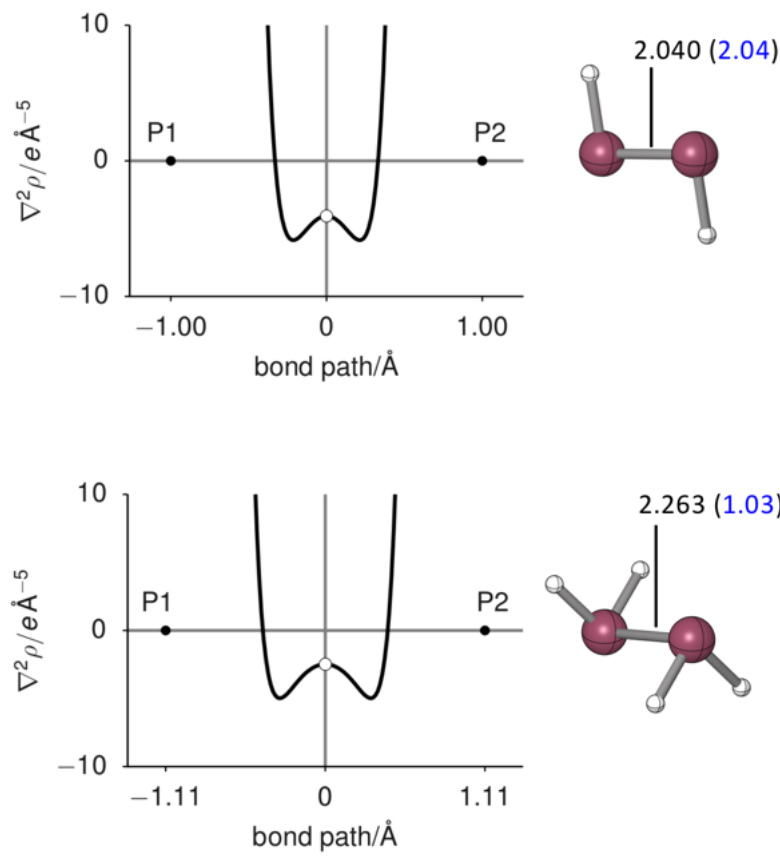

Appendix 8.3.23: Bonding analysis of $\mathrm{P}_{2}$ (a), $\mathrm{P}_{2} \mathrm{H}_{2}$ (b) and $\mathrm{P}_{2} \mathrm{H}_{4} \quad$ (c, $\omega \mathrm{B} 97 \mathrm{XD} / \mathrm{BS} 2 / / \omega \mathrm{B} 97 \mathrm{XD} / \mathrm{BS} 1)$. Left: $2 \mathrm{D}$ plots of $\nabla^{2} \rho(\mathrm{r})$; charge concentration (blue) and depletion (red), bond paths (black lines), and bond critical points (black dots). Middle: 1D Laplacian profiles along the P-P bond paths. Right: P-P bond lenghts and Wiberg bond indices (in blue). 

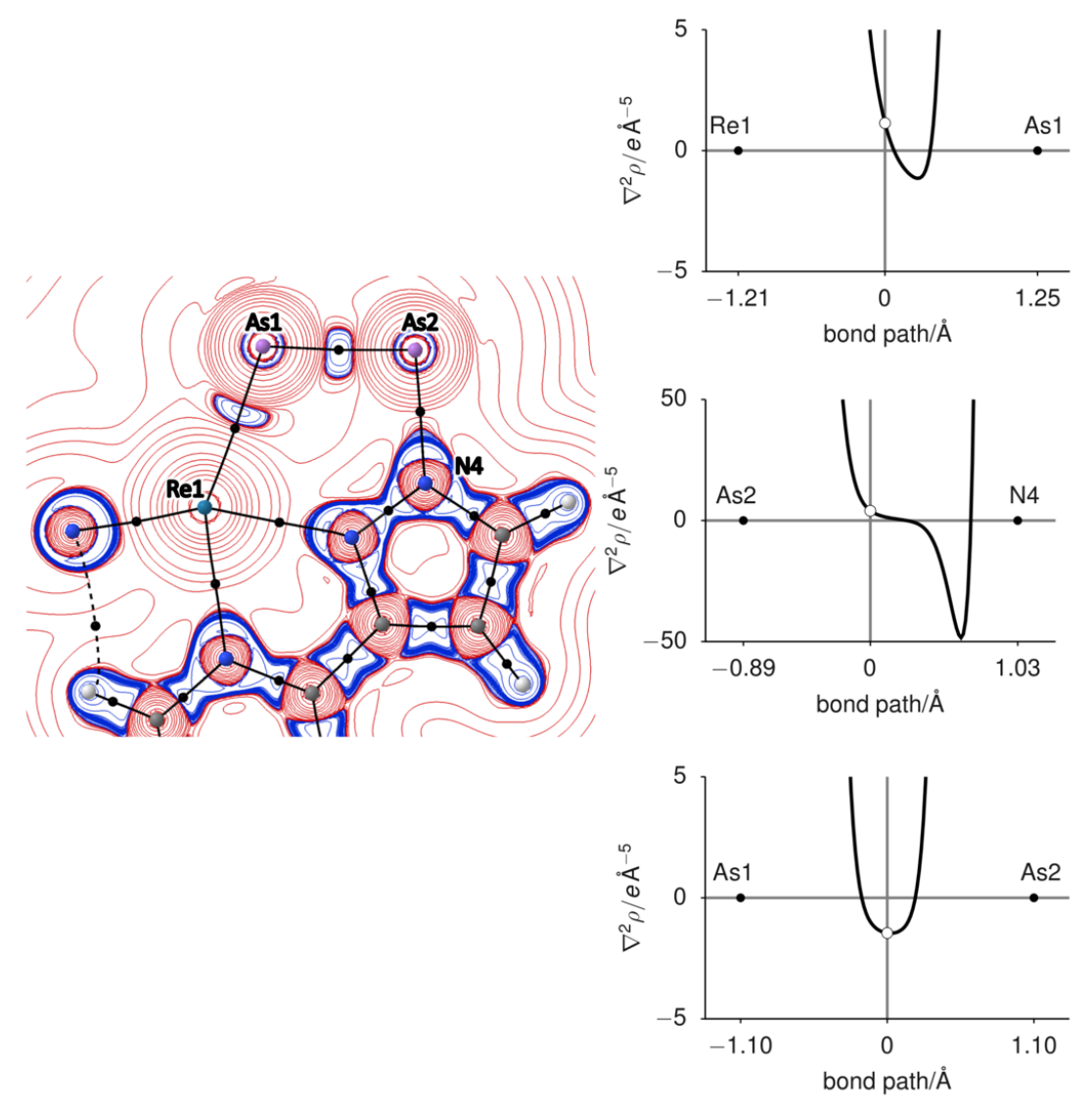

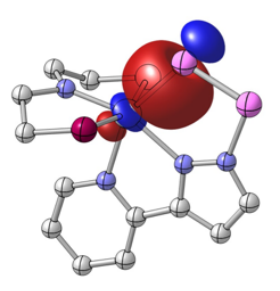

(37\%) Re1-As1 (58\%)

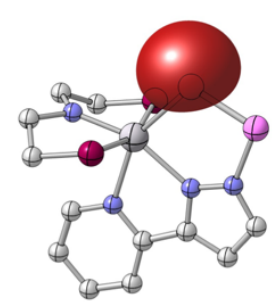

(95\%) As1 sp 0.43

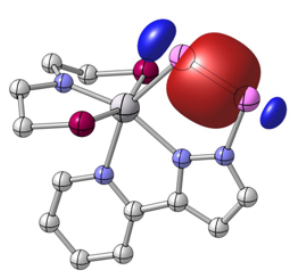

(49\%) As1-As2 (48\%)

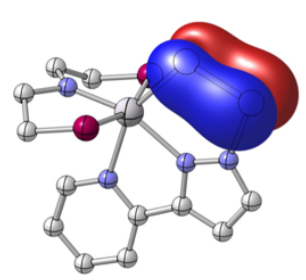

(37\%) As1-As2 (61\%)

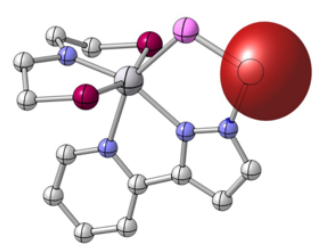

(99\%) As2 sp 0.27

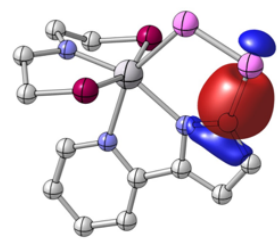

(18\%) As2 - N4 (80\%)

Appendix 8.3.24: Top: Bonding analysis of 30 ( $\omega$ B97XD/BS2// $\omega$ B97XD/BS1). Left: 2D plots of $\nabla^{2} \rho(\mathrm{r})$; charge concentration (blue) and depletion (red), bond paths (black lines), and bond critical points (black dots). Right: 1D Laplacian profiles along the Re1-As1 (top), As1-As2 (middle) and As2-N4 (bottom) bond paths; Bottom: NLMOs representing the Re1-As1, As1=As2 and As2-N4 bonds and the two lone pairs at As1 and As2 (isosurfaces at $\pm 0.05 \mathrm{a}_{0}^{3 / 2}$ 


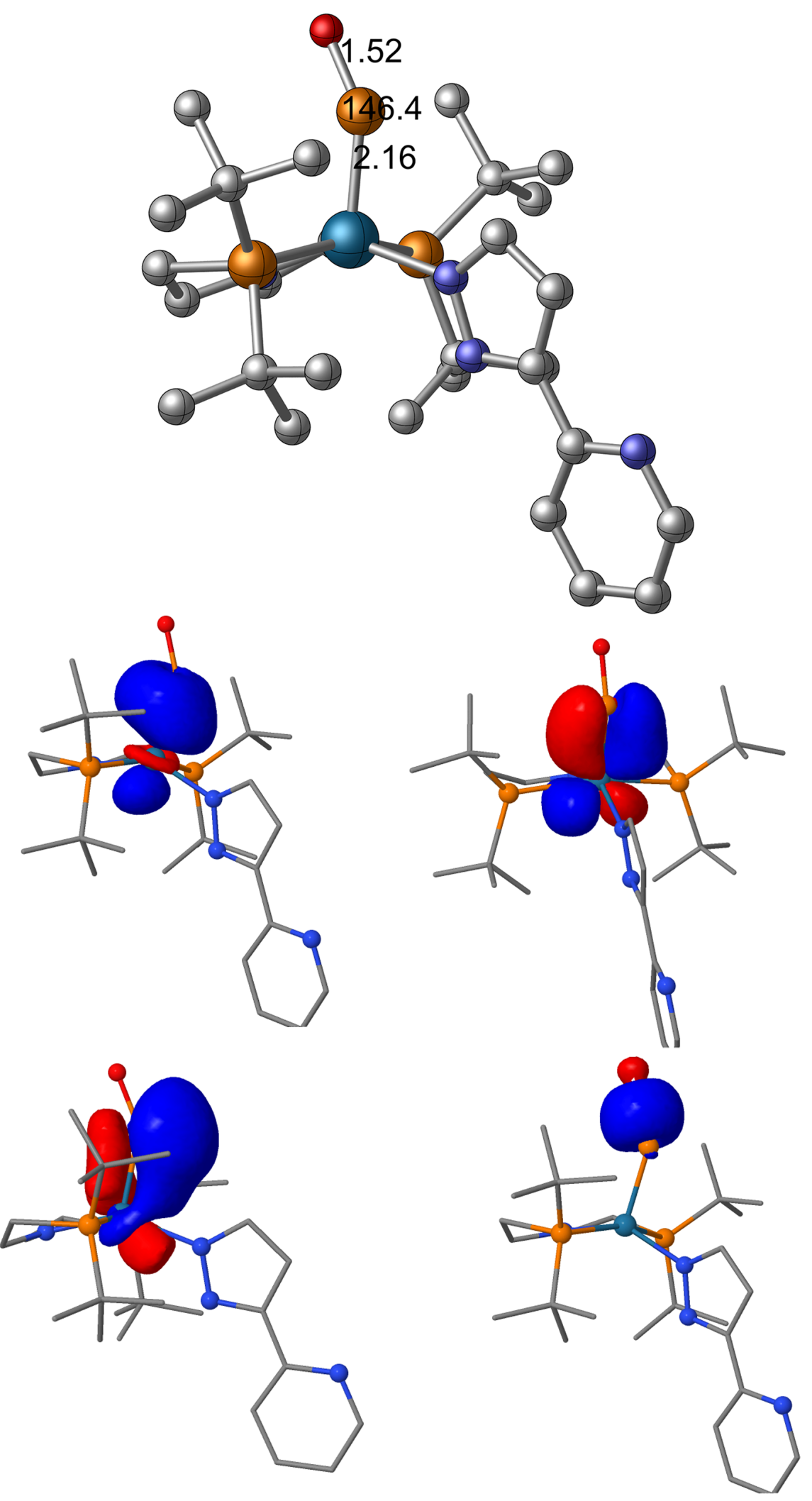

Appendix 8.3.25: Computed structure of 32 and the computed NBOs of the ${ }^{+} \mathrm{Re}=\mathrm{P}^{-} \mathrm{O}^{-}$unit. 


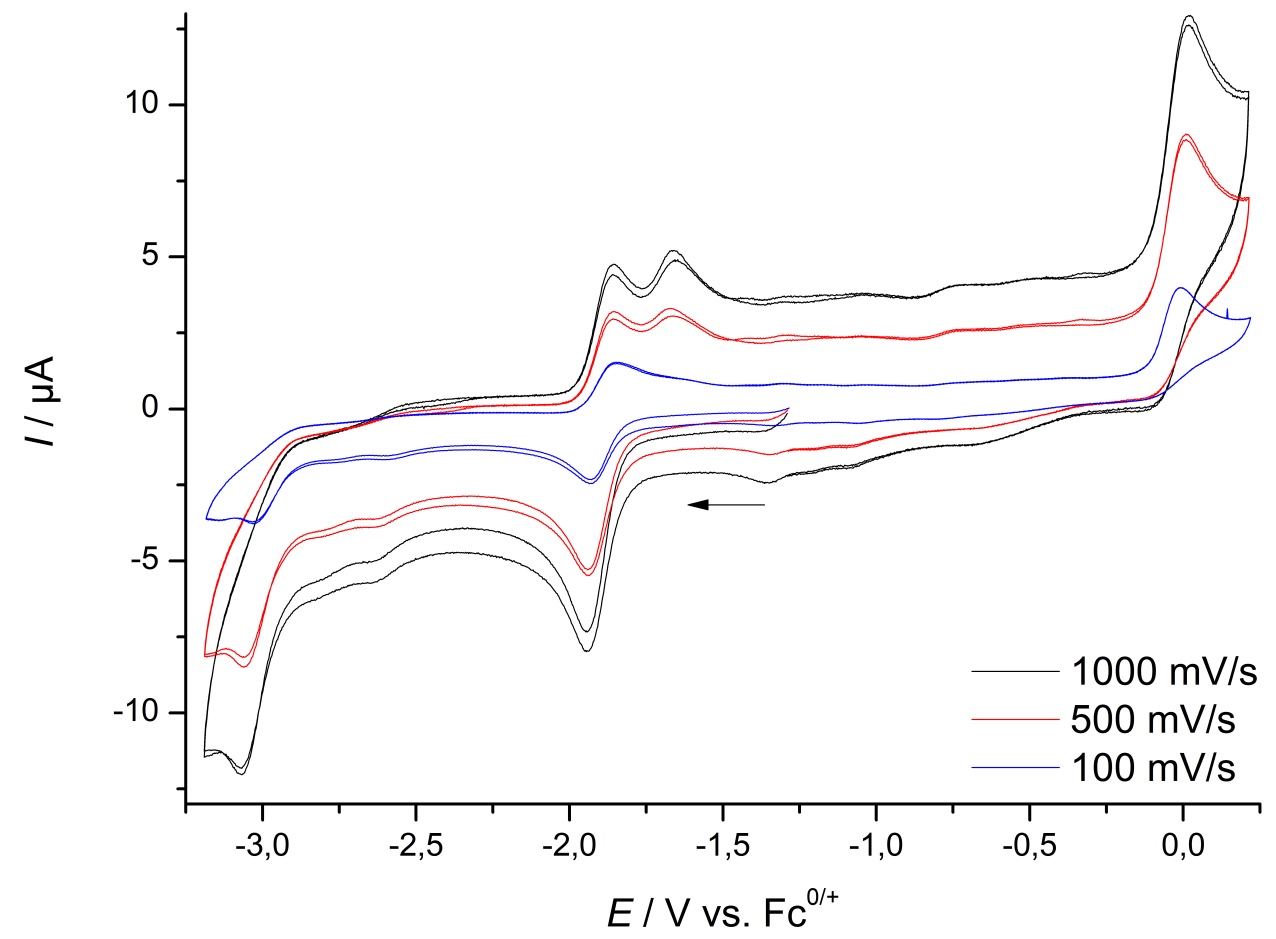

Appendix 8.3.26: Cyclic voltammogram of $31,0.7 \mathrm{mM}, 0.1 \mathrm{M} \mathrm{NBu}_{4} \mathrm{PH}_{6}, 2$ scans, THF, RT.

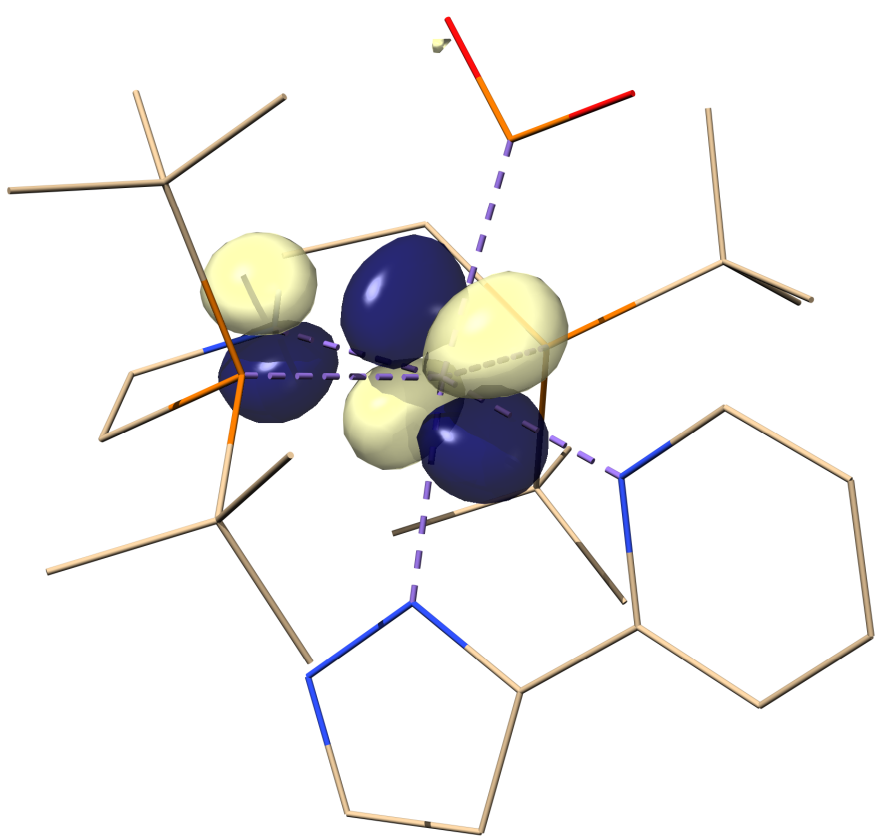

Appendix 8.3.27: Computed SOMO of $\mathbf{3 4 .}$ 

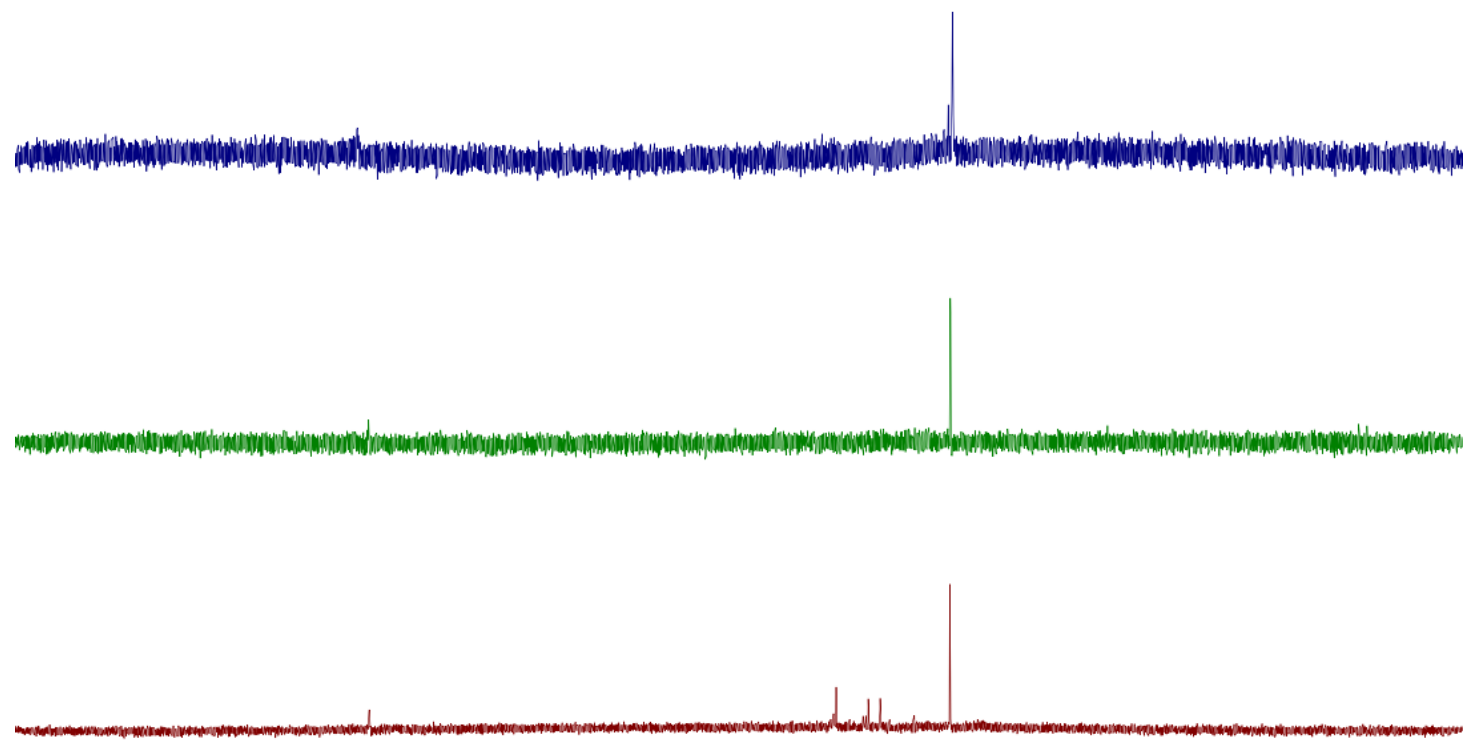

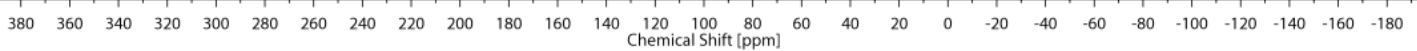

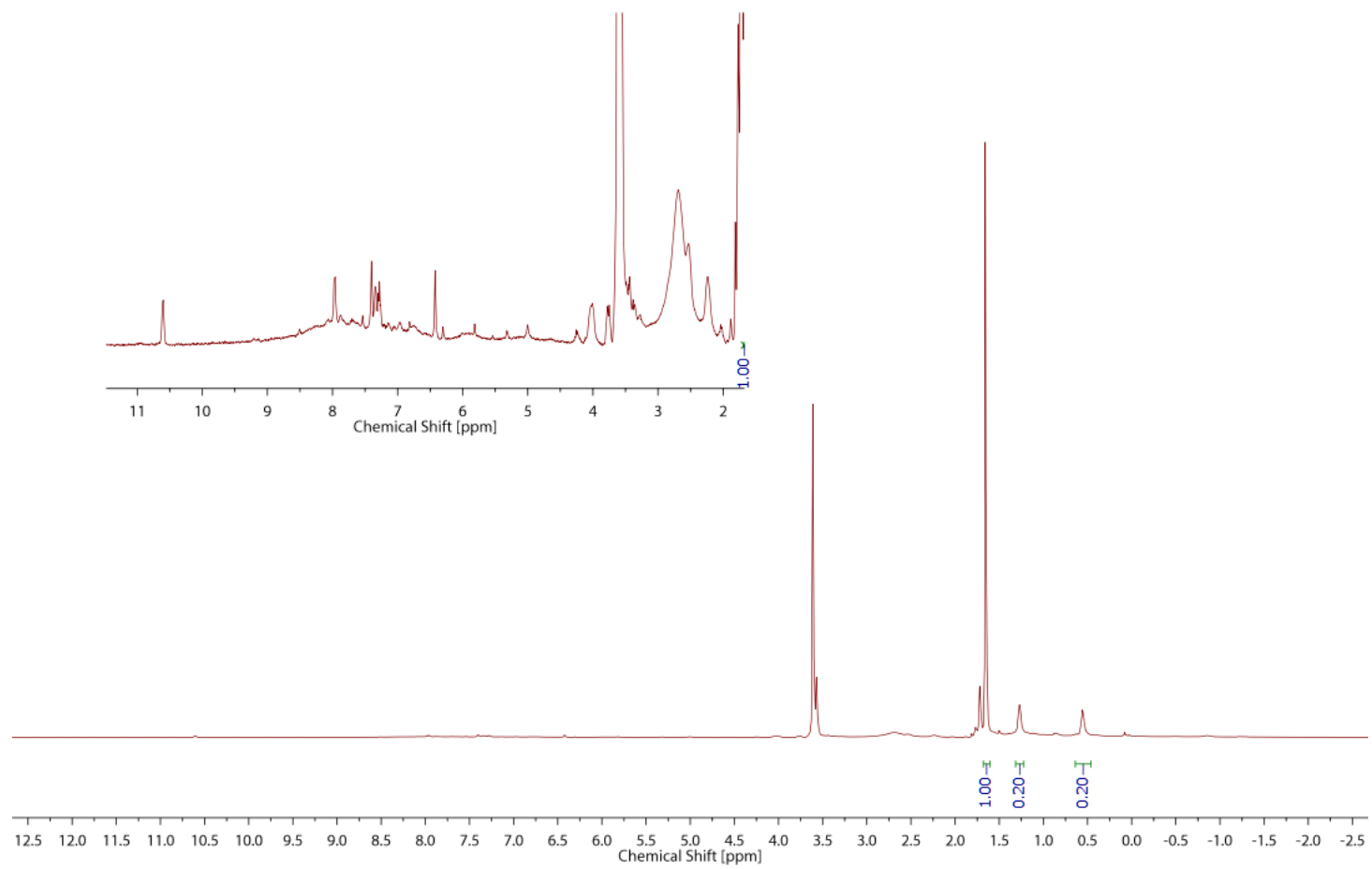

Appendix 8.3.28: Top: ${ }^{31} \mathrm{P}\left\{{ }^{1} \mathrm{H}\right\} \quad$ NMR spectra of the oxidation of $\mathbf{3 4}$ with $\left[\mathrm{Fe}\left(\mathrm{C}_{5} \mathrm{Me}_{5}\right)_{2}\right]\left[\mathrm{Al}\left(\mathrm{O}\left(\mathrm{C}\left(\mathrm{CF}_{3}\right)_{3}\right)_{4}\right]\right.$, THF-d $\mathrm{d}_{8},-85^{\circ} \mathrm{C}$ (blue), $0^{\circ} \mathrm{C}$ (green), $25^{\circ} \mathrm{C}$ (red); Bottom: ${ }^{1} \mathrm{H}$ NMR spectrum of the oxidation of $\mathbf{3 4}$ with $\left[\mathrm{Fe}\left(\mathrm{C}_{5} \mathrm{Me}_{5}\right)_{2}\right]\left[\mathrm{Al}\left(\mathrm{O}\left(\mathrm{C}\left(\mathrm{CF}_{3}\right)_{3}\right)_{4}\right]\right.$, THF-d $8,0^{\circ} \mathrm{C}$. 


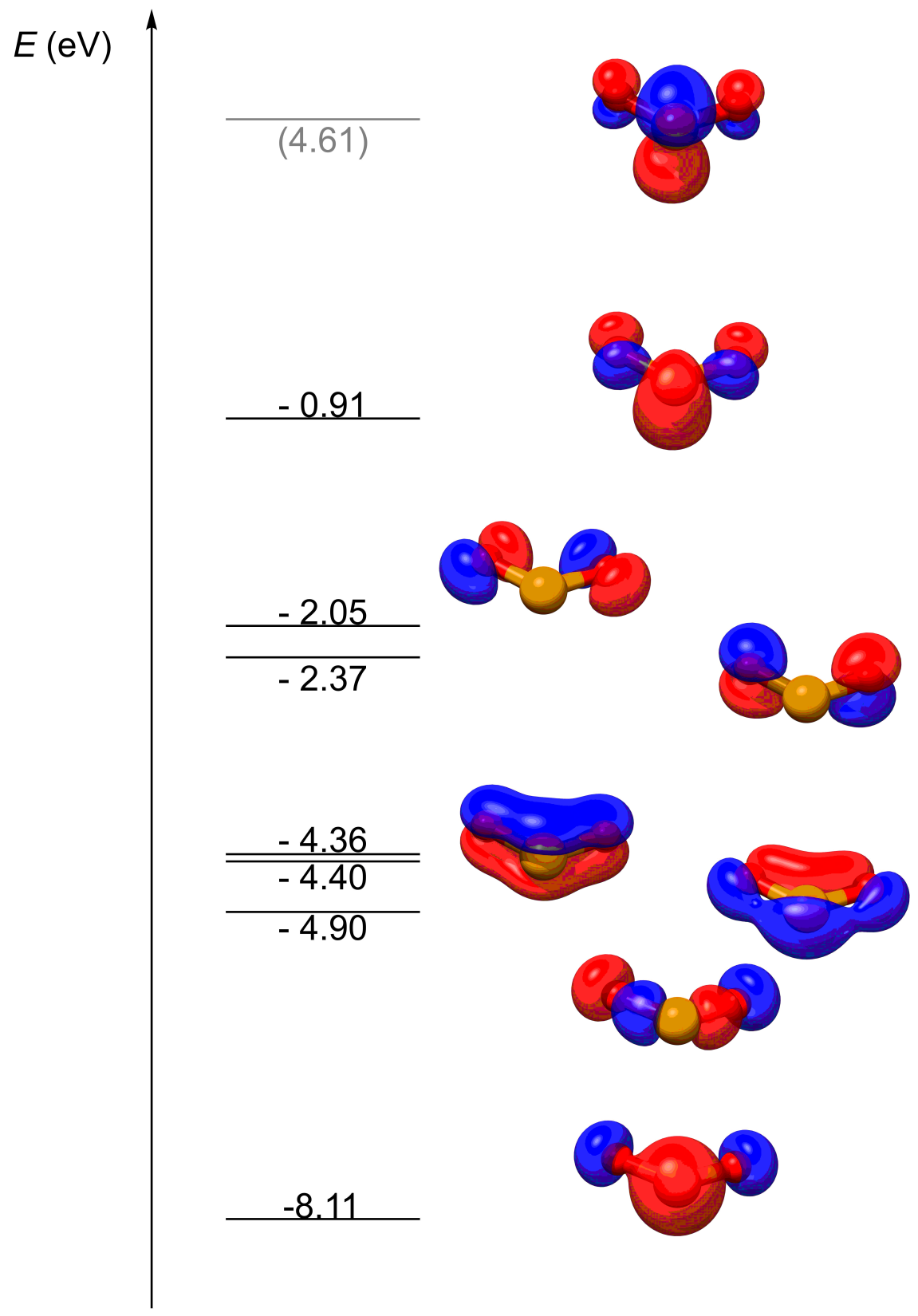

Appendix 8.3.29: Molecular orbital diagram of free $\mathrm{PO}_{2}^{-}$, unoccupied orbitals in grey. 


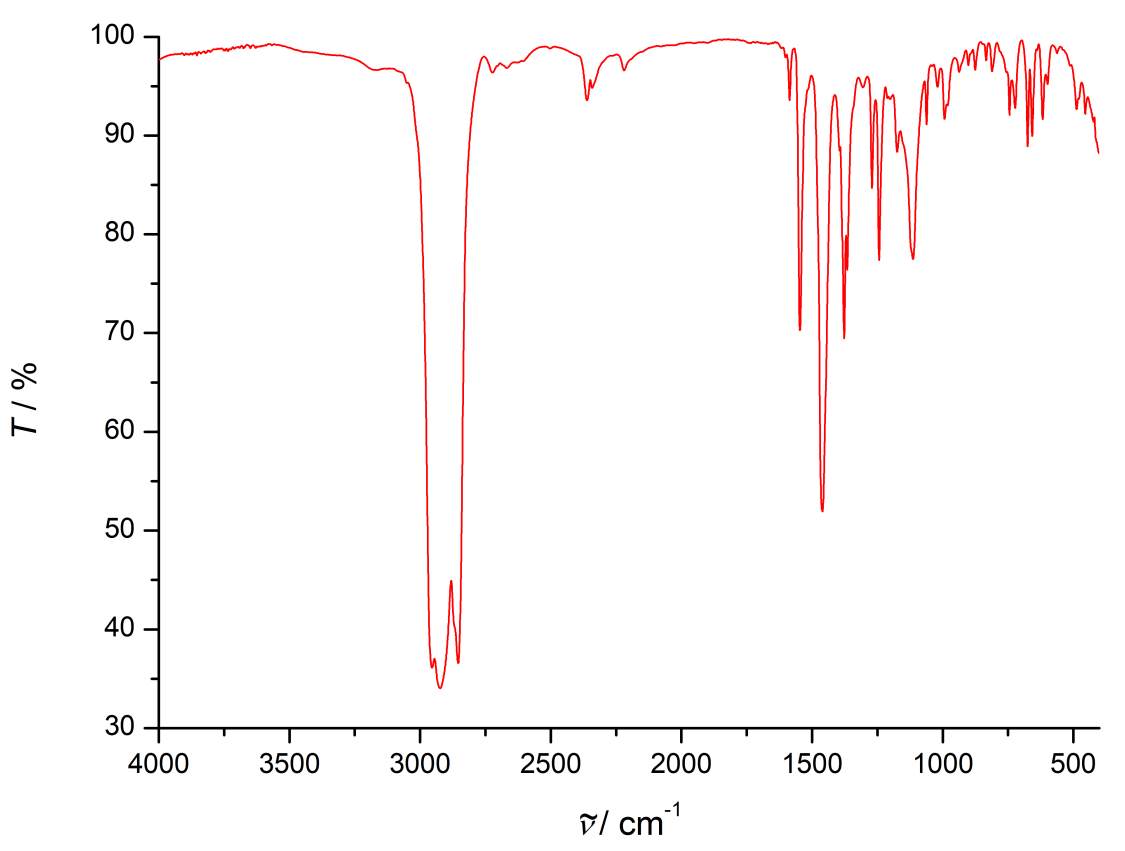

Appendix 8.3.30: IR spectrum of 37, Nujol, RT.

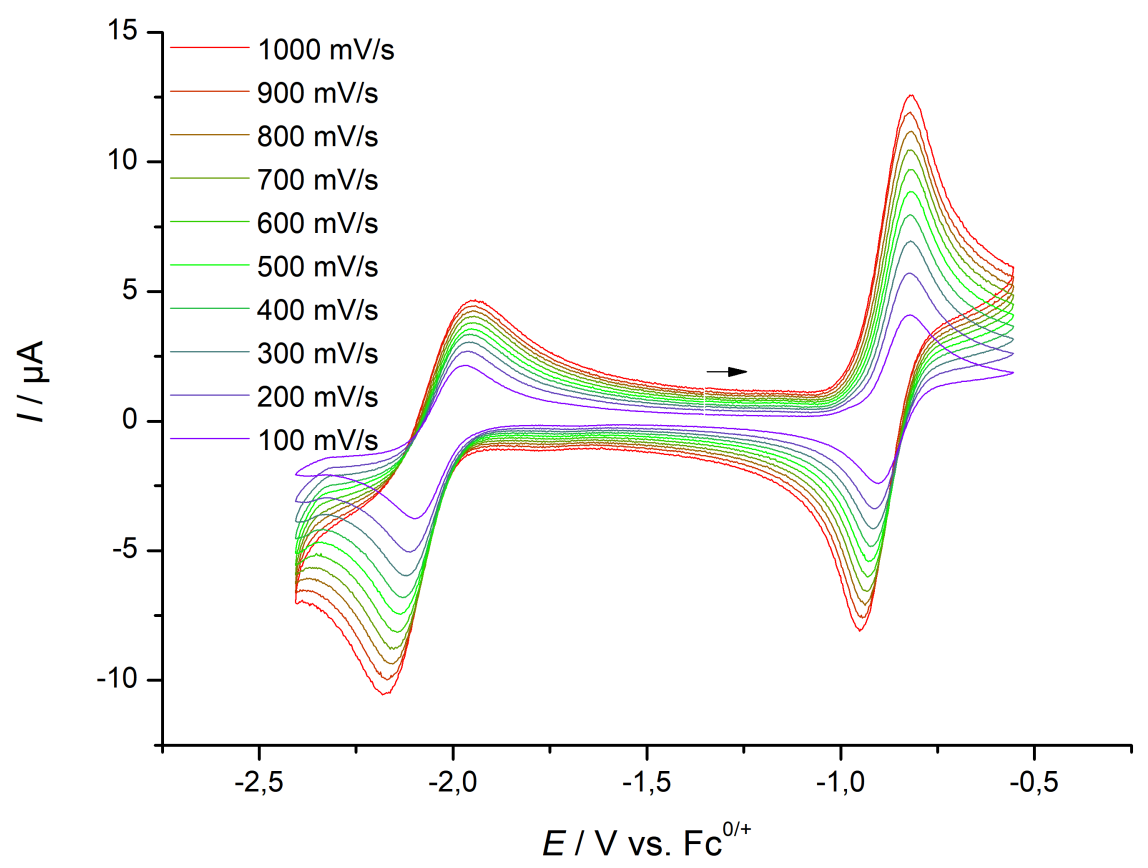

Appendix 8.3.31: Cyclovoltammogram of $38,2^{\text {nd }}$ scan, $1 \mathrm{mM}, 0.1 \mathrm{M} \mathrm{NBu}_{4} \mathrm{PF}_{6}$, THF, RT. 


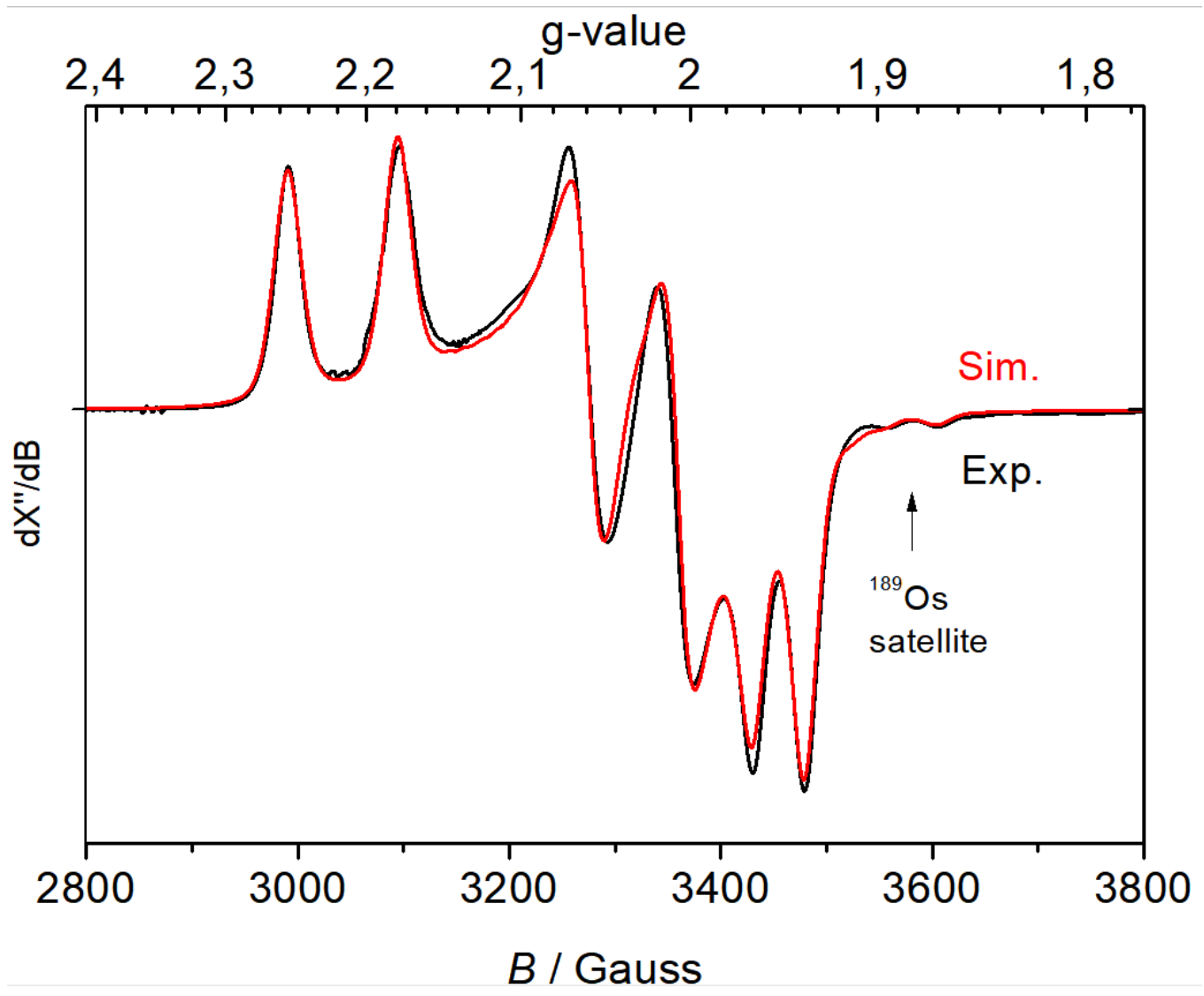

Appendix 8.3.32: EPR spectrum of complex 38, toluene, $148 \mathrm{~K}, 9.436620 \mathrm{GHz}$, simulation assuming coaxial g- and ${ }^{31} \mathrm{P}$ A-tensors (blue): $\mathrm{g}_{11}=2.215, \mathrm{~g}_{22}=2.032, \mathrm{~g}_{33}=1.951, \mathrm{~A}_{11}\left({ }^{31} \mathrm{P}\right)$ $=322 \mathrm{MHz}, \mathrm{A}_{22}\left({ }^{31} \mathrm{P}\right)=243 \mathrm{MHz}, \mathrm{A}_{33}\left({ }^{31} \mathrm{P}\right)=136 \mathrm{MHz}, \mathrm{A}_{11}\left({ }^{189} \mathrm{Os}\right)=230 \mathrm{MHz}, \mathrm{A}_{22}\left({ }^{189} \mathrm{Os}\right)$ and $\mathrm{A}_{33}\left({ }^{189} \mathrm{Os}\right)$ not resolved. The spectrum reveals fully resolved ${ }^{31} \mathrm{P}$ hyperfine interactions (HFI) and partially resolved ${ }^{189} \mathrm{Os}$ (16\% natural abundance) HFI satellites. Simulation of the spectrum assuming coaxial $\mathrm{g}$ and ${ }^{31} \mathrm{P}$ hyperfine A-tensors (W95EPR) yields ${ }^{31} \mathrm{P}$ hyperfine values that deviate largely from the DFT calculated ones.

However, closer inspection of the DFT calculated EPR parameters reveals that the gtensor of complex $\mathbf{3 8}$ is strongly tilted with respect to the molecular symmetry axes. This is most likely caused by predominant spin-orbit coupling involving the SOMO and HOMO of $\mathbf{3 8}$ along these g-tensor axes. 


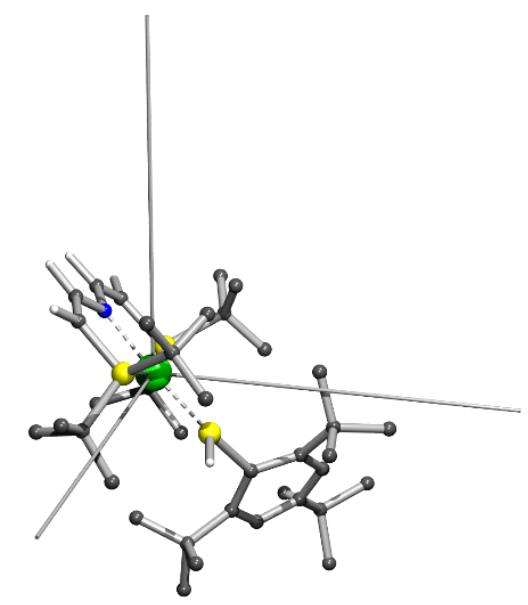

Appendix 8.3.33: Electronic g-tensor directions of 38 which are strongly tilted with respect to the molecular axis.

In contrast, the ${ }^{31} \mathrm{P}$ hyperfine A-tensor is closely aligned with the molecular symmetry axes. In combination with the abovementioned strongly tilted g-tensor directions with respect to the molecular symmetry axes, the g- and A-tensors of complex $\mathbf{3 8}$ are not coaxial, with large DFT computed Euler angles of $\alpha=-74.3^{\circ}, \beta=+142.7^{\circ}, \gamma=-7.5^{\circ}$.

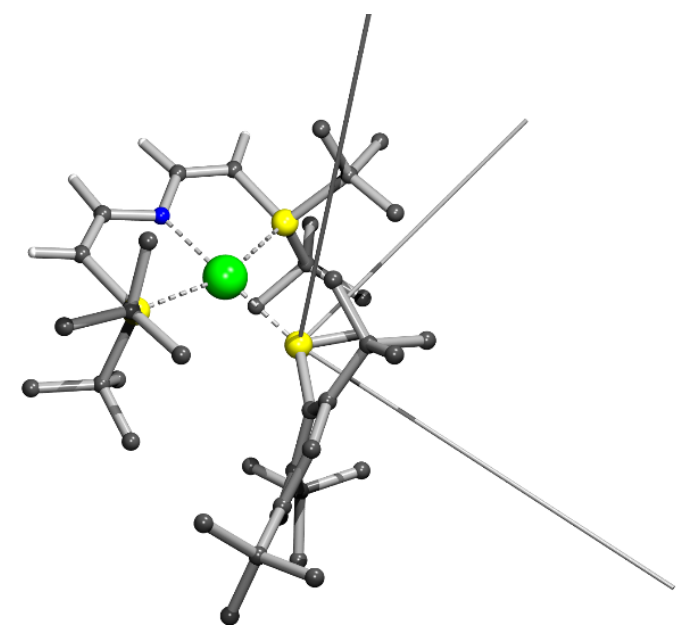

Appendix 8.3.34: HFI A-tensor of 38 which follows the molecular axis.

Similarly, the ${ }^{189}$ Os hyperfine interaction (HFI) tensor (16\% natural abundance; HFI tensor roughly aligned along the symmetry axes of the molecule) is rotated with respect to the electronic g-tensor, with computed Euler angles of $\alpha=+90.5^{\circ}, \beta=+51.7^{\circ}$, $\gamma=+106.5^{\circ}$. It is therefore clear that the experimental spectrum needs to be simulated using non-coaxial g- and A-tensors, the thus obtained simulation provides an excellent fit with g- and A-tensor values and Euler angles that closely match the DFT calculated EPR parameters. 


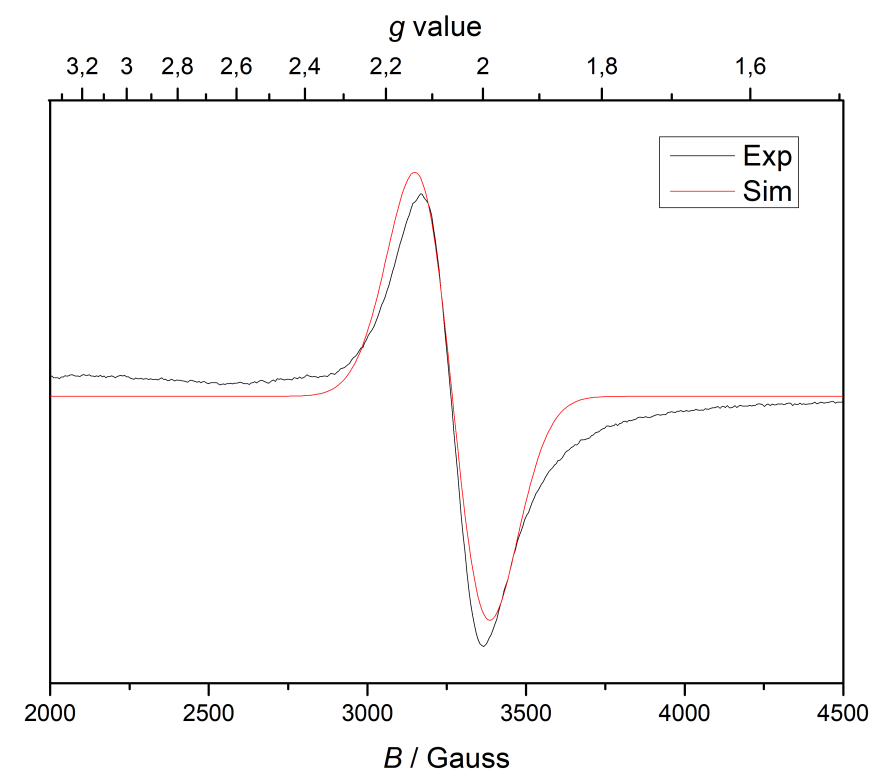

Appendix 8.3.35: EPR spectrum of 38 (black), toluene, $293 \mathrm{~K}, 9.4262 \mathrm{GHz}$, simulation (red) using parameters $g_{\text {iso }}=2.0611$.

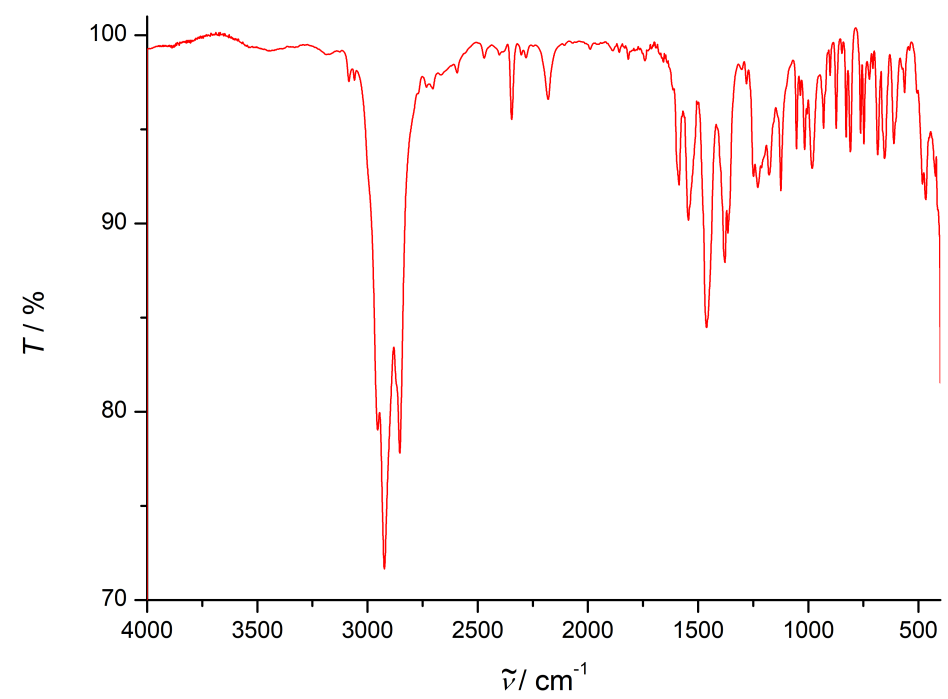

Appendix 8.3.36: IR spectrum of 38, Nujol, RT. 


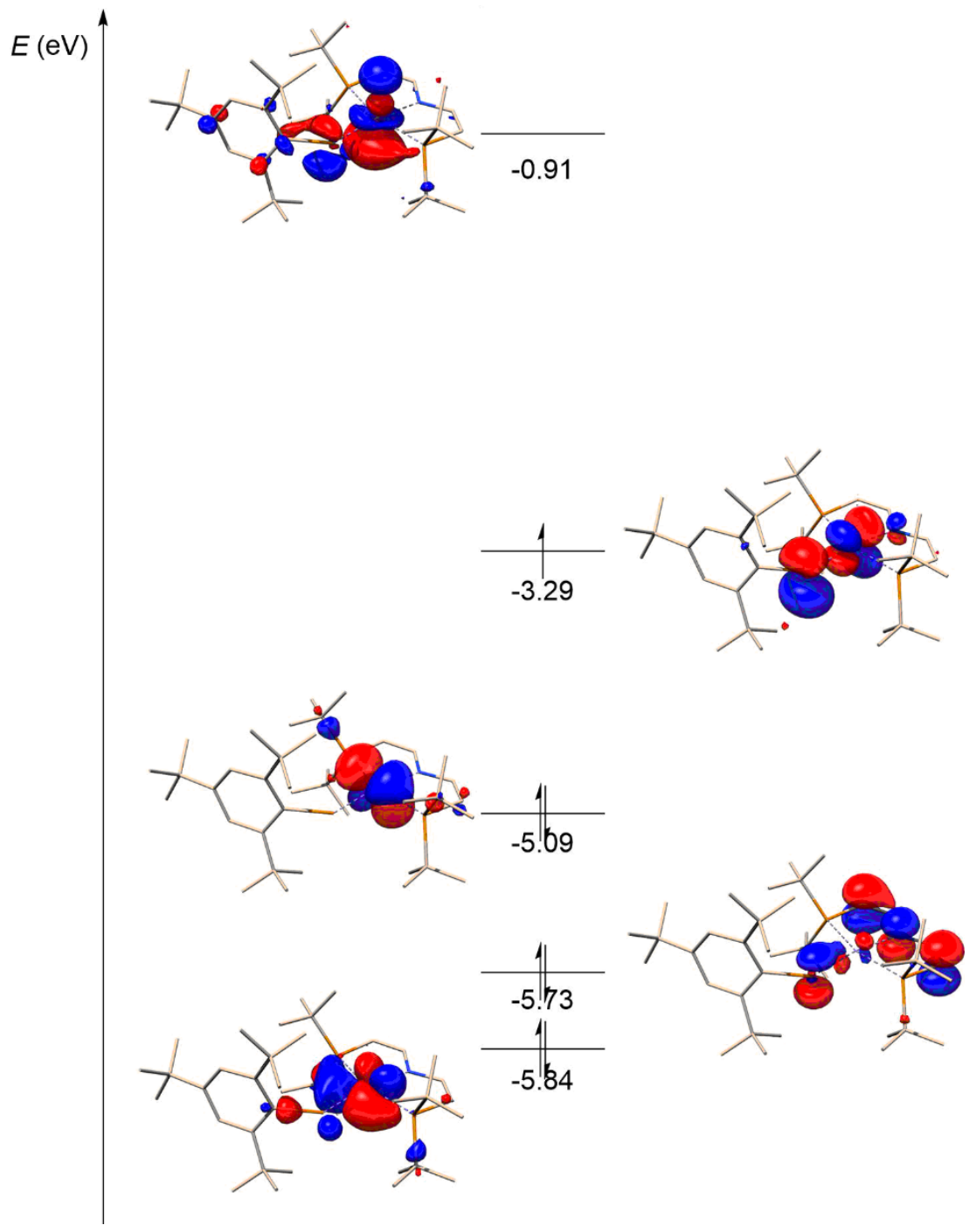

Appendix 8.3.37: Frontier molecular orbital scheme (quasi-restricted orbitals) of 38 (D3BJPBE0-RIJCOSX/def2-TZVP//D3BJ-PBE-RI/def2-SVP). 


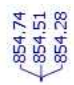
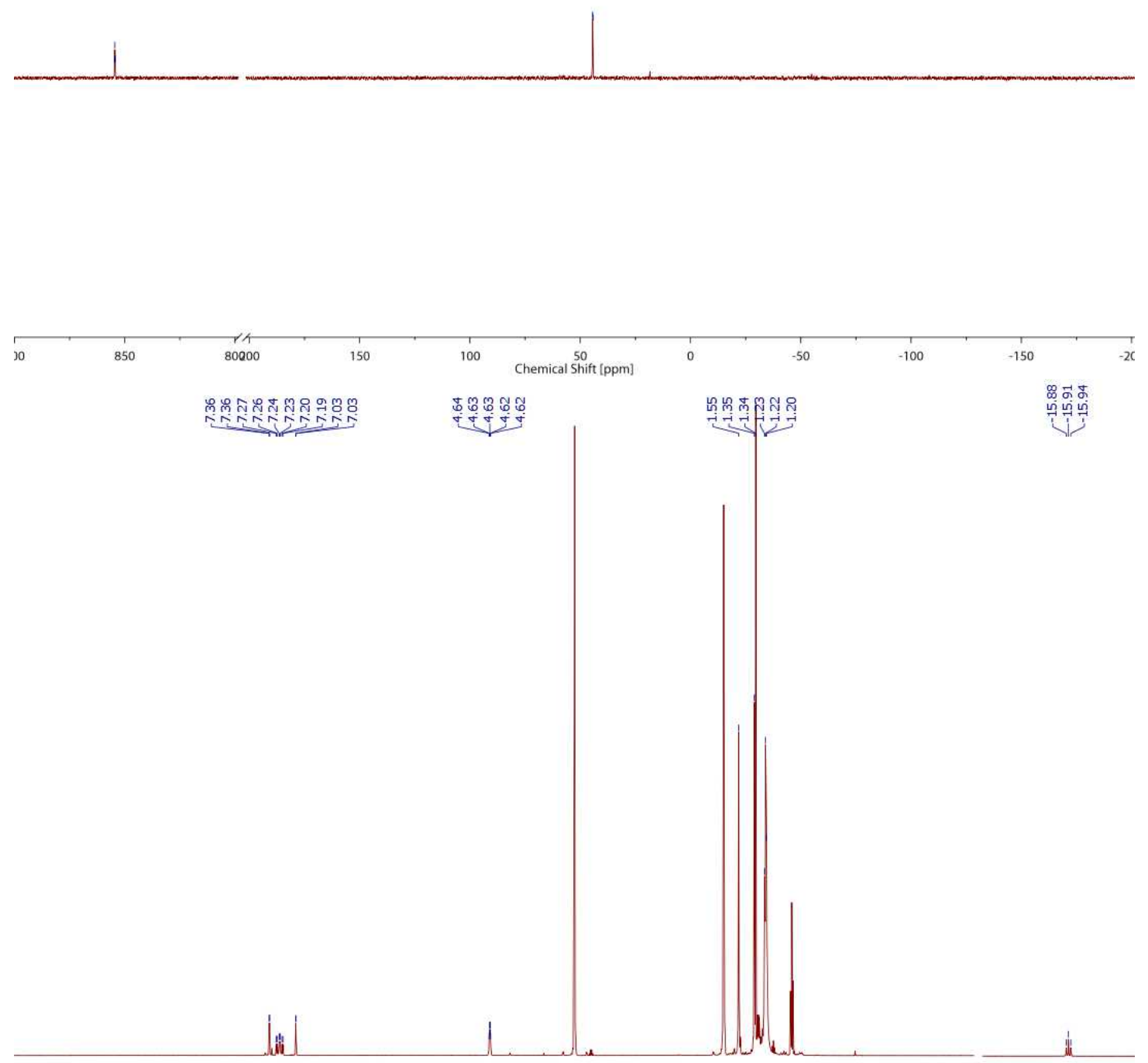

$\begin{array}{llllllllllllllllllllllllllllllllll}1.5 & 10.0 & 9.5 & 9.0 & 8.5 & 8.0 & 7.5 & 7.0 & 6.5 & 6.0 & 5.5 & 5.0 & 4.5 & 4.0 & 3.5 & 3.0 & 2.5 & 1.0 & 1.5 & 1.0 & 0.5 & 0.0 & -0.5 & -1.0 & -15.0 & -15.5 & -16.0 & -16.5\end{array}$

Appendix 8.3.38: Top: ${ }^{31} \mathrm{P}\left\{{ }^{1} \mathrm{H}\right\}$ NMR spectrum of 39, THF- $\mathrm{d}_{8},-30{ }^{\circ} \mathrm{C}$; Bottom: ${ }^{1} \mathrm{H}$ NMR spectrum of $\mathbf{3 9}, \mathrm{THF}-\mathrm{d}_{8},-30^{\circ} \mathrm{C}$. 


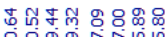

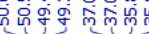

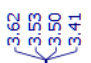
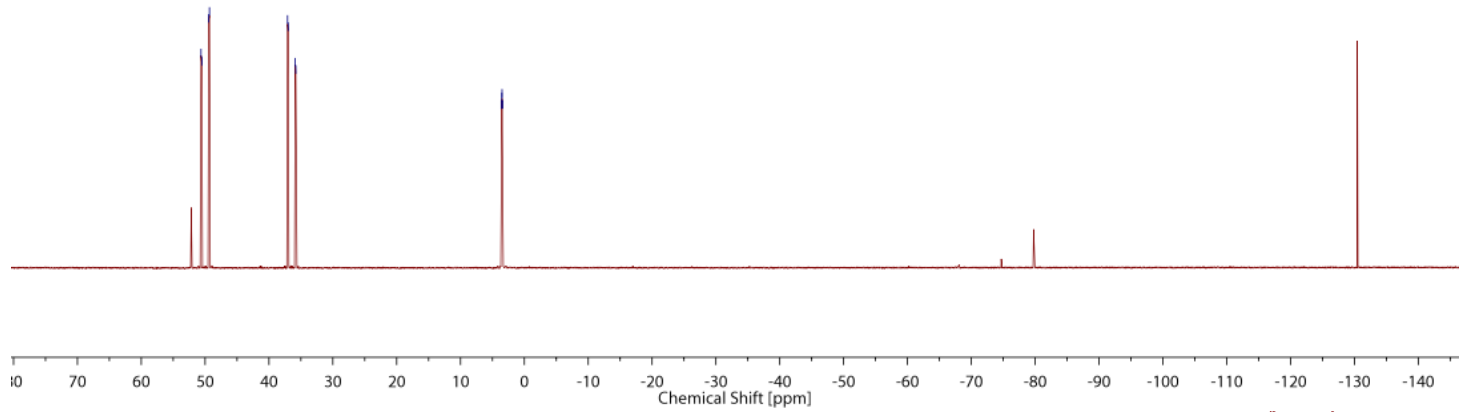

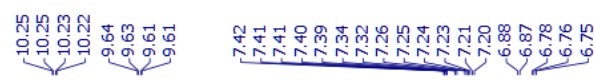

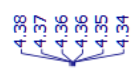

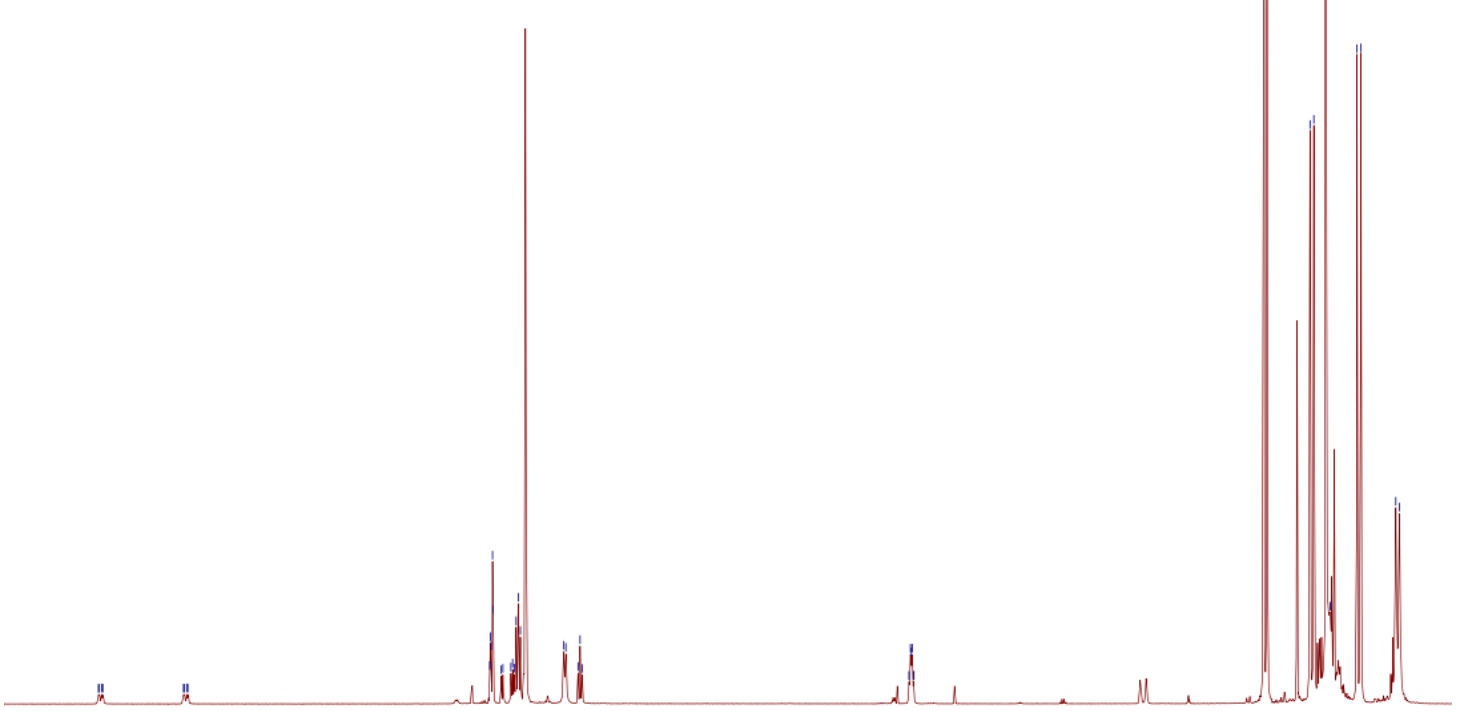

$\begin{array}{lllllllllllllllllllllllll}10.5 & 10.0 & 9.5 & 9.0 & 8.5 & 8.0 & 7.5 & 7.0 & 6.5 & \begin{array}{c}6.0 \\ \text { Chemical Shift [ppm] }\end{array} & 5.0 & 4.5 & 4.0 & 3.5 & 3.0 & 2.5 & 2.0 & 1.5 & 1.0 & 0.5\end{array}$

Appendix 8.3.39: Top: ${ }^{31} \mathrm{P}\left\{{ }^{1} \mathrm{H}\right\}$ NMR spectrum of 42, $\mathrm{C}_{6} \mathrm{D}_{6}$, RT; Bottom: ${ }^{1} \mathrm{H}$ NMR spectrum of $42, \mathrm{C}_{6} \mathrm{D}_{6}, \mathrm{RT}$. 


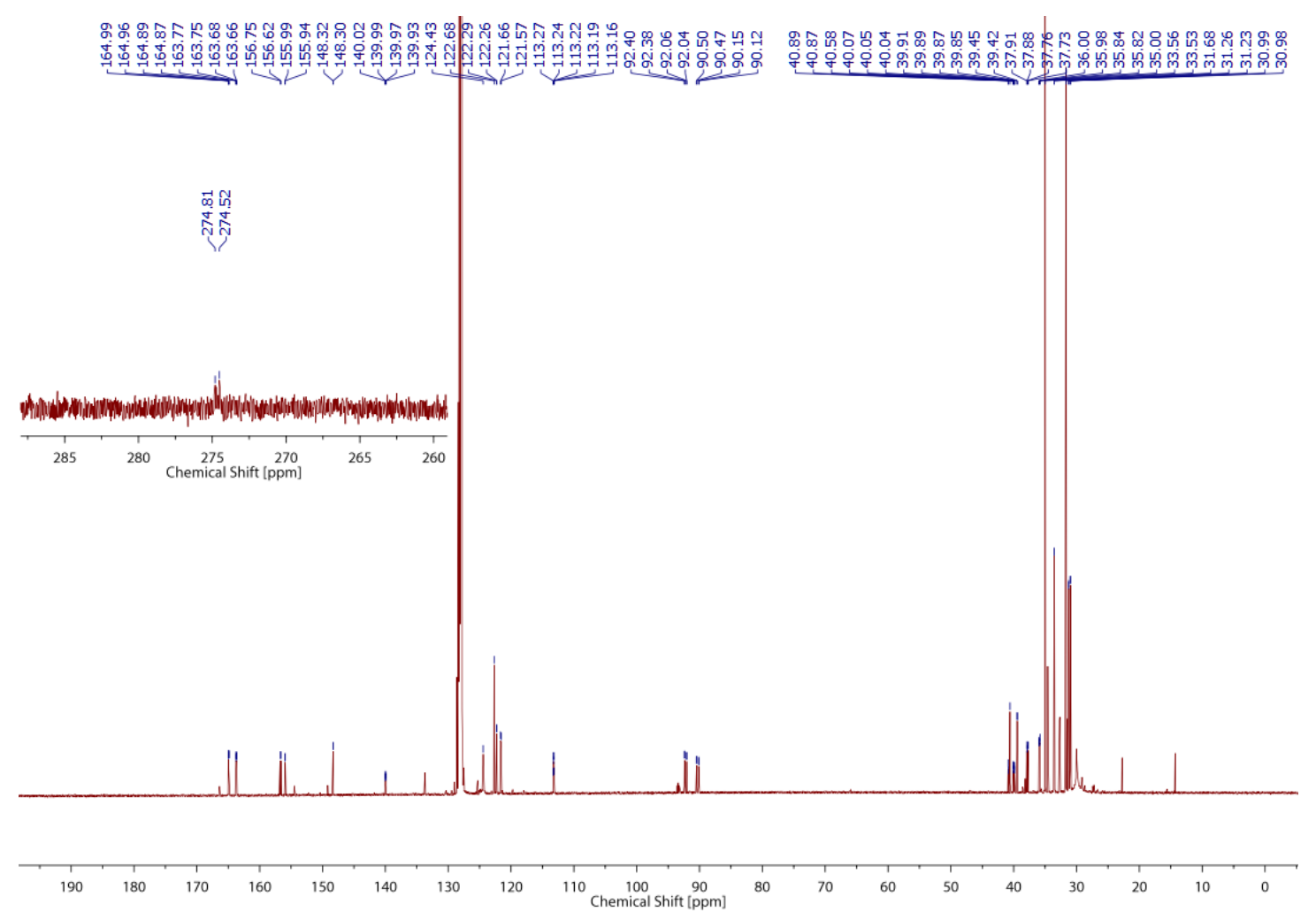

Appendix 8.3.40: ${ }^{13} \mathrm{C}\left\{{ }^{1} \mathrm{H}\right\}$ NMR spectrum of $42, \mathrm{C}_{6} \mathrm{D}_{6}$, RT. 


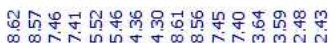

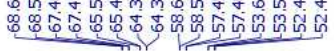

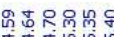

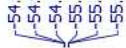
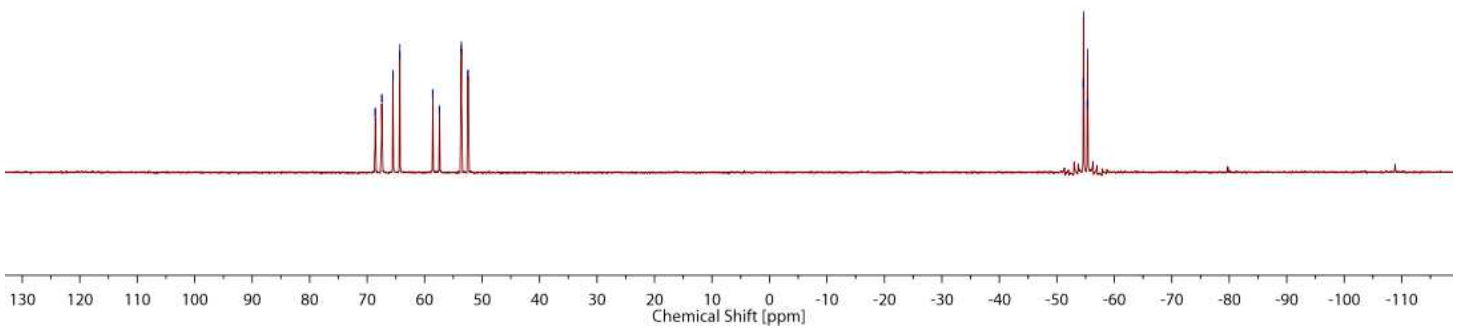

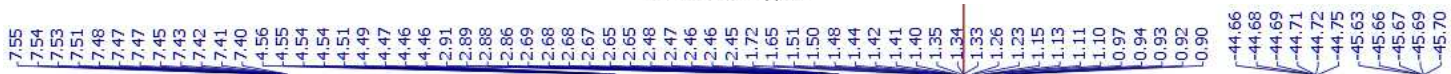

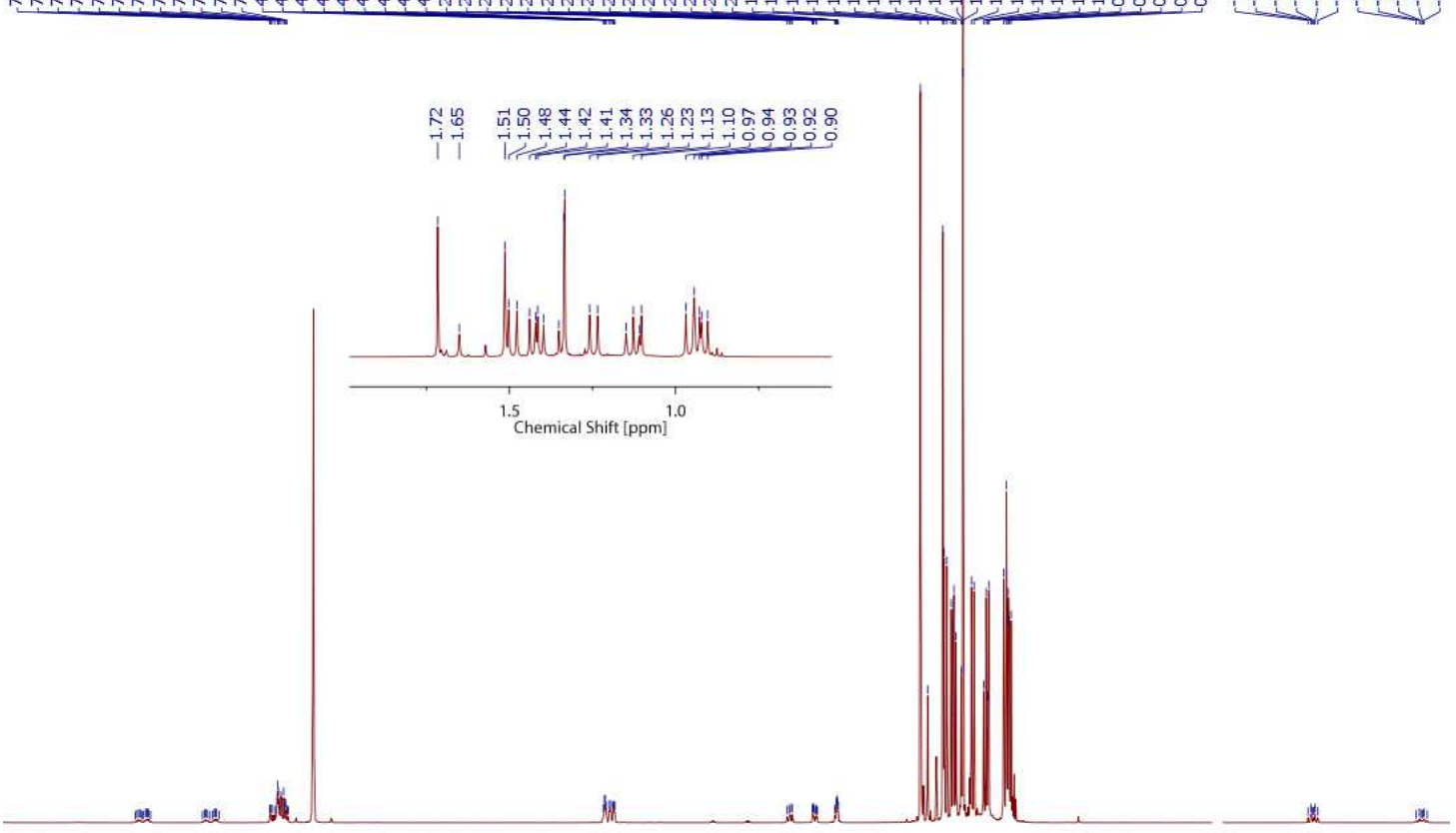

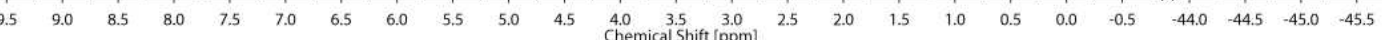

Appendix 8.3.41: Top: ${ }^{31} \mathrm{P}\left\{{ }^{1} \mathrm{H}\right\}$ NMR spectrum of 40, $\mathrm{C}_{6} \mathrm{D}_{6}$, RT; Bottom: ${ }^{1} \mathrm{H}$ NMR spectrum of $40, \mathrm{C}_{6} \mathrm{D}_{6}, \mathrm{RT}$. 


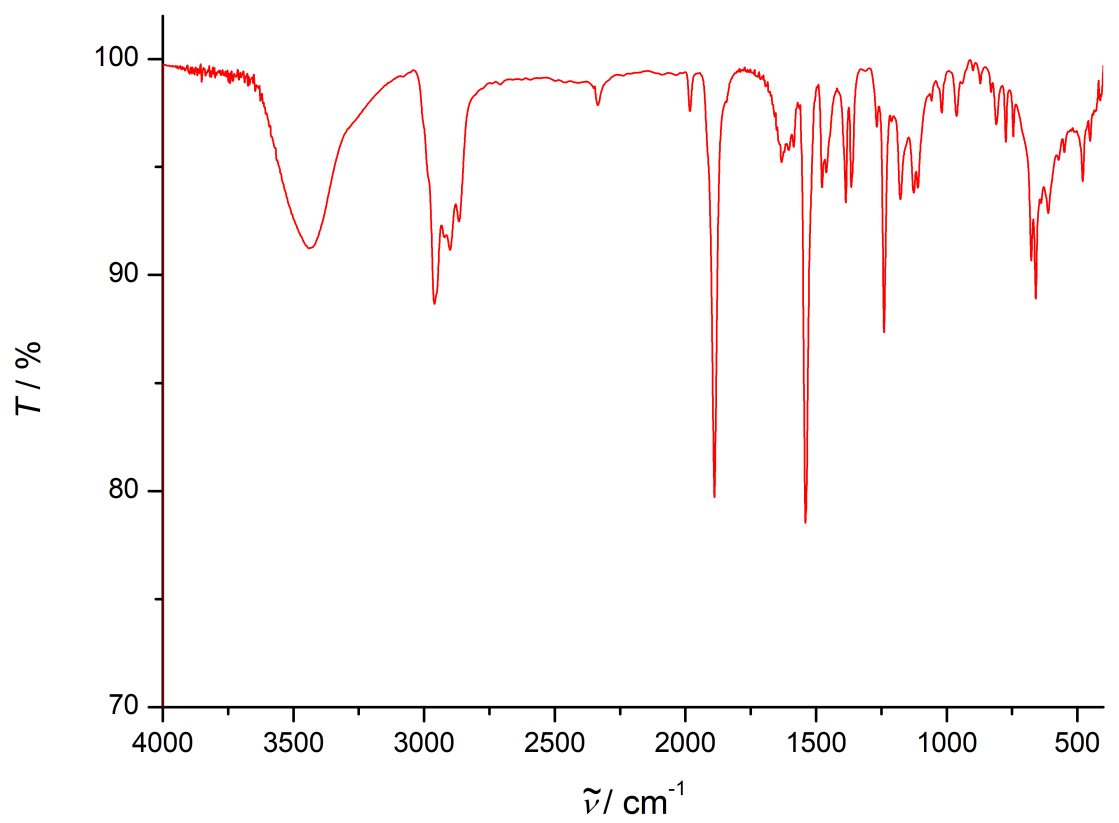

Appendix 8.3.42: IR spectrum of 38, $\mathrm{KBr}, \mathrm{RT}$.

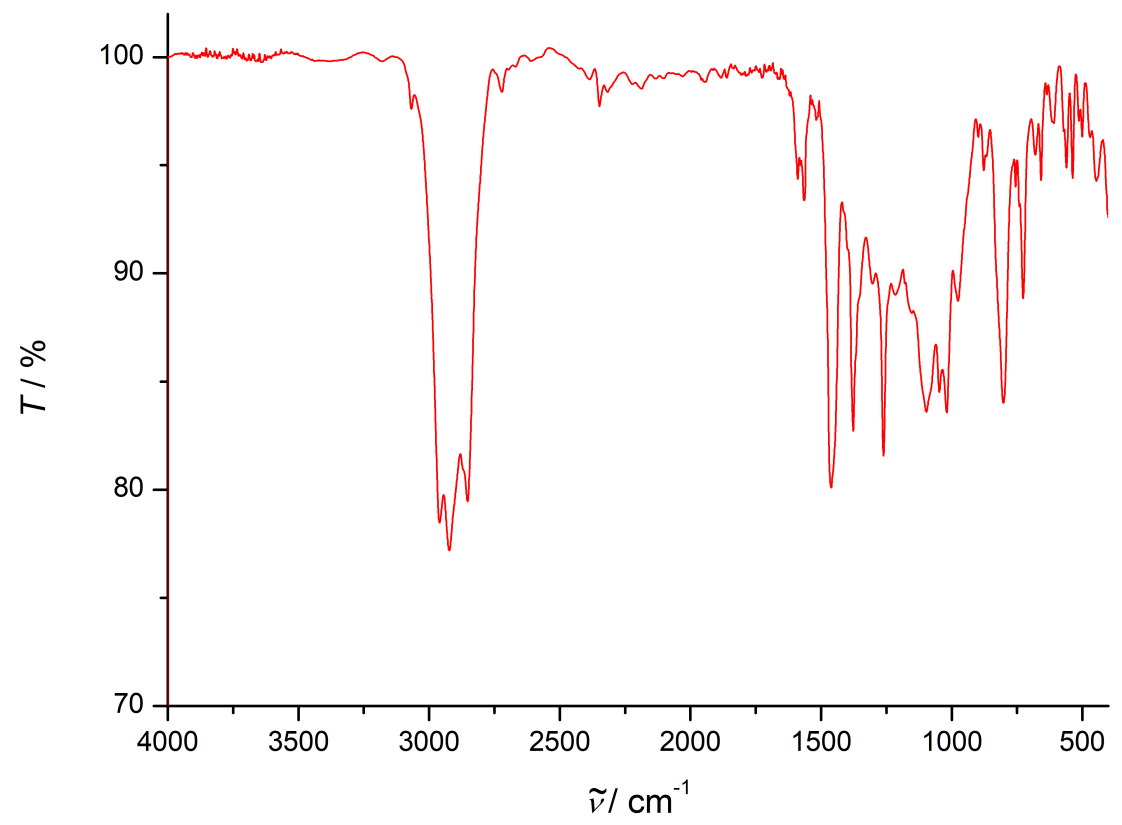

Appendix 8.3.43: IR spectrum of 43, Nujol, RT. 

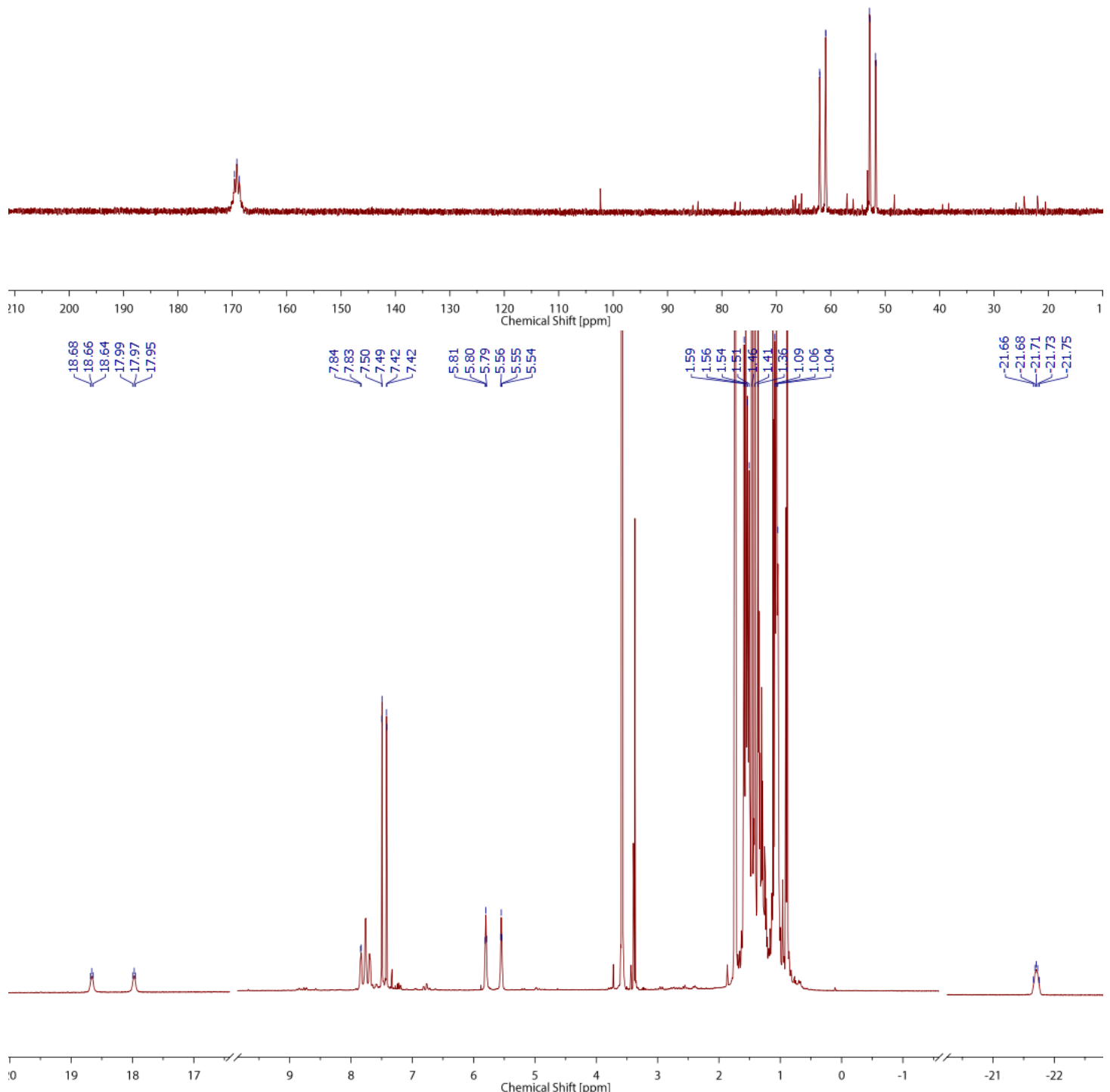

Appendix 8.3.44: Top: ${ }^{31} \mathrm{P}\left\{{ }^{1} \mathrm{H}\right\}$ NMR spectrum of 43, THF- $\mathrm{d}_{8},-30{ }^{\circ} \mathrm{C}$; Bottom: ${ }^{1} \mathrm{H}$ NMR spectrum of $43, \mathrm{THF}-\mathrm{d}_{8},-30^{\circ} \mathrm{C}$. 


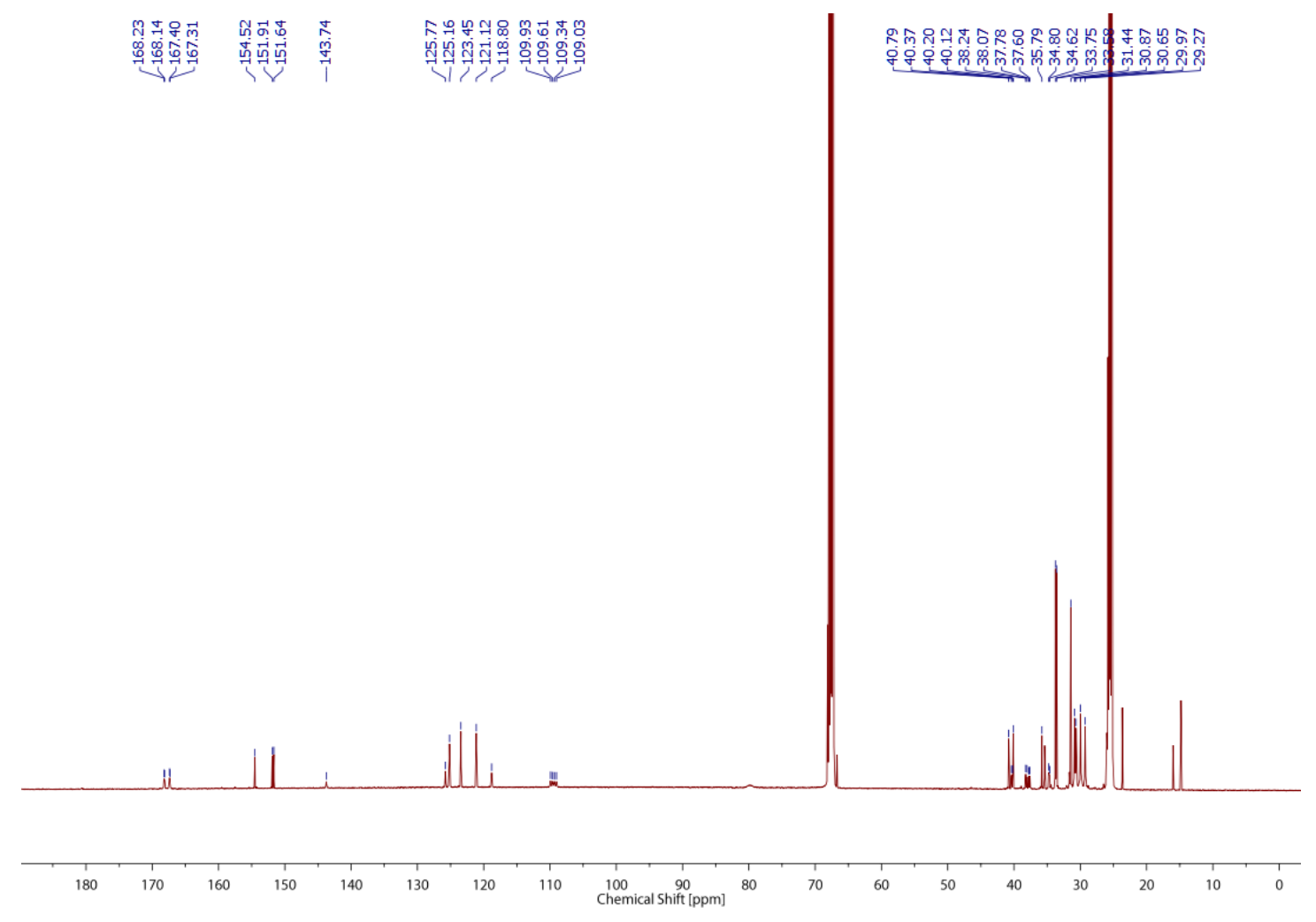

Appendix 8.3.45: ${ }^{13} \mathrm{C}\left\{{ }^{1} \mathrm{H}\right\}$ NMR spectrum of 43 , THF- $\mathrm{d}_{8},-30^{\circ} \mathrm{C}$. 
8.4 Proton-Induced Splitting of Dinitrogen by Dimeric Mo PNP Pincer COMPLEXES

\subsection{Proton-Induced Splitting of Dinitrogen by Dimeric Mo PNP Pincer Complexes}
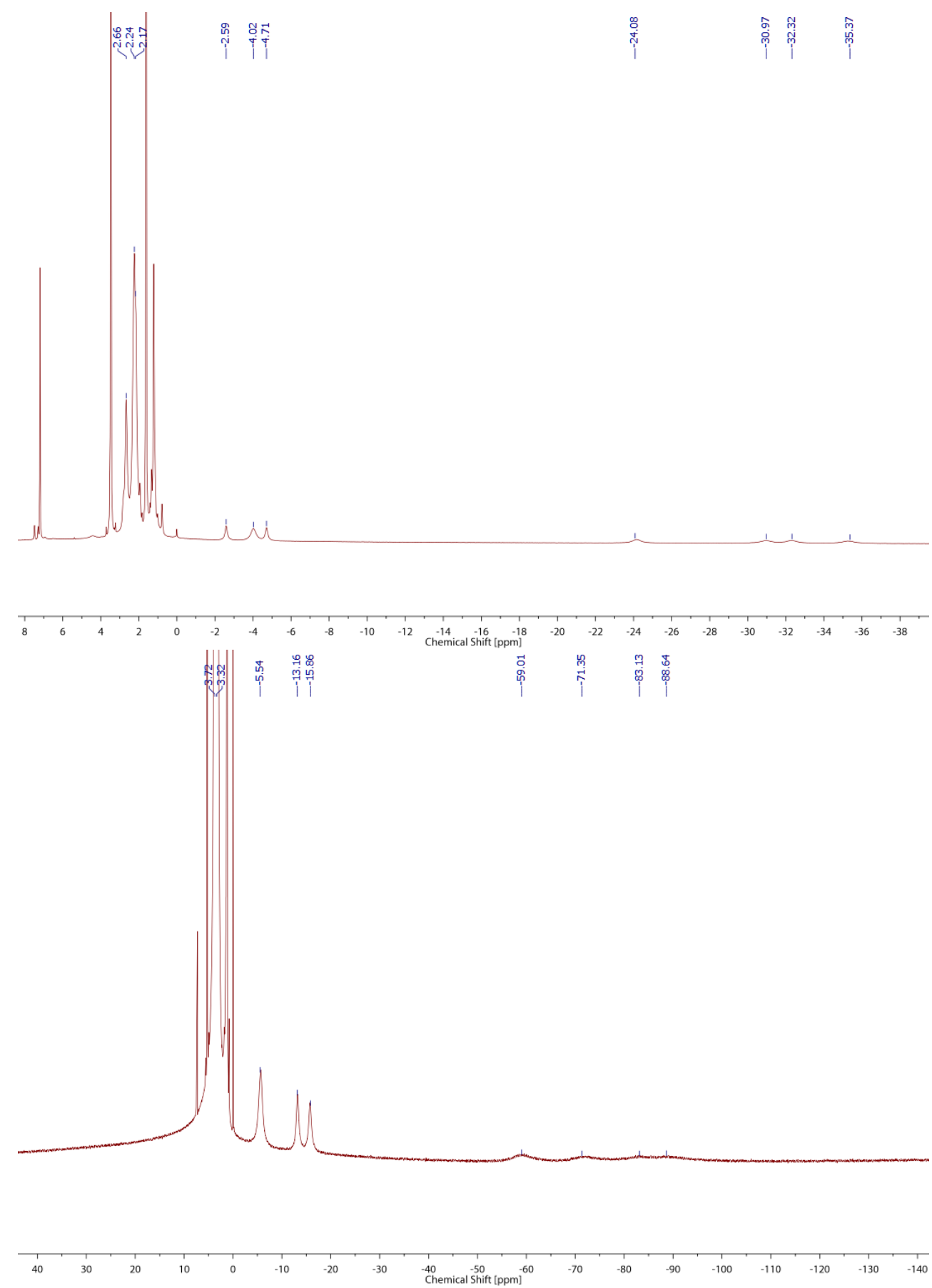

Appendix 8.4.1: Top: ${ }^{1} \mathrm{H}$ NMR spectrum of 48, THF-d 8 , RT; Bottom: ${ }^{1} \mathrm{H}$ NMR spectrum of $49, \mathrm{CD}_{2} \mathrm{Cl}_{2}, \mathrm{RT}$. 


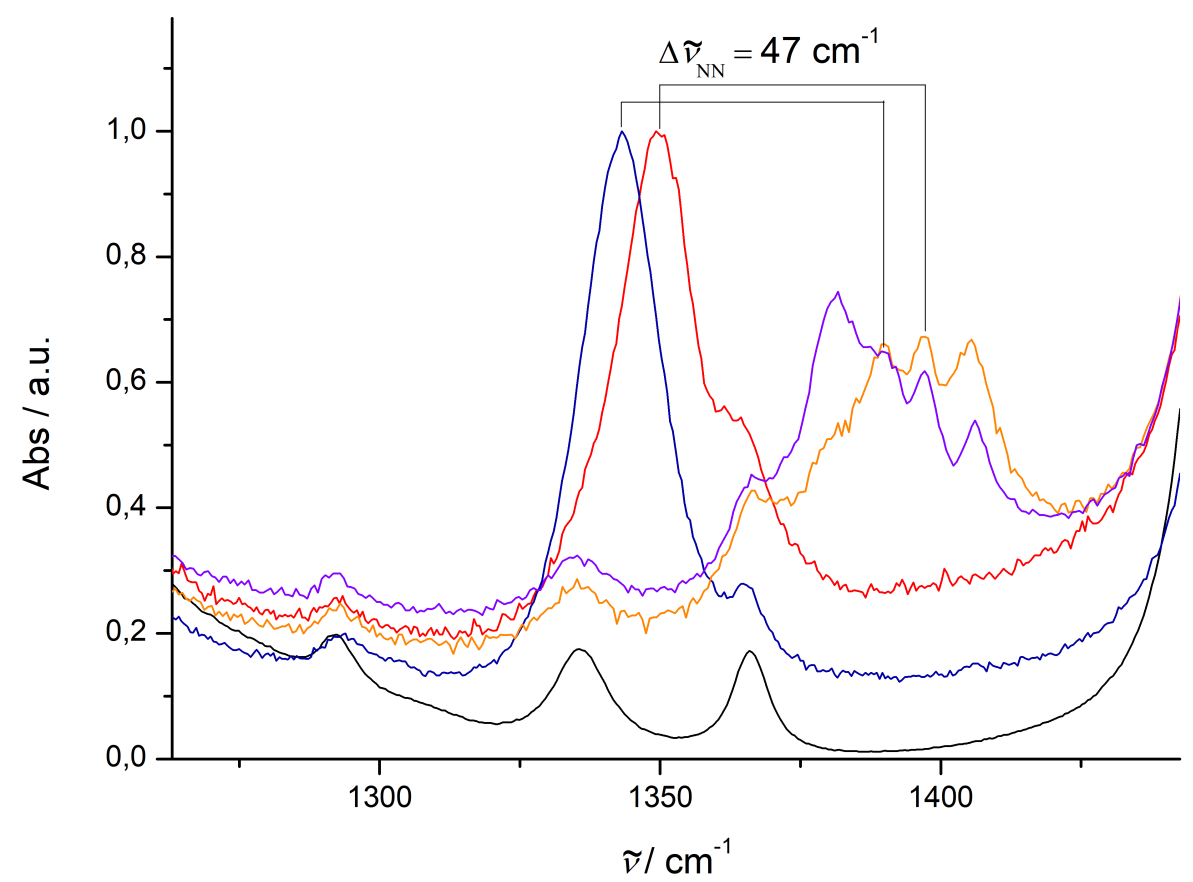

Appendix 8.4.2: rRaman spectra of 48 (orange), $48-{ }^{15} \mathrm{~N}_{2}$ (red), 49 (purple), $49-{ }^{15} \mathrm{~N}_{2}$ (purple), THF (black), $100 \mathrm{~K}, \lambda_{\mathrm{ex}}=514 \mathrm{~nm}$.

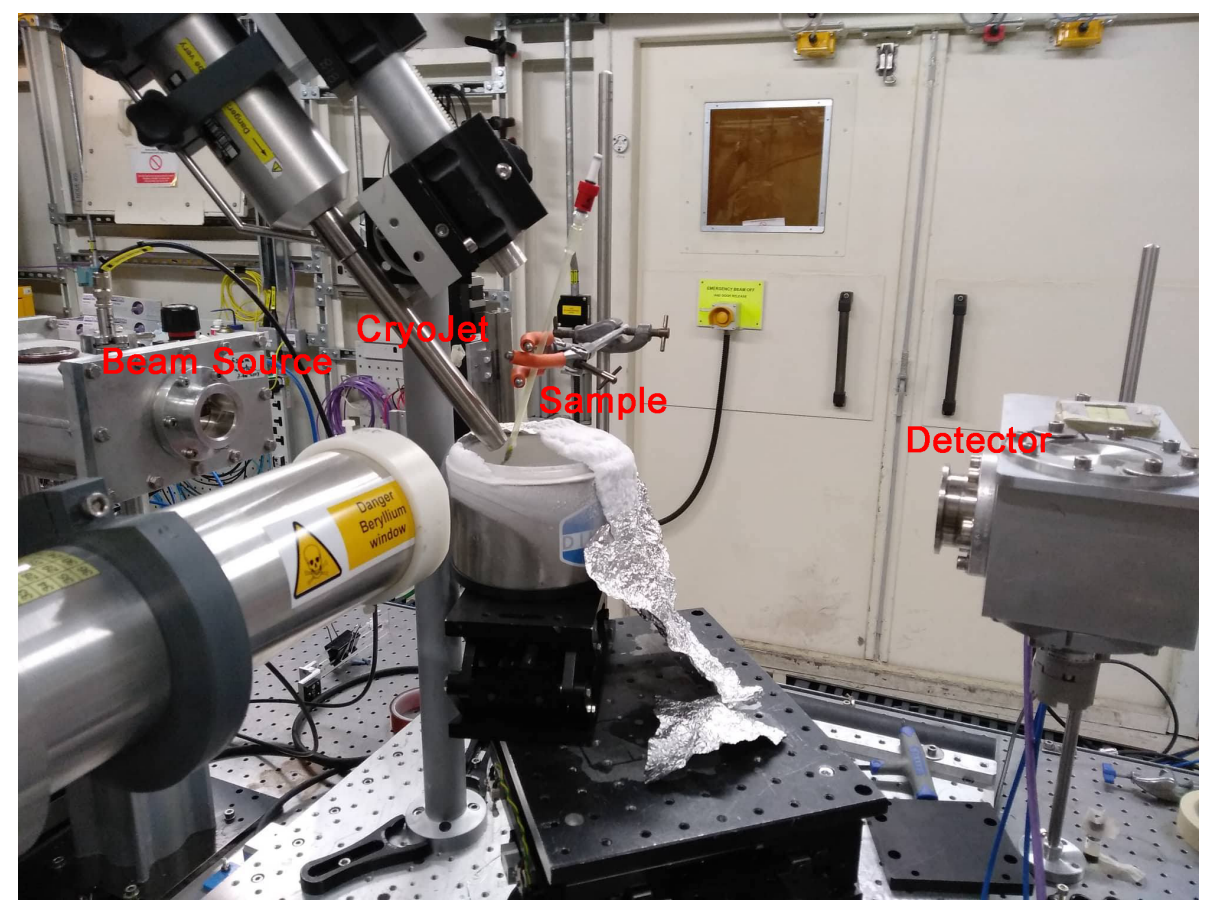

Appendix 8.4.3: Experimental setup of the XAS measurement performed at the B18 Beamline (Diamond Light Source). The sample is partly emerged in liquid nitrogen and additionally cooled by a CryoJet keeping the solution near $-100^{\circ} \mathrm{C}$. The measurements were perfomed in transmission mode. 
8.4 Proton-Induced Splitting of Dinitrogen by Dimeric Mo PNP Pincer COMPLEXES
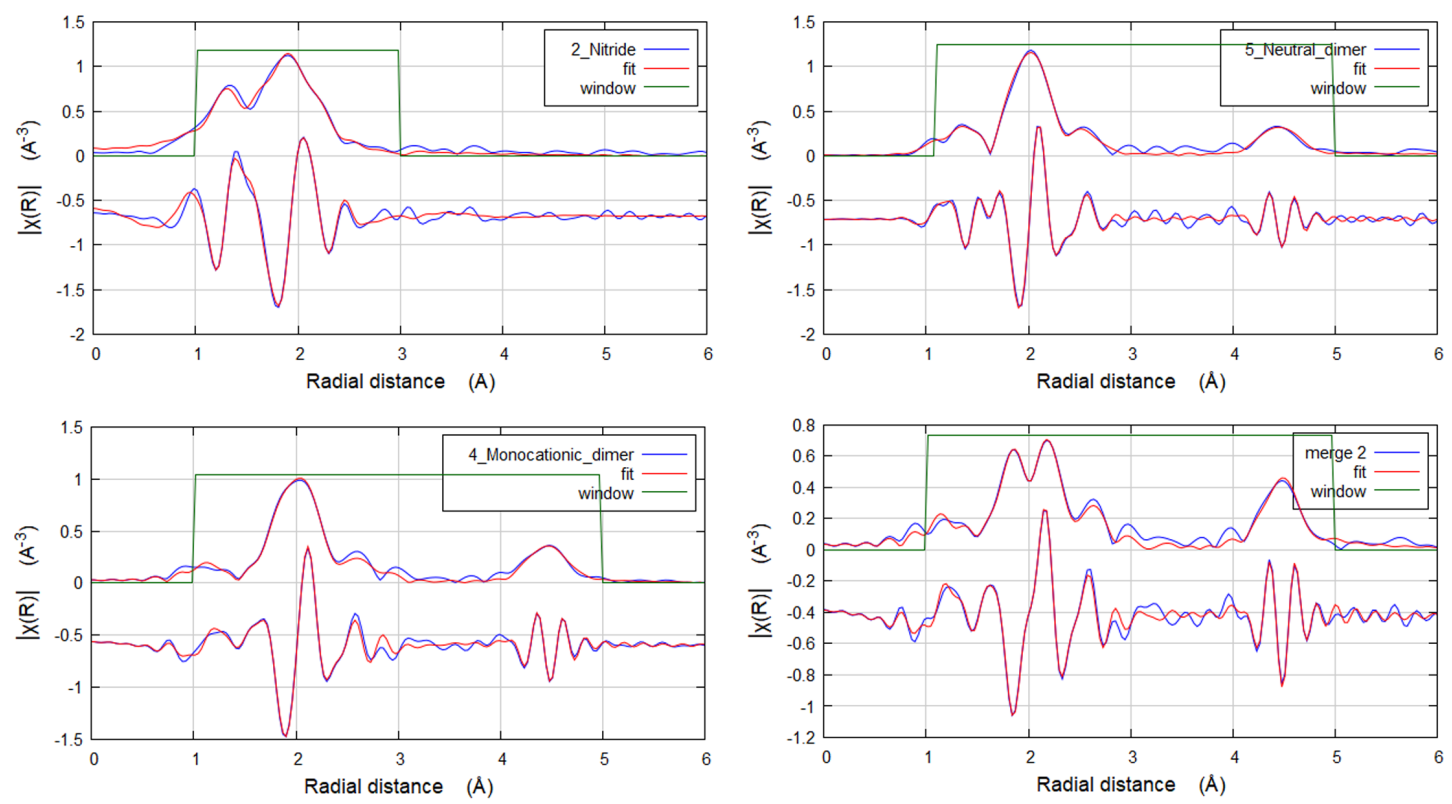

Appendix 8.4.4: Mo-K Edge EXAFS spectra XCIX, XCVII, 48 and 49, R space.

Table 8.2: Structural parameters of XCIX, XCVII, 48, 49 and XCVIII derived by EXAFS spectroscopy, [a]: For XCIX the nitride bond length is given.

\begin{tabular}{clllllll}
\hline Complex & Mo-N $\mathrm{PNP}$ & ${\mathrm{Mo}-\mathrm{N}_{2}}{ }^{\mathrm{a}]}$ & $\mathrm{Mo}-\mathrm{P}$ & $\mathrm{Mo}-\mathrm{Cl}$ & $\mathrm{Mo}-\mathrm{Mo}$ & $\mathrm{N}-\mathrm{N}$ & Mo-C \\
\hline XCIX & $2.11(3)$ & $1.62(1)$ & $2.54(3)$ & $2.37(1)$ & & & \\
XCVII & $1.99(2)$ & $1.80(1)$ & $2.50(2)$ & $2.44(2)$ & $4.74(2)$ & $1.27(4)$ & $3.11(3)$ \\
$\mathbf{4 8}$ & $1.99(2)$ & $1.82(3)$ & $2.50(2)$ & $2.41(2)$ & $4.74(1)$ & $1.22(4)$ & $3.00(8)$ \\
$\mathbf{4 9}$ & $1.92(4)$ & $1.80(4)$ & $2.55(1)$ & $2.37(1)$ & $4.74(8)$ & $1.20(1)$ & $2.99(1)$ \\
XCVIII & $2.30(9)$ & $1.87(2)$ & $2.60(8)$ & $2.48(6)$ & $5.17(3)$ & n.a. & n.a. \\
\hline
\end{tabular}


Table 8.3: Experimental and computational structural parameters of XCVII, 48 and 49 in solution derived by Mo-K edge EXAFS spectroscopy and single crystal X-Ray diffraciton in $\AA$, $\mathrm{THF}, 100^{\circ} \mathrm{C} \cdot{ }^{[280]}$

\begin{tabular}{|c|c|c|c|c|c|c|c|c|c|}
\hline & \multicolumn{3}{|c|}{ Mo- $\mathrm{N}_{\mathrm{PNP}}$} & \multicolumn{3}{|c|}{ Mo-N ${ }_{2}$} & \multicolumn{3}{|c|}{ Mo-Mo } \\
\hline & XRD & XAS & DFT & XRD & XAS & DFT & XRD & XAS & DFT \\
\hline XCVII & $1.917(7)$ & $1.99(2)$ & 2.05 & $1.799(49$ & $1.80(1)$ & 1.84 & $4.846(1)$ & $4.74(2)$ & 4.93 \\
\hline 48 & $\begin{array}{l}1.974(4) \\
1.974(4)\end{array}$ & $1.99(2)$ & $\begin{array}{l}1.97 \\
1.99\end{array}$ & $\begin{array}{l}1.825(4) \\
1.804(4)\end{array}$ & $1.82(3)$ & $\begin{array}{l}1.81 \\
1.77\end{array}$ & $4.846(1)$ & $4.74(1)$ & 4.81 \\
\hline 49 & $\begin{array}{l}1.958(8) \\
1.963(8)\end{array}$ & $1.99(2)$ & $\begin{array}{l}1.97 \\
1.97\end{array}$ & $\begin{array}{l}1.803(8) \\
1.810(8)\end{array}$ & $1.82(3)$ & $\begin{array}{l}1.79 \\
1.79\end{array}$ & $4.847(2)$ & $4.74(8)$ & 4.80 \\
\hline
\end{tabular}



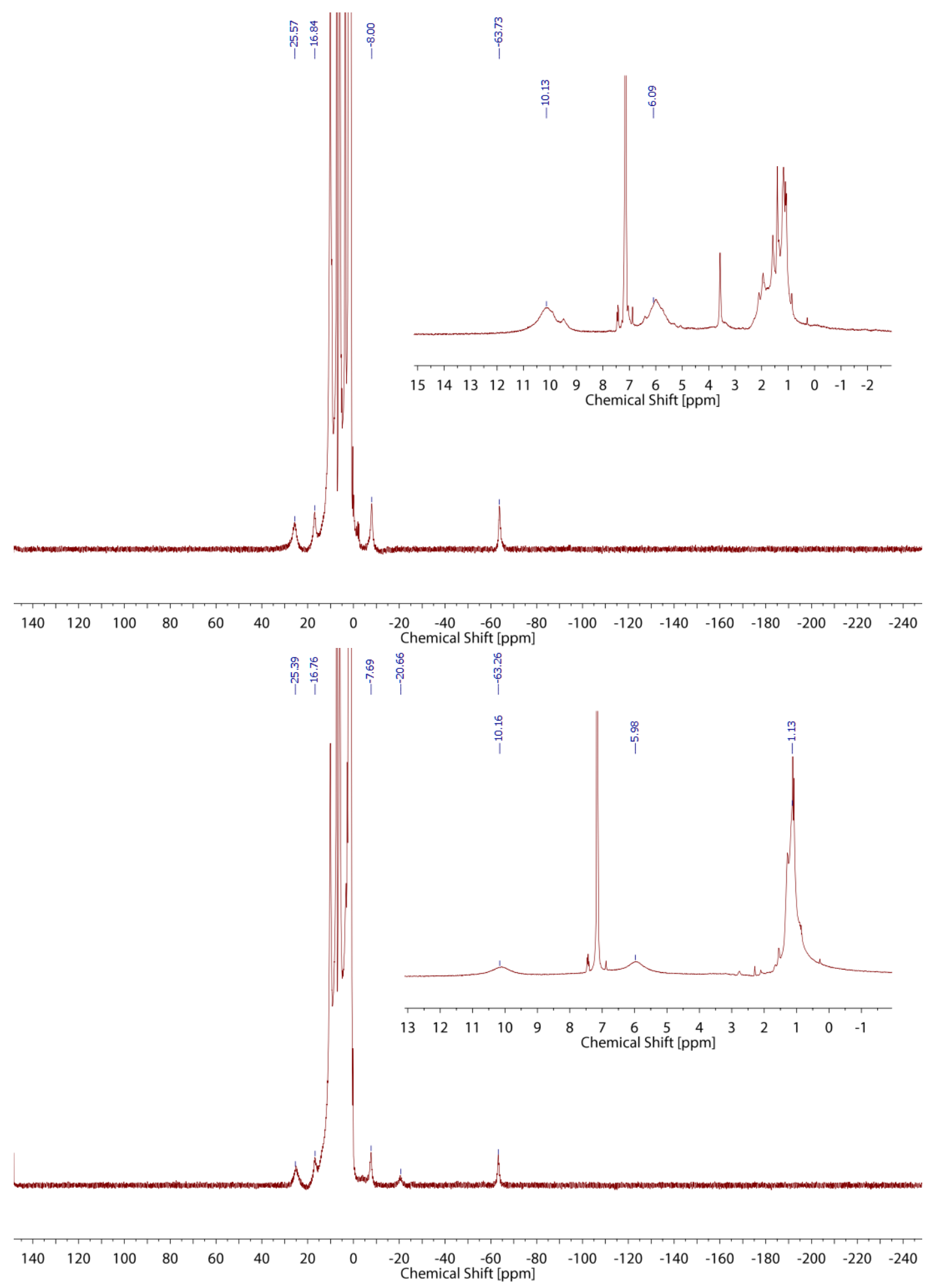

Appendix 8.4.5: Top: ${ }^{1} \mathrm{H}$ NMR spectrum of the attempted complexation of $\left[\mathrm{MoCl}_{3}(\mathrm{thf})_{3}\right]$ by ${ }^{\mathrm{Me}} \mathrm{L}, \mathrm{DCM} / \mathrm{THF}$ 1:2 as solvents, $\mathrm{C}_{6} \mathrm{D}_{6}$, RT; Bottom: ${ }^{1} \mathrm{H}$ NMR spectrum of the attempted complexation of $\left[\mathrm{MoCl}_{3}(\mathrm{thf})_{3}\right]$ by ${ }^{\mathrm{Me}} \mathrm{L}, \mathrm{PhCl}$ as solvent, $\mathrm{C}_{6} \mathrm{D}_{6}, \mathrm{RT}$. 


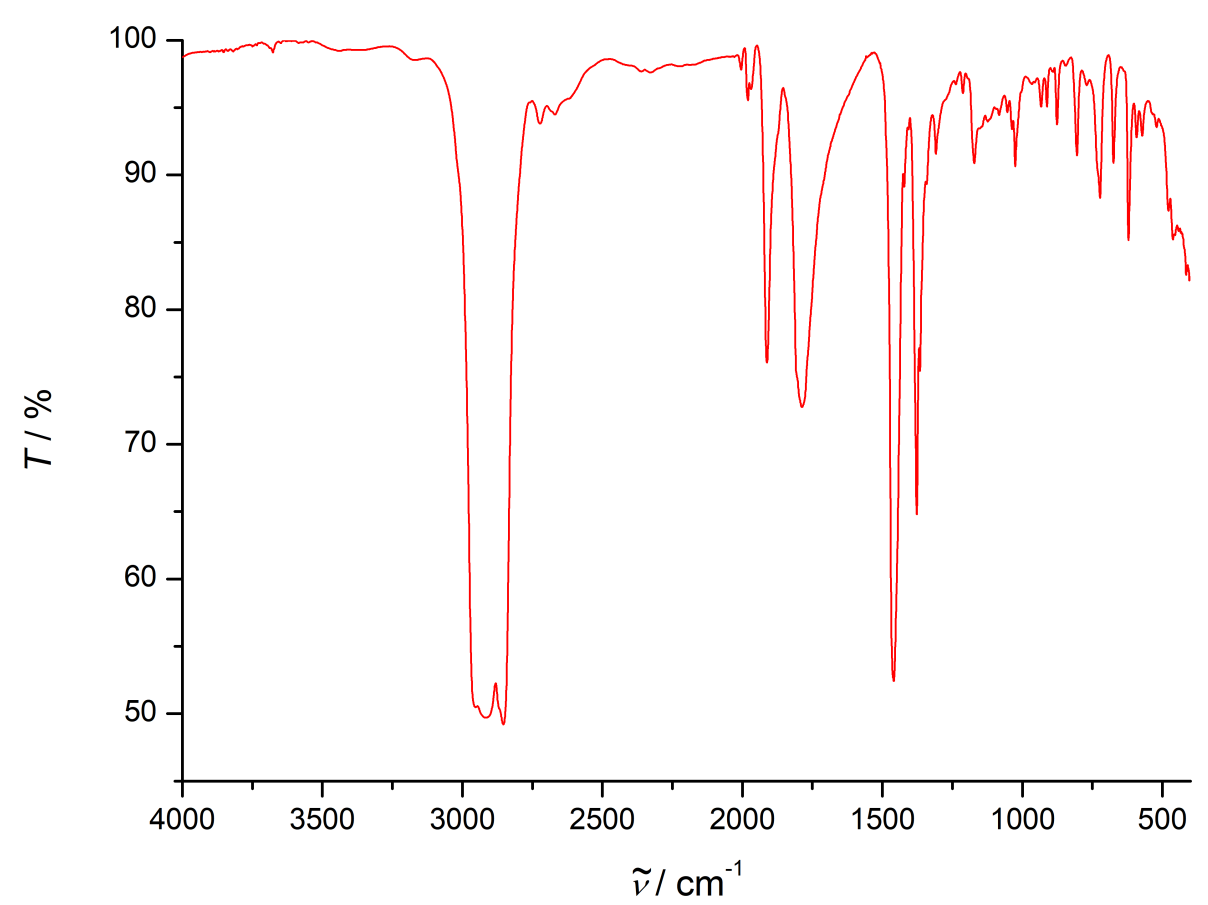

Appendix 8.4.6: IR spectrum of 51, ATR, RT.

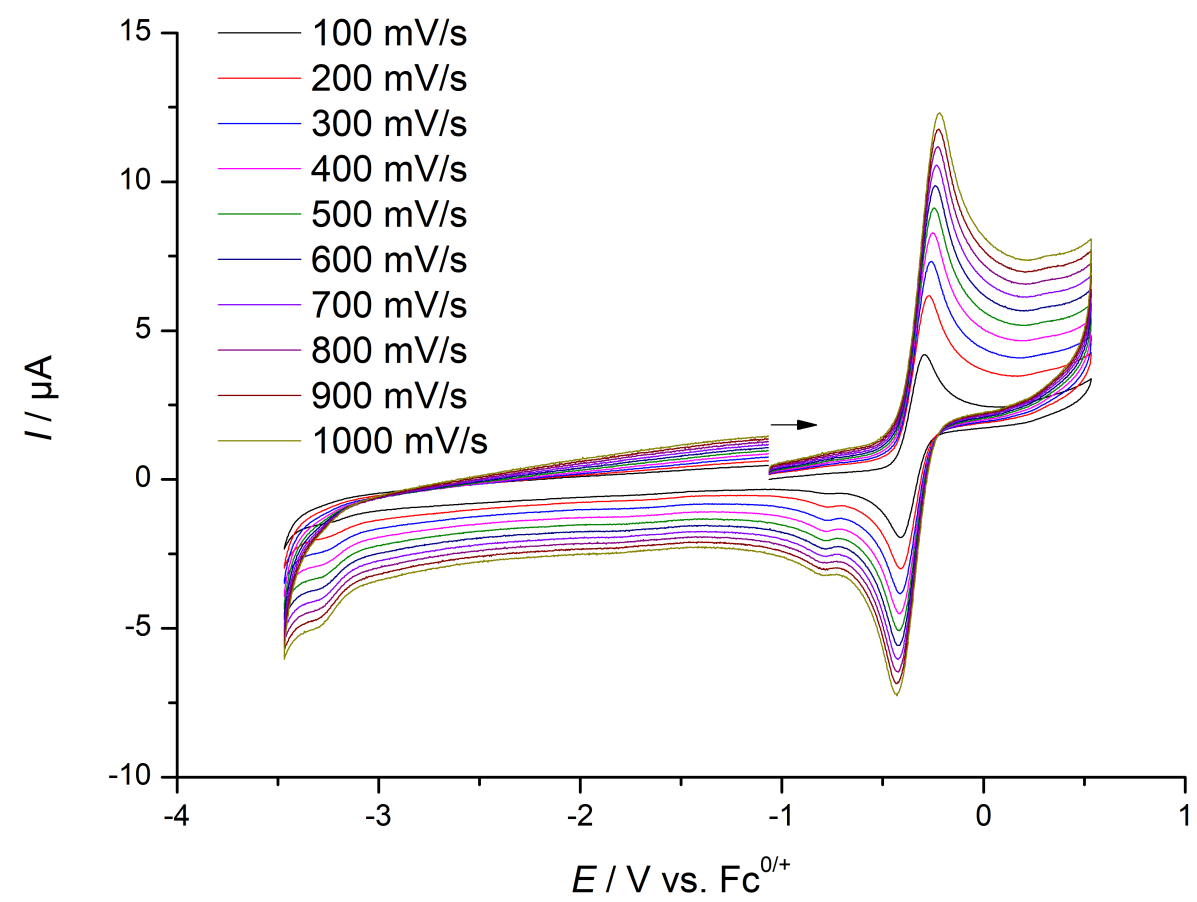

Appendix 8.4.7: Cyclic voltammetry of 51, $1 \mathrm{mM}, 0.1 \mathrm{M} \mathrm{NBu}_{4} \mathrm{PF}_{6}$, THF, RT. 


\section{Used Abbreviations}




\section{Used Abbreviations}

\begin{tabular}{|c|c|}
\hline a.u. & Atomic units / arbitrary units \\
\hline ATP & Adenosine triphosphate \\
\hline bipy & 2,2'-Bipyridine \\
\hline $\mathrm{BD}(\mathrm{F}) \mathrm{E}$ & Bond dissociation (free) energy \\
\hline CAAC & Cyclic alkyl amino carbenes \\
\hline CASSCF & Complete active space self constistent field \\
\hline ClPA & chloro-substituted dibenzo- $7 \lambda^{3}$-phosphanorbornadiene \\
\hline COSY & Correlated spectroscopy \\
\hline $\mathrm{Cp}$ & Cyclopentadienyl- \\
\hline Cys & Cysteine \\
\hline$\delta$ & Chemical shift \\
\hline $\mathrm{DCM}$ & $\mathrm{CH}_{2} \mathrm{Cl}_{2}$ \\
\hline DFT & Density functional theory \\
\hline Dipp & $2,6-{ }^{i} \mathrm{Pr}_{2} \mathrm{C}_{6} \mathrm{H}_{3}$ \\
\hline diox & 1,4-Dioxane \\
\hline DMAD & Acetylenedicarboxylate \\
\hline EDA & Energy decomposition analysis \\
\hline ESI & Electron spray ionisation \\
\hline EPR & Electron paramagnetic resonance \\
\hline$E_{1 / 2}$ & Half-cell potential \\
\hline$E_{\text {p.a. }}$ & Anodic peak current \\
\hline$E_{\text {p.c. }}$ & Cathodic peak current \\
\hline EXAFS & Extended X-ray absorption fine structure \\
\hline $\mathrm{Fc} / \mathrm{FeCp} /[\mathrm{FeCp}]$ & Ferrocene, $\left[\mathrm{Fe}\left(\mathrm{C}_{5} \mathrm{H}_{5}\right)_{2}\right]$ \\
\hline FT-ICR & Fourier-transform ion cyclotron resonance mass spectrometry \\
\hline GB & Gray-Ballhausen \\
\hline GVB & Generalized valence bond \\
\hline HAT & Hydrogen atom transfer \\
\hline HFI & Hyperfine interaction \\
\hline His & Histidin \\
\hline HMBC & Heteronuclear multiple bond correlation \\
\hline $\mathrm{HOMO}$ & Highest occupied molecular orbital \\
\hline${ }^{\mathrm{H}} \mathrm{PyrPz}$ & 3 -(1H-pyrazol-3-yl)-pyridine \\
\hline HSQC & Heteronuclear single quantum coherence \\
\hline${ }^{i} \operatorname{Pr}$ & iso-propyl \\
\hline ITC & Isothermal titration calorimetry \\
\hline$K_{\mathrm{a}}$ & Acid dissociation constant \\
\hline LIFDI & Liquid injection field desorption ionization \\
\hline LUMO & Lowest unoccupied molecular orbital \\
\hline $\mathrm{L}^{1}$ & $\mathrm{~N}\left(\mathrm{CH}_{2} \mathrm{CH}_{2} \mathrm{P}^{t} \mathrm{Bu}_{2}\right)_{2}$ \\
\hline${ }^{\mathrm{H}_{\mathrm{L}}}{ }^{1}$ & $\mathrm{HN}\left(\mathrm{CH}_{2} \mathrm{CH}_{2} \mathrm{P}^{t} \mathrm{Bu}_{2}\right)_{2}$ \\
\hline${ }^{\mathrm{Me}} L^{1}$ & $(\mathrm{Me}) \mathrm{N}\left(\mathrm{CH}_{2} \mathrm{CH}_{2} \mathrm{P}^{t} \mathrm{Bu}_{2}\right)_{2}$ \\
\hline $\mathrm{L}^{2}$ & $\mathrm{~N}\left(\mathrm{CH}_{2} \mathrm{CH}_{2} \mathrm{P}^{t} \mathrm{Bu}_{2}\right)\left(\mathrm{CHCHP}^{t} \mathrm{Bu}_{2}\right)$ \\
\hline
\end{tabular}




\begin{tabular}{|c|c|}
\hline $\mathrm{L}^{3}$ & $\mathrm{~N}\left(\mathrm{CHCHP}^{t} \mathrm{Bu}_{2}\right)_{2}$ \\
\hline $\mathrm{L}^{\mathrm{Si}}$ & $\mathrm{N}\left(\mathrm{SiMe}_{2} \mathrm{CH}_{2} \mathrm{P}^{t} \mathrm{Bu}_{2}\right)_{2}$ \\
\hline $\mathrm{L}^{\mathrm{SiO}}$ & $\mathrm{O}\left(\mathrm{SiMe}_{2} \mathrm{CH}_{2} \mathrm{P}^{t} \mathrm{Bu}_{2}\right)_{2}$ \\
\hline$\mu_{\mathrm{B}}$ & Bohr magneton \\
\hline$\mu_{\text {eff }}$ & Effective magnetic moment \\
\hline $\mathrm{Me}$ & Methyl \\
\hline Mes & $2,4,6-\mathrm{Me}_{3} \mathrm{C}_{6} \mathrm{H}_{2}$ \\
\hline Mes* & $2,4,6-{ }^{t} \mathrm{Bu}_{3} \mathrm{C}_{6} \mathrm{H}_{2}$ \\
\hline $\mathrm{Mes}^{*} \mathrm{O}$ & $2,4,6-{ }^{t} \mathrm{Bu}_{3} \mathrm{C}_{6} \mathrm{H}_{2} \mathrm{O}$ \\
\hline MO & Molecular orbital \\
\hline MS & Mass spectrometry \\
\hline NBO & Natural bonding orbital \\
\hline NEVPT & N-electron valence state perturbation theory \\
\hline $\mathrm{NHC}$ & N-heterocyclic carbene \\
\hline NMR & Nuclear magnetic resonance \\
\hline NPA & Natural population analysis \\
\hline OTf & Triflate \\
\hline $\operatorname{PBE}(0)$ & Perdew-Burke-Ernzerhof \\
\hline PCET & Proton coupled electron transfer \\
\hline PDI & Pyridinediimine \\
\hline $\mathrm{Ph}$ & Phenyl \\
\hline $\mathrm{Ph}_{\mathrm{T} p y}$ & 4'-phenyl-2,2':6',2"'-terpyridine \\
\hline PI & Paramagnetic impurity \\
\hline $\mathrm{p} K_{\mathrm{a}}$ & $-\log 10\left(K_{\mathrm{a}}\right)$ \\
\hline ppm & Parts per million \\
\hline Pyr & Pyridine \\
\hline${ }^{\mathrm{H}} \mathrm{PyrPz}$ & 3-(1H-pyrazol-3-yl)-pyridine \\
\hline QDPT & Quasi-degenerate pertubation theory \\
\hline rRaman & Resonance Raman \\
\hline RT / r.t. & Room temperature \\
\hline SOC & Spin-orbit coupling \\
\hline $\mathrm{SOMO}$ & Singly occupied molecular orbital \\
\hline SQUID & Superconducting quantum interference device \\
\hline SVP & Split valence polarization \\
\hline THF & Tetrahydrofurane \\
\hline TBA & Tetra-butyl ammonium \\
\hline${ }^{t} \mathrm{Bu}$ & tert-butyl \\
\hline TEMPO & 2,2,6,6-Tetramethylpiperidinyloxyl \\
\hline TIP & Temperature independent paramagnetism \\
\hline Tpy & Terpyridine \\
\hline TZVP & Triple $\zeta$ valence plus polarization \\
\hline vs. & versus \\
\hline WBI & Wiberg bond index \\
\hline XAS & X-ray absorption \\
\hline ZORA & Zeroth order regular approximation \\
\hline
\end{tabular}





\section{References}




\section{References}

[1] G. S. Girolami, J. Chem. Educ. 2009, 86, 1200.

[2] B. M. Hoffman, D. Lukoyanov, Z. Y. Yang, D. R. Dean, L. C. Seefeldt, Chem. Rev. 2014, 114, 4041.

[3] M. Appl, Ullmann's Encyclopedia of Industrial Chemistry, 7. Aufl., WILEY-VCH Verlag GmbH, Weinheim, 2001.

[4] F. Schüth, R. Palkovits, R. Schlögl, D. S. Su, Energy Environ. Sci. 2012, 5, 6278.

[5] A. Hellman, K. Honkala, I. N. Remediakis, Á. Logadóttir, A. Carlsson, S. Dahl, C. H. Christensen, J. K. Nørskov, Surf. Sci. 2006, 600, 4264.

[6] G. Ertl, Angew. Chem. Int. Ed. 2008, 47, 3524.

[7] B. M. Hoffman, D. Lukoyanov, Z.-Y. Yang, D. R. Dean, L. C. Seefeldt, Chem. Rev. 2014, 114, 4041.

[8] T. Spatzal, M. Aksoyoglu, L. Zhang, S. L. Andrade, E. Schleicher, S. Weber, D. C. Rees, O. Einsle, Science 2011, 334, 940.

[9] K. M. Lancaster, M. Roemelt, P. Ettenhuber, Y. Hu, M. W. Ribbe, F. Neese, U. Bergmann, S. DeBeer, Science 2011, 334, 974.

[10] O. Einsle, F. A. Tezcan, S. L. Andrade, B. Schmid, M. Yoshida, J. B. Howard, D. C. Rees, Science 2002, 297, 1696.

[11] B. M. Hoffman, D. R. Dean, L. C. Seefeldt, Acc. Chem. Res. 2009, 42, 609.

[12] N. N. Greenwood, A. Earnshaw, Chemistry of the Elements, 2. Aufl., ButterworthHeinemann, Oxford, 1997.

[13] E. Wiberg, N. Wiberg, Lehrbuch der anorganische Chemie, 102. Aufl., Walter de Gruyter \& Co., Berlin, New York, 2007.

[14] F. Wolfe-Simon, J. Switzer Blum, T. R. Kulp, G. W. Gordon, S. E. Hoeft, J. PettRidge, J. F. Stolz, S. M. Webb, P. K. Weber, P. C. W. Davies, A. D. Anbar, R. S. Oremland, Science 2011, 332, 1163.

[15] J. T. Waber, D. T. Cromer, J. Chem. Phys. 1965, 42, 4116.

[16] K. P. Huber, G. Herzberg, Molecular Spectra and Molecular Structure of Diatomic Molecules, Nostrand, New York, 1979.

[17] W. Kutzelnigg, Angew. Chem. Int. Ed. 1984, 23, 272.

[18] P. Jerabek, G. Frenking, Theor. Chem. Acc. 2014, 133, 1.

[19] M. von Hopffgarten, G. Frenking, Wiley Interdiscip. Rev. Comput. Mol. Sci. 2012, 2, 43 . 
[20] L. T. Xu, T. H. Dunning, J. Chem. Theory Comput. 2015, 11, 2496.

[21] W. A. Goddard, T. H. Dunning, W. J. Hunt, P. J. Hay, Acc. Chem. Res. 1973, 6, 368.

[22] R. A. Eikey, M. Abu-Omar, Coord. Chem. Rev. 2003, 243, 83.

[23] C. E. Laplaza, W. M. Davis, C. C. Cummins, Angew. Chem. Int. Ed. 1995, 34, 2042 .

[24] M. Scheer, Coord. Chem. Rev. 1997, 163, 271.

[25] G. Becker, W. Becker, R. Knebl, H. Schmidt, U. Weber, W. M, Nov. Acta Leopoldina 1985, 59, 55 .

[26] G. Becker, W. Becker, R. Knebl, H. Schmidt, U. Hildenbrand, M. Westerhausen, Phosphorous Sulfur Relat. Elem. 1987, 30, 349.

[27] M. Scheer, K. Schuster, T. A. Budzichowski, M. H. Chisholm, William E. Streib, J. Chem. Soc., Chem. Comm. 1995, 1671.

[28] M. L. Ziegler, H.-P. Neumann, Chem. Ber. 1989, 122, 25.

[29] C. Caballero, D. Lehne, B. Nuber, M. L. Ziegler, Chem. Ber. 1991, 124, 1327.

[30] F. P. Arnold, D. P. Ridge, A. L. Rheingold, J. Am. Chem. Soc. 1995, 117, 4427.

[31] I. Klopsch, E. Yuzik-Klimova, „Functionalization of N2 by Mid to Late Transition Metals via N-N Bond Cleavage" in Top. Organomet. Chem., 2017.

[32] M. F. Espada, S. Bennaamane, Q. Liao, N. Saffon-Merceron, S. Massou, E. Clot, N. Nebra, M. Fustier-Boutignon, N. Mézailles, Angew. Chem. Int. Ed. 2018, 57, 12865 .

[33] I. Klopsch, F. Schendzielorz, C. Volkmann, C. Würtele, S. Schneider, Z. anorg. allg. Chem. 2018, 644, 916.

[34] L. M. Duman, L. R. Sita, J. Am. Chem. Soc. 2017, 139, 17241.

[35] F. Schendzielorz, M. Finger, J. Abbenseth, C. Würtele, V. Krewald, S. Schneider, Angew. Chem. Int. Ed. 2018, 2, 840.

[36] Y. Ashida, K. Arashiba, K. Nakajima, Y. Nishibayashi, Nature 2019, 568, 536.

[37] Z.-j. Lv, Z. Huang, W.-x. Zhang, Z. Xi 2019, DOI: 10.10.

[38] Y. Nishibayashi, Transition Metal-Dinitrogen Complexes - Preparation and Reactivity, WILEY-VCH Verlag GmbH, Weinheim, 2019.

[39] S. M. Bhutto, P. L. Holland, Eur. J. Inorg. Chem. 2019, 1861.

[40] B. M. Lindley, R. S. Van Alten, M. Finger, F. Schendzielorz, C. Würtele, A. J. Miller, I. Siewert, S. Schneider, J. Am. Chem. Soc. 2018, 140, 7922. 
[41] M. J. Chalkley, T. J. Del Castillo, B. D. Matson, J. C. Peters, J. Am. Chem. Soc. 2018, 140,6122 .

[42] T. J. Del Castillo, N. B. Thompson, J. C. Peters, J. Am. Chem. Soc. 2016, 138, 5341.

[43] R. Cai, S. D. Minteer, M. Pita, D. Leech, A. L. De Lacey, R. D. Milton, S. Abdellaoui, Angew. Chem. Int. Ed. 2017, 56, 2680.

[44] E. Y. Jeong, C. Y. Yoo, C. H. Jung, J. H. Park, Y. C. Park, J. N. Kim, S. G. Oh, Y. Woo, H. C. Yoon, ACS Sustain. Chem. Eng. 2017, 5, 9662.

[45] N. Ostermann, I. Siewert, Curr. Opin. Electrochem. 2019, DOI: 10.1016/j.coelec.2019.04.024.

[46] C. E. Laplaza, C. C. Cummins, R. E. T. Hill, A. L. Boettcher, Science 1995, 268, 861.

[47] J. J. Curley, T. R. Cook, S. Y. Reece, P. Müller, C. C. Cummins, J. Am. Chem. Soc. 2008, 130, 9394.

[48] C. E. Laplaza, M. J. Johnson, J. C. Peters, A. L. Odom, E. Kim, C. C. Cummins, G. N. George, I. J. Pickering, J. Am. Chem. Soc. 1996, 118, 8623.

[49] D. V. Yandulov, R. R. Schrock, Science 2003, 301, 76.

[50] G. Christian, J. Driver, R. Stranger, Faraday Discuss. 2003, 124, 331.

[51] G. Chrstian, R. Stranger, B. F. Yates, D. C. Graham, Dalton Trans. 2005, 962.

[52] I. Klopsch, M. Finger, C. Würtele, B. Milde, D. B. Werz, S. Schneider, J. Am. Chem. Soc. 2014, 136, 6881.

[53] I. Klopsch, M. Kinauer, M. Finger, C. Würtele, S. Schneider, Angew. Chem. Int. Ed. 2016, 55, 4786 .

[54] W. A. Nugent, R. J. Mckinney, V. Kasowski, F. A. Van-Catledge, Inorganica Chim. Acta 1982, 65, 91.

[55] M. J. Bezdek, S. Guo, P. J. Chirik, Inorg. Chem. 2016, 55, 3117.

[56] J. J. Curley, N. A. Piro, C. C. Cummins, Inorg. Chem. 2009, 48, 9599.

[57] M. Scheer, J. Miiller, M. Haser, Angew. Chem. Int. Ed. 1996, 35, 2492.

[58] J. S. Figueroa, C. C. Cummins, J. Am. Chem. Soc. 2003, 125, 4020.

[59] J. S. Figueroa, C. C. Cummins, Angew. Chem. Int. Ed. 2004, 43, 984.

[60] O. J. Scherer, Acc. Chem. Res. 1999, 32, 751.

[61] B. P. Johnson, G. Balázs, M. Scheer, Coord. Chem. Rev. 2006, 250, 1178.

[62] C. C. Cummins, Angew. Chem. Int. Ed. 2006, 45, 862. 
[63] G. Balázs, L. J. Gregoriades, M. Scheer, Organometallics 2007, 26, 3058.

[64] J. A. Buss, P. H. Oyala, T. Agapie, Angew. Chem. Int. Ed. 2017, 56, 14502.

[65] M. Joost, W. J. Transue, C. C. Cummins, Chem. Commun. 2017, 53, 10731.

[66] G. Balázs, M. Sierka, M. Scheer, Angew. Chem. Int. Ed. 2005, 44, 4920.

[67] A. Velian, M. Nava, M. Temprado, Y. Zhou, R. W. Field, C. C. Cummins, J. Am. Chem. Soc. 2014, 136, 13586.

[68] J. M. Goicoechea, H. Grützmacher, Angew. Chem. Int. Ed. 2018, 57, 16968.

[69] A. Hinz, J. M. Goicoechea, Angew. Chem. Int. Ed. 2016, 55, 8536.

[70] L. N. Grant, B. Pinter, B. C. Manor, R. Suter, H. Grützmacher, D. J. Mindiola, Chem. Eur. J. 2017, 23, 6272.

[71] L. Weber, Eur. J. Inorg. Chem. 2018, 2175.

[72] Z. J. Quan, X. C. Wang, Org. Chem. Front. 2014, 1, 1128.

[73] G. Hierlmeier, A. Hinz, R. Wolf, J. M. Goicoechea, Angew. Chem. Int. Ed. 2017, $57,431$.

[74] L. Liu, D. A. Ruiz, F. Dahcheh, G. Bertrand, R. Suter, A. M. Tondreau, H. Grützmacher, Chem. Sci. 2016, 7, 2335.

[75] Y. Lu, H. Wang, Y. Xie, H. Liu, H. F. Schaefer, Inorg. Chem. 2014, 53, 6252.

[76] G. L. Hou, B. Chen, W. J. Transue, Z. Yang, H. Grützmacher, M. Driess, C. C. Cummins, W. T. Borden, X. B. Wang, J. Am. Chem. Soc. 2017, 139, 8922.

[77] C. J. Ballhausen, H. B. Gray, Inorg. Chem. 1962, 1, 111.

[78] J. R. Winkler, H. B. Gray, Electronic Structures of Oxo-Metal Ions, Berlin, Heidelberg, 2011, S. 17.

[79] M. G. Scheibel, J. Abbenseth, M. Kinauer, F. W. Heinemann, C. Würtele, B. De Bruin, S. Schneider, Inorg. Chem. 2015, 54, 9290.

[80] M. G. Scheibel, B. Askevold, F. W. Heinemann, E. J. Reijerse, B. de Bruin, S. Schneider, Nature Chem. 2012, 4, 552.

[81] B. Askevold, J. T. Nieto, S. Tussupbayev, M. Diefenbach, E. Herdtweck, M. C. Holthausen, S. Schneider, Nature Chem. 2011, 3, 532.

[82] M. G. Scheibel, Y. Wu, A. C. Stückl, L. Krause, E. Carl, D. Stalke, B. De Bruin, S. Schneider, J. Am. Chem. Soc. 2013, 135, 17719.

[83] F. S. Schendzielorz, M. Finger, C. Volkmann, C. Würtele, S. Schneider, Angew. Chem. Int. Ed. 2016, 55, 11417. 
[84] J. Schöffel, A. Y. Rogachev, S. D. George, P. Burger, Angew. Chem. Int. Ed. 2009, 48,4734 .

[85] J. Schöffel, N. Šušnjar, S. Nückel, D. Sieh, P. Burger, Eur. J. Inorg. Chem. 2010, 4911.

[86] A. Walstrom, M. Pink, X. Yang, J. Tomaszewski, M. H. Baik, K. G. Caulton, J. Am. Chem. Soc. 2005, 127, 5330.

[87] D. R. Lide, CRC Handbook of CHemistry and Physics, 90. Aufl., Francis, CRC Press/Taylor and Francis, Boco Raton, FL, 2010.

[88] K. K. Pandey, G. Frenking, Eur. J. Inorg. Chem. 2004, 3, 4388.

[89] J. M. Smith, Prog. Inorg. Chem. 2014, 58, 417.

[90] M. G. Scheibel, I. Klopsch, H. Wolf, P. Stollberg, D. Stalke, S. Schneider, Eur. J. Inorg. Chem. 2013, 3454.

[91] D. Sieh, J. Schöffel, P. Burger, Dalton Trans. 2011, 40, 9512.

[92] T. J. Crevier, J. M. Mayer, J. Am. Chem. Soc. 1998, 120, 5595.

[93] T. J. Crevier, B. K. Bennett, J. D. Soper, J. A. Bowman, A. Dehestani, D. A. Hrovat, S. Lovell, W. Kaminsky, J. M. Mayer, J. Am. Chem. Soc. 2001, 123, 1059 .

[94] T. A. Betley, J. C. Peters, J. Am. Chem. Soc. 2004, 126, 6252.

[95] J. J. Scepaniak, M. D. Fulton, R. P. Bontchev, E. N. Duesler, M. L. Kirk, J. M. Smith, J. Am. Chem. Soc. 2008, 130, 10515.

[96] A. Walstrom, H. Fan, M. Pink, K. G. Caulton, Inorganica Chim. Acta 2010, 363, 633.

[97] N. Tsvetkov, M. Pink, H. Fan, J.-H. Lee, K. G. Caulton, Eur. J. Inorg. Chem. 2010, 4790 .

[98] H. Kunkely, A. Vogler, Angew. Chem. Int. Ed. 2010, 49, 1591.

[99] M. M. Konnick, S. M. Bischof, D. H. Ess, R. A. Periana, B. G. Hashiguchi, J. Mol. Catal. A Chem. 2014, 382, 1.

[100] N. A. Piro, J. S. Figueroa, J. T. McKellar, C. C. Cummins, Science 2006, 313(September), 1276-1279.

[101] M. J. A. Johnson, A. L. Odom, C. C. Cummins, Chem. Comm 1997, 1523.

[102] N. A. Piro, C. C. Cummins, J. Am. Chem. Soc. 2009, 131, 8764.

[103] G. Balázs, J. C. Green, M. Scheer, Chem. Eur. J. 2006, 12, 8603.

[104] C. Che, H. Lam, W.-o. Tong, T. Lai, T. Lau, J. Chem. Soc., Chem. Comm. 1989, 1883. 
[105] O. Krahe, E. Bill, F. Neese, Angew. Chem. Int. Ed. 2014, 53, 8727.

[106] L. A. Kane-Maguire, P. S. Sheridan, F. Basolo, R. G. Pearson, J. Am. Chem. Soc. 1970, 92, 5865 .

[107] K. D. Demadis, T. J. Meyer, P. S. White, Inorg. Chem. 1997, 36, 5678.

[108] J. D. Buhr, H. Taube, Inorg. Chem. 1979, 18, 2208.

[109] D. C. Ware, H. Taube, Inorg. Chem. 1991, 30, 4605.

[110] R. M. Clarke, T. Storr, J. Am. Chem. Soc. 2016, 138, 15299.

[111] V. Krewald, L. González, Chem. Eur. J. 2018, $24,5112$.

[112] T. Chantarojsiri, A. H. Reath, J. Y. Yang, Angew. Chem. Int. Ed. 2018, 57, 14037.

[113] J. Abbenseth, M. Finger, C. Würtele, M. Kasanmascheff, S. Schneider, Inorg. Chem. Front. 2016, 3, 469.

[114] D. Tofan, C. C. Cummins, Angew. Chem. Int. Ed. 2010, 49, 7516.

[115] Y. Wang, Y. Xie, P. Wei, R. B. King, H. F. Schaefer, P. V. R. Schleyer, G. H. Robinson, J. Am. Chem. Soc. 2008, 130, 14970.

[116] M. Y. Abraham, Y. Wang, Y. Xie, P. Wei, H. F. Schaefer, P. R. Von Schleyer, G. H. Robinson, Chem. Eur. J. 2010, 16, 432.

[117] O. Back, G. Kuchenbeiser, B. Donnadieu, G. Bertrand, Angew. Chem. Int. Ed. 2009, 48, 5530.

[118] L. Liu, D. A. Ruiz, D. Munz, G. Bertrand, Chem 2016, 1, 147.

[119] A. Hinz, J. M. Goicoechea, Angew. Chem. Int. Ed. 2016, 55, 15515.

[120] S. Yao, Y. Xiong, T. Szilvási, H. Grützmacher, M. Driess, Angew. Chem. Int. Ed. 2016, 55, 4781.

[121] Y. Xiong, S. Yao, T. Szilvási, E. Ballestero-Martínez, H. Grützmacher, M. Driess, Angew. Chem. Int. Ed. 2017, 56, 4333.

[122] T. Krachko, A. W. Ehlers, M. Nieger, M. Lutz, J. C. Slootweg, Angew. Chem. Int. Ed. 2018, 5\%, 1683.

[123] B. M. Cossairt, N. A. Piro, C. C. Cummins, Chem. Rev. 2010, 110, 4164.

[124] M. Caporali, L. Gonsalvi, A. Rossin, M. Peruzzini, Chem. Rev. 2010, 110, 4178.

[125] S. Marque, P. Tordo, Top. Curr. Chem. 2005, 250, 43.

[126] P. P. Power, Chem. Rev. 2003, 103, 789.

[127] A. Armstrong, T. Chivers, M. Parvez, R. T. Boeré, Angew. Chem. Int. Ed. 2004, 43,502 . 
[128] C. D. Martin, M. Soleilhavoup, G. Bertrand, Chem. Sci. 2013, 4, 3020.

[129] V. Nesterov, D. Reiter, P. Bag, P. Frisch, R. Holzner, A. Porzelt, S. Inoue, Chem. Rev. 2018, 118, 9678.

[130] U. Schmidt, F. Geiger, A. Müller, K. Markau, Angew. Chem. 1963, $13,640$.

[131] S. K. Wong, J. K. Wan, Spectrosc. Lett. 1970, 3, 135.

[132] W. T. Cook, J. S. Vincent, I. Bernal, F. Ramirez, J. Chem. Phys. 1974, 61, 3479.

[133] B. W. Fullam, S. P. Mishra, M. C. Symons, J. Chem. Soc. Dalt. Trans. 1974, 2145 .

[134] M. Geoffroy, E. Lucken, C. Mazeline, Mol. Phys. 2006, 28, 839.

[135] T. Berclaz, M. Geoffroy, Helv. Chim. Acta 1978, 61, 684.

[136] Y. Sakaguchi, Chem. Phys. Lett. 1995, 245, 591.

[137] P. Tordo, M. Boyer, F. Vila, L. Pujol, Phosphorous Sulfur Relat. Elem. 2007, 3, 43.

[138] D. Ajo, M. B. Biagini Cingi, I. Fragala, G. Granozzi, Spectrosc. Lett. 1977, 10, 757.

[139] A. Albert, C. N. R. Composti, C. Eteroatcmi, Tetrahedron 1986, 42, 2533.

[140] R. Leardini, A. Tundo, G. Zanardi, G. F. Peduiii, J. Chem. Soc. Perkin Trans. II 1985, 1117.

[141] B. Ndiaye, S. Bhat, A. Jouaiti, T. Berclaz, G. Bernardinelli, M. Geoffroy, J. Phys. Chem. A 2006, 110, 9736 .

[142] Y. Sueishi, Y. Miyake, Bull. Chem. Soc. Jpn. 1997, 70, 397.

[143] Y. Sueishi, Y. Nishihara, J. Chem. Res. 2001, S, 84.

[144] S. L. Hinchley, C. A. Morrison, D. W. Rankin, C. L. Macdonald, R. J. Wiacek, A. Voigt, A. H. Cowley, M. F. Lappert, G. Gundersen, J. A. Clyburne, P. P. Power, J. Am. Chem. Soc. 2001, 123, 9045.

[145] J. Bezombes, K. B. Borisenko, P. B. Hitchcock, M. F. Lappert, J. E. Nycz, W. H. Rankin, H. E. Robertson, Dalton Trans. 2004, 1980.

[146] S. Ito, M. Kikuchi, M. Yoshifuji, A. J. Arduengo, T. A. Konovalova, L. D. Kispert, Angew. Chem. Int. Ed. 2006, 45, 4341.

[147] R. Kinjo, B. Donnadieu, G. Bertrand, Angew. Chem. Int. Ed. 2010, 49, 5930.

[148] O. Back, M. A. Celik, G. Frenking, M. Melaimi, B. Donnadieu, G. Bertrand, J. Am. Chem. Soc. 2010, 132, 10262. 
[149] O. Back, B. Donnadieu, M. von Hopffgarten, S. Klein, R. Tonner, G. Frenking, G. Bertrand, Chem. Sci. 2011, 2, 858.

[150] S. Ishida, F. Hirakawa, T. Iwamoto, J. Am. Chem. Soc. 2011, 133, 12968.

[151] X. Pan, X. Weng, Y. Zhao, Y. Sui, X. Wang, J. Am. Chem. Soc. 2014, 136, 9834.

[152] K. Schwedtmann, S. Schulz, F. Hennersdorf, T. Strassner, E. Dmitrieva, J. J. Weigand, Angew. Chem. Int. Ed. 2015, 54, 11054.

[153] K. Schwedtmann, G. Zanoni, J. J. Weigand, Chem. Asian J. 2018, 13, 1388.

[154] P. Agarwal, N. A. Piro, K. Meyer, P. Müller, C. C. Cummins, Angew. Chem. Int. Ed 2007, 46, 3111.

[155] M. Scheer, C. Kuntz, M. Stubenhofer, M. Linseis, R. F. Winter, M. Sierka, Angew. Chem. Int. Ed. 2009, 48, 2600.

[156] G. Tan, J. Li, L. Zhang, C. Chen, Y. Zhao, X. Wang, Y. Song, Y. Q. Zhang, M. Driess, Angew. Chem. Int. Ed. 2017, 56, 12741.

[157] U. Fischbach, M. Trincado, H. Grützmacher, Dalton Trans. 2017, 46, 3443.

[158] Y.-E. Kim, Y. Lee, Angew. Chem. Int. Ed 2018, 57, 14159.

[159] M. M. Hansmann, R. Jazzar, G. Bertrand, J. Am. Chem. Soc. 2016, 138, 8356.

[160] K. Lammertsma, Top. Curr. Chem. 2003, 229, 95.

[161] H. Aktaş, J. Chris Slootweg, K. Lammertsma, Angew. Chem. Int. Ed. 2010, 49, 2102 .

[162] R. Waterman, Dalton Trans. 2009, 18.

[163] M. J. Amme, A. B. Kazi, T. R. Cundari, Int. J. Quant. Chem. 2010, 110, 1702.

[164] K. Pal, O. B. Hemming, B. M. Day, T. Pugh, D. J. Evans, R. A. Layfield, Angew. Chem. Int. Ed. 2016, 55, 1690.

[165] J. K. Pagano, B. J. Ackley, R. Waterman, Chem. Eur. J. 2018, 24, 2554.

[166] G. Dequirez, V. Pons, P. Dauban, Angew. Chem. Int. Ed. 2012, 51, 7384.

[167] J. L. Roizen, M. E. Harvey, J. D. Bois, Acc. Chem. Res. 2012, 45, 911.

[168] P. F. Kuijpers, J. I. van der Vlugt, S. Schneider, B. de Bruin, Chem. Eur. J. 2017, 23, 13819.

[169] N. V. Barkovskii, V. I. Tsirel'nikov, A. M. Emel'yanov, Y. S. Khodeev, Teplofiz. Vys. Temp. 1991, 29, 474.

[170] J. Berkowitz, L. A. Curtiss, S. T. Gibson, J. P. Greene, G. L. Hillhouse, J. A. Pople, J. Chem. Phys. 1986, 84, 375. 
[171] J. D. Cox, D. D. Wagman, V. A. Medvedev, CODATA Key Values for Thermodynamics, Hemisphere Publishing Corp., New York, 1984.

[172] M. W. Chase, NIST-JANAF Thermochemical Tables, J. Phys. Chem. Ref. Data, Monograph 9, 1998.

[173] M. J. Bezdek, S. Guo, P. J. Chirik, Science 2016, 354, 730.

[174] S. S. Kolmar, J. M. Mayer, J. Am. Chem. Soc. 2017, 139, 10687.

[175] D. J. Mindiola, G. L. Hillhouse, J. Am. Chem. Soc. 2001, 123, 4623.

[176] M. H. V. Huynh, T. J. Meyer, Proc. Natl. Acad. Sci. U. S. A. 2000, 101, 13138.

[177] R. E. Cowley, R. P. Bontchev, J. Sorrell, O. Sarracino, Y. Feng, H. Wang, J. M. Smith, J. Am. Chem. Soc. 2007, 129, 2424.

[178] I. Nieto, F. Ding, R. P. Bontchev, H. Wang, J. M. Smith, J. Am. Chem. Soc. 2008, 130, 2716.

[179] R. E. Cowley, N. A. Eckert, S. Vaddadi, T. M. Figg, T. R. Cundari, P. L. Holland, J. Am. Chem. Soc. 2011, $133,9796$.

[180] V. M. Iluc, A. J. Miller, J. S. Anderson, M. J. Monreal, M. P. Mehn, G. L. Hillhouse, J. Am. Chem. Soc. 2011, 133, 13055.

[181] S. Wiese, J. L. McAfee, D. R. Pahls, C. L. McMullin, T. R. Cundari, T. H. Warren, J. Am. Chem. Soc. 2012, 134, 10114.

[182] C. Milsmann, S. P. Semproni, P. J. Chirik, J. Am. Chem. Soc. 2014, 136, 12099.

[183] I. Pappas, P. J. Chirik, J. Am. Chem. Soc. 2016, 138, 13379.

[184] D. M. Spasyuk, S. H. Carpenter, C. E. Kefalidis, W. E. Piers, M. L. Neidig, L. Maron, Chem. Sci. 2016, 7, 5939.

[185] P. J. Chirik, M. Bezdek, Angew. Chem. Int. Ed. 2018, 24, 1.

[186] R. Melenkivitz, D. J. Mindiola, G. L. Hillhouse, J. Am. Chem. Soc. 2002, 124, 3846 .

[187] V. M. Iluc, G. L. Hillhouse, J. Am. Chem. Soc. 2010, 132, 15148.

[188] C. Gunanathan, D. Milstein, Acc. Chem. Res. 2011, 44, 588.

[189] L. C. Liang, Coord. Chem. Rev. 2006, 250, 1152.

[190] S. Schneider, J. Meiners, B. Askevold, Eur. J. Inorg. Chem. 2012, 412.

[191] B. Askevold, M. M. Khusniyarov, W. Kroener, K. Gieb, P. Müller, E. Herdtweck, F. W. Heinemann, M. Diefenbach, M. C. Holthausen, V. Vieru, L. F. Chibotaru, S. Schneider, Chem. Eur. J. 2015, 21, 579. 
[192] P. O. Lagaditis, B. Schluschaß, S. Demeshko, C. Würtele, S. Schneider, Inorg. Chem. 2016, 55, 4529 .

[193] J. Meiners, M. G. Scheibel, M.-H. Lemée-Cailleau, S. A. Mason, M. B. Boeddinghaus, T. F. Fässler, E. Herdtweck, M. M. Khusniyarov, S. Schneider, Angew. Chem. Int. Ed. 2011, 50, 8184.

[194] B. Askevold, M. M. Khusniyarov, E. Herdtweck, K. Meyer, S. Schneider, Angew. Chem. Int. Ed. 2010, 49, 7566.

[195] M. A. Esteruelas, F. J. Modrego, E. Oñate, E. Royo, J. Am. Chem. Soc. 2003, $125,13344$.

[196] J. T. Anhaus, T. P. Kee, M. H. Schofield, R. R. Schrock, J. Am. Chem. Soc. 1990, 112, 1642.

[197] M. L. Buil, J. J. Cardo, M. A. Esteruelas, E. Oñate, J. Am. Chem. Soc. 2016, 138,9720 .

[198] B. H. Werner, A. Michenfelder, Angew. Chem. Int. Ed. 1991, 30, 596.

[199] D. Coskun, S. V. Jerome, R. A. Friesner, J. Chem. Theory Comput. 2016, 12, 1121.

[200] F. Wätjen, Dissertation, Georg-August-Universität Göttingen, 2019.

[201] F. Schneck, M. Finger, M. Tromp, S. Schneider 2017, 23, 33.

[202] A. W. Addison, T. N. Rao, J. Reedijk, J. van Rijn, G. C. Verschoor, J. Chem. Soc., Dalt. Trans. 1984, 1349.

[203] A. Soncini, W. Van Den Heuvel, J. Chem. Phys. 2013, 138, 021103.

[204] D. M. D'Alessandro, P. H. Dinolfo, M. S. Davies, J. T. Hupp, F. R. Keene, Inorg. Chem. 2006, 45, 3261 .

[205] J. Chatt, G. J. Leigh, D. M. P. Mingos, J. Chem. Soc. A 1969, 2972.

[206] M. Atanasov, D. Aravena, E. Suturina, E. Bill, D. Maganas, F. Neese, Coord. Chem. Rev. 2015, 289-290, 177.

[207] S. P. Semproni, W. S. McNeil, R. A. Baillie, B. O. Patrick, C. F. Campana, P. Legzdins, Organometallics 2010, 29, 867.

[208] J. M. Narayanam, C. R. Stephenson, Chem. Soc. Rev. 2011, 40, 102.

[209] R. Ziessel, J. Am. Chem. Soc 1993, 115, 118.

[210] K. J. Watson, R. Ziessel, Inorganica Chim. Acta 1992, 197, 125.

[211] R. Ziessel, Angew. Chem. Int. Ed 1991, 30, 844.

[212] R. Ziessel, J. Chem. Soc. Chem. Commun. 1988, 16. 
[213] T. Suenobu, D. M. Guldi, S. Ogo, S. Fukuzumi, Angew. Chem. Int. Ed. 2003, 42, 5492.

[214] M. B. Chambers, D. A. Kurtz, C. L. Pitman, M. K. Brennaman, A. J. Miller, J. Am. Chem. Soc. 2016, 138, 13509.

[215] F. Schneck, J. Ahrens, M. Finger, A. C. Stückl, C. Würtele, D. Schwarzer, S. Schneider, Nat. Commun. 2018, 9, 1.

[216] M. Irwin, R. K. Jenkins, M. S. Denning, T. Krämer, F. Grandjean, G. J. Long, R. Herchel, J. E. McGrady, J. M. Goicoechea, Inorg. Chem. 2010, 49, 6160.

[217] P. Milko, M. A. Iron, J. Chem. Theory Comput. 2014, 10, 220.

[218] F. Neese, Coord. Chem. Rev. 2009, 253, 526.

[219] Y. Gloaguen, C. Rebreyend, M. Lutz, P. Kumar, M. Huber, J. I. van der Vlugt, S. Schneider, B. de Bruin, Angew. Chem. Int. Ed. 2014, 53, 6814.

[220] J. J. Scepaniak, C. G. Margarit, J. N. Harvey, J. M. Smith, Inorg. Chem. 2011, $50,9508$.

[221] J. F. Berry, Comments Inorg. Chem. 2009, 30, 28.

[222] M. H. V. Huynh, E. S. El-Samanody, K. D. Demadis, P. S. White, T. J. Meyer, Inorg. Chem. 2000, 39, 3075.

[223] M. H. V. Huynh, P. S. White, T. J. Meyer, Angew. Chem. Int. Ed. 2000, 39, 4101.

[224] M. H. V. Huynh, P. S. White, C. A. Carter, T. J. Meyer, Angew. Chem. Int. Ed 2001, 40, 3037 .

[225] M. H. V. Huynh, R. T. Baker, D. L. Jameson, A. Labouriau, T. J. Meyer, J. Am. Chem. Soc. 2002, 124, 4580.

[226] S. N. Brown, J. Am. Chem. Soc. 1999, 121, 9752.

[227] W. L. Man, W. W. Lam, T. C. Lau, Acc. Chem. Res. 2014, 47, 427.

[228] A. Glüer, B. Askevold, B. Schluschaß, F. W. Heinemann, S. Schneider, Z. anorg. allg. Chem. 2015, 641, 49.

[229] G. Rossi, M. Gardini, G. Pennesi, C. Ercolani, V. L. Goedken, J. Chem. Soc. Dalton Trans. 1989, 193.

[230] L. Bonomo, E. Solari, R. Scopelliti, C. Floriani, Angew. Chem. Int. Ed. 2001, 40, 2529 .

[231] G. Wu, D. Rovnyak, M. J. Johnson, N. C. Zanetti, D. G. Musaev, K. Morokuma, R. R. Schrock, R. G. Griffin, C. C. Cummins, J. Am. Chem. Soc. 1996, 118, 10654.

[232] R. C. Fischer, P. P. Power, Chem. Rev. 2010, 3877. 
[233] P. Macchi, A. Sironi, Coord. Chem. Rev. 2003, 238-239, 383.

[234] J. I. Schweizer, M. G. Scheibel, M. Diefenbach, F. Neumeyer, C. Würtele, N. Kulminskaya, R. Linser, N. Auner, S. Schneider, M. C. Holthausen, Angew. Chem. Int. Ed 2016, 55, 1782.

[235] D. Stalke, Chem. Eur. J. 2011, 17, 9264.

[236] L. J. Farrugia, C. S. Frampton, J. A. Howard, P. R. Mallinson, R. D. Peacock, G. T. Smith, B. Stewart, Acta Crystallogr. 2006, B62, 236.

[237] S. Mebs, J. Henn, B. Dittrich, C. Paulmann, P. Luger, J. Phys. Chem. A 2009, 113,8366 .

[238] M. E. Weeks, J. Chem. Ed. 1932, 9, 11.

[239] E. J. Zegers, E. M. Fisher, Combust. Flame 1998, 115, 230.

[240] O. P. Korobeinichev, S. B. Ilyin, V. M. Shvartsberg, A. A. Chernov, Combust. Flame 1999, 118, 718.

[241] L. B. Knight, G. C. Jones, G. M. King, R. M. Babb, A. J. McKinley, J. Chem. Phys. 1995, 103, 497.

[242] C. W. Bauschlicher, M. Zhou, L. Andrews, J. Phys. Chem. A 2002, 104, 3566.

[243] B. T. Sterenberg, L. Scoles, A. J. Carty, Coord. Chem. Rev. 2002, 231, 183.

[244] M. McCluskey, L. Andrews, J. Phys. Chem. 1991, 95, 2988.

[245] J. S. Francisco, J. Chem. Phys. 2002, 117, 3190.

[246] A. Bauzá, A. Frontera, ChemPhysChem 2017, 18, 2191.

[247] Y. Pak, J. Chem. Phys. 1996, 104, 5547.

[248] C. Xu, E. de Beer, D. M. Neumark, J. Chem. Phys. 1996, 104, 2749.

[249] L. L. Lohr, J. Phys. Chem. 1984, 88, 5569.

[250] H. B. Qian, P. B. Davies, I. K. Ahmad, P. A. Hamilton, Chem. Phys. Lett. 1995, 235, 255.

[251] L. L. Lohr, J. Phys. Chem. 1992, 96, 119.

[252] L. Andrews, M. McCluskey, Z. Mielke, R. Withnall, J. Mol. Struct. 1990, 222, 95.

[253] M. A. Hitchman, G. L. Rowbottom, Coord. Chem. Rev. 1982, 42, 55.

[254] A. J. Timmons, M. D. Symes, Chem. Soc. Rev. 2015, 44, 6708.

[255] R. Eisenberg, C. D. Meyer, Acc. Chem. Res. 1975, 8, 26.

[256] P. F. Bernath, Astrophys. J. 1987, 312, 358. 
[257] C. Cummins, Chem. Commun. 1998, 1777.

[258] Y. Xie, P. v. R. Schleyer, Y. Wang, P. Wei, H. F. Schaefer, G. H. Robinson, J. Am. Chem. Soc. 2013, 135, 19139.

[259] O. J. Scherer, S. Weigel, G. Wolmershäuser, Angew. Chem. Int. Ed 1999, 38, 3688 .

[260] A. S. Pronin, A. I. Smolentsev, S. G. Kozlova, I. N. Novozhilov, Y. V. Mironov, Inorg. Chem. 2019, DOI: 10.10 .

[261] N. Elgrishi, K. J. Rountree, B. D. McCarthy, E. S. Rountree, T. T. Eisenhart, J. L. Dempsey, J. Chem. Educ. 2018, 95, 197.

[262] D. M. Murphy, EPR (Electron Paramagnetic Resonance) Spectroscopy of Polycrystalline Oxide Systems - Chapter 1, WILEY-VCH Verlag GmbH, Weinheim, 2009.

[263] M. L. Munzarova, P. Kubacek, M. Kaupp, J. Am. Chem. Soc. 2000, 122, 11900.

[264] P. A. Haase, M. Repisky, S. Komorovsky, J. Bendix, S. P. Sauer, Chem. Eur. J. 2018, 24, 5124 .

[265] M. Bennati, eMagRes 2017, 6, 271.

[266] F. Neese, eMagRes 2017, 6, 1.

[267] J. A. McCleverty, T. J. Meyer, Comprehensive Coordination Chemistry II - Volume: Transition Metal Group 7 and 8, Elsevier, 2003.

[268] J. J. Warren, T. A. Tronic, J. M. Mayer, Chem. Rev. 2010, 110, 6961.

[269] J. D. Masuda, K. C. Jantunen, O. V. Ozerov, K. J. Noonan, D. P. Gates, B. L. Scott, J. L. Kiplinger, J. Am. Chem. Soc. 2008, 130, 2408.

[270] M. C. Puerta, P. Valerga, Coord. Chem. Rev. 1999, 193-195, 977.

[271] N. Shida, J. A. Buss, T. Agapie, Chem. Commun. 2018, 54, 767.

[272] A. Velian, C. C. Cummins, Science 2015, 348, 1001.

[273] W. J. Transue, A. Velian, M. Nava, M. A. Martin-Drumel, C. C. Womack, J. Jiang, G. L. Hou, X. B. Wang, M. C. McCarthy, R. W. Field, C. C. Cummins, J. Am. Chem. Soc. 2016, 138, 6731.

[274] M. A. Courtemanche, W. J. Transue, C. C. Cummins, J. Am. Chem. Soc. 2016, 138,16220 .

[275] M. P. Duffy, F. Mathey, J. Am. Chem. Soc. 2009, 131, 7534.

[276] K. Vaheesar, C. M. Kuntz, B. T. Sterenberg, J. Organomet. Chem. 2013, 745, 347. 
[277] J. F. Hartwig, Organotransition Metal Chemistry, from Bonding to Catalysis, New York, 2010.

[278] Y. Wang, G. H. Robinson, Dalton Trans. 2012, 41, 337.

[279] Y. Wang, G. H. Robinson, Inorg. Chem. 2014, 53, 11815.

[280] G. A. Silantyev, M. Förster, B. Schluschaß, J. Abbenseth, C. Würtele, C. Volkmann, M. C. Holthausen, S. Schneider, Angew. Chem. Int. Ed. 2017, 56, 5872.

[281] M. B. Robin, P. Day, Adv. Inorg. Chem. Radiochem. 1968, 10, 247.

[282] P. P. Borbat, A. J. Costa-Filho, K. A. Earle, J. K. Moscicki, J. H. Freed, Science 2001, 291, 266.

[283] P. Dauber, A. T. Hagler, Acc. Chem. Res. 1980, 46, 105.

[284] M. A. Buntine, V. J. Hall, F. J. Kosovel, E. R. T. Tiekink, J. Phys. Chem. A 1998, 102, 2472.

[285] A. Gavezzotti, Crystallogr. Rev. 1998, 7, 5.

[286] F. De Groot, Chem. Rev. 2001, 101, 1779.

[287] G. Vlaic, L. Olivi, Croat. Chem. Acta 2004, 77, 427.

[288] D. C. Koningsberger, R. Prins, „X-Ray Absorption: Principles, Applications, Technique of EXAFS, SEXAFS and XANES" in Chem. Anal. Vol. 92, John Wiley \& Sons, Inc., New York, 1988.

[289] J. H. Choi, N. E. Schloerer, J. Berger, M. H. Prechtl, Dalton Trans. 2014, 43, 290.

[290] M. Tamizmani, C. Sivasankar, J. Organomet. Chem. 2017, 845, 82.

[291] M. Brookhart, B. Grant, A. F. Volpe, Organometallics 1992, 11, 3920.

[292] J. Meiners, A. Friedrich, E. Herdtweck, S. Schneider, Organometallics 2009, 28, 6331.

[293] V. W. Manner, T. F. Markle, J. H. Freudenthal, J. P. Roth, J. M. Mayer, Chem. Commun. 2008, 246, 256.

[294] A. C. Coelho, S. S. Balula, S. M. Bruno, J. C. Alonso, N. Bion, P. Ferreira, M. Pillinger, A. A. Valente, J. Rocha, I. S. Gonçalves, Adv. Synth. Catal. 2010, 352, 1759 .

[295] G. S. Girolami, A. P. Sattelberger, Inorganic Synthesis 36, John Wiley \& Sons, Inc., Hoboken, New Jersey, 2014.

[296] D. Heift, Z. Benko, H. Grützmacher, Dalton Trans. 2014, 43, 831.

[297] I. Klopsch, Dissertation, Georg-August-Universität Göttingen, 2016. 
[298] G. P. Elliot, N. M. McAuley, W. R. Roper, P. A. Shapley, Inorganic Synthesis, Volume 15, John Wiley \& Sons, Inc., New York, Chichester, Brisbane, Toronto, Singapore, 1989.

[299] W. Haberditzl, Angew. Chem. Int. Ed. 1966, 5, 288.

[300] E. Bill, „julX, Program for Simulation of Molecular Magnetic Data“, 2008.

[301] I. Ivancic, D. Degobbis, Water Resour. 1984, 18, 1143.

[302] B. Ravel, M. Newville, J. Synchrotron Rad. 2005, 12, 537.

[303] „Gaussian 09, Revision D.01“.

[304] J. P. Perdew, M. Ernzerhof, K. Burke, J. Chem. Phys. 1996, 105, 9982.

[305] C. Adamo, V. Barone, J. Chem. Phys. 2008, 6158, 6158.

[306] S. Grimme, S. Ehrlich, L. Goerigk, J. Comput. Chem 2011, 32, 1456.

[307] F. Weigend, R. Ahlrichs, Phys. Chem. Chem. Phys. 2005, 7, 3297.

[308] D. Andrae, U. Häußermann, M. Dolg, H. Stoll, H. Preuss, Theor. Chim. Acta 1990, $77,123$.

[309] „MolPro 2015.1“, 2015.

[310] S. Ten-no, Chem. Phys. Lett. 2004, 398, 56.

[311] T. B. Adler, G. Knizia, H.-J. Werner, J. Chem. Phys. 2007, 127, 221106.

[312] K. A. Peterson, T. B. Adler, H. J. Werner, J. Chem. Phys. 2008, 128, 084102.

[313] K. E. Yousaf, K. A. Peterson, J. Chem. Phys. 2008, 129, 184108.

[314] S. Kritikou, J. G. Hill, J. Chem. Theory Comput. 2015, 11, 5269.

[315] D. Figgen, K. A. Peterson, M. Dolg, H. Stoll, J. Chem. Phys. 2009, 130, 164108.

[316] J. G. Hill, J. Chem. Phys. 2011, 135, 044105.

[317] J. G. Hill, K. A. Peterson, G. Knizia, H. J. Werner, J. Chem. Phys. 2009, 131, 194105.

[318] F. Neese, Wiley Interdiscip. Rev. Comput. Mol. Sci. 2012, 2, 73.

[319] C. Van Wüllen, J. Chem. Phys. 1998, 109, 392.

[320] F. Neese, F. Wennmohs, A. Hansen, U. Becker, Chem. Phys. 2009, 356, 98.

[321] C. Haettig, Phys. Chem. Chem. Phys. 2005, 7, 59.

[322] C. Angeli, R. Cimiraglia, J. P. Malrieu, Chem. Phys. Lett. 2001, 350, 297. 
[323] C. Angeli, R. Cimiraglia, S. Evangelisti, T. Leininger, J. P. Malrieu, J. Chem. Phys. 2001, 114, 10252.

[324] C. Angeli, R. Cimiraglia, J. P. Malrieu, J. Chem. Phys. 2002, 117, 9138.

[325] B. A. Heß, C. M. Marian, U. Wahlgren, O. Gropen, Chem. Phys. Lett. 1996, 251, 365.

[326] J. P. Perdew, K. Burke, M. Ernzerhof, Phys. Rev. Lett. 1996, 77, 3865.

[327] O. Treutler, R. Ahlrichs, J. Chem. Phys. 1995, 102, 346.

[328] Karin E, Oliver T, Holger O, Marco H, Reinhart A, Chem. Phys. Lett. 1995, 240, 283.

[329] K. Eichkorn, F. Weigend, O. Treutler, R. Ahlrichs, Theor. Chem. Acc. 1997, 97, 119.

[330] S. Grimme, J. Antony, S. Ehrlich, H. Krieg, J. Chem. Phys. 2010, 132, 154104.

[331] A. V. Marenich, C. J. Cramer, D. G. Truhlar, J. Phys. Chem. B 2009, 113, 6378.

[332] D. A. Pantazis, X.-Y. Chen, C. R. Landis, F. Neese, J. Chem. Theory Comput. 2008, 4, 908 .

[333] M. D. Hanwell, D. E. Curtis, D. C. Lonie, T. Vandermeersch, E. Zurek, H. G. R., J. Cheminform. 2012, 4, 17.

[334] E. F. Pettersen, T. D. Goddard, C. C. Huang, G. S. Couch, D. M. Greenblatt, E. C. Meng, T. E. Ferrin, J. Comput. Chem. 2004, 25, 1605.

[335] M. Dolg, U. Wedig, H. Stoll, H. Preuss, J. Chem. Phys. 1987, 86, 866.

[336] A. Bergner, M. Dolg, W. Küchle, H. Stoll, H. Preuß, Mol. Phys. 1993, 80, 1431.

[337] J. D. Chai, M. Head-Gordon, Phys. Chem. Chem. Phys. 2008, 10, 6615.

[338] Y. Minenkov, A. Singstad, G. Occhipinti, V. R. Jensen, Dalton Trans. 2012, 41, 5526 .

[339] K. L. Schuchardt, B. T. Didier, T. Elsethagen, L. Sun, V. Gurumoorthi, J. Chase, J. Li, T. L. Windus, J. Chem. Inf. Model. 2007, 47, 1045.

[340] D. Feller, J. Comput. Chem. 1996, 17, 1571.

[341] T. H. Dunning, P. J. Hay, Modern Theoretical Chemistry, Plenum, New York, 1977, S. 1.

[342] A. D. McLean, G. S. Chandler, J. Chem. Phys. 1980, 72, 5639.

[343] R. Krishnan, J. S. Binkley, R. Seeger, J. A. Pople, J. Chem. Phys. 1980, 72, 650.

[344] R. C. Binning, L. A. Curtiss, J. Comput. Chem. 1990, 11, 1206. 
[345] L. A. Curtiss, M. P. McGrath, J. P. Blaudeau, N. E. Davis, R. C. Binning, L. Radom, J. Chem. Phys. 1995, 103, 6104.

[346] G. Schreckenbach, T. Ziegler, J. Phys. Chem. 1995, 99, 606.

[347] S.-t. Orbitals, M. Krykunov, T. O. M. Ziegler, E. V. A. N. Lenthe, Int. J. Quantum Chem. 2009, 109, 1676.

[348] G. T. E. Velde, F. M. Bickelhaupt, E. J. Baerends, C. F. Guerra, S. J. A. V. A. N. Gisbergen, J. Comput. Chem. 2001, 22, 931.

[349] S. K. Wolff, T. Ziegler, E. Van Lenthe, E. J. Baerends, J. Chem. Phys. 1999, 110, 7689 .

[350] E. van Lenthe, J. G. Snijders, E. J. Baerends, J. Chem. Phys. 1996, 105, 6505.

[351] E. van Lenthe, E. J. Baerends, J. G. Snijders, J. Chem. Phys. 1994, 101, 9783.

[352] J. P. Perdew, K. Burke, M. Ernzerhof, Phys. Rev. Lett. 1997, 78, 1396.

[353] E. van Lenthe, E. J. Baerends, J. Phys. Chem. 2003, 24, 1142.

[354] S. O. Grimm, W. McFariane, E. F. Davidoff, J. Phys. Chem. 1966, 70, 581.

[355] A. Klamt, G. Schüürmann, J. Chem. Soc., Perkin Trans. 2 1993, 5, 799.

[356] C. C. Pye, T. Ziegler, Theor. Chem. Acc. 1999, 101, 396.

[357] R. Ahlrichs, „Turbomole 7.3.1“, 2018.

[358] I. Baker, J. Comput. Chem. 1986, 7, 385.

[359] P. H. M. Budzelaar, J. Comput. Chem. 2007, 28, 2226.

[360] A. D. Becke, Phys. Rev. A 1988, 38, 3098.

[361] J. P. Perdew, Phys. Rev. B 1986, 7, 385.

[362] M. Sierka, A. Hogekamp, R. Ahlrichs, J. Chem. Phys. 2003, 118, 9136.

[363] F. Weigend, H. Häser, H. Patzelt, R. Ahlrichs, Chem. Phys. Lett. 1998, 294, 143.

[364] S. Grimme, J. Antony, S. Ehrlich, H. Krieg, J. Chem. Phys. 2010, 132, 154104.

[365] E. van Lenthe, A. van der Avoird, P. E. S. Wormer, J. Chem. Phys. 1997, 10\%, 2488.

[366] E. van Lenthe, A. van der Avoird, P. E. S. Wormer, J. Chem. Phys. 1998, 108, 4783 .

[367] E. J. Baerends, D. E. Ellis, P. Ros, Chem. Phys. 1973, 2, 41.

[368] L. Versluis, T. Ziegler, J. Chem. Phys. 1988, 88, 322.

[369] G. te Velde, E. J. Baerends, J. Comput. Phys. 1992, 99, 84. 
[370] C. Fonseca Guerra, J. G. Smijders, G. te Velde, E. J. Baerends, Theor. Chim. Acta 1998, 99, 391.

[371] A. L. Spek, Acta Crystallogr. 2015, C7-1, 9. 